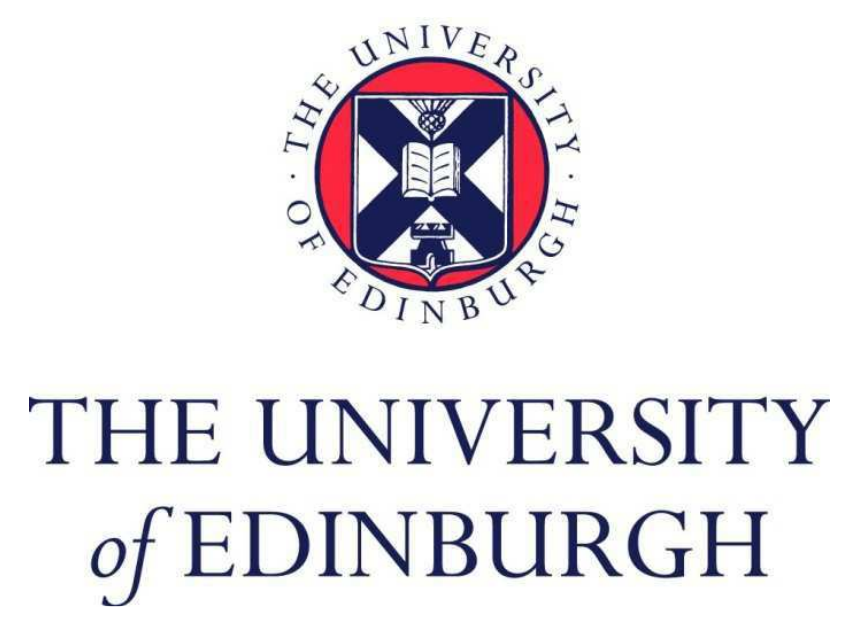

This thesis has been submitted in fulfilment of the requirements for a postgraduate degree (e.g. PhD, MPhil, DClinPsychol) at the University of Edinburgh. Please note the following terms and conditions of use:

This work is protected by copyright and other intellectual property rights, which are retained by the thesis author, unless otherwise stated.

A copy can be downloaded for personal non-commercial research or study, without prior permission or charge.

This thesis cannot be reproduced or quoted extensively from without first obtaining permission in writing from the author.

The content must not be changed in any way or sold commercially in any format or medium without the formal permission of the author.

When referring to this work, full bibliographic details including the author, title, awarding institution and date of the thesis must be given. 


\section{Total Synthesis of Phenolic Natural Products}

Irene De Silvestro

Degree of Doctor of Philosophy

School of Chemistry

The University of Edinburgh

June 2017 


\section{DECLARATION}

I declare that:

(a) this thesis has been composed by the candidate,

(b) the work contained in this thesis is the candidate's own, unless otherwise referenced,

(c) the work contained in this thesis has not been submitted for any other degree or professional qualification,

Edinburgh, 01/07/2017

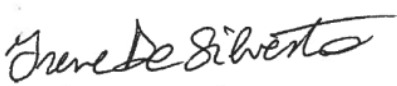

Irene De Silvestro 


\section{PUBLICATION}

De Silvestro, I.; Drew S. L.; Nichol G. S.; Duarte F.; Lawrence A. L., Angew. Chem. Int. Ed. 2017, 56, 6813.

International Edition: DOI: 10.1002/anie.201701481

German Edition: DOI: 10.1002/ange.201701481

This work is discussed in Chapter 2. 


\section{ACKNOWLEDGEMENTS}

First of all, I would like to thank my supervisor, Dr Andrew Lawrence, for giving me the opportunity to work in the Lawrence Group and providing invaluable assistance and guidance throughout my PhD. I would also like to thank Prof Giuliana Pitacco, Prof Patrizia Nitti, Prof Cristina Forzato and Prof Federico Berti at the University of Trieste, where I obtained my Bachelor and Master degrees, for their help and support in my application for this $\mathrm{PhD}$.

Next I would like to thank all my family for their support and encouragement, for welcoming me home during my short visits and for their understanding whenever I missed important family events.

I cannot forget to acknowledge all the past and present members of the Lawrence Group and the project students I have had the pleasure to supervise. In particular, I would like to thank Dr Patrick Brown for all his advice and help in the early stage of my PhD, Robert Cracknell, Peter Dabell and Dr Nicholas Green for being great lab mates. Special thanks go to Marie Thomson for her patience and encouragement during and after working hours. This thesis and the work described in it would not have been finished without her support.

Finally, I would like to thank the staff at the University of Edinburgh for their assistance. In particular Dr Gary Nichol for his work on X-ray crystallography and Dr Fernanda Duarte for computational studies. 


\section{ABBREVIATIONS}

\begin{tabular}{|c|c|}
\hline$\%$ & percentage or percentage yield \\
\hline${ }^{\circ} \mathrm{C}$ & degree/s Celsius \\
\hline$\mu$ & micro \\
\hline$\delta$ & chemical shift \\
\hline$\lambda$ & wavelength \\
\hline$v$ & absorption maxima (IR) \\
\hline Ac & acetyl \\
\hline $\mathrm{AD}$ & asymmetric dihydroxylation \\
\hline an. & anhydrous \\
\hline app. & apparent \\
\hline$a q$. & aqueous \\
\hline Ar & aryl \\
\hline ATP & adenosine triphosphate \\
\hline ATR & attenuated total reflectance \\
\hline BHT & 2,6-di-tert-butyl-4-methylphenol \\
\hline br & broad \\
\hline $\mathrm{Bu}$ & butyl \\
\hline CAN & ceric ammonium nitrate \\
\hline CBS & Corey-Bakshi-Shibata catalyst \\
\hline $\mathrm{cm}$ & centimetre \\
\hline COSY & correlated spectroscopy \\
\hline $1 \mathrm{D}$ & one dimensional \\
\hline $2 \mathrm{D}$ & two dimensional \\
\hline $\mathrm{d}$ & doublet/s \\
\hline $\mathrm{Da}$ & Dalton \\
\hline DBU & 1,8-diazabicyclo[5,4,0]undec-7-ene \\
\hline DIPEA & $N, N$-di-iso-propylethylamine \\
\hline
\end{tabular}




\begin{tabular}{|c|c|}
\hline DMDO & dimethyldioxirane \\
\hline DME & 1,2-dimethoxyethane \\
\hline DMF & dimethylformamide \\
\hline DMSO & dimethyl sulfoxide \\
\hline DMP & Dess-Martin periodinane \\
\hline d.r. & diastereomeric ratio \\
\hline EDTA & ethylenediaminetetraacetic acid \\
\hline e.e. & enantiomeric excess \\
\hline Enz & enzyme \\
\hline eq. & molar equivalents \\
\hline ESI & electrospray ionisation \\
\hline Et & ethyl \\
\hline $\mathrm{eV}$ & electron Volts \\
\hline FTIR & Fourier transform infrared spectroscopy \\
\hline $\mathrm{g}$ & gram/s \\
\hline $\mathrm{h}$ & hour/s \\
\hline HFIP & hexafluoro-2-propanol \\
\hline HMBC & heteronuclear multiple bond coherence \\
\hline HPLC & high performance liquid chromatography \\
\hline HRMS & high resolution mass spectrometry \\
\hline HSQC & heteronuclear single quantum coherence \\
\hline $\mathrm{Hz}$ & Hertz \\
\hline IR & infrared \\
\hline$i$-But & isobutyrate \\
\hline$i$-Pr & isopropyl \\
\hline$J$ & coupling constant \\
\hline LDA & lithium diisopropylamide \\
\hline$m$ & meta \\
\hline $\mathrm{m}$ & multiplet or metre/s \\
\hline
\end{tabular}




\begin{tabular}{|c|c|}
\hline M & molar \\
\hline $\mathrm{M}^{+}$ & molecular ion \\
\hline MABR & methylaluminum bis(4-bromo-2,6-di-tert-butylphenoxide) \\
\hline$m$-CPBA & meta-chloroperoxybenzoic acid \\
\hline $\mathrm{Me}$ & methyl \\
\hline $\mathrm{mg}$ & milligram/s \\
\hline $\mathrm{MHz}$ & megahertz \\
\hline $\min$ & minutes/s \\
\hline $\mathrm{mL}$ & millilitre/s \\
\hline mmol & millimole/s \\
\hline mol & mole/s \\
\hline mol. & molecular \\
\hline MOM & methoxymethyl \\
\hline m.p. & melting point \\
\hline MS & mass spectrometry \\
\hline$m / z$ & mass to charge ratio \\
\hline$n$ & normal \\
\hline NADP & nicotinamide adenine dinucleotide phosphate \\
\hline NMR & nuclear magnetic resonance \\
\hline nOe & nuclear Overhauser effect \\
\hline NOESY & nuclear Overhauser effect spectroscopy \\
\hline $\mathrm{P}$ & phosphate \\
\hline $\mathrm{Ph}$ & phenyl \\
\hline PIDA & phenyliodine (III) diacetate \\
\hline PIFA & phenyliodine (III) (bis)trifluoroacetate \\
\hline PMA & phosphomolybdic acid \\
\hline PP & pyrophosphate \\
\hline ppm & parts per million \\
\hline $\operatorname{Pr}$ & propyl \\
\hline
\end{tabular}


PTS PEG-600/ $\alpha$-tocopherol-based diester of sebacic acid

p-TSA para-toluenesulfonic acid

pyr. $\quad$ pyridine

q quartet

$\mathrm{R}_{f} \quad$ retention factor

$\mathrm{rt} \quad$ room temperature

s singlet

SAM $S$-adenosyl methionine

sat. $\quad$ saturated

SET single electron transfer

SIBX stabilised 2-iodoxybenzoic acid

$t \quad$ tert

$\mathrm{t} \quad$ triplet

TBDPS tert-butyldiphenylsilyl

TBS tert-butyldimethylsilyl

TBAF tetrabutylammonium fluoride

Tf trifluoromethanesulfonyl

TFA trifluoroacetic acid

THF tetrahydrofuran

TIPS triisopropylsilyl

TLC thin layer chromatography

TMEDA tetramethylethylenediamine

TMS trimethylsilyl

TMSE 2-(trimethylsilyl)ethane

TOF time of flight

$\mathrm{t}_{R} \quad$ retention time

UV ultraviolet

w weight

W Watt 


\section{LAY SUMMARY}

Natural products are chemical compounds which are produced by microorganisms, animals and plants and display a wide range of biological properties and structural motifs. It is sometimes impossible to study and fully understand their biological functions and chemical properties without having access to a practical synthetic route, because they are often produced in the organism in too small quantities.

Total synthesis is the name given to the preparation in the laboratory of complex natural products from commercially available and simple starting materials. Biomimetic synthesis represents one method used to develop strategies toward the total synthesis of natural products, and takes inspiration from the way these compounds are synthesised by nature inside living organisms. This branch of chemistry aims to understand the biosynthesis and use this information to develop not only feasible, but also practical, laboratory synthesis.

The first part of this thesis describes the total synthesis of a dimeric thymol derivative isolated from Arnica sachalinensis. Our investigations toward the successful synthesis of this natural product allowed us to confirm the feasibility of the proposed biosynthetic pathway and to gain insight into the reactivity of the plausible biosynthetic intermediates.

Biomimetic diversity-oriented synthesis aims to mimic the way nature synthesises groups of structurally diverse compounds from a common biosynthetic intermediate. The ability to reproduce this in the laboratory would allow synthetic chemists to rapidly and efficiently explore chemical space using minimum quantities of resources.

The second part of this thesis describes our efforts towards the development of a unified strategy to access a group of prenylated phenylpropanoid natural products from Illicium plants. We envisage that the success obtained in the preparation of a small selection of our molecular targets could be expanded on the synthesis of the most interesting members of this family of compounds. 


\begin{abstract}
This thesis is regarded with the biomimetic total synthesis of phenolic natural products and describes two different projects.

Chapter 1 introduces phenolic natural products, the most important biosynthetic pathways for their formation and some examples of relevant biomimetic syntheses. A short introduction to dimeric and pseudo-dimeric natural products can also be found in this chapter. Specific introductions can be found at the start of Chapters 2 and 3.

Chapter 2 describes the total synthesis of a dimeric thymol derivative isolated from Arnica sachalinensis (which we have named "thymarnicol"). Inspired by the biosynthesis proposed by Passreiter and co-workers, we tested and confirmed the feasibility of a key hetero-Diels-Alder dimerisation step. During our investigations, we gained significant new insights into the origin and reactivity of thymarnicol. The final oxidative cyclisation has been found to occur spontaneously upon exposure to visible light in air.

Chapter 3 discusses our efforts to develop a divergent biomimetic synthetic strategy towards a family of prenylated phenylpropanoid natural products isolated from Illicium genus plants. Our first biomimetic approach revealed the chemical instability of our proposed key intermediates. Therefore, a revised approach was trialled, allowing the total synthesis of a small set of natural products and related structures. We envisage that this strategy could be exploited by accessing a large number of members of this family of compounds.
\end{abstract}




\section{TABLE OF CONTENTS}

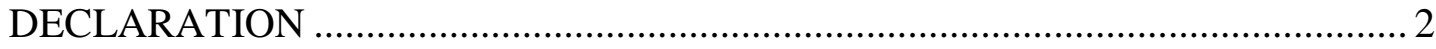

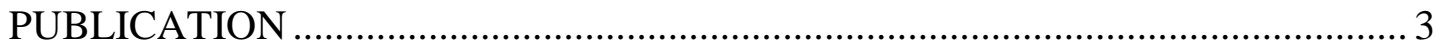

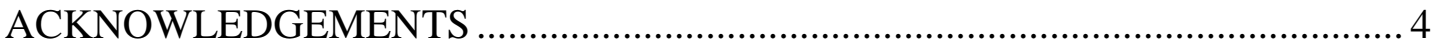

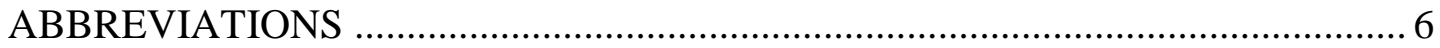

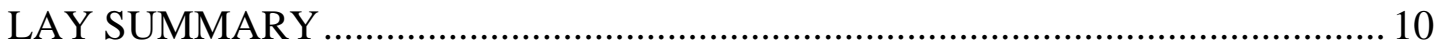

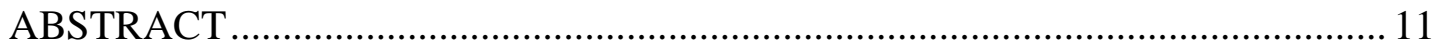

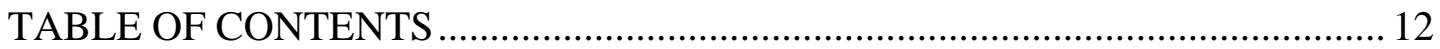

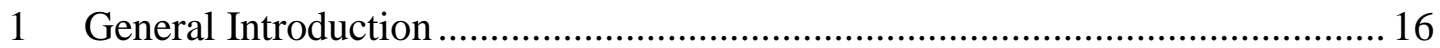

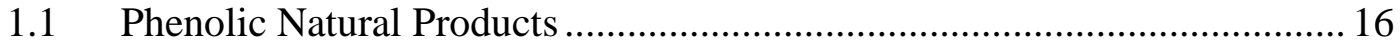

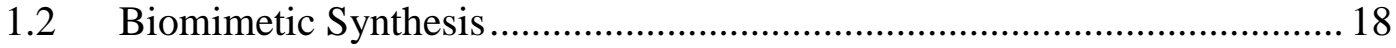

1.3 Biosynthesis of Natural Phenolic Compounds ........................................... 19

1.3.1 Polyketide Biosynthesis ………..................................................... 20

1.3.2 Biomimetic Polyketide Synthesis .................................................. 21

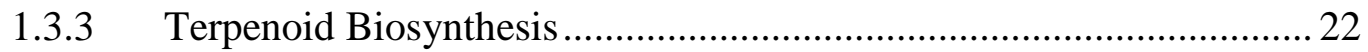

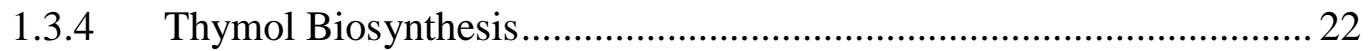

1.3.5 Biomimetic Synthesis of Terpenoids ................................................ 24

1.3.6 Shikimate Pathway: Biosynthesis of Phenylpropanoids ......................2 25

1.3.7 Biomimetic Synthesis of Phenylpropanoids ....................................... 27

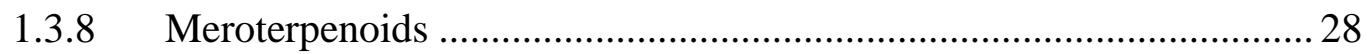

1.3.9 Biosynthesis of Meroterpenoids .......................................................... 28

1.3.10 Biomimetic Synthesis of Meroterpenoids .......................................... 30

1.4 Dimeric and pseudo-Dimeric Natural Products ...................................... 30

1.4.1 Biosynthesis of Dimeric Natural Products ........................................... 32

1.4.2 Biomimetic Synthesis of Dimeric Natural Products ........................... 32

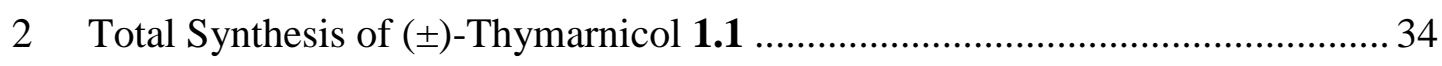

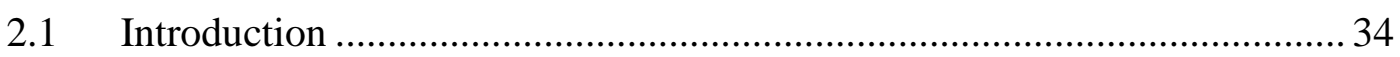

2.1.1 Thymarnicol: a Dimeric Thymol Derivative....................................... 34

2.1.2 Passreiter's Biosynthetic Proposal ....................................................... 35

2.1.3 Alternative Biosynthetic Pathways .................................................... 36

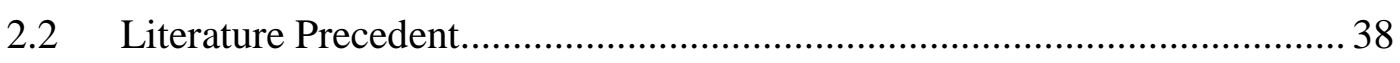

2.2.1 Dimerisation Literature Precedent .................................................... 38 
2.2.2 Oxidation/Cyclisation Literature Precedent …...................................... 40

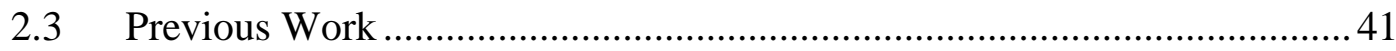

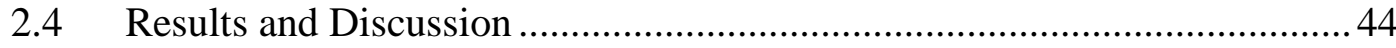

2.4.1 Repetition and Optimisation of the Previous Synthesis ...................... 44

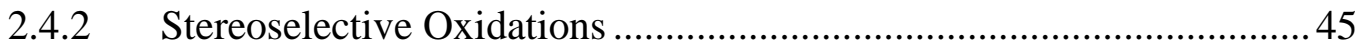

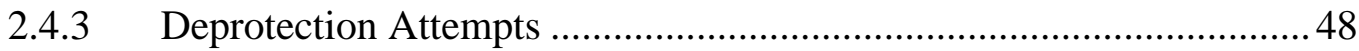

2.5 Protecting-Group Free Approach ........................................................... 53

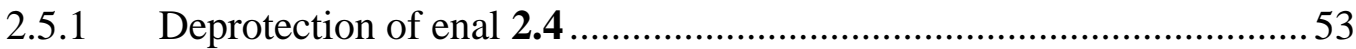

2.6 Alternative ortho-Directing Protecting Group Approach ...........................57

$2.7 \quad$ Base-Labile Protecting-Groups ................................................................ 58

2.7.1 Synthesis of Enal Monomer 2.47 ...................................................59

2.7.2 Studies on the Dimerisation of Enal Monomer 2.47 ......................... 60

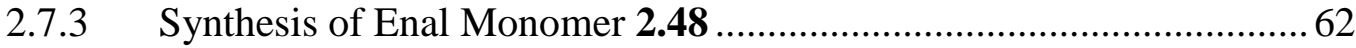

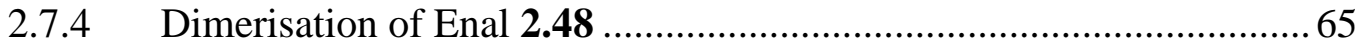

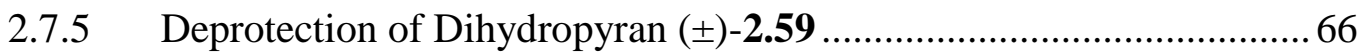

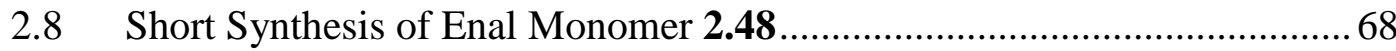

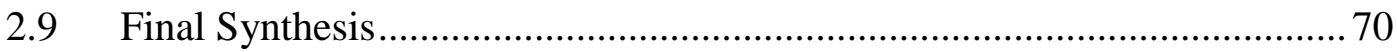

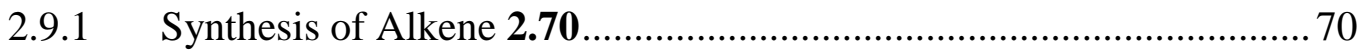

2.9.2 Allylic Oxidation of Alkene 2.70 .................................................... 71

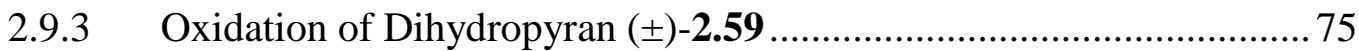

2.9.4 Preparation of $( \pm)$-Thymarnicol 1.1 …............................................ 76

2.10 Computational Studies on the Diels-Alder Dimerisation .......................... 81

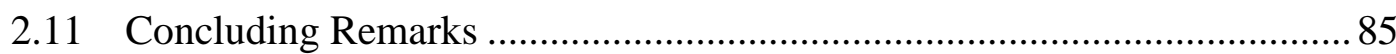

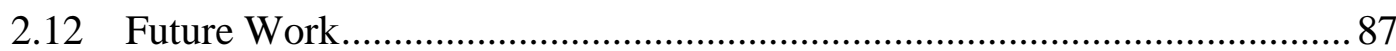

3 Biomimetic Diversity-Oriented Synthesis of Prenylated Phenylpropanoids

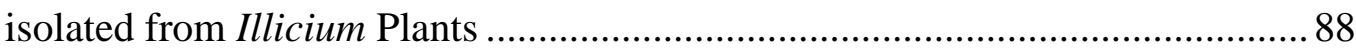

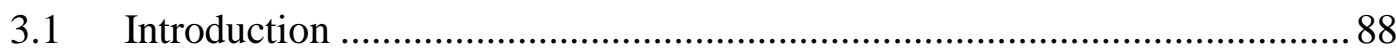

3.1.1 Biomimetic Diversity Oriented Synthesis....................................... 88

3.1.2 Prenylated Phenylpropanoids from Illicium Genus Plants................... 89

3.2 Previous Syntheses of Prenylated Phenylpropanoids from Illicium Plants.91

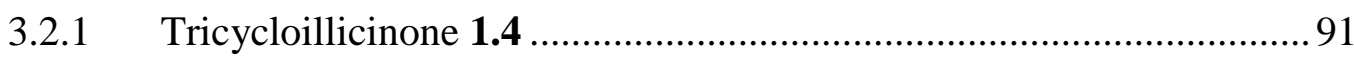

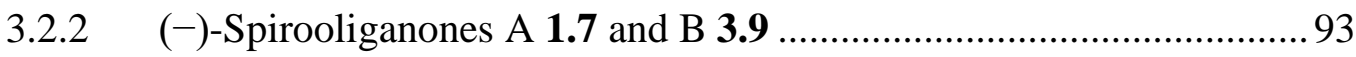

3.2.3 (+)-Illicidione A 1.5 and (+)-Illihendione A 3.23 ........................... 96

3.3 Our Biosynthetic Proposal ......................................................................... 99 


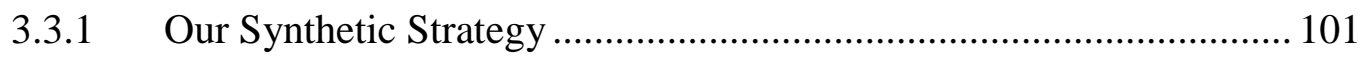

3.3.2 Synthesis of the Precursors ............................................................ 102

3.3.3 Planned Synthesis of Selected Molecular Targets ............................ 103

3.3.4 Planned Synthesis of Selected Type $\mathrm{A}_{2}$ Structures ............................ 104

3.3.5 Planned Synthesis of Selected Type $B_{2}$ Structures .......................... 105

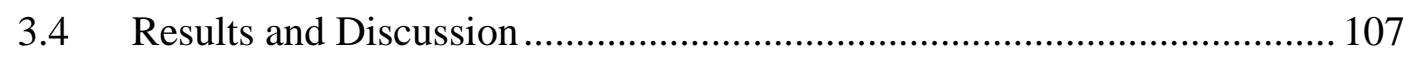

3.4.1 Synthesis of the Precursor to Type $A_{1}$ and $A_{2}$ Structures ................. 107

3.4.2 Synthesis of the Precursor to Type $B_{2}$ Structures ............................ 111

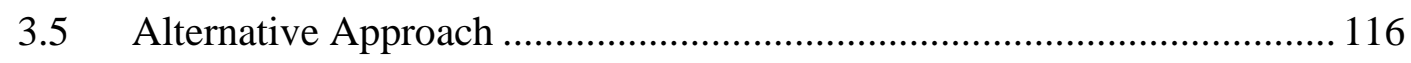

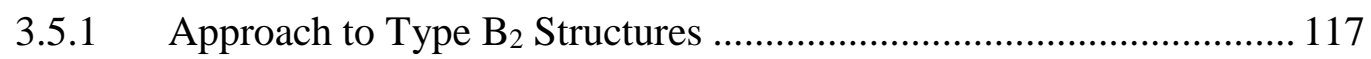

3.5.2 Total Synthesis of $( \pm)$-Cycloillicinone 1.6................................... 119

3.5.3 Synthesis of ( \pm )-Illicinone C 3.78 and ( \pm -epi-Illicinone C 3.79....... 121

3.5.4 Synthesis of ( \pm -epi-Illicinone E 3.2 and Related Structures............. 123

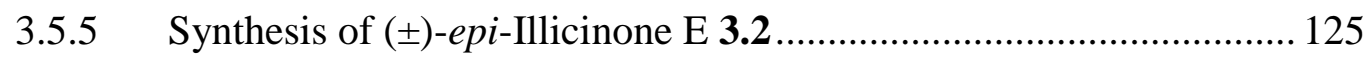

3.5.6 Synthesis of $( \pm)$-epi-13-O-Methylillicinone C 3.87 ........................ 125

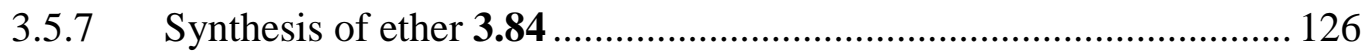

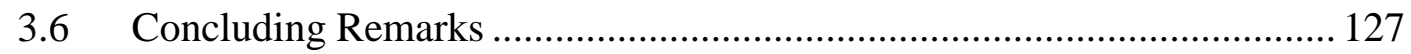

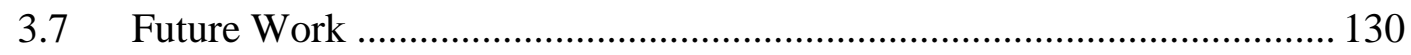

3.7.1 Illicinone E ( \pm -3.80 and Related Structures.................................... 130

3.7.2 ( \pm -Spirooliganones A 1.7 and B 3.9 ........................................... 131

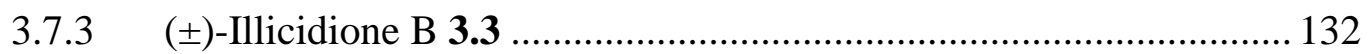

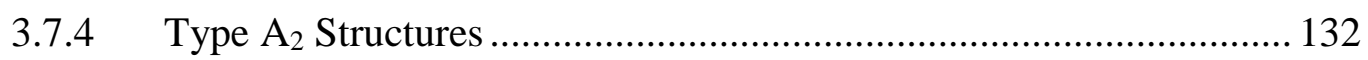

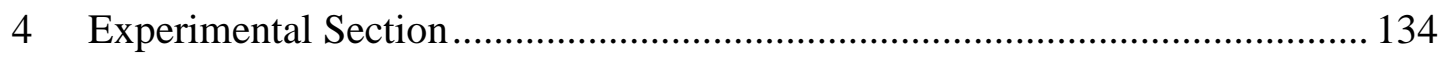

4.1 General Experimental Conditions ....................................................... 134

4.2 Specific Experimental Procedures for Chapter 2 ................................... 136

4.3 Specific Experimental Procedures for Chapter 3 ................................... 192

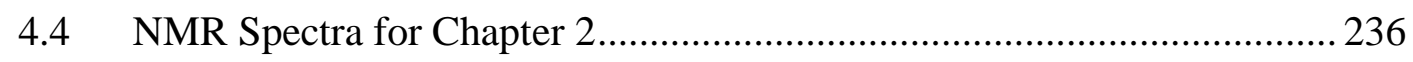

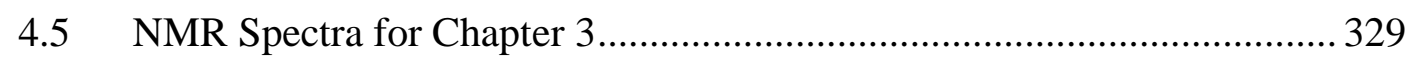

4.6 Computational Studies (Dr Fernanda Duarte) ........................................ 410

4.6.1 Computational Methods ............................................................... 410

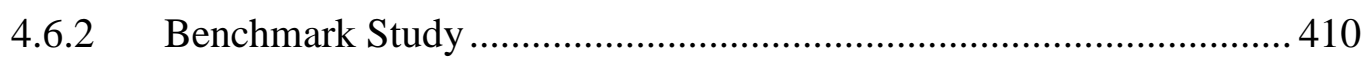

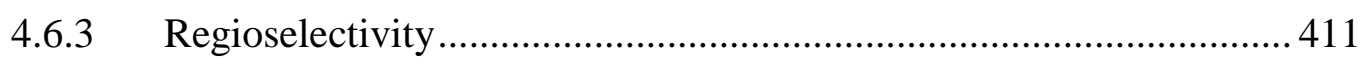

4.6.4 Substituent Effect ....................................................................... 417

4.6.5 Tables with Absolute Energies ....................................................... 420 


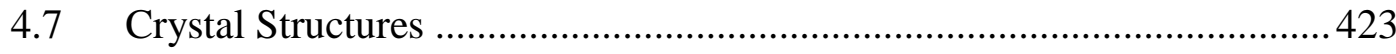

4.7.1 Crystal Structure of meso-2.31 ..................................................... 423

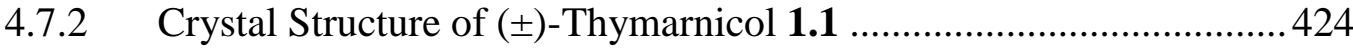

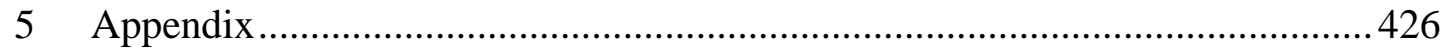

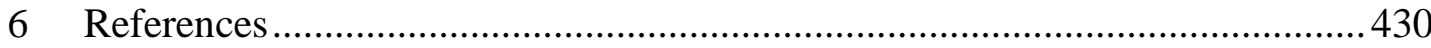




\section{General Introduction}

This thesis details the total synthesis of a dimeric thymol derivative and efforts towards developing a unified synthetic strategy toward an entire class of structurally diverse prenylated phenylpropanoids. All these molecular targets are, or can be derived from, compounds containing mono or polyhydroxylated aromatic rings. This chapter provides a general introduction to phenolic natural products, the most important biosynthetic pathways leading to their formation and some pertinent examples of biomimetic total syntheses. A short preface to dimeric and pseudo-dimeric natural products will also be given. Specific introductions can be found at the beginning of chapters 2 and 3.

\subsection{Phenolic Natural Products}

Naturally occurring phenolic compounds are a broad class of low weight organic compounds containing one or more hydroxy groups connected to an aromatic ring. According to the definition given by Haslam in 1994, this term should be used as a descriptor for water-soluble plant phenolic compounds having molecular masses from 500 to 3000-4000 Da and possessing twelve to sixteen hydroxy groups on five to seven aromatic rings per $1000 \mathrm{Da} .{ }^{1}$ However, compounds of lower molecular weight bearing multiple hydroxy substituents on one or more aromatic rings are often referred to as polyphenols. ${ }^{2}$ Although natural phenols are widely distributed in nature (animals, plants, bacteria and fungi) they are particularly common in plants, where they are the most abundant secondary metabolites. ${ }^{3}$ They are synthesised by the plant during normal development and under stressed conditions, such as infection, wounding or exposure to UV radiation. ${ }^{4}$ This huge class of compounds includes stilbenes, phenolic acids, flavonoids, anthrones, coumarins, lignans, curcuminoids, chalcones and many others (some representative members of these groups are depicted in figure 1.1). 
<smiles>Oc1ccc(/C=C/c2cc(O)cc(O)c2)cc1</smiles>

resveratrol (stilbenes)<smiles>Cc1cc(O)c2c(c1)C(=O)c1cc(O)cc(O)c1C2=O</smiles>

emodin (anthrones)<smiles>O=C(O)/C=C\c1ccc(O)c(O)c1</smiles>

caffeic acid (phenolic acids)<smiles>O=c1c(O)c(-c2ccc(O)c(O)c2)oc2ccccc12</smiles>

flavonol (flavonoids)<smiles>COc1cc2ccc(=O)oc2cc1O</smiles>

scopoletin (coumarins)<smiles>CC1C(c2ccc3c(c2)OCO3)O[C@@H](c2ccc3c(c2)OCO3)C1C</smiles>

galbacin (lignans)<smiles>COc1cc(/C=C/C(=O)/C=C(O)/C=C/c2ccc(O)c(OC)c2)ccc1O</smiles><smiles>CC(C)=CCc1c(O)ccc(C(=O)/C=C/c2ccc(O)cc2)c1O</smiles>

Figure 1.1: Representative phenolic natural products.

Phenolic compounds have been used by humans for millennia. The first reported use of them probably dates back to the ancient Greeks of the archaic period (ca. 800-500 $\mathrm{BC}$ ), when they were used in the conversion of animal skin into leather. The first mention in literature is accredited to Theophrastus of Eressus (371-286 BC), who referred to them as "vegetable tannins" in his Historia Plantarum plant encyclopedia. ${ }^{2}$ The determination of the (poly)phenolic nature of these vegetable tannins was not a simple matter prior to the development of appropriate analytical techniques. In fact, their wide distribution in nature and the diversity in their composition made it difficult to recognise them as members of this group of compounds. It wasn't until the $20^{\text {th }}$ century that this class of metabolites started to be properly studied and characterised. ${ }^{2}$ Part of the reason why there has been a growing interest in the study of phenolic natural products is their implication in various domains of commercial and social interest. In particular, phenolic compounds play a significant role in human health due to their biological activities, which includes antifungal, antioxidant, radical scavenging and anti-cancerous activity. ${ }^{5} \mathrm{~A}$ famous example is represented by resveratrol (figure 1.1) and the so-called "French paradox". 6 This refers to the apparently paradoxical observation that French people have a relatively low incidence of coronary heart 
diseases, despite having a diet very rich in saturated fats. Among the various hypotheses to explain this phenomenon, there is the relatively high levels of consumption of red wine in France. Wines, in particular red varieties, are a source of low levels of resveratrol. The average intake of wine among French people, though, would probably not provide enough resveratrol to have a significant effect and there is still a lack of medical consensus about whether there really is a connection between the moderate consumption of alcoholic beverages and low heart disease incidence. ${ }^{7}$ Nevertheless, the wine industry took advantage of the French paradox, promoting a marketing campaign which instilled in the population the idea that drinking wine leads to beneficial effects.

\subsection{Biomimetic Synthesis}

The ability of nature to produce complex molecules has fascinated and inspired synthetic chemists since the beginning of the $20^{\text {th }}$ century. Various names and definitions for the concept of biomimicry have been proposed since the late 1950s onward. ${ }^{8,9}$ With regard to the specific field of synthetic organic chemistry, Eugene Van Tamelen was the first to define biomimetic chemistry, in $1961 .{ }^{10} \mathrm{He}$ gave this name to a small yet important body of emerging work to synthesise natural products in a way which could plausibly occur in nature. He also made a distinction between the so called "biogenetic-type" and "physiological-type" syntheses. Van Tamelen defined as "biogenetic-type" a synthesis which was

"designed to follow, in at least its major aspects, biosynthetic pathways proved, or presumed to be used in the natural construction of the end product",10

He then added:

"biogenetic-type syntheses are thus to be distinguished from physiological-type syntheses, in which not only plausible bio-organic substitutes are employed, but also specific conditions of temperature, $\mathrm{pH}$, dilution etc., which supposedly compare to those obtained in a living cell"10 
According to Van Tamelen's definition, the work described in this thesis falls into the "biogenetic-type" category. Firstly, the synthetic design is inspired by a mixture of known and proposed biosynthetic pathways. Secondly, emphasis is in the overall strategy used by nature and on the key intermediates, which are prepared using conventional synthetic techniques. The starting material and the exact order of the transformations in the synthesis does not need to match the ones proposed to occur in nature. Furthermore, the key intermediates may be modified with respect to the natural ones to improve selectivity and/or reactivity, in the absence of enzymatic regio/stereocontrol in the laboratory. Finally, as our focus is on strategy, the reactions will be conducted using common synthetic techniques rather than under physiologically reasonable conditions, and the precise reagents used are not considered important.

\subsection{Biosynthesis of Natural Phenolic Compounds}

Phenolic natural products can be derived from several metabolic pathways, which perhaps explains their prevalence in nature. An overview of these biosynthetic pathways (acetate, mevalonate, methylerythritol phosphate, and shikimate) will be given in this general introduction chapter, along with a short discussion about compounds of mixed origin (scheme 1.1).

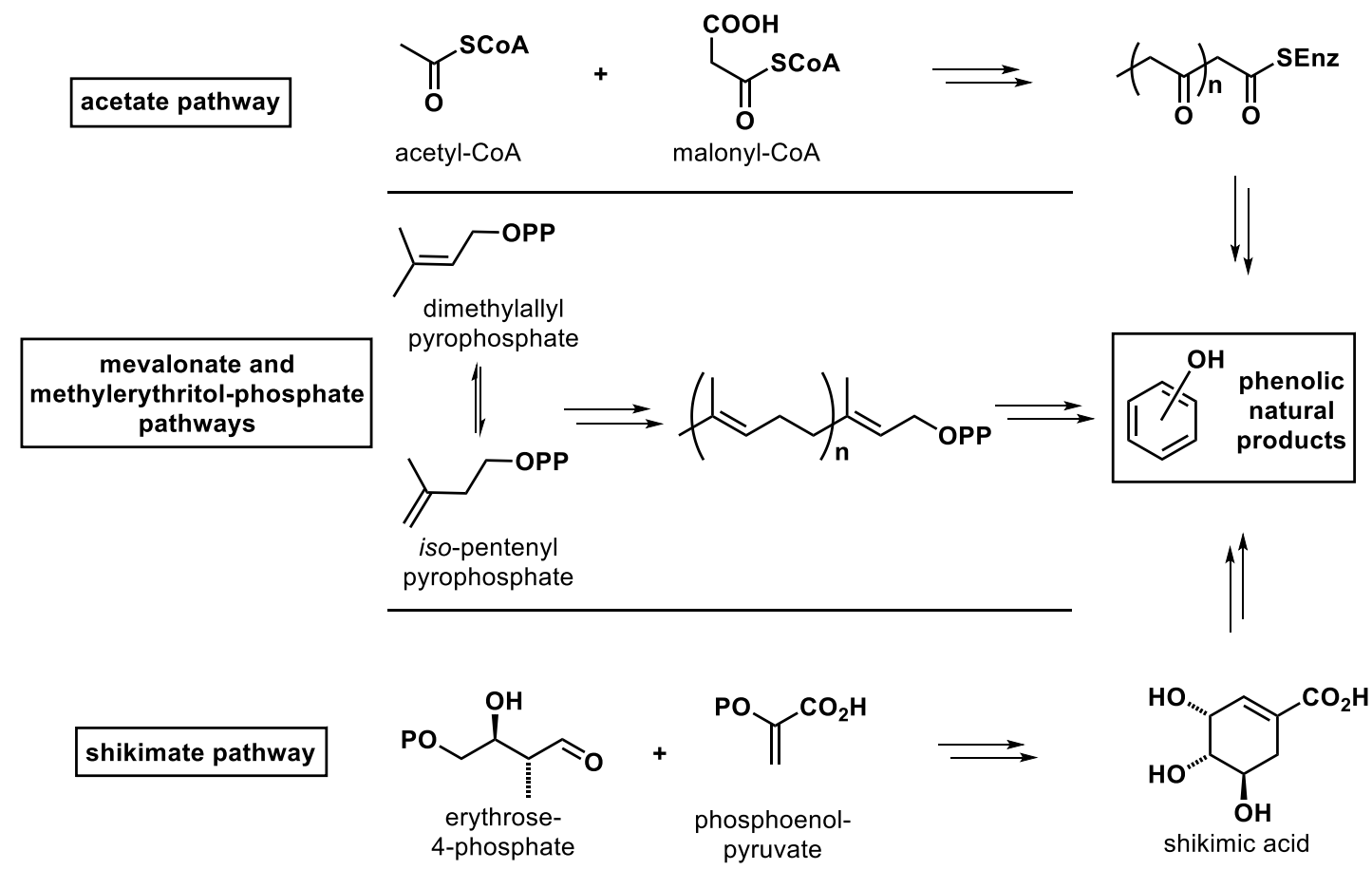

Scheme 1.1: Overview of the major biosynthetic pathways. 
Particular attention will be dedicated to the mevalonate, methylerythritol phosphate, and shikimate pathways, as they lead to the proposed biosynthetic precursors to the specific natural product targets discussed in this thesis. (A selection of our target compounds, which we aim to access in racemic form, is shown in figure 1.2).

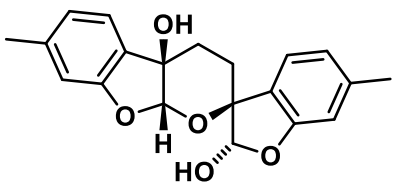

( \pm )-thymarnicol

1.1

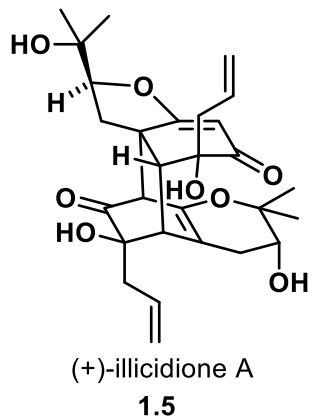

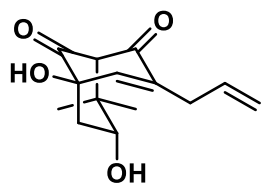

(-)-illioliganone A

1.2

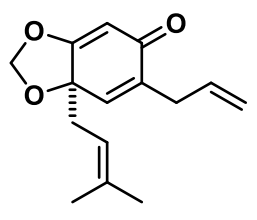

(-)-illicinone $\mathrm{A}$

1.3

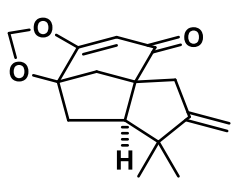

$( \pm)$-tricycloillicinone

1.4

Figure 1.2: A selection of our natural product targets.

\subsubsection{Polyketide Biosynthesis}

Phenolic natural products can be produced via the acetate pathway, the products of which are often identified from the 1,3-oxygenation pattern within the aromatic rings, resulting from the various possible intramolecular Claisen and aldol reactions of the growing poly- $\beta$-ketoester chain. Methylenes flanked by two carbonyls are activated by specific enzymes, which also fold the substrate in the correct conformation to allow reactions to occur with high regioselectivity, with a natural tendency to form strainfree six-membered rings. Tautomerisation and dehydration then allow for aromatisation of these six-membered rings. A simple example is represented by the biosynthesis of orsellinic acid. This compound is synthesised in nature from the polyketide chain resulting from the formal addition of an acetyl-CoA and three malonyl-CoA units (scheme 1.2). ${ }^{11}$ 


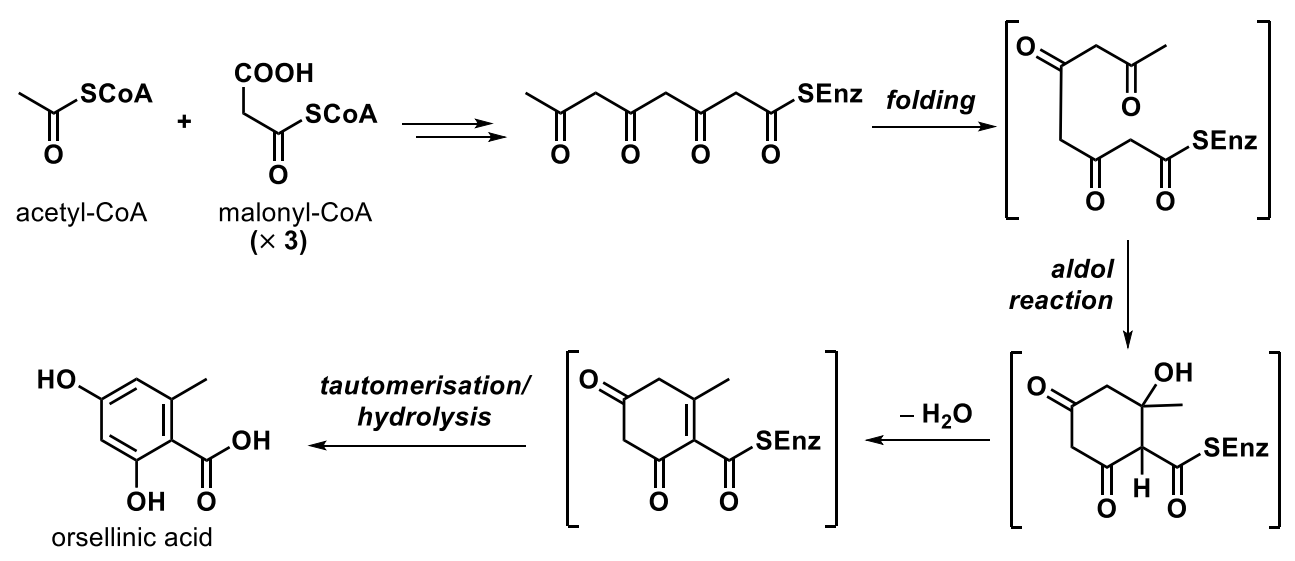

Scheme 1.2: Biosynthesis of orsellinic acid.

\subsubsection{Biomimetic Polyketide Synthesis}

The first reported biomimetic synthesis of a natural product is probably Collie's synthesis of orcinol in 1893. In this work Collie demonstrated the ability of linear polyketide chains to form cyclic aromatic frameworks through sequences of condensation reactions. ${ }^{12}$ Collie successfully synthesised orcinol from dimethylpyrone $\mathbf{1 . 8}$ by treating it with an aqueous solution of barium hydroxide to give diacetylacetone 1.9. Upon acidification of the crude reaction mixture, Collie isolated orcinol, as a result of an aldol condensation to give cyclic intermediate 1.10, which that tautomerised to afford the aromatic product orcinol (scheme 1.3).

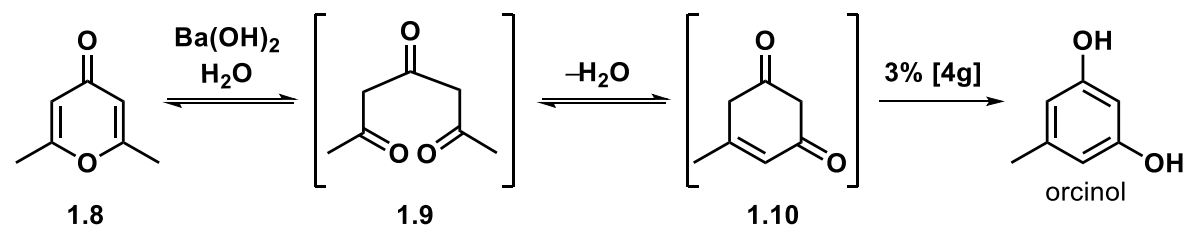

Scheme 1.3: Collie's biomimetic synthesis of orcinol.

The low overall yield of this relatively simple transformation reflects the natural tendency of polyketides to give non-selective and uncontrolled reactions. For this reason, total syntheses of this class of natural products usually involve different (nonbiomimetic) approaches. 


\subsubsection{Terpenoid Biosynthesis}

The terpenoids and steroids constitute a large and structurally diverse class of natural products derived from the combination of $\mathbf{C}_{5}$ isoprene units: isopentenyl pyrophosphate (IPP) and dimethylallyl pyrophosphate (DMAPP). The relative amounts of IPP and DMAPP are regulated via the enzyme isopentenyl diphosphate isomerase (E1), which is responsible for the isomerisation of IPP to produce DMAPP, a reactive electrophile. Regular terpenoids are the result of the head-to-tail union of isoprene fragments and are classified as hemiterpenes $\left(\mathbf{C}_{\mathbf{5}}\right)$, monoterpenes $\left(\mathbf{C}_{\mathbf{1 0}}\right)$, sesquiterpenes $\left(\mathbf{C}_{15}\right)$, diterpenes $\left(\mathbf{C}_{\mathbf{2 0}}\right)$, sesterpenes $\left(\mathbf{C}_{25}\right)$, triterpenes $\left(\mathbf{C}_{\mathbf{3 0}}\right)$ etc. The isoprene units may be derived from two different pathways: the mevalonate pathway, starting with three molecules of acetyl-CoA, and the more recently discovered methylerythritol phosphate pathway, which uses pyruvic acid and glyceraldehyde-3phosphate, as shown in scheme 1.4. ${ }^{11}$

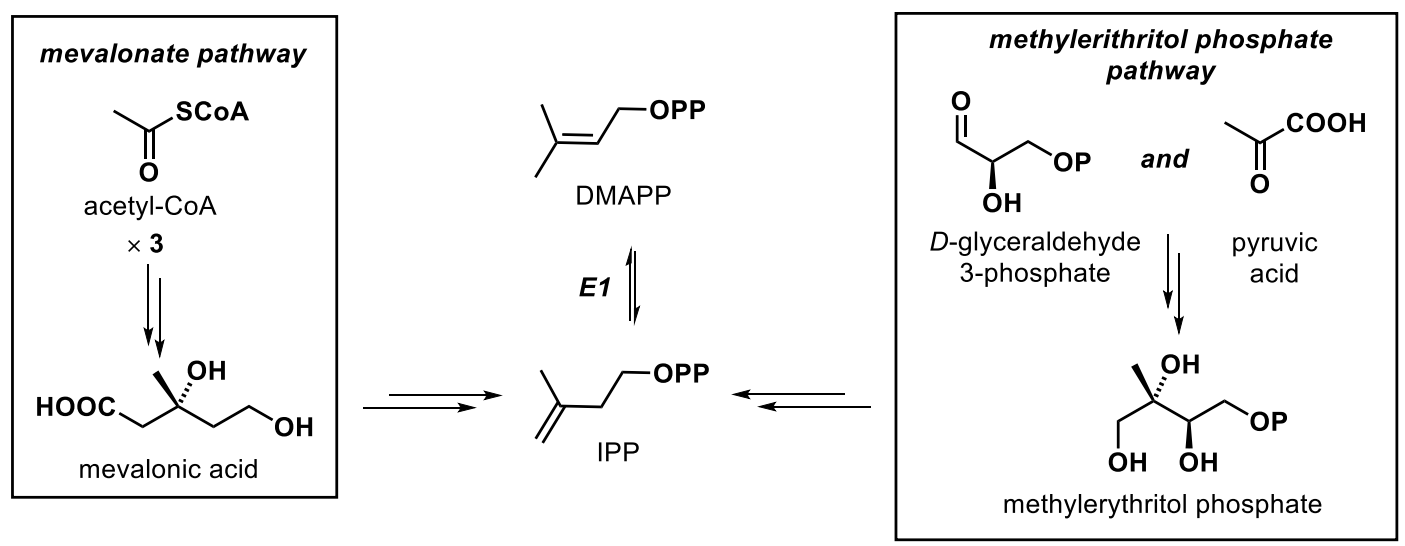

Scheme 1.4: The two routes to the formation of the isoprene units.

\subsubsection{Thymol Biosynthesis}

Thymol is a naturally occurring monoterpene found as one of the major constituents in Thymus vulgaris and many other plants as a white crystalline solid with pleasant aromatic odour. The antimicrobial and antioxidant properties of thymol have been demonstrated in numerous studies. ${ }^{13,14}$ This compound is of particular interest in this thesis as it represents the starting point in the proposed biosynthesis of $( \pm)$-thymarnicol 1.1, one of our molecular targets, discussed in chapter 2. 
The head-to-tail union of a molecule of DMAPP with an IPP fragment produces geranyl pyrophosphate (GPP), precursor to the acyclic, cyclic and aromatic monoterpenes, of which thymol is a representative member. ${ }^{11}$ The biosynthesis of thymol has been proven to start from isoprene units coming from the mevalonate pathway with an isotope labelling experiment performed by Yamazaki and coworkers. ${ }^{15}$ The synthesis of GPP is catalysed by the enzyme geranyl diphosphate synthase (E1) and it is believed to involve addition of IPP to allylic cation $\mathbf{1 . 1 1}$ formed by elimination of pyrophosphate from DMAPP and stereoselective loss of a proton, to give an E-configured alkene. Linalyl pyrophosphate (LPP) and neryl pyrophosphate (NPP) are isomers of GPP and are likely to be in equilibrium with it through elimination and recombination of pyrophosphate at different positions of allylic cation 1.12. Allylic cation $\mathbf{1 . 1 2}$ can give rise to acyclic monoterpenes, often found as components of volatile oils used in flavouring and perfumery, or undergo intramolecular electrophilic addition to produce menthyl cation 1.13, precursor to cyclic terpenoids. In the case of the $\gamma$-terpinene biosynthesis, this cation undergoes a [1,2]-hydride shift to form terpinen-4-yl cation 1.14. Finally, the loss of a proton, mediated by the enzyme $\gamma$-terpinene synthase (E2) provides $\gamma$-terpinene (scheme 1.5).

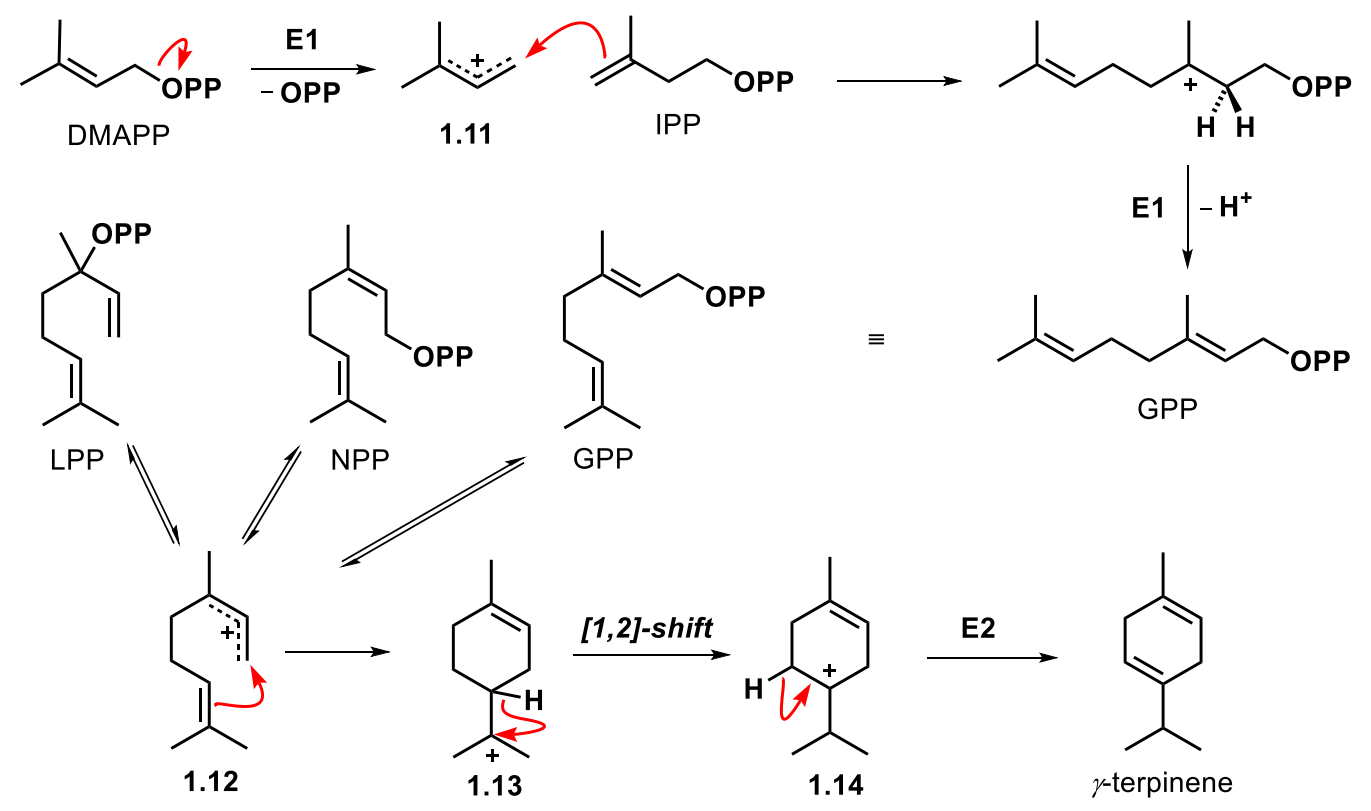

Scheme 1.5: Biogenesis of $\gamma$-terpinene.

The synthesis of thymol from $\gamma$-terpinene is somewhat speculative, but it is widely believed to involve aromatisation to $p$-cymene, which has been proved to be 
synthetically feasible, ${ }^{16}$ followed by oxidation at $\mathrm{C}_{2}$ (scheme 1.6). ${ }^{17}$ The autoxidation of $\gamma$-terpinene to $p$-cymene led Granger and co-workers to propose that it proceeds via non-enzymatic aromatisation. ${ }^{18}$ Later studies by Croteau and Poulose demonstrated that intact leaves from young thyme plants can convert isotopically labelled exogenous $\gamma$-terpinene into $p$-cymene and subsequently thymol. ${ }^{17,19}$

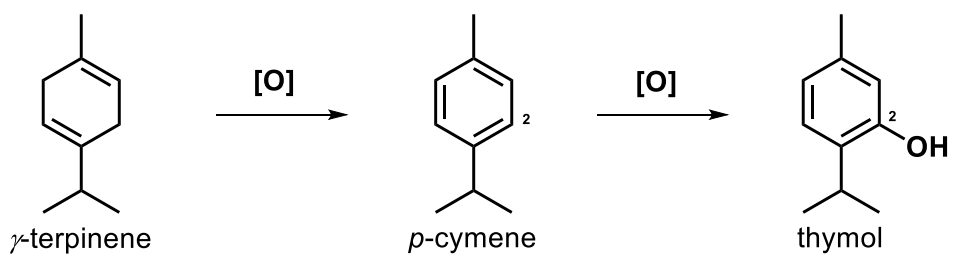

Scheme 1.6: Formation of thymol from $\gamma$-terpinene.

\subsubsection{Biomimetic Synthesis of Terpenoids}

Most biomimetic total synthesis of complex polycyclic aromatic (and non-aromatic) terpenoids take advantage of a pre-installed aromatic moiety and focus on the assembly of the aliphatic polycyclic framework..$^{20-22}$ This is not surprising, as the assembly of the aromatic ring(s) from the terpene chain requires several synthetic steps and suffers from potential selectivity issues (see thymol biosynthesis in section 1.3.4 on pages 22-23 for an example). The most used and reliable method for the biomimetic construction of the aromatic ring from aliphatic terpenoids perhaps is the oxidation of a cyclohexane ring coupled with migration of a geminal methyl group, as exemplified by the work of Cartier (scheme 1.7). ${ }^{23}$

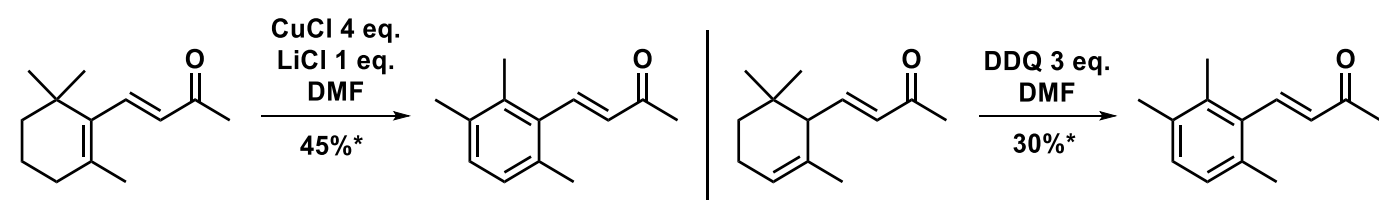

Scheme 1.7: Examples of aromatisation of terpenoid moieties. ${ }^{23} *$ Crude yield

This method, however, requires superstoichiometric amounts of oxidant and generally suffer from poor yields and very narrow substrate scope. Given the wide range of cheap and readily available aromatic frameworks, this approach is usually not the preferred one. 


\subsubsection{Shikimate Pathway: Biosynthesis of Phenylpropanoids}

Phenylpropanoids are compounds characterised by the presence of a six-membered carbon ring with a three-carbon chain $\left(\mathbf{C}_{6} \mathbf{C}_{3}\right)$. Like the closely related phenylethanoids (which feature a $\mathbf{C}_{6} \mathbf{C}_{2}$ moiety) they are biosynthetically derived from the shikimate pathway through modification of the aromatic amino acids phenylalanine or tyrosine. The shikimate pathway (scheme 1.8) starts with the coupling of phosphoenolpyruvate (PEP), a glycolysis product, and erythrose-4-phosphate 1.15, from the pentose phosphate cycle, to form 3-deoxy-D-arabino-heptulosonate-7-phosphate 1.16, with the intervention of 3-deoxy-D-arabino-heptulosonate-7-phosphatase (E1). ${ }^{11,24}$ Tetrahydropyran $\mathbf{1 . 1 6}$ is then converted into ketone $\mathbf{1 . 1 7}$ in a 3-dehydroquinate synthase (E2) mediated sequence beginning with oxidation of the $\mathrm{C} 5$ alcohol, followed by enolisation and E1cB-type elimination of phosphate. The cyclohexane ring of $\mathbf{1 . 1 7}$ is then formed through reduction of the $\mathrm{C} 5$ ketone, ring opening and aldol addition. ${ }^{24}$ 27

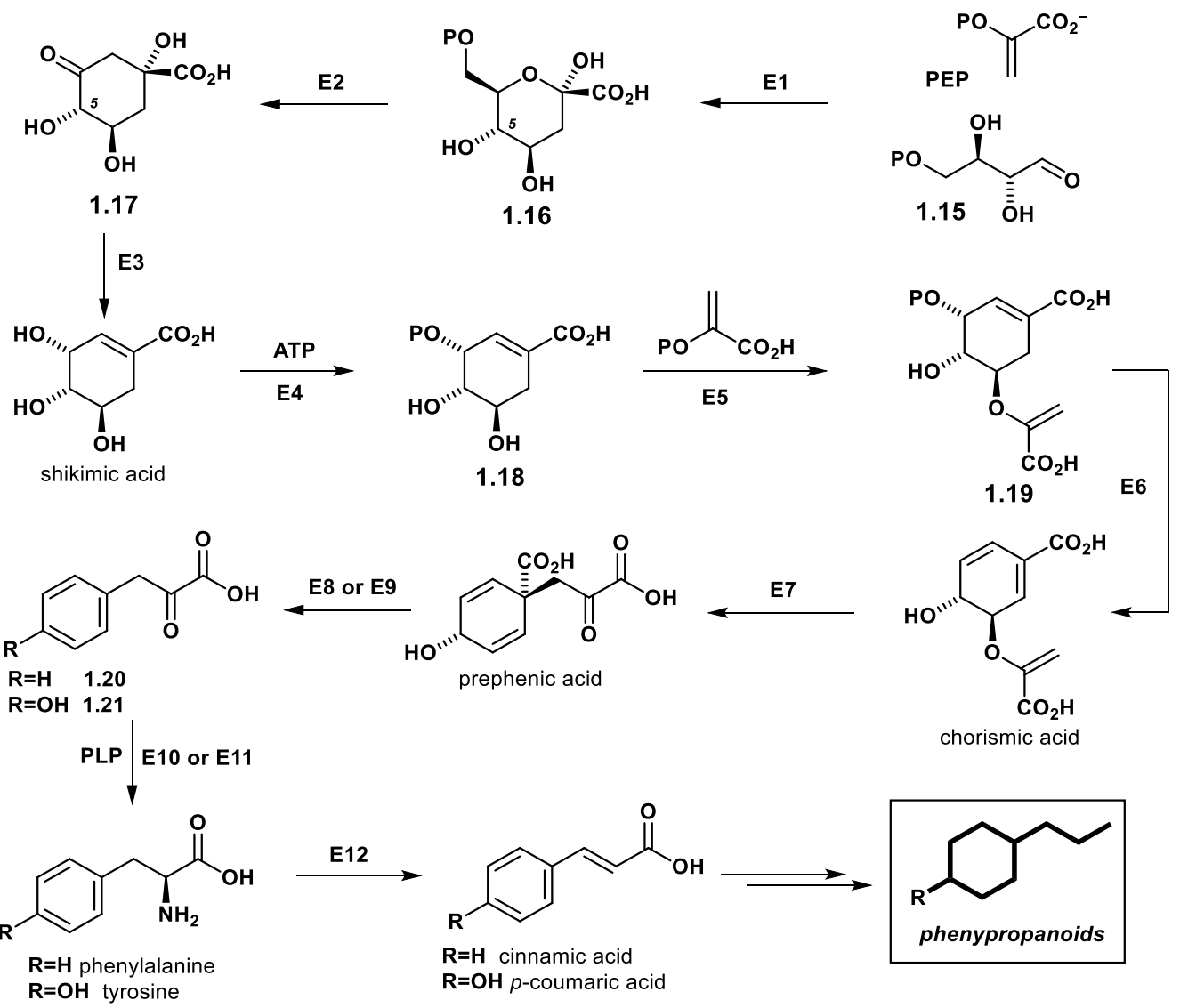

Scheme 1.8: Biogenesis of phenylpropanoids. 
Dehydration alpha to the carboxylic acid functionality of ketoacid $\mathbf{1 . 1 7}$ followed by reduction of the carbonyl group then gives shikimic acid, the first key intermediate of this pathway. In plants these two steps are catalysed by a single bifunctional 3dehydroquinate dehydratase-shikimate dehydrogenase enzyme (E3). ${ }^{24}$ Shikimic acid is then phosphorylated by ATP, reaction mediated by the shikimate kinase enzyme (E4), to give shikimic acid 3-phosphate 1.18. This is then converted into 5enolpyruvylshikimate-3-phosphate $\mathbf{1 . 1 9}$ through the addition of an enolpyruvate unit from PEP, mediated by 5-enolpyruvylshikimate-3-phosphate synthase (E5). The subsequent chorismate synthase (E6) mediated 1,4-elimination of hydrogen phosphate forms chorismic acid, the second key intermediate. Chorismic acid can then follow two divergent pathways, to ultimately give the amino acids tryptophan on the one hand and phenylalanine and tyrosine on the other. ${ }^{11,24}$ The phenylpropanoids are products of the phenylalanine/tyrosine branch of the synthesis. Chorismic acid undergoes a formal Claisen rearrangement, mediated by chorismate mutase (E7) to produce prephenic acid. The formation of phenylalanine and tyrosine from prephenic acid can then follow different pathways depending on the organism. In plants two different enzymes, prephenate dehydratase (E8) and prephenate dehydrogenase (E9), catalyse the decarboxylation of prephenic acid. In the case of $\mathrm{E} 8$ the loss of $\mathrm{CO}_{2}$ is paired with dehydration, to produce phenylpyruvate $\mathbf{1 . 2 0}$ while E9 catalyses the oxidative decarboxylation to 4-hydroxyphenylpyruvate 1.21, accompanied by the reduction of $\mathrm{NADP}^{+}$to NADPH. Finally, phenylpyruvate and 4-hydroxyphenylpyruvate aminotransferases (E9 and E10 respectively) catalyse the transamination of amino acids 1.20 and 1.21 using a PLP (pyridoxal phosphate) cofactor to produce the amino acid phenylalanine and tyrosine, precursors to a wide range of natural products. ${ }^{24} \mathrm{In}$ plants, a frequent first step is the elimination of ammonia from the side-chain to generate trans-cinnamic acid in the case of phenylalanine and 4-coumaric acid in the case of tyrosine, reaction mediated by the enzyme phenylalanine ammonia lyase (E12). These acids contain the basic $\left(\mathbf{C}_{\mathbf{6}} \mathbf{C}_{\mathbf{3}}\right)$ structure for the phenylpropanoids, which are components of several structural polymers (lignin and suberin for example) and are precursors for many classes of natural products, including flavonoids, isoflavonoids, coumarins, lignans, cinnamic aldehydes and chalcones (figure 1.3). 


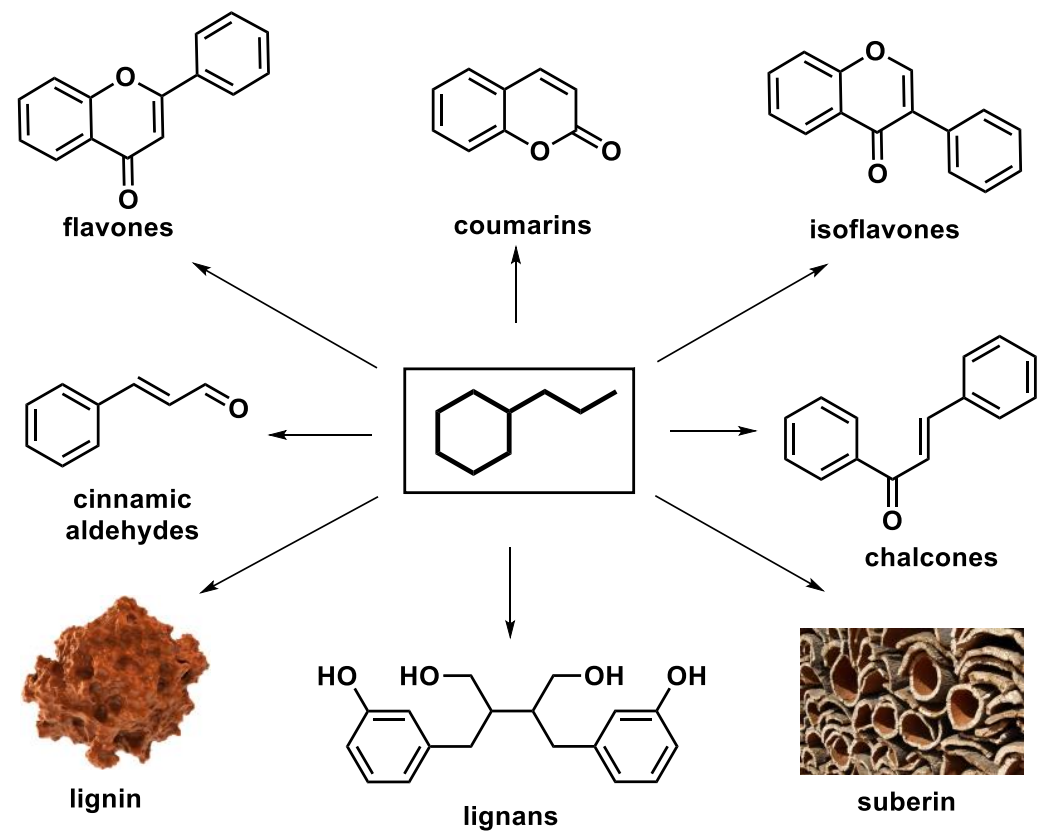

Figure 1.3: Examples of classes of classes of compounds derived from phenylpropanoids.

\subsubsection{Biomimetic Synthesis of Phenylpropanoids}

An excellent example of a biomimetic synthesis of a phenylpropanoid natural product is represented by Chapman's synthesis of carpanone. ${ }^{28}$ After isolating carpanone, Brophy and co-workers proposed a plausible biosynthetic pathway for its formation through the oxidative dimerisation of the co-isolated natural product carpacin via a $\beta \beta$-phenolic coupling followed by an intramolecular Diels-Alder cycloaddition. ${ }^{29}$ Chapman managed to mimic this hypothesis starting from demethylcarpacin $\mathbf{1 . 2 2}$ using a $\operatorname{Pd}(\mathrm{II})$ mediated oxidative coupling, which readily gave the proposed biosynthetic intermediate 1.23, which underwent biomimetic Diels-Alder reaction (scheme 1.9). ${ }^{28}$ 

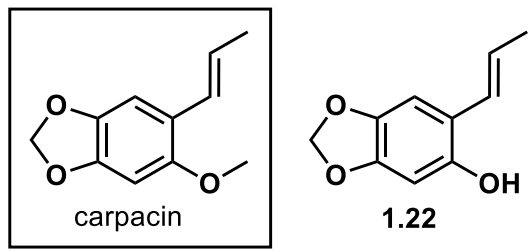<smiles>CC1C=C2C(=O)C=C3OCOC34C[C@@]2(c2cc3c(cc2O4)OCO3)[C@H]1C</smiles>

$( \pm)$-carpanone

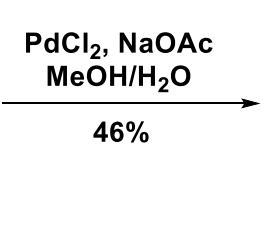

> $\beta \beta$-phenolic
coupling<smiles>CC(/C=C1\C=C2OCOC2=CC1=O)[C@@H](C)/C=C1\C=C2OCOC2=CC1=O</smiles>

Scheme 1.9: Chapman's synthesis of $( \pm)$-carpanone.

\subsubsection{Meroterpenoids}

Meroterpenoids are hybrid natural products containing a partial terpenoid structure, the prefix "mero" means "part" or "fragment", although in the literature this term is sometimes used solely for compounds of mixed polyketide and terpenoid origin. ${ }^{30}$ This class of secondary metabolites includes numerous compounds derived from plants, animals, bacteria and fungi. ${ }^{30-32}$ A sub-family is represented by the terpenophenolics, that are part terpenoids and part phenols. Representative members of this group are $\alpha$ tocopherol, tetrahydrocannabinol and mycophenolic acid (figure 1.4).<smiles>CCCC(C)(CCC(C)C)CCC1(C)CCc2c(C)c(O)c(C)c(C)c2O1</smiles>

$\alpha$-tocopherol

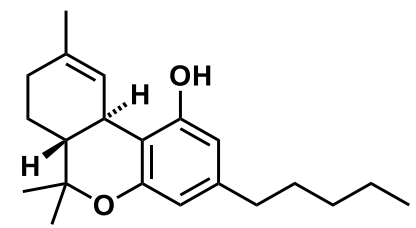

tetrahydrocannabinol<smiles>C=C(C)CC/C(C)=C/Cc1c(O)c2c(c(C)c1OC)COC2=O</smiles>

mycophenolic acid

Figure 1.4: Representative examples of meroterpenoids.

\subsubsection{Biosynthesis of Meroterpenoids}

The biosynthesis of meroterpenoids generally starts with the assembly of the polyketide chain, followed by prenyltransferase enzymes-directed prenylation of the polyketide followed by stereoselective epoxidation of a double bond in the newly introduced chain and cyclisation of the terpenoid moiety. ${ }^{30}$ There are also instances in which meroterpenoids are produced in nature by assembly of pre-cyclised units coming from the two pathways. The structural diversity within this class of compounds 
is due to the differences in the mechanism of the cyclisation reactions, which is strictly controlled by specific enzymes and usually affords a single product. Further differentiation is produced by the differences in the polyketide starting moieties and by various post-cyclisation modifications by both heme and non-heme iron dependent oxygenases. ${ }^{30}$ An example of this is represented by the meroterpenoid territrem $\mathrm{A},{ }^{33}$ the biosynthetic speculation of which ${ }^{31}$ is based on analogy with the proven one for pyripyropene A from nicotinic acid. ${ }^{34}$ The shikimate derived building block in this synthesis is proposed to be 4-hydroxybenzoic acid, which, after condensation with two molecules of malonyl-CoA would give pyranone 1.24. This could then undergo prenyltransferase-catalysed farnesylation with subsequent stereoselective epoxidation of the terminal alkene to afford epoxide 1.25. Polyene cyclisation of the terpenoid moiety would then produce compound 1.26. Finally, a series of post-cyclisation modifications, including oxidations and methylations, would generate territrem A (scheme 1.10).
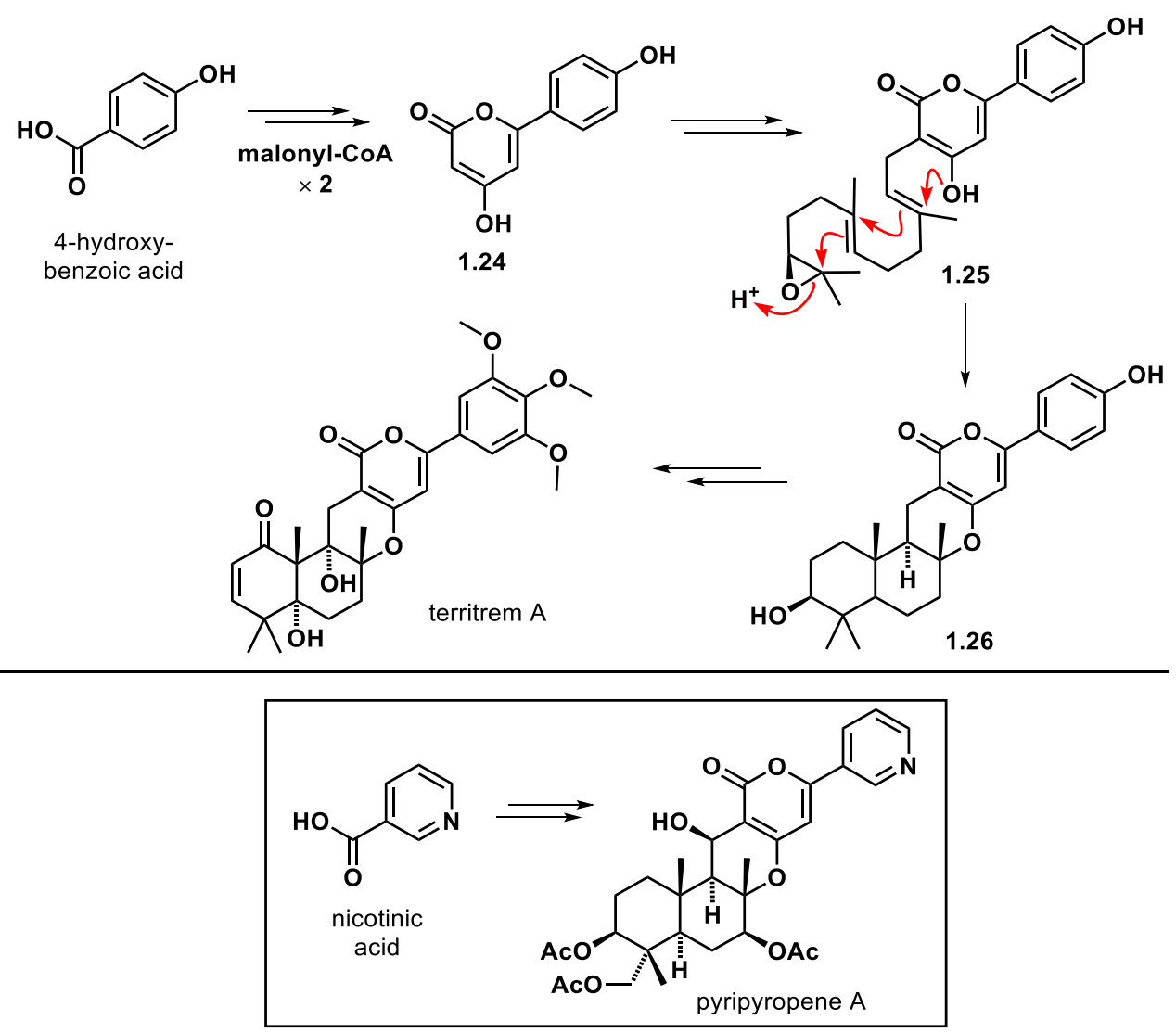

Scheme 1.10: Proposed biosynthesis of territrem A. 


\subsubsection{Biomimetic Synthesis of Meroterpenoids}

A recent example of biomimetic synthesis of a meroterpenoid, involving the coupling of pre-cyclised terpene and polyketide moieties is represented by Lee's total synthesis of guajadial and psidial A. ${ }^{35}$ Inspired by a biosynthetic hypothesis proposed by Liu and co-workers, ${ }^{36}$ Lee successfully completed a biomimetic total synthesis. Diformylphloroglucinol 1.27 was subjected to a three-component reaction along with benzaldehyde and the naturally occurring and commercially available cyclic terpene caryophyllene. After extensive screening of reaction conditions, it was found that heating a mixture of the three components at $100{ }^{\circ} \mathrm{C}$ for 15 hours in the presence of a $5 \%$ aqueous solution of PTS resulted in a $25 \%$ combined yield of guajadial and psidial A. The transformation was proposed to involve aldol condensation between diformylphloroglucinol $\mathbf{1 . 2 7}$ and benzaldehyde followed by hetero-Diels-Alder cycloaddition of the resulting $o$-quinone methide $\mathbf{1 . 2 8}$ with caryophyllene (scheme 1.11).<smiles>O=Cc1c(O)cc(O)c(C=O)c1O</smiles>

1.27

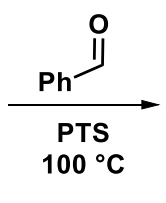

$00^{\circ} \mathrm{C}$

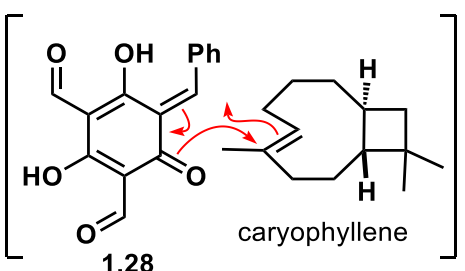

1.28

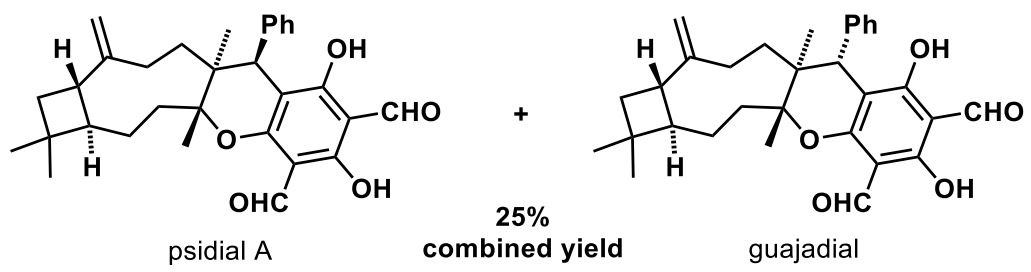

Scheme 1.11: Lee's synthesis of guajadial and psidial A.

\subsection{Dimeric and pseudo-Dimeric Natural Products}

Some of the targeted natural products in this thesis are dimers or derivatised dimers. Therefore, this section aims to give a very brief introduction to dimeric and pseudodimeric natural products and a relevant example of biomimetic synthesis. 
Greer and co-workers, in 2004, surveyed around 3000 articles about natural products and concluded that around 17\% of them included molecules that can be referred to as "dimers" or "derivatised dimers" and 7\% possess bilateral symmetry (i.e. they possess $\mathrm{C}_{2}, \mathrm{C}_{s}$ or $\mathrm{C}_{2 V}$ point groups). ${ }^{37}$ The other $10 \%$ includes molecules lacking of symmetry but formed as a result of the combination of two identical monomeric units and, sometimes, post-dimerisation modifications. The number of reported isolations of dimeric or pseudo-dimeric natural products was extremely low before 1960, but has dramatically increased in recent decades. That is because, before then, the identification of dimers, especially the ones possessing bilateral symmetry, was particularly difficult, as their spectra are usually very similar to those obtained for the corresponding monomers. ${ }^{38}$ Furthermore, most dimeric and pseudo-dimeric natural products are rather complex frameworks, making the structure assignment challenging. In the 1970s the development of 2D NMR and mass spectrometric techniques made the identification of these natural products easier, leading to a larger number of reported isolations. Following that, the number of total syntheses of such compounds has risen significantly: synthetic organic chemists are attracted by dimeric natural products due to their biological activity and often impressive complexity. In particular, biomimicry has been used to emulate the way nature generates complexity (new bonds, rings and stereocentres) in a single step through dimerisation reactions. These include radical reactions, Diels-Alder, Michael additions, esterifications, [2+2], $[4+4]$ and [6+6] cycloadditions, and many others (some selected examples are depicted in figure 1.5). 


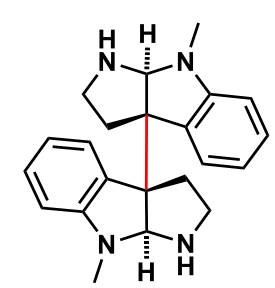

(-)-chimonanthine (radical reaction)

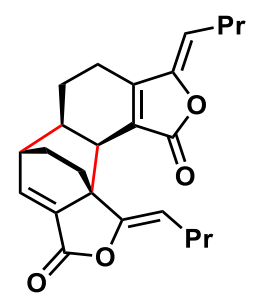

(士)-levistolide A (Diels-Alder)

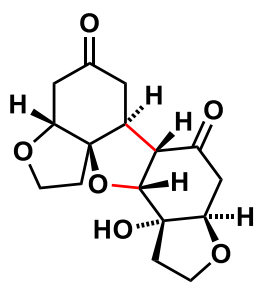

( \pm )-incarviditone (Michael)<smiles>Cc1cc(OC(=O)c2c(C)cc(O)cc2O)cc(O)c1C(=O)O</smiles>

lecanoric acid (esterification)

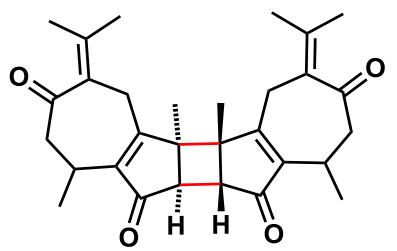

$( \pm)$-vielanin B ([2+2])

Figure 1.5: Examples of dimeric and pseudo-dimeric natural products.

\subsubsection{Biosynthesis of Dimeric Natural Products}

Nature has developed this strategy for different reasons: as mentioned, dimerisation is an efficient way of increasing complexity very rapidly, generating a new molecule by simply coupling together two identical monomers already available in the organism. Furthermore, as a result of the dimerisation, the newly formed molecule often possesses very different physical properties with respect to the starting material. This could result in different interactions with endogenous or exogenous targets, leading to a benefit for the organism itself.

\subsubsection{Biomimetic Synthesis of Dimeric Natural Products}

Nature has the ability of achieving complete regio and/or stereo-selectivity in the dimerisation, either through the use of enzymes or taking advantage of the natural reactivity in non-enzymatic reaction pathways. ${ }^{39}$ From a biomimetic point of view, in the laboratory this task is often more difficult to achieve, thus synthetic chemists often either take advantage of the inherent selectivity of the reaction or use protecting or activating groups, ${ }^{40,41}$ still retaining the overall strategy.

A nice example of biomimetic total synthesis of a dimeric natural product is represented by 2001 Nicolaou's synthesis of hybocarpone, ${ }^{42}$ a compound isolated from the lichen Lecanora hybocarpa. ${ }^{43}$ Nicolaou and co-workers identified its dimeric 
nature and proposed a possible biosynthetic pathway involving a single electron transfer (SET) mediated dimerisation. This hypothesis was plausible as Yamamoto previously reported the isolation of 6-methylcristazarin, ${ }^{44}$ a putative monomeric unit (scheme 1.12). The highly oxygenated structure of 6-methylcristazarin, though, raises questions about the feasibility of achieving a regioselective coupling. To overcome this issue, Nicolaou and co-workers chose naphthazarin $\mathbf{1 . 2 9}$ as the monomeric unit in their biomimetic synthesis. This key intermediate was prepared in 7 steps from known aromatic aldehyde $\mathbf{1 . 3 0}$ and then subjected to ceric ammonium nitrate (CAN), a single electron transfer (SET) oxidant reagent, leading to the formation of hexamethyl hybocarpone. This transformation is believed to proceed through activation of naphthazarin 1.29 via a single electron transfer process to form the very reactive radical-cationic species $\mathbf{1 . 3 1}$, which could undergo dimerisation and loss of two protons to afford bridged dimer 1.32. Selective hydration and protonation of this species were then invoked to furnish hexamethyl hybocarpone, as shown in scheme 1.12. Finally, cleavage of the six methyl-ether protecting groups completed the synthesis.

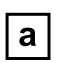

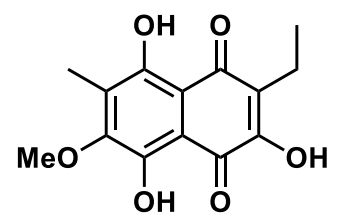

6-methylcristazarin

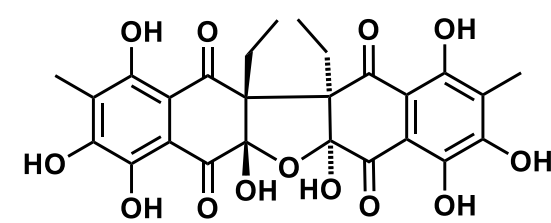

hybocarpone<smiles>C=CCc1c(C)c(OC)c(OC)c(OC)c1C=O</smiles>

1.30<smiles>CCC1=C(O)C(=O)c2c(OC)c(C)c(OC)c(OC)c2C1=O</smiles>

1.29<smiles></smiles><smiles>CCCC=C1C(=O)c2c(OC)c(C)c(OC)c(OC)c2C(=O)C1CC</smiles>

1.31

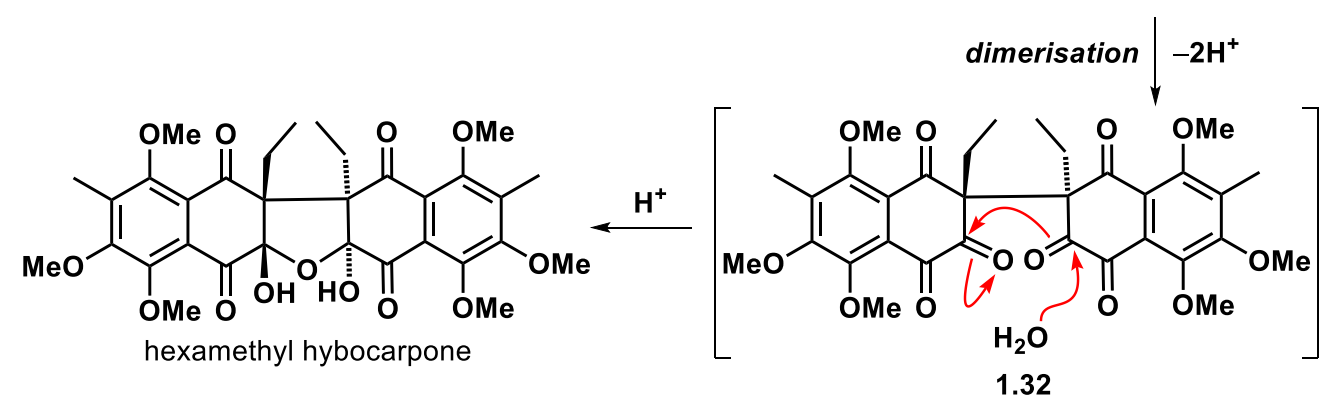

Scheme 1.12: a) Hybocarpone and its plausible monomeric precursor 6-methylcristazarin. b) Nicolaou's total synthesis of hybocarpone. 


\section{$2 \quad$ Total Synthesis of $( \pm)$-Thymarnicol 1.1}

\subsection{Introduction}

\subsubsection{Thymarnicol: a Dimeric Thymol Derivative}

Thymol derivatives are widely distributed within the Asteraceae family of plants and have been widely used for taxonomic treatment on tribal and sub-tribal level for decades. ${ }^{45}$ In 1998 Passreiter and co-workers reported the isolation of a series of simple thymol derivatives (some of which showed interesting bioactivity as contact allergens) from the flower-heads of Arnica sachalinensis, ${ }^{46}$ a plant endemic to north-east Asia (selected examples are shown in figure 2.1) ${ }^{47}$

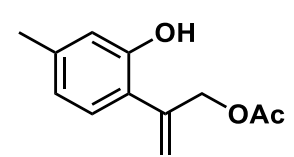

10-acetoxy-8,9dehydrothymol

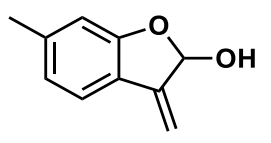

$( \pm)-2.1$

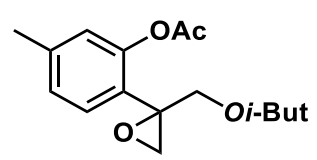

( \pm )-10-acetoxy-8,9epoxy-thymol

Figure 2.1: Selected examples of thymol derivatives.

Further investigation led to the identification of a structurally more complex racemic terpenoid, which showed promising antifeedant activity against the larvae of Spodoptera litura, a major pest for many agricultural crops. ${ }^{48}$ As this metabolite was not given a name by the isolation team, we propose "thymarnicol" a portmanteau of Arnica and thymol as a suitable name. This compound features a new spiro[benzofuran-pyranobenzofuran] ring system bearing four stereocenters and a variety of oxygen functionalities (figure 2.2), which make it a significant synthetic challenge and, therefore, a particularly attractive target for synthetic organic chemists.

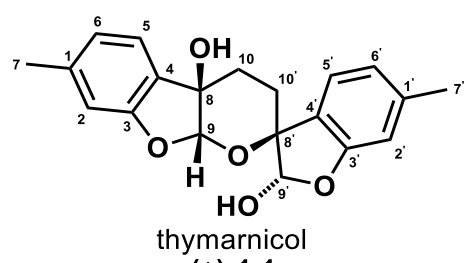

$( \pm)-1.1$

Figure 2.2: ( \pm )-Thymarnicol 1.1 .

During the analysis of the ${ }^{13} \mathrm{C}$ NMR data in the structural determination of $( \pm)$ thymarnicol 1.1, Passreiter and co-workers recognised the presence of ten pairs of 
carbons, which indicated that compound ( \pm )-1.1 could be a pseudo-dimer. Similarities between the fragmentation pattern in its mass spectrum (EI as ionisation mode) with respect to other previously isolated compounds indicated that ( \pm )-thymarnicol ${ }^{46,49} \mathbf{1 . 1}$ is likely to be biosynthetically derived from thymol. At the time of isolation the relative stereochemistry remained unclear, although it was reasonable to assume that a cis configuration at C-9 and C-8 was likely as all previously reported hydrated pyrano[2,3] benzofuran ring systems were found to be $c i s$-annelated. ${ }^{50-52}$ It was only in 2001 that the relative stereochemistry was elucidated via X-ray crystallography by Passreiter and co-workers. ${ }^{53}$

\subsubsection{Passreiter's Biosynthetic Proposal}

Besides recognising ( \pm )-thymarnicol $\mathbf{1 . 1}$ as a dimeric thymol derivative, Passreiter and co-workers also proposed a plausible biosynthetic pathway for its formation. In their hypothesis, thymol, or an acyl-protected variant of it, could undergo a series of oxidations to give alkene 2.2 and aldehyde 2.3, both known structures ${ }^{46}$. Either of these two structures could then be converted into enal 2.4, which could then undergo heteroDiels-Alder cycloaddition to provide dihydropyran $( \pm)-\mathbf{2 . 5}$. This could then, after cleavage of the acyl groups, undergo a double cyclisation to form lactol $( \pm)-\mathbf{2 . 6}$, which features the pentacyclic framework of $( \pm)$-thymarnicol 1.1. Finally, a highly selective enzyme-mediated benzylic oxidation could occur to complete the biosynthesis of $( \pm)$ thymarnicol 1.1 (scheme 2.1).

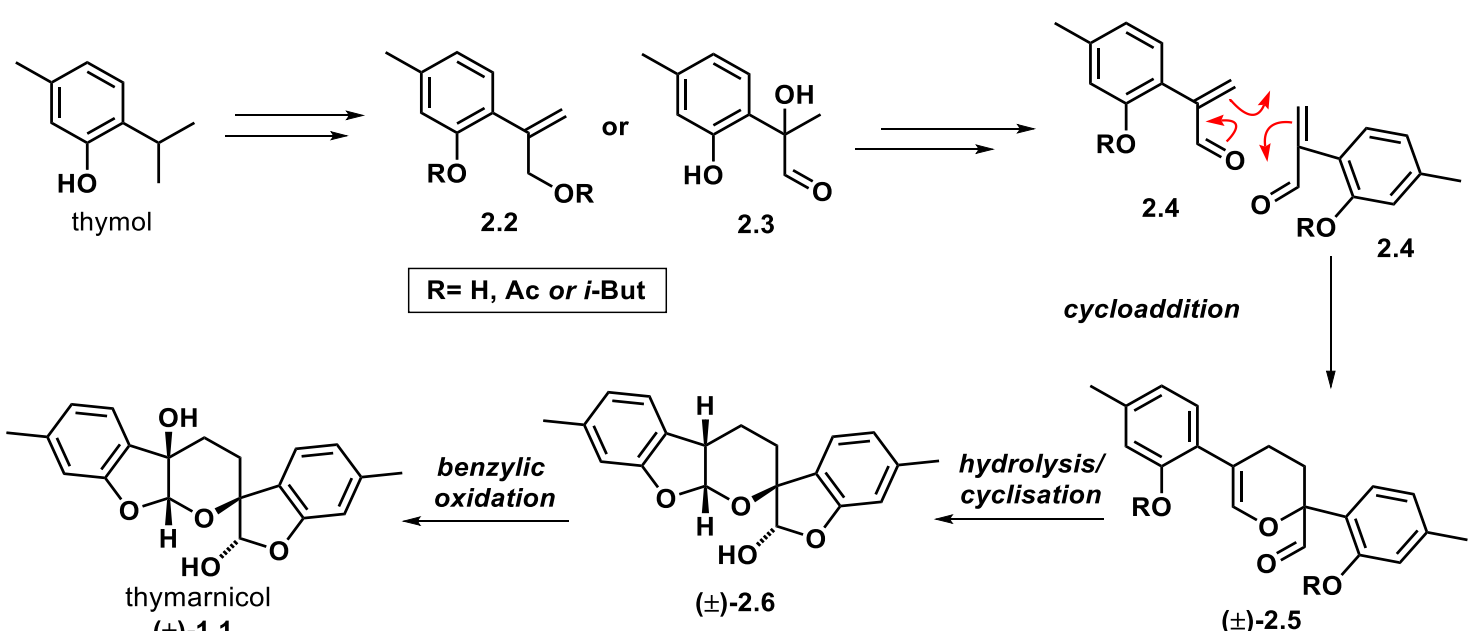

Scheme 2.1: Passreiter's biosynthetic proposal. 


\subsubsection{Alternative Biosynthetic Pathways}

Inspired by this biosynthetic hypothesis the Lawrence group started a biomimetic investigation into the first total synthesis of this dimeric natural product. Our synthetic strategy is based on the hetero-Diels-Alder dimerisation of an enal akin to compound 2.4, but it offers different possibilities in the subsequent steps. Passreiter proposed an early stage double deprotection/cyclisation of dihydropyran dimer $( \pm)-2.5$ and a late stage enzyme-mediated benzylic oxidation to install the tertiary alcohol with the desired stereoselectivity at $\mathrm{C} 8$. We reasoned that an alternative could be represented by the diastereoselective epoxidation of the double bond on dihydropyran $( \pm)-\mathbf{2 . 5}$ to give oxidised dimer $( \pm)-\mathbf{2 . 7}$, followed by the double deprotection/cyclisation sequence (scheme 2.2). A diastereoselective dihydroxylation instead of an epoxidation would give the same final result.

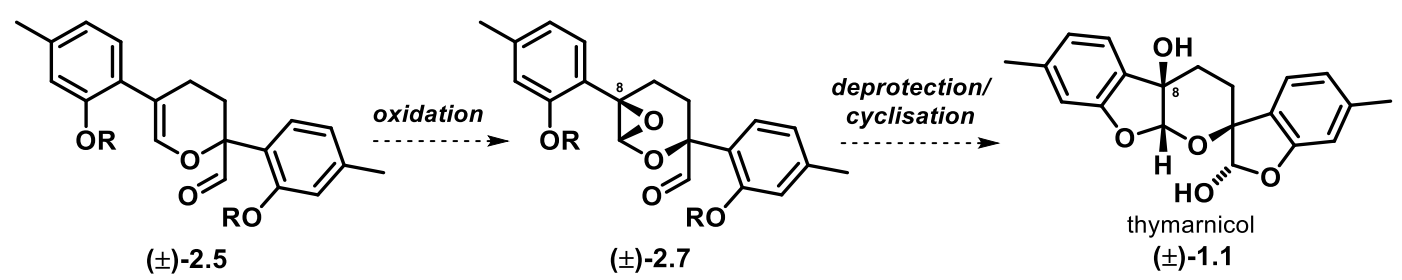

Scheme 2.2: Proposed alternative pathway for ( \pm )-thymarnicol 1.1.

The origin of the diastereoselctivity could be rationalised based on the relative stability of the two conformers of $( \pm)-\mathbf{2 . 5}$, with the conformer where the aromatic substituent is in a pseudo-equatorial position likely to be more stable (A values for $\mathrm{Ph}=3.0 \mathrm{kcal} / \mathrm{mol}$, formyl $=0.8 \mathrm{kcal} / \mathrm{mol}$ ). Approach of the oxidant to the less hindered face of this lower energy conformer would yield the configuration observed in the natural product (scheme 2.3).
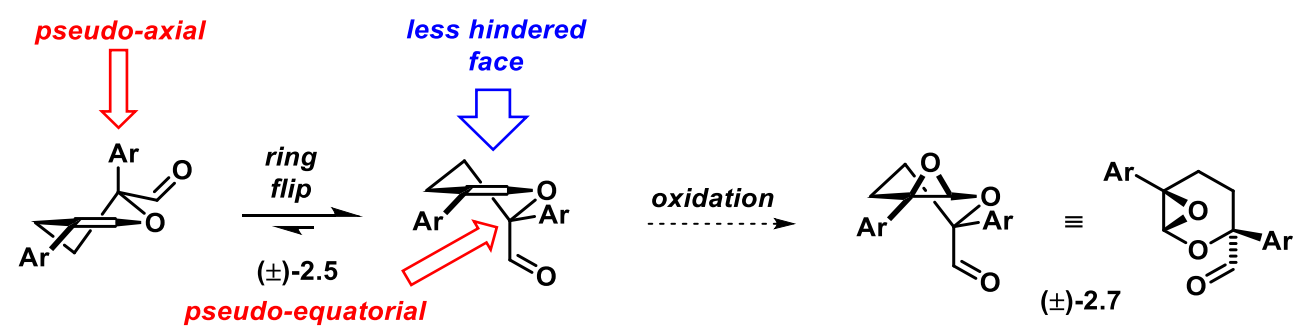

Scheme 2.3: Proposed rationalisation for the diastereoselective oxidation. 
A closer look to the literature revealed another intriguing possibility. In the discussion about the biosynthesis of $( \pm)$-thymarnicol $\mathbf{1 . 1}$ in the isolation paper, Passreiter and coworkers mentioned lactol ( \pm )-2.1 (figure 2.1 on page 34 ) to support the existence of their proposed acrolein monomer $\mathbf{2 . 4}$ (see scheme 2.1 on page 35). Lactol ( \pm )-2.1 was originally isolated in 1969 by Bohlman and co-workers from the roots of plants from the Helenium species ${ }^{47}$ and subsequently from various Asteraceae plants, the family which Arnica sachalinensis belongs to. ${ }^{54}$ This compound has been reported in the literature to be "not stable, and co-exist in equilibrium with a small amount (around $20 \%$ ) of its hemiacetal-opened aldehyde form $\mathbf{2 . 8}$, based on its ${ }^{1} \mathrm{H}$ NMR spectrum". 55 The opened aldehyde form of lactol $( \pm)-\mathbf{2 . 1}$ is the unprotected version of monomer 2.4 proposed by Passreiter, so we wondered if the instability of this compound could be toward dimerisation. If this was the case, the hetero-Diels-Alder cycloaddition would provide lactol $( \pm)$-2.9, which could then follow either of the two routes having epoxide $( \pm)-\mathbf{2 . 1 0}$ or pentacyclic compound $( \pm)-\mathbf{2 . 6}$ as intermediates to give $( \pm)$-thymarnicol 1.1. A picture of the various possibilities is shown in scheme 2.4 .

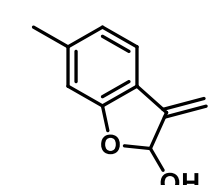

(土)-2.1

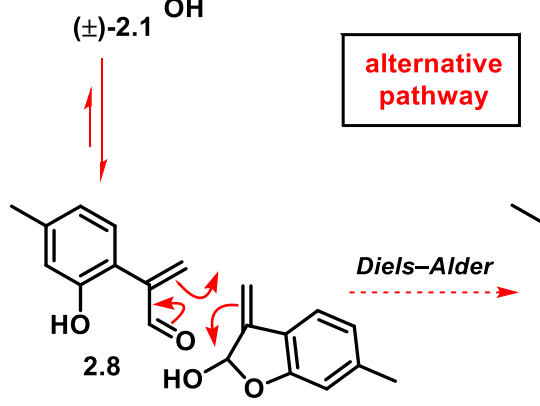

$( \pm)-2.1$

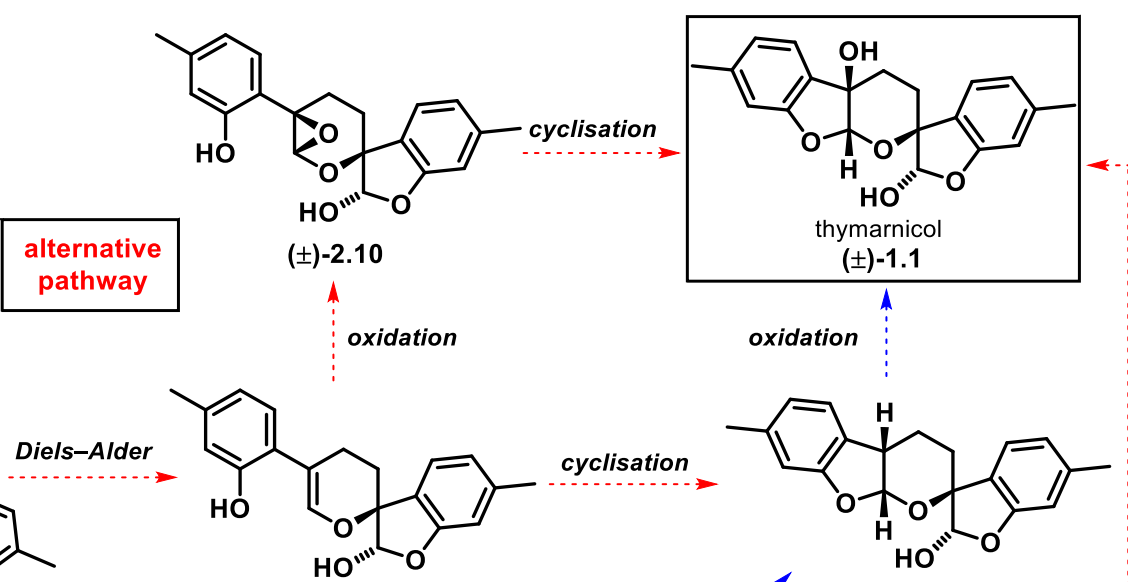

$( \pm)-2.9$

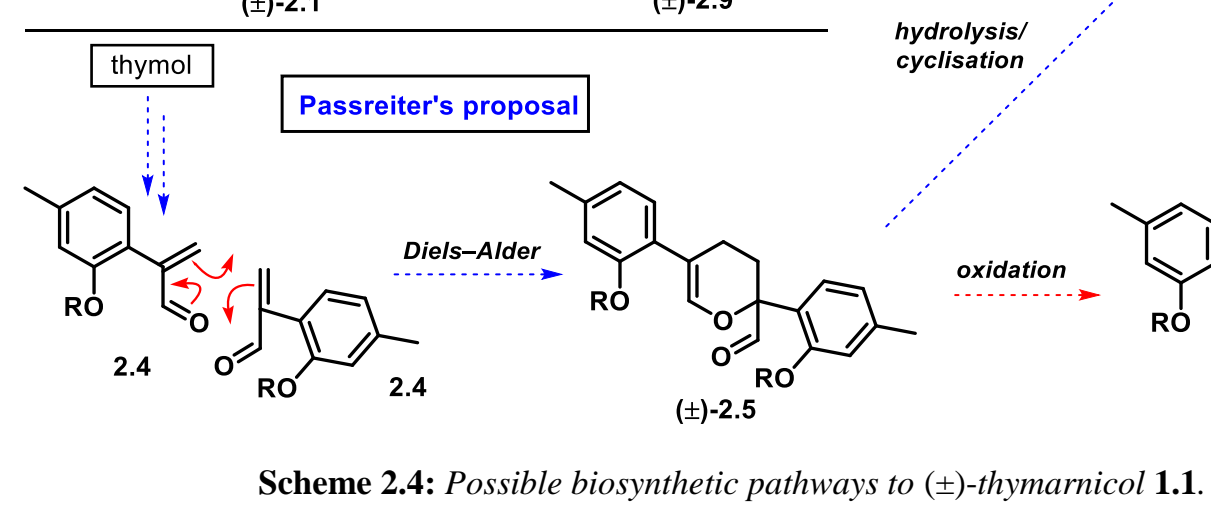

$( \pm)-2.6$

hydrolysis/ cyclisation

alternative pathway

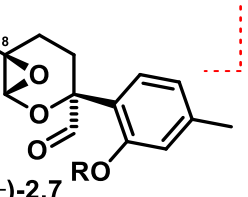

$\pm \mathbf{C O}^{\mathrm{RO}}$ 
Intrigued by this possibility, we designed a strategy which would allow us to investigate all these possible pathways. To do that, we needed to access both $( \pm)$-lactol $\mathbf{2 . 1}$ and a suitably protected enal $\mathbf{2 . 4}$, the former being in principle accessible by deprotecting the latter. Enal 2.4, in turn, could be prepared via a sequence of oxidation of primary alcohol $\mathbf{2 . 1 1}$ to the aldehyde and methylenation through a well-established procedure. ${ }^{56}$ We envisaged that primary alcohol $\mathbf{2 . 1 1}$ could be synthesised through selective $o$-metalation and ring opening of ethylene oxide from protected phenol 2.12. The choice of the phenolic protecting group in compound 2.12 would enable the required regioselectivity in this key carbon-carbon bond forming step. Protected phenol 2.12, in turn, could be accessed via protection of the commercially available and very cheap $m$-cresol (scheme 2.5).

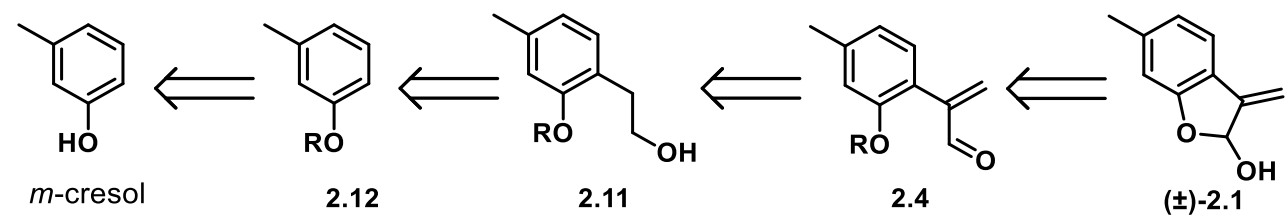

Scheme 2.5: Proposed retrosynthetic analysis to access lactol ( \pm )-2.1 and enal 2.4.

\subsection{Literature Precedent}

We envisaged that the two main challenges in our planned synthesis were the heteroDiels-Alder cycloaddition and the oxidation/cyclisation sequence. Herein we present an overview of relevant literature precedent.

\subsubsection{Dimerisation Literature Precedent}

With respect to the hetero-Diels-Alder dimerisation, there are some interesting examples in the literature with $\alpha$-aryl-enones. ${ }^{57-59}$ For example, in 1981 Overman and co-workers made an interesting observation while investigating the Diels-Alder cycloaddition of acylamino-1,3-dienes with a series of different substituted dienophiles. ${ }^{57}$ When diene carbamate $\mathbf{2 . 1 3}$ and an excess of acrylate ether $\mathbf{2 . 1 4}$ were heated under reflux in toluene, the expected cyclohexene $( \pm)-\mathbf{2 . 1 5}$ was isolated in 69\% yield. Unexpectedly, however, dihydropyran $( \pm)-\mathbf{2 . 1 6}$, which is the product of the hetero-Diels-Alder dimerisation of enal 2.14, was isolated in $43 \%$ yield (scheme 2.6). 

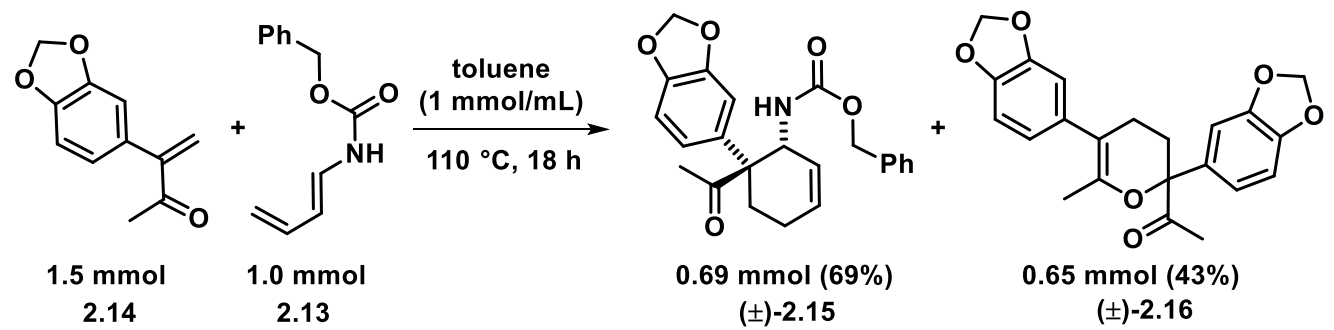

Scheme 2.6: Overman's unexpected observation.

To the best of our knowledge, the hetero-Diels-Alder dimerisation of $\alpha$-aryl-enals has been reported just twice. The first example was reported in 1993 by Laitalainen and co-workers, ${ }^{60}$ wherein atropaldehyde $\mathbf{2 . 1 7}$ (i.e., 2-phenylpropenal) was prepared via selenium dioxide allylic oxidation of styrene 2.18. They observed that the purified, neat, atropaldehyde $\mathbf{2 . 1 7}$ dimerised quantitatively within two days at room temperature to give ( \pm )-dihydropyran 2.19 (scheme 2.7 ).

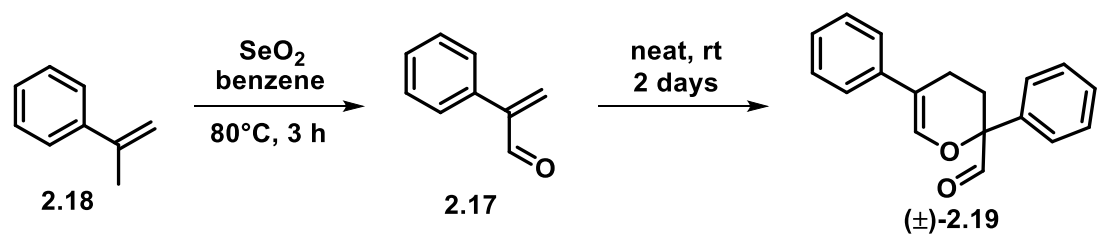

Scheme 2.7: Preparation of ( \pm -dihydropyran 2.19 .

The most promising precedent is perhaps the discovery made by Davies and coworkers. In 2005 they described the Lewis acid catalysed formal inverse electron demand hetero-Diels-Alder reaction between $\alpha$-aryl- $\alpha, \beta$-unsaturated aldehydes with cyclopentadiene. ${ }^{61}$ During their investigations they treated a series of differently substituted enals and five equivalents of cyclopentadiene with dimethylaluminium chloride as a Lewis acid catalyst (one example is shown in scheme 2.8).

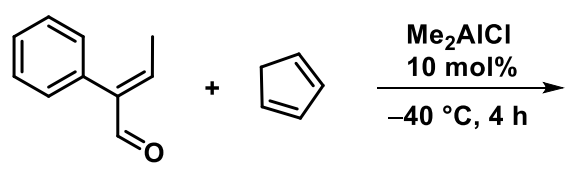

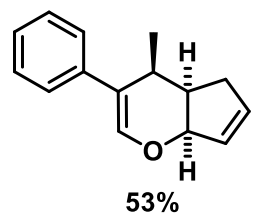

Scheme 2.8: Example of Davies' Lewis acid catalysed Diels-Alder.

A series of enals was employed to explore the substrate scope of this reaction, some of which needed to be prepared. Davies reported that enal 2.20, one of their substrates, "must be used immediately, because it is very prone to dimerisation" (scheme 2.9). 


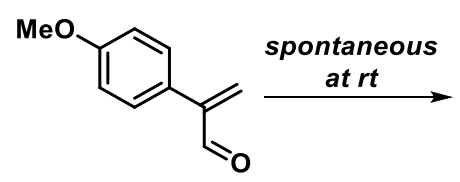

2.20<smiles>COc1ccc(C2=COCC(CO)(c3ccc(OC)cc3)CC2)cc1</smiles>

MeO<smiles>COc1ccc(C2=COC3C=CCC3C2)cc1</smiles>

Scheme 2.9: Davies'observation.

This result, along with Laitalainen's observation, indicated a natural tendency of $\alpha$ aryl-substituted enals to undergo hetero-Diels-Alder dimerisation. However, given the limited number of substrate examples, no assumption could be made with regard to the effect of different substituents on the aromatic ring.

\subsubsection{Oxidation/Cyclisation Literature Precedent}

With respect to the oxidation/cyclisation sequence there are some interesting precedents on the use of DMDO (dimethyldioxirane) in the diastereoselective epoxidation of dihydropyrans, followed by either inter or intra-molecular ring opening with oxygen-based nucleophiles. A very interesting example is represented by the work of Tan and co-workers on the stereoselective synthesis of benzannulated spiroketals. ${ }^{62}$ Spiroketal 2.21 was prepared in a one-pot procedure from glucal $\mathbf{2 . 2 2}$ through diastereoselective epoxidation with DMDO at $-78{ }^{\circ} \mathrm{C}$ to give epoxide intermediate 2.23, which readily underwent intramolecular ring opening with retention of stereochemistry at $\mathrm{C} 1$ upon warming of the reaction mixture to room temperature (scheme 2.10). This example fits very well with our planned synthesis as it encloses the oxidation and ring opening in a single step.

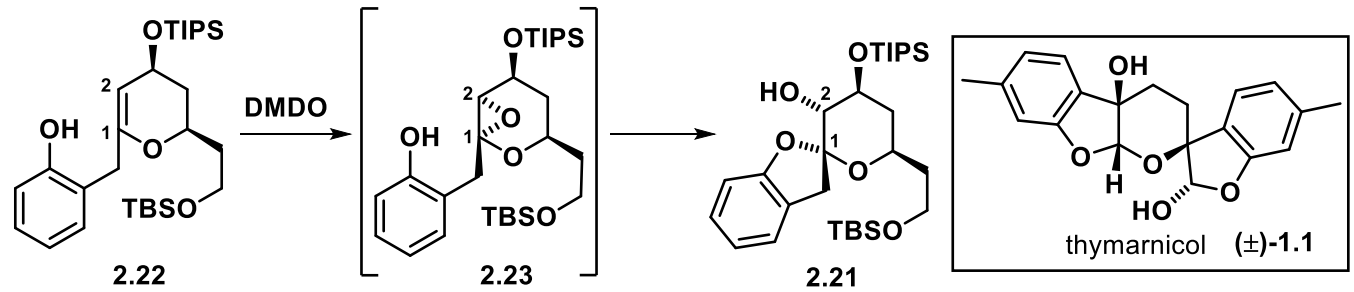

Scheme 2.10: Tan's one pot oxidation/cyclisation and comparison with ( \pm )-thymarnicol 1.1. 


\subsection{Previous Work}

In 2013, Dr Sam Drew (Australian National University) started this project by following the previously discussed proposed biomimetic strategy. The first step of the synthesis involved protecting the very cheap $(£ 0.36 / \mathrm{g}) \mathrm{m}$-cresol with a suitable orthodirecting group, as required for the next step. The MOM (methoxymethyl) group was chosen, as it has been reported to give excellent ortho regioselectivity for the alkylation upon lithiation using $n$-butyllithium and, in the specific case of $m$-cresol, tertbutyllithium. ${ }^{63,64} \mathrm{~m}$-Cresol was thus converted into its MOM-ether using a literature procedure. ${ }^{64}$ Treatment with MOM-chloride and three equivalents of Hünig's base in dichloromethane afforded MOM-ether $\mathbf{2 . 2 4}$ in $78 \%$ yield on an eleven-gram scale (scheme 2.11).

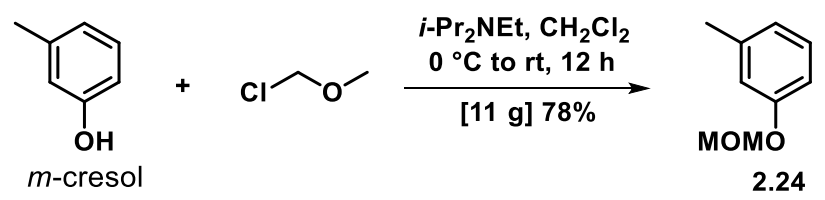

Scheme 2.11: Preparation of MOM-cresol 2.24 by Sam Drew.

MOM-ether 2.24 was then converted into primary alcohol 2.25 through directed orthometalation using a small excess of tert-butyllithium and in-situ quenching of the aryllithium with two equivalents of ethylene oxide in the presence of lithium bromide (2.12).

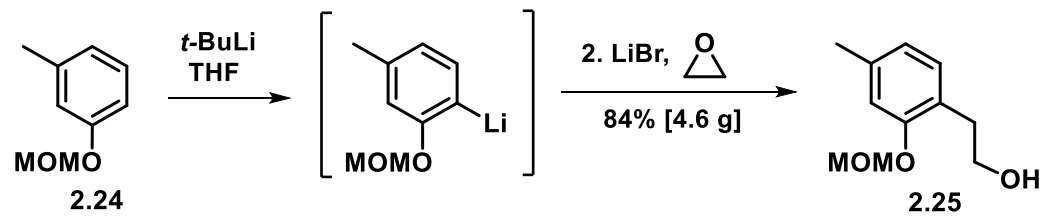

Scheme 2.12: Preparation of primary alcohol 2.25 by Sam Drew.

This reaction was performed on different scales, with the yield decreasing slightly from $90 \%$ of isolated product to $81 \%$ internal standard ${ }^{1} \mathrm{H}$ NMR yield, upon scalingup the reaction from 1 to 4.6 grams. Alcohol 2.25 was always the only isolated product, with no formation of other regioisomers observed. With this in hand, attention was then turned to the oxidation/methylenation sequence. This was a well-established onepot procedure ${ }^{56}$ involving Parikh-Doering oxidation of a primary alcohol to the 
aldehyde followed by methylenation at the $\alpha$-position using the Eschenmoser salt. Treatment of alcohol $\mathbf{2 . 2 5}$ with sulfur trioxide/pyridine complex produced aldehyde 2.26, the formation of which was detected by TLC analysis. After allowing the starting material to be fully consumed, the Eschenmoser salt was added as a solid and the reaction was left to proceed to completion (scheme 2.13).
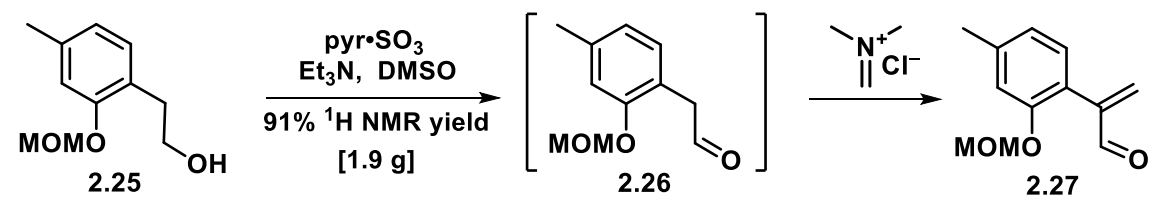

Scheme 2.13: One pot preparation of enal monomer 2.27 by Sam Drew.

This afforded 1.9 grams (75\% yield) of clean enal monomer $\mathbf{2 . 2 7}$, which was immediately used to investigate several dimerisation conditions. After extensive screening of conditions, it was found that the best conditions for the hetero-DielsAlder dimerisation are heating enal monomer 2.27 at $80{ }^{\circ} \mathrm{C}$ in the presence of $\sim 0.5$ equivalents of potassium carbonate (scheme 2.14). Reactions conducted without the base resulted in the formation of complex mixtures of compounds, presumably due to the loss of one of both the MOM-groups on the monomer and/or dimer.

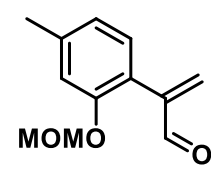

2.27

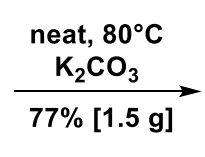

7 $1.5 \mathrm{~g}]$

Scheme 2.14: Preparation of dihydropyran ( \pm )-2.28 by Sam Drew.

This key biomimetic dimerisation afforded dihydropyran $( \pm)-\mathbf{2 . 2 8}$ in $77 \%$ yield on a 1.5-gram scale, providing material with which to investigate the two possibilities of deprotection/oxidation and oxidation/deprotection previously discussed (see section 2.1.3 on pages 36-37) (scheme 2.15). 


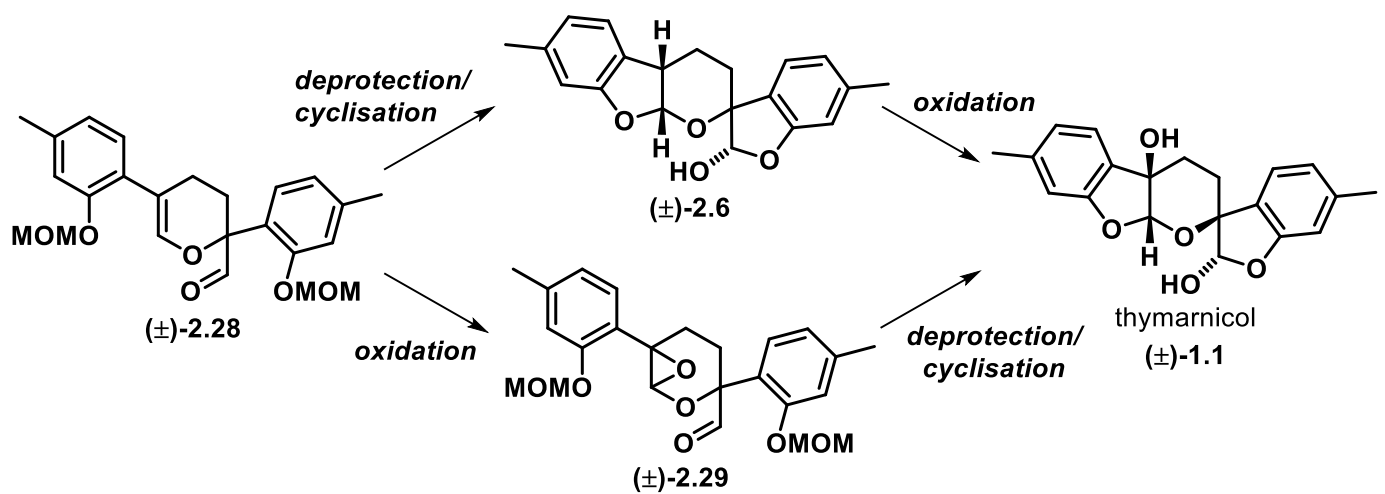

Scheme 2.15: The two alternative pathways to ( \pm )-thymarnicol $\mathbf{1 . 1}$.

The cleavage of the MOM protecting groups was first attempted with boron trifluoride diethyl etherate but this led to decomposition. ${ }^{65}$ A similar result was obtained upon treatment with trimethylsilyl (TMS)-chloride/sodium iodide ${ }^{66}$ as a way to generate TMS-iodide in situ. In this case ${ }^{1} \mathrm{H}$ NMR analysis of the crude reaction product clearly showed removal of the MOM groups; however, it was not possible to isolate any of the multiple products.

The early oxidation approach was then undertaken. Attempts to oxidise dihydropyran $( \pm)$-2.28 with $m$-CPBA met with failure, resulting in the formation of a complex mixture of unidentified products. As previously discussed (see section 2.2.2 on page 40 ), there was promising literature precedent for the DMDO epoxidation of dihydropyrans structurally related to our substrate. ${ }^{62,67}$ Thus, treating $50 \mathrm{mg}$ of dihydropyran $( \pm)-2.28$ with a freshly prepared DMDO solution afforded epoxide $( \pm)-2.29$ as a 1:1 diastereomeric mixture (scheme 2.16). The complete lack of diastereoselectivity was disappointing and therefore purification of this epoxide was not carried out.

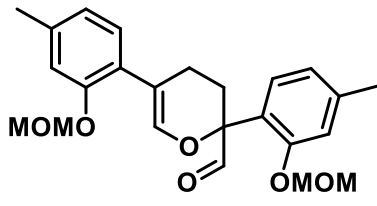

$( \pm)-2.28$

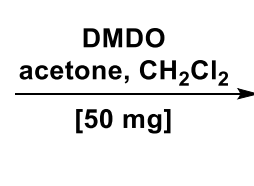

[50 mg]

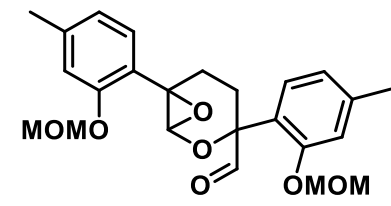

$( \pm)-2.29$

Scheme 2.16: Preparation of epoxide ( \pm )-2.29 by Sam Drew. 


\subsection{Results and Discussion}

As a result of these previous investigations within the group, when undertaking this project attention was devoted to two main areas: the oxidation of dihydropyran (士)-2.28 and the removal of the MOM-protecting groups. With respect to the oxidation, bulkier epoxidising and dihydroxylating agents needed to be tested on dihydropyran $( \pm)$-2.28 to install the desired stereochemistry required for the molecular target. Concerning the removal of the MOM groups, there was the need to test more conditions on both the unoxidised and oxidised dimers ( \pm -2.28 and ( \pm -2.29. Therefore, there was the requirement for a reasonable amount of material to be tested, thus a repetition of the entire synthesis was necessary.

\subsubsection{Repetition and Optimisation of the Previous Synthesis}

The first step of the synthesis, involving MOM-protection of $m$-cresol, was performed under the same conditions and on the same scale as previously used, with identical result (see scheme $\mathbf{2 . 1 1}$ on page 41). An improvement was achieved for the $o$-lithiation/ring-opening of ethylene oxide. This reaction was scaled up from 4.6 to 6 grams, and the yield increased from an internal standard ${ }^{1} \mathrm{H}$ NMR yield of $81 \%$ to $84 \%$ of purified product (see scheme $\mathbf{2 . 1 2}$ on page 41). The one-pot oxidation/ methylenation sequence (see scheme $\mathbf{2 . 1 3}$ on page 42) was also repeated on different scales, giving variable results, with the yield typically decreasing upon scaling up (900 mg, 90\% : $1.9 \mathrm{~g}, 75 \%$ ). Finally, the dimerisation was replicated in the optimised conditions (see scheme $\mathbf{2 . 1 4}$ on page 42 ) giving as the best result a $77 \%$ isolated yield on a 1.5-gram scale (scheme 2.17). 


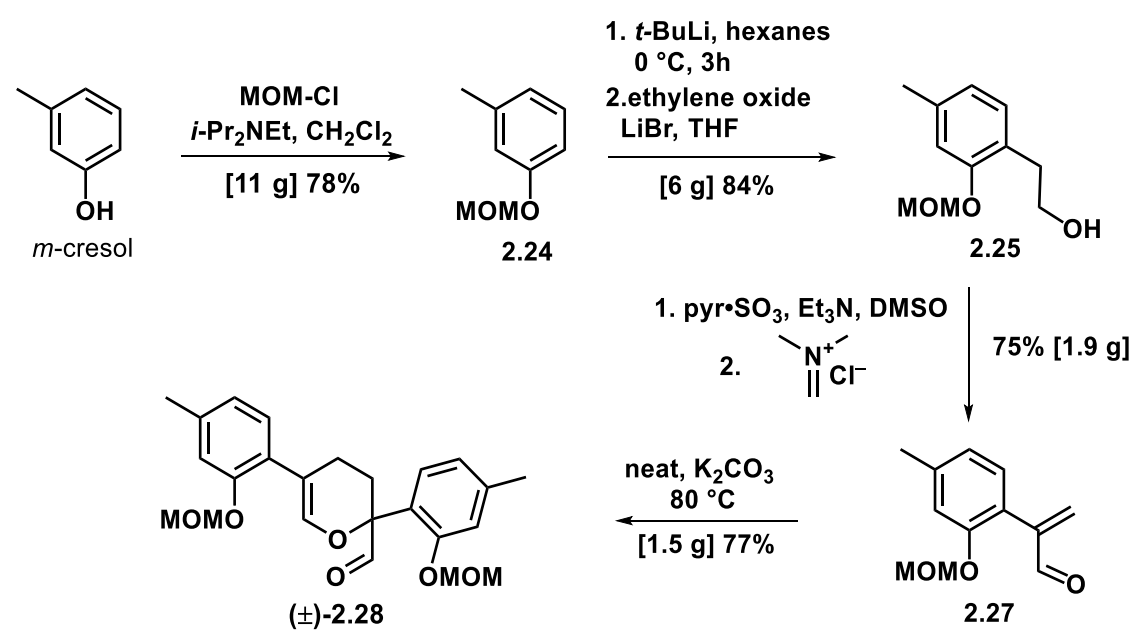

Scheme 2.17: Optimisation of the synthesis of dihydropyran ( \pm -2.28.

\subsubsection{Stereoselective Oxidations}

In the previous work the epoxidation of dihydropyran ( \pm -)-2.28 had been successfully accomplished on a small scale using freshly prepared DMDO, although with no diastereoselectivity. The preparation of DMDO, though, is typically rather inefficient, yielding dilute acetone solutions $(\sim 0.1 \mathrm{M})$ in less than $3 \%$ yield. ${ }^{68}$ Furthermore, the prepared solution needs to be stored at low temperature $\left(-10\right.$ to $\left.-20^{\circ} \mathrm{C}\right)$ for no longer than a week, and its concentration needs to be assayed immediately prior to use. ${ }^{68} \mathrm{An}$ alternative is to generate the active DMDO species in situ using acetone and Oxone (KHSO 5 ) under biphasic conditions. ${ }^{69}$ This reaction proceeds by nucleophilic addition of Oxone to the carbonyl group of acetone with subsequent loss of hydrogen sulfate. The transfer of oxygen to the substrate then forms the oxidised product and regenerates acetone (scheme 2.18), therefore the epoxidation can, in principle, be catalytic in ketone. Although this does not constitute an advantage in the case of acetone, it represents a potential for catalytic, asymmetric epoxidation using chiral (and more expensive) ketones.

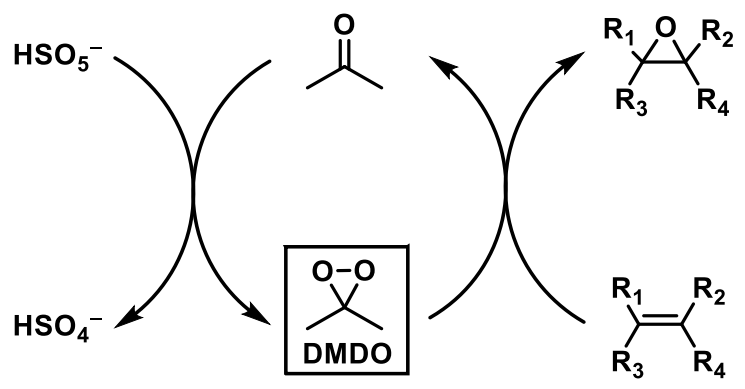

Scheme 2.18: Cycle for the epoxidation with in situ generated DMDO. 
This approach does, however, suffer from some limitations; to ensure high yields, a large excess of acetone is usually required and a slow addition of Oxone is essential to avoid its reaction with DMDO itself (scheme 2.19).

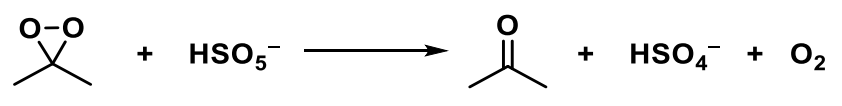

Scheme 2.19: Undesired reaction of Oxone with DMDO.

Furthermore, a careful control of the $\mathrm{pH}$ with a buffer is necessary to suppress the autodecomposition of Oxone at high $\mathrm{pH}$ and to compensate for the formation of acid during the production of DMDO. ${ }^{69}$ With this in mind, the epoxidation of dihydropyran ( \pm -2.28 was attempted with in situ generated DMDO. Using a modified literature procedure ${ }^{70}$ reaction of dihydropyran $( \pm)-\mathbf{2 . 2 8}$ with a large excess of acetone in a biphasic solvent system $\left(\mathrm{CH}_{2} \mathrm{Cl}_{2}\right.$ /aqueous $\left.\mathrm{NaHCO}_{3}\right)$ and three equivalents of slowly added Oxone successfully afforded epoxide ( \pm )-2.29. Unsurprisingly, this reaction proceeded with no diastereocontrol to afford a 1:1 mixture of diastereoisomers in $76 \%$ yield on a 45-mg scale (scheme 2.20).

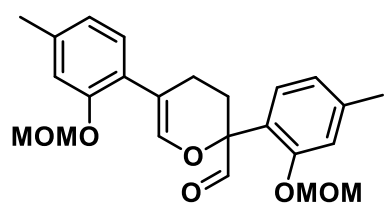

$( \pm)-2.28$

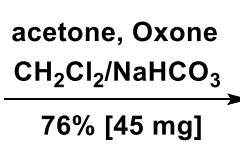

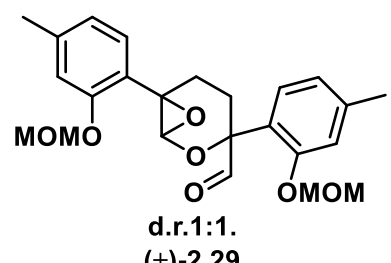

$( \pm)-2.29$

Scheme 2.20: Formation of epoxide ( \pm -2.29 with in situ generated DMDO.

Given this result, we decided to investigate the use of bulkier oxidants to achieve the desired diastereoselectivity. As discussed earlier, the in-situ generation of dioxiranes can be applied to catalytic enantioselective epoxidations. The Shi ketone, ${ }^{71}$ a fructosederived commercially available ketone, has been widely used in the epoxidation of substituted alkenes to give moderate to excellent enantioselectivity. Therefore the oxidation of dihydropyran ( \pm -2.28 using a modified literature procedure was performed. ${ }^{72}$ The outcome of this reaction proved to be highly dependent on the $\mathrm{pH}$ (controlled using a buffer), the rate of addition of Oxone, stoichiometry and concentration. With the optimised conditions in hand, the reaction afforded epoxide ( \pm -2.29 in $82 \%$ crude yield in a 5:4 diastereomeric ratio (scheme 2.21). This small 
degree of diastereoselectivity was judged to not be promising enough to pursue this route.

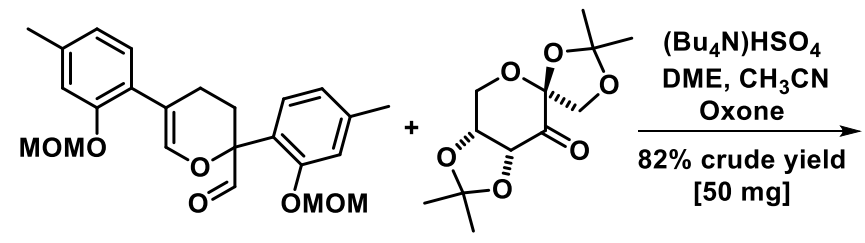

$( \pm)-2.28$

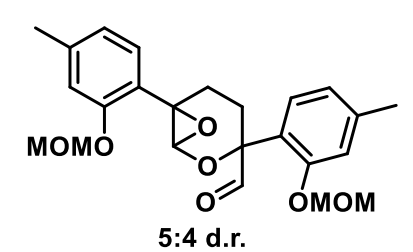

$( \pm)-2.29$

Scheme 2.21: Formation of epoxide $( \pm)-2.29$ with the in situ generated dioxirane of the Shi ketone.

We then turned our attention to the dihydroxylation of dihydropyran $( \pm)-\mathbf{2 . 2 8}$. A first trial with a small scale Sharpless asymmetric dihydroxylation using AD-mix- $\beta$ gave a mere $10 \%$ consumption of the alkene starting material after $24 \mathrm{~h}$. Thus the catalytic Upjohn oxidation was then attempted on a small scale, following a modified literature procedure. ${ }^{73}$ Treatment of dihydropyran $( \pm)$-2.28 with a substoichiometric amount of osmium tetroxide and stoichiometric quantities of $N$-methylmorpholine- $N$-oxide (NMO) as the terminal oxidant afforded lactol $( \pm)-\mathbf{2 . 3 0}$ a $2: 1$ mixture of two compounds (scheme 2.22). Based on analysis of the crude ${ }^{1} \mathrm{H}$ NMR spectrum, it is believed that these were presumably epimers at the lactol position.

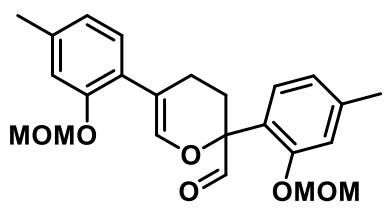

$( \pm)-2.28$

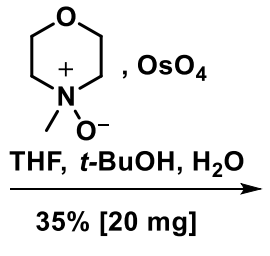

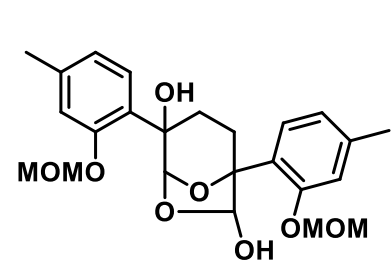

$( \pm)-2.30$

Scheme 2.22: Preparation of lactol $( \pm)-\mathbf{2 . 3 0}$.

Column chromatography afforded diastereomerically pure lactol ( \pm )-2.30 as the sole isolated product. Unfortunately, analysis of 2D NMR spectra, including NOESY experiments, did not give conclusive results on determining the relative stereochemistry. Repetitive attempts on recrystallising lactol ( \pm -2.30 using a variety of solvent systems and derivatising the two hydroxyl groups on ( \pm )-2.30 with phenyl isocyanate met with failure. Interestingly, it was noticed that simply storing a sample of lactol ( \pm )-2.30 in deuterated chloroform for five days resulted in complete loss of one of the two MOM protecting groups of lactol $( \pm)-2.30$. The same result could be 
obtained by adding a small amount of aqueous $\mathrm{HCl}$ ( 2 equivalents) to the $\mathrm{NMR}$ sample. Unfortunately, it was not possible to determine which of the two MOM-groups was removed, and increasing the temperature to $40{ }^{\circ} \mathrm{C}$ failed in removing the residual protecting group. Attempts on forcing the reaction by adding more acid resulted in decomposition.

With limited success in achieving diastereoselectivity in the oxidation of dihydropyran $( \pm)-2.28$, our attention was turned to removal of the MOM-ethers on both dihydropyran $( \pm)$-2.28 and epoxide $( \pm)-2.29$. It was envisaged that the double deprotection of the latter could potentially afford thymarnicol $( \pm)-\mathbf{1 . 1}$ through a deprotection/cyclisation sequence based on promising literature precedent, which was discussed in section

\subsection{2 on page 40 .}

\subsubsection{Deprotection Attempts}

The deprotection was initially attempted on epoxide ( \pm )-2.29. Silica-supported sodium hydrogen sulfate $\left(\mathrm{NaHSO}_{4} \cdot \mathrm{SiO}_{2}\right)$ has been reported to be a heterogeneous catalyst for "simple, efficient and selective deprotection of phenolic methoxymethyl ethers", 74 including aldehyde-containing substrates (some examples are depicted in figure 2.3).<smiles>O=Cc1ccc(O)cc1</smiles><smiles>O=Cc1ccc(O)cc1</smiles><smiles>COc1cc(C=O)ccc1O</smiles>

Figure 2.3: Examples of $\mathrm{NaHSO}_{4} \cdot \mathrm{SiO}_{2}$-mediated MOM-deprotection on aldehyde substrates. ${ }^{74}$

This appeared to be promising, as in the previous deprotection tests the immediate disappearing of the aldehyde peak in the ${ }^{1} \mathrm{H}$ NMR spectra seemed to suggest that this functional group played a role on the instability of the substrates or products of the reactions. Therefore, epoxide ( \pm )-2.29 was dissolved in anhydrous $\mathrm{CH}_{2} \mathrm{Cl}_{2}$ and treated with $\mathrm{NaHSO}_{4} \cdot \mathrm{SiO}_{2}$ freshly prepared using a literature procedure ${ }^{75}$ unfortunately, this resulted in the formation of a complex mixture of unidentified products (scheme 2.23). 


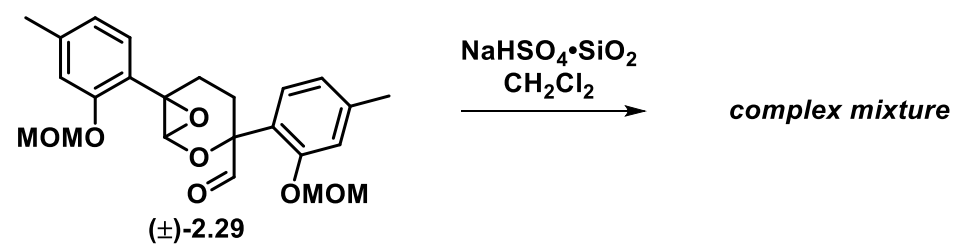

Scheme 2.23: Attempted deprotection of epoxide ( \pm )-2.29 with $\mathrm{NaHSO}_{4} \cdot \mathrm{SiO}_{2}$.

A more interesting result was obtained with the in-situ generation of TMSI. Using a modified literature procedure, ${ }^{66}$ epoxide $( \pm)$-2.29 was dissolved in a 1:1 mixture of dichloromethane and acetonitrile and treated with $\mathrm{TMSCl}$ and $\mathrm{NaI}$ (10 equivalents each) for 20 minutes at $0{ }^{\circ} \mathrm{C}$. Column chromatography afforded a colourless oil in $86 \%$ yield, which contained bis-lactone $\mathbf{2 . 3 1}$ as a 1:1 mixture of diastereoisomers based on analysis of the ${ }^{1} \mathrm{H}$ and ${ }^{13} \mathrm{C}$ NMR spectra. Bis-lactone meso-2.31 could be purified via recrystallisation to afford a colourless solid in 36\% yield (scheme 2.24), the structure of which was confirmed by X-ray crystallography. It was not possible, however, to obtain an analytically pure sample of the other diastereoisomer.

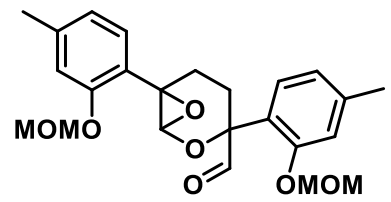

$( \pm)-2.29$

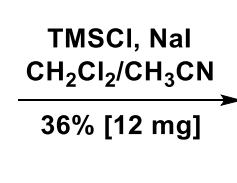

Scheme 2.24: TMSCl/NaI deprotection of epoxide ( \pm )-2.29.

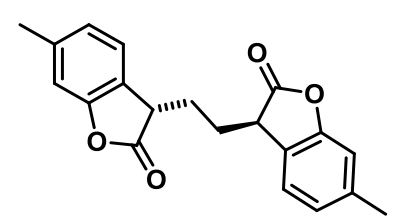

meso-2.31

The mechanism of the reaction leading to the two diastereomeric bis-lactones $\mathbf{2 . 3 1}$ is speculative, but it is proposed to initially involve cleavage of the MOM protecting groups, followed by double cyclisation to afford thymarnicol-type structure $( \pm)-\mathbf{2 . 3 2}$. Under the Lewis acidic reaction conditions cation ( \pm )-2.33 could form and then undergo a first [1,2]-hydride shift to afford oxonium ion ( \pm )-2.34, which could then, after a second [1,2]-hydride shift, produce the observed lactones $\mathbf{2 . 3 1}$ (scheme 2.25). 


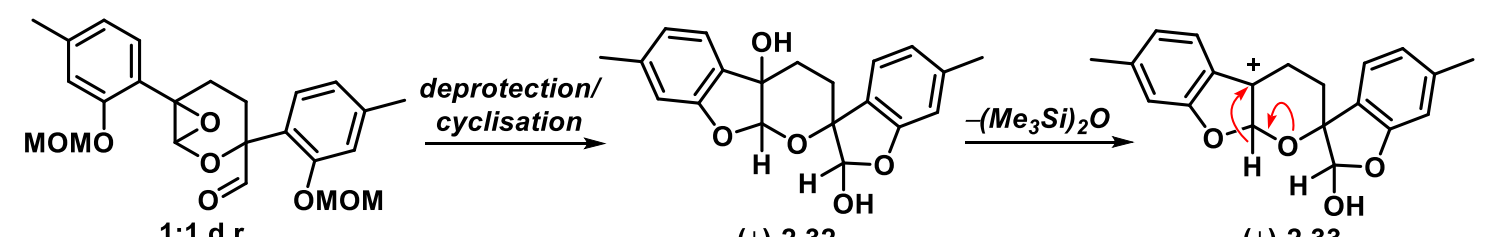

$( \pm)-2.29$

$( \pm)-2.32$

$( \pm)-2.33$

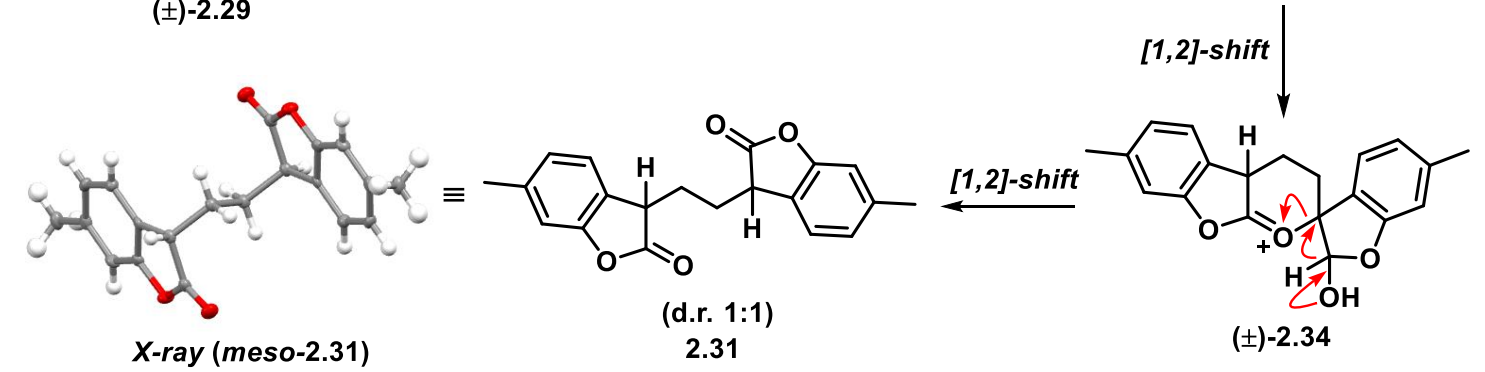

Scheme 2.25: Proposed mechanism for the formation of bis-lactones 2.31 .

The deprotection was then attempted on the unoxidised dihydropyran dimer $( \pm)-\mathbf{2 . 2 8}$ under the same reaction conditions (TMSCl/NaI) used for epoxide $( \pm)-\mathbf{2 . 2 9}$, but in this case it gave a complex mixture of compounds, with no product isolated. Another attempt was made with the milder Lewis acid TMSBr at $-78{ }^{\circ} \mathrm{C}$ in $\mathrm{CH}_{2} \mathrm{Cl}_{2}$, with the same disappointing result. ${ }^{76}$

In 1986 Hiyama and co-workers reported a mild procedure for the deprotection of alkoxymethyl aryl ethers using $\mathrm{P}_{2} \mathrm{I}_{4}{ }^{77}$ The procedure was applicable to a wide range of substrates bearing different functional groups, including esters, ethers, ketones and aldehydes. In this case, subjecting our substrate $( \pm)-\mathbf{2 . 2 8}$ to the literature reaction conditions ${ }^{77}$ resulted in decomposition. We then turned our attention to deprotection conditions with the use of Brønsted acids. Treating dihydropyran ( \pm )-2.28 with glacial acetic acid in toluene at $90{ }^{\circ} \mathrm{C},{ }^{78}$ or trifluoroacetic acid $^{79}$ led to the formation of several unidentified products in which the MOM groups appeared to stay intact and immediate loss of the aldehyde peak was observed on the crude ${ }^{1} \mathrm{H}$ NMR spectra. Subjecting dihydropyran ( \pm -2.28 to hydrolysis using both catalytic and stoichiometric $\mathrm{HCl}$ in acetone, isopropanol or tetrahydrofuran had the same result. Finally, an interesting result was obtained using methanol as the solvent: reacting dihydropyran ( \pm )-2.28 with two equivalents of $\mathrm{HCl}$ at room temperature resulted in the formation of acetal ( \pm )-2.35 as a 1:1 mixture of diatereoisomers in 92\% isolated yield (scheme 2.26). 
<smiles>COc1cc(C)ccc1C1=COC(C=O)(c2ccc(C)cc2OC)CC1</smiles>

$( \pm)-2.28$
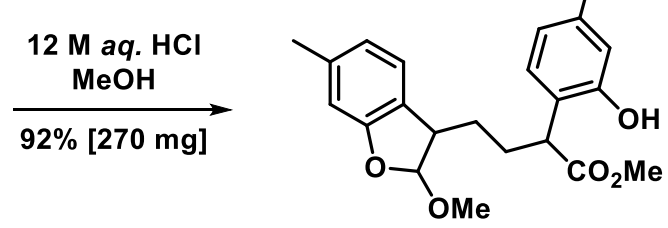

(d.r. 1:1)

$( \pm)-2.35$

Scheme 2.26: Deprotection of dihydropyran ( \pm )-2.28 using $\mathrm{HCl}$ in methanol.

A possible mechanism for the formation of acetal $( \pm)-2.35$ is shown in scheme 2.27. We suggest that initial removal of the MOM protecting groups and mono-cyclisation could form lactol ( \pm )-2.9, which under Brønsted acidic conditions could undergo protonation to give oxonium ion $( \pm)$-2.36. A [1,2]-hydride shift could then occur, forming a second ring-opened oxonium $( \pm)-2.37$, which, after cyclisation and the addition of two molecules of solvent would afford the observed products.

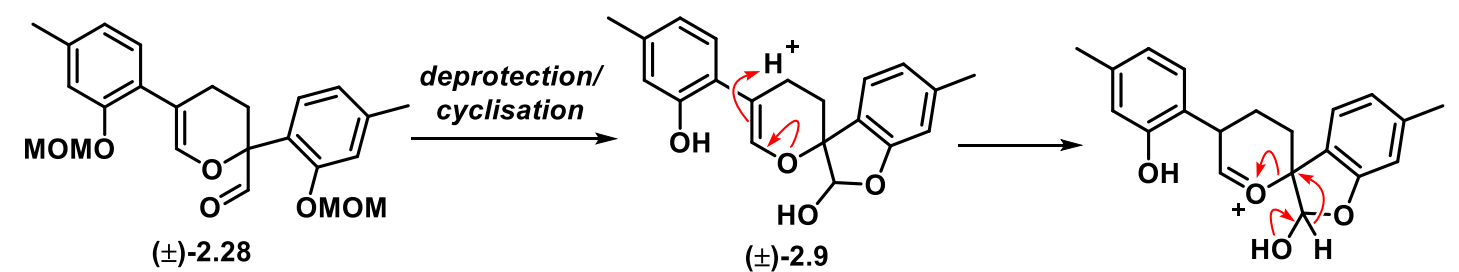

$( \pm)-2.36$

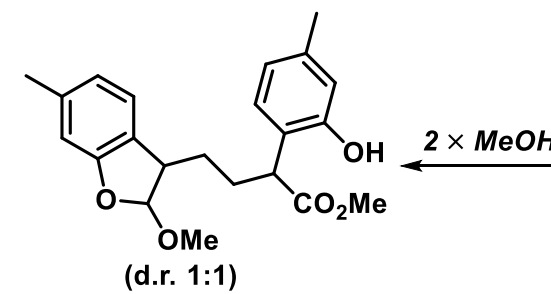

$( \pm)-2.35$

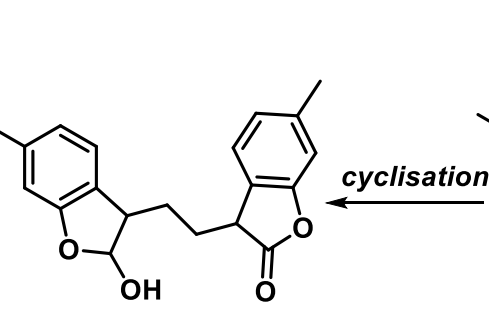

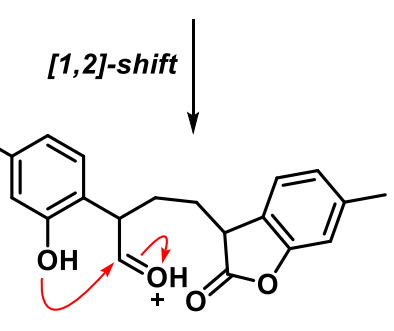

$( \pm)-2.37$

Scheme 2.27: Proposed mechanism for the formation of acetal ( \pm )-2.35.

The results obtained in the attempted deprotection of dihydropyran $( \pm)-\mathbf{2 . 2 8}$ and epoxide ( \pm )-2.29 demonstrated the acid instability of the products of their hydrolysis. The immediate loss of the aldehyde peak observed in some cases in the ${ }^{1} \mathrm{H}$ NMR spectra after addition of the acid suggested that the aldehyde functionality could play a role in this instability. 
With these results in hand, we reasoned that if we could mask the aldehyde functional group on epoxide $( \pm)-\mathbf{2 . 2 9}$ and/or dihydropyran $( \pm)-\mathbf{2 . 2 8}$, this would prevent the formation of lactols ( \pm )-2.32 (see scheme 2.25 on page 50 ) and ( \pm )-2.9 (see scheme 2.27 on page 51), thus avoiding the hydride shifts ultimately leading to the previously discussed undesired reactions. A possible approach could involve olefination of dihydropyran ( \pm )-2.28 through Wittig reaction. At this point both the MOMdeprotection/oxidation or oxidation/deprotection sequences could be investigated, before revealing the aldehyde to complete the synthesis.

To test if this alternative approach was feasible, we first performed a Wittig olefination on aldehyde $( \pm)-\mathbf{2 . 2 8}$ using a modified literature procedure. ${ }^{80}$ A THF solution of dihydropyran ( \pm )-2.28 was treated with a suspension of deprotonated Wittig reagent at $0^{\circ} \mathrm{C}$ for three hours. The reaction was quenched before complete consumption of the starting material, as TLC analysis indicated the formation of several products. Column chromatography afforded an isolated yield of $14 \%$ of alkene ( \pm - $\mathbf{2 . 3 8}$, along with $9 \%$ of recovered starting material $( \pm$ )-2.28 (scheme 2.28). This alternative route to $( \pm)$-thymarnicol 1.1 was pursued no further, as the identification of the side-products of this reaction was not possible and the yield was not considered to be sufficient.

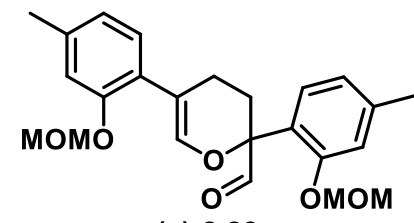

$( \pm)-2.28$

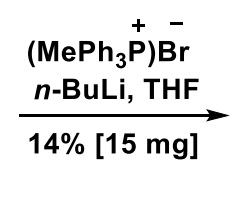

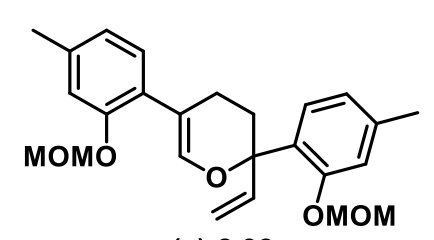

$( \pm)-2.38$

Scheme 2.28: Wittig olefination on dihydropyran $( \pm)-2.28$. 


\subsection{Protecting-Group Free Approach}

At this point it was clear that the choice of the MOM protecting group had seriously undermined the synthesis, therefore we decided to investigate the alternative proposed biomimetic strategy discussed in section 2.1.3 on page 37. This involved the known natural product lactol $( \pm)-\mathbf{2 . 1}$ as the monomeric precursor to $( \pm)$-thymarnicol 1.1. This alternative approach was based on the hypothesis that the reported instability of lactol $( \pm)-2.1^{55}$ could be due to dimerisation. This possibility was very appealing, as it would allow both the oxidation/cyclisation and cyclisation/oxidation alternative pathways to $( \pm$ )-thymarnicol 1.1 from dihydropyran dimer $( \pm)-2.9$, the former potentially occurring in one-pot (scheme 2.29).

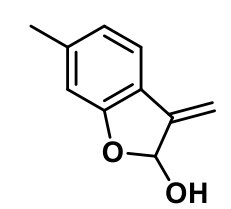

$( \pm)-2.1$

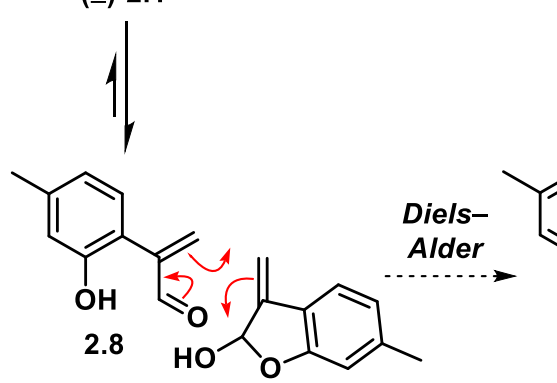

$( \pm)-2.1$

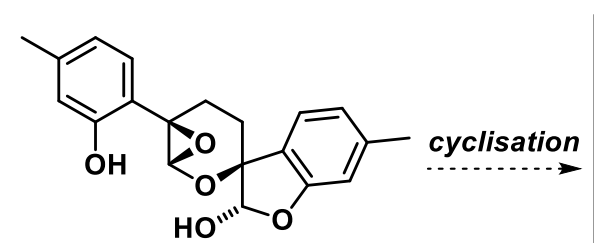

$( \pm)-2.10$

oxidation

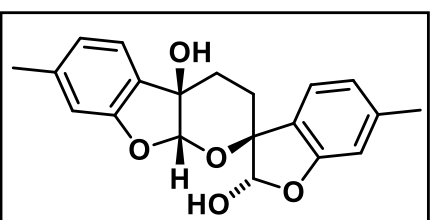

thymarnicol

$( \pm)-1.1$

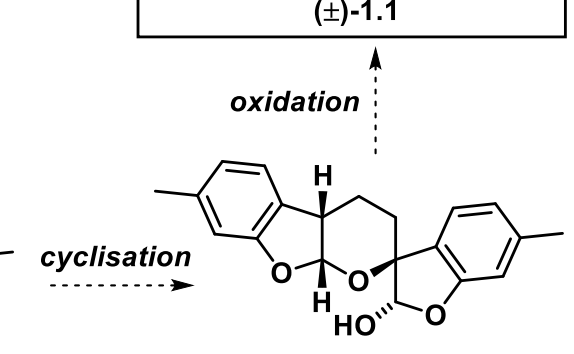

$( \pm)-2.6$

Scheme 2.29: Protecting group-free biomimetic approach.

\subsubsection{Deprotection of enal 2.4}

We started our investigation with an attempt to access lactol ( \pm )-2.1 directly through deprotection of MOM protected enal monomer 2.27 (scheme 2.30). ${ }^{55}$

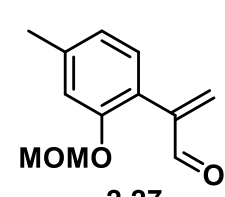

2.27

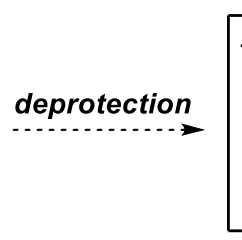

[

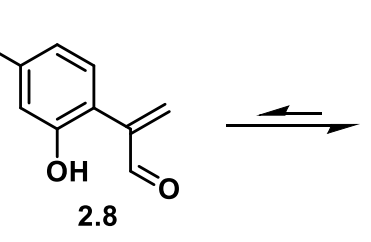

2.8

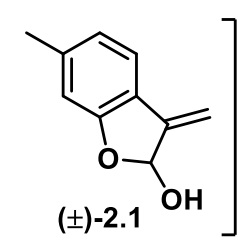

Scheme 2.30: Initial approach to access lactol ( \pm )-2.1. 
Initial attempts on removing the MOM group on enal 2.27 using $\mathrm{P}_{2} \mathrm{I}_{4},{ }^{77}$ silica supported $\mathrm{NaHSO}_{4}{ }^{74} \mathrm{TMSBr}^{76}$ and acetic acid ${ }^{78}$ gave complex mixtures of unidentified products. Different reaction conditions were then tested on the HCl-mediated deprotection: using DMSO as solvent failed to remove the MOM group, while THF gave a complex mixture of products. Finally, cleavage of the MOM-ether on 2.27 was carried out using concentrated $\mathrm{HCl}$ in methanol at room temperature. This resulted in the formation of methyl acetal ( \pm )-2.39 and dimethyl acetal 2.40, the latter formed through the addition of two molecules of solvent to starting material 2.27 (scheme 2.31).

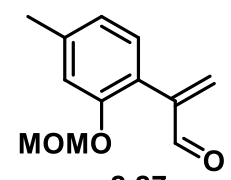

2.27

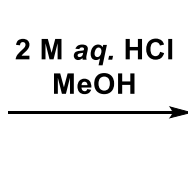$$
{ }_{400}
$$

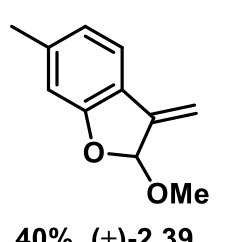

$( \pm)-2.39$

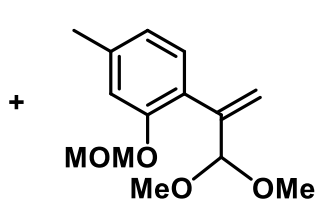

$18 \% 2.40$

Scheme 2.31: $\mathrm{HCl}$ deprotection of enal 2.27.

Although this reaction failed to give lactol $( \pm)-\mathbf{2 . 1}$ directly, there was literature precedent for its formation via acidic deprotection of acetal ( \pm )-2.39. Unfortunately the specific reaction conditions are not clearly stated in the literature. ${ }^{47,55,81}$ Therefore we carried out attempts to deprotect the acetal using $\mathrm{HCl}$ at different concentrations in THF, however no formation of expected product $( \pm)-\mathbf{2 . 1}$ was observed.

\subsubsection{Alternative Approach for the Synthesis of Lactol ( \pm )-2.1}

Having failed to access lactol ( \pm )-2.1 by directly deprotecting enal $\mathbf{2 . 2 7}$, we decided to try an alternative approach. It was envisaged that phenol $\mathbf{2 . 4 1}$, potentially prepared via the deprotection of MOM-ether $\mathbf{2 . 2 5}$, could be subjected to an oxidation/methylenation sequence converting it into enal $\mathbf{2 . 8}$ which, as previously discussed, has been reported to co-exist in equilibrium with lactol ( \pm )-2.1 (scheme 2.32). 


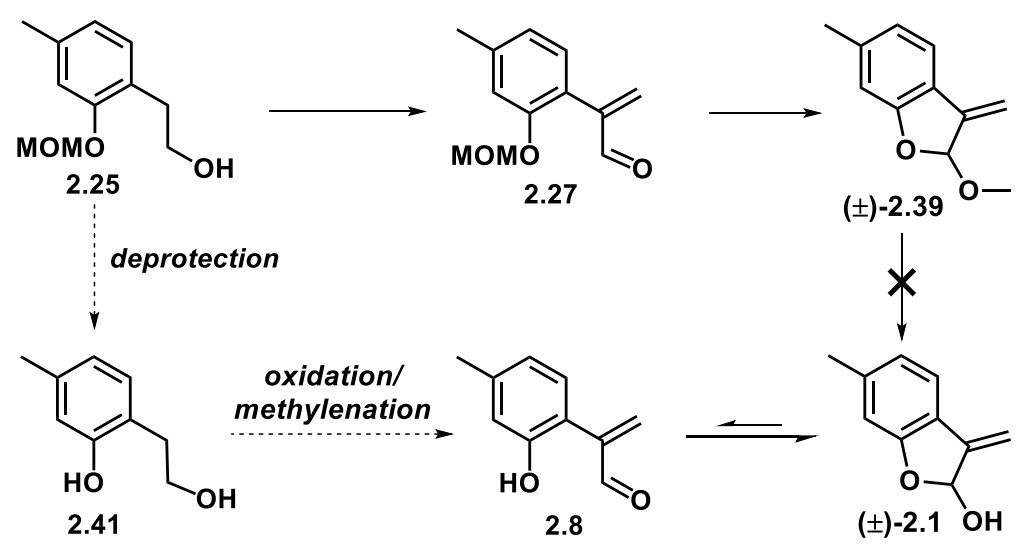

Scheme 2.32: Alternative pathway for the formation of lactol $( \pm)-2.1$.

Diol 2.41 was successfully prepared by simple hydrolysis of MOM-ether 2.25 with $6 \mathrm{M}$ aqueous $\mathrm{HCl}$ in a 4:1 mixture of methanol and dichloromethane. This reaction was conducted on a variety of scales, giving reproducible high yields, up to $97 \%$ when scaled up to $2 \mathrm{~g}$ of product (scheme 2.33).
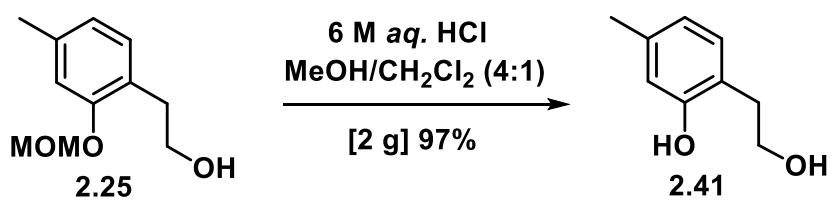

Scheme 2.33: Deprotection of MOM-ether 2.25.

With diol 2.41 in hand, the same oxidation/methylenation sequence used for the synthesis of MOM-protected enal 2.27 was performed. The first trials using the onepot procedure gave complex mixtures of products, therefore it was decided to quench an aliquot of the reaction mixture after 30 minutes, before adding the Eschenmoser salt, in order to identify potential issues. Column chromatographic purification of the first aliquot gave acetal $( \pm)-\mathbf{4 2}$ in $16 \%$ yield. Column chromatography purification of the remaining crude mixture after reaction with the Eschenmoser salt afforded a small quantity of targeted lactol ( \pm )-2.1 in 14\% yield (scheme 2.34). 


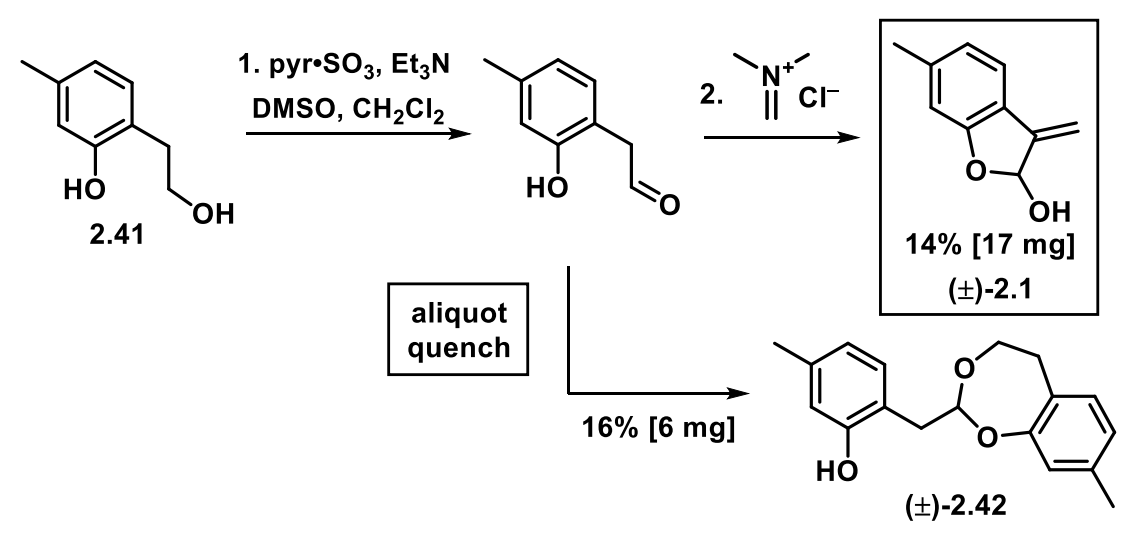

Scheme 2.34: Preparation of lactol $( \pm)-2.1$.

The formation of acetal ( \pm )-2.42 explains, at least in part, the low yield of lactol $( \pm)$-2.1 obtained in this reaction. The conditions were not optimised, because, as reported in literature, lactol $( \pm)-\mathbf{2 . 1}$ proved to be unstable. ${ }^{55}$ It was not possible to obtain any other characterisation data than the ${ }^{1} \mathrm{H}$ NMR spectrum (see page 263), which matched the one reported in literature (see page 264 for a comparison). When lactol ( \pm -2.1 was stored at room temperature, multiple new peaks appeared in the ${ }^{1} \mathrm{H}$ NMR spectrum. Lactol dimer $( \pm)-\mathbf{2 . 9}$ was identified as a component in the mixture. (scheme 2.35).

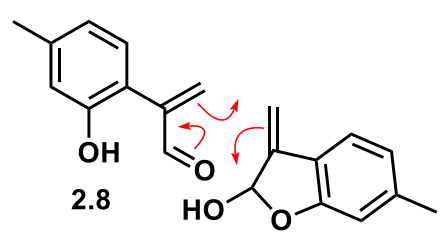

$( \pm)-2.1$

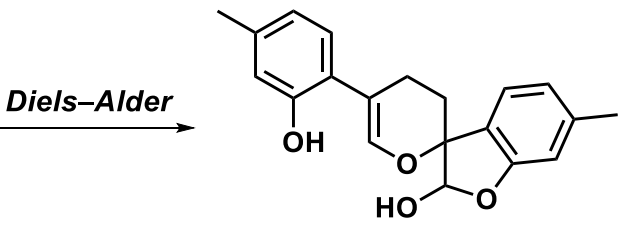

$( \pm)-2.9$

identifiable in the

${ }^{1} \mathrm{H}$ NMR spectrum

Scheme 2.35: Dimerisation of lactol ( \pm )-2.1.

However, the conversion after five days was very poor ( 82:18 starting material $( \pm)-2.1$ : dimer ( \pm -2.9) and lactol $( \pm)-2.9$ was not the only new compound in the mixture (see stacked ${ }^{1} \mathrm{H}$ NMR spectra on page 265). Therefore, although this result demonstrates the chemical feasibility of this pathway, this approach was not considered synthetically practical, due to the low efficiency of the dimerization and the difficulties on accessing monomeric precursor $( \pm)-2.1$.

This led us to re-consider the initial biomimetic strategy, bearing in mind the instability of the thymarnicol $( \pm)$-1.1 polycyclic framework to acids. Thus, we turned our attention to protecting groups which do not require acidic conditions for their removal. 


\subsection{Alternative ortho-Directing Protecting Group Approach}

A sensible alternative choice of protecting group appeared to be the carbamate group: besides being a known ortho-directing group for the metalation of arenes, ${ }^{82-84}$ it can be cleaved under basic, ${ }^{82,83}$ and reductive conditions. ${ }^{84,85}$ This would potentially allow us to use a synthetic sequence analogous to the one already developed with the MOMprotected material. This would involve phenol protection of $m$-cresol to afford carbamate $\mathbf{2 . 4 3}$ followed by ortho-lithiation and ring opening of ethylene oxide to give primary alcohol $\mathbf{2 . 4 4}$ (scheme 2.36).

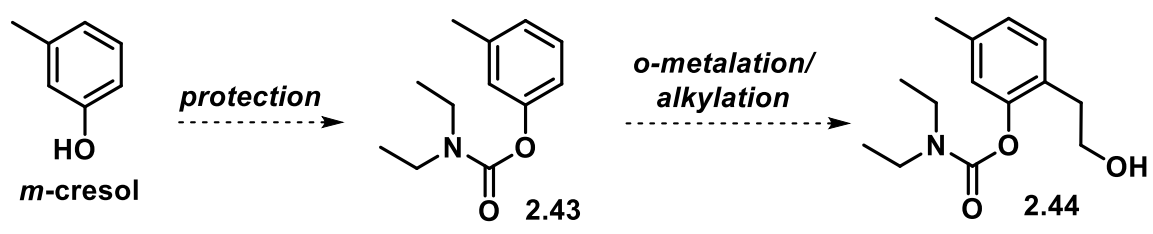

Scheme 2.36: Planned approach with the carbamate protecting group.

Protection of $m$-cresol was smoothly achieved with $N, N$-diethylcarbamoyl chloride under standard conditions, affording $N, N$-diethylcarbamate $\mathbf{2 . 4 3}$ in $91 \%$ yield on a 5-gram scale (scheme 2.37).

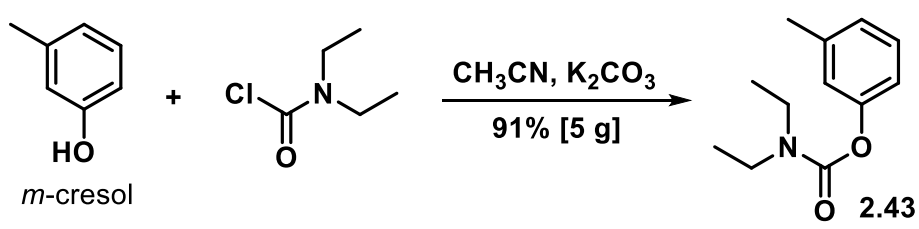

Scheme 2.37: Preparation of N,N-diethylcarbamate $\mathbf{2 . 4 3}$.

With this in hand, a modified literature procedure was used to attempt the ortholithiation and quenching of the aryl lithium with ethylene oxide. Unfortunately, the only product isolated from this reaction was phenol $\mathbf{2 . 4 5}$, presumably resulting from an anionic equivalent of the Fries rearrangement, which had been previously observed on the same substrate (scheme 2.38). ${ }^{86}$ This is proposed to proceed via the intramolecular nucleophilic addition of aryl carbanion $\mathbf{2 . 4 6}$ which results from the ortho-lithiation into the carbonyl group of the carbamate, with consequent transferring of the amide group (scheme 2.38). Therefore, this approach was abandoned. 


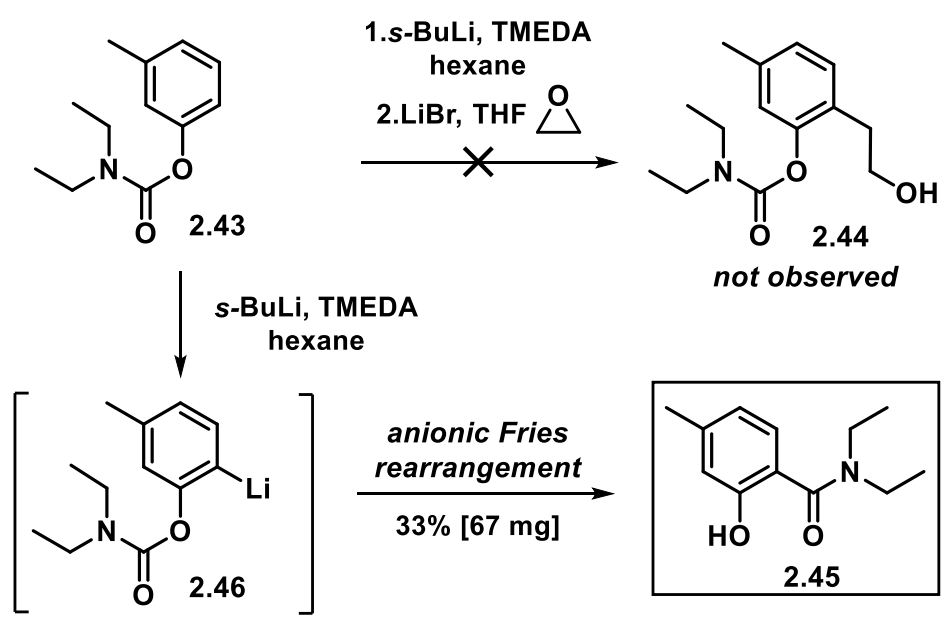

Scheme 2.38: Formation of amide $\mathbf{2 . 4 5}$.

\subsection{Base-Labile Protecting-Groups}

Given our failure to identify a phenol protecting group which is both an ortho-director and removable under non-acidic conditions, we decided to design a new synthetic strategy toward the proposed monomeric precursor 2.4 to ( \pm )-thymarnicol 1.1.

Silyl ethers are known to be readily cleaved under mild acidic and basic conditions, as well as by fluoride. Catalytic palladium (II) can also be used, particularly in the deprotection of $t$-butyldimethylsilyl- (TBS) ethers. ${ }^{87}$ This vast array of different possible deprotection conditions led us to select the TBS group as a suitable protecting group to proceed with our investigation toward the total synthesis of ( \pm )-thymarnicol 1.1.

Another option was represented by the acetyl group. This is present in a number of natural products, and in particular it has been found in several compounds extracted from plants of the Asteraceae family, some of which have been co-isolated with ( \pm )-thymarnicol 1.1. ${ }^{46,88}$ Furthermore, as discussed in the introduction to this chapter, ( \pm )-thymarnicol 1.1 has been proposed by Passreiter to be biosynthetically derived from an acyl (acetyl or isobutyrate) protected thymol 2.4. These characteristics, as well as the fact that it is a base-labile protecting group, made us consider it a suitable candidate for its use in our biomimetic synthesis. 
Our new synthetic strategy to enals $\mathbf{2 . 4 7}$ and $\mathbf{2 . 4 8}$ is depicted in scheme 2.39: we hypothesised that both TBS and acetyl-protected alcohols 2.49 and 2.50 could be accessible via selective mono-protection of diol 2.41. This was a compound we already had access to, as it was an intermediate in the preparation of lactol $( \pm)-\mathbf{2 . 1}$ (see scheme 2.33 on page 55). Primary alcohols $\mathbf{2 . 4 9}$ and $\mathbf{2 . 5 0}$ could then be converted into enals $\mathbf{2 . 4 7}$ and $\mathbf{2 . 4 8}$ through the well-established oxidation/methylenation sequence.

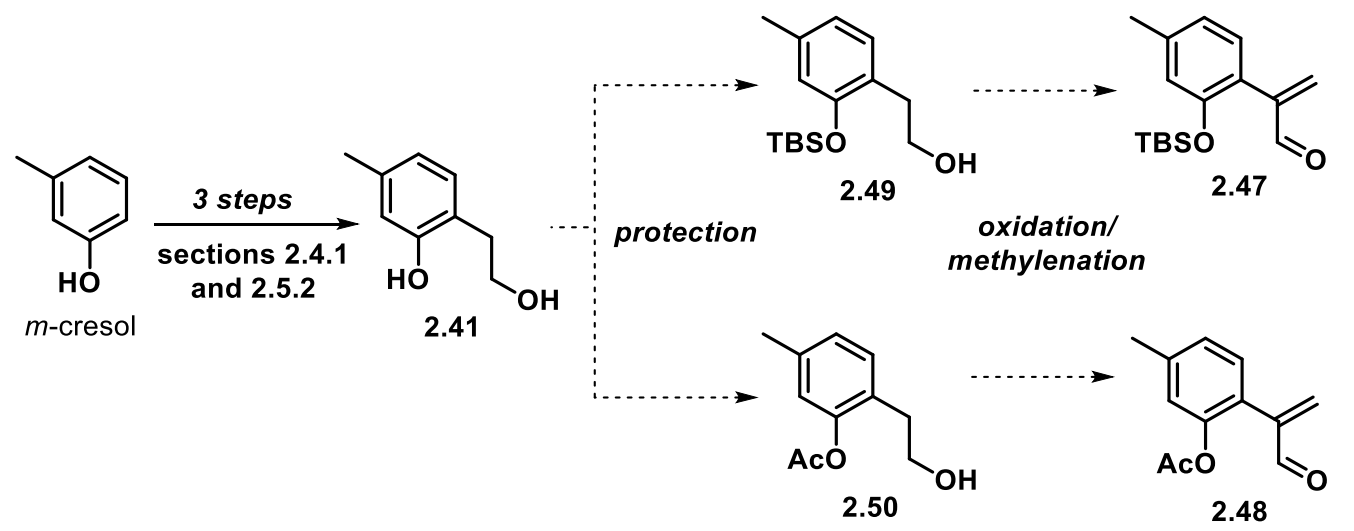

Scheme 2.39: Proposed approach for the synthesis of TBS and acetyl-protected enals 2.47 and 2.48.

\subsubsection{Synthesis of Enal Monomer 2.47}

The TBS-protected enal $\mathbf{2 . 4 7}$ was first targeted.

Initial attempts to access primary alcohol $\mathbf{2 . 4 9}$ in one step via selective monoprotection of diol 2.41 with one equivalent of TBS-Cl failed. Thus a two-step procedure was trialled. ${ }^{89}$ Both hydroxyl groups on diol $\mathbf{2 . 4 1}$ were protected using 2.5 equivalents of TBS chloride under standard conditions. This afforded bis-ether $\mathbf{2 . 5 1}$ in 95\% yield on the largest scale attempted ( 1 gram of purified product). The primary alcoholic group of phenolic ether $\mathbf{2 . 5 1}$ was then revealed using iodine in methanol, a very selective procedure for the TBS-deprotection of primary and secondary alcohols, ${ }^{90}$ affording mono-protected compound $\mathbf{2 . 4 9}$ in $92 \%$ yield on a 300-mg scale (scheme 2.40). 


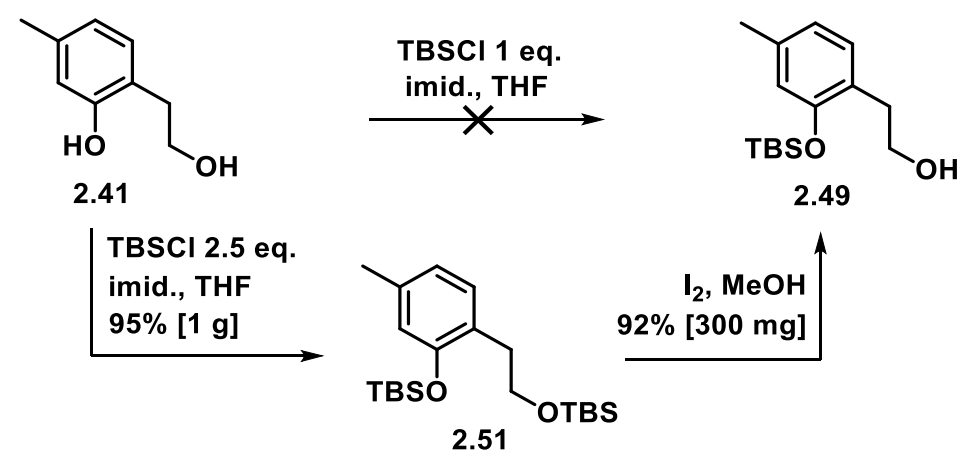

Scheme 2.40: Preparation of primary alcohol 2.49 .

Alcohol 2.49 was then converted into enal $\mathbf{2 . 4 7}$, using the previously employed onepot Parikh-Doering oxidation followed by methylenation using the Eschenmoser salt. This transformation afforded $170 \mathrm{mg}$ of enal monomer $\mathbf{2 . 4 7}$ in an unoptimised $51 \%$ yield (scheme 2.41).
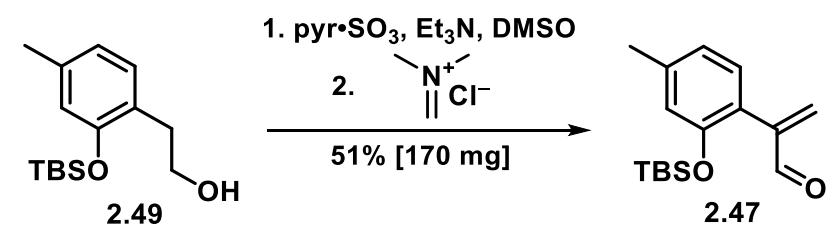

Scheme 2.41: One-pot synthesis of enal 2.47.

\subsubsection{Studies on the Dimerisation of Enal Monomer 2.47}

With this material in hand, several dimerisation conditions were screened with varying reaction temperature: attempts to dimerise enal 2.47 by heating it at $80{ }^{\circ} \mathrm{C}$ in the absence of $\mathrm{K}_{2} \mathrm{CO}_{3}$ for two days resulted in almost complete consumption of the starting material. However, a yield of only $41 \%$ of dihydropyran dimer $( \pm)-2.52$ was calculated from the crude ${ }^{1} \mathrm{H}$ NMR spectrum (with inclusion of dimethyl sulfone as an internal standard), as the formation of other unidentified and undesired products was observed (entry 1). Increasing the temperature to $140{ }^{\circ} \mathrm{C}$ and reducing the reaction time to $1 \mathrm{~h}$ gave a promising $\sim 1: 4$ ratio between starting material 2.47 and product $( \pm)-2.52$, which was formed in a $53 \%{ }^{1} \mathrm{H}$ NMR yield (entry 2). Unfortunately, this result proved difficult to reproduce on a larger scale $(140 \mathrm{mg})$ : in this case the formation of acetal $( \pm)-2.53$ in a 67:33 d.r. was observed (entry 3), far before complete consumption of the starting material, presumably through migration of one of the TBS protecting 
groups to the carbonyl group either before or after the Diels-Alder reaction (paths a and $b$ respectively, scheme $\mathbf{2 . 4 2}$ ).

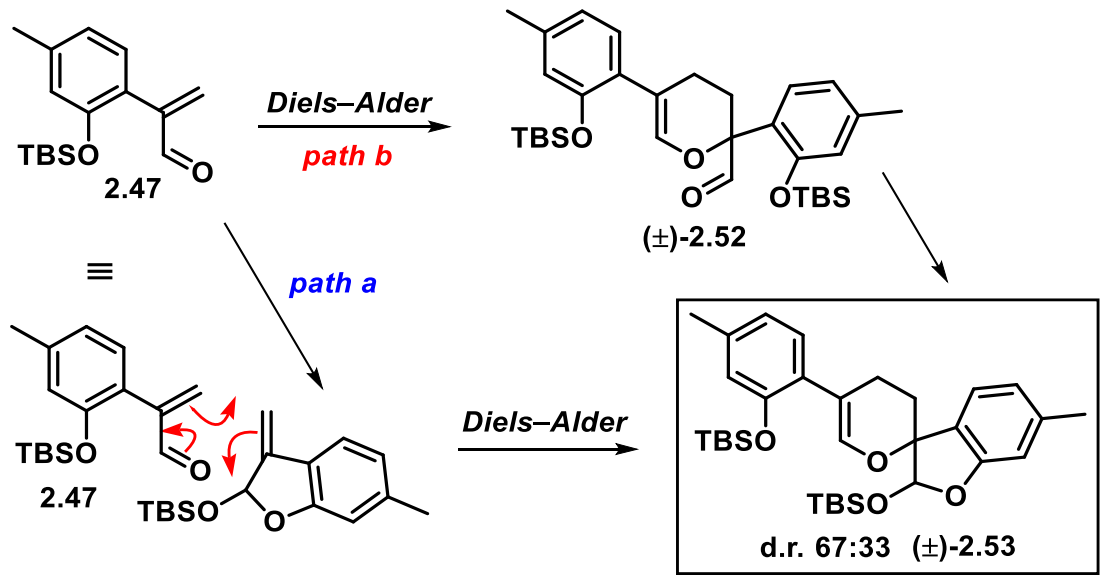

Scheme 2.42: Proposed pathways for the formation of acetal ( \pm )-2.53.

Although it was envisaged that acetal $( \pm)-\mathbf{2 . 5 3}$ could still be used in our synthesis, more test reactions were performed in an attempt to obtain selective conditions for the formation of either dihydropyran $( \pm)-\mathbf{2 . 5 2}$ or acetal $( \pm)-2.53$. The effect of solvent and additives was then investigated. Running the reaction in a small volume of deuterated toluene failed to suppress the formation of acetal ( \pm )-2.53 (entry 3 ). The reaction was then performed in the presence of two equivalents of $\mathrm{K}_{2} \mathrm{CO}_{3}$ at different temperatures. Lowering the temperature from 140 to $80{ }^{\circ} \mathrm{C}$ and prolonging the reaction time, gradually resulted in better selectivity toward dihydropyran $( \pm)-\mathbf{2 . 5 2}$ (entries 4-6) (table 2.1).<smiles>C=C(C=O)c1ccc(C)cc1OS(C)(C)C</smiles>

2.47 conditions<smiles>C1CCCCC1</smiles>

Solvent/ additive

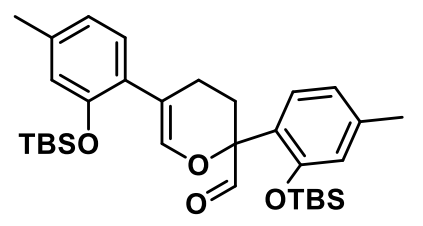

( \pm )-2.52

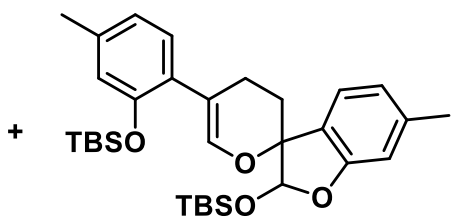

( \pm )-2.53 d.r. $67: 33$

\begin{tabular}{|c|c|c|c|c|}
\hline Entry & $\begin{array}{l}\text { Solvent/ } \\
\text { additive }\end{array}$ & $\begin{array}{c}\text { Temperature } \\
\left({ }^{\circ} \mathrm{C}\right)\end{array}$ & Time (h) & $(\mathrm{RSM}: 2.52: 2.53)^{\mathrm{a}}$ \\
\hline 1 & - & 80 & 46 & $4: 41: 0^{b}$ \\
\hline 2 & - & 140 & 3 & $48: 20: 31$ \\
\hline 3 & toluene- $\mathrm{d}_{8}(750 \mu \mathrm{M})$ & 100 & 17 & $12: 30: 58$ \\
\hline 4 & $\mathrm{~K}_{2} \mathrm{CO}_{3}$ (2 eq.) & 140 & 2 & $16: 26: 56$ \\
\hline 5 & $\mathrm{~K}_{2} \mathrm{CO}_{3}$ (2 eq.) & 110 & 16 & $23: 39: 37$ \\
\hline 6 & $\mathrm{~K}_{2} \mathrm{CO}_{3}$ (2 eq.) & 80 & 40 & 13:62:24 \\
\hline
\end{tabular}

${ }^{\mathrm{a}}$ Products ratio. ${ }^{\mathrm{b}}$ Internal standard yield.

Table 2.1: Screening of dimerisation conditions for enal ( \pm )-2.47. 
Using these optimised conditions, a larger scale reaction was performed. Enal monomer 2.47 was stirred, neat, at $80{ }^{\circ} \mathrm{C}$ in the presence of two equivalents of $\mathrm{K}_{2} \mathrm{CO}_{3}$ for 48 hours. Unfortunately, only small quantities of pure dihydropyran ( \pm )-2.52 (21\% yield) and acetal ( \pm )-2.53 (17\% yield) as a 67:33 mixture of diastereoisomers were isolated after chromatographic purification in this case (scheme 2.43).

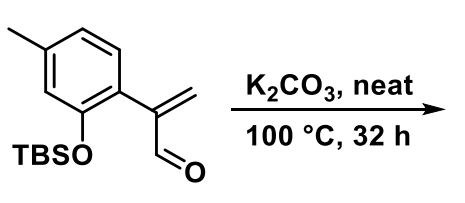

2.47

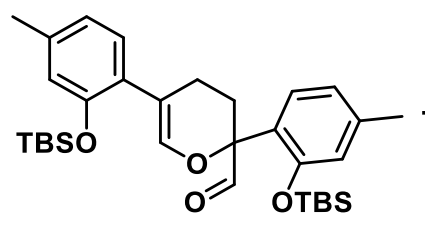

$( \pm)-2.52$

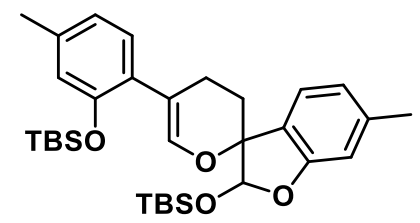

(土)-2.53 (d.r. 67:33)

Scheme 2.43: Dimerisation of TBS-protected enal 2.47 under optimised conditions.

Having failed to find efficient conditions for the key dimerisation step with the TBSprotected enal 2.47, we investigated the problem from a biomimetic standpoint, and turned our attention to the acetyl group.

\subsubsection{Synthesis of Enal Monomer 2.48}

Similarly to the TBS-protected enal $\mathbf{2 . 4 7}$, our initially planned synthesis for the acetyl protected enal monomer $\mathbf{2 . 4 8}$ involved selective phenolic protection of diol $\mathbf{2 . 4 1}$ followed by one-pot oxidation/methylenation of primary alcohol 2.50 (see scheme 2.39 on page 59). There was promising literature precedent for the selective acetyl protection of phenols in the presence of primary alcohols using 1-acetyl-v-triazolo [4,5-b]pyridine. ${ }^{91}$ Therefore we attempted the mono-protection of diol $\mathbf{2 . 4 1}$ following a modified literature procedure ${ }^{91}$ using one equivalent of triazol 2.54. TLC analysis of the crude reaction mixture showed the immediate formation of two different products, with increasing intensity of the corresponding spots. The reaction was monitored and quenched when no further change was observed ( 3 hours); however, the expected product was not observed in the crude ${ }^{1} \mathrm{H}$ NMR spectrum. Column chromatography afforded only mono acetate $\mathbf{2 . 5 5}$ and diacetate $\mathbf{2 . 5 6}$ in 33 and $20 \%$ isolated yield respectively (scheme 2.44). 


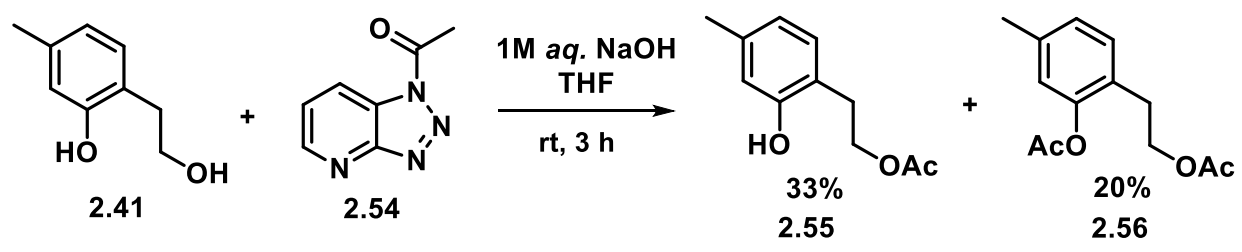

Scheme 2.44: Attempted selective mono-protection of diol $\mathbf{2 . 4 1}$.

The inability to directly access primary alcohol $\mathbf{2 . 5 0}$ led us to undertake an alternative approach which, even if not elegant, would have allowed us to use reliable chemistry to rapidly access material to investigate the subsequent steps. This involved TBSprotection of primary alcohol $\mathbf{2 . 4 1}$ followed by acetylation of the phenol hydroxyl group of 2.57. Finally, cleavage of the silyl ether of acetate $\mathbf{2 . 5 8}$ would afford targeted primary alcohol $\mathbf{2 . 5 0}$, as shown in scheme $\mathbf{2 . 4 5}$.

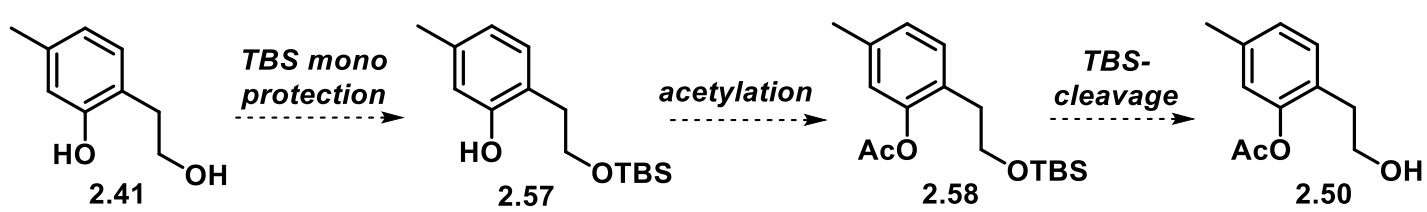

Scheme 2.45: Planned alternative synthesis of alcohol $\mathbf{2 . 5 0}$.

Thus, primary alcohol $\mathbf{2 . 4 1}$ was protected as the TBS-ether under standard conditions using one equivalent of TBS-chloride, affording phenol $\mathbf{2 . 5 7}$ in $79 \%$ yield on a gram scale. This was then acetylated using acetic anhydride in pyridine, reaction which proceeded smoothly to give more than one gram of orthogonally protected compound 2.58 in $97 \%$ yield (scheme 2.46).

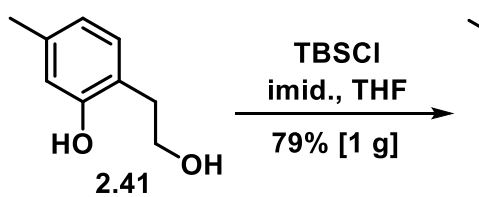

2.41

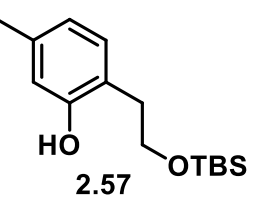

2.57

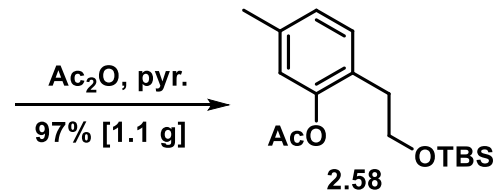

Scheme 2.46: Preparation of orthogonally protected compound $\mathbf{2 . 5 8}$

A preliminary attempt on the cleavage of the TBS group using TBAF as a fluoride source on a small scale gave as the only isolable product phenol $\mathbf{2 . 5 5}$. This is proposed to be formed via initial removal of the TBS group on compound 2.58 followed by transfer of the acetyl group to the alkyl chain on phenol $\mathbf{2 . 5 0}$, as shown in scheme 2.47 . 


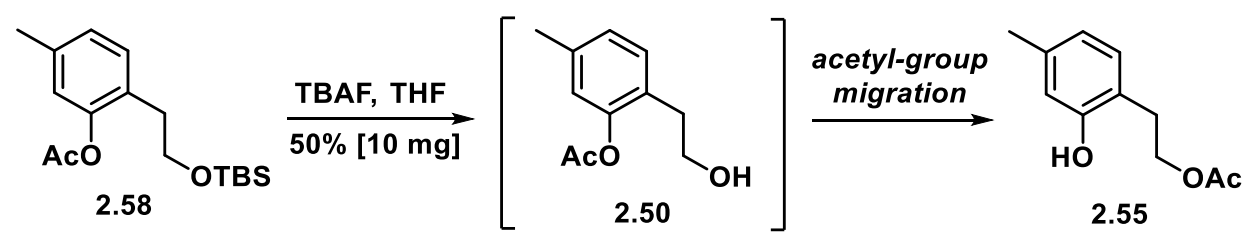

Scheme 2.47: TBAF cleavage of TBS-ether 2.58 .

It was hypothesised that phenol $\mathbf{2 . 5 5}$ and alcohol $\mathbf{2 . 5 0}$ could co-exist in equilibrium, thus allowing us to perform the oxidation/methylenation sequence on the mixture. Therefore, phenol 2.55 was subjected to the Parikh-Doering oxidation/methylenation sequence, in the same conditions used previously. Unfortunately, though, only starting material was recovered (scheme 2.48), leading us to assume that either there isn't an equilibrium taking place, or its position is almost completely toward phenol 2.55. This conclusion led us to attempt different conditions for the cleavage of the TBS group, with the aim of preventing the acetyl group migration.
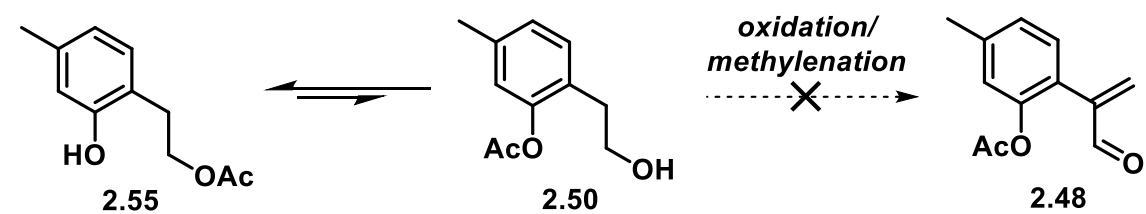

Scheme 2.48: Attempt of oxidation/methylenation of phenol $\mathbf{2 . 5 5}$.

The TBS-deprotection of compound $\mathbf{2 . 5 8}$ using iodine in methanol at room temperature was then investigated by quenching the reaction after different times. Waiting after complete consumption of the starting material was observed resulted in the isolation of both esters $\mathbf{2 . 5 0}$ and $\mathbf{2 . 5 5}$ in a 1:1.5 ratio, while stopping the reaction too early resulted in minimal formation of unwanted phenol $\mathbf{2 . 5 5}$, but poor conversion. After optimisation it was found that the ideal reaction time was 4.5 hours for 1 gram of starting material, affording more than half a gram of alcohol $\mathbf{2 . 5 0}$ in $77 \%$ isolated yield. To prevent the migration of the acetyl group, which tended to occur slowly, even when storing the compound at low temperatures, alcohol $\mathbf{2 . 5 0}$ was immediately subjected to the Parikh-Doering oxidation followed by methylenation with the Eschenmoser salt. This reaction provided more than $300 \mathrm{mg}$ of enal $\mathbf{2 . 4 8}$ to test the key dimerisation reaction (scheme 2.49). 


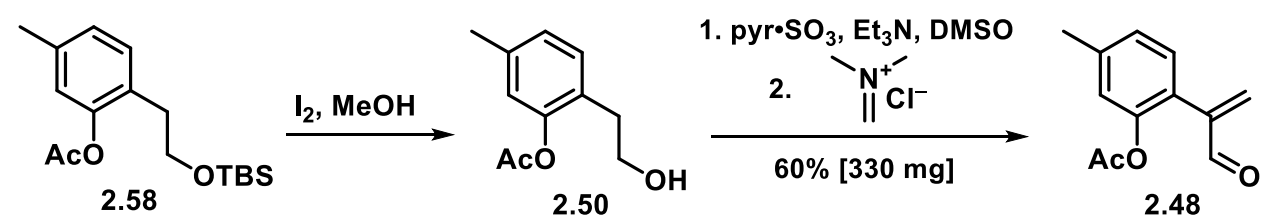

Scheme 2.49: Preparation of enal monomer 2.48.

The yield of this final transformation (60\%) was not as good as the one obtained for MOM protected enal 2.27: that is due to the acetyl transfer previously discussed, which is occurring during the oxidation of alcohol 2.50, thus competing with it in the consumption of the starting material.

\subsubsection{Dimerisation of Enal 2.48}

Enal 2.48 proved to be quite prone to Diels-Alder dimerisation to form dihydropyran ( \pm )-2.59 (see scheme 2.52 on page 66 ) when stored neat, even at $-20{ }^{\circ} \mathrm{C}$. However, the reaction in those conditions was not synthetically useful as the conversion was very poor even after several days. Increasing the temperature to $20{ }^{\circ} \mathrm{C}$ led to a faster reaction which, however, tended to reach a plateau at around 50-60\% conversion after 5 days. The same conditions used for the MOM and TBS-protected enals were then applied: heating neat acetyl-protected enal monomer 2.48 at $80{ }^{\circ} \mathrm{C}$ for 20 hours with two equivalents of potassium carbonate resulted in complete consumption of the starting material and the formation of several compounds. The expected product was not observed in this case, and column chromatography afforded only small quantities of acetal $( \pm)$-2.60 and dihydropyran $( \pm)-\mathbf{2 . 6 1}$ in a 63:37 diastereomeric ratio (scheme 2.50).

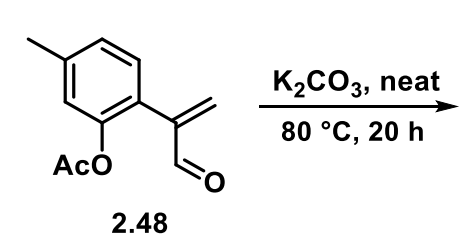

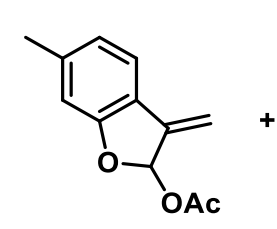

$( \pm)-2.60$

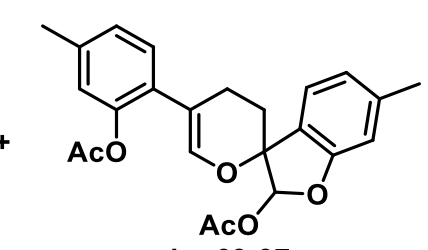

d.r. $63: 37$

$( \pm)-2.61$

Scheme 2.50: Initial attempt on the dimerisation of enal $( \pm)-\mathbf{2 . 4 8}$.

While acetal 2.60 is presumably formed via intramolecular transfer of the phenolic acetyl group on enal $\mathbf{2 . 4 8}$ into its carbonyl, acetal $( \pm)-\mathbf{2 . 6 1}$ is proposed to be formed in a similar way to TBS protected $( \pm)-\mathbf{2 . 5 3}$, (see scheme $\mathbf{2 . 4 2}$ on page 61 ), where the 
transfer could occur either before (path a) or after the Diels-Alder cycloaddition (path b) (scheme 2.51).

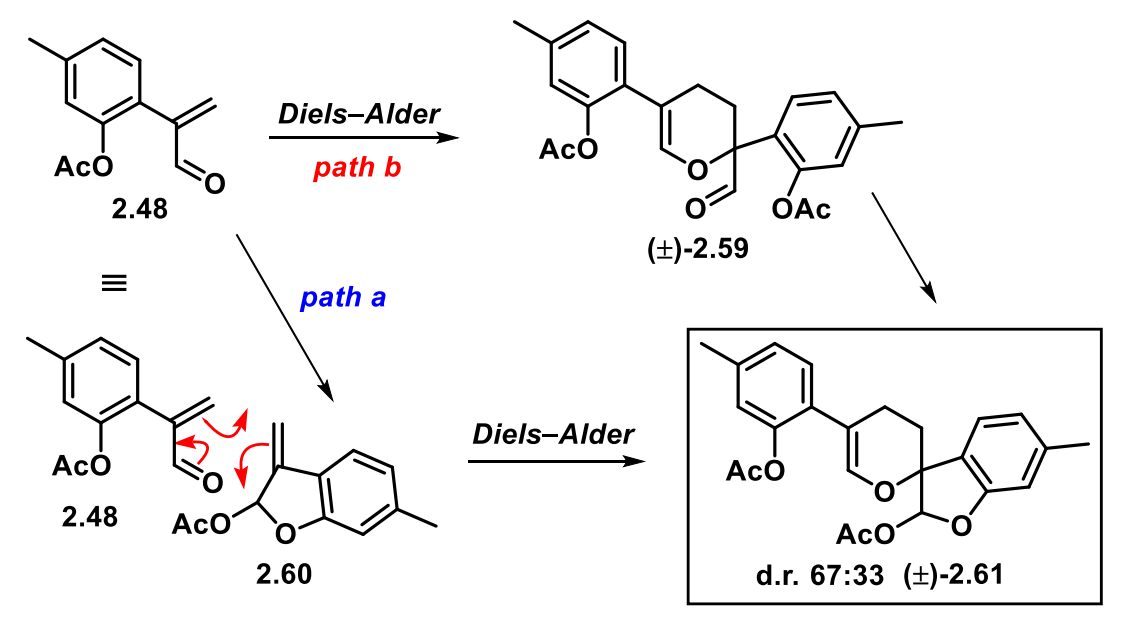

Scheme 2.51: Proposed pathways for the formation of acetal ( \pm -2.61.

The dimerisation was then carried out at a series of temperatures ranging from 30 to $110{ }^{\circ} \mathrm{C}$ in the absence of a base. The optimal temperature was found to be $80{ }^{\circ} \mathrm{C}$, giving the best compromise between reaction rate and selectivity in the formation of dihydropyran ( \pm )-2.59. Stirring $500 \mathrm{mg}$ of enal $\mathbf{2 . 4 8}$ at $80{ }^{\circ} \mathrm{C}$ for 42 hours gave complete conversion to dihydropyran $( \pm)-\mathbf{2 . 5 9}$, based on internal standard ${ }^{1} \mathrm{H}$ NMR (scheme 2.52).

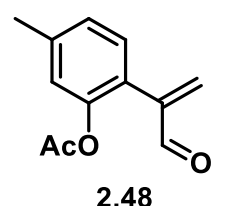

2.48

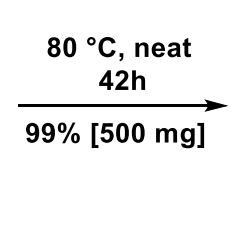

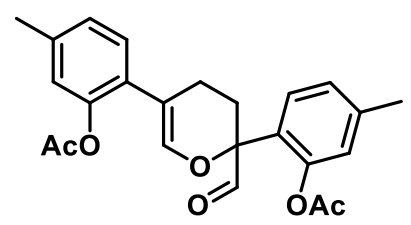

$( \pm)-2.59$

Scheme 2.52: Optimised dimerisation conditions.

With the optimised conditions for the key biomimetic dimerization in hand, we immediately focused on exploring the deprotection step.

\subsubsection{Deprotection of Dihydropyran $( \pm)-2.59$}

We started our investigation on the pivotal deprotection of dihydropyran $( \pm)-\mathbf{2 . 5 9}$ using mildly basic hydrolytic conditions: stirring dihydropyran $( \pm)-\mathbf{2 . 5 9}$ at room temperature until complete consumption of the starting material (4 hours) afforded 
mono protected lactol ( \pm )-2.62 as a 67:33 diastereomeric mixture in 57\% yield, along with acetal ( \pm )-2.61 (63:37 d.r.) in 22\% yield (scheme 2.53).

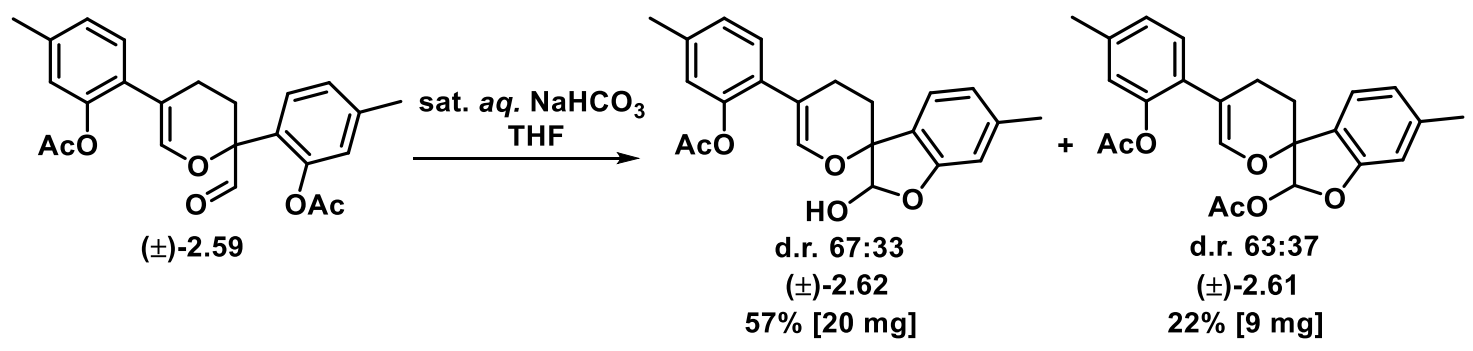

Scheme 2.53: $\mathrm{NaHCO}_{3}$ attempted deprotection of dihydropyran ( \pm )-2.59.

As the use of a mild base failed on removing both the acetyl groups, more forcing conditions were attempted. The hydrolysis was successfully accomplished by stirring substrate ( \pm -2.59 in THF with a $2 \mathrm{M}$ sodium hydroxide aqueous solution. This reaction was performed in a variety of scales, giving as the best result $270 \mathrm{mg}$ of unprotected lactol ( \pm -2.9 as a 78:22 mixture of diastereoisomers in $75 \%$ yield, calculated via ${ }^{1} \mathrm{H}$ NMR spectroscopy using dimethylsulfone as an internal standard, as shown in scheme 2.54 .

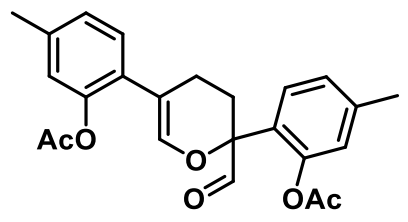

$( \pm)-2.59$

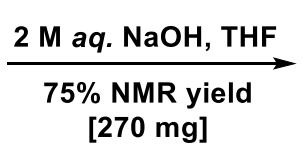

[270 mg]

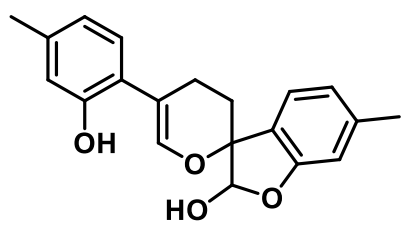

d.r. $78: 22$

$( \pm)-2.9$

Scheme 2.54: NaOH deprotection of dihydropyran ( \pm )-2.59.

Attempts on purifying the product with column chromatography, including with neutralised silica gel, resulted in a dramatic loss of material and failure to recover the product. Interestingly, though, the target molecule ( \pm )-thymarnicol 1.1 was observed as a minor component in one of the fractions.

With these results in hand, it was clear that the acetyl protecting group was a suitable choice: the dimerisation step, which could not be optimised for the TBS protected material, proved to work very efficiently and the deprotection, a major problem in the synthesis with the MOM group, was smoothly accomplished. With that in mind, we decided to design a shorter and more elegant synthesis for enal monomer $\mathbf{2 . 4 8}$. 


\subsection{Short Synthesis of Enal Monomer 2.48}

Although most steps in our first synthesis of the acetyl protected enal monomer $\mathbf{2 . 4 8}$ were high yielding, the step economy was poor in comparison to our previous synthesis of the MOM-protected enal monomer 2.27 (7 steps vs 4 steps). This encouraged us to re-consider the entire strategy. We envisaged that acetyl protected enal $\mathbf{2 . 4 8}$ could instead be accessed by a route commencing with acetylation of the commercially available 2-hydroxy-4-methylacetophenone 2.63. Ester 2.64 could then be converted into alkenyl triflate $\mathbf{2 . 6 5}$ to allow for a final palladium-catalysed formylation reaction to give enal monomer $\mathbf{2 . 4 8}$, as depicted in scheme $\mathbf{2 . 5 5}$. $^{92-94}$ This would represent a very short (3 steps) synthesis for our monomeric precursor $\mathbf{2 . 4 8}$.

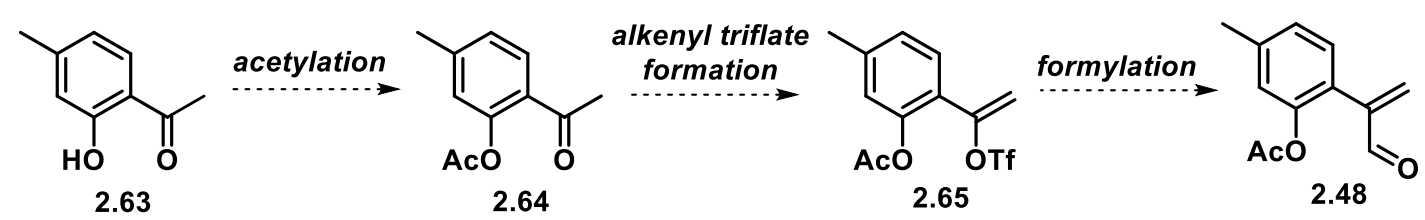

Scheme 2.55: Proposed alternative synthesis of enal monomer 2.48.

Accordingly, acetylation of phenol 2.63 under standard conditions proceeded smoothly to afford ketone $\mathbf{2 . 6 4}$ in $92 \%$ yield on a gram scale (scheme 2.56).<smiles>CCC(=O)c1ccc(C)cc1O</smiles>

2.63

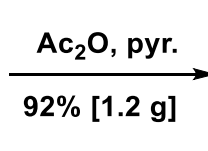

$92 \%[1.2 \mathrm{~g}]$

6: Acetylation of phenol $\mathbf{2 . 6 3 .}$

A modified literature procedure ${ }^{95}$ was used in an attempt to access alkenyl triflate $\mathbf{2 . 6 5}$. Unfortunately, treatment of acetyl protected phenol 2.64 with sulphonamide $\mathbf{2 . 6 6}$ (known as Hendrickson-McMurry reagent) using lithium diisopropylamide (LDA) as a base resulted in a mixture of unidentified products (scheme 2.57).

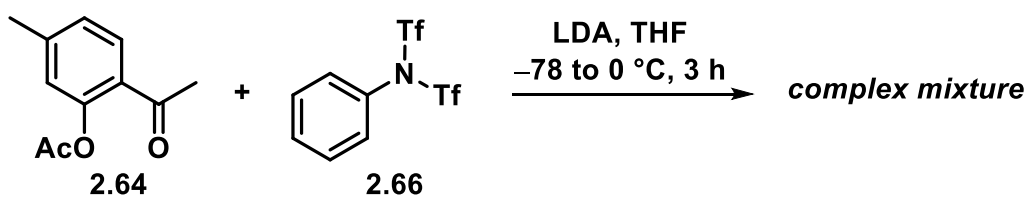

Scheme 2.57: Attempted preparation of alkenyl triflate $\mathbf{2 . 6 5}$. 
An alternative route was considered. In 2003 Vetelino and co-workers reported the unexpected migration and oxidative cyclisation of substituted 2-acetophenone triflate 2.67 under basic conditions. ${ }^{96}$ The migration was shown to occur very efficiently at low temperatures, while the subsequent cyclisation was achieved when raising the temperature to more than $50{ }^{\circ} \mathrm{C}$ (scheme 2.58).

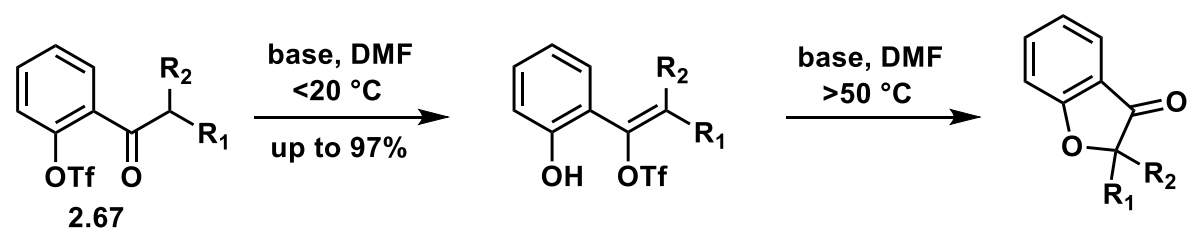

Scheme 2.58: Migration and oxidative cyclisation of triflate ester 2.67.

We envisaged we could take advantage of this reactivity for the preparation of acetyl protected alkenyl triflate $\mathbf{2 . 6 5}$. Initial protection of starting material $\mathbf{2 . 6 3}$ as the triflate ester would afford ketone $\mathbf{2 . 6 8}$, followed by the triflyl group migration reaction performed at low temperatures to give alkenyl triflate $2.69 .{ }^{96}$ Finally, acetylation of the phenolic group on $\mathbf{2 . 6 9}$ would provide substrate $\mathbf{2 . 6 5}$ to attempt the formylation reaction (scheme 2.59).

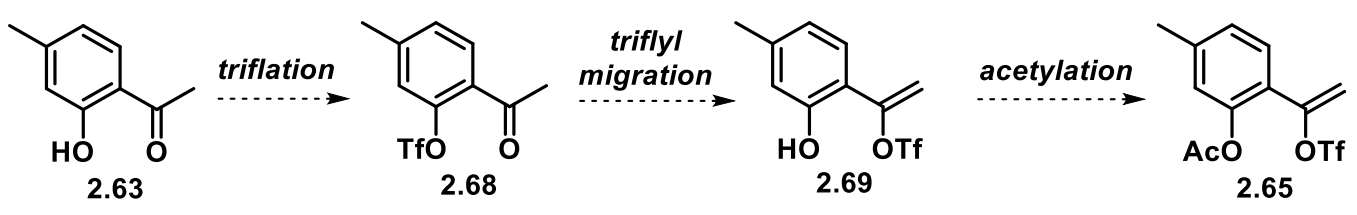

Scheme 2.59: Alternative pathways for the formation of alkenyl triflate $\mathbf{2 . 6 5}$

The protection of phenol $\mathbf{2 . 6 3}$ as the triflate ester under standard conditions proceeded smoothly affording 2 grams of product $\mathbf{2 . 6 8}$ in near quantitative yield, as shown in scheme 2.60.

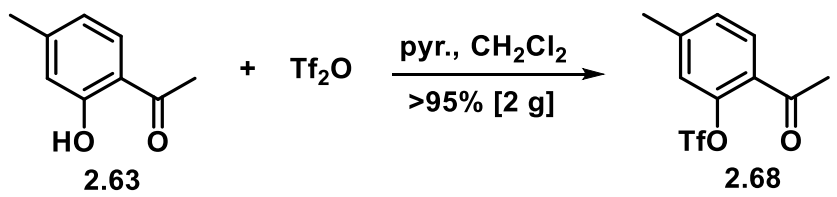

Scheme 2.60: Triflation of phenol $\mathbf{2 . 6 3}$.

We then started our investigation on the triflyl group migration using a modified literature procedure: treatment of ketone $\mathbf{2 . 6 8}$ with potassium tert-butoxide at $0{ }^{\circ} \mathrm{C}$ resulted, after optimisation, in a 53\% yield of enol ether 2.69. An alternative procedure 
was employed in an effort to improve this result using DBU as base, leading to a slight improvement on the yield (61\% on an 85-mg scale) (scheme 2.61).

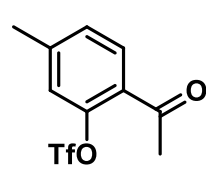

2.68
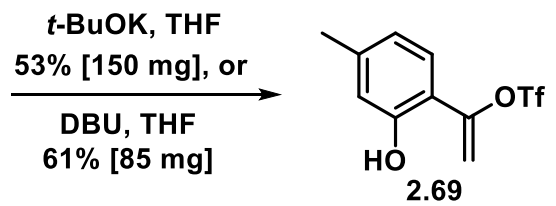

Scheme 2.61: Potassium tert-butoxide mediated triflyl group migration.

However, the scaling up of this reaction resulted in a dramatic drop in the yield, from $61 \%$ for $140 \mathrm{mg}$ to $25 \%$ for $400 \mathrm{mg}$ of starting material used. Keeping in mind the additional step, this sequence was not considered sufficiently high yielding. As we were concurrently developing a new three-step synthetic approach to acetyl-protected enal monomer $\mathbf{2 . 4 8}$ from the same ketone starting material $\mathbf{2 . 6 3}$, this route was abandoned without attempting further optimisation.

\subsection{Final Synthesis}

\subsubsection{Synthesis of Alkene 2.70}

We envisaged we could access our monomeric precursor $\mathbf{2 . 4 8}$ via oxidation of alkene 2.70. A conventional two-step oxidation, involving initial oxidation to the allylic alcohol 2.71 followed by further oxidation to the aldehyde, would likely be problematic due to the facile acyl transfer to give undesired ether $\mathbf{2 . 7 2}$ (scheme 2.62).
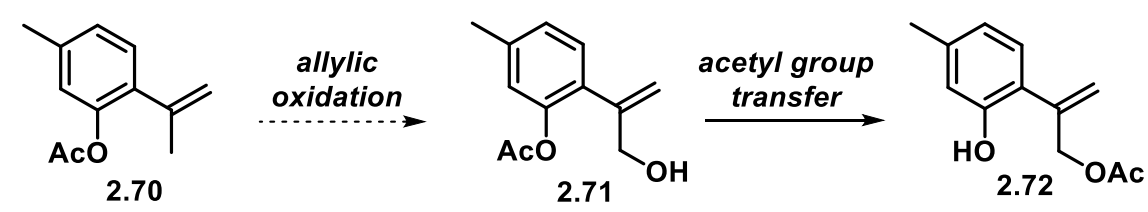

Scheme 2.62: Potential problem in a two-steps oxidation process.

We envisaged we could overcome this issue by directly accessing enal $\mathbf{2 . 4 8}$ without revealing primary alcohol 2.71. Alkene $\mathbf{2 . 7 0}$ in turn could be prepared from the commercially available ketone $\mathbf{2 . 6 3}$ through initial acetylation of the phenolic hydroxyl group, already achieved in $92 \%$ yield (see scheme 2.56 on page 68), followed by methylenation. Alternatively, ketone $\mathbf{2 . 6 3}$ could be subjected to methylenation to give alkene $\mathbf{2 . 7 3}$ prior to phenolic protection, as shown in scheme 2.63. 


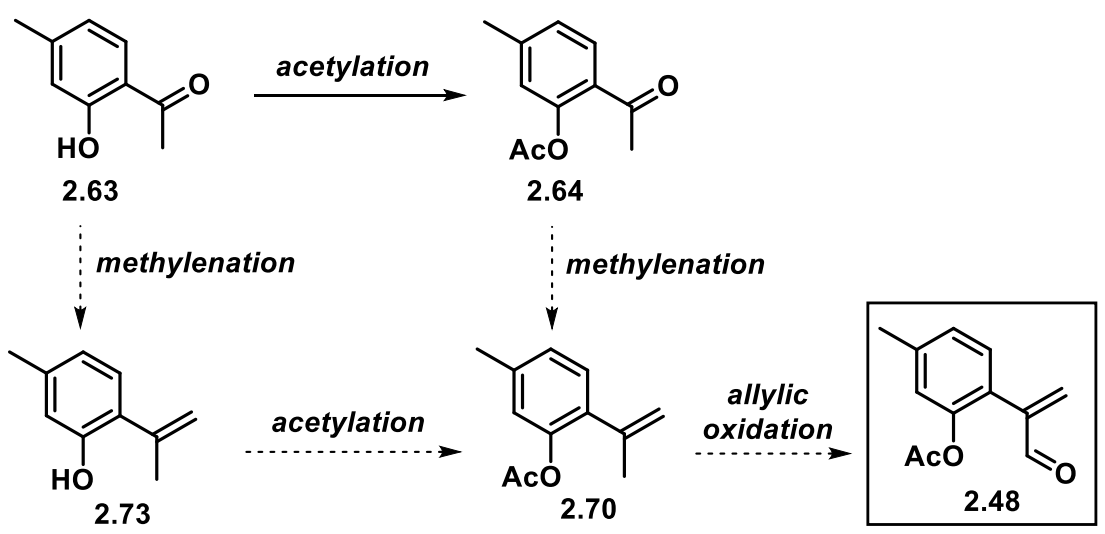

Scheme 2.63: Proposed alternative synthesis of acetyl-protected enal monomer 2.48 .

Initial attempts to methylenate the previously prepared ketone $\mathbf{2 . 6 4}$ through a Wittig reaction gave a complex mixture of products. Therefore, we applied the same reaction conditions to unprotected phenol 2.63; treatment of ketone 2.63 with excess methyl triphenylphosphonium bromide and sodium hydride smoothly provided 9.7 grams of phenol $\mathbf{2 . 7 3}$ in $94 \%$ isolated yield. This was then protected as the acetyl ester under standard conditions affording up to three grams of alkene 2.70 (scheme 2.64).

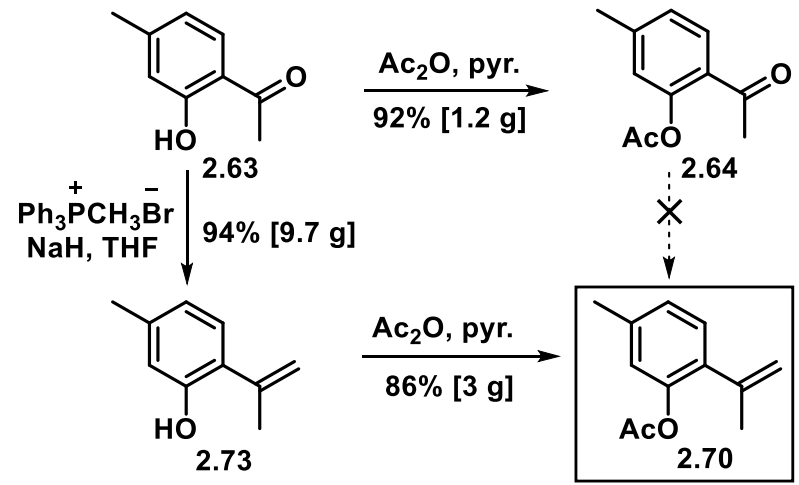

Scheme 2.64: Preparation of alkene 2.70.

\subsubsection{Allylic Oxidation of Alkene 2.70}

With alkene 2.70 in hand, we set out to investigate the allylic oxidation. The use of the chromium trioxide/dimethylpyrazole complex has been reported to be an operationally simple method for oxidising alkenes at the allylic position. ${ }^{97}$ Aldehydes could be accessed directly, without revealing the corresponding primary alcohol at any point. Therefore, this transformation was attempted following a modified literature procedure. Alkene $\mathbf{2 . 7 0}$ was added to a preformed solution of the chromium trioxide/dimethylpyrazole complex. Unfortunately, this reaction resulted in the 
formation of a complex mixture of compounds, with no targeted enal $\mathbf{2 . 4 8}$ observable in the crude ${ }^{1} \mathrm{H}$ NMR spectrum (scheme 2.65).

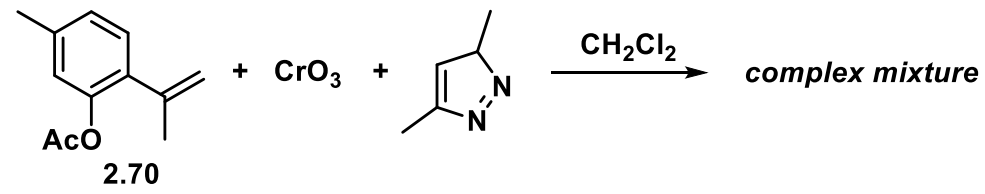

Scheme 2.65: Attempted $\mathrm{CrO}_{3} \bullet$ dimethylpyrazole allylic oxidation of alkene $\mathbf{2 . 7 0}$.

Selenium dioxide was then considered as an alternative reagent for the allylic oxidation. The first attempt was made using catalytic selenium dioxide and tert-butyl hydroperoxide as the terminal oxidant. ${ }^{98}$ Thus, alkene $\mathbf{2 . 7 0}$ was treated with $s u b$ stoichiometric amounts of selenium dioxide and excess tert-butyl hydroperoxide. This reaction proved to be very sluggish and was quenched after 5.5 hours at $40{ }^{\circ} \mathrm{C}$, as no formation of the expected product was detected by TLC analysis. Column chromatography afforded recovered starting material $\mathbf{2 . 7 0}$ in $51 \%$ and undesired phenol 2.72 in $21 \%$ isolated yield (scheme 2.66).

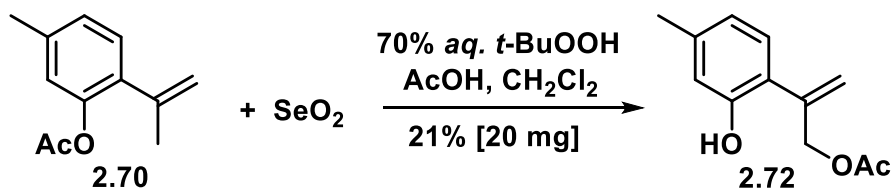

Scheme 2.66: Attempted allylic oxidation of alkene 2.70 with catalytic $\mathrm{SeO}_{2}$.

We reasoned that it could be possible to prevent the unwanted acetyl group migration by adding to the same mixture a further reagent capable of oxidising alcohols to aldehydes. Surveying the literature we identified manganese as a potentially compatible oxidant. ${ }^{99}$ Therefore, the previous reaction was repeated under similar conditions in the presence of a large excess of manganese dioxide. Unfortunately, no formation of targeted enal $\mathbf{2 . 4 8}$ was observed in the crude ${ }^{1} \mathrm{H}$ NMR spectrum after 24 hours.

We considered the use of super-stoichiometric selenium dioxide. Its use for the direct conversion of alkenes to enals with similar structure to our target $\mathbf{2 . 4 8}$ has been reported several times, although with invariably low yield $(\leq 50 \%) .{ }^{60,61,100,101}$ The mechanism of the allylic oxidation has been proposed to involve initial ene-type reaction (which could be non-concerted) between the substrate (in scheme $\mathbf{2 . 6 7}$ alkene 2.70 is used as an example) and selenium dioxide. A [2,3]-sigmatropic rearrangement then affords ester 2.74. ${ }^{102}$ At this point if under aqueous conditions hydrolysis occurs 
to give the correspondent primary alcohol. Either the free alcohol or the selenyl-ester 2.74 can undergo a second ene-type reaction/[2,3]-sigmatropic rearrangement sequence to give acetal $\mathbf{2 . 7 5}$, which would then be hydrolysed in the workup to afford enal 2.48 (scheme 2.67).

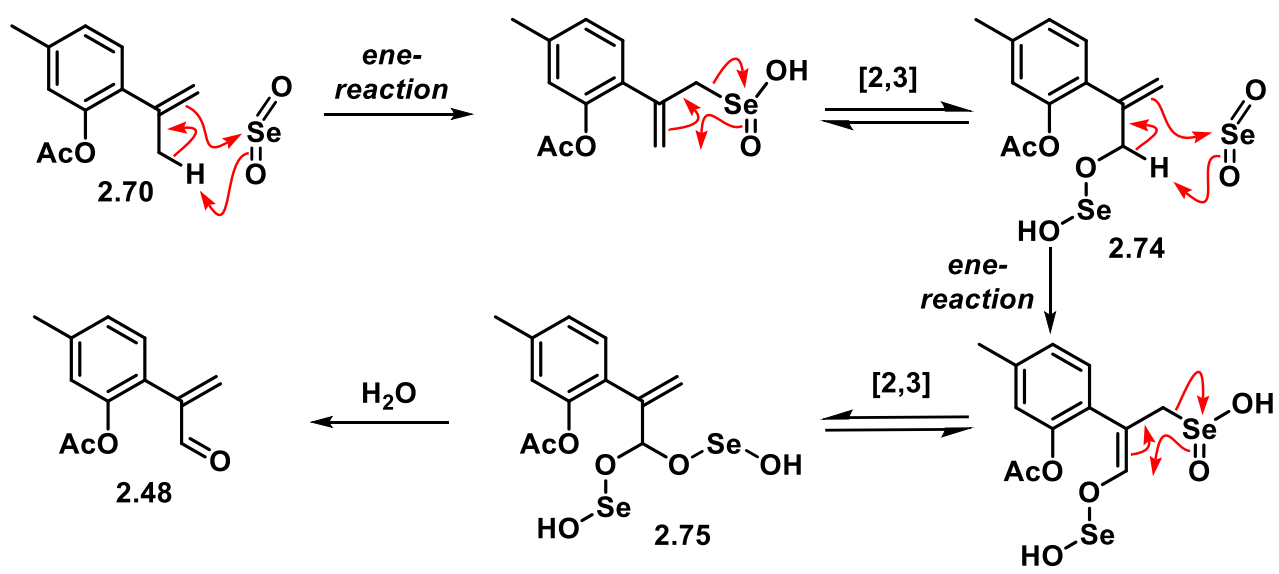

Scheme 2.67: Proposed mechanism of the allylic oxidation with selenium dioxide. ${ }^{103}$

It was therefore reasoned that performing the reaction in anhydrous conditions in the presence of an excess of selenium dioxide could lead to targeted aldehyde $\mathbf{2 . 4 8}$ without revealing primary alcohol 2.71 (see scheme 2.62 on page 70 ). We then screened a series of reaction conditions; summarised in table 2.2. Initial attempts at $60{ }^{\circ} \mathrm{C}$ in benzene in the presence of molecular sieves resulted in a sluggish reaction (entry 1). The use of ground activated molecular sieves proved to be essential, as the formation of complex mixtures of unidentified products was observed when reactions were performed without it. A $20^{\circ} \mathrm{C}$ increase on the temperature lead to a significantly faster reaction (entry 2). The effect of time was investigated for reactions performed at $110^{\circ} \mathrm{C}$ with toluene as the solvent. The relative amounts of the two unwanted products lactone $\mathbf{2 . 7 6}$ and benzofuran $\mathbf{2 . 7 7}$ where found to increase with time (entries 3 and 4). Adding potassium carbonate in an attempt to compensate for the acidity of the molecular sieves resulted in complete suppression of reactivity (entry 5). The effect of the $\mathrm{SeO}_{2}$ stoichiometry was then investigated; using 1.5 equivalents of oxidant resulted in a faster and cleaner reaction (entry 6). Unfortunately, attempts to scale up this transformation resulted in a dramatic drop in the yield (entries 7 and 8).

It was noticed that for scales larger than $100 \mathrm{mg}$ run at $110^{\circ} \mathrm{C}$ a $50 \%$ loss of mass was observed after chromatographic purification. This led us to hypothesise that decomposition at high temperatures had a big impact on the isolated yield. A slight 
decrease in the temperature in a small scale reaction appeared to be beneficial, suppressing the formation of benzofuran side-product 2.77 (entry 9). It was found that the ideal temperature range to be $95-100{ }^{\circ} \mathrm{C}$. Finally, the best compromise between yield, scale and reaction time was achieved using 1.5 equivalents of selenium dioxide in toluene at $100{ }^{\circ} \mathrm{C}$ in the presence of molecular sieves (entry 10).

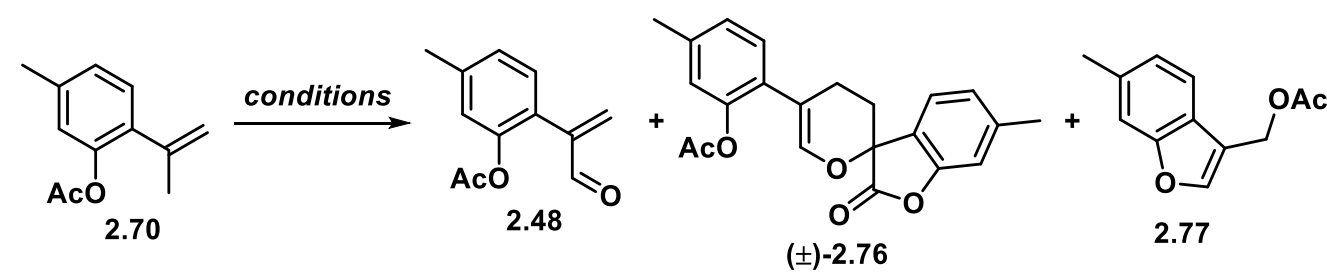

\begin{tabular}{|c|c|c|c|c|c|c|}
\hline Entry & $\begin{array}{l}\mathrm{SeO}_{2} \\
\text { (Eq.) }\end{array}$ & $\begin{array}{c}\text { Temperature } \\
\left({ }^{\circ} \mathbf{C}\right)\end{array}$ & $\begin{array}{l}\text { Scale } \\
(\mathbf{m g})^{\mathrm{a}}\end{array}$ & Solvent/additive & $\begin{array}{c}\text { Time } \\
\text { (h) }\end{array}$ & $\begin{array}{c}\% \\
(2.48: 2.76: 2.77)^{b}\end{array}$ \\
\hline 1 & 1.2 & 60 & 110 & $\begin{array}{c}\text { benzene/ } \\
\text { molecular sieves }\end{array}$ & 70 & $25: 8: 6^{c}$ \\
\hline 2 & 1.2 & 80 & 75 & $\begin{array}{c}\text { benzene/ } \\
\text { molecular sieves }\end{array}$ & 24 & $42: 6: 0^{c}$ \\
\hline 3 & 1.2 & 110 & 110 & $\begin{array}{l}\text { toluene/ } \\
\text { molecular sieves }\end{array}$ & 3.5 & $58: 16: 26$ \\
\hline 4 & 1.2 & 110 & 110 & $\begin{array}{l}\text { toluene/ } \\
\text { molecular sieves }\end{array}$ & 5 & $39: 36: 25$ \\
\hline 5 & 1.2 & 110 & 115 & $\begin{array}{l}\text { toluene } / \mathrm{K}_{2} \mathrm{CO}_{3} \\
\text { molecular sieves }\end{array}$ & 4 & No reaction \\
\hline 6 & 1.5 & 110 & 60 & $\begin{array}{l}\text { toluene/ } \\
\text { molecular sieves }\end{array}$ & 3.5 & $50: 4: 0^{c}$ \\
\hline 7 & 1.5 & 110 & 190 & $\begin{array}{l}\text { toluene/ } \\
\text { molecular sieves }\end{array}$ & 5 & $23: 0: 9^{c}$ \\
\hline 8 & 1.5 & 110 & 500 & $\begin{array}{c}\text { toluene/ } \\
\text { molecular sieves }\end{array}$ & 5 & $21: 10: 11^{\mathrm{c}}$ \\
\hline 9 & 1.5 & 95 & 40 & toluene & 3.5 & $75: 25: 0$ \\
\hline 10 & 1.5 & 100 & 415 & $\begin{array}{c}\text { toluene/ } \\
\text { molecular sieves }\end{array}$ & 6 & $24: 6: 6^{c}$ \\
\hline
\end{tabular}

${ }^{\mathrm{a}}$ The scale is referred to the amount of starting material used. ${ }^{\mathrm{b}}$ Product ratio. ${ }^{\mathrm{c}}$ Isolated yield.

Table 2.2: Screening of $\mathrm{SeO}_{2}$ allylic oxidation conditions for alkene $\mathbf{2 . 7 0}$.

With the optimised conditions in hand, we performed a series of reactions with $\sim 1$ gram of starting material, which reproducibly afforded our monomeric precursor 2.48 in 20-25\% internal standard crude ${ }^{1} \mathrm{H}$ NMR yield. The best result obtained on this 
scale after chromatographic purification was a $21 \%$ isolated yield of enal $\mathbf{2 . 4 8}$, with only small amounts of lactone $\mathbf{2 . 7 6}$ and benzofuran $\mathbf{2 . 7 7}$ isolated. (scheme 2.68).

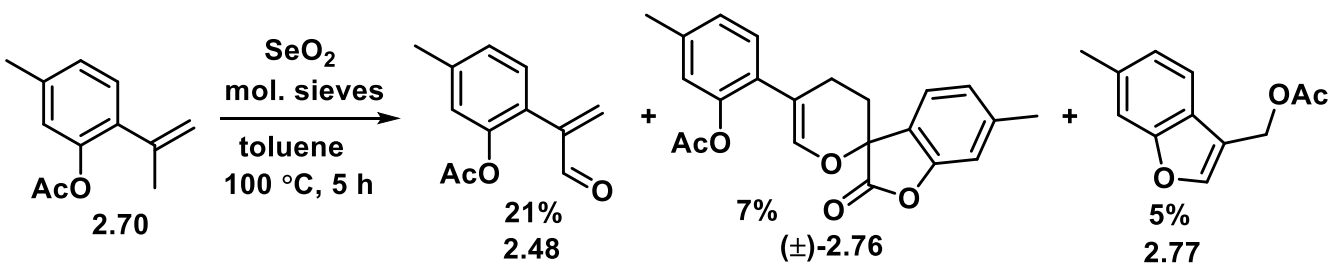

Scheme 2.68: Stoichiometric $\mathrm{SeO}_{2}$ allylic oxidation of alkene $\mathbf{2 . 7 0 .}$

Although the yield for this transformation was poor, it was possible to access more than 2 grams of monomer $\mathbf{2 . 4 8}$ through multiple batches by combining the crudes from different reactions and purifying them together. This represents a very short three steps synthesis for this key intermediate, thus there was the potential for a six or seven-step synthesis of the targeted natural product $( \pm)$-thymarnicol $\mathbf{1 . 1}$ from commercially available ketone 2.63. In fact, our previous investigations (see scheme 2.52 on page 56) demonstrated that the Diels-Alder dimerisation of enal $\mathbf{2 . 4 8}$ proceeds smoothly and quantitatively to afford up to $500 \mathrm{mg}$ of dihydropyran ( \pm -2.59 in a single batch. Furthermore, we had found suitable conditions for its deprotection, allowing us to access lactol ( \pm )-2.9 (see scheme 2.54 on page 67 ). With the optimised conditions in hand, we set out to investigate the final transformations into natural product $( \pm)$-thymarnicol 1.1. With the two alternatives of oxidation/cyclisation and cyclisation/oxidation available, we decided to attempt the oxidation of dihydropyran ( \pm -2.59 first.

\subsubsection{Oxidation of Dihydropyran $( \pm)-2.59$}

With dimer ( \pm -2.59 in hand, a small scale epoxidation was performed in the same reaction conditions previously developed: unsurprisingly, the in-situ generation of DMDO afforded epoxide ( \pm )-2.78 as a 1:1 diastereomeric mixture, in 97\% crude yield (scheme 2.69). A diastereomerically pure sample was isolated through chromatographic purification; however, attempts to determine the relative stereochemistry through 2D NMR analysis were unsuccessful. 


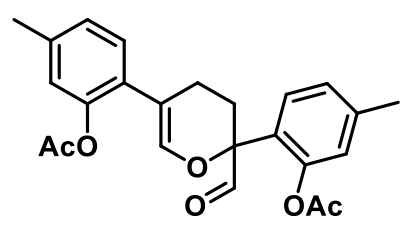

$( \pm)-2.59$

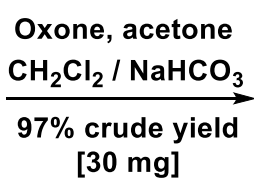

[30 mg]

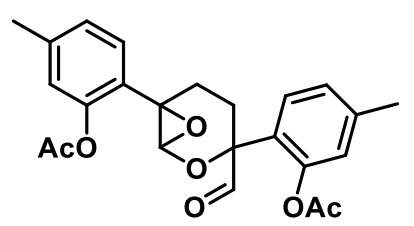

d.r. 1.1

$( \pm)-2.78$

Scheme 2.69: In-situ generated DMDO epoxidation of dihydropyran ( \pm )-2.59.

Due to the lack of diastereoselectivity in this reaction, this approach was abandoned.

\subsubsection{Preparation of $( \pm)$-Thymarnicol 1.1}

We hypothesised that the removal of the acyl groups prior to the oxidation could lead to conformational changes favouring the approach of the oxidant to the less hindered face. If so, oxidation of the double bond of lactol ( \pm )-2.9, followed by cyclisation, could afford ( \pm )-thymarnicol 1.1. Based on the literature precedent discussed in section 2.2.2 on page 40, the oxidation and cyclisation could occur in a single step. Alternatively, ( \pm )-thymarnicol 1.1 could be prepared via cyclisation followed by benzylic oxidation, as proposed by Passreiter (scheme 2.70).

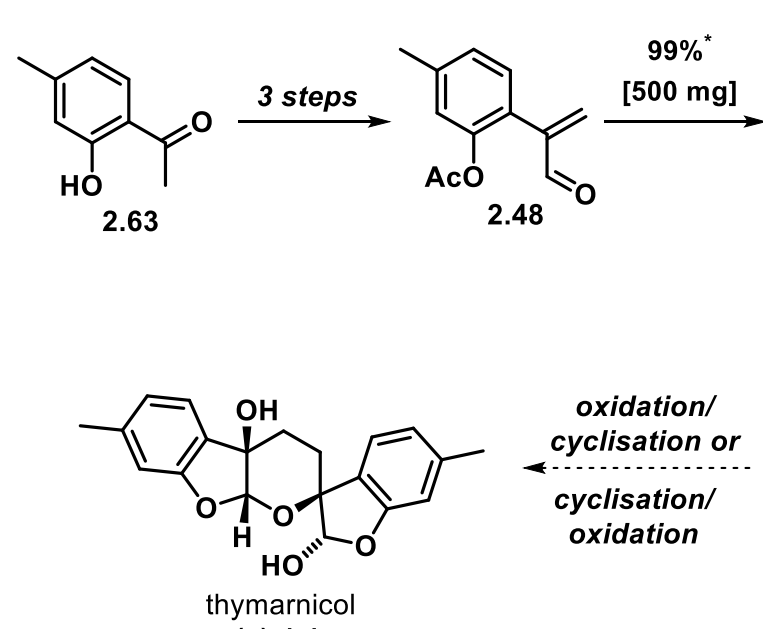

$( \pm)-1.1$

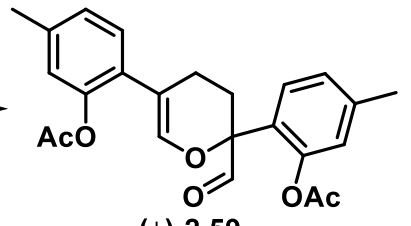

$( \pm)-2.59$

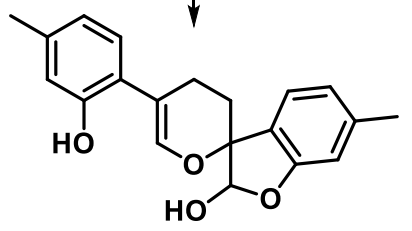

d.r. $72: 28$

$( \pm)-2.9$

*internal standard ${ }^{1} \mathrm{H}$ NMR yield.

Scheme 2.70: Potential six or seven-step synthesis of ( \pm )-thymarnicol 1.1.

As mentioned in section $\mathbf{2 . 7 . 5}$ on page 67 , attempts to purify lactol ( \pm )-2.9 by column chromatography met with failure. However, the presence of a small amount of $( \pm)$-thymarnicol 1.1 was detected in one of the fractions collected. Furthermore, the relative quantity of $( \pm)$-thymarnicol $\mathbf{1 . 1}$ with respect to the other components seemed to increase over time. This interesting observation suggests that in nature 
$( \pm)$-thymarnicol 1.1 could be formed as the result of autoxidation of lactol ( \pm )-2.9. In an effort to find a synthetically suitable procedure for the formation of $( \pm)$-thymarnicol 1.1, crude lactol $( \pm)-2.9$ was subjected to conditions which aimed to reproduce the attempted purification, when the formation of $( \pm)$-thymarnicol 1.1 was first observed. Lactol ( \pm )-2.9 was stirred in an 80:20 mixture of hexane and ethyl acetate at room temperature with an equal amount in weight of silica gel. This resulted, after 48 hours, in a $45 \%$ conversion (entry 1). A fifteen-fold increase on the amount of silica gel gave a far slower reaction, with only $10 \%$ consumption of starting material ( \pm )-2.9 after 44 hours (entry 2). Attempts on increasing the reaction rate by performing by performing the reaction under oxygen atmosphere were unsuccessful (entry 3). The effects of light and solvent were then investigated (entries 4-9). No reaction was observed in the dark, both in the presence and absence of silica gel. Irradiating the reaction mixture with a lamp $(11 \mathrm{~W}, 220-240 \mathrm{~V}, 50-60 \mathrm{~Hz}, 85 \mathrm{~mA})$ resulted in faster conversion in the absence of silica gel than in its presence, and changing the solvent to methanol resulted in a slower transformation. All the information obtained were combined to determine the final reaction conditions; irradiating lactol ( \pm -2.9 in an 80:20 mixture of hexane/ethyl acetate at room temperature resulted in internal standard ${ }^{1} \mathrm{H}$ NMR yield of $53 \%$ of ( \pm -thymarnicol 1.1. A $37 \%$ yield was calculated of an unknown compound, the structure of which was tentatively assigned as peroxide ( \pm )-2.79 (vide infra) (entry 10). Finally, it was noticed that the ratio between ( \pm )-thymarnicol 1.1 and compound ( \pm )-2.79 changed with time (entries 11 and 12) (table 2.3), suggesting the latter could be an intermediate in the formation of $( \pm)$-thymarnicol 1.1. 


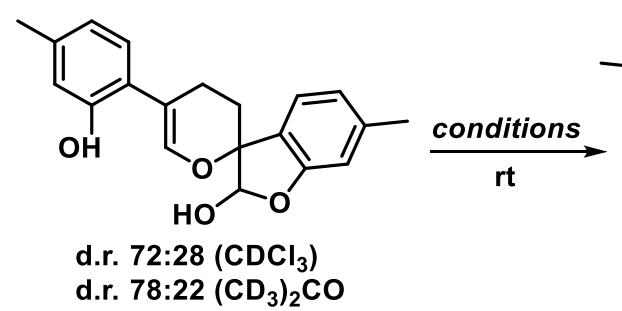

$( \pm)-2.9$

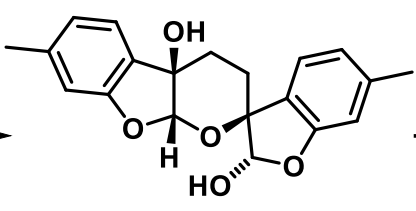

d.r. $78: 22\left(\mathrm{CDCl}_{3}\right)$

d.r. $88: 12\left(\mathrm{CD}_{3}\right)_{2} \mathrm{CO}$

$( \pm)-1.1$

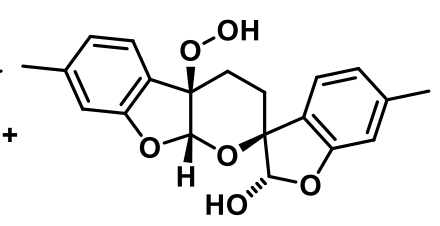

d.r. $88: 12\left(\mathrm{CD}_{3}\right)_{2} \mathrm{CO}$

$( \pm)-2.79$

\begin{tabular}{|c|c|c|c|c|c|}
\hline Entry & Additive & Scale $(\mathbf{m g})^{\mathrm{a}}$ & Solvent & $\begin{array}{c}\text { Time } \\
\text { (h) }\end{array}$ & $\begin{array}{c}\% \\
(2.9: 1.1: 2.79)^{b}\end{array}$ \\
\hline 1 & Silica gel x1 & 20 & hexane/EtOAc & 48 & $45: 45: 10$ \\
\hline 2 & Silica gel x15 & 20 & hexane/EtOAc & 44 & 90:10:0 \\
\hline 3 & Silica gel, $\mathrm{O}_{2}$ & 20 & hexane/EtOAc & 48 & $87: 13: 0$ \\
\hline 4 & NO LIGHT & 10 & pet spirit/EtOAc & 42 & 100:0:0 \\
\hline 5 & $\begin{array}{l}\text { Silica gel x1 } \\
\text { NO LIGHT }\end{array}$ & 10 & pet spirit/EtOAc & 42 & 100:0:0 \\
\hline 6 & LIGHT & 10 & pet spirit/EtOAc & 46 & $25: 50: 25$ \\
\hline 7 & $\begin{array}{c}\text { Silica gel x2 } \\
\text { LIGHT }\end{array}$ & 10 & pet spirit/EtOAc & 46 & 49:40:11 \\
\hline 8 & LIGHT & 10 & methanol & 46 & 82:18:0 \\
\hline 9 & $\begin{array}{c}\text { Silica gel x2 } \\
\text { LIGHT }\end{array}$ & 10 & methanol & 46 & 90:10:0 \\
\hline 10 & LIGHT/air & 10 & hexane/EtOAc & 40 & $0: 57: 35^{\mathrm{c}}$ \\
\hline 11 & LIGHT/air & 130 & hexane/EtOAc & 64 & $0: 66: 34$ \\
\hline 12 & LIGHT/air & 130 & hexane/EtOAc & 90 & $0: 74: 26$ \\
\hline
\end{tabular}

${ }^{\mathrm{a}}$ The scale is referred to the amount of starting material used. ${ }^{\mathrm{b}}$ Product ratio. ${ }^{\mathrm{c}}$ Internal standard yield.

Table 2.3: Screening of conditions for the formation of ( \pm )-thymarnicol $\mathbf{1 . 1}$.

With these optimised conditions in hand, a larger scale reaction was performed using $250 \mathrm{mg}$ of lactol ( \pm )-2.9 starting material. Analysis of the crude reaction mixture by ${ }^{1} \mathrm{H}$ NMR spectroscopy, with the inclusion of dimethyl sulfone as internal standard, 
indicated a 57\% yield of ( \pm )-thymarnicol 1.1 and $21 \%$ of compound $( \pm)-\mathbf{2 . 7 9}$ (scheme 2.71).

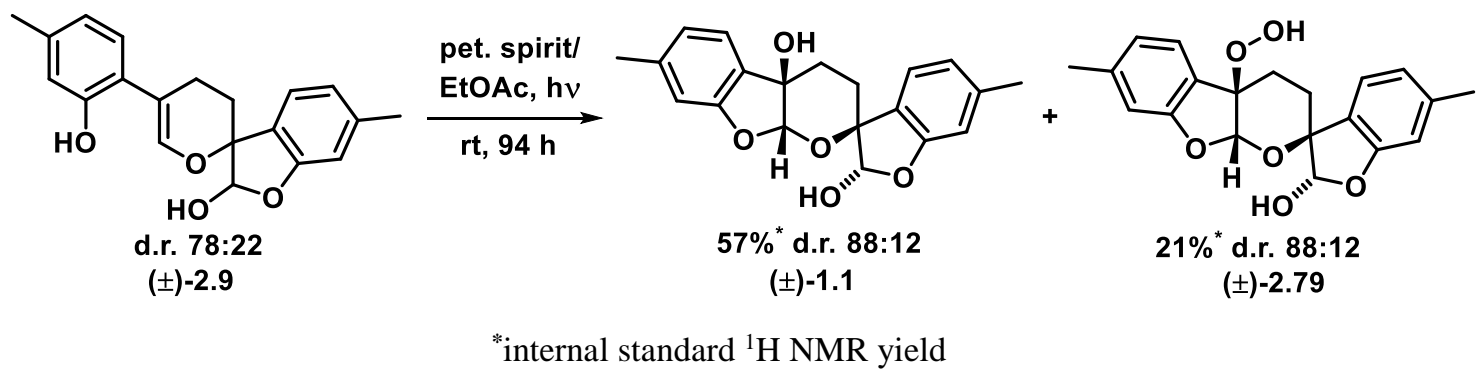

Scheme 2.71: Formation of $( \pm)$-thymarnicol 1.1.

Analytically pure samples of ( \pm )-thymarnicol 1.1 and compound ( \pm )-2.79 were obtained by preparative HPLC. After characterisation, the structure of peroxide ( \pm -2.79 was attributed to this unknown compound. Its molecular ion in the mass spectrum $\left(\mathrm{ESI}^{+}\right)$registered at $\mathrm{m} / \mathrm{z} 357.13$ was consistent with the molecular formula $\mathrm{C}_{20} \mathrm{H}_{20} \mathrm{O}_{6}$. The ${ }^{1} \mathrm{H}$ and ${ }^{13} \mathrm{C}$ NMR spectra showed similarities with the ones of $( \pm)$-thymarnicol 1.1, with a major difference. The signals in the ${ }^{13} \mathrm{C}$ NMR spectra corresponding to the major epimers of $( \pm)$-thymarnicol 1.1 and compound $( \pm)$-2.79 differ by $13.8 \mathrm{ppm}$. This is in good accordance with the difference in chemical shift reported for other systems where a benzylic hydroxyl group was replaced by a hydroperoxy group (figure 2.4). ${ }^{104}$

a)
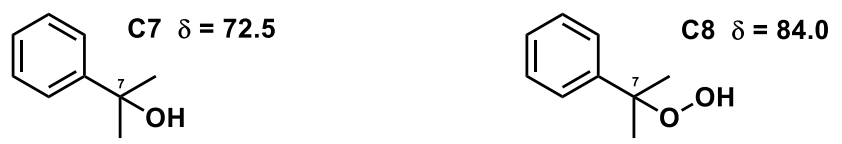

b)

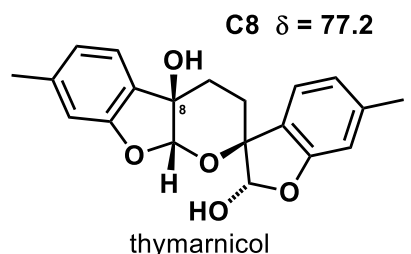

$( \pm)-1.1$

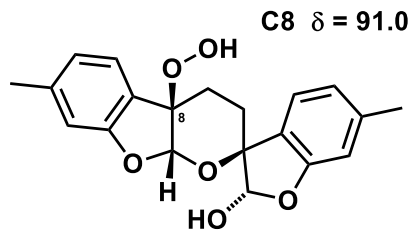

(土)-2.79

Figure 2.4: a) Comparison of carbon chemical shifts of a benzylic alcohol and hydroperoxide. ${ }^{104}$ b) Chemical shift for C8 in ( \pm )-thymarnicol 1.1 and compound ( \pm )-2.79.

It is worth noting that both ( \pm )-thymarnicol 1.1 and lactol $( \pm)-2.9$ were isolated as inseparable mixtures of epimers at the lactol position. It was found that the diastereomeric ratios calculated from the ${ }^{1} \mathrm{H}$ NMR spectra were dependent on the 
deuterated solvent used for the experiment. Some of the results are summarised on table 2.4.

\begin{tabular}{|c|c|c|}
\hline Solvent & lactol ( \pm )-2.9 (d.r.) & ( \pm )-thymarnicol 1.1 (d.r.) \\
\hline $\mathrm{CDCl}_{3}$ & $72: 28$ & $78: 22$ \\
\hline$\left(C D_{3}\right)_{2} \mathrm{CO}$ & $78: 22$ & $88: 12$ \\
\hline $\mathrm{CD}_{3} \mathrm{CN}$ & $66: 34$ & $77: 23$ \\
\hline$T H F-d_{8}$ & $75: 25$ & $87: 13$ \\
\hline$D M S O-d_{6}$ & $83: 17$ & - \\
\hline
\end{tabular}

Table 2.4: Diastereomeric ratios for ( \pm )-thymarnicol 1.1 and lactol ( \pm -2.9 in different deuterated solvents.

A diastereomerically pure sample of ( \pm )-thymarnicol $\mathbf{1 . 1}$ was obtained via recrystallisation from hot acetonitrile, and the structure of the natural product was confirmed by X-ray crystallography (figure 2.5).

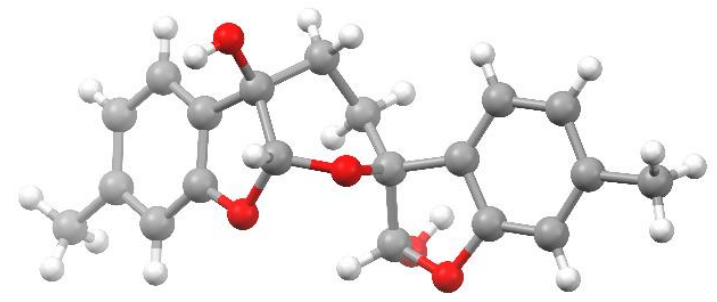

Figure 2.5: Crystal structure of ( \pm )-thymarnicol 1.1.

However, when the crystals were re-dissolved in deuterated acetone, reversion to the previously observed 78:22 d.r. was observed, thus ( \pm )-thymarnicol 1.1 also exists as a mixture of diastereoisomers at the lactol position. Attempts to obtain authentic samples of natural ( \pm )-thymarnicol $\mathbf{1 . 1}$ or the FID raw data from the isolation team in order to confirm this hypothesis were unsuccessful.

The mechanism of the formation of $( \pm)$-thymarnicol 1.1 remains unclear, but the conditions in which it occurs strongly suggest that it is the result of an autoxidation process. Autoxidation upon exposure to light and atmospheric oxygen is not an uncommon process in nature. ${ }^{105-108}$ It is therefore hypothesised that $( \pm)$-thymarnicol 1.1 could also be formed naturally as a result of a non-enzymatic oxidation. This obviously puts into question the credentials of ( \pm )-thymarnicol $\mathbf{1 . 1}$ as a natural product. 
The possibility that $( \pm)$-thymarnicol $\mathbf{1 . 1}$ could be an artefact of isolation offers different possible scenarios. For example, unprotected lactol ( \pm )-2.9 could undergo cyclisation to afford the pentacyclic framework of ( \pm )-thymarnicol $\mathbf{1 . 1}$ followed by benzylic oxidation (path a). Alternatively, an oxidation could occur initially, forming oxonium ( \pm )-2.80, which could then undergo cyclisation (path b) (scheme 2.72).<smiles>Cc1ccc(C2=CO[C@]3(CC2)c2ccc(C)cc2O[C@@H]3O)c(O)c1</smiles>

$( \pm)-2.9$

oxidation path b

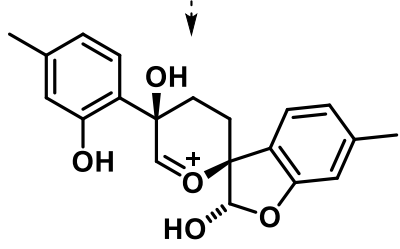

$( \pm)-2.80$

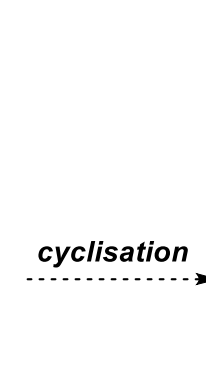

(1)

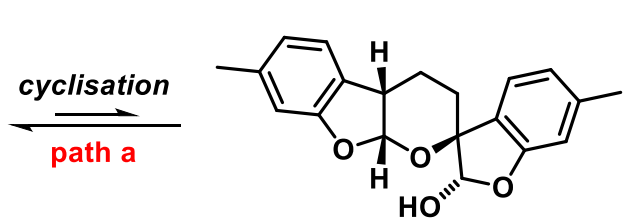

$( \pm)-2.6$

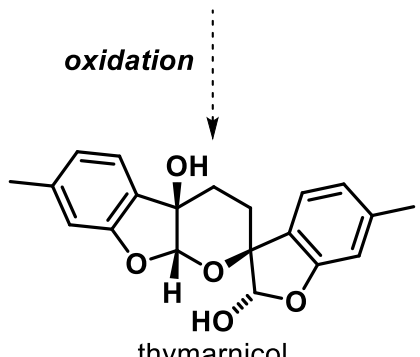

$( \pm)-1.1$

Scheme 2.72: Possible pathways for the formation of ( \pm )-thymarnicol 1.1 from lactol $( \pm)-2.9$.

We tested the possibility that the oxidative process might occur on the acylated lactol ( \pm -2.9 by subjecting acetyl protected dimer to the same conditions. Both in the presence of air or molecular oxygen we observed no reaction (scheme 2.73).

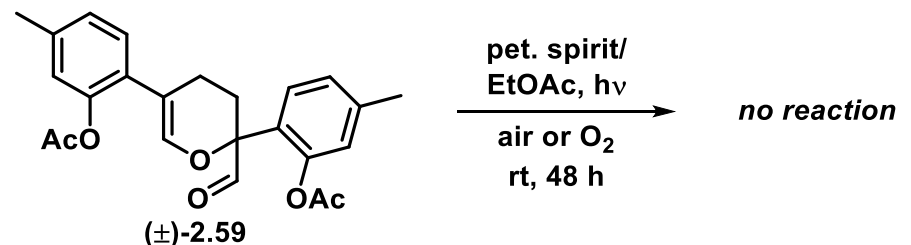

Scheme 2.73: Attempted formation of ( \pm )-thymarnicol $\mathbf{1 . 1}$ from protected dihydropyran ( \pm )-2.59.

It is therefore reasoned that the formation of ( \pm )-thymarnicol $\mathbf{1 . 1}$ is likely to occur in nature after cleavage of the acyl groups.

\subsection{Computational Studies on the Diels-Alder Dimerisation}

The key step in our synthetic sequence toward ( \pm )-thymarnicol 1.1 is the hetero-DielsAlder dimerisation. This reaction was performed with different enal starting materials, and in all cases the only product observed was the one resulting from a para- 
regiochemical orientation between the two monomeric partners; the formation of the meta-regioisomer was never detected (scheme 2.74).

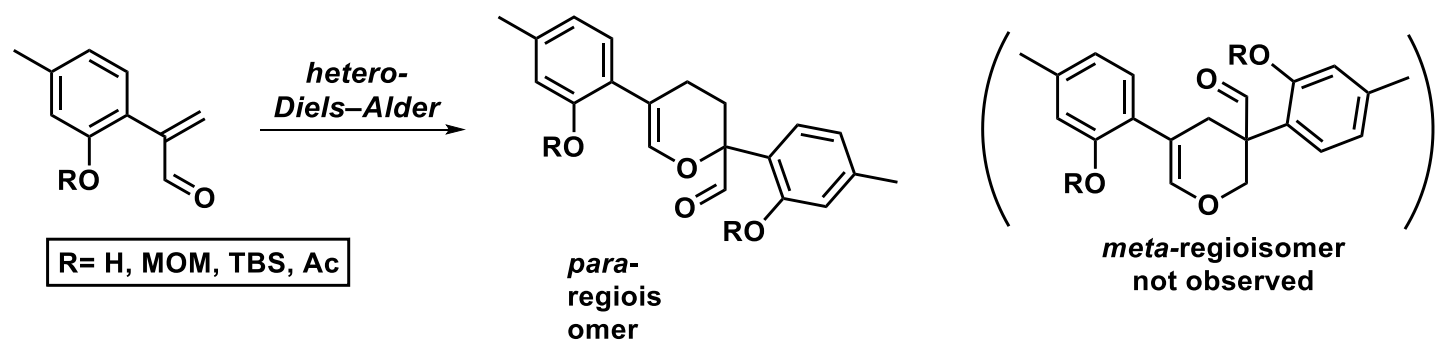

Scheme 2.74: Hetero-Diels-Alder dimerisation of differently substituted enals.

Intrigued by the degree of selectivity observed in the dimerisation, we decided to investigate this aspect further. Dr Fernanda Duarte, a newly appointed computational chemist at the University of Edinburgh, performed DFT (Density Functional Theory) calculations at the $\omega \mathrm{B} 97 \mathrm{X}-\mathrm{D} / 6-31+\mathrm{G}$ level of theory on our systems. For a simplified system (2.81) bearing no ortho-substituent there are eight different transition structures where the Alder-Stein mode is exo or endo, the dienophile adopts an s-cis or s-trans conformation and the regiochemical orientation is meta or para. With no exception, the para TSs have been found to be significantly lower in energy with respect to the meta TSs, due to lower distortion penalties and better orbital overlap. Surprisingly, the exo selectivity is observed for the majority of the transition states, due to better noncovalent interactions between the two aromatic rings (see section 4.6.3 on pages 411416 for a more detailed discussion). However, the lowest energy TS in all the systems analysed (including the differently substitutes enals) was found to be the para-endo-scis (scheme 2.75). 


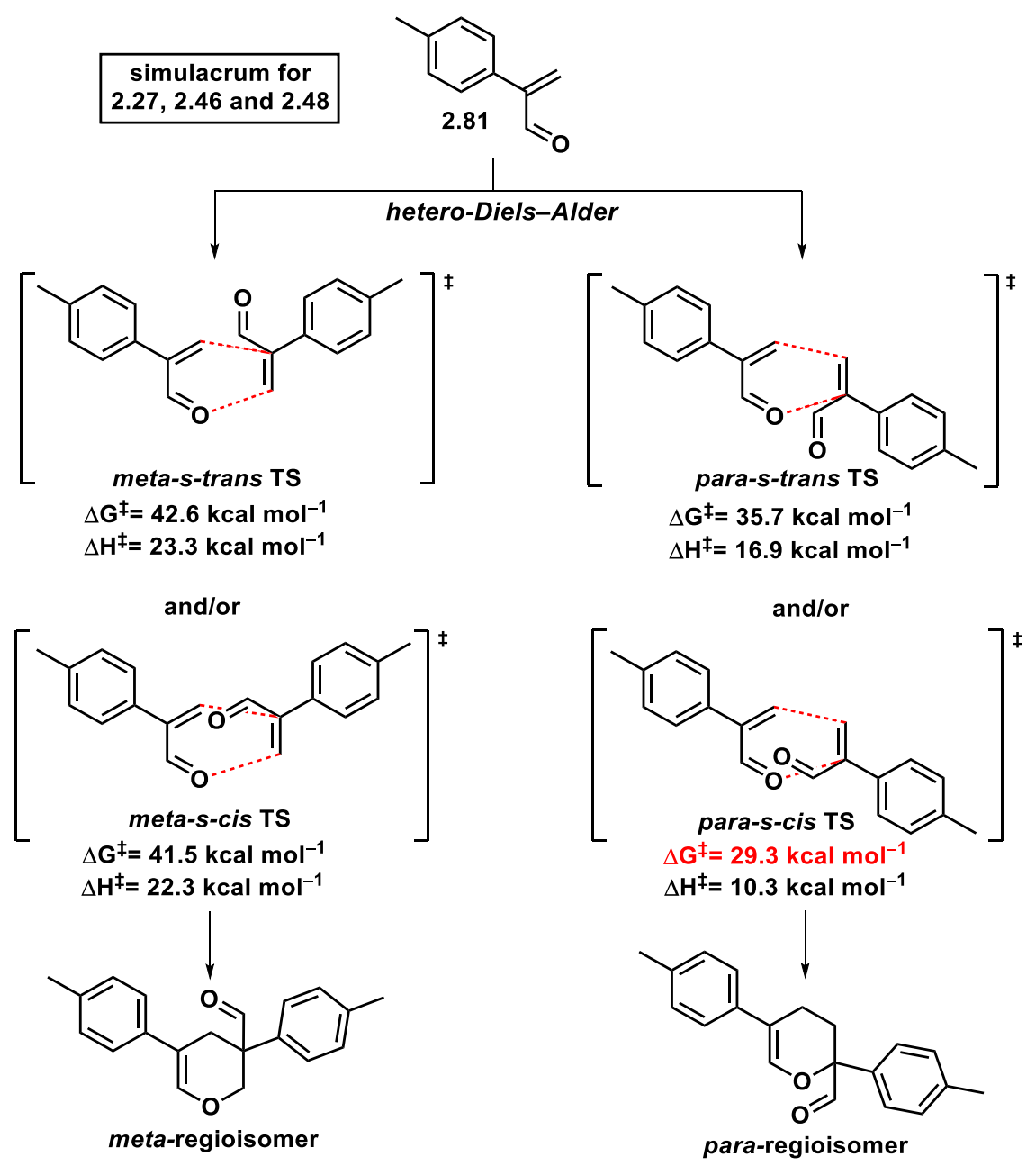

Scheme 2.75: The four endo TSs for the hetero-Diels-Alder dimerisation of our simulacrum. Energies calculated at $353 \mathrm{~K}$.

In the case of the simplified system 2.81, the para-endo-s-cis TS represents a rare example of $\mathrm{C}_{2}$-symmetric bis-pericyclic TS. The TS for the hetero-Diels-Alder dimerization of enal $\mathbf{2 . 4 8}$, although not $C_{2}$-symmetric, still has bis-pericyclic character (figure 2.6). 


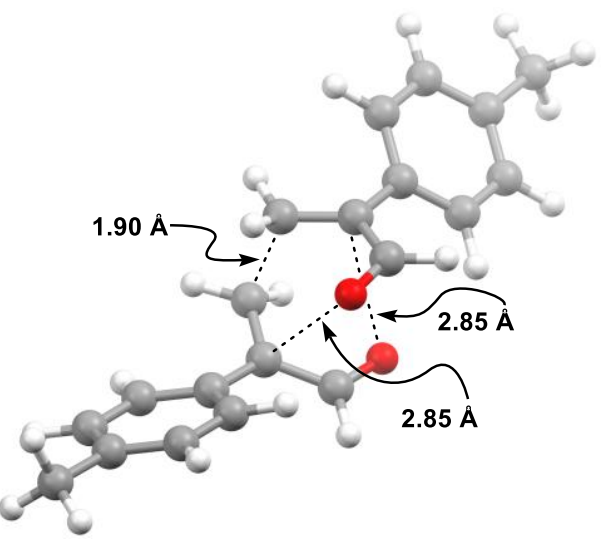

para-s-cis TS

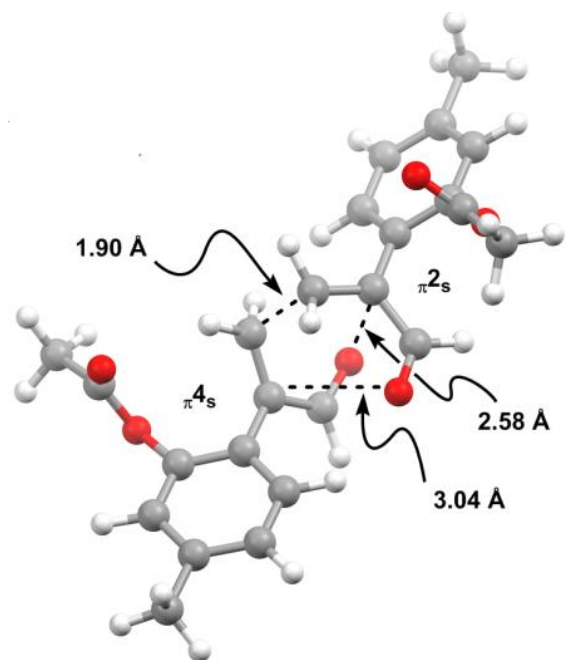

para-s-cis TS for the dimerisation of enal $\mathbf{2 . 4 8}$

Figure 2.6: para-s-cis TSs for the dimerisation of simplified simulacrum and enal 2.48.

The details of the full computational analysis performed by Dr Fernanda Duarte can be found in section 4.6 starting on page 410 . 


\subsection{Concluding Remarks}

Inspired by Passreiter's biosynthetic proposal we designed and explored alternative biomimetic pathways to $( \pm)$-thymarnicol 1.1. Investigations toward its total synthesis allowed us to confirm the chemical feasibility of the key biomimetic hetero-Diels-Alder dimerisation of unprotected lactol ( \pm )-2.1. A more synthetically practical dimerisation was achieved using suitably protected enals, in particular acetylprotected enal 2.59. After cleavage of the acyl groups on dimer $( \pm)-\mathbf{2 . 5 9}$ the final oxidation to ( \pm )-thymarnicol $\mathbf{1 . 1}$ was found to occur spontaneously in air (scheme 2.76).

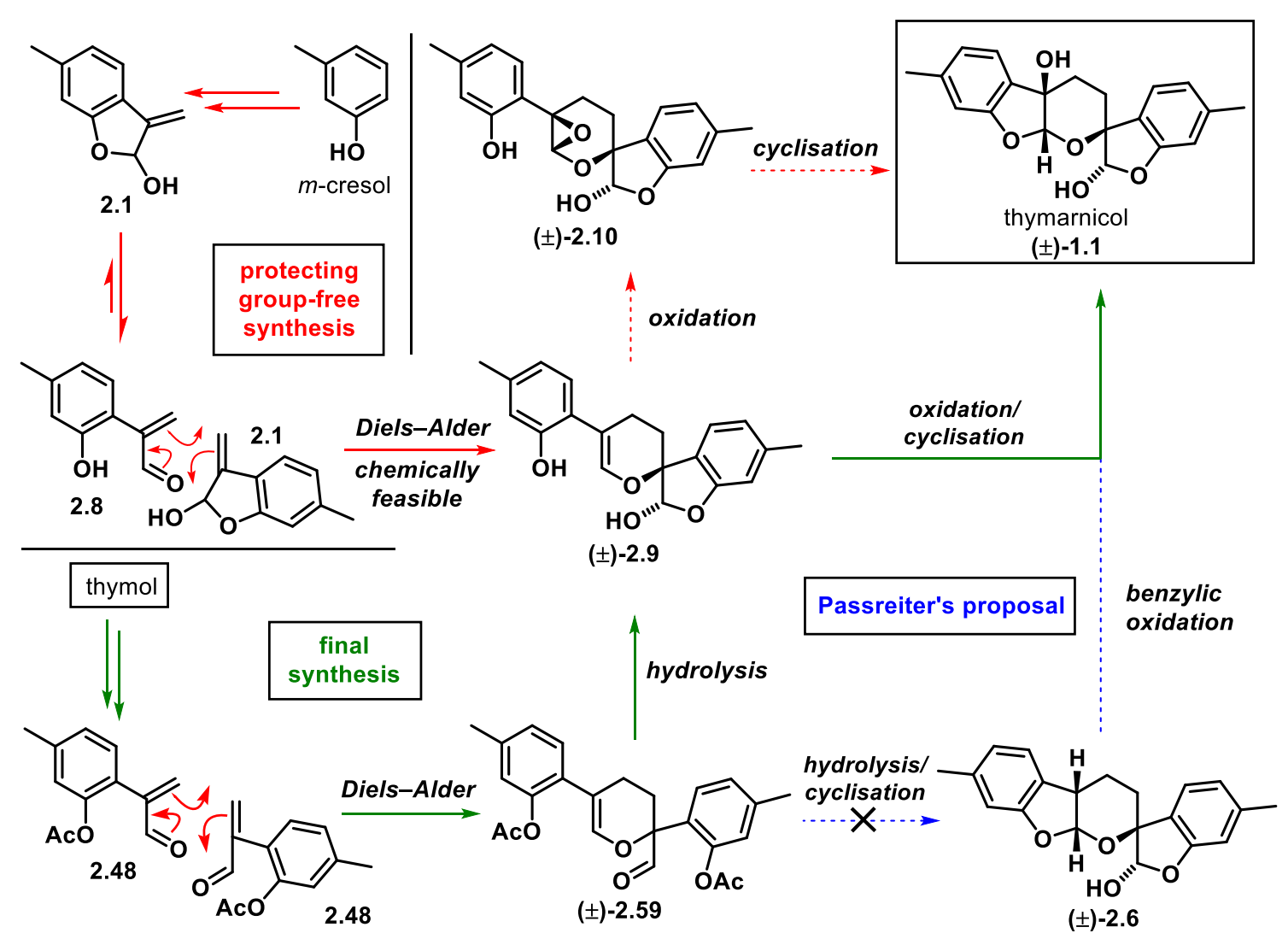

Scheme 2.76: Investigated routes toward ( \pm )-thymarnicol $\mathbf{1 . 1}$.

In conclusion, we accomplished the first biomimetic total synthesis of ( \pm )-thymarnicol 1.1 in 6-steps from commercially available 2-hydroxy-3-methyl acetophenone $\mathbf{2 . 6 3}$. The synthesis started with a high yielding Wittig methylenation, followed by phenol protection with acetic anhydride and allylic oxidation with selenium dioxide to afford enal 2.48. The subsequent hetero-Diels-Alder cycloaddition afforded dihydropyran $( \pm)-2.59$ in near quantitative yield. Cleavage of the acetyl groups on dimer $( \pm)-\mathbf{2 . 5 9}$ 
proceeded smoothly to give lactol $( \pm)-2.9$ as a mixture of epimers. Finally, the targeted natural product ( \pm )-thymarnicol $\mathbf{1 . 1}$ was shown to be produced via a remarkably regio and stereo-selective oxidation process upon exposure to air and visible light (scheme 2.77).
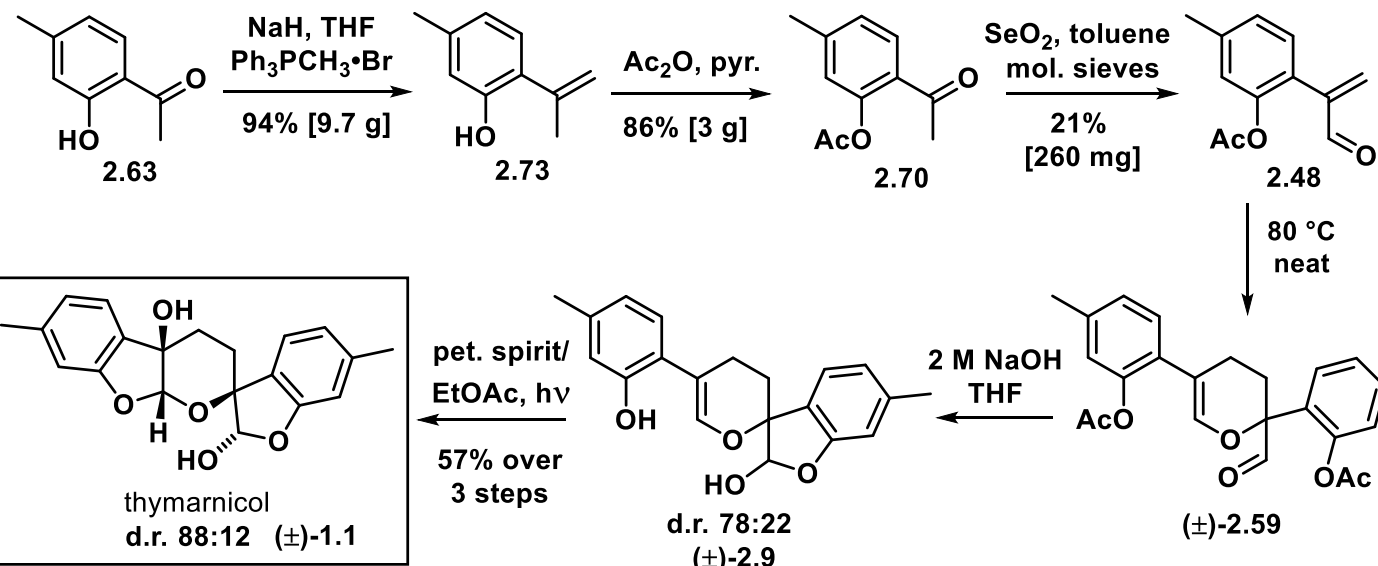

$( \pm)-2.9$

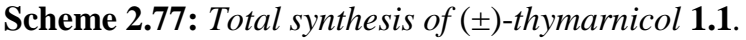

$( \pm)$-Thymarnicol 1.1 was found to be stereodynamic; it exists as a mixture of lactolepimers in solution but can exist as a single epimer in the solid state, as confirmed by single crystal X-ray diffraction studies.

The unexpected reactivity observed in our attempts to cleave the MOM-groups on protected dihydropyran ( \pm -2.28 and epoxide ( \pm -2.29 gave insights about the instability of the framework of the natural product toward acidic conditions. Finally, the autoxidation process leading to the formation of $( \pm)$-thymarnicol 1.1 raised questions about the natural product credentials of our molecular target. 


\subsection{Future Work}

The main aspect of the project which needs to be investigated further is the likely mechanism of the final transformation leading to ( \pm )-thymarnicol 1.1. Currently research is on-going to be able to identify some of the minor products of the autoxidation and to confirm the structure of compound ( \pm )-2.79. The fact that the final oxidation requires irradiation with visible light suggests a radical mechanism involving molecular oxygen. ${ }^{108}$ A possible method to verify this hypothesis is the use of an automatically controlled manostat to measure the oxygen consumed during the reaction. It should be noted, however, that the inclusion of a small quantity of BHT decreased the rate of the reaction but did not suppress it. The addition to the reaction mixture of rose bengal as a photosensitiser did not result in an increase in the reaction rate, thus suggesting singlet oxygen is not involved in the process. As no conclusive evidence has been obtained so far there is the need to access larger quantities of lactol ( \pm -2.9 to investigate this final step. Therefore, a scale-up and optimisation of the synthesis, together with a better HPLC method for the purification of ( \pm )-thymarnicol 1.1, are needed. With regard to the synthetic sequence, efforts will focus on the optimisation of the existing approach more than on the design of a shorter one. In particular, the allylic oxidation needs to be revised. An alternative to selenium dioxide could be the use of pentafluorobenzene selenic acid (figure 2.7), which has been reported to be an efficient reagent for the oxidation of alkenes at the allylic position to afford enals. The main advantage in the use of this reagent is its increased eneophilicity and therefore reactivity due to the per-fluorination of the benzene ring. However, the oxidation requires 2 equivalents of selenium reagent, for which there are no commercial sources and therefore needs to be prepared. ${ }^{109}$ Another possible alternative for the synthesis of enal $\mathbf{2 . 4 8}$ from alkene $\mathbf{2 . 7 0}$ would be the use of the Etard reaction, involving chromyl chloride as the oxidant ${ }^{110}$ (figure 2.7). Both these reagents allow the direct allylic oxidation of alkenes to enals without revealing the primary alcohol, and could therefore be suitable for our purposes.
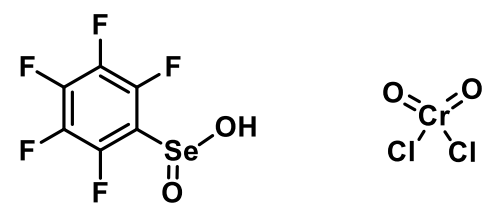

Figure 2.7: Pentafluorobenzene selenic acid and chromyl chloride. 


\section{Biomimetic Diversity-Oriented Synthesis of Prenylated Phenylpropanoids isolated from Illicium Plants}

\subsection{Introduction}

\subsubsection{Biomimetic Diversity Oriented Synthesis}

Bohacek et al. in 1996 estimated that the number of possible organic compounds with a molecular weight smaller than 500 Dalton is over $10^{60} . .^{111}$ It is clear that there is an enormous area of chemical space which has not yet been explored. Although it seems to be not possible to access all these structures, efforts have been made to develop strategies to rapidly explore chemical space. An approach to this problem is the use of Diversity Oriented Synthesis (DOS), a strategy to generate molecular diversity from common starting materials. DOS aims to allow the synthesis of collections of compounds which differ in their molecular structure. ${ }^{112}$ This can be obtained with the use of different strategies. The most straightforward is the use of "appendage decoration", which involves the use of coupling reactions to differently modify a common molecular framework. Another approach takes advantage of regio- and/or stereoselective reactions to generate different product isomers. Finally, the use of divergent reaction pathways allows the generation of structural diversity through different regio- and/or chemoselective transformations. Thus, a single starting material is converted into one or more intermediates which, in turn, are modified further to generate a selection of products possessing diverse molecular architectures, as exemplified in scheme 3.1.

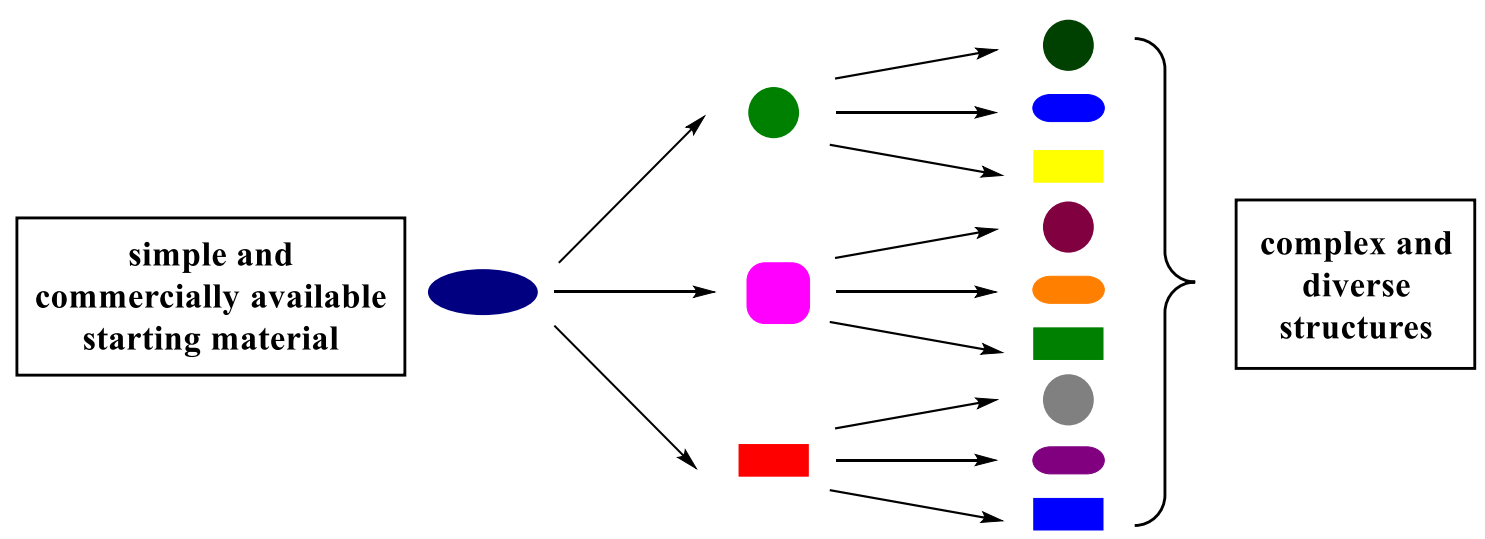

Scheme 3.1: Schematisation of DOS. 
Nature has the ability to rapidly access an incredible number of diverse structures starting from a relatively small number of simple building blocks. It is technically very challenging to try and imitate divergent biosynthetic pathways, but there exist recent successful examples of biomimetic divergent syntheses, for example by Tan, ${ }^{113}$ Snyder ${ }^{114}$ and Shair. ${ }^{115,116}$ Shair and co-workers, for instance, exploied the potential of the divergent biomimetic approach in the synthesis of a group of Lycopodium alkaloids, accessed in a small number of steps from common intermediate $\mathbf{3 . 1}$ (scheme 3.2). ${ }^{116}$ The unified strategy was based on a proposed biosynthetic network connecting the natural product targets.

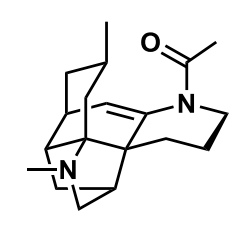

(+)-fastigiatine

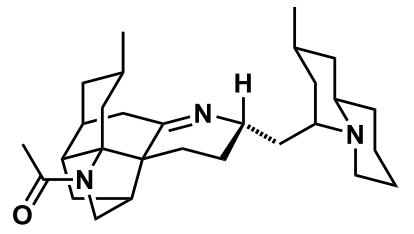

(-)-himeraldine A

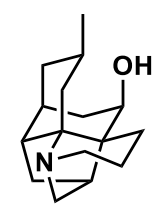

(-)-lycopecurine

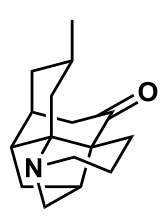

(-)-dehydro-lycopecurin

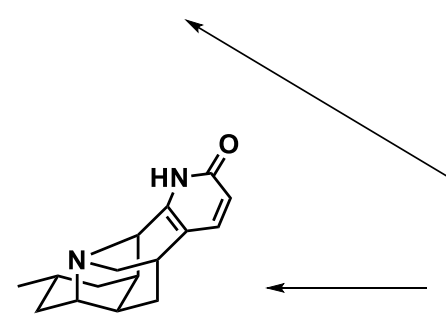

$(+)$-lyconadin A

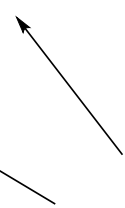<smiles>CC1CC=CCC1</smiles>

steps

steps

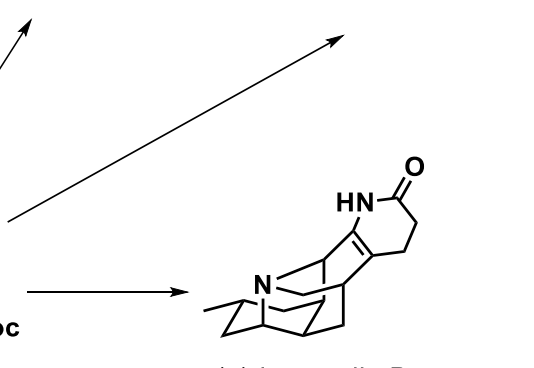

(-)-lyconadin B

Scheme 3.2: Shair's biomimetic divergent synthesis of Lycopodium alkaloids.

This work shows how several complex natural products featuring diverse carbon skeletons can be rapidly accessed from the same synthetic intermediate. The rapid generation of complexity and diversity provides a significant advantage with respect to target oriented synthesis in terms of time and resources needed.

\subsubsection{Prenylated Phenylpropanoids from Illicium Genus Plants}

This project is concerned with the total synthesis of naturally occurring prenylated phenylpropanoids from Illicium plants. A large number of these compounds have been isolated starting from the early 80 's, with an increase in the number and complexity of new compounds discovered in the last five years. ${ }^{117-134}$ A small selection of prenylted 
phenylpropanoids isolated from plants belongin to the Illicium genus are depicted in figure 3.1.

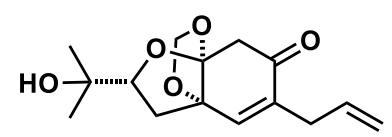

(+)-epi-illicinone E

3.2

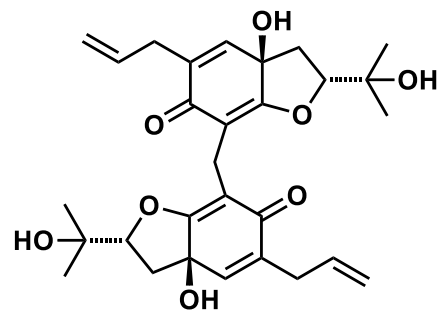

(+)-illicidione B

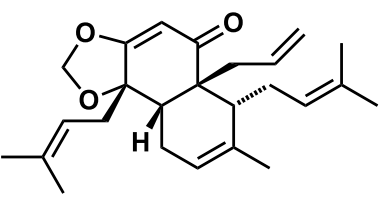

$(+)$-cycloillicinone

1.6

3.3

Figure 3.1: Selected examples of prenylated phenylpropanoids.

In the last few years there has been a growing interest in the prenylated phenylpropanoids. The natural abundance and structural complexity of these compounds, along with the wide range of bioactivities they display make them particularly attractive targets. Reported biological activities include anti-oxidant, ${ }^{131}$ anti-viral, ${ }^{129}$ anti-inflammatory properties, ${ }^{122,126,127,130}$ significant cytotoxicity against some human cancer cell lines, ${ }^{124,129}$ proliferative activity in primary cell culture of osteoblast cells, ${ }^{133}$ and moderate neurite-outgrowth promoting activity in primary cultured rat cortical neurons. ${ }^{127}$ Interestingly, the reported specific optical rotation for some Illicium natural products have small absolute values $\left([\alpha]_{\mathrm{D}}^{20}<10^{119,121,126,134}\right.$ or even $<5^{126,127,132}$ ). It is therefore possible that some of them could actually exist in nature as scalemic mixtures, with minor impurities affecting the observed optical rotation values. The interest of the scientific community toward this class of natural products resulted in the total synthesis of some of the most interesting members of this group of compounds, which will be discussed in the next section. 


\subsection{Previous Syntheses of Prenylated Phenylpropanoids from Illicium Plants}

This section describes work which has been done in other research groups on the synthesis of prenylated phenylpropanoids isolated from Illicium plants.

\subsubsection{Tricycloillicinone 1.4}

(-)-Tricycloillicinone $\mathbf{1 . 4}$ was a metabolite isolated in 1995 by Fukuyama and coworkers from the wood of Illicium tashiroi. ${ }^{134}$ This compound features a tetracyclic framework containing three stereogenic centres, the relative stereochemistry of which was elucidated from nOe experiments. Fukuyama proposed that (-)-tricycloillicinone 1.4 could be biosynthesised from the co-isolated natural product (-)-illicinone A $\mathbf{1 . 3}$ by sequential cyclisation of the prenyl side-chain. Interestingly, the first semisynthesis of (-)-tricycloillicinone $\mathbf{1 . 4}$ was accomplished in 1984 Furukawa and collaborators eleven years before its first isolation from natural sources. They were investigating the reactivity of (-)-illicinone A $\mathbf{1 . 3}$, a natural product they had recently isolated, ${ }^{135}$ by irradiating it with a $400 \mathrm{~W}$ high pressure mercury lamp. ${ }^{136}$ The major product of this reaction was found to be a novel polycyclic compound, later named (-)-tricycloillicinone $\mathbf{1 . 4}$ by Fukuyama. ${ }^{134}$ The first total synthesis (as opposed to semisynthesis) of ( \pm )-tricycloillicinone 1.4 was accomplished in 1998 by Danishefsky and co-workers from commercially available sesamol. This synthesis started with a highly efficient $O$-allylation to afford ether 3.4, followed by regioselective thermal Claisen rearrangement to give phenol 3.5, which was subsequently subjected to $O$-prenylation to provide the known natural product illicinole 3.6. The transformation of illicinole 3.6 into $( \pm$ )-illicinone A 1.3 was then investigated using a number of different catalysts. It was found that the use of the bulky Lewis acid catalyst MABR (methylaluminum bis(4-bromo-2,6-di-tert-butylphenoxide)), developed by Yamamoto and co-workers, ${ }^{137}$ gave the best results in terms of regioselectivity, affording ( \pm )-illicinone A $\mathbf{1 . 3}$ in $70 \%$ yield on a $40-\mathrm{mg}$ scale. ${ }^{120}$ The final photochemical oxidation was performed under similar conditions to the ones reported in the literature, with an optimised yield of $35 \%$ (scheme 3.3). 


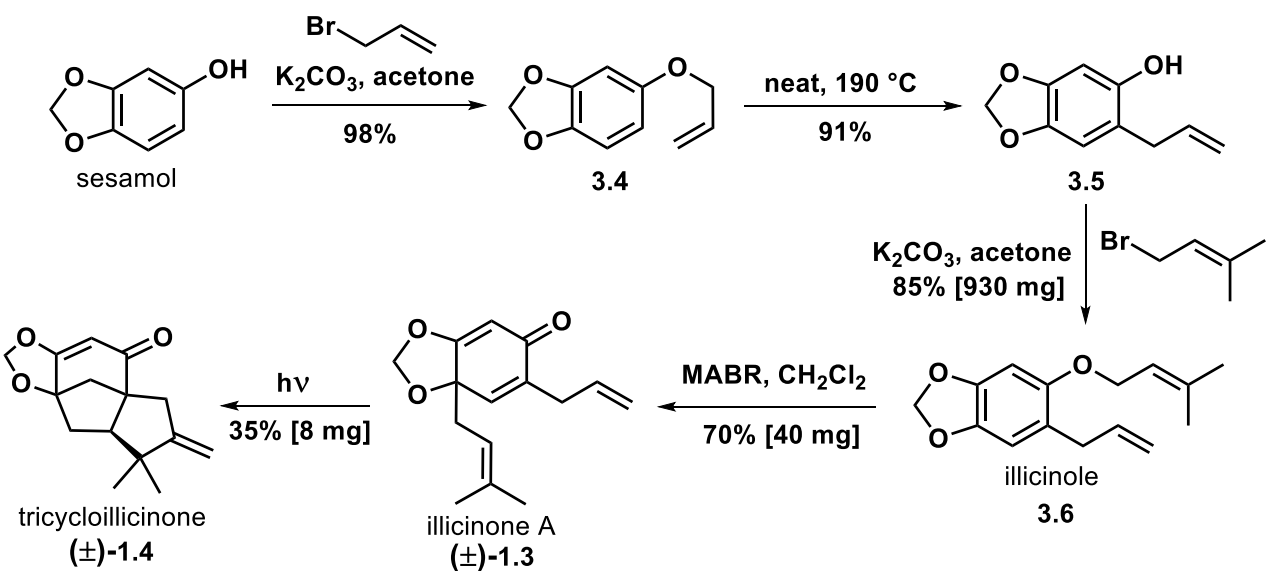

Scheme 3.3: Danishefsky's synthesis of $( \pm)$-tricycloillicinone 1.4.

The mechanism of the reaction leading to $( \pm)$-illicinone A 1.3 was also investigated, using an isotopically labelled prenyl group, but no conclusive results were obtained. Different plausible mechanisms have been proposed by Danishefsky for this MABRmediated transformation. ${ }^{120}$ One possibility involves a dissociation/reassociation pathway, resulting overall in the migration of the prenyl group from the oxygen to $\mathrm{C} 4$. Alternatively, a Claisen rearrangement was proposed to occur in either the $\mathrm{C} 2$ or $\mathrm{C} 6$ positions, to produce reverse-prenylated products 3.7 or 3.8. At this point the steric hindrance of the bulky catalyst, as well as the relative instability of the reverse-prenyl group, would play a role in favouring the Cope rearrangement, which in both cases would direct the prenyl group to the C4 position (scheme 3.4).

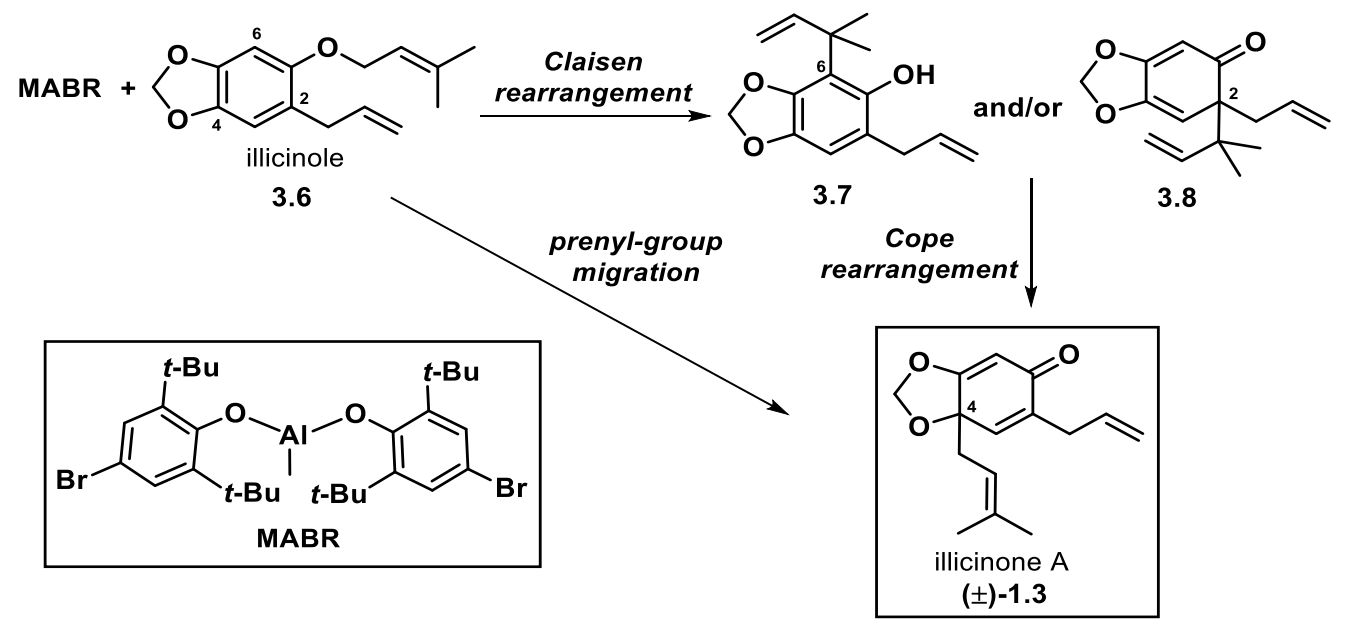

Scheme 3.4: Proposed mechanism for the formation of $( \pm)$-illicinone A 1.3. ${ }^{120}$ 
Illicinone A 1.3 has been isolated multiple times from different Illicium plants, and has been found to exist as a single enantiomer in nature. It is noteworthy that the absolute stereochemistry varies depending on the species. For example, Illicinone A 1.3 exists as the levorotatory enantiomer in Illicium arborescens ${ }^{128}$ and some Illicium tashiroi $^{134,136}$ species, while the opposite stereochemistry is encountered in Illicium anisatum. $^{121}$

\subsection{2 (-)-Spirooliganones A 1.7 and B 3.9}

At the time we started our efforts to develop a unified synthetic strategy towards the synthesis of our molecular targets, only the synthesis of (-)-tricycloillicinone $\mathbf{1 . 4}$ had been reported. During our investigation, the synthesis of other prenylated phenylpropanoids was published, including the spirooliganones A 1.7 and B 3.9. (-)-Spirooliganones A 1.7 and B 3.9 were isolated in 1993 by Yu and co-workers from the roots of Illicium oligandrum and were found to display antiviral activity against a variety of viral strains. ${ }^{129}$ Spirooliganones A 1.7 and B 3.9 are a pair of epimers at C17, one of the two spiro carbons present in their rare dioxaspiro skeleton. $\mathrm{Yu}$ and collaborators proposed a biosynthetic pathway for the formation of these two compounds from allylated polyhydroxylated phenol 3.10. In their hypothesis, this compound could be regioselectively prenylated to afford dearomatised compound 3.11, which would then undergo [1,3]-shift of the prenyl side-chain and diastereoselective epoxidation to give compound $\mathbf{3 . 1 2}$ with the required stereochemistry at C11. Spirocyclic compound $\mathbf{3 . 1 3}$ would then be formed via intramolecular cyclisation. SAM-mediated methylation would then install the required carbon atom for the subsequent formation of enone 3.14 through oxidation and dehydration of the newly introduced methyl side-chain. (-)-Spirooliganones A 1.7 and B 3.9 would then be produced via a hetero-Diels-Alder cycloaddition between enone 3.14 and (-)-sabinene, a naturally occurring monoterpene. This last step is proposed to occur via a non-enzymatic process with high regio- but lack of stereo-control to produce the (-)-spirooliganones as a pair of epimers at C17 (scheme 3.5). 


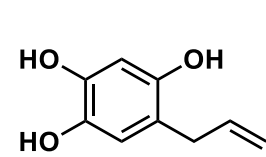

3.10<smiles>C=CCC1(C=C(C)C)C=C(O)C(O)=CC1=O</smiles>

[1,3]-migration/ epoxidation<smiles>C=CCC1=CC(O)(CC2OC2(C)C)C(O)=CC1=O</smiles>

cyclisation<smiles>C=CCC1=CC2(CC(O)C(C)(C)O2)C(=O)C(CO)C1=O</smiles>

oxidation<smiles>CC1OC2(C=C(CC=CC=CC(C)(C)C)C(=O)C2C)C(=O)C(C)C1=O</smiles><smiles>C=CCC1=CC2(CC(O)C(C)(C)O2)C(O)=CC1=O</smiles>

dehydration

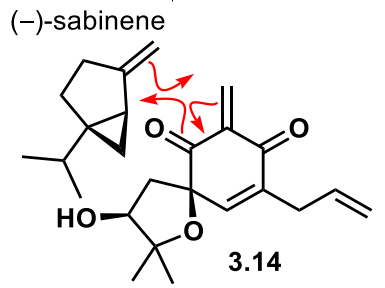

hetero-DielsAlder

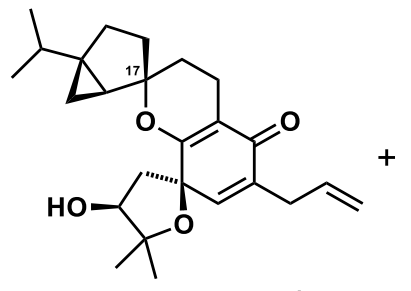

(-)-spirooliganone A 1.7

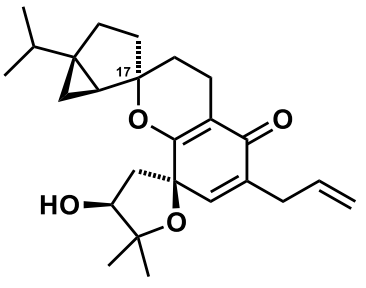

(-)-spirooliganone B 3.9

Scheme 3.5: Yu's proposed biosynthesis of (-)-spirooliganones $A$ 1.7 and B 3.9.

To date the asymmetric total synthesis of (-)-spirooliganones A 1.7 and B 3.9 has been reported three times. The first total synthesis was accomplished by Xie and co-workers in 2014 in eight steps. ${ }^{138}$ The synthetic sequence involved a three component heteroDiels-Alder cycloaddition as the first step, rather than having this transformation at a later stage, as proposed by the isolation team in their biosynthetic hypothesis. Reaction between 1,3-cyclohexadione, formalin and (-)-sabinene afforded two diastereomeric tetracycles (17S)-3.15 and (17R)-3.15 with very little stereopreference. Each one of the two epimers was then subjected separately to the same sequence involving aromatisation, $O$-prenylation of the resulting phenol 3.16, Claisen-Cope rearrangements and $O$-allylation to afford ether 3.17. Highly diastereoselective Sharpless dihydroxylation of the prenyl side-chain gave diol 3.18, which underwent a Claisen rearrangement of the allylic group to produce phenol 3.19. The synthesis of both (-)-spirooliganones A 1.7 and B 3.9 was finally achieved in $2 \%$ and $6 \%$ overall yield respectively via an intramolecular PIDA-mediated oxidative dearomatisation (scheme 3.6). 

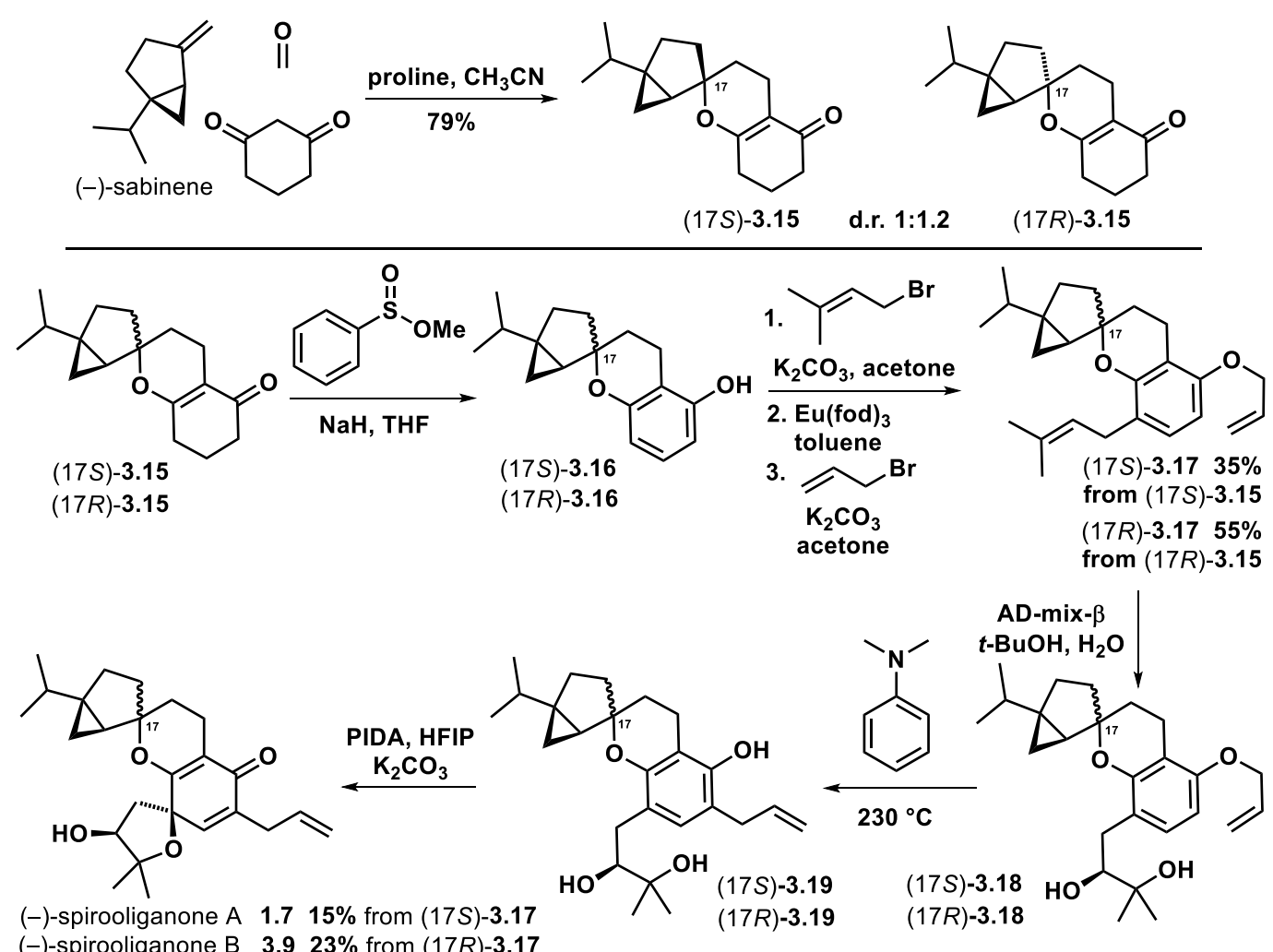

Scheme 3.6: Xie's total synthesis of (-)-spirooliganones $A$ 1.7 and B 3.9.

In the same year, Tong and co-workers reported the enantioselective synthesis of (-)-spirooliganones A 1.7 and B 3.9 in twelve steps. ${ }^{139}$ The highlight in this synthesis was a one-pot Claisen rearrangement/hetero-Diels-Alder cycloaddition to construct the central tetracyclic core of the natural products. The synthesis started from commercially available 2,6-dihydroxybenzoic acid with acetonide formation and $O$-reverse prenylation to give acetal 3.20. Reduction of the acetonide followed by double protection with acetic anhydride afforded ether 3.21, which was then subjected to the one-pot Claisen rearrangement/ortho-quinone methide formation/hetero-DielsAlder sequence. This reaction cascade afforded spirocyclic compounds (17S)-3.22 and (17R)-3.22 in a 1:1 diastereomeric ratio. The mixture was subjected to highly diastereoselective Sharpless dihydroxylation, after which the two epimers were separated. The two diols were then converted separately into the corresponding spirooliganones in seven steps via installation of the allyl group, and oxidative dearomatisation through intramolecular spirocyclisation, with conditions similar to the ones employed by Xie and collaborators (scheme 3.7). 

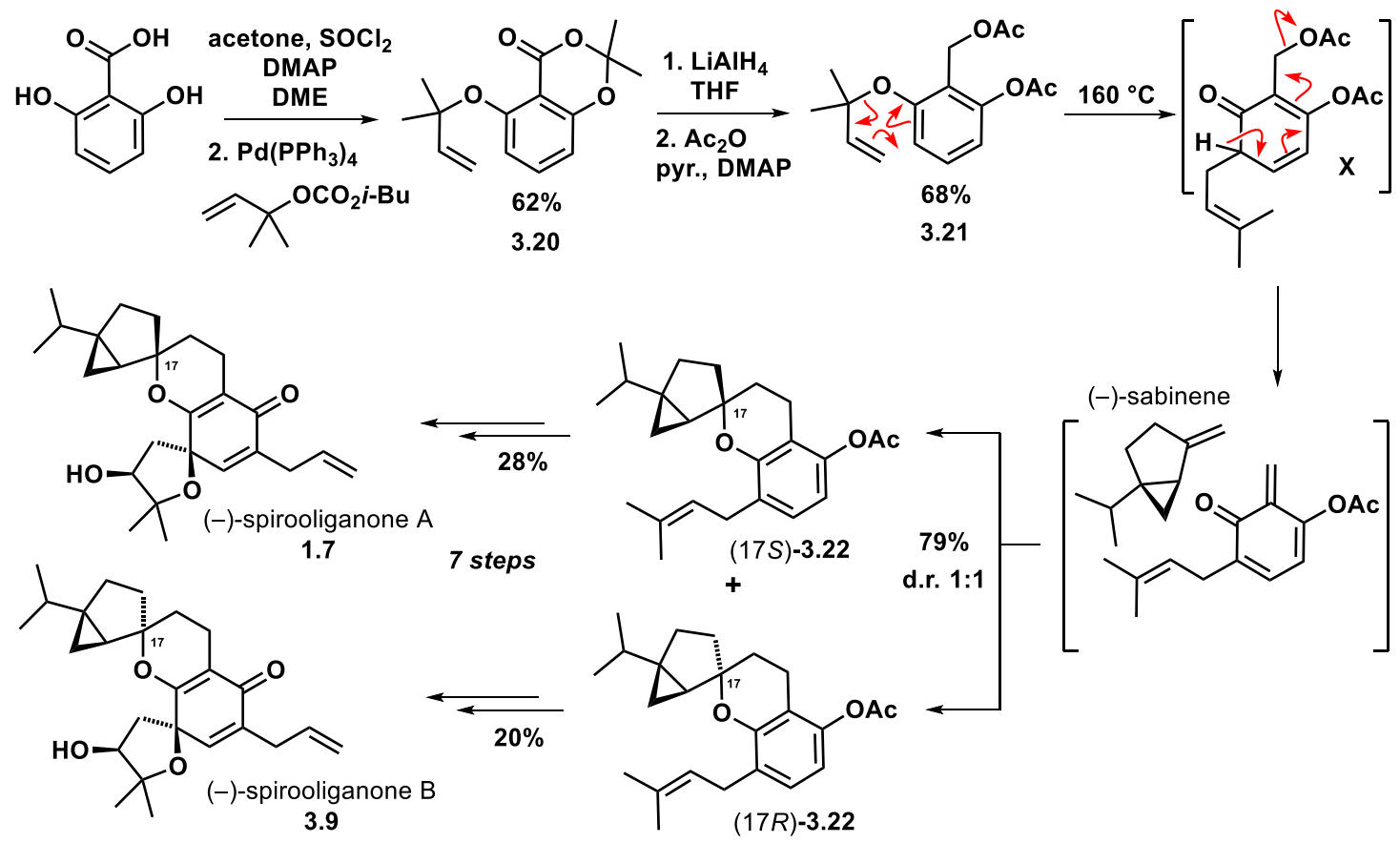

Scheme 3.7: Tong's total synthesis of (-)-spirooliganones $A$ 1.7 and B 3.9.

The most recent synthesis of spirooliganones A 1.7 and B 3.9 was achieved in 2015 by $\mathrm{Yu}$ and co-workers in eleven steps. ${ }^{140}$ The approach followed was very similar to the one employed by Tong, and mainly differed with regard to the installation of the allyl group, which was performed earlier in the synthesis. Yu and collaborators turned the poor diastereoselectivity achieved in their dihydroxylation to their advantage to prepare all eight possible diastereoisomers accessible when using $(-)$-sabinene as the dienophile.

\subsection{3 (+)-Illicidione A 1.5 and (+)-Illihendione A 3.23}

(+)-Illicidione A 1.5 was isolated by Liu and co-workers in 2011 from the stem bark of Illicium oligandrum and represents the first reported prenylated $\mathrm{C}_{6}-\mathrm{C}_{3}$ dimer from the Illicium plants. ${ }^{126}$ Two years later, (+)-illicidione A 1.5 was isolated for the second time by Hou and collaborators from the roots of Illicium henryi, together with (+)-illihendione A 3.23, a closely related new dimeric structure. ${ }^{131}$ Hou and collaborators also co-isolated the simpler natural products (-)-illihenryfunone C 3.24 and (+)-illihenrypyranone A 3.25. The former can be considered as a hydrated building block for (+)-illicidione A $\mathbf{1 . 5}$ and the latter is a hydrated diastereoisomer of the plausible monomeric unit for (+)-illihendione A 3.23. It is likely that in nature the two 
dimeric structures are the result of a hetero- (1.5) and homo (3.23) Diels-Alder cycloaddition reaction of dienes 3.26 and 3.27 (scheme 3.8).

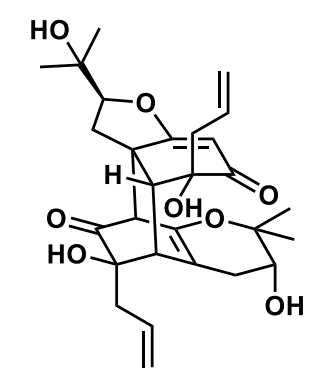

(+)-illicidione A

1.5<smiles>C1=CC=C1</smiles>

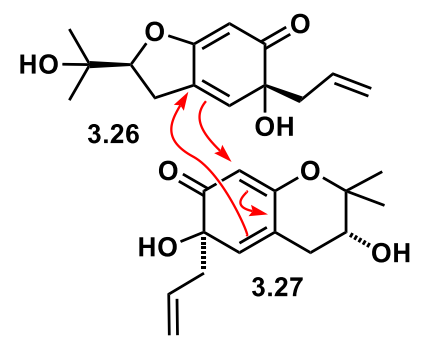

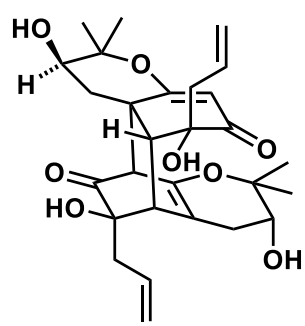

(+)-illihendione A

3.23

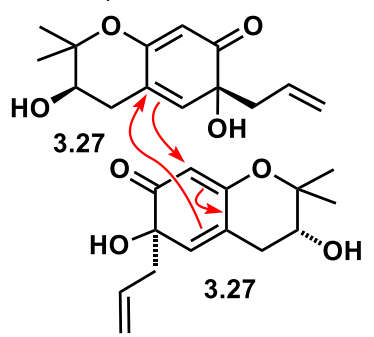

Compounds co-isolated

with (+)-illicidione A $\mathbf{1 . 5}$

and (+)-illihendione A 3.23

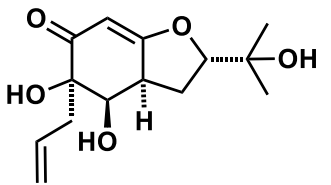

(-)-illihenryfunone C

3.24

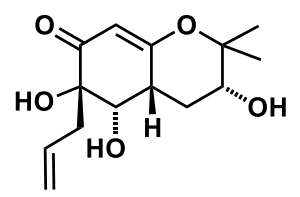

(+)-illihenrypyranone A

3.25

Scheme 3.8: (+)-Illicidione A 1.5, (+)-illihendione A 3.23, and co-isolated compounds 3.24 and 3.25.

When we started working on this project, the total synthesis of these compounds had not been reported. However, in $2015 \mathrm{Yu}$ and co-workers accomplished the first total synthesis of the two dimeric natural products (+)-illicidione A 1.5 and (+)-illihendione A 3.23 in eight and nine steps respectively ${ }^{141}$ from known compound 3.28. Initial phenol double-protection as the TBS-ether and Shi epoxidation afforded epoxide 3.29 with good enantioselectivity. Acid and base-promoted intramolecular cyclisations then gave bicycles $\mathbf{3 . 3 0}$ and $\mathbf{3 . 3 1}$ respectively. These were separately converted into their corresponding allylated analogues $\mathbf{3 . 3 2}$ and $\mathbf{3 . 3 3}$ via a sequence of iodination, protection of the hydroxyl groups, Suzuki coupling to install the allyl group and phenol deprotection. By subjecting equimolar quantities of two phenols 3.32 and 3.33 to SIBX oxidative dearomatisation conditions, $\mathrm{Yu}$ and co-workers found that the Diels-Alder cycloadditions occur spontaneously at room temperature to produce the framework of the two natural products. Final benzyl-ether deprotection afforded (+)-illicidione A $\mathbf{1 . 5}$ and (+)-illihendione A $\mathbf{3 . 2 3}$ in $8 \%$ and $13 \%$ overall yield respectively (scheme 3.9). 


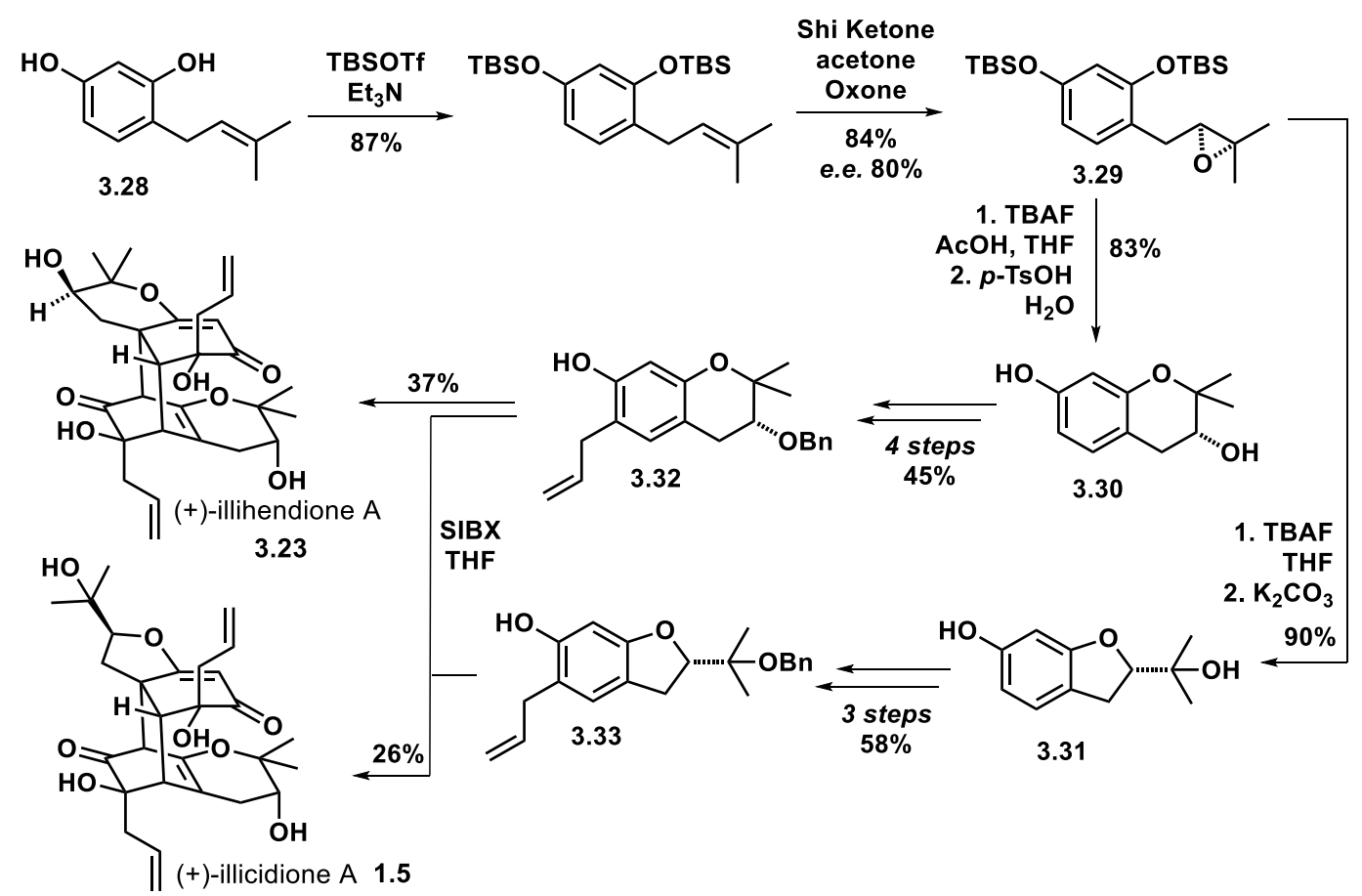

Scheme 3.9: Yu's total synthesis of (+)-illicidione A 1.5, (+)-illihendione A 3.23.

The facile nature of this final transformation could explain why the two putative monomeric units 3.26 and 3.27 (see scheme 3.8 on page 97) have never been isolated from natural sources. Interestingly, when subjecting benzyl-ether $\mathbf{3 . 3 3}$ to the same dearomatisation conditions, no homo-dimerisation was observed, thus suggesting enone 3.33 cannot serve as a diene, but solely as a dienophile (scheme 3.10).

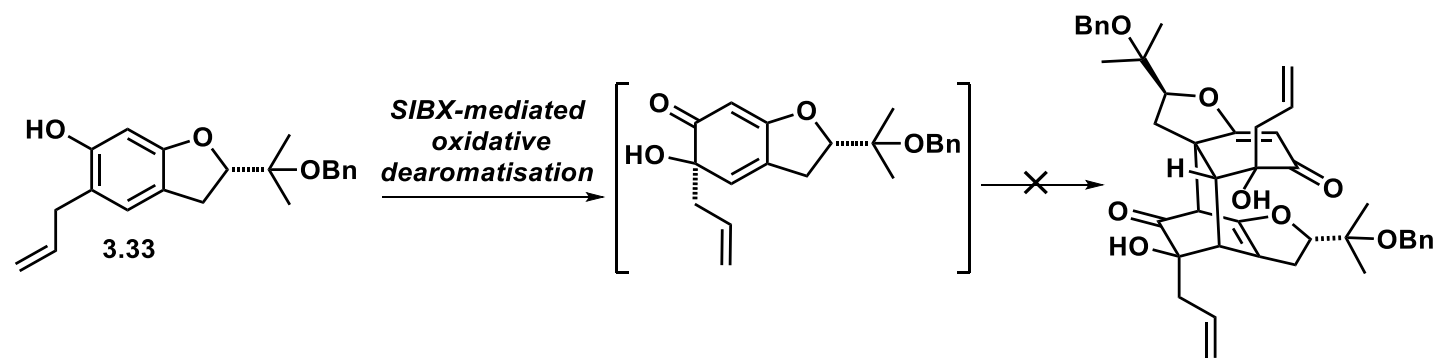

Scheme 3.10: Attempted dimerisation of enone 3.33. 


\subsection{Our Biosynthetic Proposal}

To date, more than 100 different prenylated phenylpropanoids have been isolated from plants of the illicium genus, differing with regard to the position of the allyl and prenyl groups on the phenolic ring and variations in their oxidation state. Most of these compounds (81) can be seen as biosynthetically derived from a common precursor. The structural diversity present in the core frameworks of the prenylated phenylpropanoids can be, formally at least, explained through two divergent biosynthetic pathways distinguished by either a normal or reverse $O$-prenylation. In an effort to compartmentalise this structural diversity we propose that an oxygenated phenyl propanoid moiety, derived from the shikimate pathway, could undergo $O$-prenylation or $O$-reverse-prenylation, leading to what we herein classify as type A0 and type $\mathbf{B}_{0}$ structures. Subsequently, a Claisen rearrangement could occur, leading to type $A_{1}$ and type $\mathbf{B}_{1}$ structures, with twelve known natural products belonging to the type A1. It should be noted that no examples of type $\mathbf{A}_{0}$ and $\mathbf{B}_{1}$ prenylated phenylpropanoids have been isolated to date. Finally, a Cope rearrangement would give type $\mathbf{A}_{2}$ and type $\mathbf{B}_{2}$ structures, with type $\mathbf{B}_{2}$ being the most commonly encountered framework for natural prenylated phenylpropanoids. Our proposed conceptual biosynthetic network with examples of known natural products possessing type $B_{0}$, type $A_{1}$, type $A_{2}$ and type $B_{2}$ structures is shown in scheme 3.11.

It is important to note that this proposal is provided principally as a conceptual framework through which we can better understand the molecular diversity of prenylated phenylpropanoid natural products. In reality, there might be specific prenyl transferase enzymes ${ }^{142}$ that can install the prenyl group selectively and directly at all relevant positions. Indeed, there may be prenyl transferase enzymes that install the prenyl group at one position and then catalyse a sigmatropic rearrangement. 


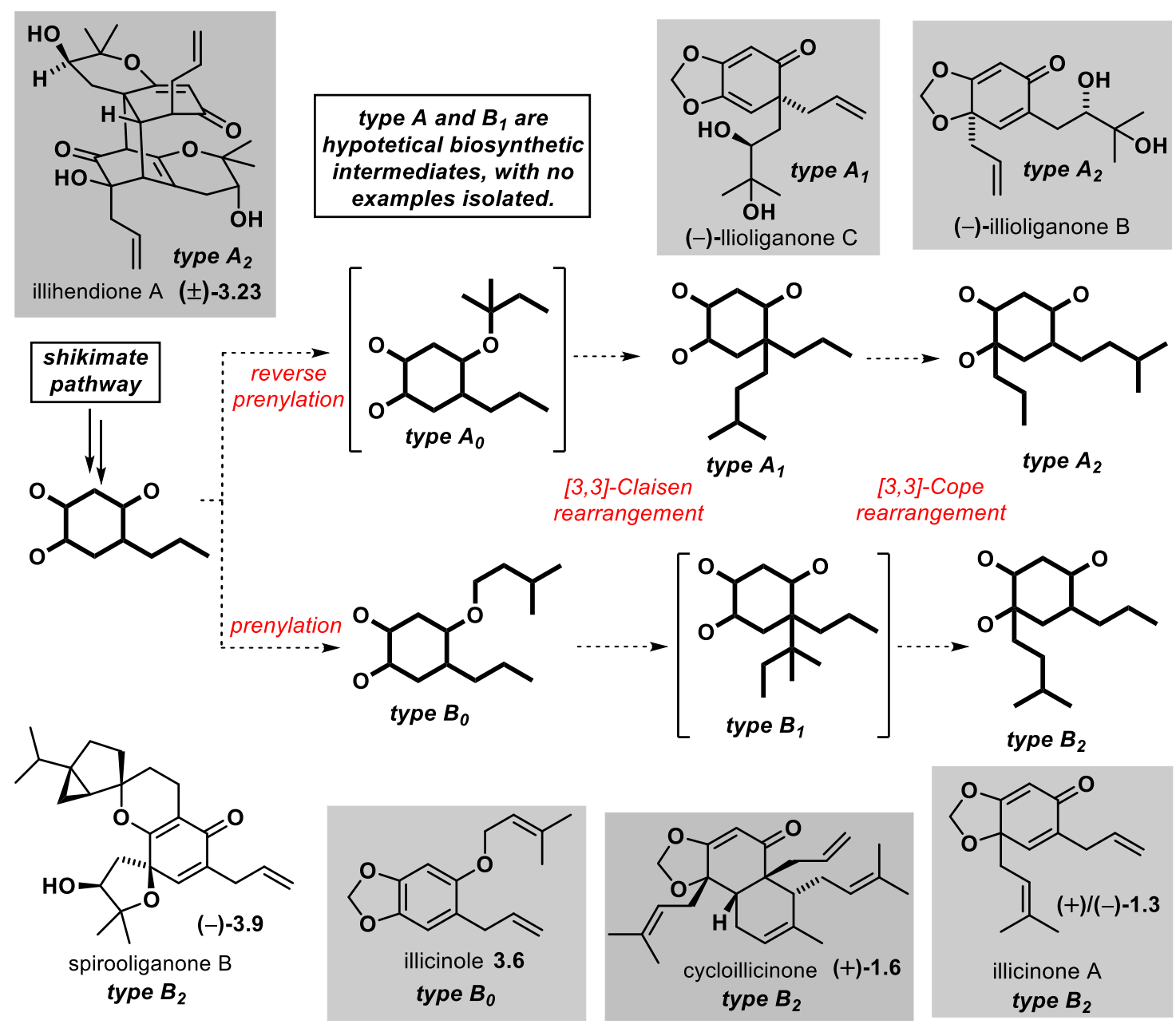

Scheme 3.11: Our proposed biosynthetic network and some example of natural products.

Most members of this family of compounds can be related through redox processes. However, there are higher order structures that result, for example, from intramolecular carbocyclisations, as (-)-illioliganone A 1.2 and $( \pm)$-tricycloillicinone 1.4, or Diels-Alder reactions (-)-spirooliganone A 1.7, (-)-spirooliganone B 3.9 (see section 3.2.2 on pages 93-94), (+)-cycloillicinone 1.6, (+)-illihendione A 3.23 and (+)-illicidione A 1.5 (see section 3.2.3 on pages 96-97). Some examples are depicted in scheme 3.12. 

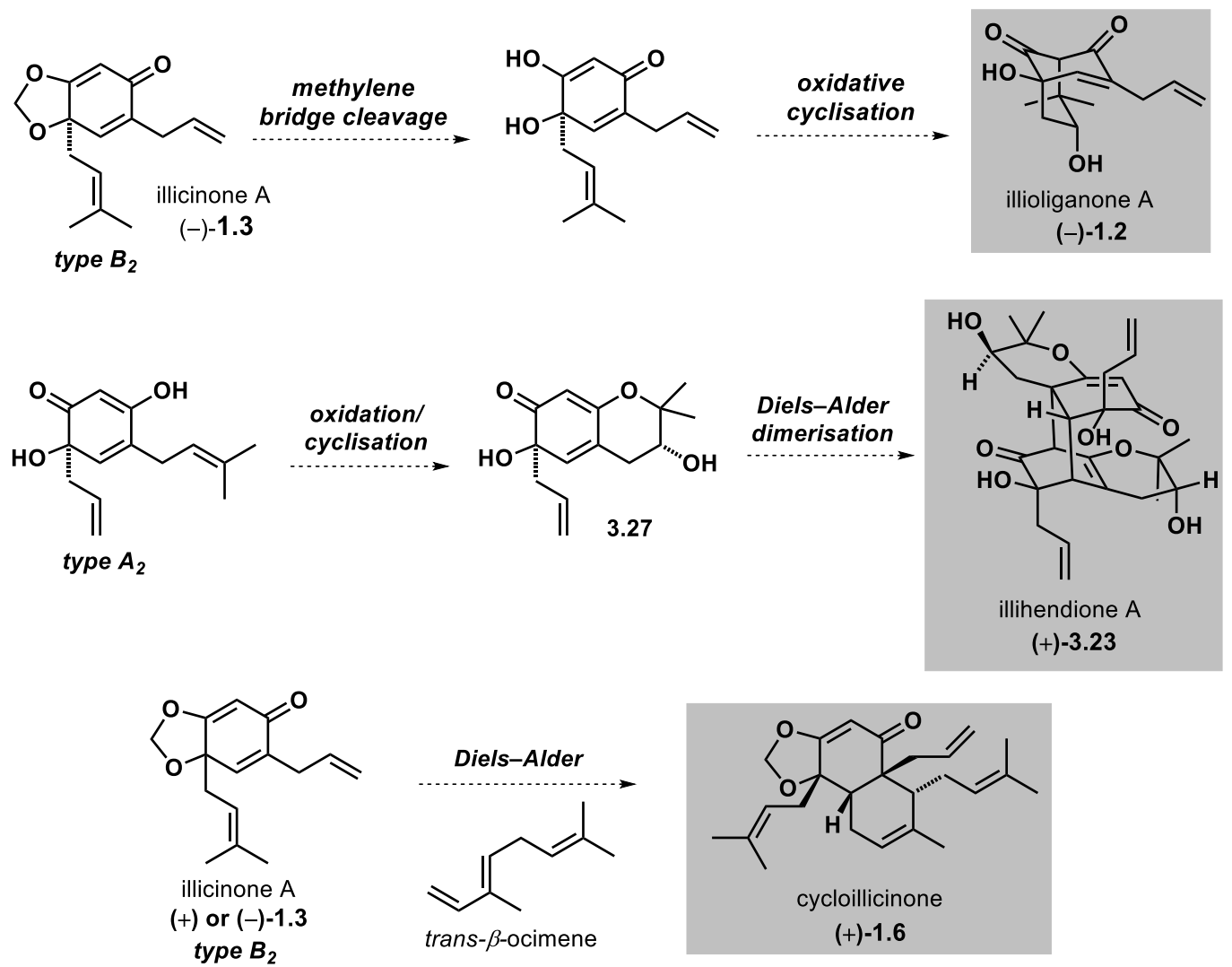

Scheme 3.12: Proposed biosyntheses of select higher-order prenylated phenylpropanoids.

\subsubsection{Our Synthetic Strategy}

Nature, over billions of years, has developed very efficient strategies to achieve selectivity in the synthesis of organic compounds. We aim to achieve selectivity in the laboratory with the standard techniques employed by organic chemists such as the use of protecting groups and the careful choice of reagents and reactions conditions. Thus, our synthetic plan is slightly different from our proposed biosynthesis, but has incorporated its strategic implications, in accordance with the biogenetic-type philosophy described by Van Tamelen (discussed in section 1.2 on page 18). Therefore, we will make extensive use of specific biomimetic reactions such as $[3,3]$ sigmatropic rearrangements, prenyl side-chain oxidations, cyclisations and DielsAlder reactions. 


\subsubsection{Synthesis of the Precursors}

To be able to access all our targets, we will need two different precursors $(( \pm)-3.34$ or ( \pm -3.35, and $( \pm)-3.36$ or $( \pm)$-3.37) which differ in the relative positions of the allyl and prenyl side chains. Precursors ortho-quinol ( \pm )-3.35 and para-quinol ( \pm )-3.36 possess the framework of the structures we classified as type A2, while enone ( \pm )-3.38, potentially accessible from ortho-quinol $( \pm)-3.35$, belongs to type A1. Finally, paraquinol ( \pm )-3.34 and ortho-quinol ( \pm )-3.37 would lead to type $\mathbf{B}_{2}$ structures. We propose that all precursors could be accessed from the same commercially available starting material (4-bromo-3-methoxyphenol, 3.39) through a sequence of allyl or reverseprenyl ether formations, [3,3]-sigmatropic rearrangements, allylation or prenylation and then regio and chemo-selective oxidative dearomatisations. It is worth noting that, in principle, both type $\mathbf{A}_{2}$ and type $\mathbf{B}_{2}$ frameworks could potentially be accessed from the same phenol (3.40 or 3.41) via deprotection of the methyl ether group, provided selective conditions were found for both ortho and para oxidative dearomatisations (scheme 3.13). 


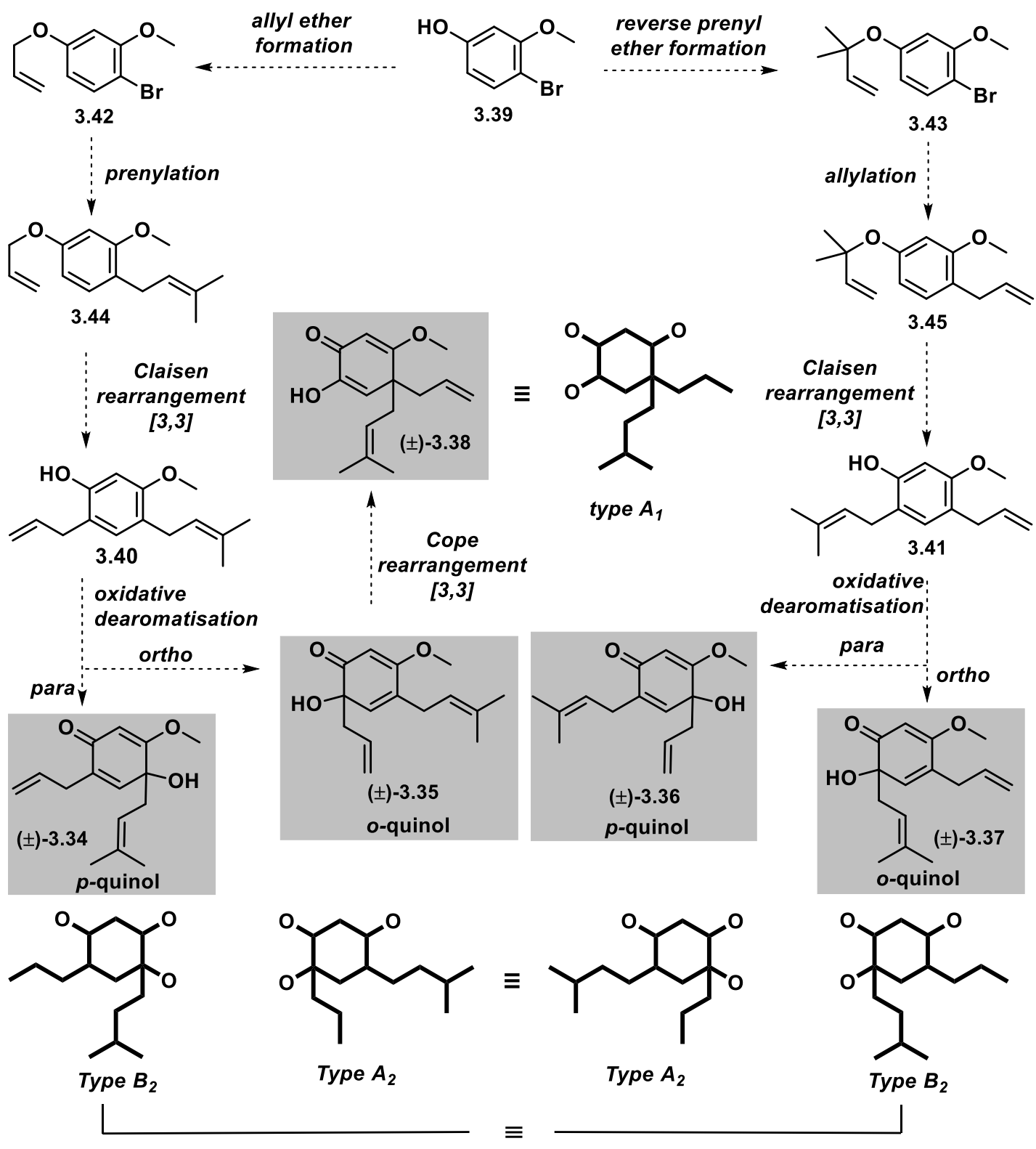

Scheme 3.13: Our proposed synthesis for the precursors to type $\boldsymbol{A}_{1}, \boldsymbol{A}_{2}$ and $\boldsymbol{B}_{2}$ structures.

If successful, this approach would potentially allow access to all the framework types via a unified strategy, and would therefore exploit even more the divergent nature of our strategy.

\subsubsection{Planned Synthesis of Selected Molecular Targets}

Given the large number of isolated prenylated phenylpropanoid natural products, focus will be devoted to the total synthesis of a group of them. To demonstrate the generality of our strategy we aim to prepare a selection of targets having diverse molecular 
scaffolds. Given the non-enantioselective nature of the oxidative dearomatisation we are planning to use, our targets will be prepared in racemic form. We envisage that the use of carefully selected reagents and reaction conditions could allow us to control the relative stereochemistry in the subsequent transformations. Our proposed synthetic plan for the most interesting and complex structures involves a relatively small number of transformations: deprotections, Knoevenagel condensations, prenyl side-chain oxidations, cyclisations and Diels-Alder cycloadditions, as shown below (schemes 3.14-3.16, sections 3.3.4 and 3.3.5).

\subsubsection{Planned Synthesis of Selected Type A2 Structures}

The synthesis of $( \pm)$-illihendione A $\mathbf{3 . 2 3}$ and ( \pm )-illicidione A 1.5 starting from type A2 precursor $( \pm)$-3.35 would involve a diastereoselective epoxidation of the prenyl sidechain to give quinol $( \pm)$-3.46, followed by a cyclisation leading to the formation of either a five or six-membered ether $( \pm)$-3.26 or $( \pm)$-3.27. A Diels-Alder cycloaddition could then occur giving the homo-dimer $( \pm)$-illihendione A $\mathbf{3 . 2 3}$ and the hetero-dimer $( \pm$ )-illicidione A 1.5 (scheme 3.14). Based on the literature precedent discussed in section 3.2.3 on pages $97-98$, it is reasonable to expect this final transformation to occur spontaneously at room temperature. ${ }^{141}$<smiles>C=CCC1(O)C=C(CC=C(C)C)C(OC)=CC1=O</smiles>

$( \pm)-3.35$<smiles>C=CC[C@]1(O)C=C(CCC2OC2(C)C)C(OC)=CC1=O</smiles>

$( \pm)-3.46$

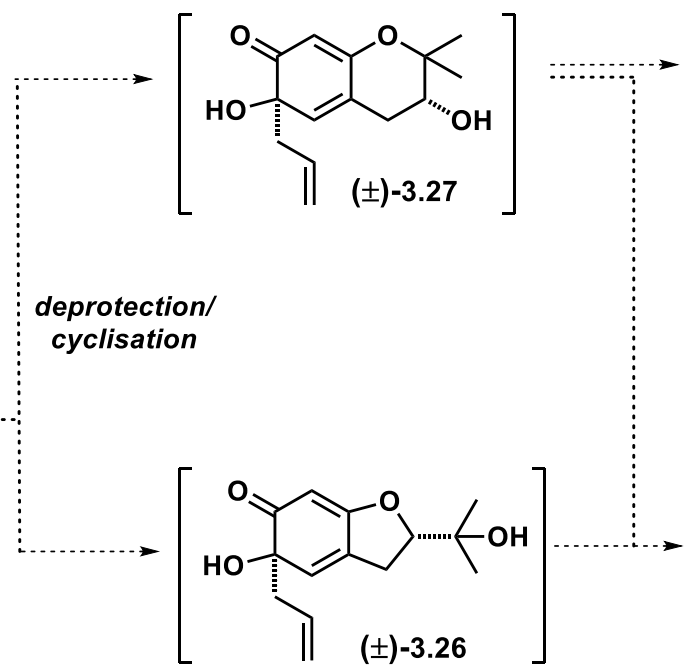

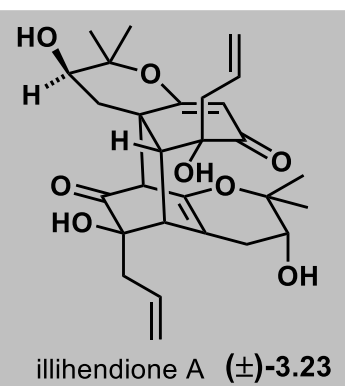

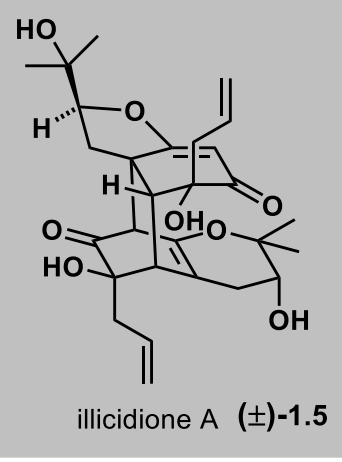

Scheme 3.14: Proposed synthesis of $( \pm)$-illihendione A 3.23 and ( \pm )-illicidione A 1.5. 


\subsubsection{Planned Synthesis of Selected Type $B_{2}$ Structures}

The majority of our targets belong to the type $\mathbf{B}_{2}$ framework. For example, we propose then that ( \pm )-illioliganone A $\mathbf{1 . 2}$ could be accessed by deprotection followed by oxidative carbocyclisation from type $\mathbf{B}_{2}$ precursor $( \pm)-3.37$. Alternatively, this natural product could be accessed from diol $( \pm)$-3.47 via a sequence of diastereoselective epoxidation and intramolecular epoxide-ring opening. Intermediate $( \pm)$-3.46 could also be methylenated to give the bridged natural product illicinone A $\mathbf{1 . 3}$ in racemic form. This could then be reacted with trans- $\beta$-ocimene (a naturally occurring and commercially available monoterpene) through a Diels-Alder cycloaddition leading to $( \pm$ )-cycloillicinone 1.6, or cyclise to give $( \pm)$-tricycloillicinone 1.4 (scheme 3.15), as shown by Fukuyama ${ }^{118}$ and Danishefsky ${ }^{120}$ (see section 3.2.1 on pages 91-92).

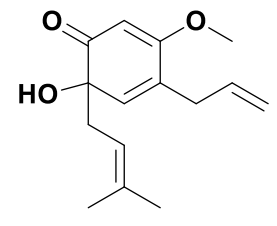

type $B_{2}$

$( \pm)-3.37$

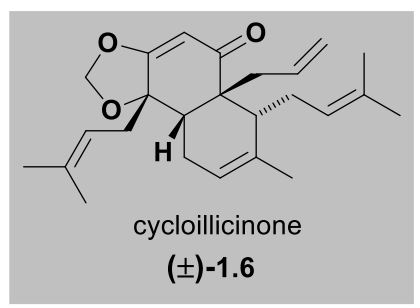

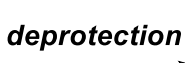
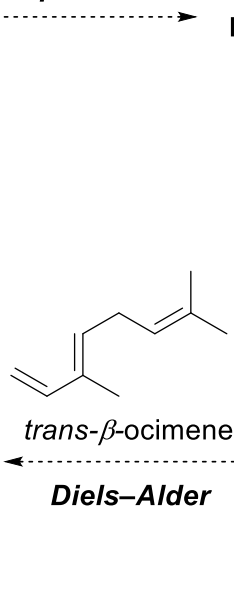

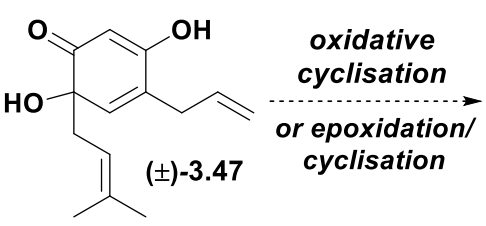

methylenation

$\dot{v}$

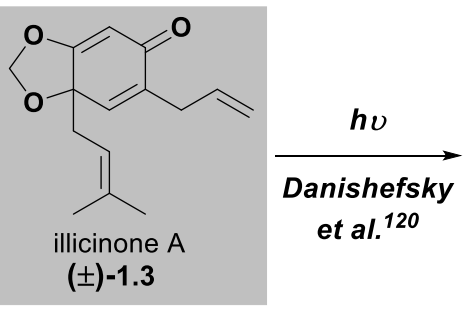

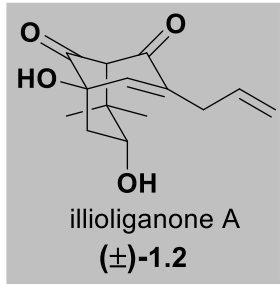

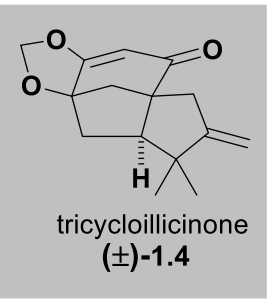

Scheme 3.15: Proposed synthesis of ( \pm )-illioliganone A 1.2, ( \pm -cycloillicinone 1.6 and $( \pm)-$ tricycloillicinone $\mathbf{1 . 4 .}$

We propose that $( \pm)$-illicidione B 3.3 and spirooliganones A $( \pm)-1.7$ and $B( \pm)-3.9$ could also be accessed from the same type $\mathbf{B}_{2}$ precursor $( \pm)$-3.37. ( \pm )-Illicidione B 3.9 could be prepared through a sequence involving deprotection, Knoevenagel condensation with formaldehyde (or a synthetic analogue) and Michael addition leading to dimer $( \pm)$-3.48. We anticipate this transformation could be challenging, as there are issues of selectivity (homo vs. hetero dimers) to address. Dimer $( \pm)$-3.48 could be subjected to diastereoselective epoxidation of the prenyl side-chains and 
finally intramolecular double cyclisation. Alternatively, precursor $( \pm)-3.37$ could be diastereoselectively epoxidised first to give alcohol ( \pm -3.49. Spirocyclic compound $( \pm)$-3.50 could then be formed via intramolecular epoxide ring opening and, after deprotection and Knoevenagel condensation with formaldehyde, would be converted into enone $( \pm)$-3.51. Based on literature precedent (see section 3.2.2 on pages 94-96), we envisage this could undergo a Diels-Alder cycloaddition with (-)-sabinene (a natural occurring monoterpene) to give $( \pm$ )-spirooliganones A 1.7 and B 3.9 (scheme 3.16).
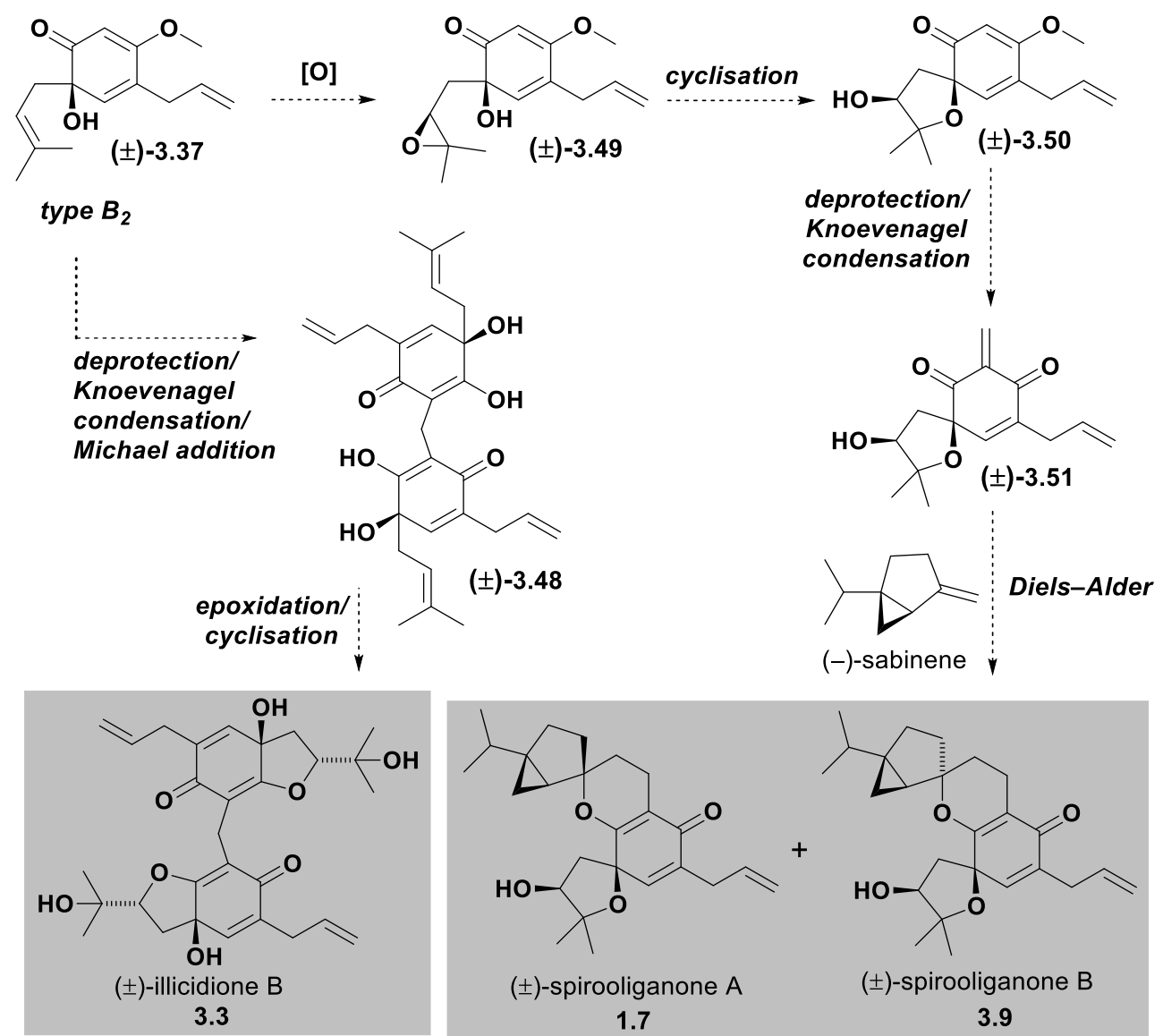

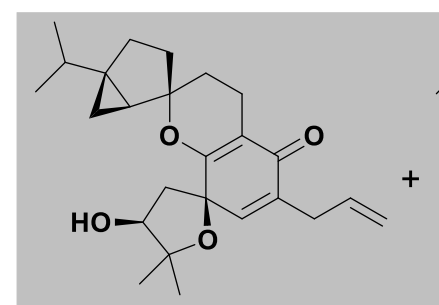

$( \pm)$-spirooliganone A 1.7

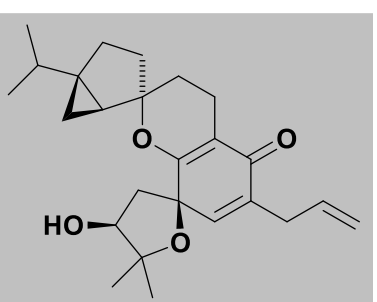

( \pm -spirooliganone B 3.9

Scheme 3.16: Proposed syntheses of $( \pm)$-illicidione B 3.3 and ( \pm )-spirooliganones A 1.7 and B 3.9.

The order in which these reactions are performed could be modified to overcome regio- and/or stereo-selectivity issues which could arise during the synthesis. For example, removal of the methyl ether functionality on epoxide ( \pm )-3.49 could be performed after the hetero-Diels-Alder cycloaddition to avoid formation of undesired regioisomers. 


\subsection{Results and Discussion}

\subsubsection{Synthesis of the Precursor to Type A1 and A2 Structures}

Our planned synthesis for precursor $( \pm)-3.35$ to type $\mathbf{A}_{1}$ and $\mathbf{A}_{2}$ structures from commercially available phenol $\mathbf{3 . 3 9}$ involved the formation of allyl ether $\mathbf{3 . 4 2}$, which would be prenylated to give arene 3.44. A Claisen rearrangement would afford phenol 3.40, which would then be subjected to regioselective oxidative dearomatisation to give ortho-quinol ( \pm )-3.35 (scheme 3.17).

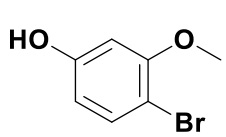

3.39

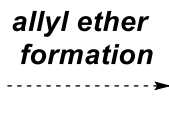

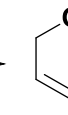

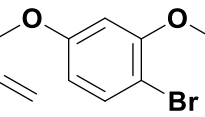

3.42
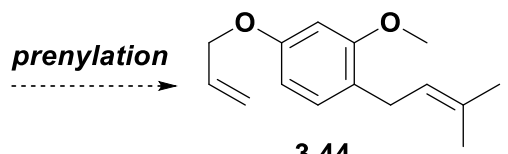

3.44

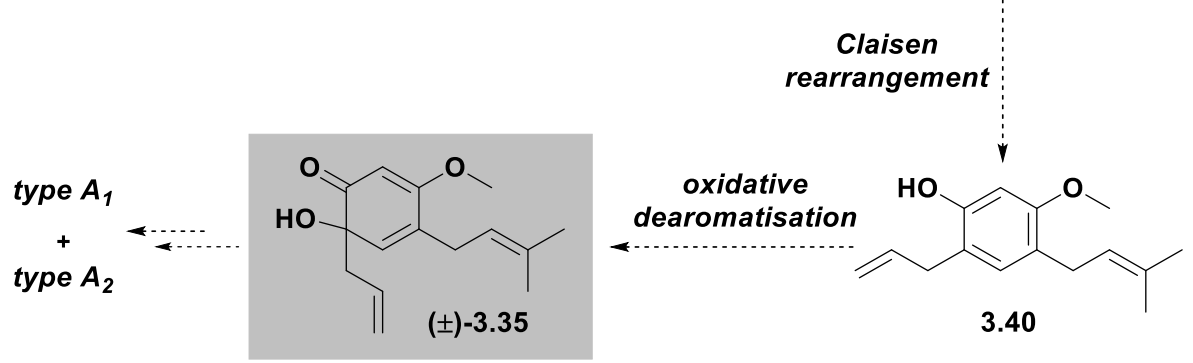

Scheme 3.17: Our planned synthesis for the precursor ( \pm )-3.35 to type $A_{1}$ and $A_{2}$ structures.

The synthesis of compound ( \pm )-3.35 began with the formation of allyl ether 3.39, ${ }^{143}$ which proceeded smoothly to give expected product $\mathbf{3 . 4 2}$ in $92 \%$ isolated yield on a 5-gram scale (scheme 3.18).

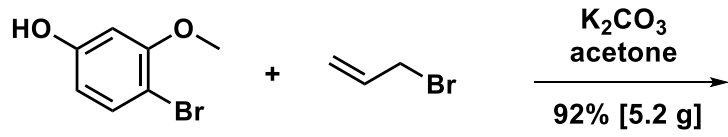

3.39<smiles>C=CCOc1ccc(Br)c(OC)c1</smiles>

3.42

Scheme 3.18: Formation of allyl ether 3.42.

The next step, involving prenylation of aryl bromide $\mathbf{3 . 4 2}$ to give compound $\mathbf{3 . 4 4}$, proved to be more challenging. The first attempts were performed using the Stille reaction. ${ }^{144}$ This palladium catalysed coupling between aryl bromide $\mathbf{3 . 4 2}$ and tributyl(3-methyl-2-butenyl)tin proved to be unsuccessful, giving just reverse-prenylated compound $\mathbf{3 . 5 2}$ in a very low isolated yield, along with $40 \%$ of recovered starting material (scheme 3.19). 


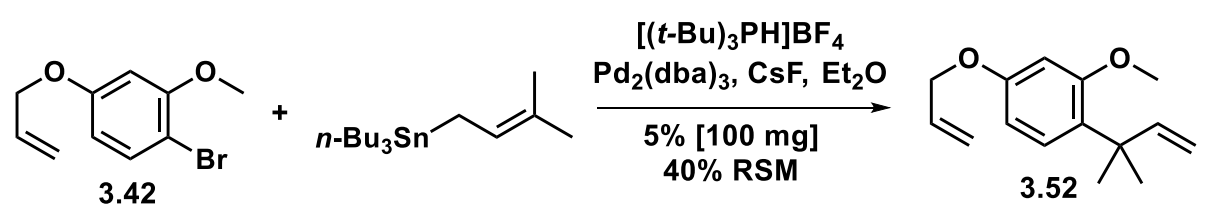

Scheme 3.19: Attempted prenylation of arene $\mathbf{3 . 4 2}$ with Stille reaction.

The prenylation of arene $\mathbf{3 . 4 2}$ was then attempted following a procedure involving a lithium-halogen exchange to give the aryl lithium species 3.53, which would then be quenched with 3,3-dimethylallyl bromide ${ }^{145}$ to give product 3.44. ${ }^{1} \mathrm{H}$ NMR analysis of the crude reaction mixture indicated a $76 \%$ yield of the expected product along with $15 \%$ of protonated species $\mathbf{3 . 5 4}$ and $5 \%$ of compound $\mathbf{3 . 5 5}$, the result of allylic double bond isomerisation. Following flash chromatography targeted compound 3.44 was isolated in 50\% yield (scheme 3.20). It is proposed that the proton source could either be water present in the reaction mixture or the methylene protons in the allyl group, as suggested by the isomerisation of the double bond. Nevertheless, larger quantities of product $\mathbf{3 . 4 4}$ could be isolated by repeating the purification.

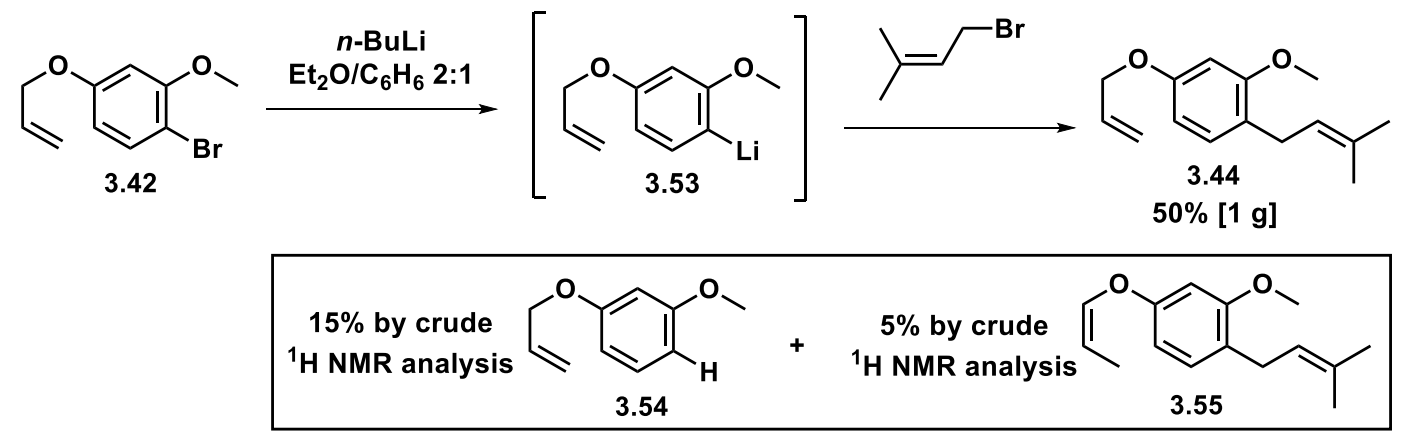

Scheme 3.20: Prenylation of allyl ether 3.42.

We then focused on the Claisen rearrangement of allyl ether 3.44, targeting phenol 3.40. Thermal Claisen rearrangements are known in the literature to be not particularly regioselective, ${ }^{146}$ we therefore decided to use allyl ether $\mathbf{3 . 4 2}$ as a model system in an attempt to find suitable conditions. Ishikawa and co-workers in 2007 reported the use of boron trichloride as an efficient reagent for the regioselective Claisen rearrangement of resorcinol derivatives, with a strong preference for the C6 position. ${ }^{147}$ Treatment of compound 3.42 with three equivalents of boron trichloride gave a mixture of the two possible regioisomers $\mathbf{3 . 5 6}$ and 3.57, allylated at $\mathrm{C} 6$ or at $\mathrm{C} 2$, with a 20:1 ratio in favour of desired regioisomer 3.56 (scheme 3.21). 
<smiles>C=CCOc1ccc(Br)c(OC)c1</smiles>

3.42

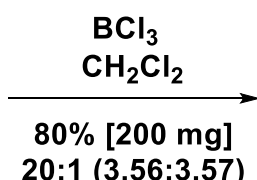

$20: 1(3.56: 3.57)$<smiles>C=CCc1cc(Br)c(OC)cc1O</smiles>

3.56<smiles>C=CCc1c(O)cccc1Br</smiles>

3.57

Scheme 3.21: $\mathrm{BCl}_{3}$ mediated Claisen rearrangement of allyl ether 3.42.

Given these promising results, the same conditions were applied to prenylated allyl ether 3.44, but this resulted in decomposition. Thermal conditions were therefore investigated. It was found that simply heating the neat ether 3.44 at $190{ }^{\circ} \mathrm{C}$ overnight resulted in the formation of a 6:1 mixture of $\mathrm{C} 6$ allylated phenol 3.40 and $\mathrm{C} 2$ allylated phenol 3.58. Pleasingly, the two regioisomers were found to be separable by flash chromatography and targeted phenol $\mathbf{3 . 4 0}$ was obtained in $71 \%$ isolated yield (scheme 3.22).
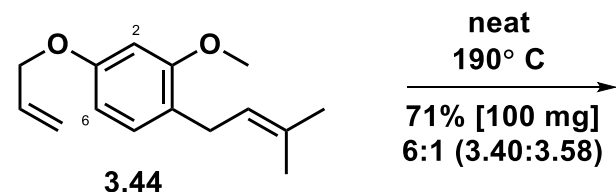<smiles>C=CCc1cc(CC=C(C)C)c(OC)cc1O</smiles>

3.40<smiles>C=CCc1c(O)ccc(CC=C(C)C)c1OC</smiles>

Scheme 3.22: Thermal Claisen rearrangement of ether 3.44.

Several reaction conditions were screened for the oxidative dearomatisation of phenol 3.40. Reactions using Oxone $e^{148}$ and [bis-(trifluoacetoxy)-iodo]benzene (PIFA) $)^{149}$ as the oxidants gave mixture of products which we could not identify, while lead tetraacetate ${ }^{150}$ gave ortho-quinol acetate $( \pm)$-3.59 in 30\% isolated yield (scheme 3.23). Analysis of the crude ${ }^{1} \mathrm{H}$ NMR spectrum for this reaction suggested formation of both ortho- and para-quinol acetates $( \pm)-3.59$ and $( \pm)-3.60$ in an approximately $1: 1$ ratio, but only the former could be isolated.<smiles>C=CCc1cc(CC=C(C)C)c(OC)cc1O</smiles>
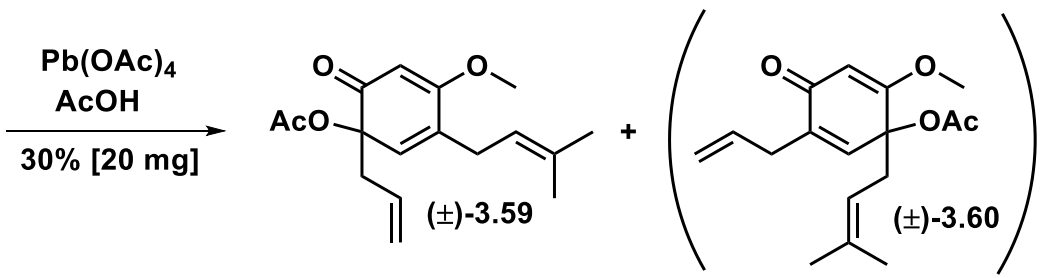

Scheme 3.23: $\mathrm{Pb}(\mathrm{OAc})_{4}$-mediated oxidative dearomatisation of phenol 3.40. 
Then (diacetoxyiodo)benzene (PIDA) was tested using a modified literature procedure, ${ }^{151}$ but the only identified and isolated compound among the complex mixture of products was epoxide $( \pm)-3.61$, in low yield (scheme 3.24).<smiles>C=CCc1cc(CC=C(C)C)c(OC)cc1O</smiles><smiles>CC(=O)OCCCCCO</smiles><smiles>C=CCc1cc(CC2OC2(C)C)c(OC)cc1O</smiles>

Scheme 3.24: Oxidative dearomatisation of phenol 3.40 with PIDA to give epoxide ( \pm )-3.61.

Stabilised 2-iodoxybenzoic acid (SIBX) is a known reagent for the regioselective formation of ortho-quinols from phenols. ${ }^{152}$ Therefore, a reaction was performed using phenol 3.40 and 2.4 equivalents of SIBX as the oxidant, with ortho-quinol ( \pm )-3.35 being the only observed product in the crude ${ }^{1} \mathrm{H}$ NMR spectrum (scheme 3.25).

Attempts to separate ortho-quinol $( \pm)$-3.35 from the residual unreacted IBX and the stabilisers by flash chromatography failed. When the crude reaction mixture was washed with aqueous sodium bicarbonate solutions ortho-quinol $( \pm)$-3.35 proved to be highly prone toward Cope rearrangement to give dienone $( \pm)-\mathbf{3 . 3 8}$. However, the crude material was sufficiently pure that analysis of $1 \mathrm{D}$ and 2D NMR spectra allowed us to unambiguously determine the structure of compound ( \pm -3.35. The facile rearrangement of ortho-quinol $( \pm)$-3.35 would enable direct investigation into the synthesis of type A1 structures. Dienone ( \pm )-3.38, in fact, possesses the correct framework for this group and can also conveniently be prepared with the same procedure used for ortho-quinol $( \pm)-\mathbf{3 . 3 8}$, by letting the reaction proceed for longer (scheme 3.25).
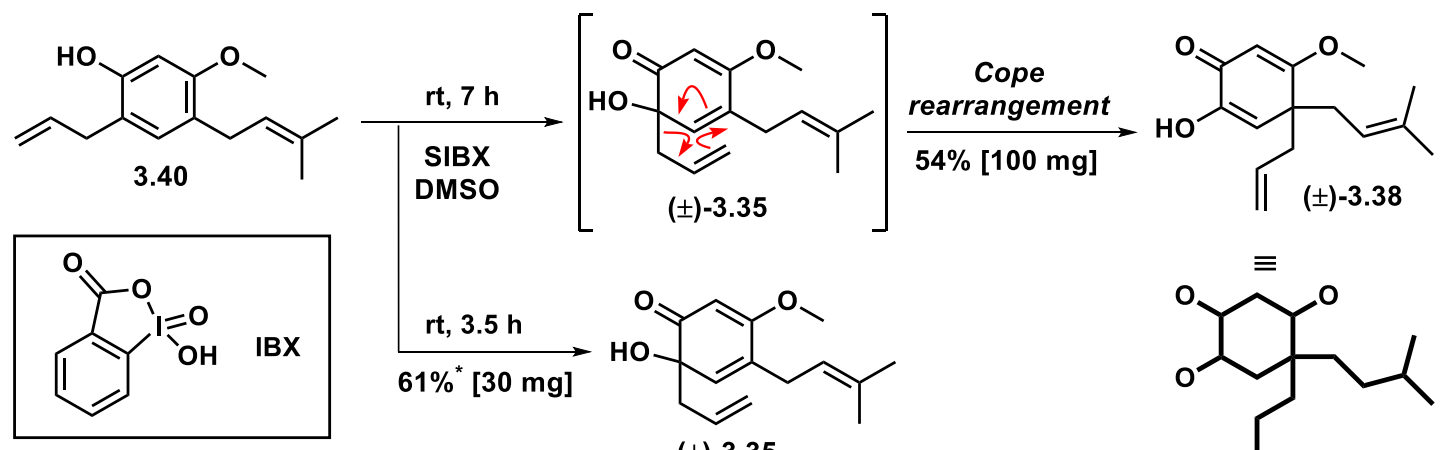

$( \pm)-3.35$

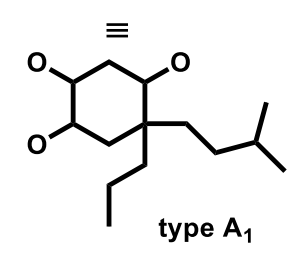

*crude ${ }^{1} H$ NMR yield.

Scheme 3.25: SIBX oxidative dearomatisation of phenol $( \pm)-3.40 .{ }^{*}$ crude ${ }^{1} H$ NMR yield. 
Considering the small number and complexity of natural product targets belonging to type A1 frameworks, their synthesis was not pursued. To the best of our knowledge there are just twelve known prenylated phenylpropanoids isolated from Illicium plants, and they differ only for subtle modifications of the prenyl side-chain (see appendix on page 426). The inability to access the precursor to type $\mathbf{B}_{2}$ structures, which would require selective oxidative dearomatisation of phenol $\mathbf{3 . 4 0}$ in the para-position (see section 3.3.2 on page 103), and the instability of ortho-quinol $( \pm)-\mathbf{3 . 3 5}$, precursor to type $\mathbf{A}_{2}$ structures, led us to abandon this route. However, we envisaged that the results obtained so far could provide useful information about the reactivity of these structures and inspiration for the synthesis of the precursor to type $\mathbf{B}_{2}$ frameworks.

\subsubsection{Synthesis of the Precursor to Type $B_{2}$ Structures}

In our proposed biosynthetic network the majority of our molecular targets could be seen as derived from type $\mathbf{B}_{2}$ precursor $( \pm)-3.37$. We initially hypothesised that it was possible to access this compound through a short sequence involving a direct calciumcatalysed mild Friedel-Crafts prenylation ${ }^{153}$ at C6, allylation at $\mathrm{C} 4$ to afford phenol 3.41 and SIBX-mediated oxidative dearomatisation (scheme 3.26).

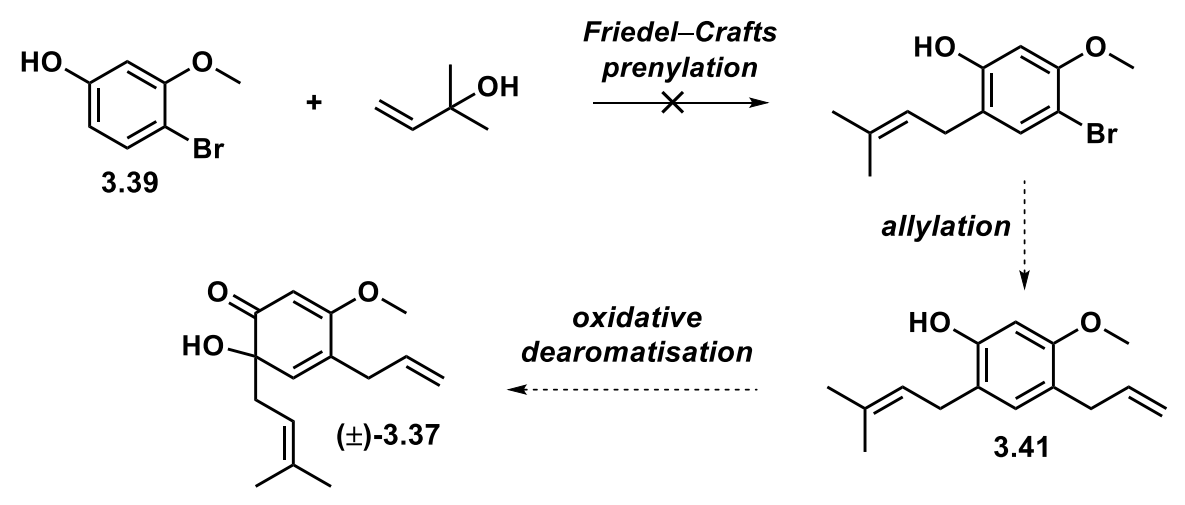

Scheme 3.26: Our initial proposed synthesis of precursor ( \pm )-3.37 to type $\boldsymbol{B}_{2}$ structures.

The direct $C$-prenylation of arene 3.39 was attempted under the conditions recently developed by Niggemann and co-workers, ${ }^{153}$ involving the use of calcium triflimide as a mild Lewis-acid catalyst. This methodology was applied to couple a series of alcohols and arenes, some of which similar to our substrates. An example is shown in scheme 3.27. 


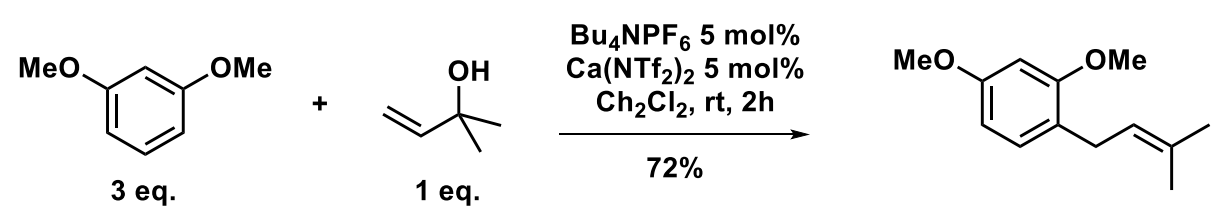

Scheme 3.27: Example of Niggemann'calcium-catalysed Friedel-Crafts prenylation. ${ }^{153}$

Unfortunately, after several trials varying the reaction conditions, no desired prenylation was observed. It was, therefore, decided to undertake the slightly longer approach akin to the one used for type $\mathbf{B}_{2}$ precursor. Our new planned synthesis for the synthesis of precursor $( \pm)-3.37$ from phenol 3.39 involved a reverse-prenyl ether formation to give bromoarene 3.43. Direct allylation would then afford ether 3.45, which could be subjected to Claisen rearrangement to produce phenol 3.41. Finally, an ortho-oxidative dearomatisation would provide ortho-quinol ( \pm )-3.37 (scheme 3.28).<smiles>COc1cc(O)ccc1Br</smiles>

3.39

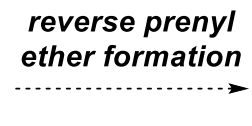

reverse prenyl
ether formation<smiles>C=CC(C)(C)Oc1ccc(Br)c(OC)c1</smiles>

3.43 allylation<smiles>C=CCc1ccc(OC(C)(C)C=C)cc1OC</smiles>

3.45

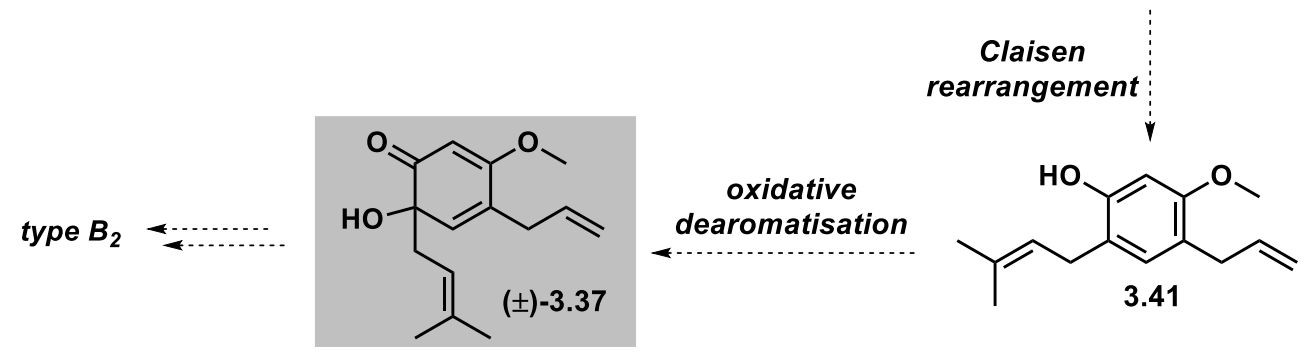

Scheme 3.28: Alternative proposed synthesis for precursor ( \pm )-3.37 to type $\boldsymbol{B}_{2}$ structures.

The preparation of reverse-prenyl ether 3.43 was initially attempted by reacting phenol 3.39 with carbonate $\mathbf{3 . 6 2}$, which could be prepared following a literature procedure. ${ }^{154}$ Unfortunately, palladium-catalysed reverse $O$-prenylation of phenol 3.39 proved to be unselective. ${ }^{1} \mathrm{H}$ NMR analysis of the crude reaction mixture revealed reverse-prenyl ether $\mathbf{3 . 4 3}$ to be present as the major product. However, unwanted C4 prenylated phenol 3.63 and prenyl ether $\mathbf{3 . 6 4}$ were produced in significant amounts (scheme 3.29). 


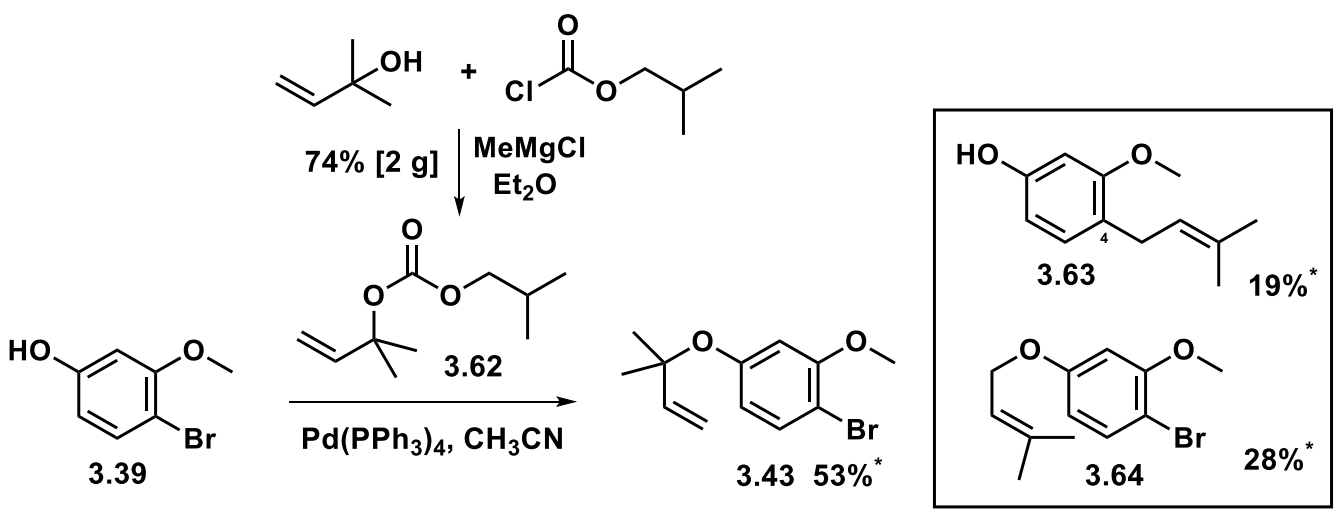

Scheme 3.29: Attempted O-reverse prenylation of phenol 3.39. ${ }^{*} H$ NMR yield.

Attempts to isolate reverse-prenyl ether 3.43 from the other components of the reaction mixture through flash chromatography met with failure. and varying the reaction conditions proved unproductive. It was therefore decided to undertake a more secure albeit less elegant route to access reverse-prenyl ether 3.43, to avoid selectivity issues. A two-step sequence involving $O$-propargylation ${ }^{155}$ of phenol 3.39 followed by partial hydrogenation of alkyne $\mathbf{3 . 6 5}$ using Lindlar's catalyst ${ }^{156}$ proved to be a reliable method to access alkene $\mathbf{3 . 4 3}$ (scheme 3.30 ).<smiles>COc1cc(O)ccc1Br</smiles>

3.39<smiles>C#CC(C)(Cl)[C@H](C)OCCCC(C)(C)Oc1ccc(Br)c(OC)c1</smiles><smiles>C=CC(C)(C)OC(=O)OCC(C)C</smiles>

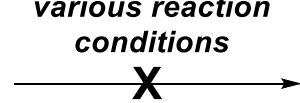
$X$<smiles>C=CC(C)(C)Oc1ccc(Br)c(OC)c1</smiles>

Scheme 3.30: Preparation of reverse-prenyl ether $\mathbf{3 . 4 3}$.

The moderate yield for the propargyl ether formation is ascribed to difficulties in the separation of the product from allene 3.66, which, under optimised conditions, was formed only as a minor product $(<5 \%)$. This is proposed to be formed from deprotonation of propargyl ether $\mathbf{3 . 6 5}$, followed by a $\mathrm{S}_{\mathrm{N}} 2^{\prime}$ reaction with another molecule of alkyne electrophile (scheme 3.31). 
<smiles>C#CC(C)(C)Oc1ccc(Br)c(OC)c1</smiles>

3.65
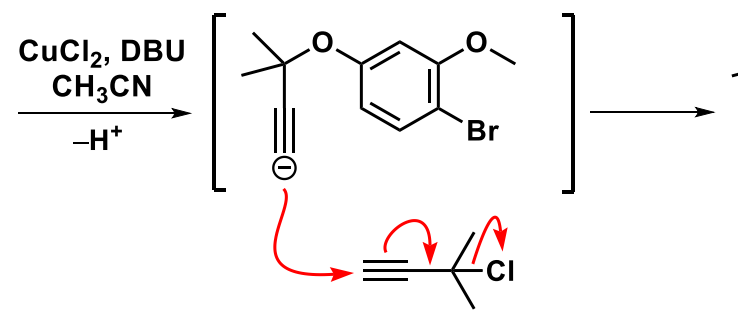<smiles>COc1cc(OC(C)(C)C#CC=C(C)C)ccc1Br</smiles>

Scheme 3.31: Proposed mechanism for the formation of allene 3.66.

The subsequent attempt of allylation ${ }^{145}$ of alkene 3.43 via lithium-halogen exchange and quenching with allyl bromide proved difficult. The formation of a mixture of products, some of them with loss of the prenyl group, was observed in the crude ${ }^{1} \mathrm{H}$ NMR spectrum. Protonation at $\mathrm{C} 4$ was also observed, thus suggesting we were facing the same issues we had encountered in the prenylation of allyl ether $\mathbf{3 . 4 2}$ (see scheme 3.20 on page 108) (scheme 3.32).
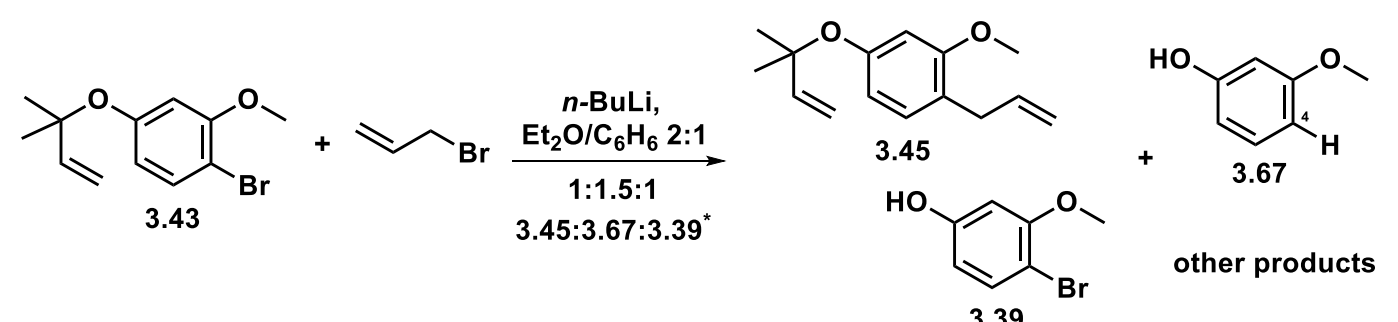

"Ratios based on ${ }^{1} H$ NMR spectrum of the crude reaction mixture.

Scheme 3.32: Attempted allylation of reverse-prenyl ether 3.43.

We therefore decided to investigate the allylation process further, with a different starting material. It was envisaged that a longer sequence involving protection of the phenolic hydroxyl functionality as the MOM-ether followed by allylation of arene 3.68 would allow us to overcome the issues related to the protonation at $\mathrm{C} 4$. Subsequent cleavage of the MOM group on $\mathbf{3 . 6 9}$ to give phenol 3.70, followed by $O$-propargylation and partial reduction of alkyne 3.71 would then afford prenyl-ether

\subsection{5 (scheme 3.33).}<smiles>COc1cc(O)ccc1Br</smiles>

3.39

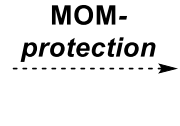$$
\text { (1) }
$$

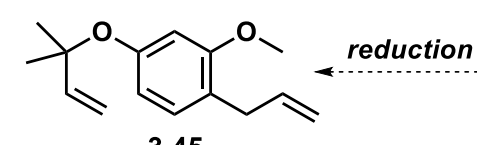

3.45<smiles>COc1ccc(Br)c(OC)c1</smiles>

3.68

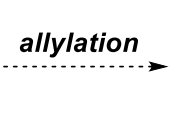

MOMO<smiles>C=CCc1ccc(C)cc1OC</smiles>

3.69

MOM-

deprotection

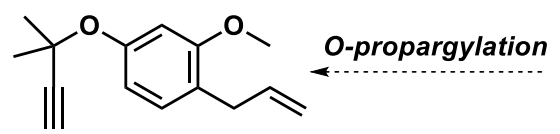

3.71

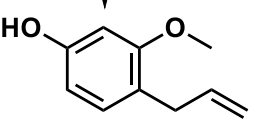

3.70

Scheme 3.33: Proposed alternative synthesis of ether 3.45. 
MOM-ether 3.68 was easily prepared under standard conditions ${ }^{157}$ in $86 \%$ yield on a 10 -gram scale and subjected to the same allylation conditions used with prenyl-ether 3.43 (see scheme 3.42 on page 114). ${ }^{145}$ Lithium-halogen exchange followed by quenching with allyl bromide proceeded smoothly, affording MOM-ether 3.69 in 95\% yield on a 480-mg scale (attempts on scaling up the allylation resulted in a far less clean reaction). This was then subjected to hydrolytic cleavage of the MOM-ether to afford phenol 3.70 in $84 \%$ yield on a $370-\mathrm{mg}$ scale (scheme 3.34).

The $O$-propargylation/reduction sequence was then employed to access reverse-prenyl ether 3.45. Etherification of phenol $\mathbf{3 . 7 0}$ using the previously employed conditions with phenol 3.39 (see scheme $\mathbf{3 . 3 0}$ on page 113), gave compound $\mathbf{3 . 7 1}$ in $68 \%$ yield on a 100-mg scale, along with a small amount of allene 3.72. Subsequent partial hydrogenation of alkyne $\mathbf{3 . 7 1}$ to the alkene using the Lindlar's catalyst afforded ether 3.45 in $94 \%$ yield on a $110-\mathrm{mg}$ scale.

Ether 3.45 was then subjected to thermal Claisen rearrangement; heating ether 3.45, neat, to $190{ }^{\circ} \mathrm{C}$ resulted in the formation of two isomeric products. Phenol 3.41 was isolated in $45 \%$ yield, along with a small amount of its regioisomer 3.73 (6\% yield) (scheme 3.34).<smiles>COc1cc(O)ccc1Br</smiles>

3.39

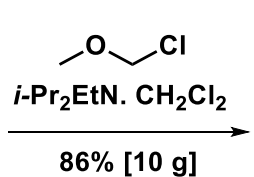

$86 \%[10 \mathrm{~g}]$<smiles>COc1ccc(Br)c(OC)c1</smiles>

3.68

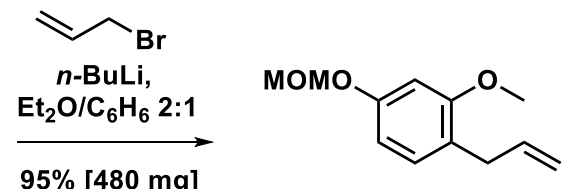

3.69
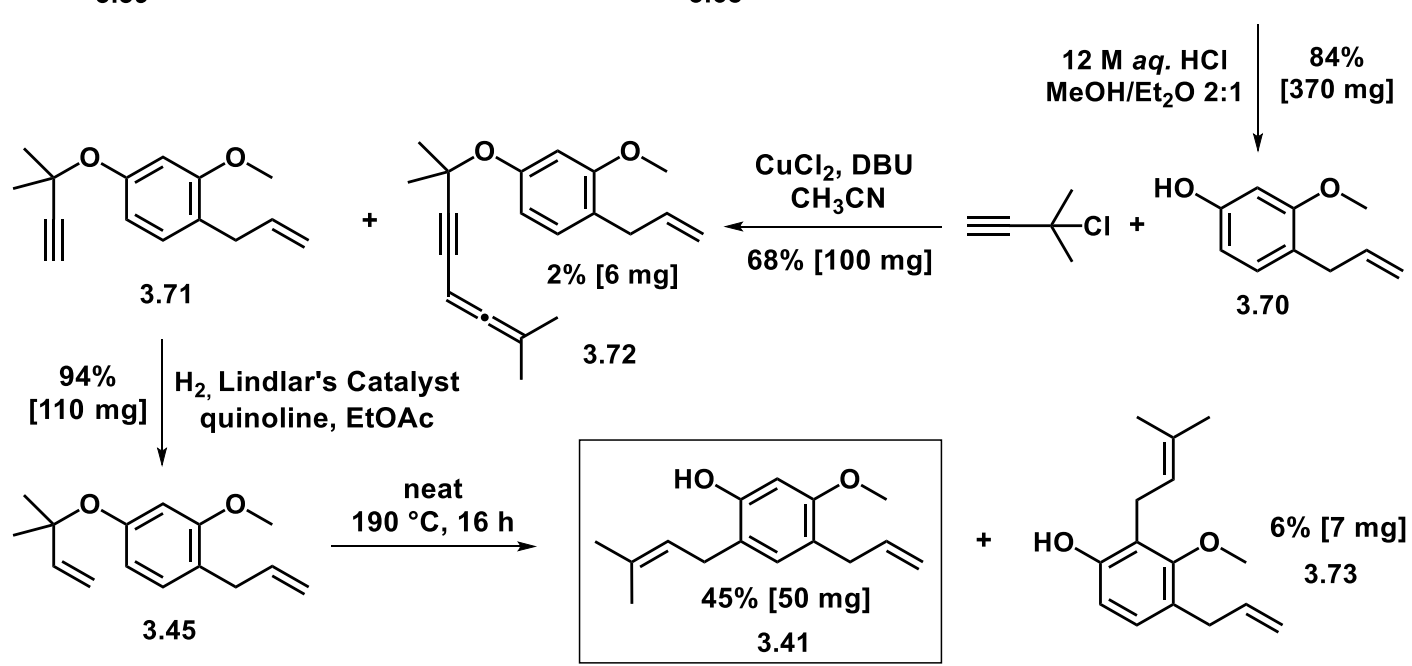

Scheme 3.34: Synthesis of phenol 3.41. 
The SIBX-mediated oxidative dearomatisation was then attempted on phenol 3.41 on a small scale (6 $\mathrm{mg}$ of starting material) in deuterated DMSO. The reaction was monitored by ${ }^{1} \mathrm{H}$ NMR spectroscopy and was found to reach completion in between two to four hours (see page 378 for stacked ${ }^{1} \mathrm{H}$ NMR spectra) to afford ortho-quinol $( \pm)-3.37$. Unfortunately, attempts on separating the residual SIBX from the product by repetitive washings with saturated aqueous $\mathrm{NaHCO}_{3}$ resulted in the formation of a complex mixture of products (scheme 3.35).

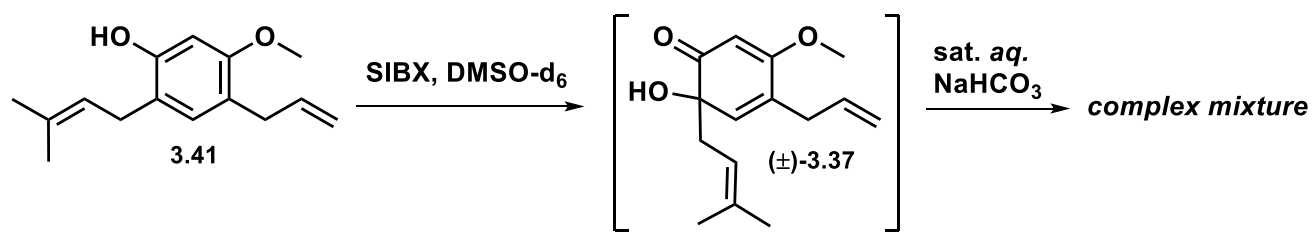

Scheme 3.35: SIBX-mediated oxidative dearomatisation of phenol 3.41.

The apparent instability of ortho-quinol $( \pm)-3.37$, along with the step-count and the overall lack of efficiency and scalability of the steps led us to completely re-consider our biomimetic approach.

\subsection{Alternative Approach}

Inspired by Danishefsky's previously discussed synthesis of ( \pm )-tricycloillicinone, ${ }^{120}$ we envisaged we could access both type $\mathbf{A}_{2}$ and type $\mathbf{B}_{2}$ structures from the two alternative precursors $( \pm$ )-illicinone A 1.3 and compound ( \pm )-3.74, prepared following an analogous approach. Starting from the commercially available and cheap sesamol ( $11.5 / \mathrm{g}$ ), type $\mathbf{B}_{2}$ structures could be accessed via cleavage of the methylene bridge on ( \pm )-illicinone A 1.3, which could be prepared using literature procedures. ${ }^{120}$ The preparation of type $\mathbf{A}_{2}$ framework ( \pm )-3.74 would involve the use of the same reaction types, but in a different order. $O$-reverse-prenylation of sesamol would give ether $\mathbf{3 . 7 5}$, which could undergo a Claisen rearrangement to afford phenol 3.76. $O$-allylation would then produce ether 3.77, which would then be subjected to the MABR-mediated Claisen-Cope rearrangement to produce dienone $( \pm)-3.74$. Finally, cleavage of the methylene bridge would provide the type A2 framework (scheme 3.36). 


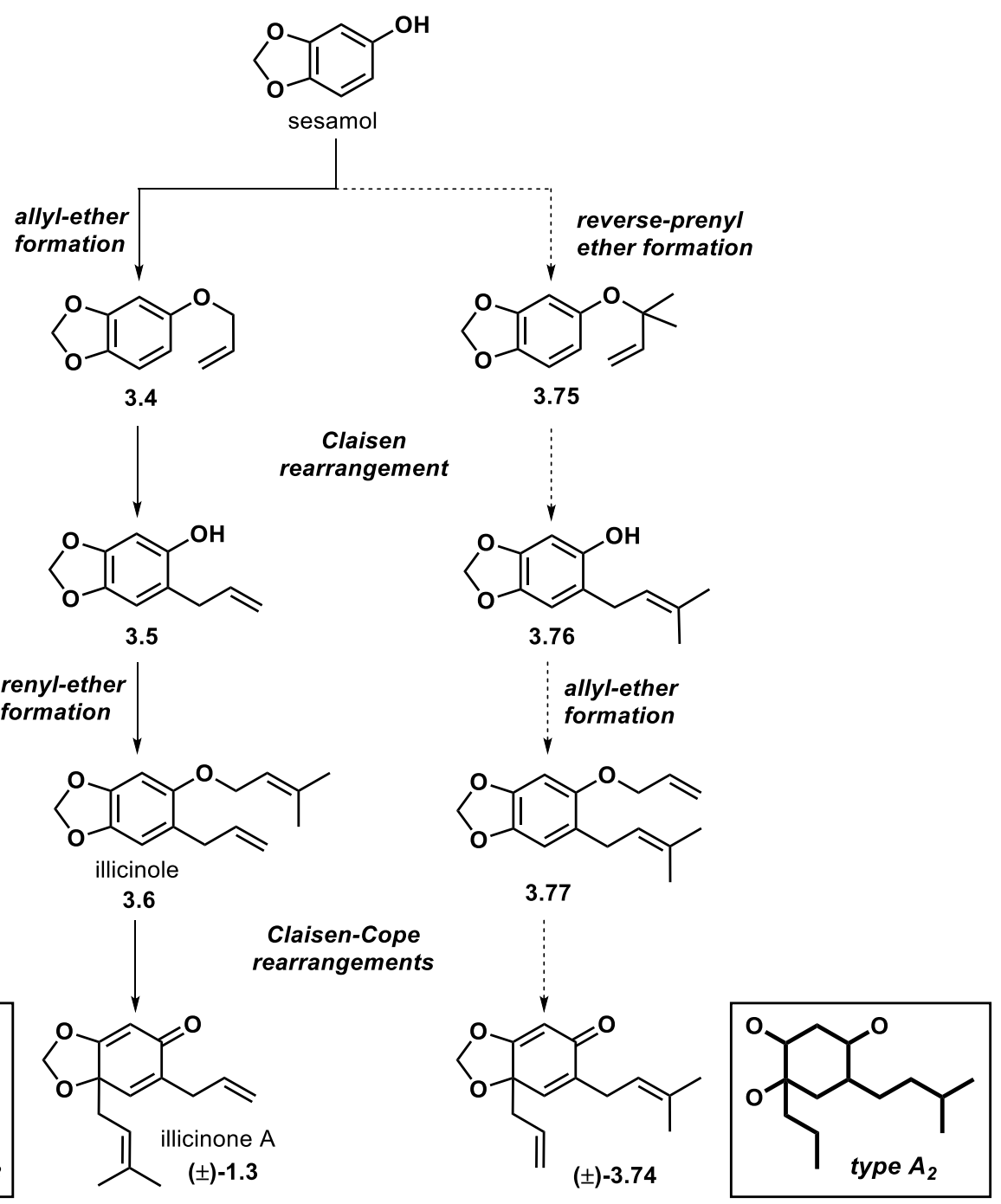

Scheme 3.36: Revised approach to access type $\boldsymbol{A}_{2}$ and type $\boldsymbol{B}_{2}$ frameworks.

\subsubsection{Approach to Type $\mathbf{B}_{2}$ Structures}

It was envisaged that our investigation toward the total synthesis of the most interesting members of the prenylated phenylpropanoids family would require a substantial amount of material. Thus, we repeated the known sequence leading to ( \pm )-illicinone A $\mathbf{1 . 3}$ in a far larger scale than previously reported. Allylation of sesamol proceeded smoothly to afford ether $\mathbf{3 . 4}$ in nearly quantitative yield on a 25-gram scale, which was used directly in the next step, without purification. The subsequent thermal Claisen rearrangement afforded 25 grams of crude reaction mixture containing phenol 3.5, which was directly subjected to prenyl-ether formation conditions (affording 22 grams of illicinole 3.6 in $65 \%$ isolated yield over 3 steps), with final purification by 
column chromatography required. This represents a $>20$-fold scale-up with respect to the previous synthesis, which was accomplished in $75 \%$ yield on a $930-\mathrm{mg}$ scale. The smaller yield in our case has to be ascribed to the longer times required for the Claisen rearrangement in this scale, which led to some decomposition occurring. The conditions developed by Danishefsky and co-workers were then applied for the preparation of ( \pm )-illicinone A 1.3. Treatment of illicinole $\mathbf{3 . 6}$ with in situ generated MABR, a bulky Lewis acid, resulted in a 75\% yield on a 1.7-gram scale (scheme 3.37).

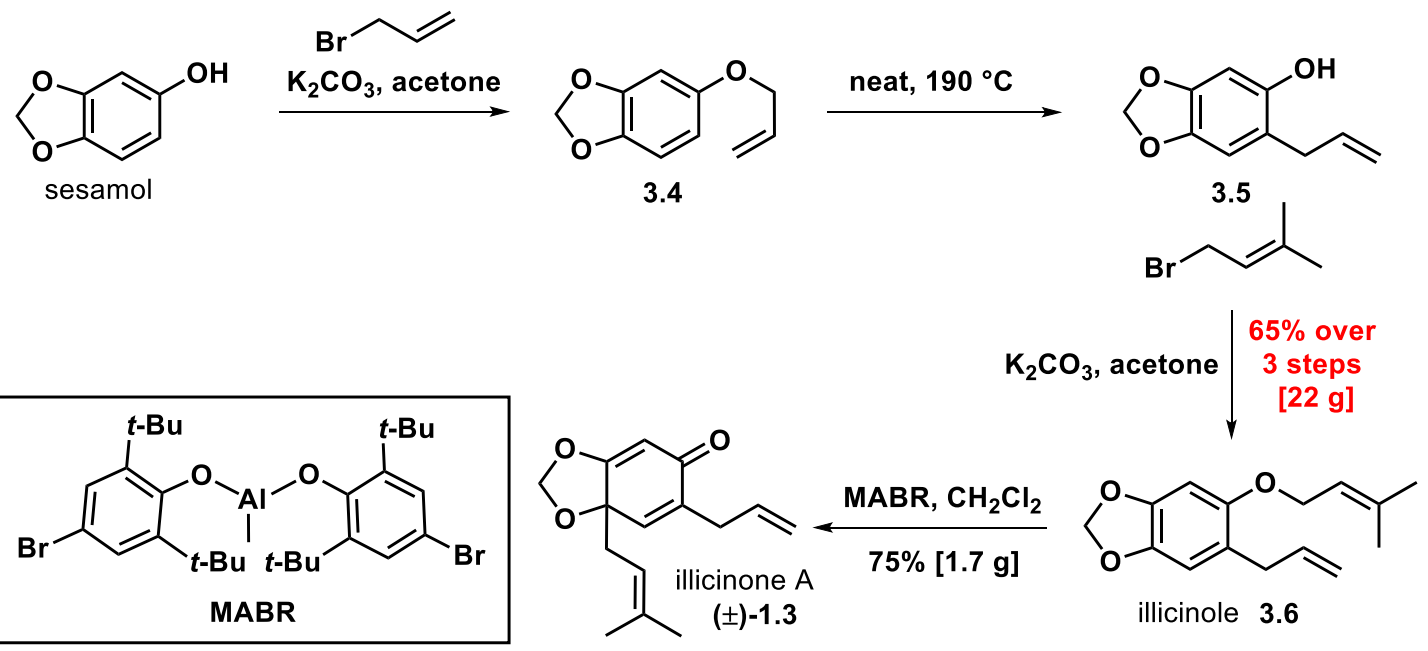

Scheme 3.37: Our scale-up of Danishefsky's synthesis of ( \pm -illicinone A 1.3.

With ( \pm )-illicinone A 1.3 in hand, we started our investigation into the synthesis of more complex phenylpropanoid natural products. As discussed previously, $( \pm)$-illicinone A $\mathbf{1 . 3}$ is the putative precursor to the majority of our molecular targets. It was decided to initially focus on those retaining the methylene bridge, as there are a number of structures closely related to one another containing that functionality (a few examples are depicted in figure 3.2).

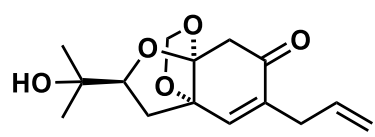

(+)-illicinone $\mathrm{E}$ 3.80

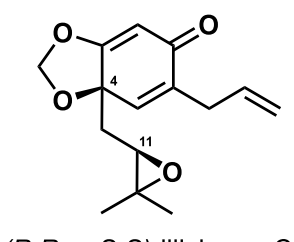

$(+)$ or $(-)-3.78$

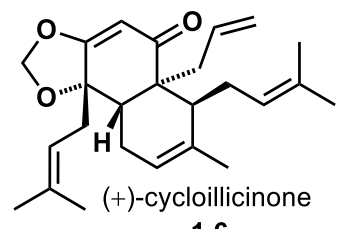

1.6

Figure 3.2: Examples of targeted bridged prenylated phenylpropanoids. 


\subsubsection{Total Synthesis of ( \pm )-Cycloillicinone 1.6}

(+)-Cycloillicinone $\mathbf{1 . 6}$ was isolated for the first time in 2011 by Fukuyama and coworkers from the twigs of Illicium anisatum. ${ }^{127}$ Preliminary studies showed that this compound showed moderate neurite-outgrowth promoting activity in rat neurons. This compound features a tricyclic framework and 4 contiguous stereogenic centers. The relative stereochemistry was elucidated with nOe experiments and polarimetric measurements indicated natural cycloillicinone $\mathbf{1 . 6}$ to be the dextrorotatory enantiomer. However, it was not possible to establish its absolute configuration. Fukuyama and collaborators proposed two divergent biosynthetic pathways for its formation, both starting from allylated sesamol 3.5. Initial direct $C$-prenylation of allylated sesamol 3.5 would produce the known natural product illicinone A $\mathbf{1 . 3}$, the common intermediate in these two biosynthetic hypotheses. At this point two alternative routes were proposed. The first one involved geranylation of illicinone A $\mathbf{1 . 3}$ to afford a geranylated intermediate, which would then undergo a cyclisation to provide (+)-cycloillicinone $\mathbf{1 . 6}$ (path a), with no detail on the exact mechanism of this transformation provided by the authors. Given the non-nucleophilic character of illicinone A 1.3 at $\mathrm{C} 3$, we consider the second proposed pathway, a Diels-Alder cycloaddition between illicinone A $\mathbf{1 . 3}$ and trans- $\beta$-ocimene (path b), to be a more likely possibility (scheme 3.38).
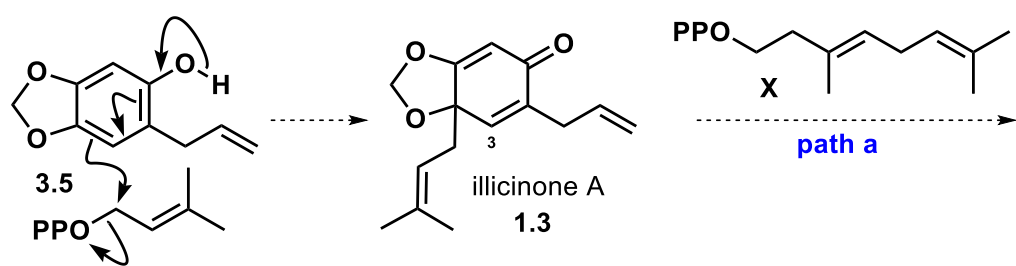

III

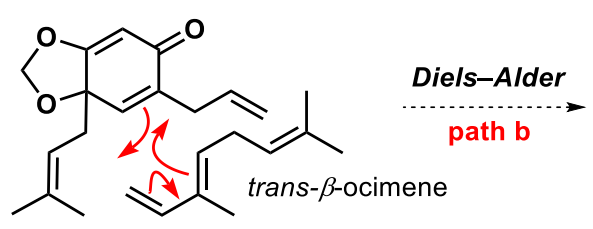

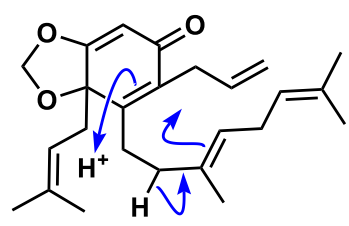

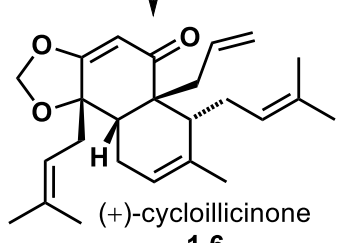

1.6

Scheme 3.38: Fukuyama's proposed biosynthesis of $(+)$-cycloillicinone 1.6. ${ }^{127}$

Intrigued by this second hypothesis, we set out to investigate the feasibility of this highly selective Diels-Alder cycloaddition. Heating ( \pm )-illicinone A 1.3 at $140{ }^{\circ} \mathrm{C}$ for 
2 hours in the presence of ten equivalents of $\beta$-ocimene (as a 6:4 mixture of trans/cis isomers) resulted in the formation of a small amount $(\sim 8 \%)$ of targeted $( \pm)$-cycloillicinone 1.6, along with a complex mixture of unidentified products and recovered starting material $(\sim 6 \%)$, based on the ${ }^{1} \mathrm{H}$ NMR spectrum of the crude reaction mixture. As Diels-Alder cycloadditions are known to be catalysed by Lewis acids, we speculated that a one-pot synthesis of $( \pm)$-cycloillicinone $\mathbf{1 . 6}$ from illicinole 3.6 was possible. Thus, the conditions previously employed for the formation of $( \pm)$-illicinone A 1.3 were used until complete conversion of the starting material to give $( \pm)$-illicinone A 1.3 was observed by TLC analysis. Five equivalents of $\beta$-ocimene were then added and the reaction mixture was allowed to slowly warm to room temperature and stirred overnight before being quenched. To our delight, the DielsAlder cycloaddition proceeded with complete control of regio and stereo-selectivity, affording ( \pm )-cycloillicinone $\mathbf{1 . 6}$ as the sole product, isolated in $43 \%$ yield from illicinole 3.6 (scheme 3.39).

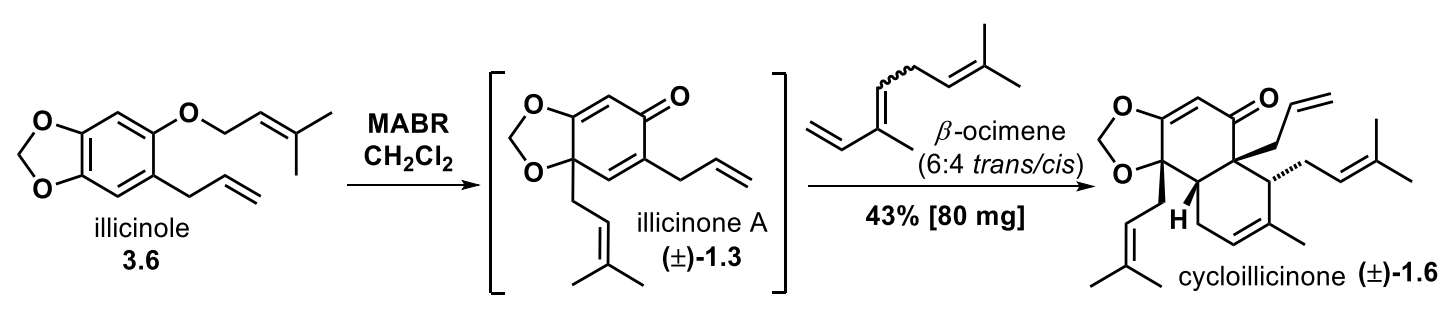

Scheme 3.39: Our synthesis of $( \pm)$-cycloillicinone 1.6.

The relative stereochemistry proposed by Fukuyama and co-workers was confirmed to be correct by X-ray crystallography. When achieved, this represented the first total synthesis of $( \pm)$-cycloillicinone $\mathbf{1 . 6}$ at the time, accomplished in $28 \%$ yield over 4 steps. Unfortunately, shortly after this accomplishment, Rahman and co-workers published the synthesis of this compound employing the same strategy. ${ }^{158}$ However, in this slightly longer and less efficient synthesis ( 5 steps, $24 \%$ yield from sesamol), the Diels-Alder cycloaddition was performed as an additional step after the MABRpromoted prenyl-group migration. A different Lewis acid was used to catalyse the Diels-Alder reaction: upon extensive screening of conditions and catalysts, they found that treatment of $( \pm)$-illicinone A $\mathbf{1 . 3}$ with $20 \mathrm{~mol} \%$ of $(S)$-o-tolyl-CBSoxazaborolidine in $\mathrm{CH}_{2} \mathrm{Cl}_{2}$ at $-40{ }^{\circ} \mathrm{C}$ for 8 hours gave the best results, affording $( \pm)$-cycloillicinone $\mathbf{1 . 6}$ in 55\% yield from $\left( \pm\right.$ )-illicinone A 1.3 (scheme 3.40). ${ }^{158}$ 


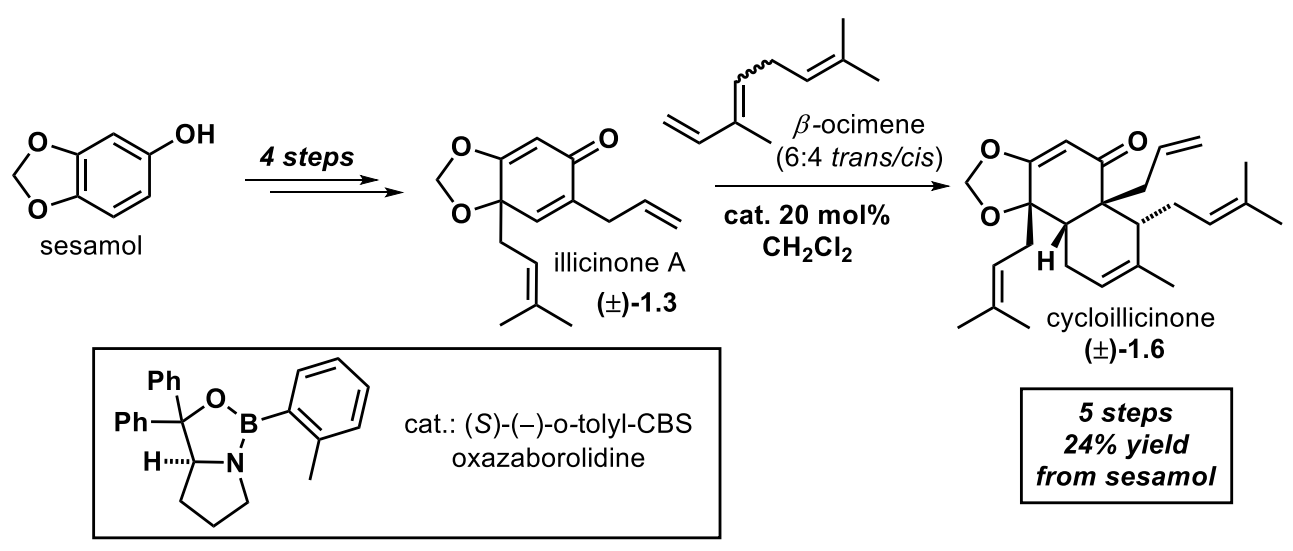

Scheme 3.40: Rahman's 2015 synthesis of $( \pm)$-cycloillicinone 1.6. ${ }^{158}$

Although disappointing to see this synthesis published, it is important to remember that the aim of our project is not to access a particular compound. We aim to achieve the synthesis of a selection of natural product targets featuring diverse molecular frameworks. The emphasis is in the number and structural diversity of molecules prepared using a unified strategy.

\subsubsection{Synthesis of ( \pm )-Illicinone C 3.78 and ( \pm )-epi-Illicinone C 3.79}

(+)-Illicinone E 3.80, (-)-epi-illicinone E 3.2 and the closely related natural products (+)-13-O-methylillicinone E $\mathbf{3 . 8 1}$ and (-)-illioliganfunone A $\mathbf{3 . 8 2}$ have been isolated from Illicium oligandrum and Illicium tashiroi between 1992 and 2011. ${ }^{117,119,125}$ These are part of a large group of compounds extracted from different plants belonging to the Illicium genus, some in enantiopure form and others as racemic mixtures. A number of permutations with regard to the relative stereochemistry have also been encountered for these frameworks, in compounds isolated from different species. ${ }^{135}$ We envisaged that these structures could be accessed from either epoxide ( \pm )-illicinone C 3.78 or ( \pm )-epi-illicinone C 3.79 (figure 3.3). 


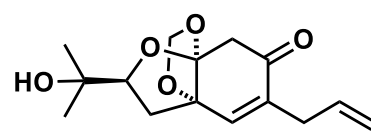

illicinone $\mathrm{E}$

(+) -3.80

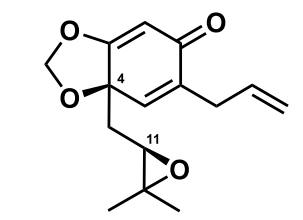

(R,R or $S, S)$-illicinone $\mathrm{C}$

$(+)$ or $(-)-3.78$

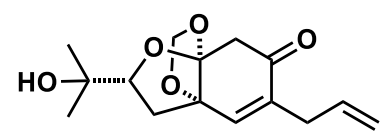

epi-illicinone $\mathrm{E}$

$(-)-3.2$

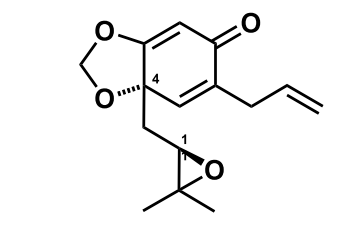

(S,R or $R, S)$-epi-illicinone C

$(+)$ or $(-)-3.79$

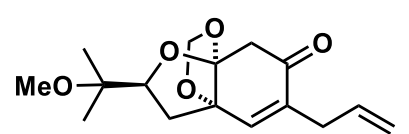

13-O-methylillicinone $\mathrm{E}$

(+)-3.81

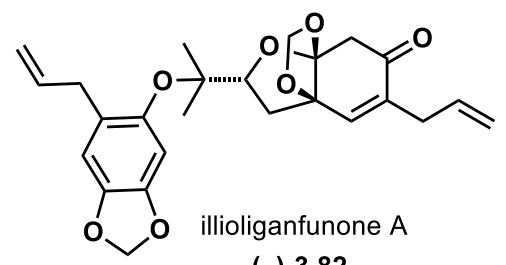

(-) -3.82

Figure 3.3: (+)-Illicinone E $\mathbf{3 . 8 0}$ and related structures.

It should be noted that (+)-Illicinone C 3.78 and (-)-epi-illicinone $C \mathbf{3 . 7 9}$ are both known natural products, isolated from Illicium tashiroi and Illicium arborescens respectively. ${ }^{135} \mathrm{We}$ propose that both of these compounds could be prepared in racemic form via non-diastereoselective epoxidation of $( \pm)$-illicinone A 1.3. Therefore, our investigation toward the total synthesis of this group of natural products began with the epoxidation of $( \pm)$-illicinone A 1.3. Treatment with $m$-CPBA in dichloromethane at room temperature smoothly afforded a nearly 1:1 mixture of diastereoisomeric epoxides ( \pm )-illicinone C 3.78 and ( \pm )-epi-illicinone C 3.79 in $94 \%$ yield on a 1.8 gram scale (scheme 3.41).

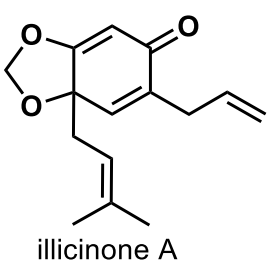

$( \pm)-1.3$

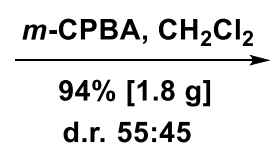

$( \pm)-3.79:( \pm)-3.78$

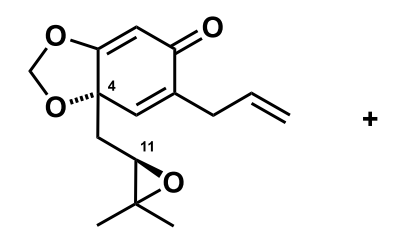

$(S, R$ or $R, S)$-epi-illicinone $\mathrm{C}$ $( \pm)-3.79$

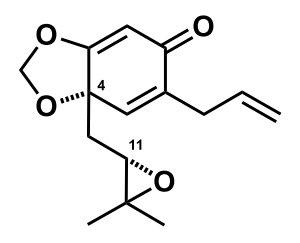

$(R, R$ or $S, S)$-illicinone $\mathrm{C}$

$( \pm)-3.78$

Scheme 3.41: Epoxidation of $( \pm)$-illicinone A 1.3.

The lack of diastereoselectivity was not considered a problem, as both stereoisomers were potential precursors to known natural products. More disappointing was the difficulty encountered in separating the two diastereoisomers; the $(4 S-11 R / 4 R-11 S)$ diastereoisomer ( $( \pm)$-epi-illicinone C 3.79) was isolated in a modest yield of $34 \%$, whereas $( \pm$ )-illicinone C $\mathbf{3 . 7 8}$ was isolated in only $17 \%$ yield following flash chromatography. 


\subsubsection{Synthesis of ( \pm )-epi-Illicinone E 3.2 and Related Structures}

Due to the low yield of $( \pm$ )-illicinone $C$ 3.78, resulting from the difficulties in purification, we decided to proceed with our investigations using its diastereoisomer $( \pm)$-epi-illicinone $\mathrm{C} \mathrm{3.79}$, which we could access in larger quantities. A first attempt to access ( \pm )-epi-illicinone E 3.2 was performed by reacting ( \pm )-epi-illicinone C 3.79 with a catalytic amount of $p$-toluenesulfonic acid in a 95:5 mixture of ethanol and water. The reaction was quenched after complete consumption of the starting material was observed by TLC analysis. Chromatographic purification afforded four different compounds, one of which was identified as ( \pm )-epi-illicinone E 3.2, a known natural product. Another fraction consisted of the ethyl ether of ( \pm -epi-illicinone E $\mathbf{3 . 2}$ $\left( \pm\right.$-epi-13-O-ethylillicinone E 3.83, while ${ }^{1} \mathrm{H}$ NMR analysis of the other two compounds revealed the absence of the methylene bridge. Comparison with the NMR data reported for related natural products isolated from Illicium genus plants suggested these compounds corresponded to structures ( \pm )-3.84 and ( \pm )-3.85 (scheme 3.42). Ethyl ether ( \pm )-3.84 was fully characterised, while diol $( \pm)-3.85$ was not deemed sufficiently pure for these purposes. However, by comparing their ${ }^{1} \mathrm{H}$ NMR spectra with that reported for the known natural product 2,3-dehydroillifunone $\mathrm{C} \mathrm{3.86}$, it is reasonable to assign the structures shown in scheme 3.42, which possess a different relative stereochemistry with respect to the known natural product.

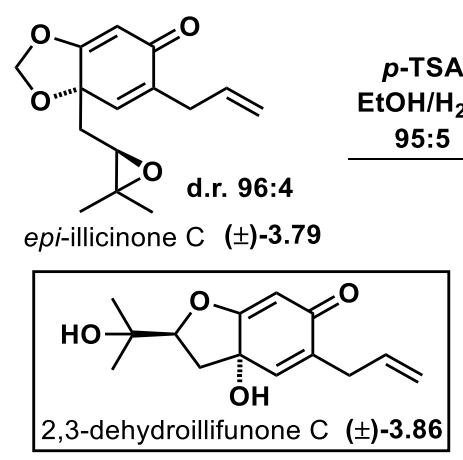

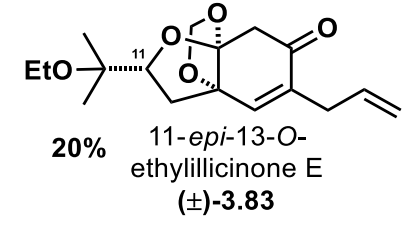

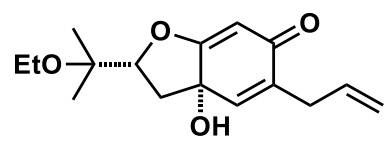

$8 \%( \pm)-3.84$

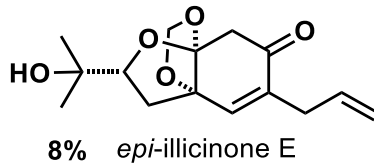

$( \pm)-3.2$

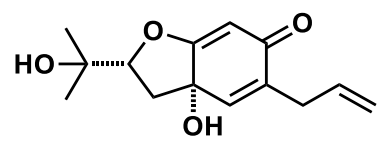

$5 \%( \pm)-3.85$

Scheme 3.42: Preliminary attempt on accessing ( \pm )-epi-illicinone E 3.2 and related structures.

We propose that these structures could be produced via acid-catalysed regioselective ring-opening of the epoxide by either ethanol or water. Intramolecular addition of the free secondary alcohol functionality to $\mathrm{C} 5$ would then produce the oxa-Michael 
reaction products. This sequence would lead to ( \pm )-epi-illicinone E 3.2 and ( \pm )-epi-13$O$-ethylillicinone E 3.83 (scheme 3.43).
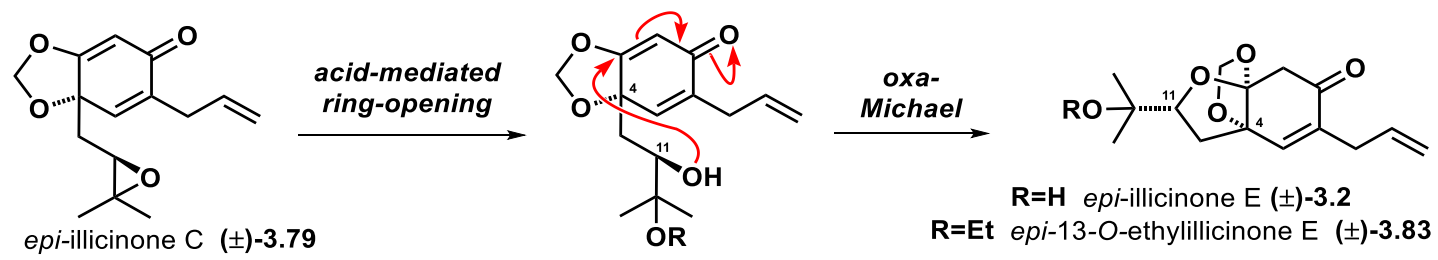

Scheme 3.43: Proposed mechanism for the formation of (土)-epi-illicinone E 3.2 and

(士)-epi-13-O-ethylillicinone E $\mathbf{3 . 8 3}$.

It is envisaged that an analogous sequence could be used to access ( \pm )-illicinone $\mathrm{E}$ and the related natural products with the same relative stereochemistry at C4 and C11.

We then attempted to rationalise the formation of tertiary alcohols $( \pm)-\mathbf{3 . 8 4}$ and $( \pm)-3.85$, which lack of methylene bridge. Diol $( \pm)-3.85$ has not yet been extracted from natural sources, but its diastereoisomer 2,3-dehydroillifunone $\mathrm{C}$ has been coisolated with both (+)-illicinone E 3.80 and (-)-epi-illicinone E 3.2. It is therefore reasonable to assume that ( \pm )-epi-2,3-dehydroillifunone $C \mathbf{3 . 8 5}$ could also exist in nature. The formation of ( \pm )-epi-2,3-dehydroillifunone C 3.85 and ( \pm )-epi-13-O-ethyl2,3-dehydroillifunone C $\mathbf{3 . 8 4}$ was detected via TLC analysis of the crude reaction mixture, taken at various times, well before complete consumption of the starting material. With regard to ( \pm -epi-2,3-dehydroillifunone $C \mathbf{3 . 8 5}$, we hypothesise that it could be formed via acetal hydrolysis and elimination from ( \pm )-epi-illicinone E 3.2 (scheme 3.44). An analogous mechanism would account for the formation of ( \pm )-epi13-O-ethyl-2,3-dehydroillifunone C $\mathbf{3 . 8 4}$ from ( \pm )-13- $O$-ethylillicinone C $\mathbf{3 . 8 3}$.

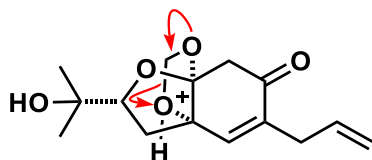

(土)-epi-illicinone $\mathrm{E}$ 3.2

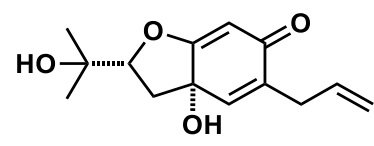

$( \pm)$-epi-2,3dehydroillifunone $C$
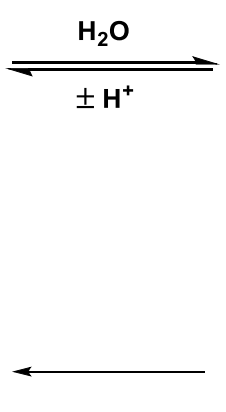
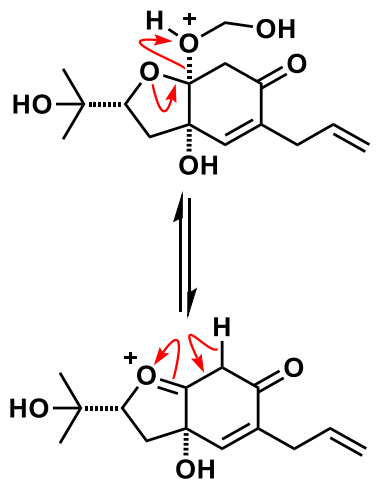

Scheme 3.44: Proposed mechanism for the formation of (土)-epi-2,3-dehydroillifunone C $\mathbf{3 . 8 5}$. 


\subsubsection{Synthesis of ( \pm )-epi-Illicinone $\mathbf{E} 3.2$}

It was envisaged that ( \pm -epi-illicinone $\mathrm{E} \mathbf{3 . 2}$ could be accessed selectively by reacting ( \pm )-epi-illicinone C 3.2 with water as the nucleophile in the presence of a catalytic amount of acid. Therefore, epoxide $\mathbf{3 . 7 9}$ was dissolved in a mixture of water and THF, for solubility reasons, and a small amount of $p$-toluenesulfonic acid was added. Pleasingly, $( \pm)$-epi-illicinone E $\mathbf{3 . 2}$ was isolated as a product of this reaction in $19 \%$ yield. Unfortunately, attempts to isolate analytically pure samples of ( \pm )-epi-2,3dehydroillifunone $\mathrm{C} \mathbf{3 . 8 5}$, the presence of which was detected in the ${ }^{1} \mathrm{H}$ NMR spectrum of the crude reaction mixture, failed (scheme 3.45).
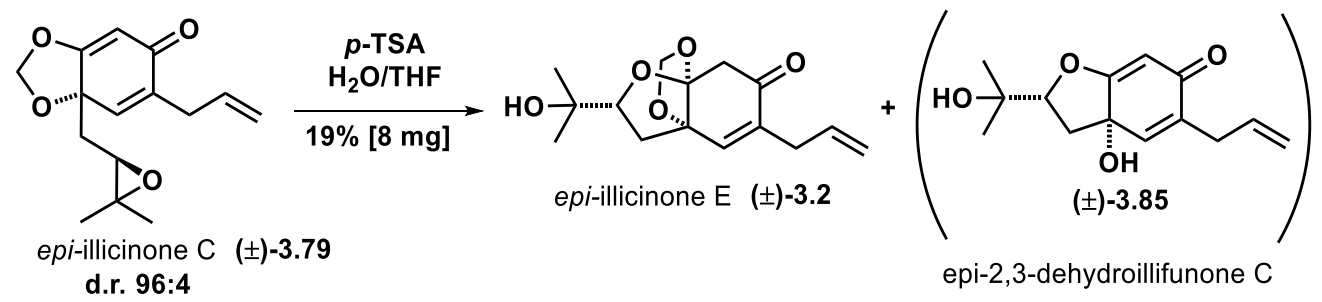

Scheme 3.45: Synthesis of ( \pm -epi-illicinone E 3.2.

\subsubsection{Synthesis of ( \pm )-epi-13-O-Methylillicinone C 3.87}

It was reasoned that ( \pm )-epi-13-O-methylillicinone $\mathrm{C} \mathrm{3.87}$, the diastereoisomer of the known natural product 13-O-methylillicinone C 3.81 (see figure 3.3 on page 122), ${ }^{117}$ could be accessed using a similar approach, using methanol as the nucleophile. Therefore, the reaction of epoxide ( \pm )-epi-illicinone C $\mathbf{3 . 7 9}$ in anhydrous methanol with a small amount of $p$-toluenesulfonic acid was attempted. As a result, $( \pm)$-epi-13$O$-methylillicinone C $\mathbf{3 . 8 7}$ was isolated, along with alcohol ( \pm -3.88, in 40 and $13 \%$ yield respectively (scheme 3.46).

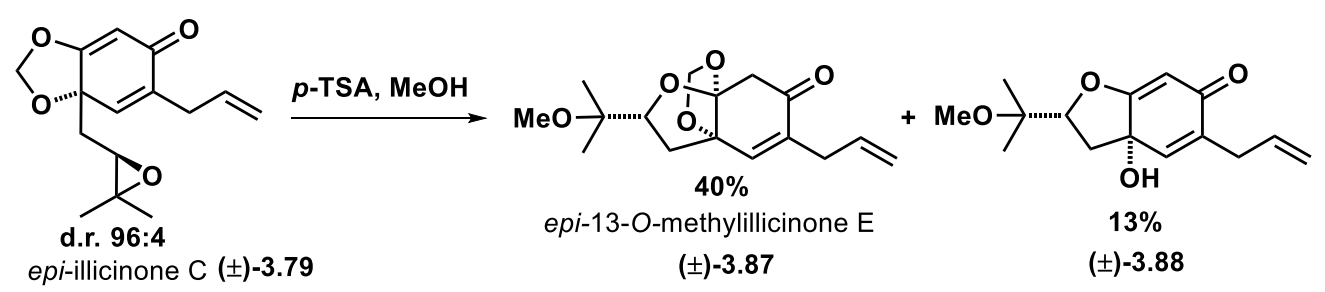

Scheme 3.46: Synthesis of ( \pm )-epi-13-O-methylillicinone E 3.87 and alcohol ( \pm )-3.88. 
Alcohol ( \pm )-3.88 was detected by TLC analysis of the crude reaction mixture as soon as the formation of ( \pm -epi-13-O-methylillicinone E 3.87 was detected. Attempts to suppress its formation by lowering the temperature were unsuccessful.

Similarly to ( \pm )-epi-2,3-dehydroillifunone C $\mathbf{3 . 8 5}$, we propose alcohol ( \pm )-3.88 to be the result of the solvent-mediated cleavage of the methylene bridge on the corresponding ether $( \pm)$-3.87. To confirm this hypothesis, we re-subjected $( \pm)$-epi-13$O$-methylillicinone C $\mathbf{3 . 8 7}$ to the same conditions. Deuterated methanol was used as the solvent to enable monitoring of the reaction by ${ }^{1} \mathrm{H}$ NMR. A first control after 30 minutes indicated the presence of trace amounts of alcohol ( \pm -3.89, which reached $21 \%$ conversion after another hour (scheme 3.47), thus supporting our mechanistic proposal (see scheme 3.44 on page 124).

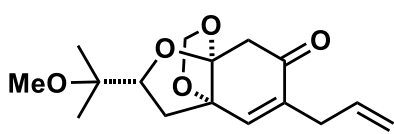

epi-13-Omethylillicinone $\mathrm{C}$

$( \pm)-3.87$

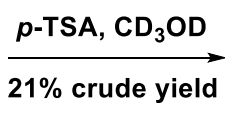

Scheme 3.47: Formation of alcohol ( \pm )-3.89 from $( \pm)$-epi-13-O-methylillicinone E 3.87.

\subsubsection{Synthesis of ether 3.84}

It was envisaged that this methodology could be used to access a wide range of differently substituted analogues. We therefore expanded the scope of this transformation by performing a similar reaction using ethanol as the solvent. The only product isolated after 24 hours in this case $( \pm)$-epi-13-O-ethyl-2,3-dehydroillifunone C 3.84, in $20 \%$ yield (scheme 3.48).

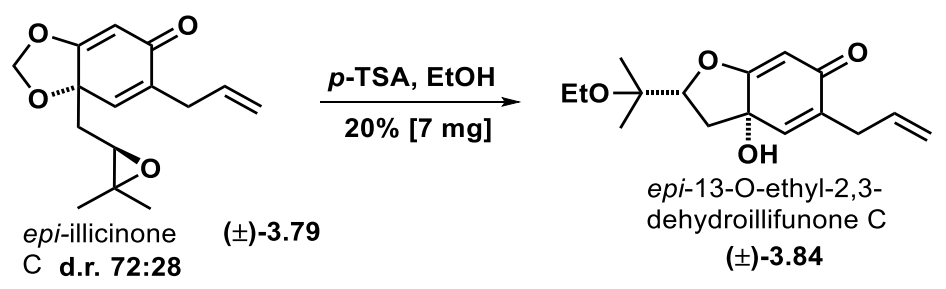

Scheme 3.48: Preparation of ( \pm )-epi-13-O-ethyl-2,3-dehydroillifunone C 3.84. 


\subsection{Concluding Remarks}

In conclusion, we designed and implemented two biomimetic approaches toward the total synthesis of a family of prenylated phenylpropanoids from plants of the Illicium genus based on our proposed biosynthetic pathway. The first approach involved the oxidative dearomatisation of two already assembled prenylated phenylpropanoid moieties $\mathbf{3 . 4 0}$ and $\mathbf{3 . 4 1}$ which differed by the relative position of the allyl and prenyl group in the aromatic ring. Different routes were investigated in an attempt to access these two aromatic intermediates in our synthesis. Phenol 3.40 was prepared in three steps from commercially available arene 3.39 via a sequence involving allyl-ether formation, prenylation of the aromatic ring and Claisen rearrangement. Ortho-quinol ( \pm )-3.35, our putative precursor to the natural products we classified as type $\mathbf{A}_{2}$ structures, was prepared via oxidative dearomatisation of phenol 3.40. However, ortho-quinol ( \pm )-3.35 underwent facile Cope rearrangement to produce dienone (士)-3.38, precursor to type $A_{1}$ frameworks (scheme 3.49).<smiles>COc1cc(O)ccc1Br</smiles>

3.39

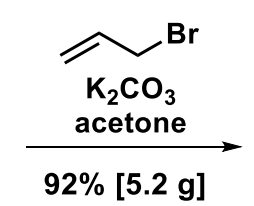

(5.2 g]<smiles>C=CCOc1ccc(Br)c(OC)c1</smiles>

3.42<smiles>CCCCC(C)=CCBr</smiles>

$\mathrm{Et}_{2} \mathrm{O} / \mathrm{C}_{2} \mathrm{H}_{6} 2: 1$<smiles>C=CCOc1ccc(CC=C(C)C)c(OC)c1</smiles>

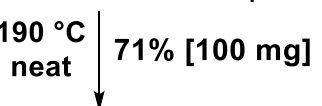<smiles>C=CCC1(O)C=C(CC=C(C)C)C(OC)=CC1=O</smiles><smiles>C=CCc1cc(CC=C(C)C)c(OC)cc1O</smiles>
SIBX, DMSO

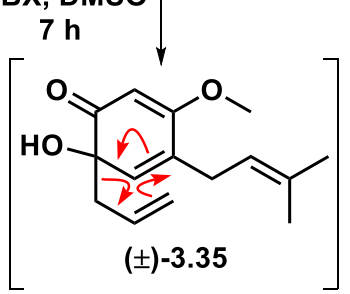<smiles>C=CCC1(CC=C(C)C)C=C(O)C(=O)C=C1OC</smiles>

$54 \%[100 \mathrm{mg}]$

$( \pm)-3.35$

"Crude yield.

Scheme 3.49: Preparation of ortho-quinol ( \pm )-3.35 and dienone ( \pm )-3.38.

Phenol 3.41 was accessed following a different approach from the same starting material. The successful sequence began with phenolic MOM-protection, followed by allylation and propargyl-ether formation. Partial reduction of alkyne $\mathbf{3 . 7 1}$ and Claisen 
rearrangement of the resulting alkene 3.45 then afforded phenol 3.41 in six steps from arene 3.39. Oxidative dearomatisation of prenylated phenylpropanoid 3.41 produced the corresponding ortho-quinol ( \pm )-3.37, precursor to the type $\mathbf{B}_{2}$ frameworks, however, this compound was found to be not stable (scheme 3.50).
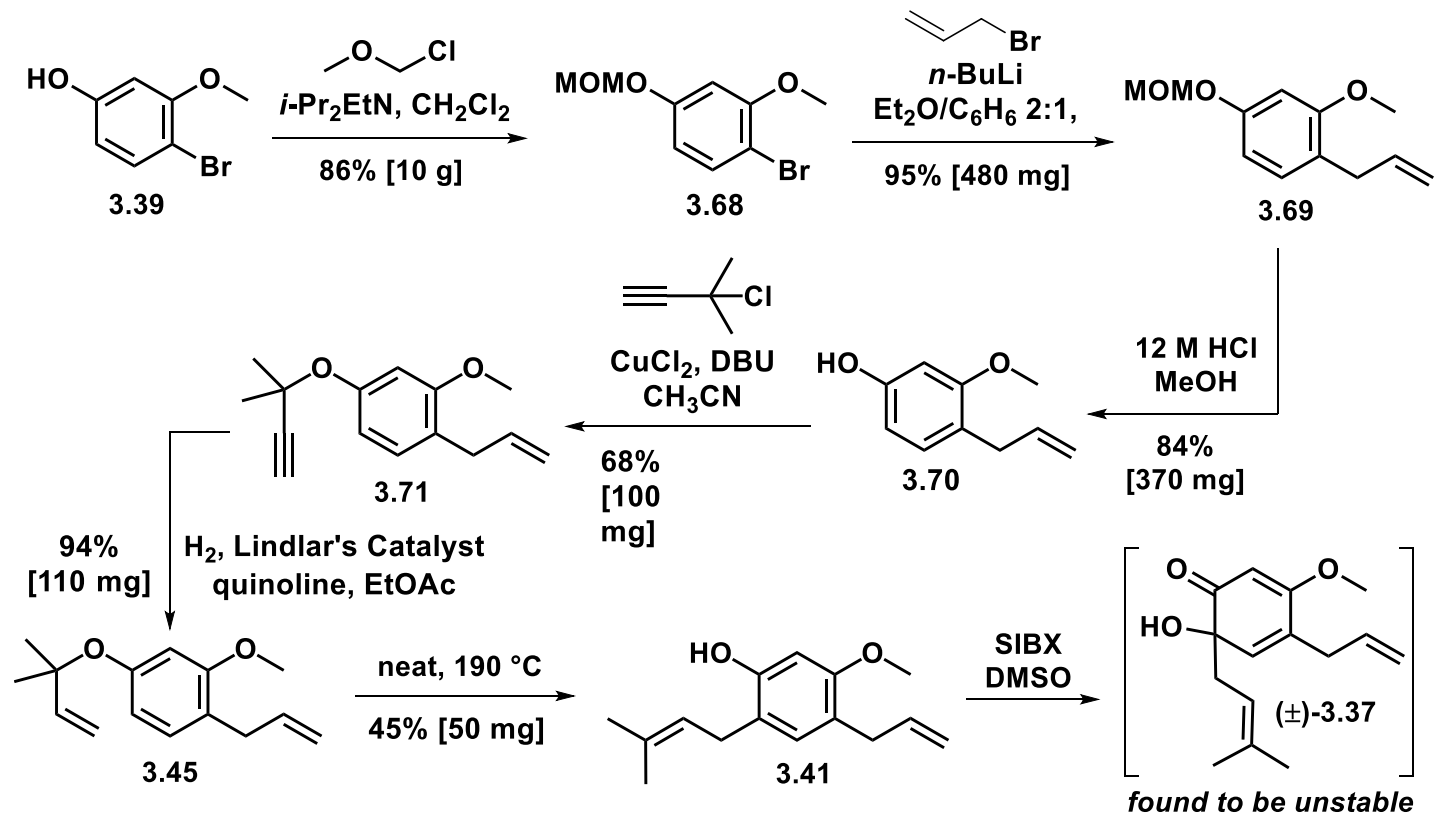

Scheme 3.50: Preparation of phenol ortho-quinol ( \pm$)-3.37$.

The instability of intermediates $( \pm)-3.35$ and $( \pm)-3.37$, along with the overall low efficiency of the synthetic sequences, especially with regard to the precursor to type $\mathbf{B}_{2}$ structures ( \pm )-3.37, lead us to undertake our second biomimetic approach. We therefore adopted Danishefsky's previous synthesis of ( \pm )-illicinone A $\mathbf{1 . 3}$ from sesamol, discussed in section 3.2.1. This involved a sequence of $O$-allylation, thermal Claisen rearrangement, $O$-prenylation and MABR-mediated prenyl migration. This afforded the alkylative dearomatisation product $( \pm)$-illicinone A 1.3 in four steps from sesamol (scheme 3.51).
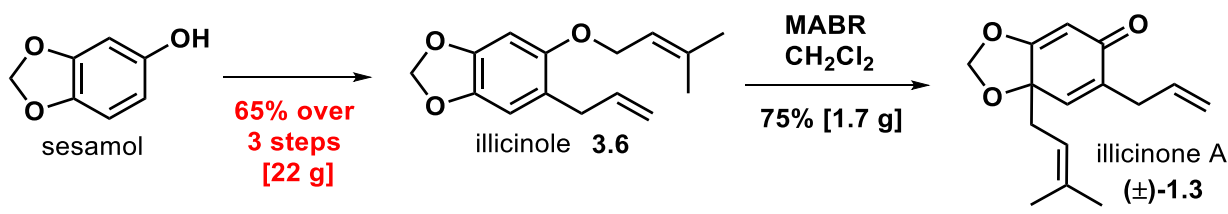

Scheme 3.51: Our synthesis of $( \pm)$-illicinone A 1.3.

$( \pm)$-Illicinone A $\mathbf{1 . 3}$ possesses a type $\mathbf{B}_{2}$ structure, therefore we started our investigation toward the total synthesis of more complex natural products belonging 
to this group. We prepared $( \pm$ )-cycloillicinone $\mathbf{1 . 6}$ in 4 steps and $28 \%$ yield from sesamol via a one-pot sequence of migration of the prenyl group on illicinole $\mathbf{3 . 6}$ and Diels-Alder cycloaddition with the natural occurring terpene $\beta$-ocimene (scheme 3.52). $m$-CPBA epoxidation of ( \pm -)-illicinone A $\mathbf{1 . 3}$ produced a $1: 1$ mixture on diastereomeric epoxides illicinone $C( \pm)-\mathbf{3 . 7 8}$ and $( \pm)$-epi-illicinone $C$ 3.79. As both epoxides are precursors to natural products, this could enable a stereodivergent synthesis of our molecular targets. ( \pm )-epi-Illicinone C $\mathbf{3 . 7 9}$ was subjected to a onepot epoxide ring-opening/oxa-Michael reaction in different nucleophilic solvents. This reaction produced ( \pm )-epi-illicinone $\mathrm{E} 3.2,( \pm)$-epi-13-O-methylillicinone $\mathrm{E} 3.87$, and ethyl-ether ( \pm )-3.83 along with small amounts of ( \pm )-epi-2,3-dehydroillifunone C 3.85 and related structures (scheme 3.51). We envisage that ( \pm )-illicinone $\mathrm{C} \mathbf{3 . 7 8}$ could be used to prepare other natural products, epimers of compounds ( \pm )-epi-illcinone $\mathbf{3 . 2}$ and ( \pm -3.87 at $\mathrm{C} 11$.
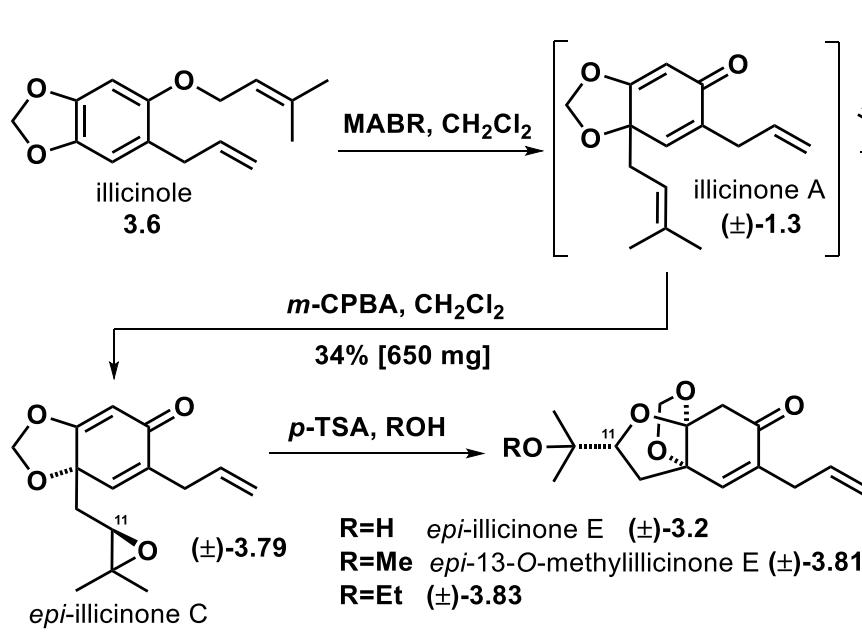
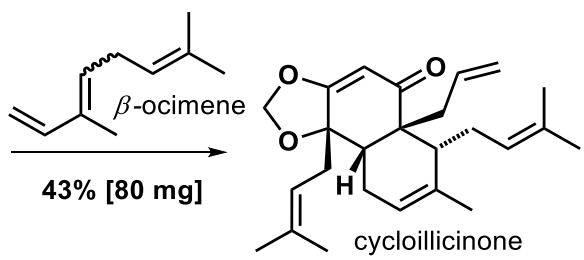

$( \pm)-1.6$

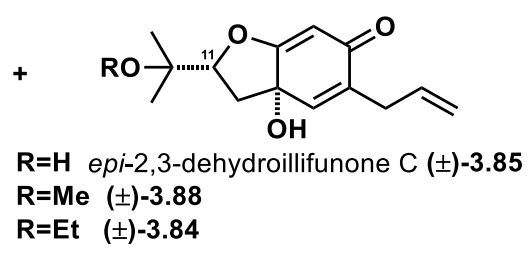

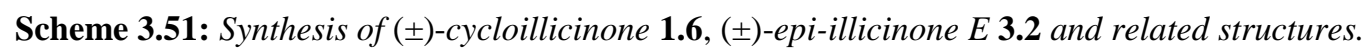




\subsection{Future Work}

\subsubsection{Illicinone E ( \pm )-3.80 and Related Structures}

Future work on this group of natural products will focus on the optimisation of the reaction conditions in an attempt to improve yield and selectivity. Furthermore, there are other natural products potentially accessible from epoxides ( \pm )-illicinone C 3.78 and ( \pm )-epi-illicinone C 3.79 under similar reaction conditions (see section 3.5.4). In particular, ( \pm )-illicinone E 3.80, ( \pm )-2,3-dehydroillifunone $\mathrm{C} 3.86$ and 13-Omethylillicinone E $\mathbf{3 . 8 1}$ could be accessed using ( \pm )-illicinone C 3.78 as the starting material. Furthermore, the more complex $( \pm)$-illioliganfunones A 3.90 and B 3.91 could be prepared by reacting epoxide ( \pm )-illicinone C $\mathbf{3 . 7 8}$ with allylated sesamol $\mathbf{3 . 5}$ in a non-nucleophilic solvent. Finally, a group of natural products containing the core structure of $( \pm)-3.92^{159}$ could be accessed in racemic form via hydrogenation of ( \pm )-2,3-dehydroillifunone C $\mathbf{3 . 8 6}$ and related frameworks. A selection of targets is depicted in figure 3.4.

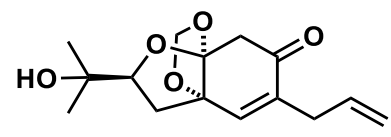

illicinone $\mathrm{E}$ $( \pm)-\mathbf{3 . 8 0}$

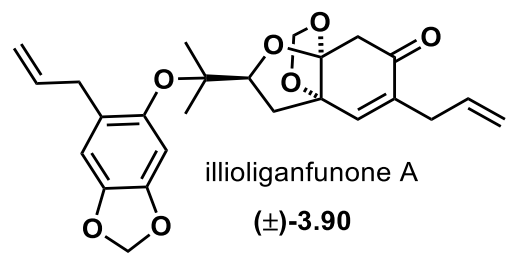

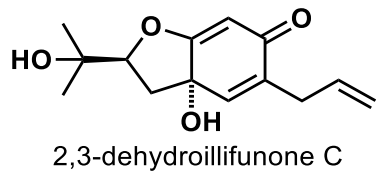

$( \pm)-3.86$

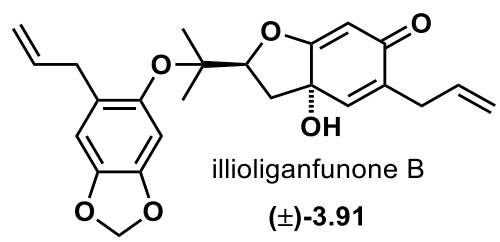

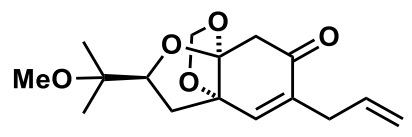

13-O-methylillicinone $\mathrm{E}$

$( \pm)-3.81$

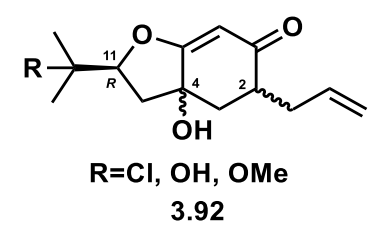

Figure 3.4: Selection of targets potentially accessible from ( \pm -illicinone C $\mathbf{3 . 7 8}$.

It is envisaged that large quantities of epoxide ( \pm )-illicinone $\mathrm{C} \mathbf{3 . 7 8}$ will be required to investigate and optimise the reaction conditions to selectively access each one of these structures. In this regard, priority will be given to the optimisation of the purification process used to separate the two diastereomeric epoxides ( \pm )-illicinone C $\mathbf{3 . 7 8}$ and ( \pm -epi-illicinone C 3.79. We propose that preparative HPLC could be a suitable method for this purpose. 


\subsection{2 ( \pm )-Spirooliganones A 1.7 and B 3.9}

As discussed in section 3.2.2 on pages 93-96, to date the asymmetric total synthesis of (-)-spirooliganones A 1.7 and B 3.9 has been reported three times. ${ }^{138-140}$ However, we aim to exploit our divergent approach by targeting these natural products as well. We hypothesise that we could access them as racemates along with two of their diastereoisomers (the four stereochemical combinations at $\mathrm{C} 4$ and $\mathrm{C} 17$ ) in five steps from illicinole 3.6. This could be achieved via initial cleavage of the methylene bridge of illicinole 3.6 to produce catechol 3.93. Knoevenagel condensation with formaldehyde or a synthetic equivalent would then afford ortho-quinone methide intermediate 3.94, which could undergo hetero-Diels-Alder cycloaddition as suggested by literature precedent. ${ }^{138-140}$ This would afford a mixture of epimers at C17, which would then be subjected to prenyl group migration (sequence of Claisen-Cope rearrangements), generating a new stereocenter at $\mathrm{C} 4$. Diastereoselective epoxidation of the prenyl side-chain would then produce a mixture of four diastereomeric epoxides, which could undergo intramolecular ring-opening upon addition of the hydroxyl group at $\mathrm{C} 4$ (scheme 3.52).

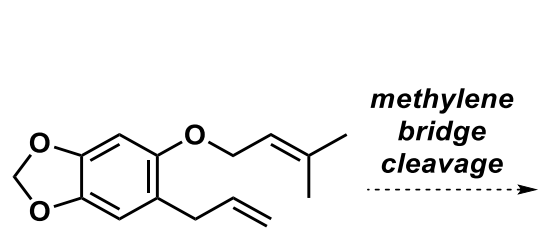

illicinole 3.6<smiles>C=CCc1cc(O)c(O)cc1OCC=C(C)C</smiles>

$( \pm)-3.93$<smiles>C=CCC1=C(OCC=C(C)C)C(=C)C(=O)C(O)=C1</smiles>

heteroDiels-Alder

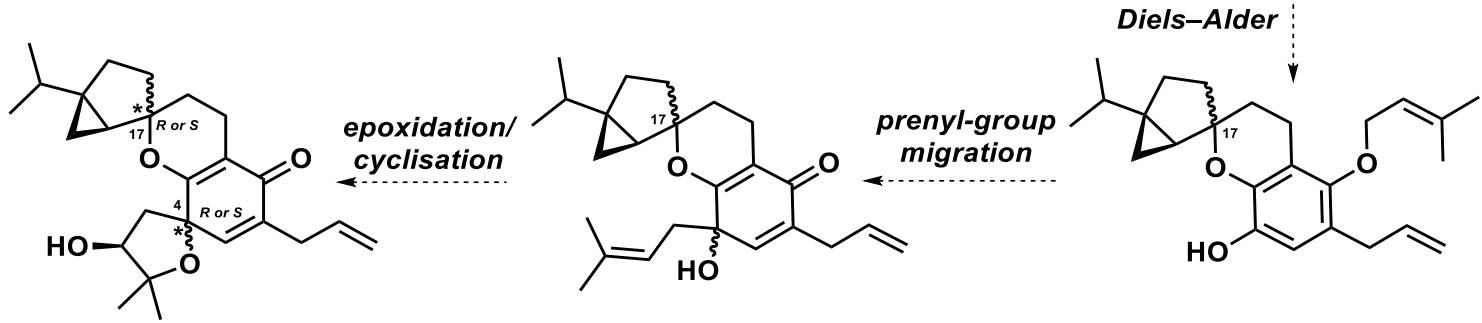

Scheme 3.52: Our proposed synthesis of ( \pm -spirooliganones $A 1.7$ and B 3.9 and other diastereoisomers.

If successful, this could represent the shortest (seven steps $v$ s. eight, twelve and eleven steps $)^{138-140}$ synthesis of these two natural products and two natural product-like related structures. 


\subsection{3 ( \pm )-Illicidione B 3.3}

(-)-Illicidione B 3.3 was isolated in 2011 by Liu and co-workers from the stem bark of Illicium oligandrum. ${ }^{126}$ We propose we could synthesise it in racemic form from the ready accessible ( \pm )-illicinone A $\mathbf{1 . 3}$ through initial cleavage of the methylene bridge, followed by Knoevenagel condensation of the resulting dienone ( \pm )-3.95 with formaldehyde. The so formed dienone ( \pm )-3.96 could then undergo Michael addition with another molecule of dienone ( \pm )-3.95 followed by tautomerisation. Finally, diastereoselective double epoxidation of bis-ketone $( \pm)-3.97$ to install the correct relative stereochemistry between $\mathrm{C} 4$ and $\mathrm{C} 11$ (and $\mathrm{C} 4$ ' and $\mathrm{C} 11^{\prime}$ ) and a final double cyclisation would produce ( \pm )-illicidione B $\mathbf{3 . 3}$ (scheme 3.53).

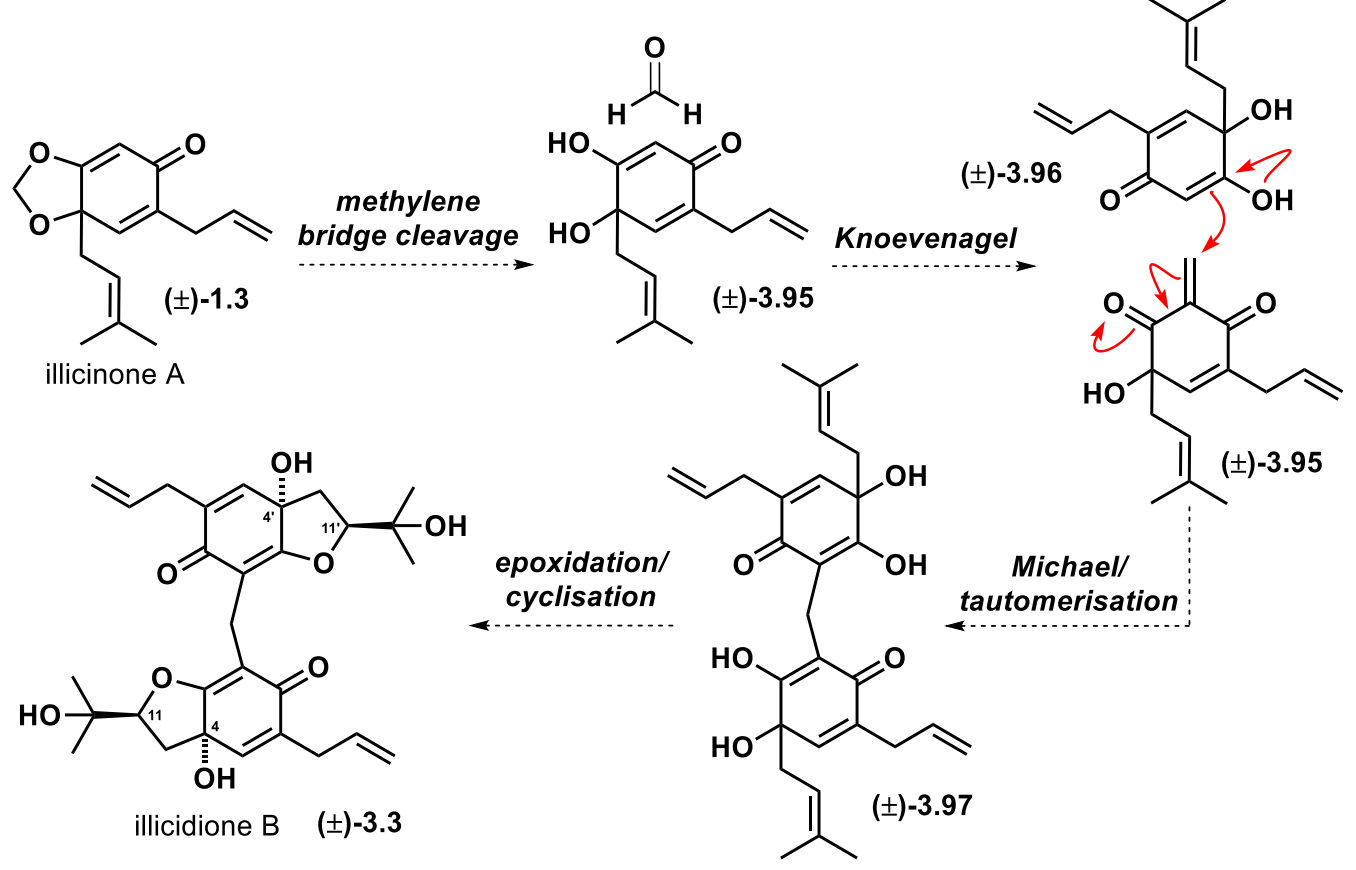

Scheme 3.53: Our proposed synthesis of ( \pm -)-illicidione B 3.3.

\subsubsection{Type A2 Structures}

We propose that the precursor ( \pm )-3.74 to the type $\boldsymbol{A}_{2}$ frameworks could be accessed in four steps from sesamol. The sequence would mirror the preparation of ( \pm )-illicinone A 1.3 (scheme 3.54). 


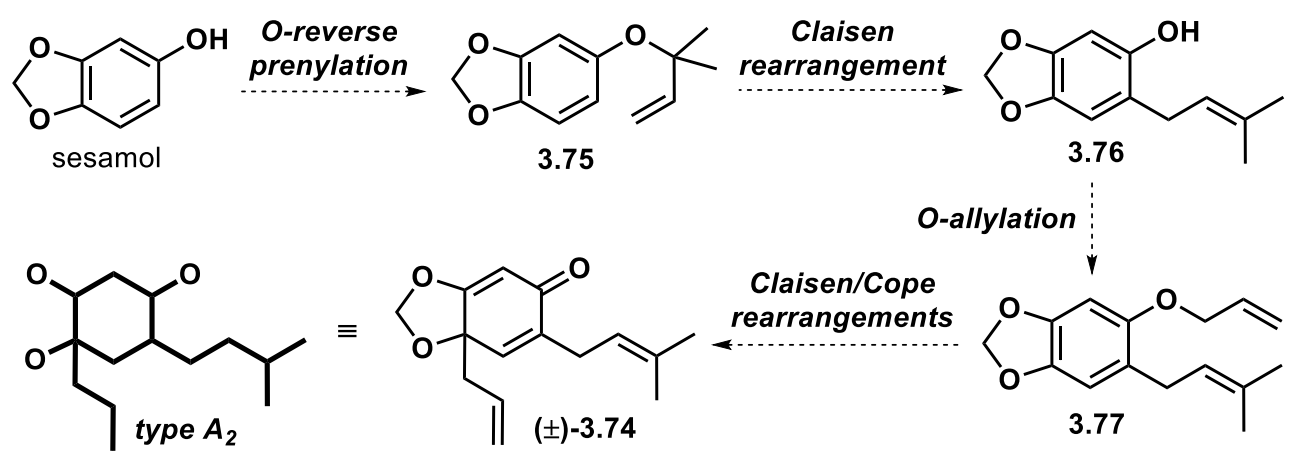

Scheme 3.54: Our proposed synthesis of precursor ( \pm -3.74 to type A2 structures.

Perhaps the most interesting members of this group are (+)-illicidione A $\mathbf{1 . 5}$ and (+)-illihendione A 3.23, the first asymmetric total syntheses of which has been reported in 2015 by $\mathrm{Yu}$ and co-workers (see section 3.2.3 on pages 96-98). ${ }^{141}$ These syntheses were accomplished in eight and nine steps respectively from a known starting material, which, however, needed to be prepared in two steps. We envisage there is potential to access ( \pm )-illicidione A $\mathbf{1 . 5}$ and ( \pm )-illihendione A $\mathbf{3 . 2 3}$ in two steps from precursor $( \pm)-3.74$, six steps from commercially available sesamol (see section 3.3.4 on page 104) (scheme 3.55).

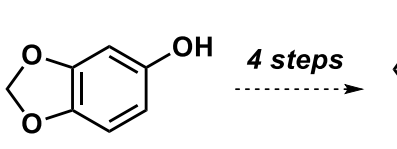

sesamol

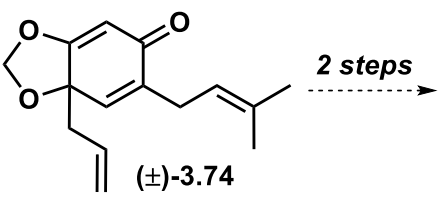

$( \pm)-3.74$

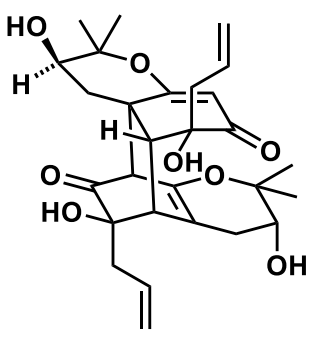

illihendione A $( \pm)-3.23$

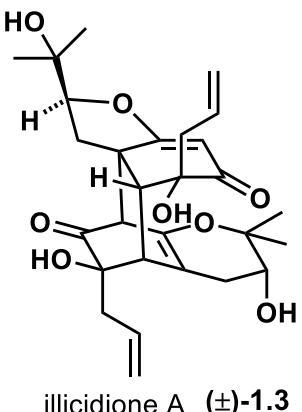

illicidione A $( \pm)-1.3$

Scheme 3.55: Our proposed synthesis of ( \pm )-illicidione 1.5 and ( \pm -)-illihendione 3.23.

The full picture of all the prenylated phenylpropanoids isolated to date from Illicium plants potentially accessible via our unified strategy can be found in the appendix on pages 426-428. The synthesis of all of them would represent a considerable challenge and would necessitate huge amounts of time and resources with limited benefits. The aim of this project is to demonstrate the power and the generality of our biomimetic divergent approach by accessing the most complex and structurally diverse members of type $\mathbf{A}_{2}$ and type $\mathbf{B}_{2}$ natural products. Therefore, we reason that accessing the molecular targets described in this section would fulfil our aim. 


\section{Experimental Section}

\subsection{General Experimental Conditions}

\section{NMR spectra}

${ }^{1} \mathrm{H}$ NMR spectra were recorded at $600 \mathrm{MHz}, 500 \mathrm{MHz}$, and $400 \mathrm{MHz}$ using a Bruker AVANCE 600, Bruker AVANCE 500, Bruker PRO 500, Varian INOVA 500 or Bruker AVANCE 400 spectrometer. Residual solvent peaks were used as an internal reference for ${ }^{1} \mathrm{H} \mathrm{NMR}$ spectra $\left(\mathrm{CDCl}_{3} \delta 7.26 \mathrm{ppm},\left(\mathrm{CD}_{3}\right)_{2} \mathrm{CO} \delta 2.05 \mathrm{ppm},\left(\mathrm{CD}_{3}\right)_{2} \mathrm{SO}\right.$ $\delta 2.50 \mathrm{ppm})$. Coupling constants $(J)$ are quoted to the nearest $0.1 \mathrm{~Hz}$. Assignment of proton signals was assisted by ${ }^{1} \mathrm{H}^{1}{ }^{1} \mathrm{H}$ COSY, HSQC, HMBC and NOESY experiments. ${ }^{13} \mathrm{C}$ NMR spectra were recorded at $151 \mathrm{MHz}, 126 \mathrm{MHz}$ or $100 \mathrm{MHz}$ using a Bruker AVANCE 600, Bruker AVANCE 500, Bruker PRO 500, Varian INOVA 500 or Bruker AVANCE 400 spectrometer. Solvent peaks were used as an internal reference for ${ }^{13} \mathrm{C} \mathrm{NMR}$ spectra $\left(\mathrm{CDCl} 3 \delta 77.16 \mathrm{ppm},\left(\mathrm{CD}_{3}\right)_{2} \mathrm{CO} \delta 206.13\right.$ ppm). Assignment of carbon signals was assisted by ${ }^{1} \mathrm{H}-{ }^{1} \mathrm{H}$ COSY, HSQC, HMBC and NOESY experiments.

\section{IR spectra}

IR spectra of solids and liquids were recorded as neat samples on a Shimadzu IRAffinity-1 FTIR spectrometer fitted with an ATR attachment.

\section{Melting Point}

Melting points were measured on a Gallenkamp Melting Point System or a Stanford Research Systems OptiMelt MPA100.

\section{Mass spectrometry}

High resolution mass spectra were recorded on a Bruker microTOF instrument using 136 electrospray ionisation $\left(\mathrm{ESI}^{+}\right)$. 


\section{Analytical TLC}

Analytical TLC was performed with Merck silica gel plates, precoated with silica gel 60 F254 $(0.2 \mathrm{~mm})$. Visualisation was effected by quenching of UV fluorescence $\left(\lambda_{\max }=\right.$ $254 \mathrm{~nm}$ ) and by staining with $p$-anisaldehyde or $\mathrm{KMnO}_{4}$ standard TLC stain solutions, followed by heating.

\section{Flash chromatography}

Flash chromatography employed Merck Kiesegel 60 (230-400 mesh) silica gel. When mentioned, the silica gel was pre-neutralised using a $1 \%$ solution of trimethylamine in the solvent system used for the column.

\section{Preparative HPLC}

Preparative HPLC was conducted on a Shimadzu system using a LC-20AP pump, a DGU-20A 3 R degassing unit, an FRC-10A fraction collector and monitored by an SPD20A UV-Vis detector. An ACE-321-2520 C18 preparative column was used.

\section{Experimental procedures, reagents and glassware}

Reactions were conducted under a positive pressure of dry argon or nitrogen and the glassware was dried with a heat gun. Anhydrous solvents were obtained from commercial sources. Commercially available chemicals were used as purchased. The yields reported in this paper are shown as isolated yields unless otherwise stated. We provide experimental details for the largest scale procedures conducted (Note: the stated scale corresponds to the quantity of product isolated). 


\subsection{Specific Experimental Procedures for Chapter 2}

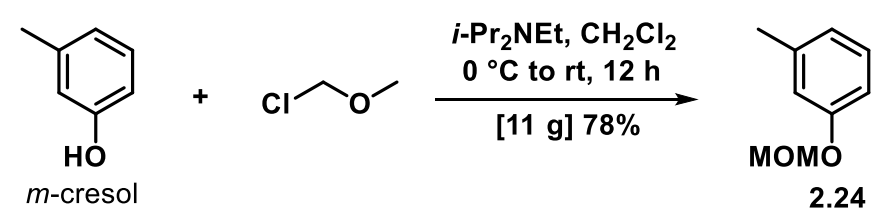

Compound 2.24 was prepared using a modified literature procedure. ${ }^{64}$

$m$-Cresol $(10.0 \mathrm{~mL}, 94.7 \mathrm{mmol})$ was dissolved in anhydrous $\mathrm{CH}_{2} \mathrm{Cl}_{2}(200 \mathrm{~mL})$. $i$ - $\operatorname{Pr}_{2} \mathrm{NEt}(49.5 \mathrm{~mL}, 284 \mathrm{mmol})$ was then added at $\mathrm{rt}$. The mixture was cooled to $0{ }^{\circ} \mathrm{C}$ and MOM-Cl (18.1 mL, $237 \mathrm{mmol})$ was added dropwise. The mixture was stirred at 0 ${ }^{\circ} \mathrm{C}$ for $30 \mathrm{~min}$, then allowed to warm to $\mathrm{rt}$ and stirred for $11 \mathrm{~h}$. After this time the reaction was quenched with sat. $a q . \mathrm{NaHCO}_{3}(150 \mathrm{~mL})$, diluted with diethyl ether (200 $\mathrm{mL}$ ) and stirred at $0{ }^{\circ} \mathrm{C}$ for another $30 \mathrm{~min}$. The mixture was then extracted with diethyl ether $(3 \times 150 \mathrm{~mL})$, the combined organic layers were washed with sat. $a q . \mathrm{NH}_{4} \mathrm{Cl}$ (400 mL), brine (400 mL), $1 \mathrm{M}$ aq. $\mathrm{NaOH}\left(400 \mathrm{~mL}\right.$ ) and dried over anhydrous $\mathrm{Na}_{2} \mathrm{SO}_{4}$. The solvent was removed under reduced pressure to afford a yellow oil (11.2 g, 73.7 mmol, 78\% yield). The crude material was deemed sufficiently pure (see ${ }^{1} \mathrm{H}$ NMR spectrum on page 236) to be used directly in the next step. All data for compound $\mathbf{2 . 2 4}$ matched literature values. ${ }^{64}$

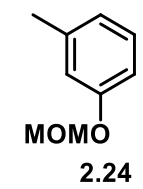

$\mathbf{R}_{\mathbf{f}} 0.61$ (70:30 petroleum spirit 40-60 / ethyl acetate);

${ }^{1} \mathbf{H}$-NMR $\left(500 \mathrm{MHz}, \mathrm{CDCl}_{3}\right) \delta 7.18$ (apparent t, $\left.J=7.8 \mathrm{~Hz}, 1 \mathrm{H}\right), 6.92-6.85(\mathrm{~m}, 3 \mathrm{H})$, 5.17 (s, 2H), 3.49 (s, 3H), 2.34 (s, 3H) ppm;

${ }^{13}$ C-NMR $\left(126 \mathrm{MHz}, \mathrm{CDCl}_{3}\right) \delta 157.4,139.7,129.3,122.8,117.1,113.4,94.5,56.1$, $21.6 \mathrm{ppm}$. 


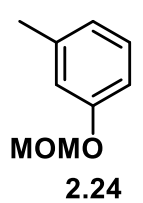

1. $t$-BuLi, hexanes

$0^{\circ} \mathrm{C}, 3 \mathrm{~h}$

2. $\mathrm{LiBr}$, ethylene oxide

THF, $-78^{\circ} \mathrm{C}$ to $\mathrm{rt}, 1.5 \mathrm{~h}$

[6 g] $84 \%$

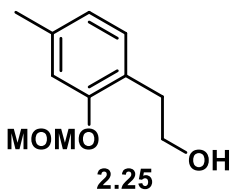

In a 3-necked round bottom flask MOM-protected cresol 2.24 (5.49 g, $36.1 \mathrm{mmol})$ was dissolved in anhydrous hexane $(24.0 \mathrm{~mL})$. The solution was cooled to $0{ }^{\circ} \mathrm{C}$ then $t$-BuLi (23.3 mL, $39.7 \mathrm{mmol}, 1.7 \mathrm{M}$ in pentane) was added slowly. The mixture was stirred at $0{ }^{\circ} \mathrm{C}$ for $3 \mathrm{~h}$ then cooled to $-78^{\circ} \mathrm{C}$. Ethylene oxide $(30.0 \mathrm{~mL}, 75.8 \mathrm{mmol}, 2.5 \mathrm{M}$ in THF) and a solution of $\operatorname{LiBr}(3.45 \mathrm{~g}, 39.7 \mathrm{mmol})$ in anhydrous THF (24.0 mL) were added and the mixture was allowed to warm to $\mathrm{rt}$ and stirred for $1.5 \mathrm{~h}$. The reaction was then quenched adding sat. $a q . \mathrm{NH}_{4} \mathrm{Cl}$ and extracted with diethyl ether $(3 \times 60 \mathrm{~mL})$. The combined organic layers were washed with brine, dried over anhydrous $\mathrm{MgSO}_{4}$ and the solvent was removed under reduced pressure. Flash chromatography (75:25 petroleum spirit 40-60 / ethyl acetate) gave alcohol $\mathbf{2 . 2 5}$ as a colourless oil (5.92 g, $0.30 \mathrm{mmol}, 84 \%$ yield).

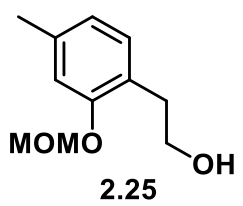

Rf 0.21 (75:25 petroleum spirit 40-60 / ethyl acetate);

${ }^{1} \mathbf{H}-\mathbf{N M R}\left(500 \mathrm{MHz}, \mathrm{CDCl}_{3}\right): \delta 7.06(\mathrm{~d}, J=7.6 \mathrm{~Hz}, 1 \mathrm{H}), 6.93-6.91(\mathrm{~m}, 1 \mathrm{H}), 6.78(\mathrm{dm}$, $J=7.6,1 \mathrm{H}), 5.20(\mathrm{~s}, 2 \mathrm{H}), 3.82$ (apparent q, $J=6.3 \mathrm{~Hz}, 2 \mathrm{H}), 3.49$ (s, 3H), 2.89 (t, $J=$ $6.6 \mathrm{~Hz}, 2 \mathrm{H}), 2.32(\mathrm{~m}, 3 \mathrm{H}), 1.82$ (s, broad, 1H) ppm;

${ }^{13}$ C-NMR (126 MHz, $\left.\mathrm{CDCl}_{3}\right): \delta 155.3,137.9,130.8,124.5,122.6,115.0,94.5,63.0$, 56.2, 33.9, $21.5 \mathrm{ppm}$;

HRMS $\left(\mathrm{ESI}^{+}\right)$197.1176, (calculated $[\mathrm{M}+\mathrm{H}]^{+}$197.1217);

IR (film, cm ${ }^{-1}$ ) 3373 (broad), 2952, 2924, 2361, 1614, 1580. 

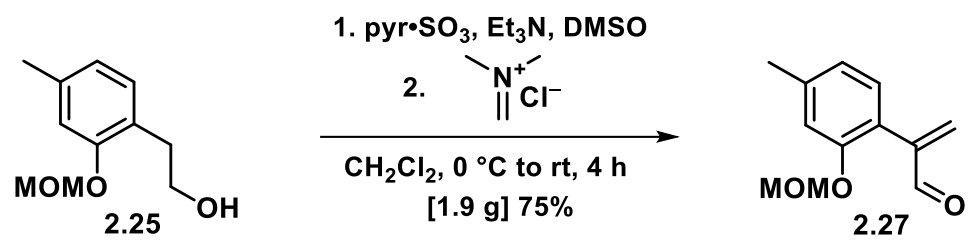

Alcohol 2.25 (2.38 g, $12.1 \mathrm{mmol})$ was dissolved in anhydrous $\mathrm{CH}_{2} \mathrm{Cl}_{2}(28 \mathrm{~mL}) . \mathrm{Et}_{3} \mathrm{~N}$ (10.5 mL, $75.6 \mathrm{mmol})$, DMSO (5.37 mL, $75.6 \mathrm{mmol})$ and pyr $\bullet \mathrm{SO}_{3}(4.81 \mathrm{~g}, 30.3 \mathrm{mmol})$ were added at $0{ }^{\circ} \mathrm{C}$. The reaction was allowed to warm to $\mathrm{rt}$ and stirred for $2 \mathrm{~h}$. The Eschenmoser salt $(2.02 \mathrm{~g}, 21.8 \mathrm{mmol})$ was added and the mixture was stirred for another $2 \mathrm{~h}$. The reaction was then quenched with sat. $a q . \mathrm{CuSO}_{4}(30 \mathrm{~mL})$ and extracted with ethyl acetate $(3 \times 60 \mathrm{~mL})$. The combined organic layers were washed with $1 \mathrm{Maq}$. $\mathrm{HCl}(150 \mathrm{~mL})$ and brine $(150 \mathrm{~mL})$ and then dried over anhydrous $\mathrm{Na}_{2} \mathrm{SO}_{4}$. The solvent was removed under reduced pressure. Flash chromatography (70:30 to 50:50 petroleum spirit 40-60/ $\mathrm{CH}_{2} \mathrm{Cl}_{2}$ ) gave enal $\mathbf{2 . 2 7}$ as a pale-yellow oil (1.88 g, $9.03 \mathrm{mmol}, 75 \%$ yield).

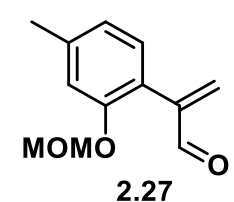

$\mathbf{R}_{\mathbf{f}}$ 0.29 (70:30 petroleum spirit 40-60 / ethyl acetate);

${ }^{1}$ H-NMR $\left(500 \mathrm{MHz}, \mathrm{CDCl}_{3}\right) \delta 9.73(\mathrm{~d}, J=0.6 \mathrm{~Hz}, 1 \mathrm{H}), 7.06(\mathrm{~d}, J=7.6 \mathrm{~Hz}, 1 \mathrm{H}), 6.99$ $6.98(\mathrm{~m}, 1 \mathrm{H}), 6.85(\mathrm{dm}, J=7.6 \mathrm{~Hz}, 1 \mathrm{H}), 6.36(\mathrm{~d}, J=0.9 \mathrm{~Hz}, 1 \mathrm{H}), 6.27$ (d, $J=0.8 \mathrm{~Hz}$, $1 \mathrm{H}), 5.11(\mathrm{~s}, 2 \mathrm{H}), 3.44$ (s, 3H), $2.36(\mathrm{~m}, 3 \mathrm{H}) \mathrm{ppm}$;

${ }^{13}$ C-NMR (126 MHz, $\left.\mathrm{CDCl}_{3}\right) \delta 192.6,154.7,147.4,140.7,133.8,130.3,122.8,122.2$, 115.5, 94.8, 56.3, 21.7 ppm;

HRMS $\left(\mathrm{ESI}^{+}\right) 229.0837$ (calculated $[\mathrm{M}+\mathrm{Na}]^{+} 229.0835$ );

IR (film, cm ${ }^{-1}$ ) 2953, 2922, 2826, 1699, 1611. 

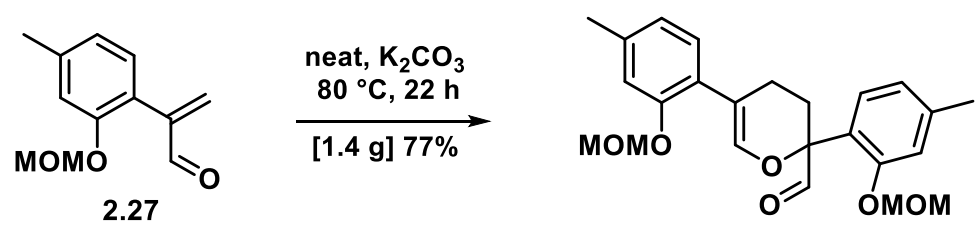

$( \pm)-2.28$

Enal 2.27 (1.878 g, $9.10 \mathrm{mmol})$, neat, was placed in a round bottom flask. $\mathrm{K}_{2} \mathrm{CO}_{3}(570$ $\mathrm{mg}, 4.12 \mathrm{mmol}$ ) was added and the mixture was stirred at $80{ }^{\circ} \mathrm{C}$ under an argon atmosphere for $22 \mathrm{~h}$. The mixture was then allowed to cool to rt, then diluted with $\mathrm{CH}_{2} \mathrm{Cl}_{2}(100 \mathrm{~mL})$ and filtered through a short pad of celite. The solvent was removed under reduced pressure. Flash chromatography using neutralised silica gel (see general experimental) (85:15 to $75: 25$ petroleum spirit 40-60 / diethyl ether) afforded dihydropyran dimer ( \pm )-2.28 as a pale-yellow oil (1.45 g, $3.51 \mathrm{mmol}, 77 \%$ yield).

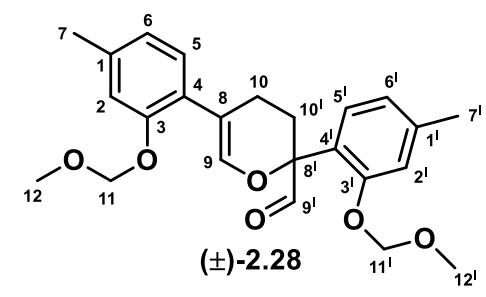

$\mathbf{R}_{\mathbf{f}}$ 0.54 (70:30 petroleum spirit 40-60 / ethyl acetate);

${ }^{1} \mathbf{H}$ NMR $\left(500 \mathrm{MHz}, \mathrm{CDCl}_{3}\right) \delta 9.76(\mathrm{~d}, J=0.8 \mathrm{~Hz}, 1 \mathrm{H}, \mathrm{H} 9)$ ) $7.44(\mathrm{~d}, J=7.9 \mathrm{~Hz}, 1 \mathrm{H}$, H5'), 7.02 (d, $J=7.7 \mathrm{~Hz}, 1 \mathrm{H}, \mathrm{H} 5), 6.97-6.96$ (m, 1H, H2'), 6.92-6.91 (m, 1H, H9), 6.90-6,87 (m, 2H, H2 + H6'), 6.78 (dm, J = 7.7 Hz, 1H, H6), 5.20-5.16 (m, 2H, H11 or H11'), 5.13 (s, 2H, H11 or H11'), 3.47 (s, 3H, H12 or H12'), 3.46 (s, 3H, H12 or H12'), 2.64-2.57 (m, 1H, H10'), 2.55-2.45 (m, 1H, H10), 2.42-2.36 (m, 1H, H10'), 2.35 (s, 3H, H7'), 2.32 (s, 3H, H7), 2.29-2.26 (m, 1H, H10) ppm;

${ }^{13}$ C NMR (126 MHz, $\mathrm{CDCl}_{3}$ ) $\delta 198.6$ (C9'), 154.8 (C3), 153.9 (C3'), 141.2 (C9), 140.5 (C1 or C1'), 138.0 (C1 or C1'), 129.2 (C5), 127.8 (C5'), 126.5 (C4), $124.6(\mathrm{C} 4$ '), 122.9 (C6'), 122.8 (C6), 116.0 (C2), 115.3 (C2'), 113.0 (C8), 94.8 (C11 or C11'), 94.5 (C11 or C11'), 82.0 (C8'), 56.6 (C12 or C12'), 56.3 (C12 or C12'), 26.2 (C10'), 21.9 (C10), $21.6\left(\mathrm{C}^{\prime}\right), 21.5(\mathrm{C} 7) \mathrm{ppm}$;

HRMS $\left(\mathrm{ESI}^{+}\right) 435.1778$ (calculated $[\mathrm{M}+\mathrm{Na}]^{+}$435.1778);

IR (film, cm cm $^{-1}$ 2922, 2653, 2361, 2342, 1724, 1670, 1609. 


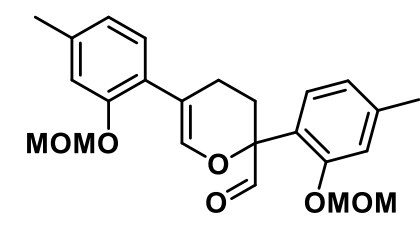

$( \pm)-2.28$

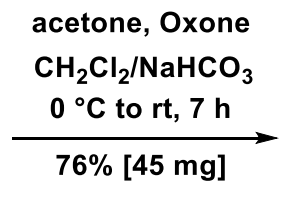

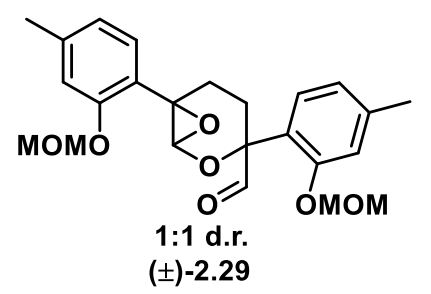

$( \pm)-2.29$

Compound ( \pm )-2.29 was prepared using a modified literature procedure. ${ }^{70}$

A solution of oxone ( $256 \mathrm{mg}, 0.42 \mathrm{mmol}$ ) in $\mathrm{H}_{2} \mathrm{O}(1.2 \mathrm{~mL}$ ) was slowly added (25 min) to a vigorously stirred solution of dihydropyran $( \pm)-2.28(57 \mathrm{mg}, 0.14 \mathrm{mmol})$ in $\mathrm{CH}_{2} \mathrm{Cl}_{2}(1.7 \mathrm{~mL})$, sat. aq. $\mathrm{NaHCO}_{3}(2.75 \mathrm{~mL})$ and acetone $(0.55 \mathrm{~mL})$ at $0{ }^{\circ} \mathrm{C}$. The mixture was stirred at $0{ }^{\circ} \mathrm{C}$ for $1 \mathrm{~h}$ and then allowed to slowly warm to $\mathrm{rt}$ and stirred for another $6 \mathrm{~h}$. After this time the mix ture was transferred into a separating funnel and extracted with $\mathrm{CH}_{2} \mathrm{Cl}_{2}(3 \times 4 \mathrm{~mL})$. The combined organic layers were dried over anhydrous $\mathrm{Na}_{2} \mathrm{SO}_{4}$. Removal of the solvent under reduced pressure afforded of epoxide $( \pm)-2.29$ as a $1: 1$ diastereomeric mixture (colourless oil, $45 \mathrm{mg}, 0.11 \mathrm{mmol}, 76 \%$ yield). The crude material was deemed sufficiently pure (see ${ }^{1} \mathrm{H}$ NMR spectrum on page 244) to be used in the next step.

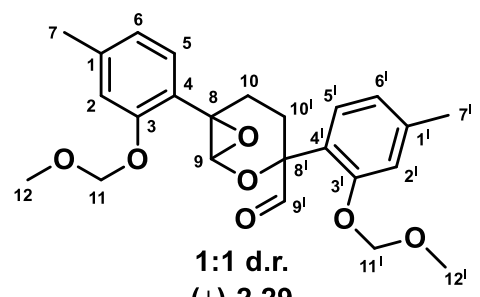

(土)-2.29

$\mathbf{R}_{\mathbf{f}}$ 0.56 (50:50 petroleum spirit 40-60 / ethyl acetate);

${ }^{1} \mathbf{H}$ NMR $\left(500 \mathrm{MHz}, \mathrm{CDCl}_{3}\right) \delta 9.88\left(\mathrm{~d}, J=1.3 \mathrm{~Hz}, 1 \mathrm{H}, \mathrm{H} 9^{\prime \dagger}\right), 9.53$ (s, 1H, H9 $\left.{ }^{\prime \dagger}\right), 7.60$ $\left(\mathrm{d}, J=7.9 \mathrm{~Hz}, 1 \mathrm{H}, \mathrm{H} 5^{\dagger}\right), 7.51\left(\mathrm{~d}, J=8.0 \mathrm{~Hz}, 1 \mathrm{H}, \mathrm{H} 5^{\dagger}\right), 7.19\left(\mathrm{~d}, J=7.7 \mathrm{~Hz}, 1 \mathrm{H}, \mathrm{H} 5^{\dagger \dagger}\right)$, $7.16\left(\mathrm{~d}, J=7.7 \mathrm{~Hz}, 1 \mathrm{H}, \mathrm{H} 5^{\prime \dagger}\right), 6.97-6.96\left(\mathrm{~m}, 1 \mathrm{H}, \mathrm{H} 2^{\prime \dagger}\right), 6.95-6.90\left(\mathrm{~m}, 3 \mathrm{H}, \mathrm{H} 6^{\dagger}+\mathrm{H}^{\dagger}+\right.$ $\left.\mathrm{H} 2^{\dagger}\right), 6.91-6.88\left(\mathrm{~m}, 1 \mathrm{H}, \mathrm{H} 6^{\dagger}\right), 6.88-6.87\left(\mathrm{~m}, 1 \mathrm{H}, \mathrm{H} 2^{\dagger}\right), 6.82-6.78$ (m, 2H, H6 $\left.6^{\dagger}\right), 5.27-$ $5.23\left(\mathrm{~m}, 2 \mathrm{H}, \mathrm{H} 11^{\dagger}\right.$ or $\left.\mathrm{H} 11^{\dagger \dagger}\right), 5.17-5.14\left(\mathrm{~m}, 2 \mathrm{H}, \mathrm{H} 11^{\dagger}\right.$ or $\left.\mathrm{H} 11^{\prime \dagger}\right), 5.13\left(\mathrm{~s}, 2 \mathrm{H}, \mathrm{H} 11^{\dagger}\right.$ or H1 $\left.1^{\prime \dagger}\right), 5.08-4.99$ (m, 4H, H1 $1^{\ddagger}$ or H1 $1^{\dagger}, \mathrm{H} 9^{\ddagger}, \mathrm{H} 9^{\dagger}$ ), 3.52 (s, 3H, H12 $2^{\dagger}$ or H12 $2^{\dagger \dagger}$ ), 3.47 (s, $3 \mathrm{H}, \mathrm{H} 12^{\dagger}$ or $\left.\mathrm{H} 12^{\prime \dagger}\right), 3.44$ (s, 3H, H12 $2^{\dagger}$ or $\left.\mathrm{H} 12^{\prime \dagger}\right), 3.36$ (s, 3H, H12 $2^{\dagger}$ or H12 $2^{\prime \dagger}$ ), 2.60$2.46\left(\mathrm{~m}, 3 \mathrm{H}, \mathrm{H} 10^{\ddagger}\right.$ or $\mathrm{H} 10^{\ddagger}+\mathrm{H} 10^{\dagger}$ or $\left.\mathrm{H} 10^{\prime \dagger}\right), 2.37$ (s, 3H, $\mathrm{H} 7^{\dagger}$ or $\left.\mathrm{H} 7^{\prime \dagger}\right), 2.33(\mathrm{~s}, 3 \mathrm{H}$, $\mathrm{H} 7^{\dagger}$ or $\left.\mathrm{H}^{\prime \dagger}\right), 2.33\left(\mathrm{~s}, 3 \mathrm{H}, \mathrm{H} 7^{\dagger}\right.$ or $\left.\mathrm{H}^{\dagger \dagger}\right), 2.31$ (s, 3H, $\mathrm{H}^{\dagger}$ or $\left.\mathrm{H} 7^{\dagger \dagger}\right), 2.36-1.30(\mathrm{~m}, 1 \mathrm{H}$, $\mathrm{H} 10^{\dagger}$ or $\left.\mathrm{H} 10^{\prime \dagger}\right), 2.29-2.19$ (m, $4 \mathrm{H}, \mathrm{H} 10^{\ddagger}$ or $\left.\mathrm{H} 10^{\prime *}\right) \mathrm{ppm}$; 
${ }^{13} \mathbf{C}$ NMR $\left(126 \mathrm{MHz}, \mathrm{CDCl}_{3}\right) \delta 199.5^{\dagger}\left(\mathrm{C}^{\prime}\right), 196.2^{\dagger}\left(\mathrm{C}^{\prime}\right), 154.1^{\dagger}\left(\mathrm{C} 3\right.$ or $\left.\mathrm{C}^{\prime}\right), 154.0^{\dagger}$ (C3 or $\left.\mathrm{C}^{\prime}\right), 154.0^{\dagger}$ (C3 or $\left.\mathrm{C}^{\prime}\right), 153.6^{\dagger}\left(\mathrm{C} 3\right.$ or $\left.\mathrm{C}^{\prime}\right), 140.5^{\dagger}(\mathrm{C} 1), 140.3^{\dagger}(\mathrm{C} 1), 139.6^{\dagger}$ (C1'), 139.6 $6^{\dagger}\left(\mathrm{C}^{\prime}\right), 128.5^{\dagger}(\mathrm{C} 5), 127.6^{\dagger}(\mathrm{C} 5), 127.4^{\dagger}\left(\mathrm{C}^{\prime}\right), 127.3^{\dagger}\left(\mathrm{C}^{\prime}\right), 125.6^{\dagger}(\mathrm{C} 4$ or $\left.\mathrm{C}^{\prime}\right), 125.5^{\dagger}\left(\mathrm{C} 4\right.$ or $\left.\mathrm{C}^{\prime}\right), 125.3^{\dagger}$ (C4 or $\left.\mathrm{C}^{\prime}\right), 123.7^{\dagger}\left(\mathrm{C} 4\right.$ or $\left.\mathrm{C}^{\prime}\right), 123.2^{\dagger}(\mathrm{C} 6), 122.7^{\dagger}$ (C6), 122.4 $4^{\ddagger}\left(\mathrm{C}^{\prime}\right), 115.3^{\ddagger}\left(\mathrm{C}^{\prime}\right), 114.3^{\dagger}(\mathrm{C} 2), 114.1^{\dagger}(\mathrm{C} 2), 94.5^{\dagger}\left(\mathrm{C} 11\right.$ or $\left.\mathrm{C} 11^{\prime}\right), 94.3^{\dagger}$ (C11 or $\left.\mathrm{C}_{1} 1^{\prime}\right), 93.8^{\dagger}\left(\mathrm{C} 11\right.$ or $\left.\mathrm{C} 11^{\prime}\right), 93.7^{\dagger}\left(\mathrm{C} 11\right.$ or $\left.\mathrm{C} 11^{\prime}\right), 82.4^{\dagger}(\mathrm{C} 9), 82.4^{\dagger}(\mathrm{C} 9), 81.3^{\dagger}$ (C8'), $81.1^{\dagger}\left(\mathrm{C} 8^{\prime}\right), 60.1^{\dagger}(\mathrm{C} 8), 59.0^{\dagger}(\mathrm{C} 8), 56.5^{\dagger}\left(\mathrm{C} 12\right.$ or $\left.\mathrm{C} 12^{\prime}\right), 56.5^{\dagger}$ (C12 or C12'), 56.3 (C12 or C12'), 56.0 (C12 or C12'), $26.4^{\dagger}\left(\mathrm{C} 10\right.$ or $\left.\mathrm{C} 10^{\prime}\right), 24.8^{\dagger}$ (C10 or $\left.\mathrm{C} 10^{\prime}\right), 24.4^{\dagger}(\mathrm{C} 10$ or $\left.\mathrm{C} 10^{\prime}\right), 23.5^{\dagger}\left(\mathrm{C} 10\right.$ or $\left.\mathrm{C} 10^{\prime}\right), 21.7^{\dagger}$ (C7 or $\left.\mathrm{C} 7^{\prime}\right), 21.6^{\dagger}\left(\mathrm{C} 7\right.$ or $\left.\mathrm{C} 7{ }^{\prime}\right), 21.5^{\ddagger}(\mathrm{C} 7$ or $\mathrm{C} 7$ ) ppm;

HRMS (ESI $\left.{ }^{+}\right) 451.1733$ (calculated [M+Na] $]^{+}$451.1733).

$\dagger$ One diastereoisomer, $₫$ both diastereoisomers. 
<smiles>COc1cc(C)ccc1C1=COC(C=O)(c2ccc(C)cc2OC)CC1</smiles>

$( \pm)-2.28$

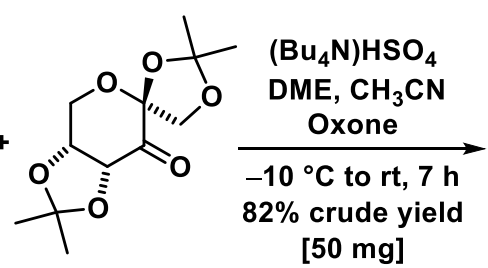

[50 mg]

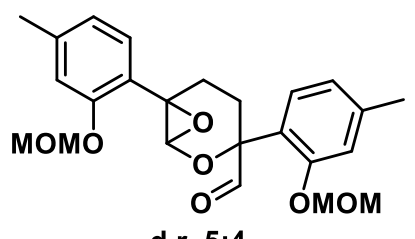

d.r. $5: 4$

$( \pm)-2.29$

Compound ( \pm -2.29 was prepared using a modified literature procedure. ${ }^{72}$

Dihydropyran ( \pm )-2.28 (57 mg, $0.13 \mathrm{mmol})$, Shi lactone (11 mg, $0.04 \mathrm{mmol}$ ) and $\left(\mathrm{Bu}_{4} \mathrm{~N}\right) \cdot \mathrm{HSO}_{4}(0.7 \mathrm{mg}, 2 \mu \mathrm{mol})$ were dissolved in a 2:1 mixture of dimethoxyethane and acetonitrile $(1.2 \mathrm{~mL})$. An aqueous $\mathrm{pH} 9.3$ buffer $(0.75 \mathrm{~mL})$ was added. The mixture was cooled to $-10{ }^{\circ} \mathrm{C}$, then a solution of Oxone $(123 \mathrm{mg}, 0.20 \mathrm{mmol})$ in aq. $\mathrm{Na}_{2}$ (EDTA) $\left(4 \times 10^{-3} \mathrm{M}, 0.55 \mathrm{~mL}\right)$ and a solution of $\mathrm{K}_{2} \mathrm{CO}_{3}(107 \mathrm{mg}, 0.77 \mathrm{mmol})$ in water $(0.55 \mathrm{~mL})$ were added at the same time within $2 \mathrm{~h}$. The reaction was allowed to warm to rt and vigorously stirred for another $1.5 \mathrm{~h}$, then $\mathrm{CH}_{2} \mathrm{Cl}_{2}(2.5 \mathrm{~mL})$ and water $\left(2 \mathrm{~mL}\right.$ ) were added and the aqueous layer was extracted with $\mathrm{CH}_{2} \mathrm{Cl}_{2}(3 \times 5 \mathrm{~mL})$. The combined organic layers were washed with brine $(15 \mathrm{~mL})$, dried over anhydrous $\mathrm{Na}_{2} \mathrm{SO}_{4}$ and the solvent was removed under reduced pressure. ${ }^{1} \mathrm{H} \mathrm{NMR}$ analysis of the crude mixture indicated a 5:4 diastereomeric ratio of epoxide ( \pm -2.29. 


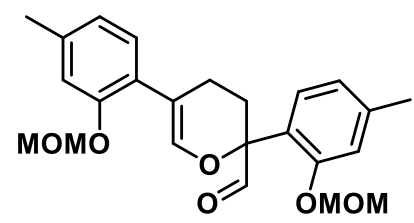

$( \pm)-2.28$

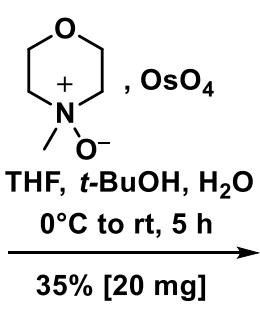

$35 \%$ [20 mg]

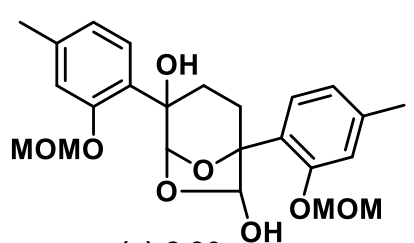

$( \pm)-2.30$

Lactol ( \pm )-2.30 was prepared using a modified literature procedure. ${ }^{73}$

Dihydropyran ( \pm )-2.28 (50 mg, $0.12 \mathrm{mmol})$ was dissolved in a mixture of THF (1.5 $\mathrm{mL})$, tert-butanol $(50 \mu \mathrm{L})$ and water $(30 \mu \mathrm{L})$. The mixture was cooled to $0{ }^{\circ} \mathrm{C}$ then $N$ methylmorpholine- $\mathrm{N}$-oxide $(22 \mathrm{mg}, 0.20 \mathrm{mmol})$ and a catalytic amount of $\mathrm{OsO}_{4}(2$ small crystals) were added. The mixture was allowed to warm to rt and stirred for $5 \mathrm{~h}$, then the reaction was quenched adding solid $\mathrm{Na}_{2} \mathrm{~S}_{2} \mathrm{O}_{5}(80 \mathrm{mg}, 0.41 \mathrm{mmol})$. The mixture was stirred for $1 \mathrm{~h}$ at rt, filtered through a short pad of silica gel and extracted with ethyl acetate $(3 \times 5 \mathrm{~mL})$. The combined organic layers were washed with brine $\left(15 \mathrm{~mL}\right.$ ), dried over anhydrous $\mathrm{Na}_{2} \mathrm{SO}_{4}$ and the solvent was removed under reduced pressure. Flash chromatography (70:30 petroleum spirit 40-60 / ethyl acetate) afforded lactol ( \pm )-2.30 as a single diastereoisomer (amorphous solid, $19 \mathrm{mg}, 0.041 \mathrm{mmol}, 35 \%$ yield).

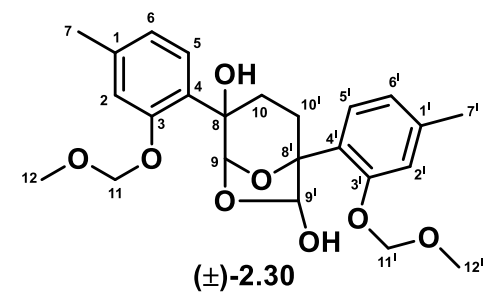

$\mathbf{R}_{\mathbf{f}} 0.20$ (50:50 petroleum spirit 40-60/ ethyl acetate);

${ }^{1} \mathbf{H}$ NMR $\left(500 \mathrm{MHz}, \mathrm{CDCl}_{3}\right) \delta 7.58\left(\mathrm{~d}, J=7.8 \mathrm{~Hz}, 1 \mathrm{H}, \mathrm{H} 5^{\prime}\right), 7.34(\mathrm{~d}, J=7.9 \mathrm{~Hz}, 1 \mathrm{H}$, H5), 6.99 (s, 1H, H2), 6.95 (s, 1H, H2'), 6.87 (d, J= 7.7 Hz, 1H, H6'), 6.85 (d, J = 8.1 Hz, 1H, H6), 5.96 (d, $J=1.4$ Hz, 1H, H9), 5.73 (d, $J=10.5$ Hz, 1H, H9'), 5.38-5.25 (m, 2H, H11 or H11'), 5.25-5.11 (m, 2H, H11 or H11'), 4.23 (broad s, 1H, OH at C9'), 3.51 (s, 3H, H12 or H12'), 3.51 (s, 3H, H12 or H12'), 2.45-2.34 (m, 2H, H10 and H10'), 2.35 (s, 3H, H7 or H7'), 2.33 (s, 3H, H7 or H7'), 2.15-2.02 (m, 2H, H10 and H10') ppm; 
${ }^{13} \mathrm{C} \mathrm{NMR}\left(126 \mathrm{MHz}, \mathrm{CDCl}_{3}\right) \delta 155.4(3 \mathrm{C}), 152.9\left(\mathrm{C}^{\prime}\right), 139.5\left(\mathrm{C} 1\right.$ or $\left.\mathrm{C}^{\prime}\right), 139.4(\mathrm{C} 1$ or C1'), 127.9 (C4), 127.3 (C5), 126.9 (C5'), 125.6 (C4'), 123.0 (C6), 122.6 (C6'), 115.5 (C2), 114.7 (C2'), 103.5 (C9), 96.8 (C9'), 94.7 (C11 or C11'), 94.6 (C11 or C11'), 86.0 (C8'), 72.2 (C8), 56.7 (C12 or C12'), 56.4 (C12 or C12'), 28.5 (C10), 28.4 (C10'), 21.6 (C7 or $\left.\mathrm{C}^{\prime}\right), 21.4\left(\mathrm{C} 7\right.$ or $\left.\mathrm{C}^{\prime}\right)$ ppm;

HRMS $\left(\mathrm{ESI}^{+}\right) 469.1833$ (calculated $[\mathrm{M}+\mathrm{Na}]^{+} 469.18330$ );

IR (film, $\mathrm{cm}^{-1}$ ) 3402 (broad), 2934, 1614, 1578, 1508;

m.p. $98-99{ }^{\circ} \mathrm{C}$ (ethyl acetate). 


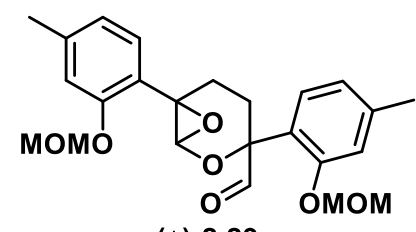

$( \pm)-2.29$

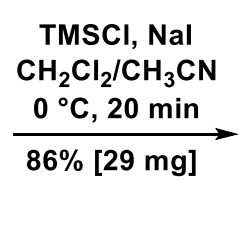

$\underset{\mathrm{CH}_{2} \mathrm{Cl}_{2} / \mathrm{CH}_{3} \mathrm{CN}}{{ }^{\circ} \mathrm{C}, 20 \mathrm{~min}}$
$\underset{86 \%[29 \mathrm{mg}]}{\longrightarrow}$

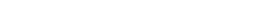

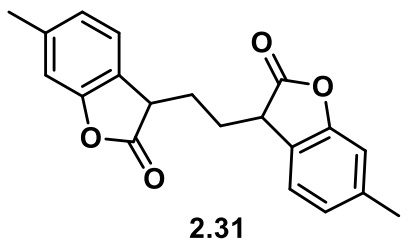

Bis-lactone 2.31 was prepared using a modified literature procedure. ${ }^{66}$

The crude diastereomeric mixture of epoxide $( \pm)-2.29$ (45 $\mathrm{mg}, 0.11 \mathrm{mmol}$ ) was dissolved in 1:1 mixture of anhydrous $\mathrm{CH}_{2} \mathrm{Cl}_{2}$ and $\mathrm{CH}_{3} \mathrm{CN}(2.8 \mathrm{~mL})$ at $\mathrm{rt}$. The solution was cooled to $0{ }^{\circ} \mathrm{C}$, then $\mathrm{TMSCl}(94 \mu \mathrm{L}, 1.05 \mathrm{mmol})$ and $\mathrm{NaI}(112 \mathrm{mg}, 1.05 \mathrm{mmol})$ were added. The mixture was stirred at $0{ }^{\circ} \mathrm{C}$ for $20 \mathrm{~min}$, then quenched with sat. $a q$. $\mathrm{Na}_{2} \mathrm{~S}_{2} \mathrm{O}_{3}(5 \mathrm{~mL})$, extracted with ethyl acetate $(3 \times 4 \mathrm{~mL})$. The combined organic layers were washed with water $(10 \mathrm{~mL})$ and brine $(10 \mathrm{~mL})$, dried over anhydrous $\mathrm{Na}_{2} \mathrm{SO}_{4}$ and the solvent was removed under reduced pressure. Flash chromatography (75:25 petroleum spirit 40-60 / diethyl ether) afforded bis-lactone $\mathbf{2 . 3 1}$ as a 1:1 mixture of diastereoisomers (colourless oil, $29 \mathrm{mg}$, 86\% yield) (see ${ }^{1} \mathrm{H}$ NMR spectrum on page 248). Recrystallisation from petroleum spirit 40-60 / ethyl acetate gave meso-bislactone 2.31 as a single diastereoisomer (white solid, $12 \mathrm{mg}, 36 \%$ yield) (CCDC 1530570) (see page 423 for X-ray crystal structure).

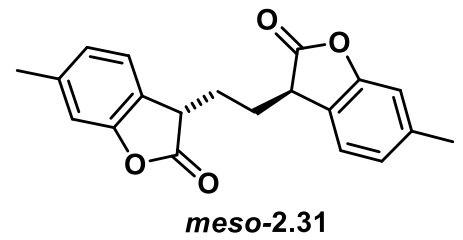

$\mathbf{R}_{\mathbf{f}}$ 0.56 (75:25 petroleum spirit 40-60 / diethyl ether);

${ }^{1} \mathbf{H}$ NMR $\left(500 \mathrm{MHz}, \mathrm{CDCl}_{3}\right) \delta$ 7.14-7.12 (m, 2H), 7.02-6.94 (m, 2H), $6.93(\mathrm{~m}, 2 \mathrm{H})$, 3.75-3.60 (m, 2H), 2.39-2.38 (m, 6H), 2.13-1.91 (m, 4H) ppm;

${ }^{13} \mathrm{C}$ NMR $\left(126 \mathrm{MHz}, \mathrm{CDCl}_{3}\right) \delta 177.1,154.0,139.8,125.2,124.0,123.4,111.6,43.1$, 27.2, $21.8 \mathrm{ppm}$;

HRMS $\left(\mathrm{ESI}^{+}\right) 323.1263$ (calculated $\left.[\mathrm{M}+\mathrm{H}]^{+} 323.1278\right)$;

IR (film, cm ${ }^{-1}$ ) 2957, 2924, 2857, 1802, 1623. 


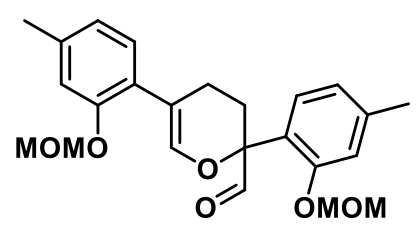

$( \pm)-2.28$

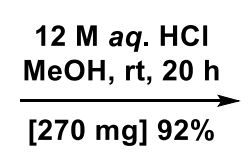

)-2.35 (d.r. 1:1)

MOM-protected dihydropyran ( \pm )-2.28 (332 $\mathrm{mg}, 0.806 \mathrm{mmol})$ was dissolved in methanol $(12 \mathrm{~mL}) .12 \mathrm{M} a q . \mathrm{HCl}(130 \mu \mathrm{L})$ was added and the mixture was stirred at $\mathrm{rt}$ for $20 \mathrm{~h}$. After complete consumption of starting material, judged by TLC analysis $(20 \mathrm{~h})$, the reaction was quenched by adding sat. $a q . \mathrm{NaHCO}_{3}(5.0 \mathrm{~mL})$ and extracted with $\mathrm{CH}_{2} \mathrm{Cl}_{2}(5 \times 10 \mathrm{~mL})$. The organic layers were dried over anhydrous $\mathrm{Na}_{2} \mathrm{SO}_{4}$. Flash chromatography (80:20 petroleum spirit 40-60 / ethyl acetate) gave compound (士)-2.36 as a 1:1 mixture of diastereoisomers (pale yellow oil, $273 \mathrm{mg}, 0.744 \mathrm{mmol}$, $92 \%$ yield).

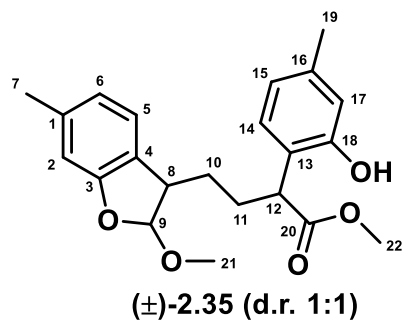

Rf 0.37 (70:30 petroleum spirit 40-60 / ethyl acetate);

${ }^{1} \mathbf{H}$ NMR $\left(500 \mathrm{MHz}, \mathrm{CDCl}_{3}\right) \delta 7.41\left(\mathrm{~s}\right.$, broad, $2 \mathrm{H}, \mathrm{OH}$ at $\left.\mathrm{C} 18^{\ddagger}\right), 7.02(\mathrm{~d}, J=7.5 \mathrm{~Hz}$, 2H, H5 $\left.5^{\ddagger}\right), 6.97\left(\mathrm{~d}, J=7.6 \mathrm{~Hz}, 1 \mathrm{H}, \mathrm{H} 14^{\dagger}\right), 6.95$ (d, $\left.J=7.6 \mathrm{~Hz}, 1 \mathrm{H}, \mathrm{H} 14^{\dagger}\right), 6.72-6.67$ (m, $\left.8 \mathrm{H}, \mathrm{H} 2^{\ddagger}+\mathrm{H}^{\ddagger}+\mathrm{H} 15^{\ddagger}+\mathrm{H} 17^{\ddagger}\right), 5.26\left(\mathrm{~d}, J=1.9 \mathrm{~Hz}, 1 \mathrm{H}, \mathrm{H} 9^{\dagger}\right), 5.22(\mathrm{~d}, J=1.9 \mathrm{~Hz}, 1 \mathrm{H}$,

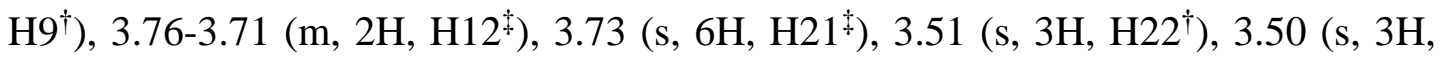
$\left.\mathrm{H} 22^{\dagger}\right), 3.13-3.10$ (m, 2H, H8 $\left.8^{\ddagger}\right), 2.32$ (s, 3H, H7 $\left.7^{\dagger}\right), 2.31$ (s, 3H, H7 $\left.7^{\dagger}\right), 2.28$ (s, 6H, H19*), 2.25-2.14 (m, 2H, H10 ), 2.06-1.94 (m, 2H, H10"), 1.60-1.43 (m, 4H, H1 $1^{\ddagger}$ ) ppm;

${ }^{13} \mathrm{C} \mathrm{NMR}\left(126 \mathrm{MHz}, \mathrm{CDCl}_{3}: \delta 177.0\left(\mathrm{C} 20^{\dagger}\right), 176.9\left(\mathrm{C} 20^{\dagger}\right), 158.1\left(\mathrm{C} 3^{\ddagger}\right), 154.6\left(\mathrm{C} 18^{\dagger}\right)\right.$,

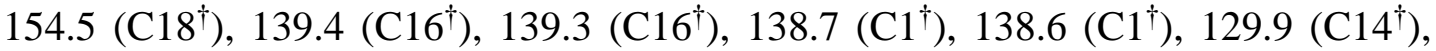
$129.8\left(\mathrm{C} 14^{\dagger}\right), 126.3\left(\mathrm{C}^{\ddagger}\right), 124.3\left(\mathrm{C}^{\star}\right), 121.8\left(\mathrm{CAr}^{\dagger}\right), 121.7\left(\mathrm{CAr}^{\dagger}\right), 121.0\left({\left.\mathrm{C} 13^{\dagger}\right),}^{\dagger}\right.$ $120.8\left(\mathrm{C} 13^{\dagger}\right), 118.6\left(\mathrm{CAr}^{\dagger}\right), 118.5\left(\mathrm{CAr}^{\dagger}\right), 112.4\left(\mathrm{C}^{*}\right), 110.8\left(\mathrm{CAr}^{\ddagger}\right), 55.9\left(\mathrm{C} 22^{\ddagger}\right), 52.7$ $\left(\mathrm{C} 21^{\ddagger}\right), 48.3\left(\mathrm{C} 12^{\ddagger}\right), 48.2\left(\mathrm{C} 8^{\ddagger}\right), 31.4\left(\mathrm{C} 11^{\dagger}\right), 31.3\left(\mathrm{C} 11^{\dagger}\right), 28.7\left(\mathrm{C} 10^{\dagger}\right), 28.5\left(\mathrm{C} 10^{\dagger}\right)$, $21.6\left(\mathrm{C} 7^{*}\right), 21.1\left(\mathrm{C} 19^{\ddagger}\right) \mathrm{ppm}$; 
IR (film, cm cm $^{-1}$ 3404, 2949, 2924, 1732, 1707, 1620, 1591;

HRMS $\left(\mathrm{ESI}^{+}\right) 393.1681$ (calculated $[\mathrm{M}+\mathrm{Na}]^{+}$393.1673).

$\dagger$ One diastereoisomer, $\$$ both diastereoisomers. 


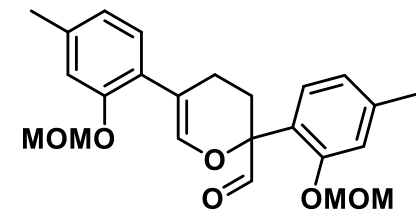

$( \pm)-2.28$

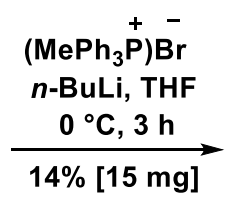

$\begin{gathered}n-B u L i, ~ T H F \\ 0{ }^{\circ} \mathrm{C}, 3 \mathrm{~h}\end{gathered}$
$\underset{4 \%[15 \mathrm{mg}]}{\longrightarrow}$

$14 \%[15 \mathrm{mg}$

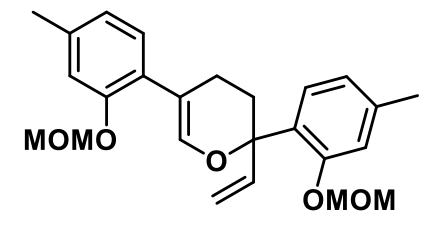

$( \pm)-2.38$

$n$-BuLi $(2.5 \mathrm{M}$ in hexanes, $146 \mu \mathrm{L}, 0.36 \mathrm{mmol})$ was added dropwise at $0{ }^{\circ} \mathrm{C}$ to a suspension of methyltriphenylphosphonium bromide (133 $\mathrm{mg}, 0.36 \mathrm{mmol}$ ) in anhydrous THF (12 mL) and the mixture was stirred at the same temperature for $1 \mathrm{~h}$. A solution of aldehyde $( \pm)-\mathbf{2 . 2 8}(100 \mathrm{mg}, 0.24 \mathrm{mmol})$ in anhydrous THF (2 mL) was then added at $0{ }^{\circ} \mathrm{C}$ and the mixture was stirred for another $2 \mathrm{~h}$. The reaction was then quenched with sat. $a q . \mathrm{NH}_{4} \mathrm{Cl}(2 \mathrm{~mL})$, then diethyl ether $(5 \mathrm{~mL})$ and water $(2 \mathrm{~mL})$ were added. The mixture was extracted with diethyl ether $(3 \times 15 \mathrm{~mL})$ and the combined organic layers were washed with water $(15 \mathrm{~mL})$, brine $(15 \mathrm{~mL})$ and dried over anhydrous $\mathrm{Na}_{2} \mathrm{SO}_{4}$. The solvent was removed under reduced pressure. Flash chromatography (75:25 petroleum spirit 40-60 / diethyl ether) gave compound (士)-2.38 as a pale-yellow oil ( $14 \mathrm{mg}, 33 \mu \mathrm{mol}, 14 \%$ yield) as well as recovered starting material ( \pm )-2.28 (9 mg, $22 \mu \mathrm{mol}, 9 \%$ yield).

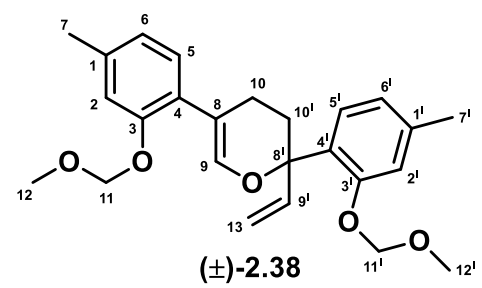

$\mathbf{R}_{\mathbf{f}} 0.37$ (75:25 petroleum spirit 40-60 / diethyl ether);

${ }^{1} \mathbf{H}$ NMR $\left(500 \mathrm{MHz}, \mathrm{CDCl}_{3}\right) \delta 7.42(\mathrm{~d}, J=7.9 \mathrm{~Hz}, 1 \mathrm{H}, \mathrm{H} 5), 6.99(\mathrm{~d}, J=7.7 \mathrm{~Hz}, 1 \mathrm{H}$, H5), 6.95-6.93 (m, 1H, H2'), 6.92-6.91 (m, 1H, H2), 6.87 - 6.83 (m, 1H, H9), 6.81$6.79\left(\mathrm{dm}, J=8.0 \mathrm{~Hz} 1 \mathrm{H}, \mathrm{H}^{\prime}\right), 6.76-6.75(\mathrm{dm}, J=7.8 \mathrm{~Hz}, 1 \mathrm{H}, \mathrm{H} 6), 6.38$ (dd, $J=17.2$, $10.7 \mathrm{~Hz}, 1 \mathrm{H}, \mathrm{H9}$ '), 5.30 (dd, $J=17.3,1.5 \mathrm{~Hz}, 1 \mathrm{H}, \mathrm{H} 13$ ), 5.21 (s, 2H, H11'), 5.11 (dd, $J=6.5 \mathrm{~Hz}, 1 \mathrm{H}, \mathrm{H} 13), 5.06(\mathrm{q}, J=6.5 \mathrm{~Hz}, 2 \mathrm{H}, \mathrm{H} 11), 3.50$ (s, 3H, H12), 3.42 (s, 3H, H12'), 2.66 - 2.61 (m, 1H, H10), 2.32 (s, 3H, H7), 2.30 (s, 3H, H7), 2.29 - 2.23 (m, $\left.2 \mathrm{H}, \mathrm{H} 10+\mathrm{H} 10^{\prime}\right), 2.14-2.04\left(\mathrm{~m}, 1 \mathrm{H}, \mathrm{H} 10^{\prime}\right) \mathrm{ppm}$; 
${ }^{13}$ C NMR (126 MHz, $\mathrm{CDCl}_{3}$ ) $\delta 154.7$ (C3), 153.6 (C3'), 141.3 (C9), 140.2 (C9'), 138.6 (C1), 137.3 (C1'), 129.0 (C5), 128.4 (C4'), 127.5 (C4), 127.4 (C5'), 122.8 (C6'), 122.5 (C6), 116.4 (C2'), 115.3 (C2), 113.5 (C13), 111.9 (C8), 94.9 (C11), 94.4 (C11'), 79.6 (C8'), 56.4 (C12 or $\left.\mathrm{C} 12^{\prime}\right), 56.2$ (C12 or C12'), 30.4 (C10'), 22.6 (C10), 21.4 (C7 or C7'), $21.4\left(\mathrm{C} 7\right.$ or $\left.\mathrm{C}^{\prime}\right) \mathrm{ppm}$;

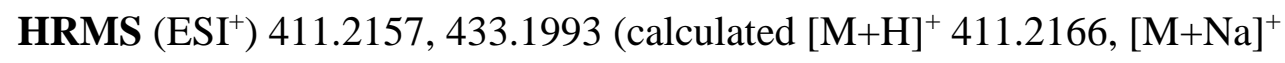
433.1985);

IR: (film, cm $\mathrm{cm}^{-1}$ ) 2951, 2922, 1636, 1611, 1503. 

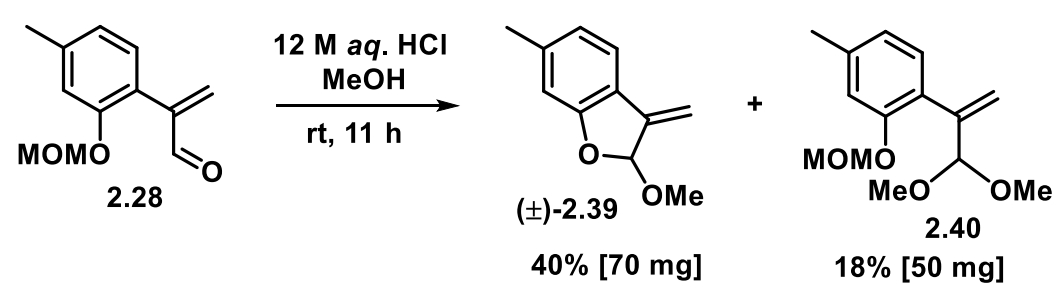

MOM-protected enal 2.28 (211 mg, $1.02 \mathrm{mmol})$ was dissolved in methanol (7.0 mL). $12 \mathrm{M}$ aq. $\mathrm{HCl}(128 \mu \mathrm{L}, 1.5 \mathrm{mmol})$ was added and the mixture was stirred at $\mathrm{rt}$ until complete consumption of the starting material, judged by TLC analysis (11 h). The reaction was then quenched by adding sat. aq. $\mathrm{NaHCO}_{3}(4 \mathrm{~mL})$ and the mixture was extracted with $\mathrm{CH}_{2} \mathrm{Cl}_{2}(5 \times 8 \mathrm{~mL})$. The organic layers were dried over anhydrous $\mathrm{Na}_{2} \mathrm{SO}_{4}$ and the solvent was removed under reduced pressure. Flash chromatography (90:10 petroleum spirit 40-60 / ethyl acetate) gave acetal ( $\mathbf{\pm}$-2.39 as a colourless oil (71 mg, $0.40 \mathrm{mmol}, 40 \%$ yield) and bis-acetal $\mathbf{2 . 4 0}$ as a colourless oil (47 mg, 0.19 mmol, $18 \%$ yield).

All data for compound 2.39 matched literature values. ${ }^{55}$

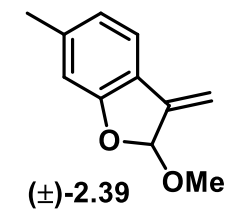

Rf 0.44 (90:10 petroleum spirit 40-60 / ethyl acetate);

${ }^{1} \mathbf{H}$ NMR $\left(500 \mathrm{MHz}, \mathrm{CDCl}_{3}\right) \delta 7.28(\mathrm{~d}, J=7.7 \mathrm{~Hz}, 1 \mathrm{H}), 6.74(\mathrm{~d}, J=7.7,1 \mathrm{H}), 6.71(\mathrm{~d}$, $J=0.7 \mathrm{~Hz}, 1 \mathrm{H}), 5.97$ (apparent t $, J=1.9 \mathrm{~Hz}, 1 \mathrm{H}), 5.62(\mathrm{~d}, J=2.1 \mathrm{~Hz}, 1 \mathrm{H}), 5.27$ (d, $J$ $=1.7 \mathrm{~Hz}, 1 \mathrm{H}), 3.51(\mathrm{~s}, 3 \mathrm{H}), 2.34(\mathrm{~s}, 3 \mathrm{H}) \mathrm{ppm}$;

${ }^{13}$ C NMR (126 MHz, $\left.\mathrm{CDCl}_{3}\right) \delta 161.4,143.2,141.6,122.2,121.8,120.8,111.1,107.5$, 105.8, 54.4, $22.0 \mathrm{ppm}$. 


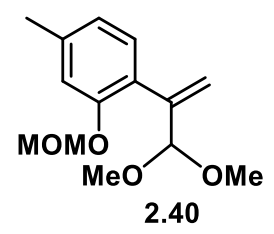

$\mathbf{R}_{\mathbf{f}}$ 0.30 (90:10 petroleum spirit 40-60 / ethyl acetate);

${ }^{1} \mathbf{H}$ NMR $\left(500 \mathrm{MHz}, \mathrm{CDCl}_{3}\right) \delta 7.11(\mathrm{~d}, J=7.7 \mathrm{~Hz}, 1 \mathrm{H}), 7.00-6.88(\mathrm{~m}, 1 \mathrm{H}), 6.80$ (ddd, $J=7.5,1.6,0.8 \mathrm{~Hz}, 1 \mathrm{H}), 5.80-5.47(\mathrm{~m}, 1 \mathrm{H}), 5.34$ (apparent q, $J=0.9 \mathrm{~Hz}, 2 \mathrm{H}), 5.16$ (s, 2H), 3.48 (s, 3H), $3.32(\mathrm{~s}, 6 \mathrm{H}), 2.33$ (d, $J=0.7 \mathrm{~Hz}, 3 \mathrm{H}) \mathrm{ppm}$;

${ }^{13}$ C NMR $\left(126 \mathrm{MHz}, \mathrm{CDCl}_{3}\right) \delta 154.3,144.1,139.1,130.3,127.1,122.8,117.7,115.5$, 102.5, 94.8, 56.2, 52.9 (2C), $21.6 \mathrm{ppm}$;

HRMS $\left(\mathrm{ESI}^{+}\right) 275.1238$ (calculated $\left.[\mathrm{M}+\mathrm{Na}]^{+} 275.1269\right)$;

IR (film, cm ${ }^{-1}$ ) 2932, 2907, 2828, 1611, 1570. 

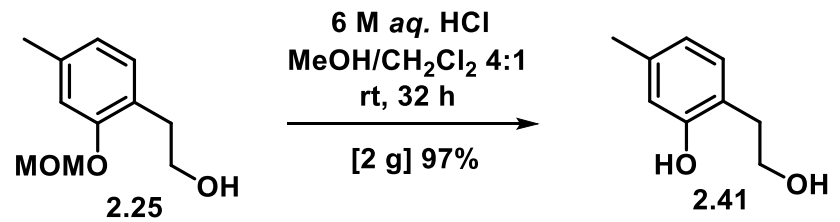

MOM-protected alcohol 2.25 (2.62 g, $13.4 \mathrm{mmol})$ was dissolved in a 4:1 mixture of methanol and $\mathrm{CH}_{2} \mathrm{Cl}_{2}(60 \mathrm{~mL}) .6 \mathrm{M} a q . \mathrm{HCl}(10 \mathrm{~mL})$ was added and the mixture was stirred at $\mathrm{rt}$ for $32 \mathrm{~h}$. Upon completion of the reaction, judged by TLC analysis, the mixture was diluted with water $(60 \mathrm{~mL})$ and extracted with $\mathrm{CH}_{2} \mathrm{Cl}_{2}(4 \times 60 \mathrm{~mL})$. The combined organic layers were washed with sat. aq. $\mathrm{NaHCO}_{3}(150 \mathrm{~mL})$ and dried over anhydrous $\mathrm{Na}_{2} \mathrm{SO}_{4}$. Removal of the solvent under reduced pressure afforded diol $\mathbf{2 . 4 1}$ as a white crystalline solid (1.98 g, $12.3 \mathrm{mmol}$, 97\% yield). The crude material was deemed sufficiently pure (see ${ }^{1} \mathrm{H}-\mathrm{NMR}$ spectrum on page 259 ) to be used directly in the next step.

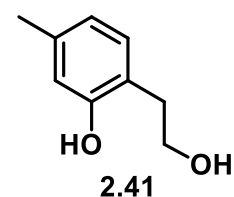

$\mathbf{R}_{\mathbf{f}} 0.37$ (50:50 petroleum spirit 40-60 / ethyl acetate);

${ }^{1}$ H-NMR $\left(500 \mathrm{MHz}, \mathrm{CDCl}_{3}\right) \delta 6.94(\mathrm{~d}, J=7.6 \mathrm{~Hz}, 1 \mathrm{H}), 6.73(\mathrm{~d}, J=1.6 \mathrm{~Hz}, 1 \mathrm{H}), 6.70$ $6.59(\mathrm{~m}, 1 \mathrm{H}), 4.97(\mathrm{~s}$, broad, $1 \mathrm{H}+1 \mathrm{H}), 4.05-3.83(\mathrm{~m}, 2 \mathrm{H}), 2.94-2.69(\mathrm{~m}, 2 \mathrm{H}), 2.28(\mathrm{~s}$, 3H) ppm;

${ }^{13}$ C-NMR $\left(126 \mathrm{MHz}, \mathrm{CDCl}_{3}\right) \delta$ 155.2, 138.5, 130.9, 123.6, 121.4, 117.7, 64.9, 34.3, $21.1 \mathrm{ppm}$

HRMS $\left(\mathrm{ESI}^{+}\right) \quad 153.0851,175.0748$ (calculated $[\mathrm{M}+\mathrm{H}]^{+}$153.0910, $[\mathrm{M}+\mathrm{Na}]^{+}$ 175.0730);

IR (film, $\left.\mathrm{cm}^{-1}\right)$ 3412, 3393, 2951, 2938, 2886, 1628;

m.p.: $70-71{ }^{\circ} \mathrm{C}\left(\mathrm{CH}_{2} \mathrm{Cl}_{2}\right)$. 


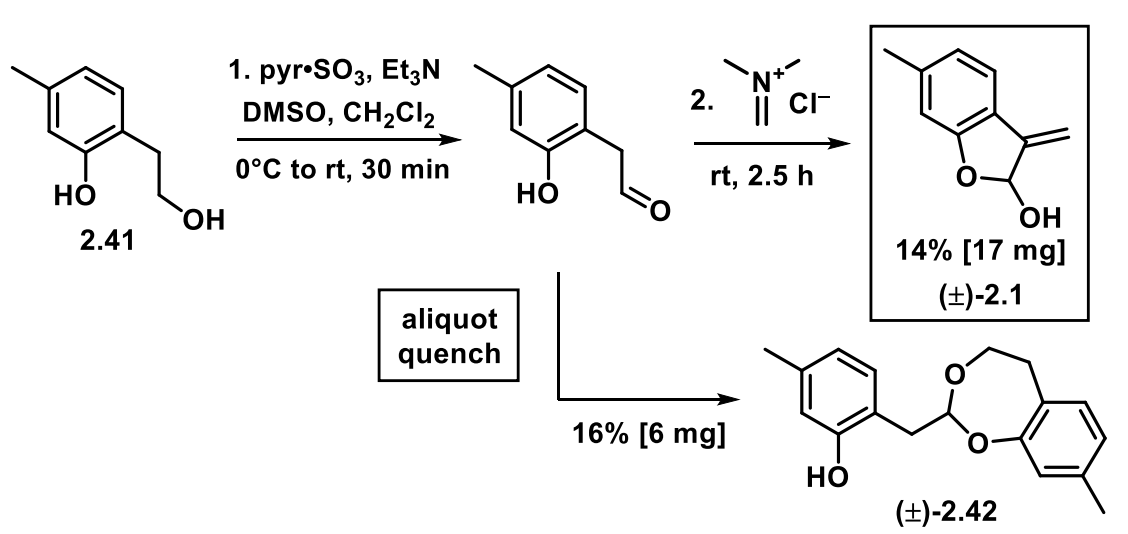

Alcohol 2.41 (189 mg, $1.24 \mathrm{mmol}$ ) was dissolved in anhydrous $\mathrm{CH}_{2} \mathrm{Cl}_{2}(8 \mathrm{~mL}) . \mathrm{Et}_{3} \mathrm{~N}$ (1.08 mL, $7.75 \mathrm{mmol})$, DMSO (0.55 mL, $7.75 \mathrm{mmol})$ and pyr• $\mathrm{SO}_{3}(459 \mathrm{mg}, 3.11$ mmol) were added at $0{ }^{\circ} \mathrm{C}$. The mixture was stirred at $\mathrm{rt}$. After complete consumption of the starting material, judged by TLC analysis (30 $\mathrm{min}$ ), a $3 \mathrm{~mL}$ aliquot of crude reaction mixture was submitted to the work up detailed below. To the remaining crude reaction mixture, the Eschenmoser salt $(148 \mathrm{mg}, 1.59 \mathrm{mmol})$ was added and the mixture was stirred for another $2.5 \mathrm{~h}$. The reaction was then quenched with sat. $a q$. $\mathrm{CuSO}_{4}(8 \mathrm{~mL})$ and the mixture was extracted with ethyl acetate $(3 \times 10 \mathrm{~mL})$. The combined organic layers were washed with $1 \mathrm{M} a q . \mathrm{HCl}$ and brine and then dried over an. $\mathrm{Na}_{2} \mathrm{SO}_{4}$ and the solvent was removed under reduced pressure. Flash chromatography of the first aliquot (80:20 petroleum spirit 40-60 / ethyl acetate) gave compound ( \pm )-2.42 as a white solid (6 mg, $35 \mu \mathrm{mol}, 16 \%$ yield based on the aliquot worked up).

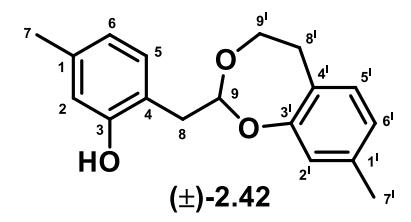

Rf 0.45 (70:30 petroleum spirit 40-60 / ethyl acetate);

${ }^{1} \mathbf{H}-\mathbf{N M R}\left(500 \mathrm{MHz}, \mathrm{CDCl}_{3}\right) \delta 7.08\left(\mathrm{~d}, J=7.4 \mathrm{~Hz}, 1 \mathrm{H}, \mathrm{H} 5^{\prime}\right), 6.95(\mathrm{~d}, J=7.5 \mathrm{~Hz}, 1 \mathrm{H}$, H5), 6.88 (s, 1H, OH at C3), 6.80-6.70 (m, 1H, H6'), 6.69 (d, J=1.6 Hz, 1H, H2'), 6.68-6.65 (m, 2H, H2 + H6), $5.68(\mathrm{dd}, J=6.6,2.0 \mathrm{~Hz}, 1 \mathrm{H}, \mathrm{H} 9), 4.24$ (ddd, $J=9.2$, 5.8, 3.7 Hz, 1H, H9'), 3.75 (apparent td, $J=9.2,3.1 \mathrm{~Hz}, 1 \mathrm{H}, \mathrm{H9}$ '), 3.32 (dm, $J=16.7$, $\mathrm{Hz}, 1 \mathrm{H}, \mathrm{H} 8$ ), 3.02 (dm, $J=16.7,1 \mathrm{H}, \mathrm{H} 8), 2.94$ (ddd, $J=15.1,9.4,3.7 \mathrm{~Hz}, 1 \mathrm{H}, \mathrm{H} 8$ '), 2.78 (ddd, $J=15.1,5.8,3.1 \mathrm{~Hz}, 1 \mathrm{H}, \mathrm{H8}$ '), 2.32 (s, 3H, H7), 2.28 (s, 3H, H7') ppm; 
${ }^{13}$ C-NMR (126 MHz, $\left.\mathrm{CDCl}_{3}\right) \delta 158.2(\mathrm{C} 3), 155.3\left(\mathrm{C}^{\prime}\right), 138.7\left(\mathrm{C} 1\right.$ or $\left.\mathrm{C}^{\prime}\right), 138.6(\mathrm{C} 1$ or C1'), 130.9 (C5), 124.7 (C5'), 123.3 (C4'), 122.3 (C6'), 121.8 (C4), 121.5 (C6), 117.8 (C2'), 110.8 (C2), 106.4 (C9), 70.6 (C9'), 36.8 (C8), 32.2 (C8'), 21.8 (C7), 21.3 (C7') ppm;

IR (film, $\mathrm{cm}^{-1}$ ) 3352, 2928, 2160, 2029, 1624, 1578;

HRMS $\left(\mathrm{ESI}^{+}\right) 307.1320$ (calculated $[\mathrm{M}+\mathrm{Na}]^{+}$307.1305);

m.p. $75-77{ }^{\circ} \mathrm{C}$ (acetonitrile).

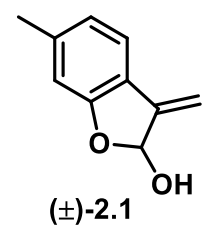

Flash chromatography of the second aliquot (85:15 petroleum spirit 40-60 / ethyl acetate) gave compound 2.1 as a white solid (17 mg, $105 \mu \mathrm{mol}, 14 \%$ yield). All data for compound ( \pm )-2.1 matched the available literature values. ${ }^{55}$

Rf 0.45 (70:30 petroleum spirit 40-60 / ethyl acetate);

${ }^{1}$ H-NMR $\left(500 \mathrm{MHz}, \mathrm{CDCl}_{3}\right) \delta 7.26(\mathrm{~s}, 1 \mathrm{H}), 6.76(\mathrm{ddd}, J=7.7,1.5,0.8 \mathrm{~Hz}, 1 \mathrm{H}), 6.71$ (s, 1H), $6.18(\mathrm{~s}, 1 \mathrm{H}), 5.59$ (s, 1H), 5.33 (s, 1H), 3.17 (s, 1H), 2.34 (s, 3H) ppm. 


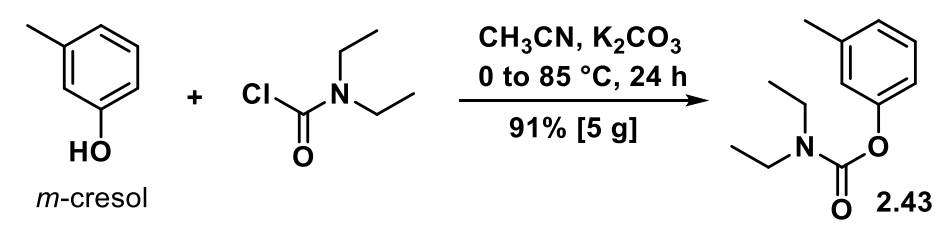

Carbamate $\mathbf{2 . 4 3}$ was prepared using a modified literature procedure. ${ }^{160}$

$m$-Cresol (4.84 mL, $45.8 \mathrm{mmol})$ was dissolved in anhydrous acetonitrile $(300 \mathrm{~mL})$ at rt. The solution was cooled to $0{ }^{\circ} \mathrm{C}$ and $\mathrm{K}_{2} \mathrm{CO}_{3}(127 \mathrm{~g}, 920 \mathrm{mmol})$ was added as a solid followed by dropwise addition of $N, N$-diethylcarbamoyl chloride $(6.59 \mathrm{~mL}, 50.4$ mmol). The mixture was warmed to $85^{\circ} \mathrm{C}$ and stirred until complete consumption of the starting material, judged by TLC analysis ( $24 \mathrm{~h})$. Then the reaction mixture was cooled to $0{ }^{\circ} \mathrm{C}$, diluted with water $(420 \mathrm{~mL})$ and the mixture was extracted with diethyl ether $(3 \times 50 \mathrm{~mL})$. The organic layer was washed with $1 \mathrm{Maq}$. $\mathrm{NaOH}(100 \mathrm{~mL})$, water $(100 \mathrm{~mL})$ and brine $(100 \mathrm{~mL})$, then dried over anhydrous $\mathrm{MgSO}_{4}$ and the solvent was removed under reduced pressure. Flash chromatography (90:10 petroleum spirit 40-60 / ethyl acetate) gave carbamate $\mathbf{2 . 4 3}$ as a colourless oil (8.65 g, $417 \mathrm{mmol}, 91 \%$ yield). All data for compound $\mathbf{2 . 4 3}$ matched literature values. ${ }^{160}$

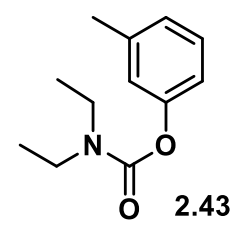

Rf 0.35 (90:10 petroleum spirit 40-60 / ethyl acetate);

${ }^{1} \mathbf{H}$ NMR $\left(500 \mathrm{MHz}, \mathrm{CDCl}_{3}\right) \delta 7.23(\mathrm{t}, J=7.0 \mathrm{~Hz}, 1 \mathrm{H}), 6.99(\mathrm{~d}, J=7.6 \mathrm{~Hz}, 1 \mathrm{H})$, $6.95(\mathrm{~d}, J=1.8 \mathrm{~Hz}, 1 \mathrm{H}), 6.92(\mathrm{dd}, J=8.1,2.3 \mathrm{~Hz}, 1 \mathrm{H}), 3.46-3.37(\mathrm{~m}, 4 \mathrm{H}), 2.35$ (s, $3 \mathrm{H}), 1.25(\mathrm{t}, J=7.3 \mathrm{~Hz}, 3 \mathrm{H}), 1.20(\mathrm{t}, J=7.3 \mathrm{~Hz}, 3 \mathrm{H}) \mathrm{ppm}$;

${ }^{13}$ C NMR $\left(126 \mathrm{MHz}, \mathrm{CDCl}_{3}\right) \delta 154.5,151.6,139.4,129.0,126.0,122.5,118.8,42.3$, 42.0, 21.4, 14.4, $13.4 \mathrm{ppm}$. 

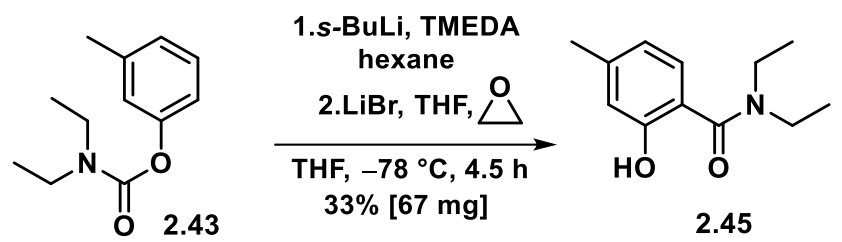

Carbamate protected phenol $\mathbf{2 . 4 3}$ (209 $\mathrm{mg}, 1.01 \mathrm{mmol})$ was dissolved in anhydrous THF ( $4 \mathrm{~mL})$. The solution was cooled to $-78^{\circ} \mathrm{C}$ then TMEDA $(167 \mu \mathrm{L}, 1.11 \mathrm{mmol})$ was added. A solution of $s$-BuLi (1.4 M in hexane, $0.79 \mathrm{~mL}, 1.11 \mathrm{mmol})$ was added dropwise and the mixture was stirred at $-78^{\circ} \mathrm{C}$ for $1 \mathrm{~h}$. Then ethylene oxide $(2.5 \mathrm{M}$ in THF $0.81 \mathrm{~mL}, 2.02 \mathrm{mmol})$ and a solution of $\mathrm{LiBr}(96 \mathrm{mg}, 1.11 \mathrm{mmol})$ in anhydrous THF $(1 \mathrm{~mL})$ were added. The mixture was stirred at $-78^{\circ} \mathrm{C}$ for $3.5 \mathrm{~h}$ then the reaction was quenched with sat. $a q$. $\mathrm{NH}_{4} \mathrm{Cl}(5 \mathrm{~mL})$ and extracted with diethyl ether $(3 \times 8 \mathrm{~mL})$. The combined organic layers were washed with brine $(20 \mathrm{~mL})$, dried over anhydrous $\mathrm{Na}_{2} \mathrm{SO}_{4}$ and the solvent was removed under reduced pressure. Flash chromatography (80:20 petroleum spirit 40-60 / ethyl acetate) gave phenol $\mathbf{2 . 4 5}$ as a pale-yellow oil (67 $\mathrm{mg}, 0.33 \mathrm{mmol}, 32 \%$ yield).

All data for compound $\mathbf{2 . 4 5}$ matched literature values. ${ }^{161}$

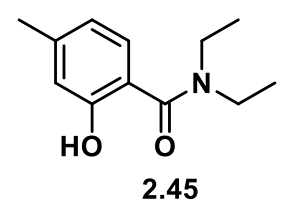

$\mathbf{R}_{\mathbf{f}} 0.08$ (90:10 petroleum spirit 40-60/ ethyl acetate);

${ }^{1} \mathbf{H}$ NMR $\left(500 \mathrm{MHz}, \mathrm{CDCl}_{3}\right) \delta 9.95(\mathrm{~s}, 1 \mathrm{H}), 7.16(\mathrm{~d}, J=7.9 \mathrm{~Hz}, 1 \mathrm{H}), 6.81(\mathrm{dd}, J=1.7$, $0.8 \mathrm{~Hz}, 1 \mathrm{H}), 6.65(\mathrm{ddd}, J=8.0,1.8,0.7 \mathrm{~Hz}, 1 \mathrm{H}), 3.52(\mathrm{q}, J=7.1 \mathrm{~Hz}, 4 \mathrm{H}), 2.32$ (s, 3H), $1.27(\mathrm{t}, J=7.1 \mathrm{~Hz}, 6 \mathrm{H}) \mathrm{ppm}$;

${ }^{13}$ C NMR (126 MHz, $\left.\mathrm{CDCl}_{3}\right) \delta 171.8,159.3,143.3,127.3,119.5,118.5,115.2,42.3$, 21.7 (2C), $13.6(2 \mathrm{C}) \mathrm{ppm}$. 


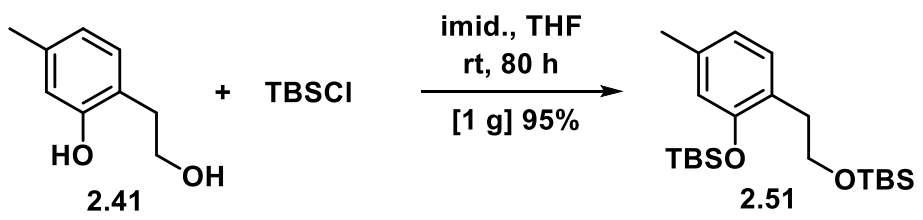

TBSCl (990 mg, $6.57 \mathrm{mmol})$ and imidazole (447 mg, $6.57 \mathrm{mmol})$ were dissolved in anhydrous THF (8.0 mL). Alcohol 2.41 (400 mg, $2.63 \mathrm{mmol}$ ) was added as a solid and the reaction was stirred at $\mathrm{rt}$ for $80 \mathrm{~h}$. Then the mixture was diluted with water $(15 \mathrm{~mL})$ and extracted with diethyl ether $(3 \times 10 \mathrm{~mL})$. The combined organic layers were washed with brine $(20 \mathrm{~mL})$ and water $(20 \mathrm{~mL})$, dried over anhydrous $\mathrm{Na}_{2} \mathrm{SO}_{4}$ and the solvent was removed under reduced pressure. Flash chromatography (95:5 petroleum spirit 40-60 / ethyl acetate) afforded compound $\mathbf{2 . 5 1}$ as a colourless oil (954 mg, 2.50 mmol, $95 \%$ yield).

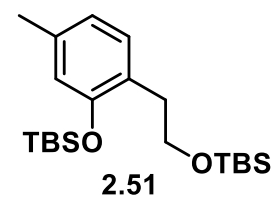

$\mathbf{R}_{\mathbf{f}}$ 0.61 (95:5 petroleum spirit 40-60 / ethyl acetate);

${ }^{1} \mathbf{H}$ NMR $\left(500 \mathrm{MHz}, \mathrm{CDCl}_{3}\right) \delta 7.04(\mathrm{dd}, J=7.7,0.9 \mathrm{~Hz}, 1 \mathrm{H}), 6.69(\mathrm{~d},, J=7.7 \mathrm{~Hz}$, $1 \mathrm{H}), 6.60(\mathrm{~s}, 1 \mathrm{H}), 3.75(\mathrm{td}, J=7.4,1.1 \mathrm{~Hz}, 2 \mathrm{H}), 2.80(\mathrm{t}, J=7.4 \mathrm{~Hz}, 2 \mathrm{H}), 2.27(\mathrm{~s}, 3 \mathrm{H})$, $1.02(\mathrm{~d}, J=1.1 \mathrm{~Hz}, 9 \mathrm{H}), 0.88(\mathrm{~s}, 9 \mathrm{H}), 0.24(\mathrm{~d}, J=1.1 \mathrm{~Hz}, 6 \mathrm{H}),-0.01(\mathrm{~d}, J=1.1 \mathrm{~Hz}$, 6H) ppm;

${ }^{13} \mathrm{C}$ NMR $\left(126 \mathrm{MHz}, \mathrm{CDCl}_{3}\right) \delta 153.8,137.1,131.1,126.4,121.7,119.3,63.4,34.1$, $26.1(3 \mathrm{C}), 26.0$ (3C), 21.3, 18.5, 18.4, -3.9 (2C), -5.2 (2C) ppm;

IR (film, $\mathrm{cm}^{-1}$ ) 2955, 2928, 2857, 1614, 1578;

HRMS $\left(\mathrm{ESI}^{+}\right) 381.2644$ (calculated $[\mathrm{M}+\mathrm{H}]^{+}$381.2640). 

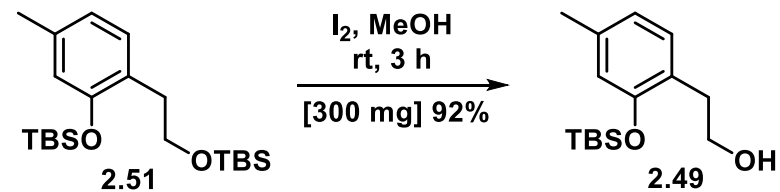

Compound 2.51 (467 mg, $1.23 \mathrm{mmol}$ ) was dissolved in anhydrous methanol (8.0 mL). $\mathrm{I}_{2}(29 \mathrm{mg}, 0.12 \mathrm{mmol})$ was added as a solid at $\mathrm{rt}$. The mixture was stirred at $\mathrm{rt}$ for $3 \mathrm{~h}$ then the reaction was quenched by adding a $10 \%$ w/w aq. $\mathrm{Na}_{2} \mathrm{~S}_{2} \mathrm{O}_{4}(5 \mathrm{~mL})$. The methanol was removed under reduced pressure and the residue was taken up in diethyl ether. The organic layer was washed with brine and dried over anhydrous $\mathrm{Na}_{2} \mathrm{SO}_{4}$. Removal of the solvent under reduced pressure gave compound 2.49 as a colourless oil (301 mg, $1.13 \mathrm{mmol}, 92 \%$ yield). The crude material was deemed sufficiently pure (see ${ }^{1} \mathrm{H}$ and spectrum on page 272) to be used directly in the next step.

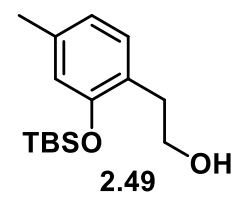

$\mathbf{R}_{\mathbf{f}}$ 0.12 (95:5 petroleum spirit 40-60 / ethyl acetate);

${ }^{1} \mathbf{H}$ NMR $\left(500 \mathrm{MHz}, \mathrm{CDCl}_{3}\right) \delta 7.04(\mathrm{~d}, J=7.5 \mathrm{~Hz}, 1 \mathrm{H}), 6.72(\mathrm{dm}, J=7.6 \mathrm{~Hz}, 1 \mathrm{H})$, 6.63-6.62 (m, 1H), $3.80(\mathrm{t}, J=6.5 \mathrm{~Hz}, 2 \mathrm{H}), 2.84(\mathrm{t}, J=6.5 \mathrm{~Hz}, 2 \mathrm{H}), 2.28(\mathrm{~m}, 3 \mathrm{H}), 1.02$ $(\mathrm{s}, 9 \mathrm{H}), 0.25$ (s, 6H) ppm;

${ }^{13}$ C NMR $\left(126 \mathrm{MHz}, \mathrm{CDCl}_{3}\right) \delta 153.9,137.6,130.9,126.0,122.1,119.6,63.1,34.0$, 25.9 (3C), 21.3, 18.4, -4.0 (2C) ppm;

IR (film, cm $\left.\mathrm{cm}^{-1}\right) 3347$ (broad), 2955, 2928, 2856, 1612, 1576;

HRMS $\left(\mathrm{ESI}^{+}\right)$267.1788 (calculated $[\mathrm{M}+\mathrm{H}]^{+}$267.1775). 

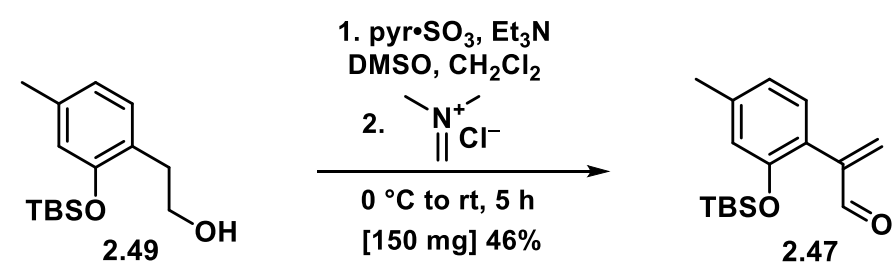

Alcohol 2.49 (311 mg, $1.17 \mathrm{mmol})$ was dissolved in anhydrous $\mathrm{CH}_{2} \mathrm{Cl}_{2}(7 \mathrm{~mL}) . \mathrm{Et}_{3} \mathrm{~N}$ (1.02 mL, $7.31 \mathrm{mmol})$, DMSO (519 $\mu \mathrm{L}, 7.31 \mathrm{mmol})$ and pyr $\bullet \mathrm{SO}_{3}(465 \mathrm{mg}, 2.92 \mathrm{mmol})$ were added at $0{ }^{\circ} \mathrm{C}$. The mixture was stirred at $\mathrm{rt}$ until complete consumption of the starting material, judged by TLC analysis $(2.5 \mathrm{~h})$, then the Eschenmoser salt (196 mg, $2.11 \mathrm{mmol}$ ) was added. The mixture was stirred at $\mathrm{rt}$ for another $2.5 \mathrm{~h}$, then the reaction was quenched by adding sat. $a q . \mathrm{CuSO}_{4}(8.0 \mathrm{~mL})$. The mixture was extracted with ethyl acetate $(3 \times 10 \mathrm{~mL})$, the combined organic layers were washed with $1 \mathrm{M} a q$. $\mathrm{HCl}$ $(20 \mathrm{~mL})$ and brine $(20 \mathrm{~mL})$ and dried over anhydrous $\mathrm{Na}_{2} \mathrm{SO}_{4}$. The solvent was removed under reduced pressure. Flash chromatography (85:15 petroleum spirit 40-60 / ethyl acetate) afforded enal $\mathbf{2 . 4 7}$ as a pale-yellow oil (166 mg, $0.53 \mathrm{mmol}, 46 \%$ yield).

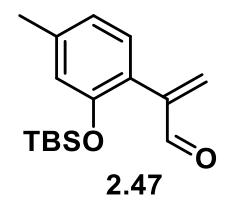

$\mathbf{R}_{\mathbf{f}} 0.59$ (70:30 petroleum spirit 40-60 / ethyl acetate);

${ }^{1} \mathbf{H}$ NMR $\left(500 \mathrm{MHz}, \mathrm{CDCl}_{3}\right) \delta 9.71(\mathrm{~s}, 1 \mathrm{H}), 7.05(\mathrm{~d}, J=7.6 \mathrm{~Hz}, 1 \mathrm{H}), 6.80(\mathrm{dm}, J=$ $7.7 \mathrm{~Hz}, 1 \mathrm{H}), 6.68(\mathrm{~m}, 1 \mathrm{H}), 6.33(\mathrm{~d}, J=1.3 \mathrm{~Hz}, 1 \mathrm{H}), 6.27$ (d, $J=1.3 \mathrm{~Hz}, 1 \mathrm{H}), 2.32(\mathrm{~m}$, $3 \mathrm{H}), 0.92(\mathrm{~s}, 9 \mathrm{H}), 0.16(\mathrm{~s}, 6 \mathrm{H}) \mathrm{ppm}$;

${ }^{13} \mathrm{C}$ NMR $\left(126 \mathrm{MHz}, \mathrm{CDCl}_{3}\right) \delta 192.9,153.1,147.4,140.1,133.6,130.6,123.8,122.2$, 120.1, 25.9 (3C), 21.5, 18.2, -4.0 (2C) ppm;

IR (film, $\mathrm{cm}^{-1}$ ) 2955, 2928, 2857, 1742, 1611;

HRMS $\left(\mathrm{ESI}^{+}\right) 277.1641$ (calculated $[\mathrm{M}+\mathrm{H}]^{+}$277.1618). 


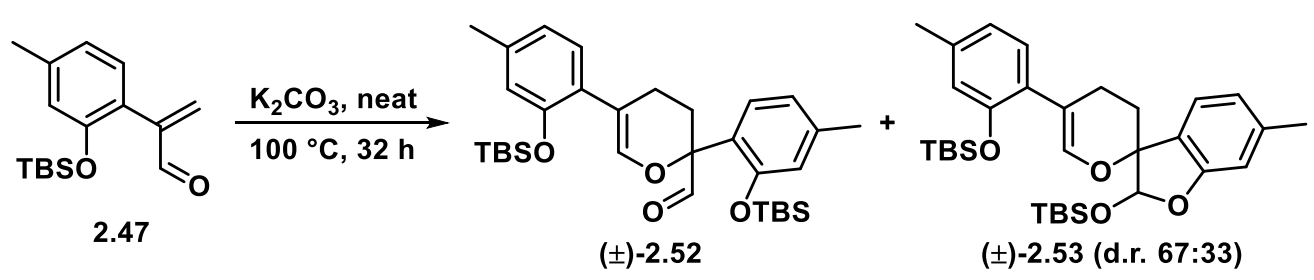

$\mathrm{K}_{2} \mathrm{CO}_{3}(69 \mathrm{mg}, 0.50 \mathrm{mmol})$ was added to neat enal monomer $2.47(87 \mathrm{mg}, 0.33 \mathrm{mmol})$. The mixture was stirred at $100{ }^{\circ} \mathrm{C}$ for $32 \mathrm{~h}$., then diluted with $\mathrm{CH}_{2} \mathrm{Cl}_{2}(10 \mathrm{~mL})$ and filtered. The solvent was removed under reduced pressure. Flash chromatography (96:4 to 90:10 petroleum spirit 40-60 / diethyl ether) gave aldehyde ( \pm )-2.52 as a paleyellow oil (18 mg, $34 \mu \mathrm{mol}, 21 \%$ yield) and side product ( $\mathbf{\pm})-\mathbf{2 . 5 3}$ as a 67:33 mixture of diastereoisomers (pale yellow oil, $15 \mathrm{mg}, 28 \mu \mathrm{mol}, 17 \%$ yield).

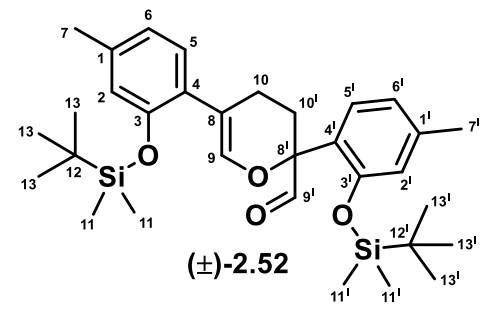

$\mathbf{R}_{\mathbf{f}}$ 0.30 (95:5 petroleum spirit 40-60 / diethyl ether);

${ }^{1} \mathbf{H}$ NMR $\left(500 \mathrm{MHz}, \mathrm{CDCl}_{3}\right) \delta 9.81(\mathrm{~d}, J=0.7 \mathrm{~Hz}, 1 \mathrm{H}, \mathrm{H9}), 7.37(\mathrm{~d}, J=7.9 \mathrm{~Hz}, 1 \mathrm{H}$, H5), 6.97 (d, $J=7.7 \mathrm{~Hz}, 1 \mathrm{H}, \mathrm{H} 5), 6.83-6.82$ (m, 1H, H9), 6.80 (dm, $J=7.9 \mathrm{~Hz}, 1 \mathrm{H}$, H6'), $6.71(\mathrm{dm}, J=7.7 \mathrm{~Hz}, 1 \mathrm{H}, \mathrm{H6}), 6.68-6.66$ (m, 1H, H2'), 6.61-6.60 (m, 1H, H2), 2.50 (m, 1H, H10'), 2.48-2.39 (m, 1H, H10), 2.38-2.32 (m, 2H , H10 + H10'), 2.30 (s, 3H, H7'), 2.27 (s, 3H, H7), 1.02 (s, 9H, H13 or H13'), 0.97 (s, 9H, H13 or H13'), 0.32 (s, 3H, 6.8Hz, H11 or H11'), 0.31 (s, 3H, H11 or H11'), 0.19 (s, 3H, 7.9 HZ?, H11 or H11'), 0.17 (s, 3H, H11 or H11') ppm;

${ }^{13}$ C NMR (126 MHz, $\mathrm{CDCl}_{3}$ ) $\delta 199.3$ (C9'), 153.2 (C3), 152.9 (C3'), 141.2 (C2), 139.9 (C1'), 137.5 (C1), 129.7 (C5), 128.1 (C4), 127.9 (C5'), 126.3 (C4'), 122.1 (C6'), 121.9 (C6), 120.7 (H2), 119.5 (C2'), 113.2 (C8), 81.9 (C8'), 26.2 (3C, C13 or C13'), 26.1 (C10'), 26.0 (3C, C13 or C13'), 21.8 (C10), 21.4 (C7'), 21.3 (C7), 18.8 (C12 or C12'), $18.5\left(\mathrm{C} 12\right.$ or $\left.\mathrm{C} 12^{\prime}\right),-3.6\left(\mathrm{C} 11\right.$ or $\left.\mathrm{C} 11^{\prime}\right),-3.9\left(\mathrm{C} 11\right.$ or $\left.\mathrm{C} 11^{\prime}\right),-4.0\left(\mathrm{C} 11\right.$ or $\left.\mathrm{C} 11^{\prime}\right),-4.0$ (C11 or $\left.\mathrm{C} 11^{\prime}\right) \mathrm{ppm}$;

IR (film, $\mathrm{cm}^{-1}$ ) 2953, 2928, 2857, 1740, 1611;

HRMS $\left(\mathrm{ESI}^{+}\right) 553.3168$ (calculated $[\mathrm{M}+\mathrm{H}]^{+}$553.3164). 


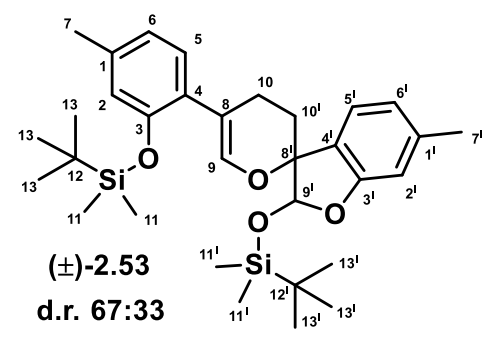

Rf 0.39 (95/5 petroleum spirit 40-60 / diethyl ether);

${ }^{1} \mathbf{H}-\mathbf{N M R}\left(500 \mathrm{MHz}, \mathrm{CDCl}_{3}\right) \delta 7.20\left(\mathrm{~d}, J=7.6 \mathrm{~Hz}, 1 \mathrm{H}, \mathrm{H} 5^{\prime \dagger}\right), 7.17(\mathrm{~d}, J=7.6 \mathrm{~Hz}, 1 \mathrm{H}$, H5 $\left.5^{\ddagger}\right), 7.02\left(\mathrm{~d}, J=7.6 \mathrm{~Hz}, 1 \mathrm{H}, \mathrm{H} 5^{\dagger}\right), 7.01\left(\mathrm{~d}, J=7.6 \mathrm{~Hz}, 1 \mathrm{H}, \mathrm{H} 5^{\ddagger}\right), 6.78$ (dm, $J=7.6$ $\left.\mathrm{Hz}, 1 \mathrm{H}, \mathrm{H}^{\dagger \dagger}\right), 6.78-6.71\left(\mathrm{~m}, 4 \mathrm{H}, \mathrm{H} 6^{\dagger}+\mathrm{H}^{\ddagger}, \mathrm{H} 6^{\ddagger}, \mathrm{H} 2^{\ddagger}\right), 6.71-6.70\left(\mathrm{~m}, 1 \mathrm{H}, \mathrm{H} 2^{\dagger \dagger}\right), 6.66-$ $6.65\left(\mathrm{~m}, 1 \mathrm{H}, \mathrm{H} 2^{\ddagger}\right), 6.64\left(\mathrm{~m}, 2 \mathrm{H}, \mathrm{H} 2^{\dagger}+\mathrm{H} 9^{\ddagger}\right), 6.60\left(\mathrm{~d}, J=1.7 \mathrm{~Hz}, 1 \mathrm{H}, \mathrm{H} 9^{\dagger}\right), 5.76(\mathrm{~s}$, $\left.1 \mathrm{H}, \mathrm{H}^{\prime \dagger}\right), 5.56$ (s, 1H, H9 $\left.9^{\dagger}\right), 2.64-2.44\left(\mathrm{~m}, 4 \mathrm{H}, \mathrm{H} 10^{\prime \dagger}+\mathrm{H} 10^{\dagger}\right), 2.35$ (s, 3H, H7 $\left.7^{\prime \dagger}\right), 2.33$ (s, 3H, H7 $\left.7^{\dagger}\right), 2.29\left(\mathrm{~s}, 6 \mathrm{H}, \mathrm{H} 7^{\dagger}+\mathrm{H} 7^{\ddagger}\right), 2.27-2.21\left(\mathrm{~m}, 2 \mathrm{H}, \mathrm{H} 10^{\dagger}\right), 2.19-2.12(\mathrm{~m}, 1 \mathrm{H}$, H10 $\left.0^{\ddagger}\right), 97$ (ddd, $\left.J=13.7,5.8,4.5 \mathrm{~Hz}, 1 \mathrm{H}, \mathrm{H} 10^{\prime}\right), 1.02$ (s, 9H, H13* or H13 $3^{\ddagger}, 1.01$

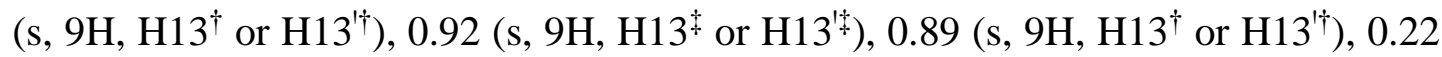

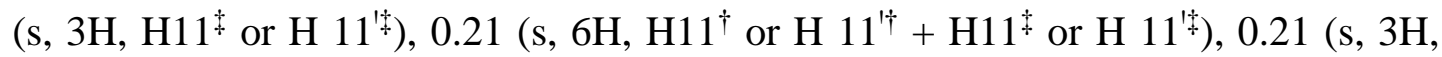
$\mathrm{H} 11^{\dagger}$ or $\left.\mathrm{H} 11^{\prime \dagger}\right), 0.19\left(\mathrm{~s}, 3 \mathrm{H}, \mathrm{H} 11^{\dagger}\right.$ or $\left.\mathrm{H} 11^{\prime \dagger}\right), 0.18\left(\mathrm{~s}, 3 \mathrm{H}, \mathrm{H} 11^{\ddagger}\right.$ or $\left.\mathrm{H} 11^{\dagger}\right), 0.15(\mathrm{~s}, 3 \mathrm{H}$, $\mathrm{H} 11^{\ddagger}$ or $\left.\mathrm{H} 11^{\ddagger}\right), 0.14\left(\mathrm{~s}, 3 \mathrm{H}, \mathrm{H} 11^{\dagger}\right.$ or $\left.\mathrm{H} 11^{\prime \dagger}\right) \mathrm{ppm}$;

${ }^{13}$ C-NMR $\left(126 \mathrm{MHz}, \mathrm{CDCl}_{3}\right) \delta 159.5^{\dagger}\left(\mathrm{C}^{\prime}\right), 157.6^{\ddagger}\left(\mathrm{C}^{\prime}\right), 153.2^{\dagger}(\mathrm{C} 3), 153.2^{\ddagger}(\mathrm{C} 3)$, 142.9 (C9), 141.6 $6^{\dagger}(\mathrm{C} 9), 141.4^{\dagger}\left(\mathrm{C}^{\prime}\right), 140.7^{\ddagger}\left(\mathrm{C} 1^{\prime}\right), 137.5^{\dagger}(\mathrm{C} 1), 137.3^{\ddagger}(\mathrm{C} 1), 129.8$ $\left(1 \mathrm{C}^{\dagger}+1 \mathrm{C}^{\ddagger}, \mathrm{C} 5\right), 129.5^{\ddagger}(\mathrm{C} 4), 128.4^{\dagger}(\mathrm{C} 4), 126.8^{\ddagger}\left(\mathrm{C} 4^{\prime}\right), 126.3^{\dagger}\left(\mathrm{C} 4^{\prime}\right), 124.1\left(1 \mathrm{C}^{\dagger}+1 \mathrm{C}^{\ddagger}\right.$, $\left.\mathrm{C}^{\prime}\right), 122.2^{\dagger}$ (C6 or $\left.\mathrm{C}^{\prime}\right), 122.2^{\ddagger}\left(\mathrm{C} 6\right.$ or $\left.\mathrm{C} 6^{\prime}\right), 122.1^{\dagger}$ (C6 or $\left.\mathrm{C} 6^{\prime}\right), 122.1^{\ddagger}\left(\mathrm{C} 6\right.$ or $\left.\mathrm{C} 6{ }^{\prime}\right)$, $120.8^{\dagger}(\mathrm{C} 2), 120.8^{\ddagger}(\mathrm{C} 2), 112.4^{\dagger}\left(\mathrm{C}^{\prime}\right), 111.4^{\dagger}\left(\mathrm{C}^{\prime}\right), 111.1^{\ddagger}\left(\mathrm{C}^{\prime}\right), 110.4^{\ddagger}\left(\mathrm{C}^{\prime}\right), 105.7^{\dagger}$

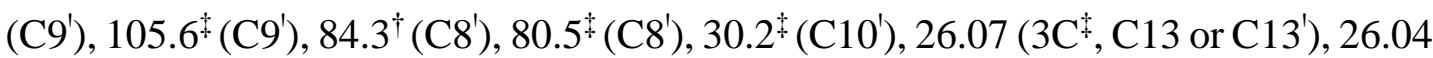

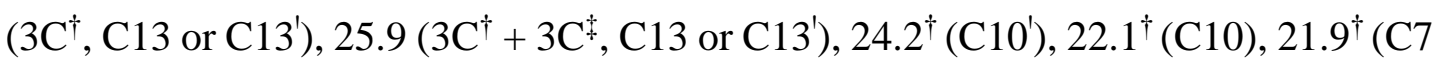
or $\left.\mathrm{C}^{\prime}\right), 21.8^{\ddagger}(\mathrm{C} 10), 21.3^{\ddagger}\left(\mathrm{C} 7\right.$ or $\left.\mathrm{C} 7^{\prime}\right), 21.3\left(1 \mathrm{C}^{\dagger}+1 \mathrm{C}^{\ddagger},(\mathrm{C} 7\right.$ or $\mathrm{C} 7), 18.5^{\ddagger}(\mathrm{C} 12$ or $\left.\mathrm{C} 12^{\prime}\right), 18.5^{\dagger}\left(\mathrm{C} 12\right.$ or $\left.\mathrm{C} 12^{\prime}\right), 18.3^{\ddagger}\left(\mathrm{C} 12\right.$ or $\left.\mathrm{C} 12^{\prime}\right), 18.1^{\dagger}\left(\mathrm{C} 12\right.$ or $\left.\mathrm{C} 12^{\prime}\right),-3.9^{\ddagger}(\mathrm{C} 11$ or $\left.\mathrm{C} 11^{\prime}\right),-3.9^{\dagger}\left(\mathrm{C} 11\right.$ or $\left.\mathrm{C} 11^{\prime}\right),-3.9^{\dagger}\left(\mathrm{C} 11\right.$ or $\left.\mathrm{C} 11^{\prime}\right),-4.0^{\dagger}\left(\mathrm{C} 11\right.$ or $\left.\mathrm{C} 11^{\prime}\right),-4.0^{\ddagger}(\mathrm{C} 11$ or $\left.\mathrm{C} 11^{\prime}\right),-4.1^{\ddagger}\left(\mathrm{C} 11\right.$ or $\left.\mathrm{C} 11^{\prime}\right),-4.6^{\ddagger}\left(\mathrm{C} 11\right.$ or $\left.\mathrm{C} 11^{\prime}\right),-5.1^{\dagger}\left(\mathrm{C} 11\right.$ or $\left.\mathrm{C} 11^{\prime}\right) \mathrm{ppm}$;

IR (film, $\mathrm{cm}^{-1}$ ) 2953, 2928, 2886, 2857, 1754, 1611;

HRMS $\left(\mathrm{ESI}^{+}\right) 553.3169$ (calculated $[\mathrm{M}+\mathrm{H}]^{+}$553.3169).

$\dagger$ Major diastereoisomer, $\$$ minor diastereoisomer. 

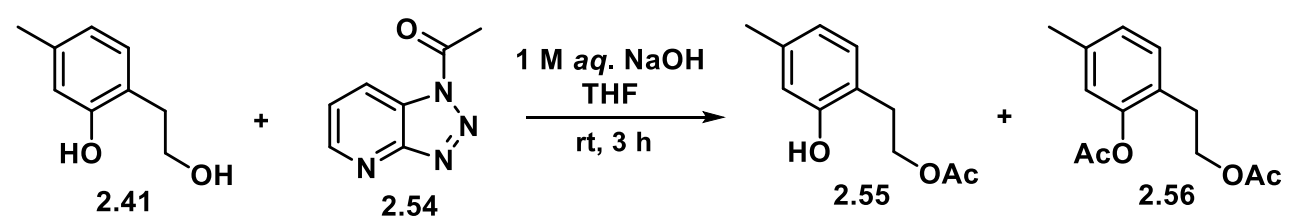

A solution of triazolopyridine $\mathbf{2 . 5 4}(88 \mathrm{mg}, 0.53 \mathrm{mmol})$ in anhydrous THF ( $1 \mathrm{~mL})$ was added to a stirred solution of diol $2.41(81 \mathrm{mg}, 0.53 \mathrm{mmol})$ in anhydrous THF (1 mL) and $1 \mathrm{Maq}$. $\mathrm{NaOH}(0.5 \mathrm{~mL})$. The mixture was stirred at $\mathrm{rt}$ until complete consumption of the starting material, judged by TLC analysis ( $3 \mathrm{~h})$, then the reaction was quenched with $2 \mathrm{M} \mathrm{aq}$. $\mathrm{HCl}(2 \mathrm{~mL})$ and extracted with diethyl ether $(3 \times 3 \mathrm{~mL})$. The combined organic layers were washed with water $(8 \mathrm{~mL})$, dried over anhydrous $\mathrm{Na}_{2} \mathrm{SO}_{4}$ and the solvent was removed under reduced pressure. Flash chromatography $\left(99: 1 \mathrm{CH}_{2} \mathrm{Cl}_{2}\right.$ / methanol) gave monoacetate $\mathbf{2 . 5 5}$ as a white solid (34 $\mathrm{mg}, 0.17 \mathrm{mmol}, 33 \%$ yield) and diacetate 2.56 as a colourless oil (24 mg, $0.10 \mathrm{mmol}, 20 \%$ yield).

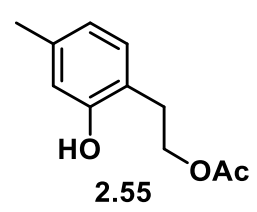

$\mathbf{R}_{\mathbf{f}} 0.42\left(99: 1 \mathrm{CH}_{2} \mathrm{Cl}_{2}\right.$ / methanol);

${ }^{1} \mathbf{H}$ NMR $\left(500 \mathrm{MHz}, \mathrm{CDCl}_{3}\right) \delta 6.98(\mathrm{~d}, J=7.5 \mathrm{~Hz}, 1 \mathrm{H}), 6.69-6.67(\mathrm{~m}, 1 \mathrm{H}), 6.66(\mathrm{~s}$, $1 \mathrm{H}), 4.25(\mathrm{t}, J=7.1 \mathrm{~Hz}, 2 \mathrm{H}), 2.91(\mathrm{t}, J=7.0 \mathrm{~Hz}, 2 \mathrm{H}), 2.28(\mathrm{~s}, 3 \mathrm{H}), 2.08(\mathrm{~s}, 3 \mathrm{H}) \mathrm{ppm}$;

${ }^{13} \mathrm{C} \mathrm{NMR}\left(126 \mathrm{MHz}, \mathrm{CDCl}_{3}\right) \delta 171.7,154.5,138.5,130.8,121.5,120.6,116.8,64.9$, 29.9, 21.2 (2C) ppm;

IR (film, cm ${ }^{-1}$ ) 3262 (broad), 2932, 1737, 1697, 1667, 1618;

HRMS $\left(\mathrm{ESI}^{+}\right) 217.0857$ (calculated $[\mathrm{M}+\mathrm{Na}]^{+} 217.0835$ );

m.p. $47-48{ }^{\circ} \mathrm{C}\left(\mathrm{CH}_{2} \mathrm{Cl}_{2}\right)$. 


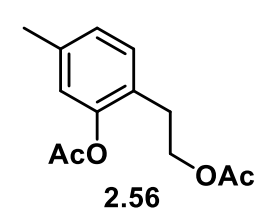

$\mathbf{R}_{\mathbf{f}} 0.52\left(99: 1 \mathrm{CH}_{2} \mathrm{Cl}_{2}\right.$ / methanol);

${ }^{1} \mathbf{H}$ NMR $\left(500 \mathrm{MHz}, \mathrm{CDCl}_{3}\right) \delta 7.15(\mathrm{~d}, J=7.7 \mathrm{~Hz}, 1 \mathrm{H}), 7.00(\mathrm{ddd}, J=7.7,1.8,0.8$ $\mathrm{Hz}, 1 \mathrm{H}), 6.87(\mathrm{dd}, J=1.8,0.8 \mathrm{~Hz}, 1 \mathrm{H}), 4.21$ (t, $J=7.2 \mathrm{~Hz}, 2 \mathrm{H}), 2.82$ (t, $J=7.2 \mathrm{~Hz}$, $2 \mathrm{H}), 2.33(\mathrm{~s}, 3 \mathrm{H}), 2.32(\mathrm{~s}, 3 \mathrm{H}), 2.03$ (s, 3H) ppm;

${ }^{13}$ C NMR $\left(126 \mathrm{MHz}, \mathrm{CDCl}_{3}\right) \delta 171.1,169.8,149.3,138.2,130.6,127.2,126.6,123.2$, 64.0, 29.5, 21.1, 21.0 (2C) ppm;

IR (film, $\mathrm{cm}^{-1}$ ) 2959, 2926, 1727, 1736, 1508;

HRMS $\left(\mathrm{ESI}^{+}\right)$237.1132, 259.0965 (calculated $[\mathrm{M}+\mathrm{H}]^{+}$237.1121, $[\mathrm{M}+\mathrm{Na}]^{+}$ 259.0941). 


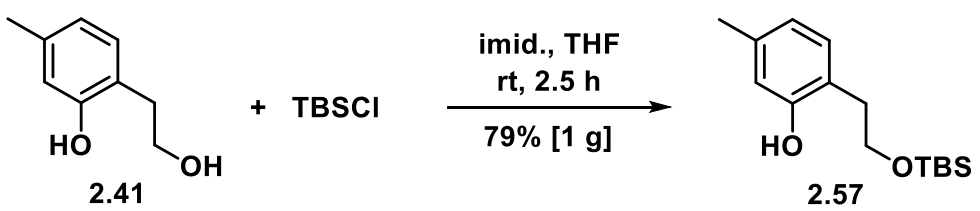

Diol 2.41 (753 mg, $4.83 \mathrm{mmol}$ ) was dissolved in anhydrous THF at rt. Imidazole (329 $\mathrm{mg}, 4.83 \mathrm{mmol}$ ) was added, then the solution was cooled to $0{ }^{\circ} \mathrm{C}$. A solution of tertbutyldimethylsilyl-chloride $(728 \mathrm{mg}, 4.83 \mathrm{mmol})$ in anhydrous THF $(2.5 \mathrm{~mL})$ was added within $1 \mathrm{~h}$ via syringe pump at $0{ }^{\circ} \mathrm{C}$. The mixture was then allowed to warm to the $\mathrm{rt}$ and stirred for another $1.5 \mathrm{~h}$. The reaction was then quenched with sat. aq. $\mathrm{NH}_{4} \mathrm{Cl}$ $(5 \mathrm{~mL})$. The aqueous layer was extracted with diethyl ether $(3 \times 6 \mathrm{~mL})$, the combined organic layers were dried over anhydrous $\mathrm{Na}_{2} \mathrm{SO}_{4}$ and the solvent was removed under reduced pressure. Flash chromatography (90:10 petroleum spirit 40-60 / ethyl acetate) gave phenol 2.57 as a colourless oil (1.01 g, $3.80 \mathrm{mmol}, 79 \%$ yield).

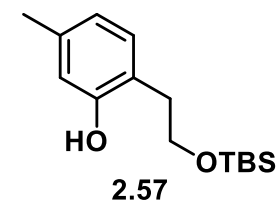

$\mathbf{R}_{\mathbf{f}} 0.54$ (70:30 petroleum spirit 40-60 / ethyl acetate);

${ }^{1} \mathbf{H}$ NMR $\left(500 \mathrm{MHz}, \mathrm{CDCl}_{3}\right) \delta 8.22(\mathrm{~s}, 1 \mathrm{H}), 6.90(\mathrm{~d}, J=7.6 \mathrm{~Hz}, 1 \mathrm{H}), 6.75(\mathrm{~s}, 1 \mathrm{H})$, $6.64(\mathrm{~d}, J=7.5 \mathrm{~Hz}, 1 \mathrm{H}), 3.92-3.89$ (m, 2H), 2.91-02.76 (m, 2H), 2.29 (s, 3H), 0.92 (s, 9H), 0.09 (s, 6H) ppm;

${ }^{13}$ C NMR $\left(126 \mathrm{MHz}, \mathrm{CDCl}_{3}\right) \delta 155.9,138.4,130.7,124.1,120.9,117.8,66.1,35.5$, 26.0 (3C), 21.2, 18.4, -5.5 (2C) ppm;

IR (film, cm cm $^{-1} 3292$ (broad), 2953, 2928, 2859, 1630;

HRMS $\left(\mathrm{ESI}^{+}\right) \quad 267.1790,289.1607$ (calculated $[\mathrm{M}+\mathrm{H}]^{+}$267.1775, $[\mathrm{M}+\mathrm{Na}]^{+}$ 289.1594). 


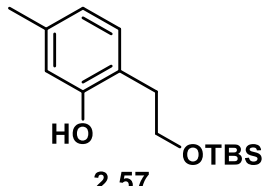

2.57

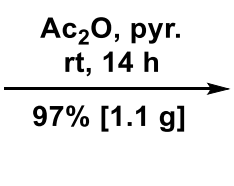

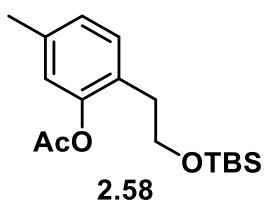

2.58

Phenol 2.57 (1.01 g, $3.8 \mathrm{mmol}$ ) was dissolved in anhydrous pyridine (4 mL). Acetic anhydride $(0.39 \mathrm{~mL}, 4.0 \mathrm{mmol})$ was added and the mixture was stirred at $\mathrm{rt}$ until complete consumption of the starting material, judged by TLC analysis (14 h). The reaction mixture was then extracted with $\mathrm{CH}_{2} \mathrm{Cl}_{2}(3 \times 5 \mathrm{~mL})$ and washed with $1 \mathrm{M} a q$. $\mathrm{HCl}(2 \times 8 \mathrm{~mL})$. The combined organic layers were dried over anhydrous $\mathrm{Na}_{2} \mathrm{SO}_{4}$ and the solvent was removed under reduced pressure to afford compound $\mathbf{2 . 5 8}$ as a colourless oil (1.14 g, $3.7 \mathrm{mmol}, 97 \%$ crude yield). The crude material was deemed sufficiently pure (see ${ }^{1} \mathrm{H}$ NMR spectrum on page 286 ) to be used directly in the next step.

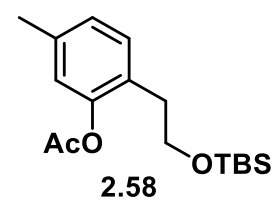

Rf 0.55 (75:25 petroleum spirit 40-60 / ethyl acetate);

${ }^{1} \mathbf{H}$ NMR $\left(500 \mathrm{MHz}, \mathrm{CDCl}_{3}\right) \delta 7.15(\mathrm{~d}, J=7.7 \mathrm{~Hz}, 1 \mathrm{H}), 6.99-6.97(\mathrm{~m}, 1 \mathrm{H}), 6.84(\mathrm{~s}$, 1H), 3.73 (t, $J=7.4 \mathrm{~Hz}, 2 \mathrm{H}), 2.71(\mathrm{t}, J=7.4 \mathrm{~Hz}, 2 \mathrm{H}), 2.32$ (s, 3H), 2.31 (s, 3H), 0.89 (s, 9H), $0.01(\mathrm{~s}, 6 \mathrm{H}) \mathrm{ppm}$;

${ }^{13} \mathrm{C}$ NMR $\left(126 \mathrm{MHz}, \mathrm{CDCl}_{3}\right) \delta 169.8,149.2,137.6,131.1,127.6,127.0,122.9,63.4$, $33.8,26.1(3 \mathrm{C}), 21.1,21.0,18.5,-5.2(2 \mathrm{C}) \mathrm{ppm}$;

IR (film, $\mathrm{cm}^{-1}$ ) 2953, 2928, 2857, 1769, 1508;

HRMS $\left(\mathrm{ESI}^{+}\right)$309.1893, 331.1761 (calculated $[\mathrm{M}+\mathrm{H}]^{+}$309.1880, $[\mathrm{M}+\mathrm{Na}]^{+}$ 331.1700). 

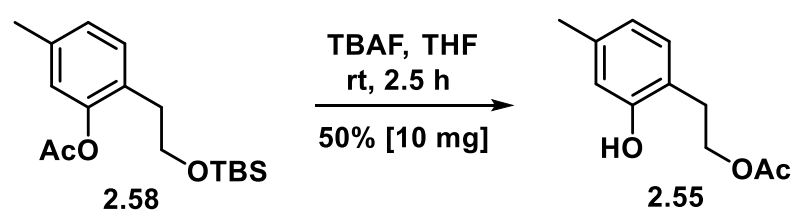

Protected diol 2.58 (32 mg, $0.10 \mathrm{mmol})$ was dissolved in anhydrous THF (0.4 mL). TBAF (1 M in THF, $0.12 \mathrm{~mL}, 0.12 \mathrm{mmol}$ ) was added at $\mathrm{rt}$ and the mixture was stirred until complete consumption of the starting material, judged by TLC analysis ( $2.5 \mathrm{~h})$. The reaction was then quenched with water $(1 \mathrm{~mL})$ and extracted with ethyl acetate (3 $\times 2 \mathrm{~mL}$ ). The combined organic layers were dried over anhydrous $\mathrm{Na}_{2} \mathrm{SO}_{4}$ and the solvent was removed under reduced pressure. Flash chromatography (50:50 petroleum spirit 40-60 / diethyl ether) afforded phenol 2.55 as a white solid (10 mg, 51 $\mu \mathrm{mol}$, $50 \%$ yield). 


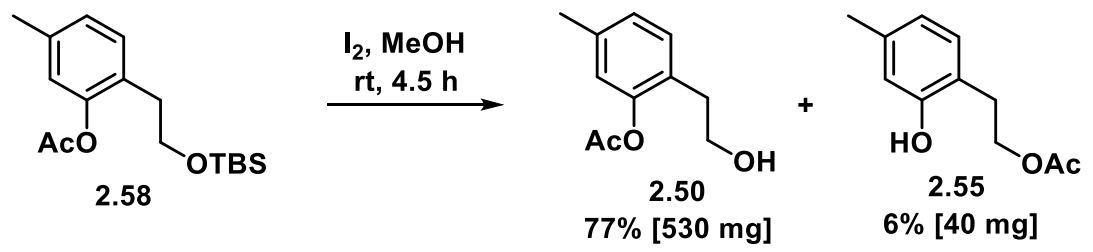

Protected alcohol 2.58 (1.10 g, $3.56 \mathrm{mmol})$ was dissolved in anhydrous methanol (20 $\mathrm{mL}) . \mathrm{I}_{2}(100 \mathrm{mg}, 0.39 \mathrm{mmol})$ was added as a solid and the mixture was stirred at $\mathrm{rt}$ for $4.5 \mathrm{~h}$. Then the reaction was quenched by adding sat. aq. $\mathrm{Na}_{2} \mathrm{~S}_{2} \mathrm{O}_{4}(20 \mathrm{~mL})$, the methanol was removed under reduced pressure and the residue was taken up in diethyl ether $(20 \mathrm{~mL})$. The organic layer was washed with brine $(20 \mathrm{~mL})$, dried over anhydrous $\mathrm{Na}_{2} \mathrm{SO}_{4}$ and the solvent was removed under reduced pressure. Flash chromatography (60:40 petroleum spirit 40-60 / ethyl acetate) gave primary alcohol 2.50 as a colourless oil (535 mg, $2.75 \mathrm{mmol}, 77 \%$ yield), along with recovered starting material 2.58 (73 mg, 0.24 mmol, 7\% yield) and phenol 2.55 (40 mg, $0.21 \mathrm{mmol}, 6 \%$ yield). 

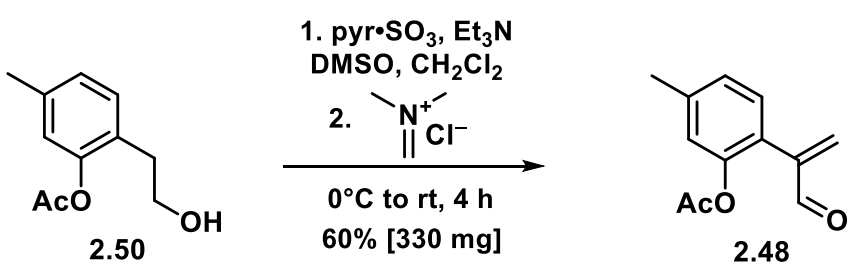

Alcohol 2.50 (530 mg, $2.73 \mathrm{mmol}$ ) was dissolved in anhydrous $\mathrm{CH}_{2} \mathrm{Cl}_{2}(10 \mathrm{~mL})$. $\mathrm{Et}_{3} \mathrm{~N}$ (2.38 mL, $17.1 \mathrm{mmol})$, and DMSO $(1.22 \mathrm{~mL}, 17.1 \mathrm{mmol})$ were added at rt. pyr $\bullet \mathrm{SO}_{3}$ $(1.09 \mathrm{~g}, 6.82 \mathrm{mmol})$ was added at $0{ }^{\circ} \mathrm{C}$ and the mixture was allowed to warm to $\mathrm{rt}$ and stirred for $1.5 \mathrm{~h}$. After complete consumption of the starting material, judged by TLC analysis $(1.5 \mathrm{~h})$, the Eschenmoser salt $(456 \mathrm{mg}, 4.91 \mathrm{mmol})$ was added at $\mathrm{rt}$ and the mixture was stirred for another $2.5 \mathrm{~h}$. The reaction was then quenched with sat. $a q$. $\mathrm{CuSO}_{4}(10 \mathrm{~mL})$ and extracted with ethyl acetate $(3 \times 20 \mathrm{~mL})$. The combined organic layers were washed with $1 \mathrm{M} a q . \mathrm{HCl}(30 \mathrm{~mL})$ and brine $(40 \mathrm{~mL})$ and dried over anhydrous $\mathrm{Na}_{2} \mathrm{SO}_{4}$. The solvent was removed under reduced pressure. Flash chromatography (60:40 petroleum spirit 40-60 / ethyl acetate) gave enal $\mathbf{2 . 4 8}$ as a paleyellow oil (337 mg, $1.63 \mathrm{mmol}, 60 \%$ yield).

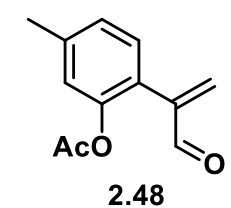

Rf 0.29 (95:5 to 70:30 petroleum ether 40-60 / ethyl acetate);

${ }^{1} \mathbf{H}$ NMR $\left(500 \mathrm{MHz}, \mathrm{CDCl}_{3}\right) \delta 9.70(\mathrm{~s}, 1 \mathrm{H}), 7.14(\mathrm{~d}, J=7.8 \mathrm{~Hz}, 1 \mathrm{H}), 7.08$ (ddd, $J=$ 7.8, 1.7, $0.8 \mathrm{~Hz}, 1 \mathrm{H}), 6.99-6.95(\mathrm{~m}, 1 \mathrm{H}), 6.44(\mathrm{~d}, J=0.9 \mathrm{~Hz}, 1 \mathrm{H}), 6.31(\mathrm{~d}, J=0.9 \mathrm{~Hz}$, 1H), 2.37 (s, 3H), 2.17 (s, 3H) ppm;

${ }^{13}$ C NMR (126 MHz, $\left.\mathrm{CDCl}_{3}\right) \delta 192.0,169.1,148.0,146.4,140.4,136.1,130.4,127.0$, 124.6, 123.4, 21.3, 21.0 ppm;

IR (film, $\mathrm{cm}^{-1}$ ) 2924, 2853, 1765, 1736, 1697, 1624;

HRMS $\left(\mathrm{ESI}^{+}\right) 227.0699$ (calculated $[\mathrm{M}+\mathrm{Na}]^{+}$227.0679). 

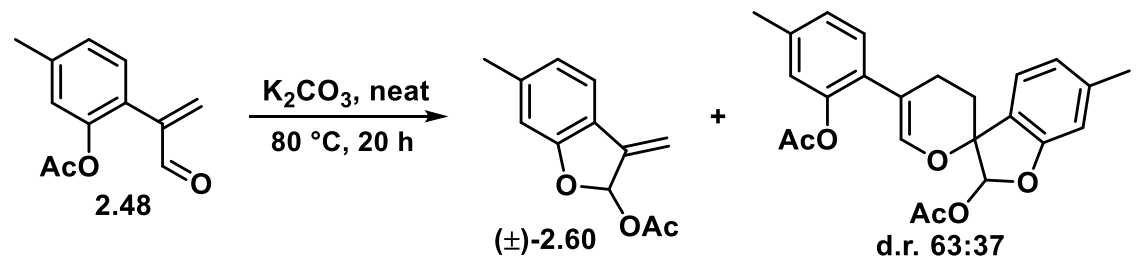

$( \pm)-2.61$

Enal 2.48 (39 mg, $0.19 \mathrm{mmol})$ was transferred in a round bottom flask. $\mathrm{K}_{2} \mathrm{CO}_{3}(47 \mathrm{mg}$, $0.34 \mathrm{mmol}$ ) was added to the neat enal. The mixture was warmed to $80{ }^{\circ} \mathrm{C}$ and stirred for $24 \mathrm{~h}$, then allowed to cool to $\mathrm{rt}$ and diluted with $\mathrm{CH}_{2} \mathrm{Cl}_{2}$. The suspension was filtered through a pad of celite and the solvent was removed under reduced pressure. Flash chromatography (80:20 petroleum spirit 40-60 / ethyl acetate) gave compound ( \pm )-2.60 as a pale-yellow oil ( $7 \mathrm{mg}, 32 \mu \mathrm{mol}, 17 \%$ yield) and compound ( \pm )-2.61 as a 63:37 diastereomeric mixture (pale brown oil, $5 \mathrm{mg}, 13 \mu \mathrm{mol}, 14 \%$ yield).

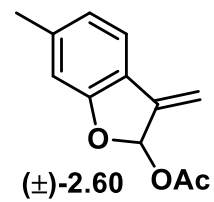

Rf 0.68 (60:40 petroleum spirit 40-60 / ethyl acetate);

${ }^{1} \mathbf{H}$ NMR $\left(500 \mathrm{MHz}, \mathrm{CDCl}_{3}\right) \delta 7.31(\mathrm{~d}, J=7.7 \mathrm{~Hz}, 1 \mathrm{H}), 7.02(\mathrm{t}, J=1.7 \mathrm{~Hz}, 1 \mathrm{H}), 6.81$ $(\mathrm{ddd}, J=7.7,1.6,0.8 \mathrm{~Hz}, 1 \mathrm{H}), 6.76-6.72(\mathrm{~m}, 1 \mathrm{H}), 5.65(\mathrm{~d}, J=1.9 \mathrm{~Hz}, 1 \mathrm{H}), 5.32(\mathrm{~d}, J$ $=1.4 \mathrm{~Hz}, 1 \mathrm{H}), 2.35(\mathrm{~s}, 3 \mathrm{H}), 2.14(\mathrm{~s}, 3 \mathrm{H}) \mathrm{ppm}$;

${ }^{13}$ C NMR (126 MHz, $\left.\mathrm{CDCl}_{3}\right) \delta 170.4,161.3,142.6,141.9,123.1,121.0,120.9,111.5$, 107.1, 98.2, 22.0, $21.3 \mathrm{ppm}$;

IR (film, $\mathrm{cm}^{-1}$ ) 2924, 2361, 1751, 1620, 1595;

HRMS $\left(\mathrm{ESI}^{+}\right) 227.0686$ (calculated $[\mathrm{M}+\mathrm{Na}]^{+}$287.0678). 


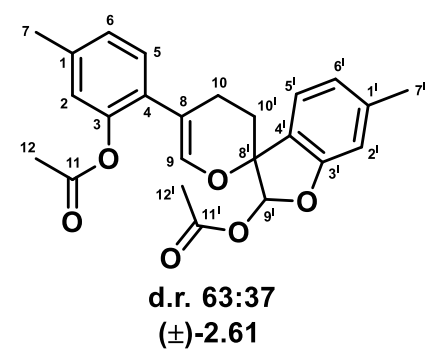

$\mathbf{R}_{\mathbf{f}}$ 0.59 (60:40 petroleum spirit 40-60 / ethyl acetate);

${ }^{1}$ H-NMR $\left(500 \mathrm{MHz}, \mathrm{CDCl}_{3}\right) \delta 7.25\left(\mathrm{~d}, J=7.7 \mathrm{~Hz}, 1 \mathrm{H}^{\dagger}, \mathrm{H} 5^{\prime}\right), 7.25\left(\mathrm{~d}, 7.5 \mathrm{~Hz}, 1 \mathrm{H}^{\ddagger}\right.$,

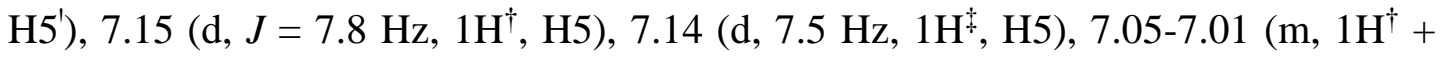
$\left.1 \mathrm{H}^{\ddagger}, \mathrm{H} 6\right), 6.89-6.84\left(\mathrm{~m}, 2 \mathrm{H}^{\dagger}+2 \mathrm{H}^{\ddagger}, \mathrm{H} 2+\mathrm{H}^{\prime}\right), 6.81-6.78\left(\mathrm{~m}, 1 \mathrm{H}^{\dagger}, \mathrm{H} 2\right), 6.75(\mathrm{~d}, J=$ $\left.1.3 \mathrm{~Hz}, 1 \mathrm{H}^{\ddagger}, \mathrm{H} 2^{\prime}\right), 6.66\left(\mathrm{~s}, 1 \mathrm{H}^{\ddagger}, \mathrm{H}^{\prime}\right), 6.55\left(\mathrm{~s}, 1 \mathrm{H}^{\dagger}, \mathrm{H}^{\prime}\right), 6.52-6.50\left(\mathrm{~m}, 1 \mathrm{H}^{\dagger}+1 \mathrm{H}^{\ddagger}, \mathrm{H} 9\right)$, 2.48 (tdd, $\left.J=10.6,3.9,2.4 \mathrm{~Hz}, 1 \mathrm{H}^{\dagger}, \mathrm{H} 10\right), 2.42-2.39$ (m, $\left.1 \mathrm{H}^{\dagger}, \mathrm{H} 10\right), 2.38-2.27$ (m,

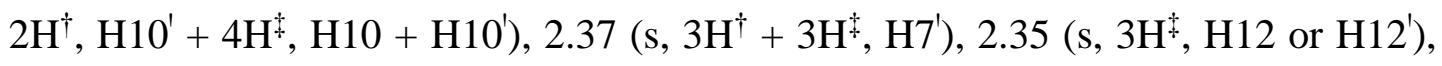

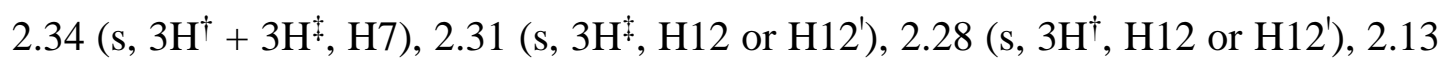
(s, $3 \mathrm{H}^{\dagger}, \mathrm{H} 12$ or $\left.\mathrm{H} 12^{\prime}\right)$ ppm;

${ }^{13} \mathrm{C}$-NMR $\left(126 \mathrm{MHz}, \mathrm{CDCl}_{3}\right) \delta 169.8^{\ddagger}\left(\mathrm{C} 11\right.$ or $\left.\mathrm{C} 11^{\prime}\right), 169.8\left(1 \mathrm{C}^{\dagger}+1 \mathrm{C}^{\ddagger}, \mathrm{C} 11\right.$ or $\left.\mathrm{C} 11^{\prime}\right)$, $169.5^{\dagger}\left(\mathrm{C} 11\right.$ or $\left.\mathrm{C} 11^{\prime}\right), 159.6^{\dagger}\left(\mathrm{C}^{\prime}\right), 157.4^{\dagger}\left(\mathrm{C} 3^{\prime}\right), 148.3^{\ddagger}(\mathrm{C} 3), 148.2^{\dagger}(\mathrm{C} 3), 142.3^{\dagger}\left(\mathrm{C} 1^{\prime}\right)$, $141.5^{\ddagger}\left(\mathrm{C} 1^{\prime}\right), 141.4\left(1 \mathrm{C}^{\dagger}+1 \mathrm{C}^{\ddagger}, \mathrm{C} 9\right), 138.3^{\dagger}(\mathrm{C} 1), 138.2^{\ddagger}(\mathrm{C} 1), 129.8\left(1 \mathrm{C}^{\dagger}+1 \mathrm{C}^{\ddagger}, \mathrm{C} 5\right)$,

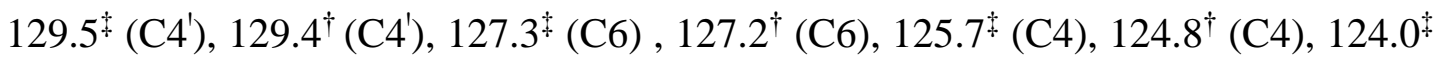
$\left(\mathrm{C}^{\prime}\right), 123.9^{\dagger}\left(\mathrm{C}^{\prime}\right), 123.5^{\ddagger}\left(\mathrm{C}^{\prime}\right), 123.4^{\ddagger}(\mathrm{C} 2), 123.4^{\dagger}\left(\mathrm{C}^{\prime}\right), 123.3^{\dagger}(\mathrm{C} 2), 111.9^{\dagger}\left(\mathrm{C} 2^{\prime}\right)$, 111.6 $6^{\ddagger}\left(\mathrm{C}^{\prime}\right), 110.6^{\dagger}(\mathrm{C} 8), 110.4^{\ddagger}(\mathrm{C} 8), 101.2^{\dagger}\left(\mathrm{C}^{\prime}\right), 100.8^{\ddagger}\left(\mathrm{C}^{\prime}\right), 83.2^{\dagger}\left(\mathrm{C}^{\prime}\right), 80.9^{\ddagger}$ $\left(\mathrm{C}^{\prime}\right), 24.6^{\dagger}\left(\mathrm{C} 10^{\prime}\right), 22.6\left(1 \mathrm{C}^{\dagger}+1 \mathrm{C}^{\ddagger}, \mathrm{C} 10\right), 21.9^{\dagger}\left(\mathrm{C}^{\prime}\right), 21.8^{\star}\left(\mathrm{C}^{\prime}\right), 21.4^{\star}\left(\mathrm{C} 10^{\prime}\right), 21.3^{\star}$ (C7), $21.2\left(1 \mathrm{C}^{\dagger}+1 \mathrm{C}^{\ddagger}, \mathrm{C} 12\right.$ or $\left.\mathrm{C} 12^{\prime}\right), 21.1^{\ddagger}\left(\mathrm{C} 12\right.$ or $\left.\mathrm{C} 12^{\prime}\right), 21.1^{\dagger}(\mathrm{C} 7), 21.0^{\dagger}(\mathrm{C} 12$ or C12') ppm;

IR (film, cm ${ }^{-1}$ ) 2924, 2359, 1755, 1647, 1628;

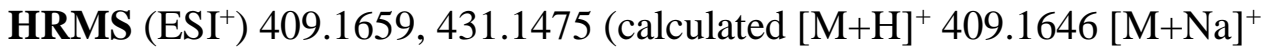
431.1465).

$\dagger$ Major diastereoisomer, $\$$ minor diastereoisomer. 

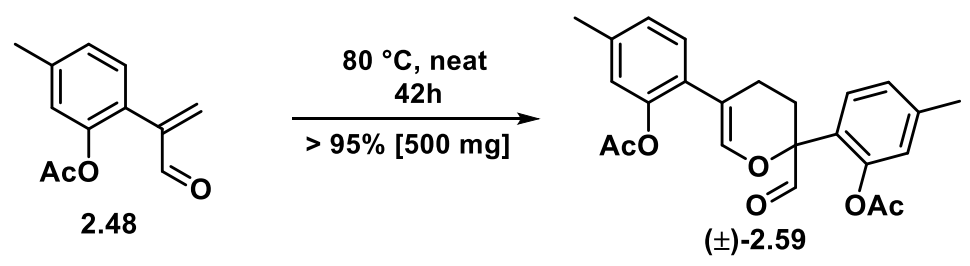

Enal 2.48 (518 $\mathrm{mg}, 2.54 \mathrm{mmol}$ ) was heated, neat, at $80{ }^{\circ} \mathrm{C}$ in a round bottom flask for $42 \mathrm{~h}$. Analysis of the crude ${ }^{1} \mathrm{H}$ NMR spectrum, with inclusion of an internal standard, indicated a $>95 \%$ yield of the acetyl-protected dimer $( \pm)-2.59$. The crude material was deemed sufficiently pure (see ${ }^{1} \mathrm{H}$ NMR spectrum on page 296) to be used directly in the next step.

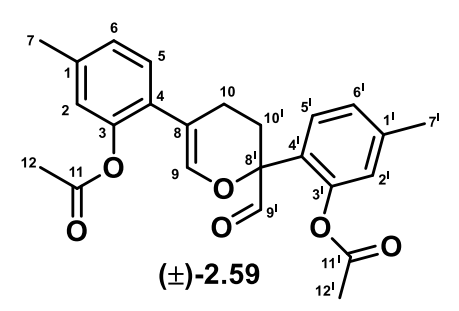

$\mathbf{R}_{\mathbf{f}} 0.36$ (70:30 petroleum spirit 40-60 / ethyl acetate);

${ }^{1} \mathbf{H}$ NMR $\left(500 \mathrm{MHz}, \mathrm{CDCl}_{3}\right) \delta 9.53(\mathrm{~d}, J=1.1 \mathrm{~Hz}, 1 \mathrm{H}, \mathrm{H} 9), 7.48(\mathrm{~d}, J=8.1 \mathrm{~Hz}, 1 \mathrm{H}$, H5'), 7.12 (dm, $J=8.0 \mathrm{~Hz}, 1 \mathrm{H}, \mathrm{H6}$ ), 7.07 (d, $J=7.8 \mathrm{~Hz}, 1 \mathrm{H}, \mathrm{H} 5), 7.00$ (dm, $J=7.8$ Hz, 1H, H6), 6.91-6.90 (m, 1H, H2'), 6.83-6.82 (m, 1H, H2), 6.69 (apparent t, $J=1.7$ Hz, 1H, H9), 2.55-2.45 (m, 1H, H10'), 2.41-2.37 (m, 1H, H10), 2.36 (s, 3H, H7 or H7'), 2.32 (s, 3H, H7 or H7'), 2.30 (s, 3H, H12 or H12'), 2.28-2.24 (m, 1H, H10'), 2.20-2.14 (m, 1H, H10), 2.17 (s, 3H, H12 or H12') ppm;

${ }^{13}$ C NMR (126 MHz, $\left.\mathrm{CDCl}_{3}\right): \delta 197.2\left(\mathrm{C}^{\prime}\right), 169.6\left(\mathrm{C} 11\right.$ or $\left.\mathrm{C} 11^{\prime}\right), 169.5\left(\mathrm{C} 11\right.$ or $\left.\mathrm{C} 11^{\prime}\right)$, 148.3 (C3), 147.8 (C3'), 141.0 (C9), 140.6 (C1'), 138.4 (C1), 129.7 (C5), 129.4 (C4), $127.9\left(\mathrm{C}^{\prime}\right), 127.4\left(\mathrm{C}^{\prime}\right), 127.1$ (C6), $126.0\left(\mathrm{C}^{\prime}\right), 124.8$ (C2'), $123.3(\mathrm{C} 2), 112.0(\mathrm{C} 8)$, $82.2\left(\mathrm{C}^{\prime}\right), 26.0$ (C10'), 22.0 (C10), 21.3 (C12 or C12'), 21.1 (C12 or C12'), 21.1 (C7 or $\mathrm{C}^{\prime}$ ), 21.0 (C7 or $\mathrm{C}^{\prime}$ ') ppm;

IR (film, $\mathrm{cm}^{-1}$ ) 2924, 1763, 1740, 1649, 1618;

HRMS $\left(\mathrm{ESI}^{+}\right) 409.1659$ (calculated $[\mathrm{M}+\mathrm{H}]^{+}$409.1646). 


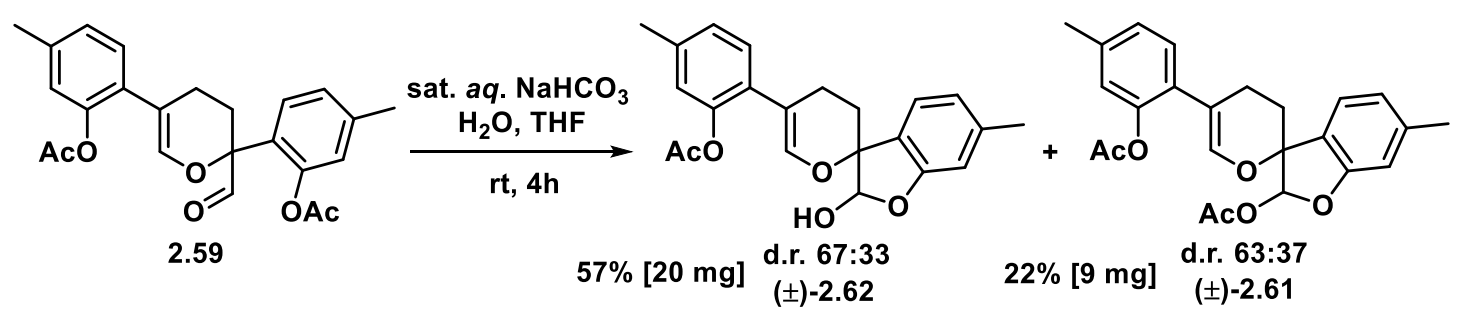

Dihydropyran ( \pm )-2.59 (39 mg, $94 \mu \mathrm{mol})$ was dissolved in THF (3 mL). Water (1 mL) and sat. $a q . \mathrm{NaHCO}_{3}(1.5 \mathrm{~mL})$ were then added and the mixture was stirred at $\mathrm{rt}$ until complete consumption of the starting material, judged by TLC analysis $(4 \mathrm{~h})$. The reaction was then quenched adding sat. $a q . \mathrm{NH}_{4} \mathrm{Cl}(2 \mathrm{~mL})$ and the mixture was extracted with $\mathrm{CH}_{2} \mathrm{Cl}_{2}(3 \times 5 \mathrm{~mL})$. The combined organic layers were dried over anhydrous $\mathrm{Na}_{2} \mathrm{SO}_{4}$ and the solvent was removed under reduced pressure. Flash chromatography $\left(100 \% \mathrm{CH}_{2} \mathrm{Cl}_{2}\right)$ afforded lactol $( \pm)-\mathbf{2 . 6 2}$ as a 67:33 mixture of diastereoisomers (pale yellow oil, $19 \mathrm{mg}, 53 \mu \mathrm{mol}, 57 \%$ yield), along with acetal ( \pm -2.61 in a 63:37 diastereomeric ratio (pale yellow oil, $9 \mathrm{mg}, 21 \mu \mathrm{mol}, 22 \%$ yield).

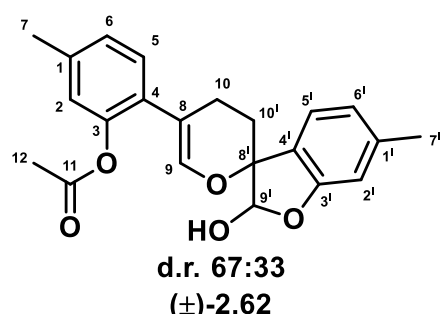

$\mathbf{R}_{\mathbf{f}} 0.11\left(100 \% \mathrm{CH}_{2} \mathrm{Cl}_{2}\right)$.

${ }^{1} \mathbf{H}$ NMR $\left(500 \mathrm{MHz}, \mathrm{CDCl}_{3}\right) \delta 7.23\left(\mathrm{~d}, J=7.6 \mathrm{~Hz}, 1 \mathrm{H}^{\dagger}, \mathrm{H} 5^{\prime}\right), 7.23\left(\mathrm{~d}, J=7.5+1 \mathrm{H}^{\ddagger}\right.$, $\left.\mathrm{H}^{\prime}\right), 7.18\left(\mathrm{~d}, J=7.8 \mathrm{~Hz}, 1 \mathrm{H}^{\ddagger}, \mathrm{H} 5\right), 7.16$ (d, $\left.J=7.8 \mathrm{~Hz}, 1 \mathrm{H}^{\dagger}, \mathrm{H} 5\right), 7.05(\mathrm{dm}, J=7.7 \mathrm{~Hz}$, $\left.1 \mathrm{H}^{\dagger}, \mathrm{H} 6\right), 7.07-7.01\left(\mathrm{~m}, 1 \mathrm{H}^{\ddagger}, \mathrm{H} 6\right), 6.87-6.87\left(\mathrm{~m}, 1 \mathrm{H}^{\dagger}, \mathrm{H} 2\right), 6.86-6.86\left(\mathrm{~m}, 1 \mathrm{H}^{\ddagger}, \mathrm{H} 2\right)$, $6.83\left(\mathrm{dm}, J=7.6 \mathrm{~Hz}, 1 \mathrm{H}^{\ddagger}, \mathrm{H}^{\prime}\right), 6.79\left(\mathrm{dm}, J=7.6 \mathrm{~Hz}, 1 \mathrm{H}^{\dagger}, \mathrm{H}^{\prime}\right), 6.76-6.75\left(\mathrm{~m}, 1 \mathrm{H}^{\ddagger}\right.$, $\left.\mathrm{H} 2^{\prime}\right), 6.72-6.71\left(\mathrm{~m}, 1 \mathrm{H}^{\dagger}, \mathrm{H} 2{ }^{\prime}\right), 6.57-6.55\left(\mathrm{~m}, 1 \mathrm{H}^{\ddagger}+1 \mathrm{H}^{\dagger}, \mathrm{H} 9\right), 5.76\left(\mathrm{~d}, J=5.3 \mathrm{~Hz}, 1 \mathrm{H}^{\ddagger}\right.$, $\left.\mathrm{H}^{\prime}\right), 5.52\left(\mathrm{~d}, J=11.8 \mathrm{~Hz}, 1 \mathrm{H}^{\dagger}, \mathrm{H}^{\prime}\right), 4.08$ (d, $J=11.8 \mathrm{~Hz}, 1 \mathrm{H}^{\dagger}, \mathrm{OH}$ at $\left.\mathrm{C}^{\prime}\right), 3.14$ (d, $J$ $=5.3 \mathrm{~Hz}, 1 \mathrm{H}^{\ddagger}, \mathrm{OH}$ at $\left.\mathrm{C}^{\prime}\right), 2.73-2.63\left(\mathrm{~m}, 1 \mathrm{H}^{\ddagger}, \mathrm{H} 10\right), 2.57$ (tdd, $J=6.1,5.0,1.7 \mathrm{~Hz}$,

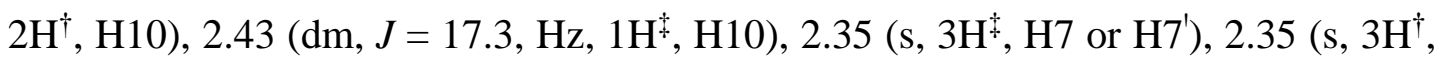
$\mathrm{H} 7$ or $\mathrm{H}^{\prime}$ ), 2.34 (s, $3 \mathrm{H}^{\dagger}+3 \mathrm{H}^{\ddagger}, \mathrm{H} 7$ or $\left.\mathrm{H}^{\prime}\right), 2.33-2.29$ (m, $\left.2 \mathrm{H}^{\ddagger}, \mathrm{H} 10^{\prime}\right), 2.28$ (s, $3 \mathrm{H}^{\ddagger}$,

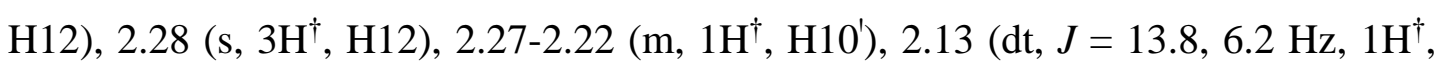
H10') ppm; 
${ }^{13} \mathrm{C}$ NMR $\left(126 \mathrm{MHz}, \mathrm{CDCl}_{3}\right) \delta 169.9^{\dagger}(\mathrm{C} 11), 169.8^{\ddagger}(\mathrm{C} 11), 159.3^{\ddagger}\left(\mathrm{C}^{\prime}\right), 157.7^{\dagger}\left(\mathrm{C}^{\prime}\right)$, $148.3^{\dagger}(\mathrm{C} 3), 148.1^{\ddagger}(\mathrm{C} 3), 142.0^{\ddagger}\left(\mathrm{C}^{\prime}\right), 141.9^{\dagger}\left(\mathrm{C}^{\prime}\right), 141.6^{\ddagger}(\mathrm{C} 9), 141.1^{\dagger}(\mathrm{C} 9), 138.4^{\dagger}$ (C1), 138.0*(C1), $129.7\left(1 \mathrm{C}^{\dagger}+1 \mathrm{C}^{\ddagger}, \mathrm{C} 5\right), 129.5^{\ddagger}(\mathrm{C} 4), 129.4^{\dagger}(\mathrm{C} 4), 127.2^{\dagger}(\mathrm{C} 6), 127.2^{\star}$ (C6), 125.3 $3^{\ddagger}\left(\mathrm{C}^{\prime}\right), 125.1^{\dagger}\left(\mathrm{C}^{\prime}\right), 124.4^{\dagger}\left(\mathrm{C}^{\prime}\right), 124.2^{\ddagger}\left(\mathrm{C}^{\prime}\right), 123.4^{\dagger}(\mathrm{C} 2), 123.4^{\ddagger}(\mathrm{C} 2)$, $122.8^{\ddagger}\left(\mathrm{C}^{\prime}\right), 122.5^{\dagger}\left(\mathrm{C}^{\prime}\right), 111.7^{\star}\left(\mathrm{C}^{\prime}\right), 111.5^{\dagger}\left(\mathrm{C}^{\prime}\right), 111.1^{\ddagger}(\mathrm{C} 8), 111.0^{\dagger}(\mathrm{C} 8), 105.0^{\dagger}$ (C9'), 104.7 $7^{\ddagger}\left(\mathrm{C}^{\prime}\right), 83.8^{\ddagger}\left(\mathrm{C}^{\prime}\right), 79.7^{\dagger}\left(\mathrm{C}^{\prime}\right), 29.3^{\dagger}\left(\mathrm{C}^{\prime} 0^{\prime}\right), 24.3^{\ddagger}\left(\mathrm{C} 10^{\prime}\right), 22.4^{\ddagger}(\mathrm{C} 10), 21.9^{\ddagger}$ (C7 or $\left.\mathrm{C}^{\prime}\right), 21.9^{\dagger}\left(\mathrm{C} 7\right.$ or $\left.\mathrm{C}^{\prime}\right), 21.7^{\dagger}(\mathrm{C} 10), 21.3^{\star}\left(\mathrm{C} 7\right.$ or $\left.\mathrm{C} 7^{\prime}\right), 21.2^{\dagger}\left(\mathrm{C} 7\right.$ or $\left.\mathrm{C} 7^{\prime}\right), 21.1^{\dagger}$ (C12), 21.1 (C12) ppm;

IR (film, cm cm $^{-1} 3433$ (broad), 2922, 1755, 1647, 1624;

HRMS $\left(\mathrm{ESI}^{+}\right) 389.1371$ (calculated $[\mathrm{M}+\mathrm{Na}]^{+}$389.1365).

$\uparrow$ Major diastereoisomer, $\ddagger$ minor diastereoisomer. 


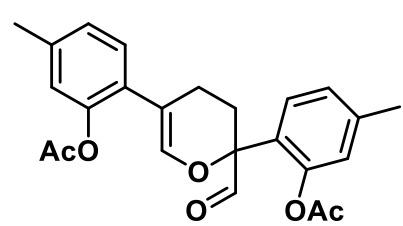

$( \pm)-2.59$

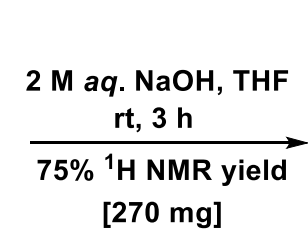

[270 mg]

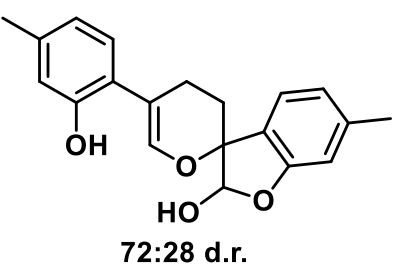

$( \pm)-2.9$

Crude acetyl-protected dimer $(\mathbf{\pm})-\mathbf{2 . 5 9}(342 \mathrm{mg}, 0.838 \mathrm{mmol})$ was dissolved in THF $(18 \mathrm{~mL}) .2 \mathrm{M} \mathrm{aq} . \mathrm{NaOH}(9.0 \mathrm{~mL})$ was added and the mixture was stirred at $\mathrm{rt}$ for $3 \mathrm{~h}$. After that time the reaction mixture was neutralised with sat. $a q . \mathrm{NH}_{4} \mathrm{Cl}$, the THF was removed under reduced pressure and the remaining aqueous layer was extracted with $\mathrm{CH}_{2} \mathrm{Cl}_{2}(3 \times 10 \mathrm{~mL})$. The combined organic layers were dried over anhydrous $\mathrm{Na}_{2} \mathrm{SO}_{4}$ and the solvent was removed under reduced pressure. Analysis of the crude ${ }^{1} \mathrm{H}$ NMR spectrum, with inclusion of an internal standard, indicated a 75\% yield (over two steps from enal 2.48) for a 78:22 diastereomeric mixture of lactols ( \pm )-2.9. The crude material was characterised and used directly in the next step (see ${ }^{1} \mathrm{H}$ and ${ }^{13} \mathrm{C}$ NMR spectra on pages 300 and 301).

Note: d.r. of lactol ( \pm )-2.9 epimers varied with solvent used for the NMR experiments (72:28 in $\mathrm{CDCl}_{3}$ and 78:22 in $\left.\left(\mathrm{CD}_{3}\right)_{2} \mathrm{CO}\right)$.

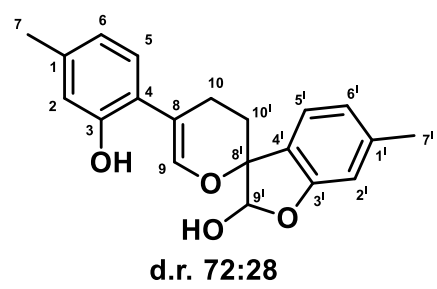

$( \pm)-2.9$

$\mathbf{R}_{\mathbf{f}}$ 0.11 (95:5 petroleum spirit 40-60 / diethyl ether);

${ }^{1} \mathbf{H}$ NMR $\left(500 \mathrm{MHz}, \mathrm{CDCl}_{3}\right) \delta 7.24\left(\mathrm{~d}, J=7.7 \mathrm{~Hz}, 1 \mathrm{H}^{\dagger}+1 \mathrm{H}^{\ddagger}, \mathrm{H} 5^{\prime}\right), 7.04(\mathrm{~d}, J=8.2$ $\left.\mathrm{Hz}, 1 \mathrm{H}^{\ddagger}, \mathrm{H} 5\right), 7.02\left(\mathrm{~d}, J=8.1 \mathrm{~Hz}, 1 \mathrm{H}^{\dagger}, \mathrm{H} 5\right), 6.82\left(\mathrm{dm}, J=7.6 \mathrm{~Hz}, 1 \mathrm{H}^{\ddagger}, \mathrm{H} 6^{\prime}\right), 6.79$ (dm, $\left.J=7.6 \mathrm{~Hz}, 1 \mathrm{H}^{\dagger}, \mathrm{H}^{\prime}\right), 6.76-6.69\left(\mathrm{~m}, 3 \mathrm{H}^{\dagger}+3 \mathrm{H}^{\ddagger}, \mathrm{H} 2, \mathrm{H} 2{ }^{\prime}, \mathrm{H} 6\right.$ '), 6.65 (apparent t, $J=1.7$

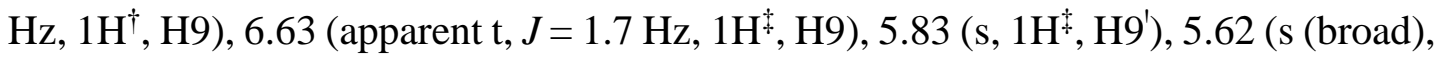

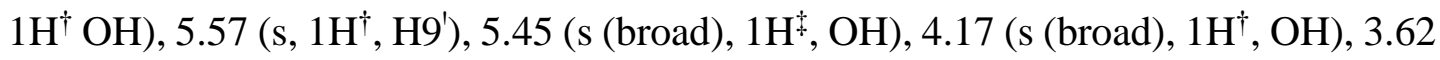

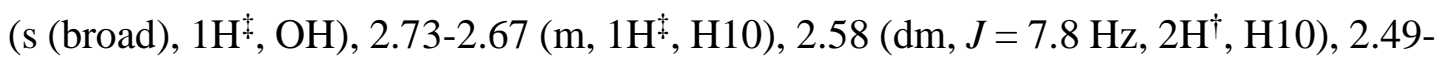

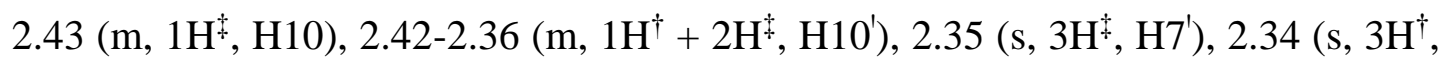
$\left.\mathrm{H}^{\prime}\right), 2.30\left(\mathrm{~s}, 3 \mathrm{H}^{\dagger}+3 \mathrm{H}^{\ddagger}, \mathrm{H} 7\right), 2.21-2.10\left(\mathrm{~m}, 1 \mathrm{H}^{\dagger}, \mathrm{H} 10^{\prime}\right) \mathrm{ppm}$; 
${ }^{13}$ C NMR $\left(126 \mathrm{MHz}, \mathrm{CDCl}_{3}\right) \delta 159.4^{\ddagger}\left(\mathrm{C}^{\prime}\right), 157.6^{\dagger}\left(\mathrm{C}^{\prime}\right), 153.4^{\dagger}(\mathrm{C} 3), 152.8^{\ddagger}(\mathrm{C} 3)$, $142.0\left(1 \mathrm{C}^{\dagger}+1 \mathrm{C}^{\dagger}, \mathrm{C}^{\prime}\right), 141.9^{\dagger}(\mathrm{C} 9), 141.8^{\ddagger}(\mathrm{C} 9), 138.9^{\dagger}(\mathrm{C} 7), 138.5^{\ddagger}(\mathrm{C} 7), 129.3^{\dagger}(\mathrm{C} 5)$, 129.2 $2^{\ddagger}(\mathrm{C} 5), 125.3^{\ddagger}\left(\mathrm{C}^{\prime}\right), 124.9^{\dagger}\left(\mathrm{C}^{\prime}\right), 124.3^{\dagger}\left(\mathrm{C}^{\prime}\right), 124.2^{\ddagger}\left(\mathrm{C}^{\prime}\right), 122.7^{\ddagger}(\mathrm{C} 4), 122.6$

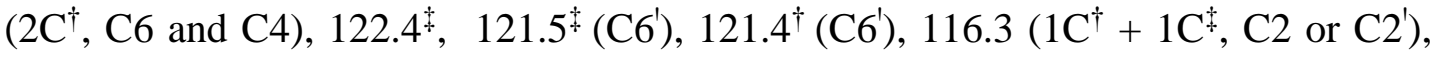
$111.7^{\ddagger}\left(\mathrm{C} 2\right.$ or $\left.\mathrm{C}^{\prime}\right), 111.4^{\dagger}\left(\mathrm{C} 2\right.$ or $\left.\mathrm{C} 2^{\prime}\right), 110.8^{\star}(\mathrm{C} 8), 110.7^{\dagger}(\mathrm{C} 8), 105.2^{\dagger}\left(\mathrm{C}^{\prime}\right), 104.8^{\star}$

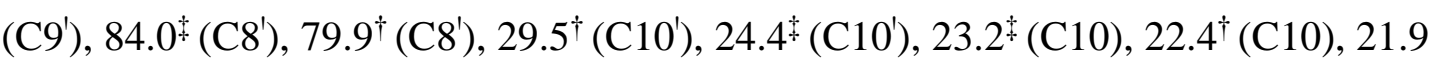
$\left(1 \mathrm{C}^{\dagger}+1 \mathrm{C}^{\dagger},\left(\mathrm{C}^{\prime}\right), 21.3^{\dagger}(\mathrm{C} 7), 21.2^{\ddagger}(\mathrm{C} 7) \mathrm{ppm} ;\right.$

IR (film, $\mathrm{cm}^{-1}$ ) 3345, 2922, 2853, 1667, 1622, 1599, 1501;

HRMS $\left(\mathrm{ESI}^{+}\right) 347.1268$ (calculated $[\mathrm{M}+\mathrm{Na}]^{+}$347.1254).

$\uparrow$ Major diastereoisomer; $\$$ minor diastereoisomer. 


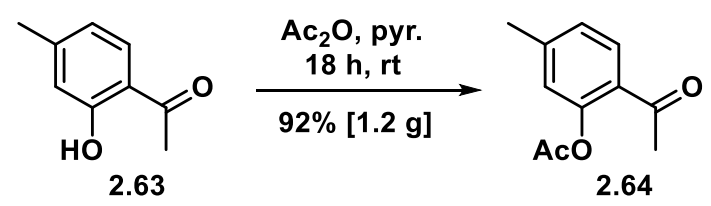

Phenol 2.63 (1.08 g, $6.98 \mathrm{mmol})$ was dissolved in anhydrous pyridine (12 mL). Acetic anhydride $(774 \mu \mathrm{L}, 8.03 \mathrm{mmol})$ was added and the mixture was stirred at rt until complete consumption of the starting material, judged by TLC analysis (18 h). The mixture was then diluted with $\mathrm{CH}_{2} \mathrm{Cl}_{2}(50 \mathrm{~mL})$ and washed with $1 \mathrm{M}$ aq. $\mathrm{HCl}(2 \times 50$ $\mathrm{mL}$ ). The organic layer was dried over anhydrous $\mathrm{Na}_{2} \mathrm{SO}_{4}$ and the solvent was removed under reduced pressure. Flash chromatography (80:20 petroleum spirit 40-60 / ethyl acetate) afforded compound $\mathbf{2 . 6 4}$ as a white solid (1.23 g, 6.39 mmol, 92\% yield).

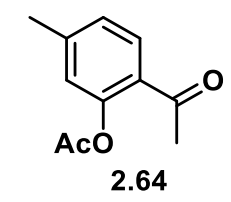

$\mathbf{R}_{\mathbf{f}} 0.39$ (70:30 petroleum ether 40-60 / ethyl acetate);

${ }^{1} \mathbf{H}$ NMR $\left(500 \mathrm{MHz}, \mathrm{CDCl}_{3}\right) \delta 7.73(\mathrm{~d}, J=7.9 \mathrm{~Hz}, 1 \mathrm{H}), 7.13-7.10(\mathrm{~m}, 1 \mathrm{H}), 6.91(\mathrm{~d}, J$ $=1.6 \mathrm{~Hz}, 1 \mathrm{H}), 2.52(\mathrm{~s}, 3 \mathrm{H}), 2.39(\mathrm{~s}, 3 \mathrm{H}), 2.34(\mathrm{~s}, 3 \mathrm{H}) \mathrm{ppm}$;

${ }^{13}$ C NMR (126 MHz, $\left.\mathrm{CDCl}_{3}\right) \delta 197.1,169.8,149.4,145.0,130.7,127.8,126.9,124.5$, 29.3, 21.5, 21.3 ppm;

IR (film, $\mathrm{cm}^{-1}$ ) 1765, 1722, 1682, 1614;

HRMS $\left(\mathrm{ESI}^{+}\right) 215.0769$ (calculated $\left.[\mathrm{M}+\mathrm{Na}]^{+} 215.0679\right)$;

m.p. $38-39{ }^{\circ} \mathrm{C}\left(\mathrm{CH}_{2} \mathrm{Cl}_{2}\right)$. 

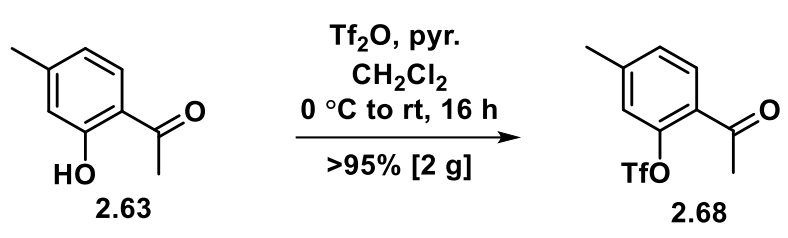

Compound 2.68 was prepared using a modified literature procedure. ${ }^{162}$

Phenol 2.63 (1.08 g, $7.42 \mathrm{mmol}$ ) was dissolved in anhydrous $\mathrm{CH}_{2} \mathrm{Cl}_{2}(20 \mathrm{~mL})$, then anhydrous pyridine $(900 \mu \mathrm{L}, 11.3 \mathrm{mmol})$ was added. The reaction mixture was cooled to $0{ }^{\circ} \mathrm{C}$, then triflic anhydride $(1.50 \mathrm{~mL}, 8.90 \mathrm{mmol})$ was added dropwise and the mixture was allowed to warm to $\mathrm{rt}$ and stirred until complete consumption of the starting material, judged by TLC analysis $(16 \mathrm{~h})$. The reaction was then quenched with $1 \mathrm{M}$ aq. $\mathrm{HCl}(20 \mathrm{~mL})$, the phases were separated and the organic layer was washed with sat. aq. $\mathrm{NaHCO}_{3}(20 \mathrm{~mL})$ and brine $(20 \mathrm{~mL})$. Removal of the solvent under reduced pressure afforded triflate $\mathbf{2 . 6 8}$ as a white solid $(2.08 \mathrm{~g}, 7.37 \mathrm{mmol}$, >95\% yield). The crude material was deemed sufficiently pure (see ${ }^{1} \mathrm{H}$ and ${ }^{13} \mathrm{C}$ NMR spectra on pages 304 and 305) to be used directly in the next step.

All data for compound $\mathbf{2 . 6 8}$ matched literature values. ${ }^{162}$

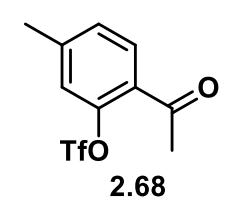

$\mathbf{R}_{\mathbf{f}}$ 0.19 (90:10 petroleum ether 40-60 / ethyl acetate);

${ }^{1} \mathbf{H}$ NMR $\left(500 \mathrm{MHz}, \mathrm{CDCl}_{3}\right) \delta 7.72(\mathrm{~d}, J=7.9 \mathrm{~Hz}, 1 \mathrm{H}), 7.28-7.25(\mathrm{~m}, 1 \mathrm{H}), 7.11(\mathrm{~s}$, $1 \mathrm{H}), 2.59$ (s, 3H), 2.43 (s, 3H) ppm;

${ }^{13}$ C NMR (126 MHz, $\left.\mathrm{CDCl}_{3}\right) \delta 196.3,147.0,145.6,131.0,129.3,129.2,123.3,118.7$ $\left(\mathrm{q}, J_{\mathrm{C}-\mathrm{F}}=320.6 \mathrm{~Hz}\right), 29.4,21.5 \mathrm{ppm}$. 

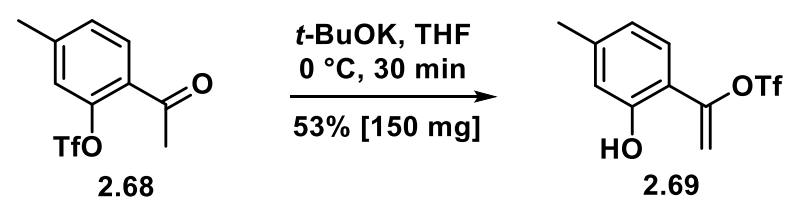

Compound 2.69 was prepared using a modified literature procedure. ${ }^{96}$

Ketone 2.68 (282 mg, $1.0 \mathrm{mmol})$ was dissolved in anhydrous THF (5 mL) at $0{ }^{\circ} \mathrm{C}$. Potassium tert-butoxide (266 mg, $2.20 \mathrm{mmol}$ ) was added as a solid. The mixture was stirred at $0{ }^{\circ} \mathrm{C}$ until complete consumption of the starting material, judged by TLC analysis (30 min). Then the reaction was quenched by adding $1 \mathrm{M} \mathrm{aq.} \mathrm{HCl}(2.5 \mathrm{~mL})$, the mixture was extracted with ethyl acetate $(3 \times 10 \mathrm{~mL})$ and the combined organic layers were washed with sat. aq. $\mathrm{NaHCO}_{3}(20 \mathrm{~mL})$ and brine $(20 \mathrm{~mL})$. The organic layer was eluted through a cotton plug and the solvent was removed under reduced pressure. The residue was dissolved in $\mathrm{CH}_{2} \mathrm{Cl}_{2}$ and filtered. The filtrate was concentrated under vacuum to afford compound 2.69 as a colourless solid (148 $\mathrm{mg}$, $0.52 \mathrm{mmol}, 53 \%$ yield). The crude material was deemed sufficiently pure (see ${ }^{1} \mathrm{H}$ and ${ }^{13} \mathrm{C}$ NMR spectra on pages 306 and 307$)$ to be characterised without further purification.

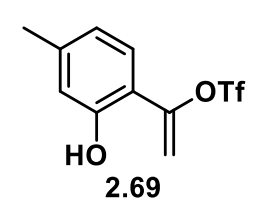

$\mathbf{R}_{\mathbf{f}} 0.42$ (60:40 petroleum ether 40-60 / ethyl acetate);

${ }^{1}$ H NMR $\left(500 \mathrm{MHz}, \mathrm{CDCl}_{3}\right) \delta 11.44(\mathrm{~s}, 1 \mathrm{H}), 7.52(\mathrm{~d}, J=8.3 \mathrm{~Hz}, 1 \mathrm{H}), 6.89-6.86(\mathrm{~d}, J$ $=1.7 \mathrm{~Hz}, 1 \mathrm{H}), 6.82(\mathrm{dd}, J=8.3,1.7 \mathrm{~Hz}, 1 \mathrm{H}), 4.79(\mathrm{~s}, 2 \mathrm{H}), 2.40(\mathrm{~s}, 3 \mathrm{H}) \mathrm{ppm}$;

${ }^{13} \mathrm{C} \mathrm{NMR}\left(126 \mathrm{MHz}, \mathrm{CDCl}_{3}\right) \delta 188.6,163.9,151.4,130.8,121.6,119.4$ (q, $J_{\mathrm{C}-\mathrm{F}}=327$ $\mathrm{Hz}), 119.3,117.1,57.0,22.5 \mathrm{ppm}$;

IR (film, cm cm $^{-1} 3117$ (broad), 2994, 2934, 2920, 1626;

HRMS $\left(\mathrm{ESI}^{+}\right)$283.0254, 305.0072 (calculated $[\mathrm{M}+\mathrm{H}]^{+}$283.0246, $[\mathrm{M}+\mathrm{Na}]^{+}$ 305.0066);

m.p. $118-119{ }^{\circ} \mathrm{C}\left(\mathrm{CH}_{2} \mathrm{Cl}_{2}\right)$. 


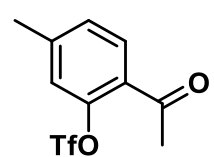

2.68

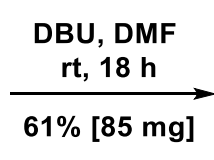

$61 \%$ [85 mg]

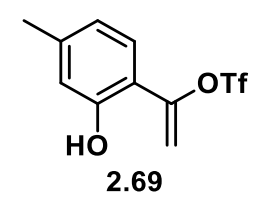

2.69

Compound 2.69 was prepared using a modified literature procedure. ${ }^{96}$

Ketone 2.68 (141 mg, $0.50 \mathrm{mmol}$ ) was dissolved in anhydrous DMF (5 mL) at rt. DBU $(150 \mu \mathrm{L}, 1.0 \mathrm{mmol})$ was then added dropwise. The reaction was stirred at $\mathrm{rt}$ until complete consumption of the starting material, judged by TLC analysis (18 h). Then the reaction was quenched by adding $1 \mathrm{M} a q . \mathrm{HCl}(13 \mathrm{~mL})$. The mixture was extracted with ethyl acetate $(3 \times 15 \mathrm{~mL})$ and the combined organic layers were washed with sat. $a q . \mathrm{NaHCO}_{3}(15 \mathrm{~mL})$, water $(15 \mathrm{~mL})$ and brine $(10 \mathrm{~mL})$. The organic layer was eluted through a cotton plug and the solvent was removed under reduced pressure. The residue was dissolved in $\mathrm{CH}_{2} \mathrm{Cl}_{2}$ and filtered. The filtrate was concentrated under vacuum to afford compound $\mathbf{2 . 6 9}$ as a colourless solid ( $85 \mathrm{mg}, 0.30 \mathrm{mmol}, 61 \%$ yield). 

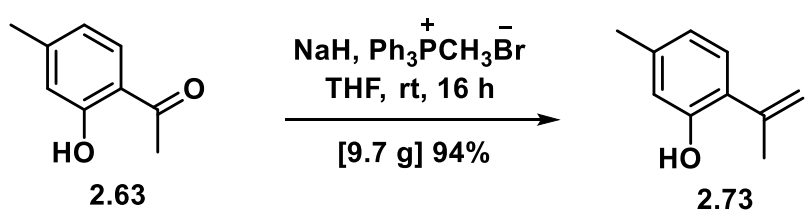

Compound 2.73 was prepared using a modified literature procedure. ${ }^{163}$

$\mathrm{NaH}(60 \%$ dispersion in mineral oil, $11.1 \mathrm{~g}, 280 \mathrm{mmol})$ was dissolved in anhydrous THF $(120 \mathrm{~mL})$. The solution was cooled to $0{ }^{\circ} \mathrm{C}$ then $\mathrm{Ph}_{3} \mathrm{PCH}_{3} \cdot \mathrm{Br}(49.9 \mathrm{~g}, 140 \mathrm{mmol})$ was added slowly as a solid. The mixture was stirred at $0{ }^{\circ} \mathrm{C}$ for $1 \mathrm{~h}$, then ketone $\mathbf{2 . 6 3}$ (10.8 g, $69.8 \mathrm{mmol}$ ) was slowly added, using a syringe pump over $45 \mathrm{~min}$. The mixture was then allowed to warm to $\mathrm{rt}$ and stirred for another $15 \mathrm{~h}$. The reaction was then quenched with $1 \mathrm{M} a q$. $\mathrm{HCl}(80 \mathrm{ml})$ and the mixture was extracted with $\mathrm{CH}_{2} \mathrm{Cl}_{2}(3 \times$ $100 \mathrm{~mL}$ ). The combined organic layers were dried over anhydrous $\mathrm{Na}_{2} \mathrm{SO}_{4}$ and the solvent was removed under reduced pressure. Flash chromatography (90:10 petroleum spirit 40-60 / ethyl acetate) gave compound 2.73 as a colourless oil (9.73 g, $65.7 \mathrm{mmol}$, $94 \%$ yield). All data for compound $\mathbf{2 . 7 3}$ matched the literature values. ${ }^{163}$

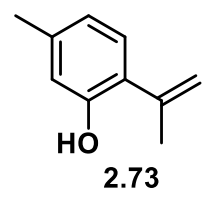

$\mathbf{R}_{\mathbf{f}} 0.44\left(100 \% \mathrm{CH}_{2} \mathrm{Cl}_{2}\right)$;

${ }^{1}$ H NMR $\left(500 \mathrm{MHz}, \mathrm{CDCl}_{3}\right) 7.03(\mathrm{~d}, J=7.7 \mathrm{~Hz}, 1 \mathrm{H}), 6.76-6.75(\mathrm{~m}, 1 \mathrm{H}), 6.72(\mathrm{dm}, J$ $=7.7 \mathrm{~Hz}, 1 \mathrm{H}), 5.64(\mathrm{~s}, 1 \mathrm{H}), 5.38$ (apparent qn, $J=1.6 \mathrm{~Hz}, 1 \mathrm{H}), 5.12(\mathrm{dd}, J=1.9,1.0$ $\mathrm{Hz}, 1 \mathrm{H}), 2.31$ (s, 3H), 2.11-2.10 (m, 3H) ppm;

${ }^{13}$ C NMR (126 MHz, $\left.\mathrm{CDCl}_{3}\right) \delta 115.9,142.3,139.0,127.7,126.0,121.1,116.3,115.4$, 24.5, $21.3 \mathrm{ppm}$. 


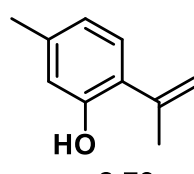

2.73

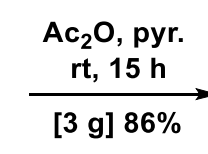

[3 g] $86 \%$

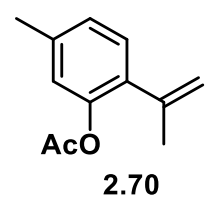

2.70

Compound 2.70 was prepared using a modified literature procedure. ${ }^{101}$

Phenol 2.73 (2.75 g, $18.6 \mathrm{mmol})$ was dissolved in anhydrous pyridine (20 mL). Acetic anhydride (2.06 mL, $21.4 \mathrm{mmol})$ was added and the mixture was stirred at rt for $15 \mathrm{~h}$. The reaction mixture was then diluted with $\mathrm{CH}_{2} \mathrm{Cl}_{2}(50 \mathrm{~mL})$ and washed with $1 \mathrm{M} a q$. $\mathrm{HCl}(2 \times 40 \mathrm{~mL})$. The organic layer was dried over anhydrous $\mathrm{Na}_{2} \mathrm{SO}_{4}$ and the solvent was removed under reduced pressure. Flash chromatography (95:5 petroleum ether 4060 / ethyl acetate) gave protected phenol $\mathbf{2 . 7 0}$ as a colourless oil (3.02 g, $15.9 \mathrm{mmol}$, $86 \%$ yield). All data for compound $\mathbf{2 . 7 0}$ matched literature values. ${ }^{101}$

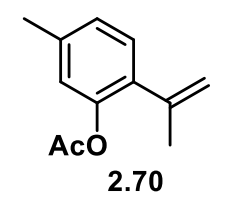

$\mathbf{R}_{\mathbf{f}} 0.61$ (80:20 petroleum ether 40-60 / ethyl acetate);

${ }^{1} \mathbf{H}$ NMR $\left(500 \mathrm{MHz}, \mathrm{CDCl}_{3}\right) \delta 7.17(\mathrm{~d}, J=7.8 \mathrm{~Hz}, 1 \mathrm{H}), 7.02(\mathrm{dm}, J=7.7 \mathrm{~Hz}, 1 \mathrm{H})$, 6.85-6.84 (m, 1H), $5.15(\mathrm{dq}, J=2.1,1.5 \mathrm{~Hz}, 1 \mathrm{H}), 5.01(\mathrm{dq}, J=1.9,0.9 \mathrm{~Hz}, 1 \mathrm{H}), 2.34-$ $2.33(\mathrm{~m}, 3 \mathrm{H}), 2.25(\mathrm{~s}, 3 \mathrm{H}), 2.05(\mathrm{dd}, J=1.5,0.9 \mathrm{~Hz}, 3 \mathrm{H}) \mathrm{ppm}$;

${ }^{13} \mathrm{C} \mathrm{NMR}\left(126 \mathrm{MHz}, \mathrm{CDCl}_{3}\right): \delta 169.7,147.5,141.7,138.4,133.4,129.1,127.0,123.2$, $115.7,23.5,21.2,21.1 \mathrm{ppm}$. 


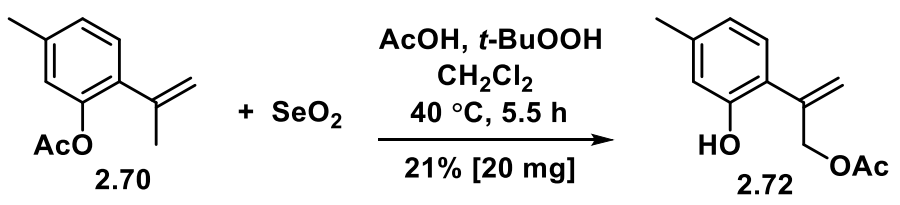

A solution of alkene $\mathbf{2 . 7 0}(104 \mathrm{mg}, 0.55 \mathrm{mmol})$ in $\mathrm{CH}_{2} \mathrm{Cl}_{2}(0.7 \mathrm{~mL})$ was added to a solution of $\mathrm{SeO}_{2}(9 \mathrm{mg}, 77 \mu \mathrm{mol})$, acetic acid $(4 \mu \mathrm{L}, 77 \mu \mathrm{mol})$ and tert-butyl hydroperoxide (70\% w/w in $\left.\mathrm{H}_{2} \mathrm{O}, 155 \mu \mathrm{L}, 1.2 \mathrm{mmol}\right)$ in $\mathrm{CH}_{2} \mathrm{Cl}_{2}(2 \mathrm{~mL})$. The mixture was heated to $40{ }^{\circ} \mathrm{C}$ and stirred for $5.5 \mathrm{~h}$. The reaction was then allowed to cool to $\mathrm{rt}$, then quenched adding sat. $a q \cdot \mathrm{Na}_{2} \mathrm{~S}_{2} \mathrm{O}_{3}(2 \mathrm{~mL})$ and stirred for another $10 \mathrm{~min}$. The phases were separated and the organic layer was washed with sat. $a q . \mathrm{NaHCO}_{3}(3.0$ $\mathrm{mL})$ and brine $(3 \mathrm{~mL})$ and the aqueous layer was back-extracted with ethyl acetate (3 $\times 5) \mathrm{mL}$. The combined organic layers were dried over anhydrous $\mathrm{Na}_{2} \mathrm{SO}_{4}$ and the solvent was removed under reduced pressure. Flash chromatography (80:20 petroleum ether 40-60 / ethyl acetate) afforded phenol 2.72 as a colourless oil (24 mg, 0.12 mmol, $22 \%$ yield) along with recovered starting material 2.70 (53 mg, $0.28 \mathrm{mmol}, 51 \%$ yield). All data for compound $\mathbf{2 . 7 2}$ matched literature values. ${ }^{46}$

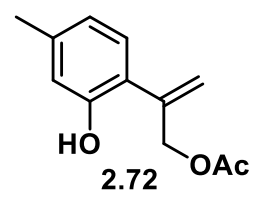

$\mathbf{R}_{\mathbf{f}}$ 0.22 (80:20 petroleum ether 40-60 / ethyl acetate);

${ }^{1}$ H NMR $\left(500 \mathrm{MHz}, \mathrm{CDCl}_{3}\right) \delta 6.98(\mathrm{~d}, J=7.6 \mathrm{~Hz}, 1 \mathrm{H}), 6.75(\mathrm{~d}, J=1.6 \mathrm{~Hz}, 1 \mathrm{H}), 6.70$ (dd, $J=7.7,1.9 \mathrm{~Hz}, 1 \mathrm{H}), 5.48$ (s, 1H), 5.29 (s, 1H), 4.77 (s, 2H), 2.30 (s, 3H), 2.14 (s, 3H) ppm;

${ }^{13}$ C NMR $\left(126 \mathrm{MHz}, \mathrm{CDCl}_{3}\right) \delta 117.8,153.4,141.8,140.1,129.3,122.6,121.1,116.8$, $116.8,66.3,21.3,21.1 \mathrm{ppm}$. 

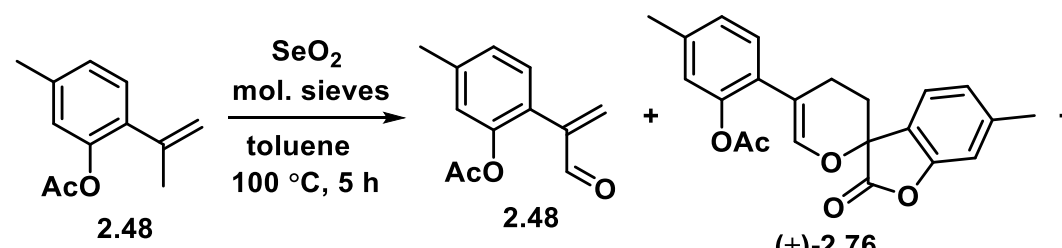

$( \pm)-2.76$<smiles>CC(=O)OCc1coc2cc(C)ccc12</smiles>

2.77

To a solution of alkene $\mathbf{2 . 7 0}(1.13 \mathrm{~g}, 5.96 \mathrm{mmol})$ in anhydrous toluene $(25 \mathrm{~mL})$ in the presence of activated grounded molecular sieves, $\mathrm{SeO}_{2}(993 \mathrm{mg}, 8.95 \mathrm{mmol}$ ) was added as a solid at room temperature. The reaction mixture was then heated to $100{ }^{\circ} \mathrm{C}$ and stirred at that temperature until complete consumption of the starting material, judged by TLC analysis ( $5 \mathrm{~h}$ ). The mixture was then filtered through a pad of celite. Water $(20 \mathrm{~mL})$ was added to the filtrate and the mixture was extracted with $\mathrm{CH}_{2} \mathrm{Cl}_{2}$ (3 $\times 20 \mathrm{~mL}$ ). The combined organic layers were dried over anhydrous $\mathrm{Na}_{2} \mathrm{SO}_{4}$ and the solvent was removed under reduced pressure. Flash chromatography (95:5 to 80:20 petroleum ether 40-60 / ethyl acetate) afforded compound 2.48 as an orange oil (259 $\mathrm{mg}, 1.27 \mathrm{mmol}, 21 \%$ yield) as well as and lactone $\mathbf{2 . 7 6}$ as a pale brown oil (70 $\mathrm{mg}$, $0.20 \mathrm{mmol}, 7 \%$ yield) and benzofuran $\mathbf{2 . 7 7}$ as a pale-yellow oil (60 mg, $0.29 \mathrm{mmol}$, $5 \%$ yield). Note: Yields of compound $\mathbf{2 . 4 8}$ were dependent on the reaction scale: from $48-50 \%$ on a $30-\mathrm{mg}$ scale and $19-21 \%$ on a $260-\mathrm{mg}$ scale.

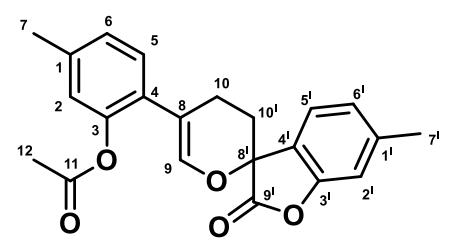

$( \pm)-2.76$

$\mathbf{R}_{\mathbf{f}} 0.38$ (70:30 petroleum ether 40-60 / ethyl acetate);

${ }^{1} \mathbf{H}$ NMR $\left(500 \mathrm{MHz}, \mathrm{CDCl}_{3}\right) \delta 7.32\left(\mathrm{~d}, J=7.7 \mathrm{~Hz}, 1 \mathrm{H}, \mathrm{H} 5^{\prime}\right), 7.19(\mathrm{~d}, J=7.8 \mathrm{~Hz}, 1 \mathrm{H}$, H5), 7.05 (dm, $J=7.8,1 \mathrm{H}, \mathrm{H} 6), 7.02$ (dm, $J=7.7,1 \mathrm{H}, \mathrm{H} 6$ '), 6.97 (d, $J=0.9 \mathrm{~Hz}, 1 \mathrm{H}$, H2'), 6.89 (d, $J=0.9 \mathrm{~Hz}, 1 \mathrm{H}, \mathrm{H} 2$ ), 6.61 (apparent t, $J=1.7 \mathrm{~Hz}, 1 \mathrm{H}, \mathrm{H} 9), 2.79$ (dddd, $J=17.3,7.5,5.9,1.9 \mathrm{~Hz}, 1 \mathrm{H}, \mathrm{H} 10), 2.53-2.45$ (m, 1H, H10), 2.41 (s, 3H, H7'), 2.36 (s, 3H, H7), 2.32 (s, 3H, H12), 2.30-2.24 (m, 1H, H10'), 2.23-2.16 (m, 1H, H10') ppm; 
${ }^{13}$ C NMR (126 MHz, CDCl 3 ) $\delta 173.7$ (C9'), 169.9 (C11), 153.3 (C3'), 148.4 (C3), 142.0 (C1'), 140.9 (C9), 138.4 (C1), 129.7 (C5), 129.3 (C4), 127.2 (C6), 125.6 (C6'), 125.3 (C4'), 124.3 (C5'), 123.5 (C2), 112.0 (C2'), 110.4 (C8), 74.9 (C8'), 29.0 (C10'), 22.0 (C7'), 21.3 (C12), 21.1 (C7), 20.5 (C10) ppm;

HRMS $\left(\mathrm{ESI}^{+}\right) \quad 365.1374, \quad 387.1205$ (calculated $[\mathrm{M}+\mathrm{H}]^{+}$365.1383, $[\mathrm{M}+\mathrm{Na}]^{+}$ 387.1203);

IR (film, cm ${ }^{-1}$ ) 2922, 1809, 1758, 1630, 1503.

All data for compound $\mathbf{2 . 7 7}$ matched literature values. ${ }^{164}$

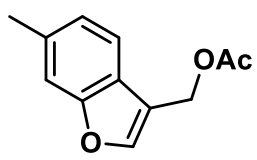

2.77

$\mathbf{R}_{\mathbf{f}} 0.47$ (70:30 petroleum ether 40-60 / ethyl acetate);

${ }^{1} \mathbf{H}$ NMR $\left.\left(500 \mathrm{MHz}, \mathrm{CDCl}_{3}\right) \delta\right) \delta 7.61(\mathrm{t}, J=0.9 \mathrm{~Hz}, 1 \mathrm{H}), 7.51(\mathrm{~d}, J=7.9 \mathrm{~Hz}, 1 \mathrm{H})$, 7.31-7.30 (m, 1H), $7.11(\mathrm{dm}, J=7.9 \mathrm{~Hz}, 1 \mathrm{H}), 5.27(\mathrm{~d}, J=0.9 \mathrm{~Hz}, 2 \mathrm{H}), 2.49-2.47(\mathrm{~m}$, $3 \mathrm{H}), 2.11(\mathrm{~s}, 3 \mathrm{H}) \mathrm{ppm}$;

${ }^{13}$ C NMR $\left(126 \mathrm{MHz}, \mathrm{CDCl}_{3}\right) \delta 171.1,156.1,143.7,135.2,124.5,124.3,119.4$, 116.0, 112.0, 56.8, 21.8, $21.1 \mathrm{ppm}$. 


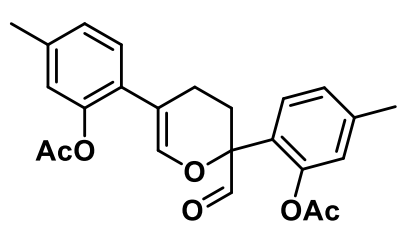

$( \pm)-2.59$

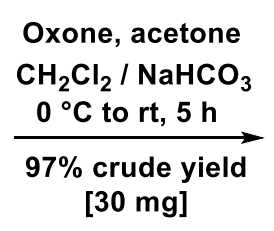

[30 mg]

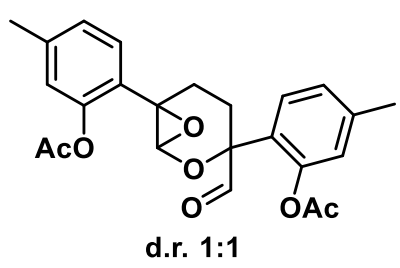

$( \pm)-2.78$

To a vigorously stirred solution of dihydropyran ( $($ ) -2.59 (30 mg, $73 \mu \mathrm{mol})$ in $\mathrm{CH}_{2} \mathrm{Cl}_{2}$ $(1.5 \mathrm{~mL})$, sat. $a q . \mathrm{NaHCO}_{3}(2 \mathrm{~mL})$ and acetone $(500 \mu \mathrm{L})$ at $0{ }^{\circ} \mathrm{C}$, a solution of oxone (135 mg, $0.22 \mathrm{mmol})$ in water $(1 \mathrm{~mL})$ was added via a syringe pump within $20 \mathrm{~min}$.. The mixture was allowed to warm to rt and stirred until complete consumption of the starting material, judged by TLC analysis $(4.5 \mathrm{~h})$. Then the reaction mixture was extracted with $\mathrm{CH}_{2} \mathrm{Cl}_{2}(3 \times 5 \mathrm{~mL})$ and the combined organic layers were dried over anhydrous $\mathrm{Na}_{2} \mathrm{SO}_{4}$. Removal of the solvent under reduced pressure afforded a colourless oil (31 mg, $71 \mu \mathrm{mol}, 97 \%$ crude yield). Analysis of the crude ${ }^{1} \mathrm{H}$ NMR spectrum indicated the presence of a 1:1 mixture of diasteroisomers epoxide $( \pm)$-2.78 (see ${ }^{1} \mathrm{H}$ NMR spectrum of the crude product on page 318 ) as the sole product. Flash chromatography $\left(100 \% \mathrm{CH}_{2} \mathrm{Cl}_{2}\right)$ afforded epoxide $( \pm)-\mathbf{2 . 7 8}$ as a single diastereoisomer (5 mg, $12 \mu \mathrm{mol}, 16 \%$ yield).

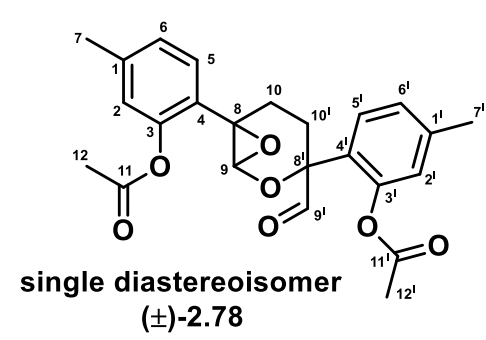

$\mathbf{R}_{\mathbf{f}} 0.13\left(100 \% \mathrm{CH}_{2} \mathrm{Cl}_{2}\right)$;

${ }^{1} \mathbf{H}$ NMR $\left(500 \mathrm{MHz}, \mathrm{CDCl}_{3}\right) \delta 9.48(\mathrm{~d}, J=1.2 \mathrm{~Hz}, 1 \mathrm{H}, \mathrm{H} 9 '), 7.54(\mathrm{~d}, J=8.1 \mathrm{~Hz}, 1 \mathrm{H}$, H5'), 7.27 (d, $J=8.0 \mathrm{~Hz}, 1 \mathrm{H}, \mathrm{H} 5), 7.13$ (dm, $J=8.1 \mathrm{~Hz}, 1 \mathrm{H}, \mathrm{H} 6$ '), 7.04 (dm, $J=7.8$ Hz, 1H, H6), 6.88-6.87 (m, 1H, H2'), 6.85-6.84 (m, 1H, H2), 5.02 (s, 1H, H9), 2.392.33 (m, 2H, H10 or H10'), 2.38 (s, 3H, H12 or H12'), 2.36 (s, 3H, H7'), 2.33 (s, 3H, H7), 2.31-2.28 (m, 1H, H10 or H10'), 2.27 (s, 3H, H12 or H12'), 2.24-2.18 (m, 1H, $\mathrm{H} 10$ or $\mathrm{H} 10$ ') ppm; 
${ }^{13}$ C NMR $\left(126 \mathrm{MHz}, \mathrm{CDCl}_{3}\right) \delta 196.5$ (C9'), 170.0 (C11 or C11'), 169.5 (C11 or C11'), 147.8 (C3), 147.6 (C3'), 140.6 (C1'), 139.8 (C1), 128.0 (2C, C5 + C4), 127.7 (C5'), 127.6 (C6'), 127.2 (C6), 126.8 (C4'), 124.5 (C2'), 123.4 (C2), 81.8 (C9), 80.4 (C8'), 59.3 (C8), 25.4 (C10 or C10'), 24.9 (C10 or C10'), 21.3 (C7, C7', C12 or C12'), 21.2 (C7, C7', C12 or C12'), 21.2 (C7, C7', C12 or C12'), 21.1 (C7, C7', C12 or C12') ppm;

IR (film, cm ${ }^{-1}$ ) 2924, 2855, 1767, 1738, 1622;

HRMS $\left(\mathrm{ESI}^{+}\right)$425.1621, 447.1450 (calculated $[\mathrm{M}+\mathrm{H}]^{+} 425.1595[\mathrm{M}+\mathrm{Na}]^{+}$ 447.1414). 


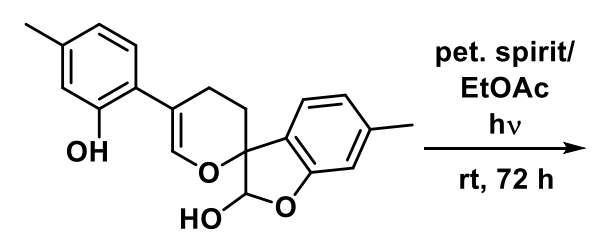

$( \pm)-2.9$

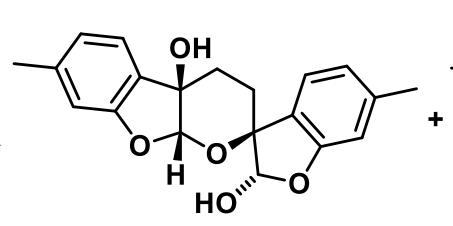

( \pm )-1.1 (d.r. 88:12)

$57 \%{ }^{1} \mathrm{H}$ NMR yield

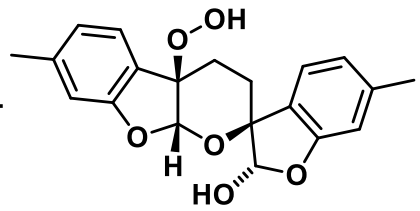

( \pm )-2.79 (d.r. 88:12)

$21 \%{ }^{1} H$ NMR yield

Crude lactol ( \pm )-2.9 (250 mg, $0.771 \mathrm{mmol})$ was dissolved in an 8:2 mixture of petroleum spirit / ethyl acetate $(40 \mathrm{~mL})$. The mixture was stirred at $\mathrm{rt}$ under the light from Kosnic Compact Fluorescent Lamp $11 \mathrm{~W}$ Ecolight bulb for $72 \mathrm{~h}$ (for further details, see: http://www.kosnic.com) in a standard desk-type lamp. Removal of the solvent under reduced pressure afforded a crude mixture as an orange powder (250 $\mathrm{mg}$ ). Analysis of this crude mixture by ${ }^{1} \mathrm{H}$ NMR analysis, with the inclusion of an internal standard, indicated a $57 \%$ yield of thymarnicol $( \pm)-1.1$ over three steps from enal 2.48 (see stacked ${ }^{1} \mathrm{H}$ NMR spectra on page 321), along with a $21 \%$ internal standard yield of peroxide ( \pm )-2.79. Analytically pure samples of thymarnicol ( \pm )-1.1 and peroxide $( \pm)-2.79$ were obtained using column chromatography followed by preparative HPLC (70:30 MeOH / $\mathrm{H}_{2} \mathrm{O}$, ACE-321-2520 C18 preparative column). Up to date we have prepared $40 \mathrm{mg}$ of analytically pure thymarnicol ( \pm )-1.1. Note: d.r. of lactol epimers varied with solvent used for the NMR experiments (78:22 in $\mathrm{CDCl}_{3}$ and 88:12 in $\left.\left(\mathrm{CD}_{3}\right)_{2} \mathrm{CO}\right)$, for both thymarnicol $( \pm)-\mathbf{1 . 1}$ and compound $( \pm)-\mathbf{2 . 7 9}$.

Recrystrallisation from hot acetonitrile gave crystalline ( \pm )-1.1, the structure of which was confirmed using single crystal X-ray crystallography (CCDC 1530569) (see page 424). 


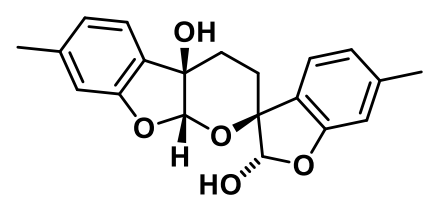

(士)-1.1 (d.r. 88:12)

tR $3.52 \mathrm{~min}$ (flow rate: $15 \mathrm{~mL} / \mathrm{min}, \lambda=254 \mathrm{~nm}$, pressure: $223 \mathrm{bar}$ ).

$\mathbf{R}_{\mathbf{f}} 0.38$ (70:30 hexane / ethyl acetate);

${ }^{1} \mathbf{H}$ NMR $\left(500 \mathrm{MHz},\left(\mathrm{CD}_{3}\right)_{2} \mathrm{CO}\right) \delta 7.43\left(\mathrm{~d}, J=7.6 \mathrm{~Hz}, 1 \mathrm{H}^{\ddagger}\right) 7.39\left(\mathrm{~d}, J=7.6 \mathrm{~Hz}, 1 \mathrm{H}^{\dagger}\right)$, $7.25\left(\mathrm{~d}, J=7.6 \mathrm{~Hz}, 1 \mathrm{H}^{\ddagger}\right), 7.23\left(\mathrm{~d}, J=7.5 \mathrm{~Hz}, 1 \mathrm{H}^{\dagger}\right), 6.83(\mathrm{ddd}, J=7.6,1.5,0.8 \mathrm{~Hz}$, $\left.1 \mathrm{H}^{\star}\right), 6.81-6.78\left(\mathrm{~m}, 1 \mathrm{H}^{*}\right), 6.80\left(\mathrm{ddd}, J=7.6,1.5,0.8 \mathrm{~Hz}, 1 \mathrm{H}^{\dagger}\right), 6.77(\mathrm{ddd}, J=7.7,1.5$, $\left.0.8 \mathrm{~Hz}, 1 \mathrm{H}^{\dagger}\right), 6.68\left(\mathrm{dd}, J=1.4,0.7 \mathrm{~Hz}, 1 \mathrm{H}^{\ddagger}\right), 6.66-6.65\left(\mathrm{~m}, 1 \mathrm{H}^{\dagger}\right), 6.65-6.64\left(\mathrm{~m}, 1 \mathrm{H}^{\dagger}+\right.$ $\left.1 \mathrm{H}^{\ddagger}\right), 6.19\left(\mathrm{~d}, J=5.8 \mathrm{~Hz}, 1 \mathrm{H}^{\dagger}\right), 5.89\left(\mathrm{~d}, J=5.5 \mathrm{~Hz}, 1 \mathrm{H}^{\dagger}\right) 5.78\left(\mathrm{~s}, 1 \mathrm{H}^{\ddagger}\right), 5.71\left(\mathrm{~s}, 1 \mathrm{H}^{\dagger}\right)$, $5.39\left(\mathrm{~d}, J=10.5 \mathrm{~Hz}, 1 \mathrm{H}^{\ddagger}\right), 5.29\left(\mathrm{~d}, J=10.3 \mathrm{~Hz}, 1 \mathrm{H}^{\ddagger}\right), 4.99\left(\mathrm{~s}, 1 \mathrm{H}^{\ddagger}\right), 4.88\left(\mathrm{~s}, 1 \mathrm{H}^{\dagger}\right), 2.43$ $\left(\mathrm{ddd}, J=13.4,11.7,3.2 \mathrm{~Hz}, 1 \mathrm{H}^{\ddagger}\right), 2.36\left(\mathrm{ddd}, J=13.3,11.7,3.4 \mathrm{~Hz}, 1 \mathrm{H}^{\dagger}\right), 2.32(\mathrm{~s}$, $\left.3 \mathrm{H}^{\star}\right), 2.31\left(\mathrm{~s}, 6 \mathrm{H}^{\dagger}\right), 2.27-2.22\left(\mathrm{~m}, 1 \mathrm{H}^{*}\right), 2.21\left(\mathrm{ddd}, J=13.3,7.0,3.4 \mathrm{~Hz}, 1 \mathrm{H}^{\dagger}\right), 2.13-$ $2.08\left(\mathrm{~m}, 1 \mathrm{H}^{\ddagger}\right), 2.11-2.05\left(\mathrm{~m}, 1 \mathrm{H}^{\dagger}\right), 2.09\left(\mathrm{~s}, 3 \mathrm{H}^{\ddagger}\right), 1.96(\mathrm{ddd}, J=14.8,11.7,3.4 \mathrm{~Hz}$, $\left.1 \mathrm{H}^{\dagger}\right), 1.64\left(\mathrm{ddd}, J=14.6,11.7,3.2 \mathrm{~Hz}, 1 \mathrm{H}^{\dagger}\right) \mathrm{ppm}$;

${ }^{13}$ C NMR $\left(126 \mathrm{MHz},\left(\mathrm{CD}_{3}\right)_{2} \mathrm{CO}\right) \delta 160.8\left(1 \mathrm{C}^{\dagger}+1 \mathrm{C}^{\ddagger}\right), 159.3^{\dagger}, 158.5^{\ddagger}, 141.9^{\ddagger}, 141.7^{\dagger}$, $141.5^{\ddagger} 141.1^{\dagger}, 129.6^{\dagger}, 129.5^{\ddagger}, 128.6^{\dagger}, 128.1^{\ddagger}, 125.0\left(1 C^{\dagger}+1 C^{\ddagger}\right), 124.6^{\ddagger}, 124.5^{\dagger}, 123.5^{\ddagger}$, $123.1^{\dagger}, 122.5^{\ddagger}, 122.5^{\dagger}, 112.1^{\dagger}, 112.1^{\ddagger}, 111.9^{\dagger}, 111.7^{\ddagger}, 111.5^{\dagger}, 111.0^{\ddagger}, 108.2^{\dagger}, 107.5^{\ddagger}$, $83.6^{\dagger}, 83.6^{\ddagger}, 77.3^{\dagger}, 77.2^{\ddagger}, 31.2^{\dagger}, 30.0^{\ddagger}, 28.5^{\ddagger}, 23.5^{\dagger}, 21.8\left(1 \mathrm{C}^{\dagger}+1 \mathrm{C}^{\ddagger}\right), 21.8\left(1 \mathrm{C}^{\dagger}+1 \mathrm{C}^{\ddagger}\right)$ ppm;

IR (film, cm ${ }^{-1}$ ) 3424, 3352, 2926, 1624, 1597;

HRMS $\left(\mathrm{ESI}^{+}\right) 341.1372$ (calculated $[\mathrm{M}+\mathrm{H}]^{+}$341.1384);

m.p. $184-185^{\circ} \mathrm{C}$ (acetonitrile).

$\dagger$ Major diastereoisomer, $\ddagger$ minor diastereoisomer. 


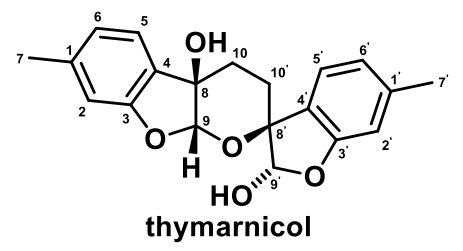

$( \pm)-1.1$

\section{Comparison of the ${ }^{1} \mathrm{H}$ and ${ }^{13} \mathrm{C}$ NMR Data for Synthetic and Natural ( $( \pm)-1.1$}

Provided below is a tabulated comparison of the NMR data for the major epimer of ( \pm )-thymarnicol 1.1.

Our ${ }^{13} \mathrm{C}$ NMR spectrum is referenced to the $\left(\mathrm{CD}_{3}\right)_{2} \mathrm{CO}$ peak at $206.26 \mathrm{ppm}$. All peaks were found to be within \pm 0.07 ppm. $^{*}$

\begin{tabular}{|c|c|c|c|c|c|c|}
\hline \multirow{2}{*}{ Atom } & \multicolumn{3}{|c|}{ Synthetic Thymarnicol $\left(\mathrm{CD}_{3}\right)_{2} \mathrm{CO}$} & \multicolumn{3}{|c|}{ Natural Thymarnicol (Passreiter) ${ }^{48}\left(\mathrm{CD}_{3}\right)_{2} \mathrm{CO}$} \\
\hline & $\delta^{1} \mathbf{H}(500 \mathrm{MHz})$ & $\mathbf{m} J(\mathbf{H z})$ & $\delta^{13} \mathrm{C}(126 \mathrm{MHz})$ & $\delta^{1}{ }^{1} \mathrm{H}(500 \mathrm{MHz})$ & $\mathbf{m} J(\mathbf{H z})$ & $\delta^{13} \mathrm{C}(126 \mathrm{MHz})$ \\
\hline 1 & - & - & 141.09 & - & - & 141.12 \\
\hline 2 & $6.65-6.64$ & $\mathrm{~m}$ & 111.93 & 6.63 & $\mathrm{~s}$ & 111.97 \\
\hline 3 & - & - & 159.25 & - & - & $159.97^{*}$ \\
\hline 4 & - & - & 129.57 & - & - & 129.57 \\
\hline 5 & 7.23 & $\mathrm{~d}, 7.5$ & 122.50 & 7.24 & $\mathrm{~d}, 7.9$ & 122.54 \\
\hline 6 & 6.80 & $\begin{array}{c}\text { ddd, } 7.6 \\
1.5,0.8\end{array}$ & 124.95 & 6.79 & $\mathrm{~d}, 7.9$ & 125.00 \\
\hline 7 & 2.31 & $\mathrm{~s}$ & 21.78 & 2.33 & $\mathrm{~s}$ & 21.85 \\
\hline 8 & - & - & 77.24 & - & - & 77.24 \\
\hline 9 & 5.71 & $\mathrm{~s}$ & 111.46 & 5.71 & $\mathrm{~s}$ & 111.50 \\
\hline $10_{a}$ & 2.36 & $\begin{array}{c}\text { ddd, } \\
13.3, \\
11.7,3.4\end{array}$ & \multirow{2}{*}{31.15} & $2.33^{\dagger}$ & $\mathrm{m}$ & \multirow{2}{*}{31.13} \\
\hline $10_{\mathrm{b}}$ & 2.21 & $\begin{array}{c}\text { ddd, } \\
13.3, \\
7.0,3.4\end{array}$ & & $2.18^{\dagger}$ & $\begin{array}{c}\mathrm{ddd}, \\
13.3,7.0, \\
3.0\end{array}$ & \\
\hline $1^{\prime}$ & - & - & 141.72 & - & - & 141.75 \\
\hline 2 & $6.66-6.65$ & $\mathrm{~m}$ & 112.11 & 6.64 & $\mathrm{~d},<1$ & 112.10 \\
\hline 3 & - & - & 160.75 & - & - & 160.78 \\
\hline 4 & - & - & 128.63 & - & - & 128.66 \\
\hline 5 & 7.39 & $\mathrm{~d}, 7.6$ & 123.14 & 7.39 & $\mathrm{~d}, 7.9$ & 123.18 \\
\hline 6 & 6.77 & $\begin{array}{c}\text { ddd, } 7.7 \\
1.5,0.8\end{array}$ & 124.51 & 6.77 & $\mathrm{~d}, 7.9$ & 124.55 \\
\hline 7 & 2.31 & $\mathrm{~s}$ & 21.80 & 2.33 & $\mathrm{~s}$ & 21.83 \\
\hline 8 & - & - & 83.62 & - & - & 83.63 \\
\hline $9^{\prime}$ & 5.89 & $\mathrm{~d}, 5.5$ & 108.15 & 5.89 & $\mathrm{~d}, 10.5^{*}$ & 108.09 \\
\hline $10_{\mathrm{a}}^{\prime}$ & $2.11-2.05^{\dagger}$ & $\mathrm{m}$ & \multirow[b]{2}{*}{23.54} & $2.05^{\dagger}$ & $\mathrm{m}$ & \multirow[b]{2}{*}{23.57} \\
\hline $10_{b}^{\prime}$ & $1.96^{\dagger}$ & $\begin{array}{c}\text { ddd, } \\
14.8, \\
11.7,3.4\end{array}$ & & $1.93^{\dagger}$ & $\begin{array}{c}\text { ddd, } \\
14.9, \\
11.8,3.0\end{array}$ & \\
\hline $\begin{array}{c}\mathrm{OH} \text { at } \\
\mathrm{C}-8\end{array}$ & 4.88 & $\mathrm{~s}$ & - & 4.94 & $\mathrm{~s}$ & - \\
\hline $\begin{array}{l}\mathrm{OH} \text { at } \\
\mathrm{C}-9\end{array}$ & 6.19 & $\mathrm{~d}, 5.8$ & - & 6.24 & $\mathrm{~d}, 10.5^{*}$ & - \\
\hline
\end{tabular}

$\dagger$ assignment interchangeable. 
* The ${ }^{13} \mathrm{C}$ NMR signal for C3 (159.97 ppm) and the coupling constant between H9 and the $\mathrm{OH}$ at $\mathrm{C}^{\prime}$ ' $(J=10.5 \mathrm{~Hz})$ reported by Passreiter and co-workers differ from what we observe $(159.25 \mathrm{ppm}$ and $J=5.5 / 5.8 \mathrm{~Hz}) .{ }^{48}$ However, given the otherwise excellent agreement between both our NMR spectra and X-ray crystal structure with that reported for the natural material, we assume these discrepancies to be the result of typographical mistakes. 


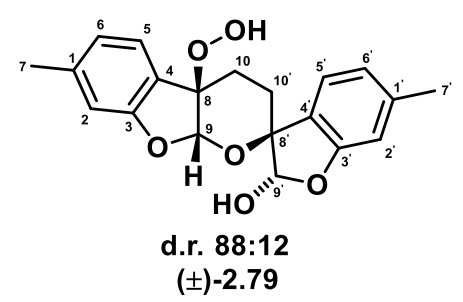

$\mathbf{R}_{\mathbf{f}} 0.38$ (70:30 hexane / ethyl acetate);

${ }^{1} \mathbf{H}$ NMR $\left(500 \mathrm{MHz},\left(\mathrm{CD}_{3}\right)_{2} \mathrm{CO}\right) \delta 7.60\left(\mathrm{~d}, J=7.6 \mathrm{~Hz}, 1 \mathrm{H}^{\ddagger}, \mathrm{H} 5^{\prime}\right), 7.56(\mathrm{~d}, J=7.7 \mathrm{~Hz}$, $\left.1 \mathrm{H}^{\dagger}, \mathrm{H}^{\prime}\right), 6.80\left(\mathrm{dd}, J=7.7,1.6 \mathrm{~Hz}, 1 \mathrm{H}^{\dagger}+1 \mathrm{H}^{\ddagger}, \mathrm{H}^{\prime}\right), 6.75\left(\mathrm{~d}, J=8.1 \mathrm{~Hz}, 1 \mathrm{H}^{\dagger}, \mathrm{H} 5\right)$, $6.74\left(\mathrm{~d}, J=8.1 \mathrm{~Hz}, 1 \mathrm{H}^{\ddagger}, \mathrm{H} 5\right), 6.71\left(\mathrm{~s}, 1 \mathrm{H}^{\dagger}, \mathrm{H} 2\right), 6.70-6.67\left(\mathrm{~m}, 1 \mathrm{H}^{\dagger}+1 \mathrm{H}^{\ddagger}, \mathrm{H} 6\right), 6.69$ $\left.\left(\mathrm{m}, 1 \mathrm{H}^{\ddagger}, \mathrm{H} 2\right)^{\prime}\right), 6.64-6.63\left(\mathrm{~m}, 1 \mathrm{H}^{\dagger}, \mathrm{H} 2\right), 6.63-6.62\left(\mathrm{~m}, 1 \mathrm{H}^{\ddagger}, \mathrm{H} 2\right), 6.33(\mathrm{~d}, J=5.9 \mathrm{~Hz}$, $1 \mathrm{H}^{\dagger}, \mathrm{OH}$ at $\left.\mathrm{C}^{\prime}\right), 6.15\left(\mathrm{~s}, 1 \mathrm{H}^{\ddagger}, \mathrm{OOH}\right.$ at $\left.\mathrm{C} 8\right), 6.11\left(\mathrm{~s}, 1 \mathrm{H}^{\dagger}, \mathrm{OOH}\right.$ at $\left.\mathrm{C} 8\right), 5.54(\mathrm{~d}, J=5.8$ $\left.\mathrm{Hz}, 1 \mathrm{H}^{\dagger}, \mathrm{H}^{\prime}\right), 5.44\left(\mathrm{~d}, J=12.7 \mathrm{~Hz}, 1 \mathrm{H}^{\ddagger}, \mathrm{OH}\right.$ at $\left.\mathrm{C}^{\prime}\right), 5.37$ (d, $\left.J=12.6 \mathrm{~Hz}, 1 \mathrm{H}^{\ddagger}, \mathrm{H}^{\prime}\right)$, $5.34\left(\mathrm{~s}, 1 \mathrm{H}^{\ddagger}, \mathrm{H} 9\right), 5.23\left(\mathrm{~s}, 1 \mathrm{H}^{\dagger}, \mathrm{H} 9\right), 2.62-2.54\left(\mathrm{~m}, 1 \mathrm{H}^{\ddagger}, \mathrm{H} 10\right.$ or H10'), $2.51(\mathrm{dd}, J=$ 13.6, $4.8 \mathrm{~Hz}, 1 \mathrm{H}^{\dagger}, \mathrm{H} 10$ or H10'), 2.44 (m, $1 \mathrm{H}^{\dagger}+1 \mathrm{H}^{\ddagger}, \mathrm{H} 10$ or $\left.\mathrm{H} 10^{\prime}\right), 2.38-2.34$ (m, $1 \mathrm{H}^{\dagger}$,

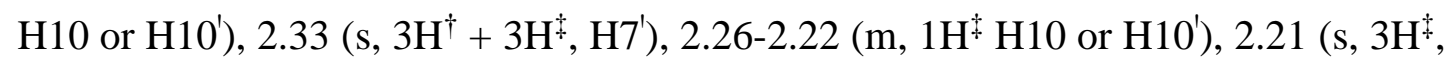

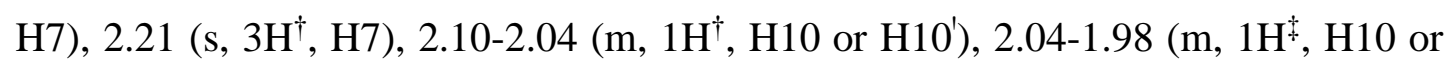
$\left.\mathrm{H} 10^{\prime}\right) \mathrm{ppm}$;

${ }^{13}$ C NMR $\left(126 \mathrm{MHz},\left(\mathrm{CD}_{3}\right)_{2} \mathrm{CO}\right) \delta 161.2\left(1 \mathrm{C}^{\dagger}+1 \mathrm{C}^{\ddagger}, \mathrm{C}^{\prime}\right), 142.1\left(1 \mathrm{C}^{\dagger}+1 \mathrm{C}^{\ddagger}, \mathrm{H} 1^{\prime}\right)$, $140.9^{\dagger}(\mathrm{C} 3), 140.8^{\ddagger}(\mathrm{C} 3), 139.9\left(1 \mathrm{C}^{\dagger}+1 \mathrm{C}^{\ddagger}, \mathrm{C} 4\right), 131.8\left(1 \mathrm{C}^{\dagger}+1 \mathrm{C}^{\ddagger}, \mathrm{C} 1\right), 126.0^{\dagger}\left(\mathrm{C}^{\prime}\right)$, $126.0^{\ddagger}(\mathrm{C} 5), 125.8^{\ddagger}\left(\mathrm{C}^{\prime}\right), 125.7^{\dagger}\left(\mathrm{C}^{\prime}\right), 123.3^{\ddagger}(\mathrm{C} 6), 123.2^{\dagger}(\mathrm{C} 6), 121.9\left(1 \mathrm{C}^{\dagger}+1 \mathrm{C}^{\ddagger}, \mathrm{C}^{\prime}\right)$, 118.0 $0^{\ddagger}(\mathrm{C} 5), 117.9\left(1 \mathrm{C}^{\dagger}+1 \mathrm{C}^{\ddagger}, \mathrm{C} 2\right), 117.8^{\dagger}(\mathrm{C} 5), 112.5\left(1 \mathrm{C}^{\dagger}+1 \mathrm{C}^{\ddagger}, \mathrm{C} 2^{\prime}\right), 107.8^{\dagger}\left(\mathrm{C}^{\prime}\right)$, 107.7* (C9'), 92.6 $6^{\dagger}(\mathrm{C} 9), 92.6^{\ddagger}(\mathrm{C} 9), 91.0^{\dagger}(\mathrm{C} 8), 90.9^{\star}(\mathrm{C} 8), 85.9^{\dagger}\left(\mathrm{C} 8^{\prime}\right), 85.8^{\ddagger}\left(\mathrm{C}^{\prime}\right)$, $32.6^{\dagger}\left(\mathrm{C} 10\right.$ or $\left.\mathrm{C} 10^{\prime}\right), 32.6^{\ddagger}\left(\mathrm{C} 10\right.$ or $\left.\mathrm{C} 10^{\prime}\right), 26.4^{\dagger}\left(\mathrm{C} 10\right.$ or $\left.\mathrm{C} 10^{\prime}\right), 26.3^{\ddagger}\left(\mathrm{C} 10\right.$ or $\left.\mathrm{C} 10^{\prime}\right), 21.6^{\ddagger}$ $\left(\mathrm{C} 7^{\prime}\right), 21.6^{\dagger}\left(\mathrm{C} 7^{\prime}\right), 20.7^{\star}(\mathrm{C} 7), 20.6^{\dagger}(\mathrm{C} 7) \mathrm{ppm}$;

IR (film, cm $\mathrm{cm}^{-1}$ ) 3219, 3211, 2922, 2359, 2342, 2324;

HRMS $\left(\mathrm{ESI}^{+}\right) \quad 357.1333, \quad 379.1149$ (calculated $[\mathrm{M}+\mathrm{H}]^{+}$357.1333, $[\mathrm{M}+\mathrm{Na}]^{+}$ 379.1152).

$\dagger$ Major diastereoisomer, $\$$ minor diastereoisomer. 


\subsection{Specific Experimental Procedures for Chapter 3}

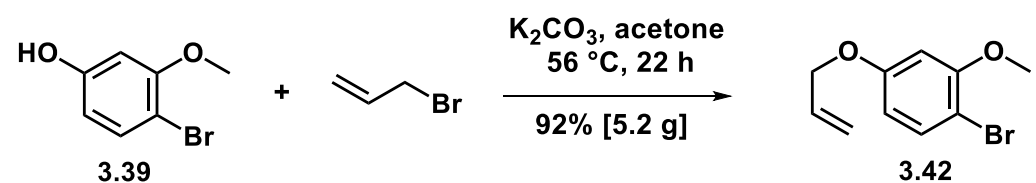

4-Bromo-3-methoxyphenol 3.39 (5.25 g, $25.8 \mathrm{mmol}$ ) was dissolved in anhydrous acetone $(100 \mathrm{~mL}) . \mathrm{K}_{2} \mathrm{CO}_{3}(3.56 \mathrm{~g}, 25.8 \mathrm{mmol})$ and allyl bromide $(3.08 \mathrm{~mL}, 35.6 \mathrm{mmol})$ were then added to this stirred solution. The mixture was heated at $56^{\circ} \mathrm{C}$ until complete consumption of the starting material, judged by TLC analysis $(22 \mathrm{~h})$, then allowed to cool to rt, diluted with $\mathrm{CH}_{2} \mathrm{Cl}_{2}(50 \mathrm{~mL})$ and washed with $2 \mathrm{M}$ aq. $\mathrm{NaOH}(2 \times 50 \mathrm{~mL})$. The organic layer was dried over anhydrous $\mathrm{Na}_{2} \mathrm{SO}_{4}$, filtered and then the solvent was removed under reduced pressure. Flash chromatography (70:30 petroleum spirit / diethyl ether) gave compound $\mathbf{3 . 4 2}$ as a colourless oil (5.48 g, $22.5 \mathrm{mmol}, 92 \%$ yield).<smiles>C=CCOc1cccc(Br)c1OC</smiles>

$\mathbf{R}_{\mathbf{f}}$ 0.41 (70:30 petroleum spirit 40-60 / diethyl ether);

${ }^{1} \mathbf{H}$ NMR $\left(500 \mathrm{MHz}, \mathrm{CDCl}_{3}\right) \delta 7.39(\mathrm{~d}, J=8.7 \mathrm{~Hz}, 1 \mathrm{H}), 6.52(\mathrm{~d}, J=2.7 \mathrm{~Hz}, 1 \mathrm{H}), 6.40$ (dd, $J=8.7,2.7 \mathrm{~Hz}, 1 \mathrm{H}), 6.04$ (ddt, $J=17.2,10.6,5.3 \mathrm{~Hz}, 1 \mathrm{H}), 5.41$ (apparent dt, $J=$ 17.2, 1.6 Hz, 1H), 5.30 (apparent dt, $J=10.5,1.5 \mathrm{~Hz}, 1 \mathrm{H}$ ), 4.53-4.49 (m, 2H), 3.86 (s, 3H) ppm;

${ }^{13}$ C NMR (126 MHz, $\left.\mathrm{CDCl}_{3}\right) \delta 159.3,156.7,133.2,133.0,118.1,106.9,102.7,100.8$, 69.3, 56.3 ppm;

IR (film, cm ${ }^{-1}$ ) 2940, 1487, 1304, 1198, 1167;

HRMS $\left(\mathrm{ESI}^{+}\right)$243.0013, 244.9991 (calculated $[\mathrm{M}+\mathrm{H}]^{+}$243.0015, $[\mathrm{M}+\mathrm{Na}]^{+}$ 244.9995). 


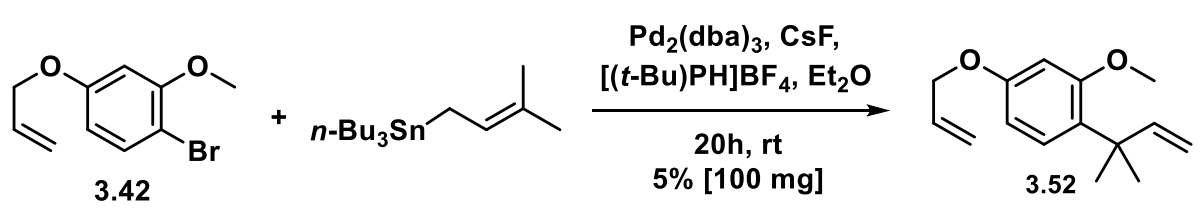

Bromoarene 3.42 (100 mg, $0.41 \mathrm{mmol}$ ) was dissolved in anhydrous diethyl ether (1.1 mL). CsF (125 mg, 0.82), [(t-Bu) $\left.)_{3} \mathrm{PH}\right] \mathrm{BF}_{4}(4 \mathrm{mg}, 14 \mu \mathrm{mol}), \mathrm{Pd}_{2}(\mathrm{dba})_{3}(6 \mathrm{mg}, 6.2$ $\mu \mathrm{mol})$ and tributyl-(3-methyl-2-butenyl)tin $(147 \mu \mathrm{L}, 0.43 \mathrm{mmol})$ were then added. The mixture was stirred at $\mathrm{rt}$ for $20 \mathrm{~h}$, then diethyl ether $(5 \mathrm{~mL})$ and $\mathrm{KF} \cdot 2 \mathrm{H}_{2} \mathrm{O}(500 \mathrm{mg})$ were added and the mixture was stirred for $30 \mathrm{~min}$. The mixture was then filtered through silica gel and the filtrate was washed with water $(5 \mathrm{~mL})$ The phases were separated and the solvent was removed under reduced pressure. Flash chromatography (90:10 petroleum spirit 40-60 / diethyl ether) afforded arene $\mathbf{3 . 5 2}$ as a light blue oil (5 $\mathrm{mg}, 5 \%$ yield) along with recovered starting material $\mathbf{3 . 4 2}$ (38 mg, $0.15 \mathrm{mmol}, 38 \%$ yield).

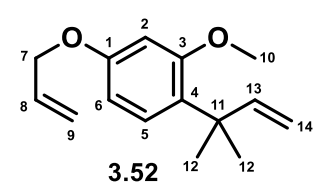

$\mathbf{R}_{\mathbf{f}} 0.34$ (80:20 petroleum spirit 40-60 / diethyl ether);

${ }^{1} \mathbf{H}$ NMR $\left(500 \mathrm{MHz}, \mathrm{CDCl}_{3}\right) \delta 7.15(\mathrm{~d}, J=8.5 \mathrm{~Hz}, 1 \mathrm{H}, \mathrm{H} 5), 6.50(\mathrm{~d}, J=2.5 \mathrm{~Hz}, 1 \mathrm{H}$, H2), 6.42 (dd, $J=8.6,2.6 \mathrm{~Hz}, 1 \mathrm{H}, \mathrm{H} 6), 6.20-6.14$ (m, 1H, H13), 6.06 (ddt, $J=17.3$, 10.6, $5.4 \mathrm{~Hz}, 1 \mathrm{H}, \mathrm{H} 8$ ), 5.42 (apparent dq, $J=17.3,1.6 \mathrm{~Hz}, 1 \mathrm{H}, \mathrm{H} 9$ ), 5.28 (appartent dq, $J=10.5,1.4$ Hz, 1H, H9), 4.93 (m, 1H, H14), 4.91 (dd, $J=5.7,1.4$ Hz, 1H, H14), 4.52 (apparent dt, $J=5.4,1.5 \mathrm{~Hz}, 2 \mathrm{H}, \mathrm{H} 7$ ), 3.77 (s, 3H, H10), 1.43 (s, 6H, H12) ppm;

${ }^{13}$ C NMR (126 MHz, $\left.\mathrm{CDCl}_{3}\right) \delta 159.3$ (C3), $158.4(\mathrm{C} 1), 148.5$ (C13), $183.6(\mathrm{C} 8), 129.4$ (C4), 127.5 (C5), 117.8 (C9), 109.7 (C14), 104.4 (C6), 100.7 (C2), 69.0 (C7), 55.2 (C10), 40.2 (C11), 27.5 (2C, C12) ppm;

IR (film, cm ${ }^{-1}$ ) 2961, 2934, 1609, 1582;

HRMS $\left(\mathrm{ESI}^{+}\right)$233.1551, $255.1372\left(\right.$ calculated $[\mathrm{M}+\mathrm{H}]^{+} 233.1536,[\mathrm{M}+\mathrm{Na}]^{+}$ 255.1355). 


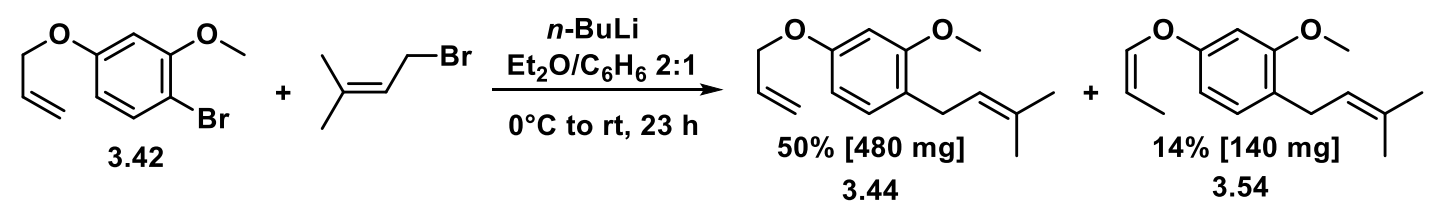

Bromo arene $3.42(1.0 \mathrm{~g}, 4.1 \mathrm{mmol})$ was dissolved in a 2:1 degassed mixture of anhydrous diethyl ether and anhydrous benzene $(40 \mathrm{~mL}) . n$-butyllithium $(3.10 \mathrm{~mL}, 1.6$ $\mathrm{M}$ in hexane, $4.85 \mathrm{mmol}$ ) was added dropwise at $0{ }^{\circ} \mathrm{C}$. After stirring for $40 \mathrm{~min}$ at $\mathrm{rt}$, 3,3-dimethylallyl bromide ( $947 \mu \mathrm{L}, 8.2 \mathrm{mmol}$ ) was added and the mixture was stirred at $\mathrm{rt}$ until complete consumption of the starting material, judged by TLC analysis (22 h). The reaction was then quenched with sat. $a q . \mathrm{NH}_{4} \mathrm{Cl}(25 \mathrm{~mL})$ and the mixture was extracted with diethyl ether $(3 \times 25 \mathrm{~mL})$. The combined organic layers were dried over anhydrous $\mathrm{Na}_{2} \mathrm{SO}_{4}$, and the solvent was removed under reduced pressure. Flash chromatography (97:3 petroleum spirit 40-60/ ethyl acetate) gave compound $\mathbf{3 . 4 4}$ as a colourless oil (480 mg, $2.06 \mathrm{mmol}, 50 \%$ yield) as well as isomer $\mathbf{3 . 5 4}$ as a colourless oil (136 mg, $0.58 \mathrm{mmol}, 14 \%$ yield).

Note: more material could be recovered via further purification of combined mixed fractions containing arene $\mathbf{3 . 4 4}$ coming from different reactions.

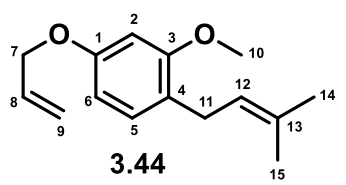

$\mathbf{R}_{\mathbf{f}} 0.58$ (70:30 petroleum spirit 40-60 / ethyl acetate);

${ }^{1} \mathbf{H}$ NMR $\left(500 \mathrm{MHz}, \mathrm{CDCl}_{3}\right) \delta 7.01(\mathrm{~d}, J=8.2 \mathrm{~Hz}, 1 \mathrm{H}, \mathrm{H} 5), 6.48(\mathrm{~d}, J=2.4 \mathrm{~Hz}, 1 \mathrm{H}$, H2), 6.43 (dd, $J=8.2,2.4 \mathrm{~Hz}, 1 \mathrm{H}, \mathrm{H} 6), 6.07$ (ddt, $J=17.3,10.6,5.4 \mathrm{~Hz}, 1 \mathrm{H}, \mathrm{H} 8$ ), 5.41 (apparent dq, $J=17.3,1.6 \mathrm{~Hz}, 1 \mathrm{H}, \mathrm{H} 9), 5.32-5.25$ (m, 2H, H9 + H12), 4.51 (apparent $\mathrm{dt}, J=5.5,1.5 \mathrm{~Hz}, 2 \mathrm{H}, \mathrm{H} 7), 3.81$ (s, 3H, H10), 3.26-3.22 (m, 2H, H11), 1.74 (d, $J=$ $1.3 \mathrm{~Hz}, 3 \mathrm{H}, \mathrm{H} 14$ or H15), 1.71-1.70 (m, 3H, H14 or H15) ppm;

${ }^{13}$ C NMR (126 MHz, $\mathrm{CDCl}_{3}$ ) $\delta 158.2(\mathrm{C} 3), 158.2$ (C1), 133.7 (C8), 132.2 (C13), 129.5 (C5), 123.1 (C12), 122.8 (C4), 117.7 (C9), 104.9 (C2), 99.4 (C6), 69.2 (C7), 55.5 (C10), 27.9 (C11), 25.9 (C14 or C15), 17.9 (C14 or C15) ppm;

IR (film, cm ${ }^{-1}$ ) 2963, 2913, 2857, 1611, 1587;

HRMS $\left(\mathrm{ESI}^{+}\right) 255.1352$ (calculated $[\mathrm{M}+\mathrm{Na}]^{+}$255.1355). 


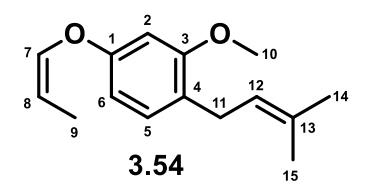

Rf 0.66 (80:20 petroleum spirit 40-60 / ethyl acetate);

${ }^{1} \mathbf{H}$ NMR (500 MHz, $\left.\mathrm{CDCl}_{3}\right) \delta 7.04(\mathrm{~d}, J=8.1 \mathrm{~Hz}, 1 \mathrm{H}, \mathrm{H} 5), 6.55$ (d, $J=2.4 \mathrm{~Hz}, 1 \mathrm{H}$, H2), 6.51 (dd, $J=8.2,2.4 \mathrm{~Hz}, 1 \mathrm{H}, \mathrm{H} 6$ ), 6.37 (dq , $J=6.1,1.7 \mathrm{~Hz} 1 \mathrm{H}, \mathrm{H} 7$ ), $5.31-5.26$ (m, $1 \mathrm{H}, \mathrm{H} 12$ ), 4.85 (dq, $J=6.9,6.1 \mathrm{~Hz}, 1 \mathrm{H}, \mathrm{H} 8$ ), 3.82 (s, $3 \mathrm{H}, \mathrm{H} 10$ ), 3.26 (dd, $J=7.4$, $1.4 \mathrm{~Hz}, 2 \mathrm{H}, \mathrm{H} 11), 1.75-1.70$ (m, 6H, H14 + H15), 1.72 (dd, $J=6.9,1.7 \mathrm{~Hz}, 3 \mathrm{H}, \mathrm{H} 9)$ ppm;

${ }^{13} \mathbf{C}$ NMR (126 MHz, $\left.\mathrm{CDCl}_{3}\right) \delta 158.2(\mathrm{C} 3), 157.0$ (C1), 141.4 (C7), 132.4 (C13), 129.6 (C5), 124.3 (C4), 122.8 (C12), 107.2 (C8), 107.1 (C5), 100.1 (C2), 55.5 (C10), 28.0 (C11), 25.9 (C14 or C15), 17.8 (C14 or C15), 9.5 (C9) ppm;

IR (film, $\mathrm{cm}^{-1}$ ) 2967, 2920, 1670, 1607, 1589;

HRMS (ESI $\left.{ }^{+}\right) 233.1532$ (calculated $[\mathrm{M}+\mathrm{H}]^{+}$233.1536). 


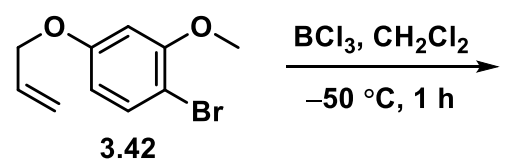

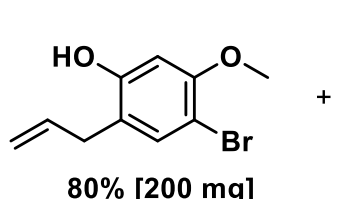

3.56

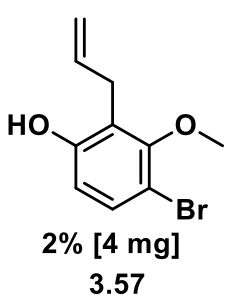

To a solution of allyl ether $\mathbf{3 . 4 2}(200 \mathrm{mg}, 0.82 \mathrm{mmol})$ in anhydrous $\mathrm{CH}_{2} \mathrm{Cl}_{2}(2.0 \mathrm{~mL})$, boron trichloride solution $\left(2.46 \mathrm{~mL}, 1 \mathrm{M}\right.$ in $\left.\mathrm{CH}_{2} \mathrm{Cl}_{2}, 2.46 \mathrm{mmol}\right)$ was added carefully at $-50{ }^{\circ} \mathrm{C}$ and the mixture was stirred at the same temperature for $1 \mathrm{~h}$. Then water $(10$ $\mathrm{mL}$ ) was added carefully, the mixture was allowed to slowly warm to $\mathrm{rt}$ and was then extracted with $\mathrm{CH}_{2} \mathrm{Cl}_{2}(3 \times 8 \mathrm{~mL})$. The organic layer was washed with brine $(20 \mathrm{~mL})$ and dried over anhydrous $\mathrm{MgSO}_{4}$. The solvent was removed under reduced pressure. Analysis of the ${ }^{1} \mathrm{H}$ NMR of the crude revealed a 20:1 ratio of compounds $\mathbf{3 . 5 6}$ and 3.57. Flash chromatography (80:20 petroleum spirit 40-60 / diethyl ether) afforded phenol 3.56 (160 mg, $0.66 \mathrm{mmol}, 80 \%$ yield), as well as its regioisomer 3.57 as a colourless oil (4 mg, $16 \mu \mathrm{mol}, 2 \%$ yield).

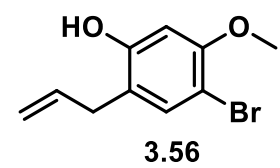

$\mathbf{R}_{\mathbf{f}} 0.15$ (70:30 petroleum spirit 40-60 / diethyl ether);

${ }^{1} \mathrm{H}$ NMR $\left(500 \mathrm{MHz}, \mathrm{CDCl}_{3}\right) \delta 7.24(\mathrm{~s}, 1 \mathrm{H}), 6.46(\mathrm{~s}, 1 \mathrm{H}), 5.97$ (ddt, $J=16.7,10.5,6.3$ Hz, 1H), 5.20-5.19 (m, 1H), 5.19-5.16 (m, 1H), 5.03 (broad s, 1H), 3.84 (s, 3H), 3.32 (apparent dt, $J=6.2,1.5,2 \mathrm{H}$ ) ppm;

${ }^{13} \mathrm{C} \mathrm{NMR}\left(126 \mathrm{MHz}, \mathrm{CDCl}_{3}\right): \delta 155.6,154.7,136.1,134.2,118.4,117.2,102.1,101.2$, 56.5, 34.6 ppm;

IR (film, $\left.\mathrm{cm}^{-1}\right) 3489$ (broad), 2974, 1610, 1583;

HRMS $\left(\mathrm{ESI}^{+}\right) 264.9841$ (calculated $[\mathrm{M}+\mathrm{H}]^{+}$264.9835). 


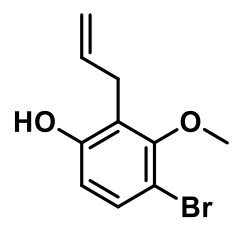

3.57

$\mathbf{R}_{\mathbf{f}} 0.25$ (70:30 petroleum spirit 40-60 / diethyl ether);

${ }^{1} \mathbf{H}$ NMR $\left(500 \mathrm{MHz}, \mathrm{CDCl}_{3}\right) \delta 7.30(\mathrm{~d}, J=8.7 \mathrm{~Hz}, 1 \mathrm{H}), 6.56(\mathrm{~d}, J=8.7 \mathrm{~Hz}, 1 \mathrm{H}), 6.1$ (ddt, $J=17.1,10.2,5.9 \mathrm{~Hz} 1 \mathrm{H}), 5.18-5.13(\mathrm{~m}, 2 \mathrm{H}), 5.01$ ( broad s, 1H), 3.80 (s, 3H), 3.52, (apparent dt, $J=5.9,1.8 \mathrm{~Hz}, 2 \mathrm{H}$ ) ppm;

${ }^{13}$ C NMR (126 MHz, $\left.\mathrm{CDCl}_{3}\right) \delta 155.9,155.1,135.8,131.6,121.1,116.7,113.6,108.3$, 61.5, $29.1 \mathrm{ppm}$;

IR (film, $\mathrm{cm}^{-1}$ ) 3410 (broad), 2941, 2359, 1638, 1580;

HRMS $\left(\mathrm{ESI}^{+}\right) 264.9831$ (calculated $[\mathrm{M}+\mathrm{H}]^{+}$264.9835). 

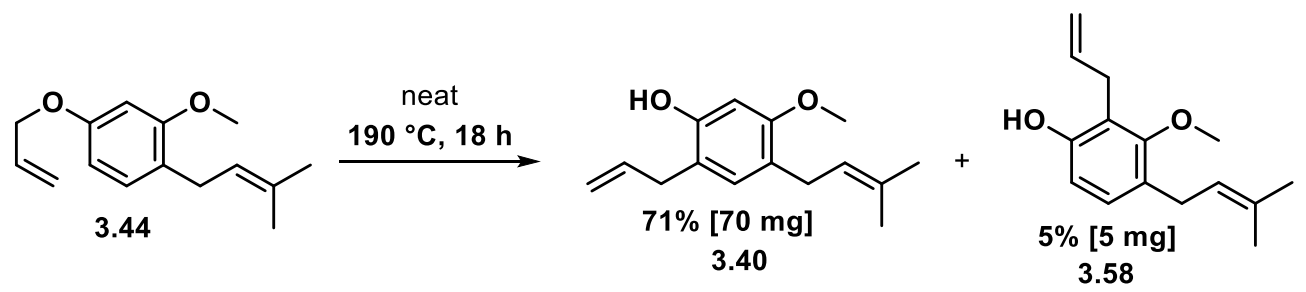

Allyl ether $3.44(100 \mathrm{mg}, 0.41 \mathrm{mmol})$ was heated, neat, at $190{ }^{\circ} \mathrm{C}$ under nitrogen atmosphere for $18 \mathrm{~h}$. Analysis of the ${ }^{1} \mathrm{H}$ NMR spectrum of the crude revealed a $6: 1$ ratio of compounds $\mathbf{3 . 4 0}$ and 3.58. Flash chromatography (80:20 petroleum spirit 4060 / diethyl ether) afforded compound $\mathbf{3 . 4 0}$ as a pale orange oil $(71 \mathrm{mg}, 0.31 \mathrm{mmol}$, $71 \%$ yield) as well as its regioisomer $\mathbf{3 . 5 8}$ as as a pale-yellow oil (5 mg, $0.021 \mathrm{mmol}$, $5 \%$ yield).

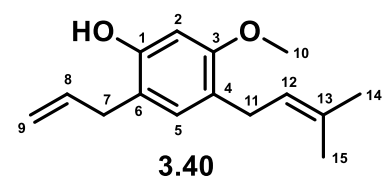

Rf 0.12 (80:20 petroleum spirit 40-60 / diethyl ether);

${ }^{1}$ H NMR $\left(500 \mathrm{MHz}, \mathrm{CDCl}_{3}\right) \delta 6.82$ (s, 1H, H5), 6.40 (s, 1H, H2), 6.01 (ddt, $J=17.4$, 10.1, 6.3 Hz, 1H, H8), 5.30-5.25 (m, 1H, H12), 5.21-5.17 (m, 2H, H9), 4.93 (broad s, $1 \mathrm{H}, \mathrm{OH}$ at C1), 3.78 (s, 3H, H10), 3.35-3.32 (m, 2H, H7), 3.24-3.21 (m, 2H, H11), 1.73 (s, 3H, H14 or H15), 1.71 (s, 3H, H14 or H15) ppm;

${ }^{13}$ C NMR (126 MHz, $\mathrm{CDCl}_{3}$ ) $\delta 157.1$ (C3), 153.3 (C1), 137.2 (C8), 132.1 (C13), 131.0 (C5), 123.3 (C12), 122.5 (C6), 116.4 (C9), 116,0 (C4), 99.7 (C2), 55.7 (C10), 35.1 (C7), 27.9 (C11), 26.0 (C14 or C15), 17.9 (C14 or C15) ppm;

IR (film, $\mathrm{cm}^{-1}$ ) 3438 (broad), 2965, 2913, 2855, 1618, 1508;

HRMS $\left(\mathrm{ESI}^{+}\right) 255.1323$ (calculated $[\mathrm{M}+\mathrm{Na}]^{+}$255.1356). 


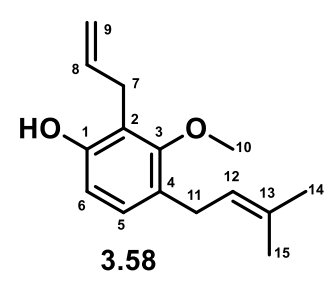

Rf 0.20 (80:20 petroleum spirit 40-60 / diethyl ether);

${ }^{1} \mathbf{H}$ NMR $\left(500 \mathrm{MHz}, \mathrm{CDCl}_{3}\right) \delta 6.95(\mathrm{~d}, J=8.3 \mathrm{~Hz}, 1 \mathrm{H}, \mathrm{H} 5), 6.60(\mathrm{~d}, J=8.3 \mathrm{~Hz}, 1 \mathrm{H}$, H6), 6.05 (ddt, $J=17.5,9.6,5.9$ Hz, 1H, H8), 5.29-5.25 (m, 1H, H12), 5.18-5.15 (m, 1H, H9), 5.14-5.13 (m, 1H, H9), 4.91 (broad s, 1H, OH at C1), 3.71 (s, 3H, H10), 3.503.48 (m, 2H, H7), 3.31-3.28 (m, 2H, H11), 1.75-1.73 (m, 3H, H14 or H15), 1.73-1.71 (m, 3H, H14 or H15) ppm;

${ }^{13}$ C NMR (126 MHz, $\left.\mathrm{CDCl}_{3}\right) \delta 157.0(\mathrm{C} 3), 153.8(\mathrm{C} 1), 136.6(\mathrm{C} 8), 132.3$ (C13), 128.6 (C5), 127.1 (C2), 123.5 (C12), 118.7 (C4), 116.2 (C9), 112.0 (C6), 61.7 (C10), 28.7 (C7), 28.1 (C11), 25.9 (C14 or C15), 18.0 (C14 or C15) ppm;

IR (film, $\mathrm{cm}^{-1}$ ) 3372 (broad), 2968, 2914, 1638, 1599;

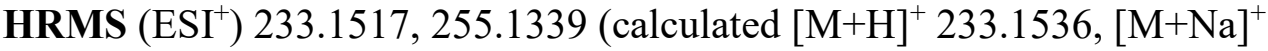
255.1356). 

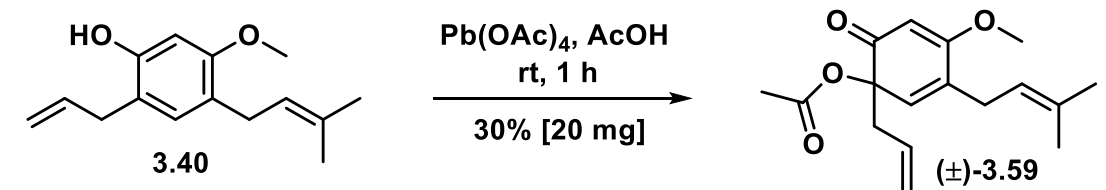

Phenol 3.40 (19 mg, $0.08 \mathrm{mmol})$ was dissolved in acetic acid $(2.5 \mathrm{~mL})$. Then $\mathrm{Pb}(\mathrm{OAc})_{4}$ (50 mg, $0.11 \mathrm{mmol}$ ) was added as a solid and the mixture was stirred at $\mathrm{rt}$ for $30 \mathrm{~min}$. Then ethyl acetate $(8 \mathrm{~mL})$ and water $(7 \mathrm{~mL})$ were added and the mixture was stirred for another $30 \mathrm{~min}$ and then was extracted with ethyl acetate $(3 \times 8 \mathrm{~mL})$ The combined organic layers were washed with brine $(3 \times 30 \mathrm{~mL})$, dried over anhydrous $\mathrm{Na}_{2} \mathrm{SO}_{4}$ and the solvent was removed under reduced pressure. Analysis of the ${ }^{1} \mathrm{H}$ NMR spectrum of the crude revealed a 1:1 mixture of two compounds. Flash chromatography (55:45 petroleum spirit 40-60 / ethyl acetate) afforded ortho-quinol acetate ( \pm )-3.59 (7 mg, 24 $\mu \mathrm{mol}, 30 \%$ yield). It was not possible to obtain a pure sample of the second compound.

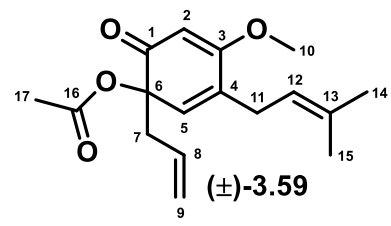

Rf 0.40 (55:45 petroleum spirit 40-60 / diethyl ether);

${ }^{1} \mathbf{H}$ NMR $\left(500 \mathrm{MHz}, \mathrm{CDCl}_{3}\right) \delta 5.91(\mathrm{t}, J=1.6 \mathrm{~Hz}, 1 \mathrm{H}, \mathrm{H} 5), 5.71$ (dddd, $J=17.0,10.2$, 7.7, $6.8 \mathrm{~Hz}, 1 \mathrm{H}, \mathrm{H} 8), 5.51$ (s, 1H, H2), 5.14-5.10 (m, 1H, H12), 5.09-5.04 (m, 2H, H9), 3.78 (s, 3H, H10), 3.03-2.88 (m, 2H, H11), 2.51-2.42 (m, 2H, H7), 2.08 (s, 3H, H17), 1.75-1.73 (m, 3H, H14 or H15), 1.61-1.59 (m, 3H, H14 or H15) ppm;

${ }^{13}$ C NMR (126 MHz, $\left.\mathrm{CDCl}_{3}\right) \delta 197.2(\mathrm{C} 1), 170.9$ (C3), 169.3 (C16), 136.5 (C5), 134.8 (C13), 133.2 (C4), 130.5 (C8), 120.1 (C12), 119.8 (C9), 100.0 (C2), 80.1 (C6), 56.2 (C10), 43.5 (C7), 28.2 (C11), 25.9 (C17), 20.9 (C14 or C15), 17.9 (C14 or C15) ppm; IR (film, $\mathrm{cm}^{-1}$ ) 2978, 2914, 2854, 1741, 1662, 1570;

HRMS $\left(\mathrm{ESI}^{+}\right) \quad 291.1605,313.1427$ (calculated $[\mathrm{M}+\mathrm{H}]^{+}$291.1591, $[\mathrm{M}+\mathrm{Na}]^{+}$ 314.1410). 

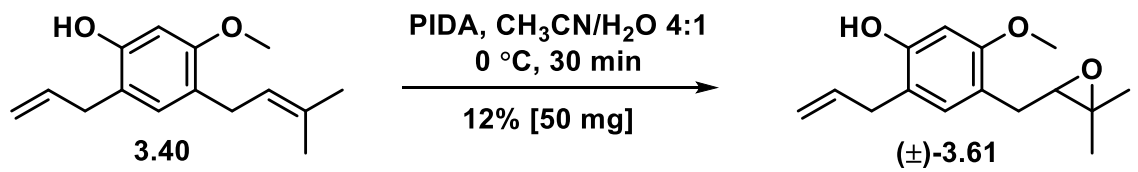

Arene 3.40 (50 mg, $0.21 \mathrm{mmol})$ was dissolved in acetonitrile $(2 \mathrm{~mL})$ and water $(0.5$ $\mathrm{mL})$ at $\mathrm{rt}$. The solution was cooled to $0{ }^{\circ} \mathrm{C}$ then $\mathrm{PIDA}\left(\mathrm{PhI}(\mathrm{OAc})_{2}\right)(83 \mathrm{mg}, 0.26 \mathrm{mmol})$ was added slowly while stirring vigorously. After 30 min at $0{ }^{\circ} \mathrm{C}$, sat. $a q . \mathrm{Na}_{2} \mathrm{SO}_{3}(2$ $\mathrm{mL})$ and diethyl ether $(2 \mathrm{~mL})$ were added, the phases were separated and the aqueous layer was extracted with diethyl ether $(2 \times 5 \mathrm{~mL})$. The combined organic layers were then dried over anhydrous $\mathrm{Na}_{2} \mathrm{SO}_{4}$ and the solvent was removed under reduced pressure. Flash chromatography (50:50 petroleum spirit 40-60 / diethyl ether) afforded epoxide ( \pm )-3.61 as an orange oil ( $6 \mathrm{mg}, 0.024 \mathrm{mmol}, 12 \%$ yield).

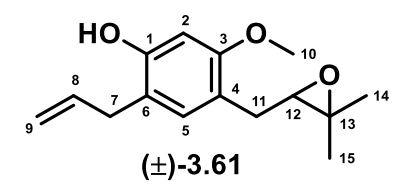

Rf 0.18 (50:50 petroleum spirit 40-60 / diethyl ether);

${ }^{1} \mathbf{H}$ NMR $\left(500 \mathrm{MHz}, \mathrm{CDCl}_{3}\right) \delta 6.89$ (s, 1H, H5), 6.42 (s,1H, H2), 5.99 (ddt, $J=17.2$, 10.1, $6.3 \mathrm{~Hz}, 1 \mathrm{H}, \mathrm{H} 8), 5.21-5.12$ (m, 2H, H9), 5.06 (broad s, 1H, OH at C1), 3.78 (s, 3H, H10), 3.34 (apparent dt, $J=6.3,1.7 \mathrm{~Hz}, 2 \mathrm{H}, \mathrm{H} 7$ ), 2.96 (apparent t, $J=6.1 \mathrm{~Hz}, 1 \mathrm{H}$, H12), 2.82-2.73 (m, 2H, H11), 1.37 (s, 3H, H14 or H15), 1.31 (s, 3H, H14 or H15) ppm;

${ }^{13}$ C NMR (126 MHz, $\left.\mathrm{CDCl}_{3}\right) \delta 157.4$ (C3), 154.0 (C1), 137.0 (C8), 131.8 (C5), 118.7 (C6), 116.7 (C9), 116.5 (C4), 99.7 (C2), 64.2 (C12), 59.0 (C13), 55.6 (C10), 34.9 (C7), 29.2 (C11), 25.1 (C14 or C15), 19.1 (C14 or C15) ppm;

IR (film, cm $\left.{ }^{-1}\right) 3390$ (broad), 2924, 1614, 1605, 1514;

HRMS $\left(\mathrm{ESI}^{+}\right) \quad 249.1492,271.1317$ (calculated $[\mathrm{M}+\mathrm{H}]^{+}$249.1485, $[\mathrm{M}+\mathrm{Na}]^{+}$ 271.1305). 


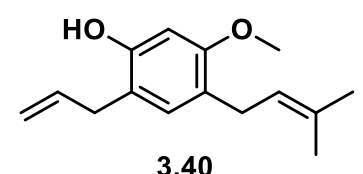

3.40
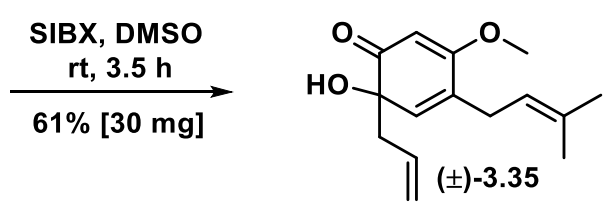

In a glass vial SIBX (183 mg, $0.30 \mathrm{mmol}, 45 \% \mathrm{w} / \mathrm{w})$ was dissolved in DMSO-d 6 (1.2 $\mathrm{mL})$. Phenol 3.40 (28 mg, $0.12 \mathrm{mmol}$ ) was then added. Aliquotes from the reaction mixture were used to monitor the reaction by ${ }^{1} \mathrm{H}$ NMR. After complete consumption of the starting material, the reaction mixture was cooled to $0{ }^{\circ} \mathrm{C}$ and quenched with water $(3 \mathrm{~mL})$. The suspension was filtered and the filtrate was extracted with diethyl ether $(2 \times 3 \mathrm{~mL})$. The combined organic layers were washed with sat. $a q . \mathrm{NaHCO}_{3}$, dried over anhydrous $\mathrm{Na}_{2} \mathrm{SO}_{4}$ and the solvent was removed under reduced pressure. Due to the inherent instability of otho-quinol $( \pm)-\mathbf{3 . 3 5}$, it was not possible to purify it, thus ${ }^{1} \mathrm{H}$ NMR and ${ }^{13} \mathrm{C}$ NMR data of the crude product are reported $\left(\right.$ see ${ }^{1} \mathrm{H}$ and ${ }^{13} \mathrm{C}$ NMR spectra on pages 345 and 346). Crude yield: $61 \%$.

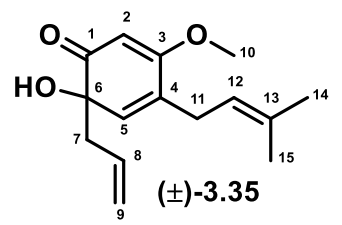

${ }^{1} \mathbf{H}$ NMR $\left(500 \mathrm{MHz}, \mathrm{CDCl}_{3}\right) \delta 6.21(\mathrm{t}, J=1.7 \mathrm{~Hz}, 1 \mathrm{H}, \mathrm{H} 5), 5.75-5.66(\mathrm{~m}, 1 \mathrm{H}, \mathrm{H} 8)$, 5.44 (s, 1H, H2), 5.16-5.12 (m, 1H, H12), 5.11-5.05 (m, 2H, H9), 3.81 (s, 3H, H10), 3.00-2.90 (m, 2H, H11), 2.44-2.33 (m, 2H, H7), 1.75-1.73 (m, 3H, H14 or H15), 1.62 (m, 3H, H14 or H15) ppm;

${ }^{13}$ C NMR (126 MHz, $\mathrm{CDCl}_{3}$ ) $\delta 203.5$ (C1), 172.3 (C3), 139.1 (C5), 134.8 (C13), 131.3 (C4), 129.5 (C8), 120.0 (C12), 119.6 (C9), 97.5 (C2), 76.6 (C6), 56.5 (C10), 47.4 (C7), 28.1 (C11), 25.9 (C14 or C15), 17.8 (C14 or C15). 

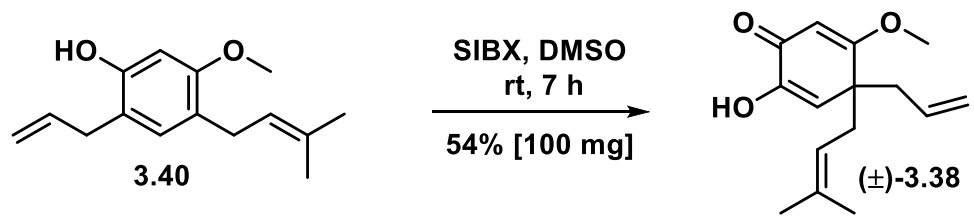

A solution of SIBX (642 mg, $1.03 \mathrm{mmol}, 45 \% \mathrm{w} / \mathrm{w})$ in DMSO (5 mL) was transferred into a flask containing phenol $3.40(100 \mathrm{mg}, 0.43 \mathrm{mmol})$ and the mixture was stirred at $\mathrm{rt}$ until complete consumption of the starting material, judged by TLC analysis ( 7 h). The reaction mixture was then cooled to $0^{\circ} \mathrm{C}$ and diethyl ether $(20 \mathrm{~mL})$ was added. The mixture was washed with sat. $a q . \mathrm{NaHCO}_{3}(3 \times 20 \mathrm{~mL})$ and water $(20 \mathrm{~mL})$. The organic layer was dried over anhydrous $\mathrm{Na}_{2} \mathrm{SO}_{4}$ and the solvent was removed under reduced pressure. Flash chromatography (80:20 petroleum spirit 40-60 / ethyl acetate) afforded compound ( \pm )-3.38 as a pale-yellow oil (58 $\mathrm{mg}, 0.23 \mathrm{mmol}, 54 \%$ yield).

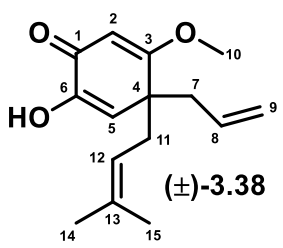

Rf 0.13 (80:20 petroleum spirit 40-60 / ethyl acetate).

${ }^{1} \mathbf{H}$ NMR $\left(500 \mathrm{MHz}, \mathrm{CDCl}_{3}\right) \delta 6.44($ broad s, 1H, OH at C6), 5.67 (s, 1H, H5), 5.63 (s, 1H, H2), 5.46 (dddd, $J=16.9,10.2,7.9,6.7 \mathrm{~Hz}, 1 \mathrm{H}, \mathrm{H} 8$ ), 5.00-4.90 (m, 2H, H9), 4.83-4.80 (m, 1H, H12), 3.72 (s, 3H, H10), 2.61-2.47 (m, 2H, H7 + H11), 2.33 (dd, $J$ $=13.4,6.7 \mathrm{~Hz}, 1 \mathrm{H}, \mathrm{H} 7), 2.25(\mathrm{dd}, J=13.9,6.9 \mathrm{~Hz}, 1 \mathrm{H}, \mathrm{H} 11), 1.60(\mathrm{~s}, 3 \mathrm{H}, \mathrm{H} 14$ or H15), 1.55 (s, 3H, H14 + H15) ppm;

${ }^{13} \mathrm{C}$ NMR $\left(126 \mathrm{MHz}, \mathrm{CDCl}_{3}\right) \delta 183.8(\mathrm{C} 1), 180.6(\mathrm{C} 3), 146.6(\mathrm{C} 6), 135.0(\mathrm{C} 13), 132.8$ (C8), 118.1 (2C, C9 + C12), 116.3 (C5), 101.5 (C2), 56.1 (C10), 48.9 (C4), 43.3 (C7), 37.8 (C11), 25.9 (C14 or C15), 18.0 (C14 or C15) ppm;

IR (film, $\left.\mathrm{cm}^{-1}\right)$ 3365, 2980, 2914, 2856, 1610, 1593;

HRMS $\left(\mathrm{ESI}^{+}\right.$) 249.1485, 271.1303 (calculated $[\mathrm{M}+\mathrm{H}]^{+}$249.1477, [M+Na] 271.1305). 

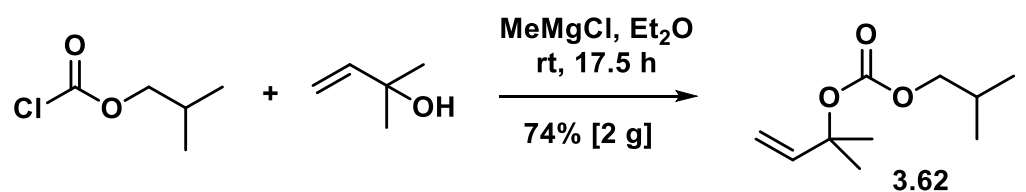

Carbonate 3.62 was prepared using a literature procedure. ${ }^{154}$

A solution of methylmagnesium chloride $(5.2 \mathrm{~mL}, 3 \mathrm{M}$ in THF, $15.6 \mathrm{mmol})$ was diluted with anhydrous diethyl ether $(20 \mathrm{~mL})$ at $0^{\circ} \mathrm{C}$. To the resulting white suspension, a solution of 2-methyl-3-buten-2-ol (1.25 mL, $12.0 \mathrm{mmol})$ in anhydrous diethyl ether $(5$ $\mathrm{mL}$ ) was added dropwise. The mixture was stirred at $0^{\circ} \mathrm{C}$ for $30 \mathrm{~min}$, then a cool solution $\left(0^{\circ} \mathrm{C}\right)$ of isobutyl choloroformate $(2.34 \mathrm{~mL}, 18.0 \mathrm{mmol})$ in diethyl ether $(25$ $\mathrm{mL}$ ) was added rapidly and the mixture was allowed to warm to $\mathrm{rt}$ and stirred for 17 h. The reaction was then quenched with sat. $a q . \mathrm{NH}_{4} \mathrm{Cl}(50 \mathrm{~mL})$, the phases were separated and the aqueous layer was extracted with diethyl ether $(3 \times 50 \mathrm{~mL})$. The combined organic layers were dried over anhydrous $\mathrm{Na}_{2} \mathrm{SO}_{4}$ and the solvent was removed under reduced pressure. Flash chromatography (92:8 petroleum spirit 40-60 / diethyl ether) gave carbonate $\mathbf{3 . 6 2}$ as a colourless oil (1.65 g, $8.88 \mathrm{mmol}, 74 \%$ yield). All data for compound 3.62 matched literature values. ${ }^{154}$

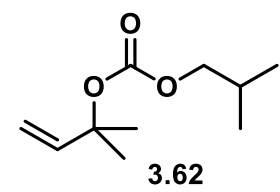

Rf 0.63 (90:10 petroleum spirit 40-60 / diethyl ether);

${ }^{1}$ H NMR $\left(500 \mathrm{MHz}, \mathrm{CDCl}_{3}\right) \delta 6.10(\mathrm{dd}, J=17.5,10.9 \mathrm{~Hz}, 1 \mathrm{H}), 5.21(\mathrm{dd}, 17.5,0.7$ Hz, 1H), 5.12 (dd, 10.9, 0.7 Hz, 1H), 3.85 (dd, $J=6.7,0.6 \mathrm{~Hz}, 2 \mathrm{H}), 1.95$ (sept, $J=6.7$ $\mathrm{Hz}, 1 \mathrm{H}), 1.55$ (s, 6H), 0.93 (d, $J=6.7 \mathrm{~Hz}, 6 \mathrm{H}) \mathrm{ppm}$;

${ }^{13} \mathrm{C}$ NMR (126 MHz, $\left.\mathrm{CDCl}_{3}\right) \delta 153.8,142.0,113.7,82.1,73.5,27.9,26.4$ (2C), 19.1 (2C) $\mathrm{ppm}$. 


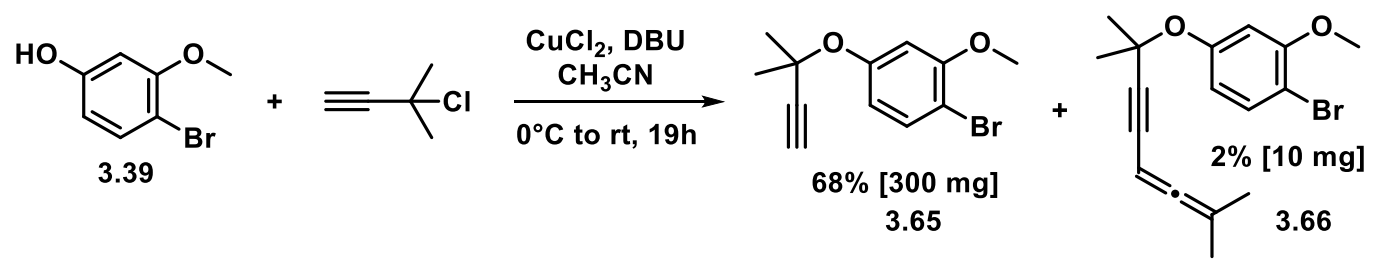

Phenol 3.39 (291 mg, $1.36 \mathrm{mmol}$ ) was dissolved in anhydrous acetonitrile $(6 \mathrm{~mL})$. DBU $(224 \mu \mathrm{L}, 1.48 \mathrm{mmol}), \mathrm{CuCl}_{2}(2 \mathrm{mg}, 15 \mu \mathrm{mol})$ and 3-chloro-3-methyl-1-butyne $(145 \mu \mathrm{L}, 1.24 \mathrm{mmol})$ were then added in portions at $0{ }^{\circ} \mathrm{C}$. The mixture was allowed to warm to $\mathrm{rt}$ and stirred for $19 \mathrm{~h}$, then diethyl ether $(15 \mathrm{~mL})$ and $1 \mathrm{M} a q . \mathrm{HCl}(3 \mathrm{~mL})$, were added. The layers were separated and the organic phase was washed with brine $(2 \times 15 \mathrm{~mL})$. The organic layer was dried over anhydrous $\mathrm{Na}_{2} \mathrm{SO}_{4}$ and the solvent was removed under reduced pressure. Flash chromatography (95:5 petroleum spirit 40-60 / diethyl ether) afforded alkyne 3.65 (228 $\mathrm{mg}, 0.85 \mathrm{mmol}, 68 \%$ yield) along with allene 3.66 ( $9 \mathrm{mg}, 0.027 \mathrm{mmol}, 2 \%$ yield).

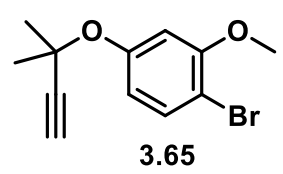

Rf 0.31 (90:10 petroleum spirit 40-60 / diethyl ether);

${ }^{1} \mathbf{H}$ NMR $\left(500 \mathrm{MHz}, \mathrm{CDCl}_{3}\right) \delta 7.40(\mathrm{~d}, J=8.6 \mathrm{~Hz}, 1 \mathrm{H}), 6.81(\mathrm{~d}, J=2.5,1 \mathrm{H}), 6.77$ (dd, $J=8.6,2.6 \mathrm{~Hz}, 1 \mathrm{H}), 3.86$ (s, 3H), 2.59 (s, 1H), 1.65 (s, 6H) ppm;

${ }^{13}$ C NMR (126 MHz, $\left.\mathrm{CDCl}_{3}\right) \delta 156.3,156.3,132.8,114.3,106.3,105.0,86.0,74.3$, 72.9, 56.3, 29.7 (2C) ppm;

IR (film, $\mathrm{cm}^{-1}$ ) 3435, 2994, 1587;

HRMS $\left(\mathrm{ESI}^{+}\right) 268.0092$ (calculated $[\mathrm{M}+\mathrm{H}]^{+}$268.0093). 


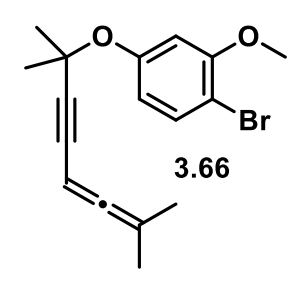

Rf 0.58 (80:20 petroleum spirit 40-60 / diethyl ether);

${ }^{1} \mathbf{H}$ NMR $\left(500 \mathrm{MHz}, \mathrm{CDCl}_{3}\right) \delta 7.38(\mathrm{~d}, J=8.6 \mathrm{~Hz}, 1 \mathrm{H}), 6.91(\mathrm{~d}, J=2.6 \mathrm{~Hz}, 1 \mathrm{H}), 6.70$ (dd, $J=8.6,2.6 \mathrm{~Hz}, 1 \mathrm{H}), 5.23$ (sept, $J=2.9 \mathrm{~Hz}, 1 \mathrm{H}), 3.86$ (s, 3H), 1.72 (d, $J=3.0 \mathrm{~Hz}$, $6 \mathrm{H}), 1.63(\mathrm{~s}, 6 \mathrm{H}) \mathrm{ppm}$;

${ }^{13}$ C NMR (126 MHz, $\left.\mathrm{CDCl}_{3}\right) \delta 210.4,156.5,156.1,132.7,114.8,106.4,104.7,98.0$, 90.5, 80.5, 73.8, 72.8, 56.2, 29.7 (2C), 20.1 (2C) ppm;

IR (film, cm ${ }^{-1}$ ) 2984, 2938, 1584, 1481, 1446;

HRMS $\left(\mathrm{ESI}^{+}\right) 334.0549$ (calculated $[\mathrm{M}+\mathrm{H}]^{+}$334.0563). 


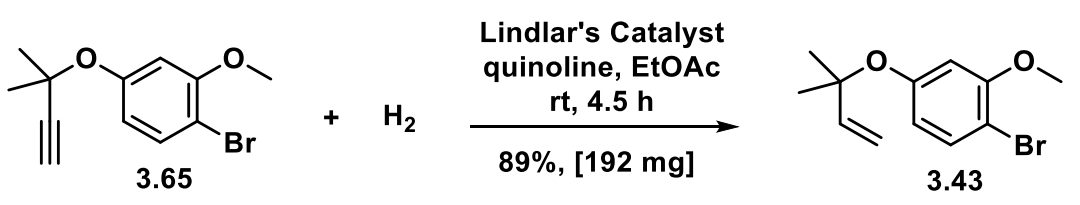

Propargylated arene $\mathbf{3 . 6 5}$ (214 mg, $1.05 \mathrm{mmol}$ ) was dissolved in ethyl acetate (12 mL). Quinoline (1.09 mL, $9.04 \mathrm{mmol})$ and Lindlar's catalyst $(30 \mathrm{mg})$ were added at rt. The suspension was frozen and the flask was evacuated and then fitted with an $\mathrm{H}_{2}$ balloon. The mixture was then allowed to warm to the rt and stirred until complete consumption of the starting material, judged by TLC analysis $(4.5 \mathrm{~h})$. The mixture was then diluted with ethyl acetate $(25 \mathrm{~mL})$, filtered through a short pad of celite and the solvent was removed under reduced pressure. Flash chromatography (90:10 petroleum spirit 40-60 / ethyl acetate) afforded alkene $\mathbf{3 . 4 3}$ (192 $\mathrm{mg}, 0.71 \mathrm{mmol}, 89 \%$ yield).

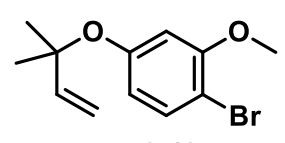

3.43

$\mathbf{R}_{\mathbf{f}}$ 0.31 (90:10 petroleum spirit 40-60 / ethyl acetate);

${ }^{1} \mathbf{H}$ NMR $\left(500 \mathrm{MHz}, \mathrm{CDCl}_{3}\right) \delta 7.32(\mathrm{~d}, J=8.6 \mathrm{~Hz}, 1 \mathrm{H}), 6.57(\mathrm{~d}, J=2.6 \mathrm{~Hz}, 1 \mathrm{H}), 6.51$ (dd, $J=8.6,2.6 \mathrm{~Hz}, 1 \mathrm{H}), 6.13(\mathrm{dd}, J=17.6,10.9 \mathrm{~Hz}, 1 \mathrm{H}), 5.21-5.14(\mathrm{~m}, 2 \mathrm{H}), 3.83$ (s, $3 \mathrm{H}), 1.46(\mathrm{~s}, 6 \mathrm{H}) \mathrm{ppm}$;

${ }^{13} \mathrm{C}$ NMR (126 MHz, $\left.\mathrm{CDCl}_{3}\right) \delta 156.8,156.2,144.3,132.6,114.4,113.9,106.4,104.1$, 80.2, 56.2, 27.1 (2C) ppm;

IR (film, $\mathrm{cm}^{-1}$ ) 2978, 2934, 1726, 1578;

HRMS $\left(\mathrm{ESI}^{+}\right.$) 270.0243, 272.0223 (calculated $[\mathrm{M}+\mathrm{H}]^{+}$270.0250, 272.0230). 


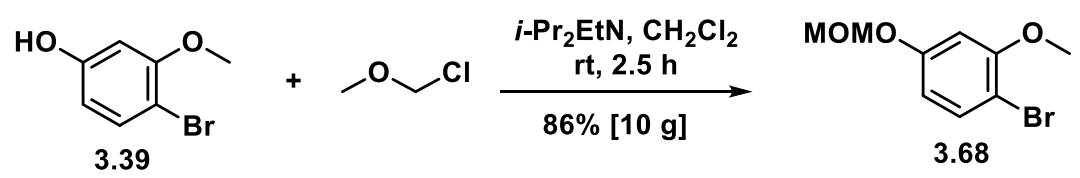

Phenol 3.39 (10.24 g, $48.3 \mathrm{mmol}$ ) was dissolved in anhydrous $\mathrm{CH}_{2} \mathrm{Cl}_{2}(200 \mathrm{~mL})$ and $i$-Pr 2 EtN (25.24 mL, $144.9 \mathrm{mmol})$ was added at rt. MOM-Cl $(9.18 \mathrm{~mL}, 121 \mathrm{mmol})$ was added dropwise at $0{ }^{\circ} \mathrm{C}$ and the mixture was stirred for 30 minute and then allowed to warm to rt and stirred for another $2 \mathrm{~h}$. The reaction was then cooled to $0{ }^{\circ} \mathrm{C}$, quenched with sat. $a q . \mathrm{NaHCO}_{3}(200 \mathrm{~mL})$, diluted with diethyl ether $(80 \mathrm{~mL})$ and stirred for 30 minutes. The phases were separated and the aqueous layer was extracted with diethyl ether $(3 \times 200 \mathrm{~mL})$. The combined organic layers were dried over anhydrous $\mathrm{Na}_{2} \mathrm{SO}_{4}$ and the solvent was removed under reduced pressure. Flash chromatography (85:15 petroleum spirit 40-60 / diethyl ether) afforded MOM-ether 3.68 as a white solid (10.26 g, $41.5 \mathrm{mmol}, 86 \%$ yield).

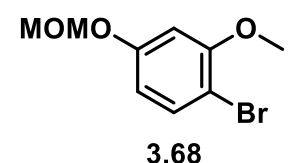

Rf 0.35 (80:20 petroleum spirit 40-60 / diethyl ether);

${ }^{1} \mathbf{H}$ NMR $\left(500 \mathrm{MHz}, \mathrm{CDCl}_{3}\right) \delta 7.40(\mathrm{~d}, J=8.7 \mathrm{~Hz}, 1 \mathrm{H}), 6.62(\mathrm{~d}, J=2.7 \mathrm{~Hz}, 1 \mathrm{H}), 6.56$ (dd, $J=8.7,2.7 \mathrm{~Hz}, 1 \mathrm{H}), 5.15$ (s, 2H), 3.87 (s, 3H), 3.48 (s, 3H) ppm;

${ }^{13}$ C NMR (126 MHz, $\left.\mathrm{CDCl}_{3}\right) \delta 158.0,156.7,133.3,109.0,103.8,101.8,94.8,56.3$, $56.2 \mathrm{ppm}$;

IR (film, $\mathrm{cm}^{-1}$ ) 2955, 2903, 2825, 1582;

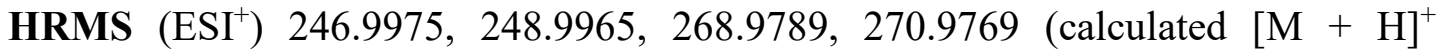
246.9964, 248.9945, [M+ Na $]^{+}$268.9784, 270.9764). 


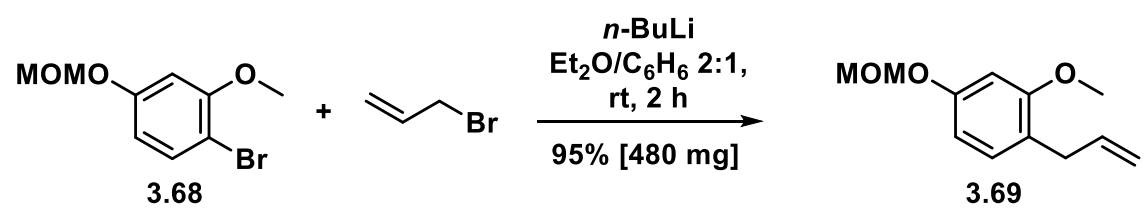

Bromo arene 3.68 (604 mg, $2.44 \mathrm{mmol})$ was dissolved in a 2:1 degassed mixture of anhydrous diethyl ether and anhydrous benzene (12 mL). $n$-butyllithium (1.69 mL, 2.6 $\mathrm{M}$ in hexane, $4.39 \mathrm{mmol}$ ) was added dropwise at rt. After $30 \mathrm{~min}$, allyl bromide (465 $\mu \mathrm{L}, 5.37 \mathrm{mmol}$ ) was added and the mixture was stirred at $\mathrm{rt}$ for $2 \mathrm{~h}$, then quenched with sat. $a q . \mathrm{NH}_{4} \mathrm{Cl}(12 \mathrm{~mL})$ and extracted with diethyl ether $(3 \times 20 \mathrm{~mL})$. The combined organic layers were dried over anhydrous $\mathrm{Na}_{2} \mathrm{SO}_{4}$ and the solvent was removed under reduced pressure. Flash chromatography (88:12 petroleum spirit 40-60 / ethyl acetate) afforded arene $\mathbf{3 . 6 9}$ as a colourless oil (485 mg, $2.33 \mathrm{mmol}, 95 \%$ yield).

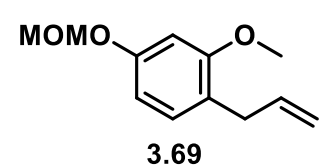

$\mathbf{R}_{\mathbf{f}}$ 0.46 (80:20 petroleum spirit 40-60 / diethyl ether);

${ }^{1} \mathbf{H}$ NMR $\left(500 \mathrm{MHz}, \mathrm{CDCl}_{3}\right) \delta 7.03(\mathrm{~d}, J=8.0 \mathrm{~Hz}, 1 \mathrm{H}), 6.62-6.57(\mathrm{~m}, 2 \mathrm{H}), 6.01-5.93$ $(\mathrm{m}, 1 \mathrm{H}), 5.16(\mathrm{~d}, J=0.7 \mathrm{~Hz}, 2 \mathrm{H}), 5.06-4.99(\mathrm{~m}, 2 \mathrm{H}), 3.81(\mathrm{~s}, 3 \mathrm{H}), 3.49$ (d, $J=0.5 \mathrm{~Hz}$, $3 \mathrm{H}), 3.32(\mathrm{~d}, J=6.6 \mathrm{~Hz}, 2 \mathrm{H}) \mathrm{ppm}$;

${ }^{13}$ C NMR (126 MHz, $\left.\mathrm{CDCl}_{3}\right) \delta 158.2,157.1,137.4,130.2,122.3,115.2,107.3,100.3$, 94.9, 56.1, 55.6, $33.8 \mathrm{ppm}$;

IR (film, cm $\mathrm{cm}^{-1}$ ) 2953, 2935, 2900, 1610, 1587;

HRMS $\left(\mathrm{ESI}^{+}\right) 208.1097$ (calculated $[\mathrm{M}+\mathrm{H}]^{+}$208.1094). 


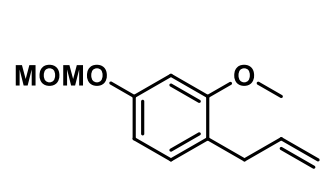

3.69

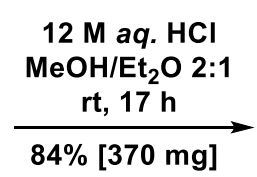

$84 \%[370 \mathrm{mg}]$

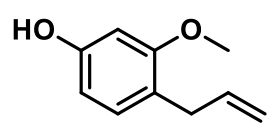

3.70

MOM-ether 3.69 (562 mg, $2.70 \mathrm{mmol})$ was dissolved in diethyl ether (20 mL). MeOH $(40 \mathrm{~mL})$ and $12 \mathrm{M} \mathrm{HCl}(1.51 \mathrm{~mL})$ were then added at $\mathrm{rt}$. The mixture was stirred at $\mathrm{rt}$ until complete consumption of the starting material, judged by TLC analysis (17 h). The reaction mixture was then diluted with water $(20 \mathrm{~mL})$ and extracted with ethyl acetate $(3 \times 30 \mathrm{~mL})$. The combined organic layers were dried over anhydrous $\mathrm{Na}_{2} \mathrm{SO}_{4}$ and the solvent was removed under reduced pressure. Flash chromatography (80:20 petroleum spirit 40-60 / ethyl acetate) afforded phenol 3.70 (371 mg, $2.26 \mathrm{mmol}, 84 \%$ yield).

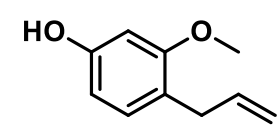

3.70

$\mathbf{R}_{\mathbf{f}} 0.30$ (80:20 petroleum spirit 40-60 / ethyl acetate);

${ }^{1} \mathbf{H}$ NMR $\left(500 \mathrm{MHz}, \mathrm{CDCl}_{3}\right) \delta 6.96(\mathrm{~d}, J=8.0 \mathrm{~Hz}, 1 \mathrm{H}), 6.41(\mathrm{~d}, J=2.4 \mathrm{~Hz}, 1 \mathrm{H}), 6.35$ (dd, $J=8.1,2.5 \mathrm{~Hz}, 1 \mathrm{H}), 5.96(\mathrm{ddt}, J=16.8,10.2,6.5 \mathrm{~Hz}, 1 \mathrm{H}), 5.06-5.01$ (m, $1 \mathrm{H})$, 5.01-4.99 (m, 1H), 4.67 (broad s, 1H), $3.79(\mathrm{~s}, 3 \mathrm{H}), 3.30(\mathrm{~d}, J=6.5 \mathrm{~Hz}, 2 \mathrm{H}) \mathrm{ppm}$;

${ }^{13}$ C NMR (126 MHz, $\left.\mathrm{CDCl}_{3}\right) \delta 158.4,155.2,137.5,130.4,121.1,115.1,106.8,99.1$, 55.6, $33.7 \mathrm{ppm}$;

IR (film, $\left.\mathrm{cm}^{-1}\right) 3308$ (broad), 2967, 2911, 1614, 1599;

HRMS $\left(\mathrm{ESI}^{+}\right) 165.0910$ (calculated $[\mathrm{M}+\mathrm{H}]^{+}$165.0910). 


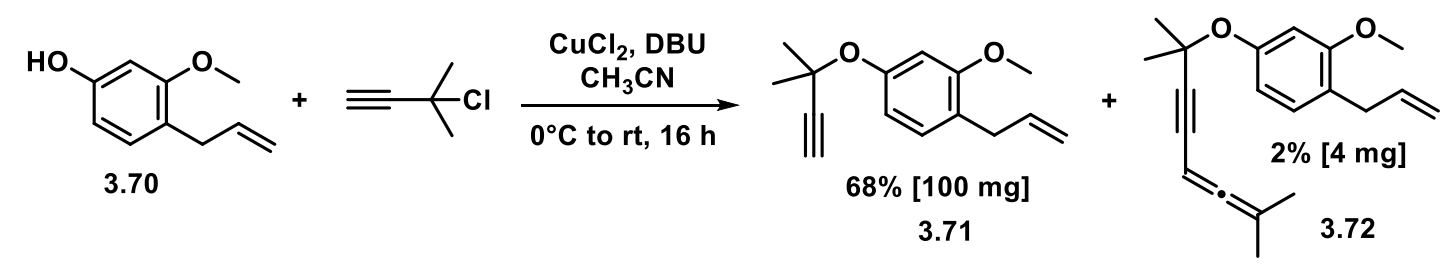

Phenol 3.70 (109 mg, $0.66 \mathrm{mmol})$ was dissolved in anhydrous acetonitrile $(3 \mathrm{~mL})$. DBU $(120 \mu \mathrm{L}, 0.79 \mathrm{mmol})$, copper chloride (1 mg, $6.0 \mu \mathrm{mol})$ and 3-chloro-3-methyl1-butyne $(67 \mu \mathrm{L}, 0.60 \mathrm{mmol})$ were added at $0{ }^{\circ} \mathrm{C}$. The mixture was stirred at $\mathrm{rt}$ for 16 $\mathrm{h}$, then diethyl ether $(7 \mathrm{~mL})$ and $1 \mathrm{Maq} . \mathrm{HCl}(1.5 \mathrm{~mL})$, were added. The layers were separated and the organic phase was washed with brine $(2 \times 8 \mathrm{~mL})$. The organic layer was dried over anhydrous $\mathrm{Na}_{2} \mathrm{SO}_{4}$ and the solvent was removed under reduced pressure. Flash chromatography (98:2 petroleum spirit 40-60 / ethyl acetate) afforded alkyne 3.71 as a pale-yellow oil ( $94 \mathrm{mg}, 0.41 \mathrm{mmol}, 68 \%$ yield) along with allene $\mathbf{3 . 7 2}$ as a pale-yellow oil (4 $\mathrm{mg}, 0.013 \mathrm{mmol}, 2 \%$ yield).

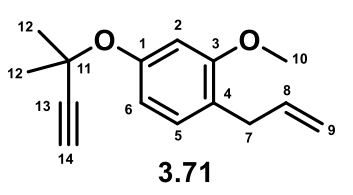

$\mathbf{R}_{\mathbf{f}}$ 0.36 (85:15 petroleum spirit 40-60 / diethyl ether);

${ }^{1} \mathbf{H}$ NMR $\left(500 \mathrm{MHz}, \mathrm{CDCl}_{3}\right) \delta 6.91(\mathrm{~d}, J=8.1 \mathrm{~Hz}, 1 \mathrm{H}, \mathrm{H} 5), 6.70(\mathrm{dd}, J=8.1,2.3 \mathrm{~Hz}$, 1H, H6), 6.66 (d, $J=2.3 \mathrm{~Hz}, 1 \mathrm{H}, \mathrm{H} 2$ ), 5.89 (ddt, $J=16.8,10.1,6.6 \mathrm{~Hz}, 1 \mathrm{H}, \mathrm{H} 8$ ), 4.974.91 (m, 2H, H9), 3.70 (s, 3H, H10), 3.23 (dd, $J=6.6,1.6$ Hz, 2H, H7), 2.46 (s, 1H, H14), 1.55 (s, 6H, H12) ppm;

${ }^{13}$ C NMR (126 MHz, $\left.\mathrm{CDCl}_{3}\right) \delta 157.6$ (C3), 155.2 (C1), 137.4 (C8), 129.4 (C5), 123.2 (C4), 115.3 (C9), 113.0 (C6), 105.0 (C2), 86.6 (C13), 73.7 (C14), 72.5 (C11), 55.5 (C10), 33.8 (C7), 29.8 (2C, C12) ppm;

IR (film, $\mathrm{cm}^{-1}$ ) 2988, 2936, 1605, 1563;

HRMS $\left(\mathrm{ESI}^{+}\right) 253.1205$ (calculated $[\mathrm{M}+\mathrm{Na}]^{+}$253.1199). 


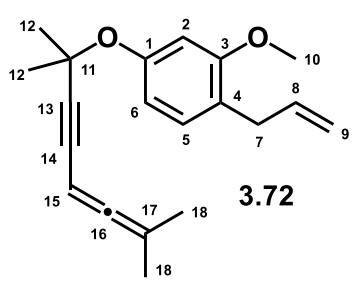

Rf 0.52 (85:15 petroleum spirit 40-60 / diethyl ether);

${ }^{1} \mathbf{H}$ NMR $\left(500 \mathrm{MHz}, \mathrm{CDCl}_{3}\right) \delta 6.99(\mathrm{~d}, J=8.2 \mathrm{~Hz}, 1 \mathrm{H}, \mathrm{H} 5), 6.84(\mathrm{~d}, J=2.3 \mathrm{~Hz}, 1 \mathrm{H}$, H2), 6.72 (dd, $J=8.2,2.3 \mathrm{~Hz}, 1 \mathrm{H}, \mathrm{H} 6$ ), 5.99 (ddt, $J=16.8,10.1,6.6 \mathrm{~Hz}, 1 \mathrm{H}, \mathrm{H} 8$ ), 5.24 (sept, $J=2.9 \mathrm{~Hz}, 1 \mathrm{H}, \mathrm{H} 15), 5.06-4.99$ (m, 2H, H9), 3.79 (s, 3H, H10), 3.34-3.31 (m, 2H, H7), 1.72 (d, $J=2.9 \mathrm{~Hz}, 6 \mathrm{H}, \mathrm{H} 18), 1.63$ (s, 6H, H12) ppm;

${ }^{13}$ C NMR (126 MHz, $\left.\mathrm{CDCl}_{3}\right) \delta 210.3$ (C16), 157.6 (C3), 155.4 (C1), 137.5 (C8), 129.4 (C5), 123.0 (C4), 115.2 (C9), 113.5 (C6), 105.2 (C2), 97.7 (C17), 91.2 (C13), 79.9 (C14), 73.4 (C11), 73.0 (C15), 55.5 (C10), 33.9 (C7), 29.8 (2C, C12), 20.1 (2C, C18) ppm;

IR (film, cm ${ }^{-1}$ ) 2987, 2936, 1958, 1604, 1568;

HRMS $\left(\mathrm{ESI}^{+}\right) 297.1844$ (calculated $[\mathrm{M}+\mathrm{H}]^{+}$297.1849). 


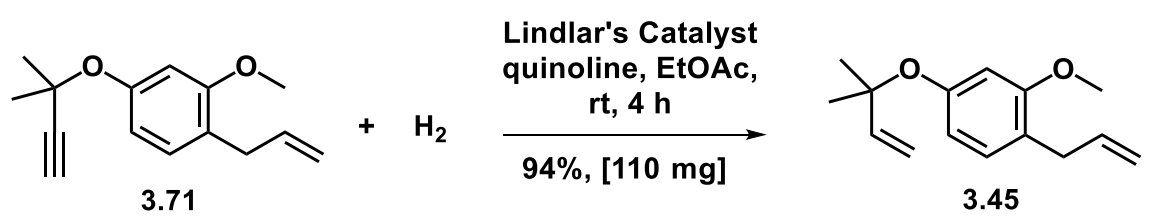

Propargyl ether 3.71 (120 mg, $0.52 \mathrm{mmol})$ was dissolved in ethyl acetate $(10 \mathrm{~mL})$. Quinoline $(530 \mu \mathrm{L}, 4.47 \mathrm{mmol})$ and Lindlar's catalyst $(28 \mathrm{mg})$ were added at rt. The suspension was frozen and the flask was evacuated and then fitted with an $\mathrm{H}_{2}$ balloon. The mixture was then allowed to warm to the rt and stirred until complete consumption of the starting material, judged by TLC analysis $(4 \mathrm{~h})$. The mixture was diluted with ethyl acetate $(15 \mathrm{~mL})$, filtered through a short pad of celite and the solvent was removed under reduced pressure. Flash chromatography (90:10 petroleum spirit 40-60 / ethyl acetate) afforded alkene $\mathbf{3 . 4 5}$ as a pale-yellow oil (114 mg, 0.49 mmol, 94\% yield).

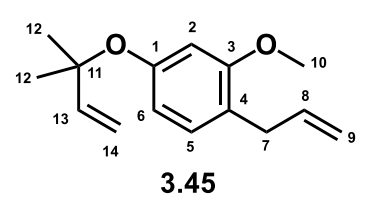

$\mathbf{R}_{\mathbf{f}}$ 0.56 (80:20 petroleum spirit 40-60 / ethyl acetate);

${ }^{1} \mathbf{H}$ NMR $\left(500 \mathrm{MHz}, \mathrm{CDCl}_{3}\right) \delta 6.94(\mathrm{~d}, J=8.1 \mathrm{~Hz}, 1 \mathrm{H}, \mathrm{H} 5), 6.55(\mathrm{dd}, J=8.2,2.3 \mathrm{~Hz}$, $1 \mathrm{H}, \mathrm{H} 6), 6.52$ (d, $J=2.3 \mathrm{~Hz}, 1 \mathrm{H}, \mathrm{H} 2), 6.16$ (dd, $J=17.6,10.9 \mathrm{~Hz}, 1 \mathrm{H}, \mathrm{H} 13), 5.97$ (ddt, $J=16.8,10.2,6.6 \mathrm{~Hz}, 1 \mathrm{H}, \mathrm{H} 8), 5.18$ (dd, $J=17.6,1.0 \mathrm{~Hz}, 1 \mathrm{H}, \mathrm{H} 14), 5.13$ (dd, $J=$ 10.9, $1.0 \mathrm{~Hz}, 1 \mathrm{H}, \mathrm{H} 14), 5.06-4.99$ (m, 2H, H9), 3.76 (s, 3H, H10), 3.32-3.27 (m, 2H, H7), 1.45 (s, 6H, H12) ppm;

${ }^{13} \mathbf{C}$ NMR (126 MHz, $\mathrm{CDCl}_{3}$ ) $\delta 157.6$ (C3), 155.7 (C1), 145.9 (C13), 137.5 (C8), 129.3 (C5), 122.4 (C4), 115.2 (C9), 113.3 (C14), 113.1 (C6), 105.2 (C2), 79.6 (C11), 55.5 (C10), 33.8 (C7), 27.2 (2C, C12) ppm;

IR (film, $\mathrm{cm}^{-1}$ ) 2978, 2934, 1607, 1584, 1501;

HRMS $\left(\mathrm{ESI}^{+}\right) 233.1536$ (calculated $[\mathrm{M}+\mathrm{H}]^{+}$233.1536). 


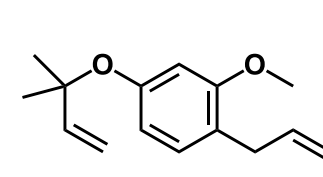

3.45
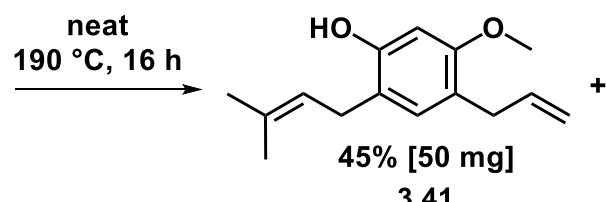

3.41

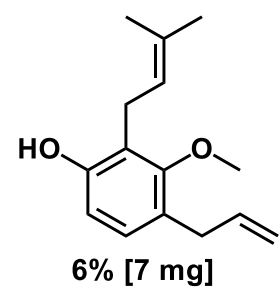

3.73

Ether 3.45 (113 mg, $0.49 \mathrm{mmol})$, neat, was heated to $190{ }^{\circ} \mathrm{C}$ for $16 \mathrm{~h}$ under nitrogen atmosphere. The crude was directly subjected to chromatographic purification. Flash chromatography (70:30 petroleum spirit 40-60 / diethyl ether) afforded phenol 3.41 as a pale-yellow oil (50 mg, $0.22 \mathrm{mmol}, 50 \%$ yield) and its isomer $\mathbf{3 . 7 3}$ as a pale-yellow oil (7 mg, $0.030 \mathrm{mmol}, 6 \%$ yield).

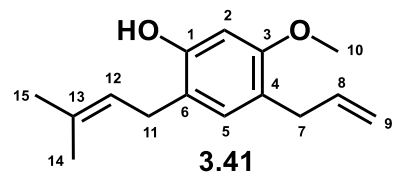

$\mathbf{R}_{\mathbf{f}}$ 0.19 (70:30 petroleum spirit 40-60 / diethyl ether);

${ }^{1} \mathbf{H}$ NMR $\left(500 \mathrm{MHz}, \mathrm{CDCl}_{3}\right) \delta 6.82(\mathrm{~s}, 1 \mathrm{H}, \mathrm{H} 5), 6.40$ (s, 1H, H2), 5.97 (ddt, $J=16.7$, 10.1, $6.5 \mathrm{~Hz}, 1 \mathrm{H}, \mathrm{H} 8), 5.32-5.28$ (m, 1H, H12), 5.10 (broad s, 1H, OH at C1), 5.054.98 (m, 2H, H9), 3.77 (s, 3H, H10), 3.29-3.27 (m, 4H, H7 + H11), 1.79 (s, 3H, H14 or H15), 1.77 (d, $J=1.4 \mathrm{~Hz}, 3 \mathrm{H}, \mathrm{H} 14$ or H15) ppm;

${ }^{13}$ C NMR (126 MHz, $\left.\mathrm{CDCl}_{3}\right) \delta 156.9$ (C3), 153.8 (C1), 137.7 (C8), 134.8 (C13), 131.1 (C5), 122.5 (C12), 120.6 (C4), 117.9 (C6), 115.0 (C9), 99.7 (C2), 55.7 (C10), 33.7 (C7 or C11), 29.6 (C7 or C11), 25.9 (C14 or C15), 18.0 (C14 or C15) ppm;

IR (film, $\mathrm{cm}^{-1}$ ) 3416 (broad), 2963, 1913, 1616, 1512;

HRMS $\left(\mathrm{ESI}^{+}\right) 233.1533$ (calculated $[\mathrm{M}+\mathrm{H}]^{+}$233.1536). 


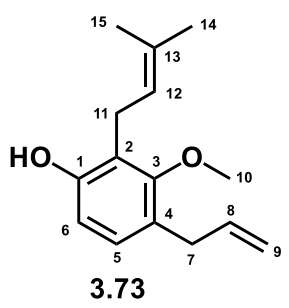

Rf 0.55 (70:30 petroleum spirit 40-60 / diethyl ether);

${ }^{1} \mathbf{H}$ NMR $\left(500 \mathrm{MHz}, \mathrm{CDCl}_{3}\right) \delta 6.93(\mathrm{~d}, J=8.4 \mathrm{~Hz}, 1 \mathrm{H}, \mathrm{H} 5), 6.59(\mathrm{~d}, J=8.3 \mathrm{~Hz}, 1 \mathrm{H}$, H6), 5.97 (ddt, $J=17.9,9.4,6.5 \mathrm{~Hz}, 1 \mathrm{H}, \mathrm{H} 8$ ), 5.26-5.22 (m, 1H, H12), 5.14 (broad s, $1 \mathrm{H}, \mathrm{OH}$ at C1), 5.08-5.03 (m, 2H, H9), 3.70 (s, 3H, H10), 3.45-3.41 (m, 2H, H11), 3.37-3.34 (m, 2H, H7), 1.84-1.83 (m, 3H, H14 or H15), 1.76-1.75 (m, 3H, H14 or H15) ppm;

${ }^{13} \mathbf{C}$ NMR $\left(126 \mathrm{MHz}, \mathrm{CDCl}_{3}\right) \delta 156.8(\mathrm{C} 3), 154.5$ (C1), 137.9 (C8), 135.1 (C13), 128.6 (C5), 125.1 (C4), 122.2 (C12), 120.5 (C2), 115.5 (C9), 112.1 (C6), 61.7 (C10), 33.9 (C7), 25.9 (C14 or C15), 23.6 (C11), 18.1 (C14 or C15) ppm;

IR (film, cm ${ }^{-1}$ ) 3406, 2965, 2913, 1599, 1458, 1423;

HRMS $\left(\mathrm{ESI}^{+}\right.$) 233.1527 (calculated $[\mathrm{M}+\mathrm{H}]^{+}$233.1536). 


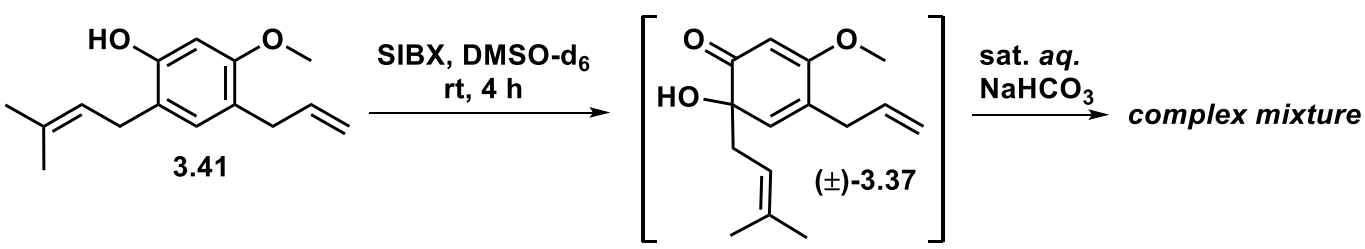

Phenol 3.41 (6 mg, $27 \mu \mathrm{mol}$ ) was dissolved in DMSO-d 6 (0.6 mL). SIBX (40 mg, 64 $\mu \mathrm{mol}, 45 \% \mathrm{w} / \mathrm{w}$ ) was then added in one portion. The reaction was monitored by ${ }^{1} \mathrm{H}$ NMR and judged to be complete after $4 \mathrm{~h}$ (see page 378 for stacked ${ }^{1} \mathrm{H}$ NMR spectra). Attempts on separating ortho-quinol $( \pm)$-3.37 from the residual resulted in the formation of a mixture of unidentified products.

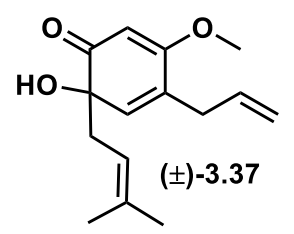

${ }^{1} \mathbf{H}$ NMR $\left(500 \mathrm{MHz}, \mathrm{CDCl}_{3}\right) \delta 6.07(\mathrm{~d}, J=1.3 \mathrm{~Hz}, 1 \mathrm{H}), 5.87-5.73(\mathrm{~m}, 1 \mathrm{H}), 5.39(\mathrm{~s}$, 1H), 5.10-4.98 (m, 2H), 4.97-4.89 (m, 1H), 3.74 (s, 3H), 3.01-2.88 (m, 2H), 2.34 (dd, $J=13.7,7.9 \mathrm{~Hz}, 1 \mathrm{H}), 2.18(\mathrm{dd}, J=13.8,7.3 \mathrm{~Hz}, 1 \mathrm{H}), 1.58(\mathrm{~s}, 3 \mathrm{H}), 1.46(\mathrm{~s}, 3 \mathrm{H}) \mathrm{ppm}$. 


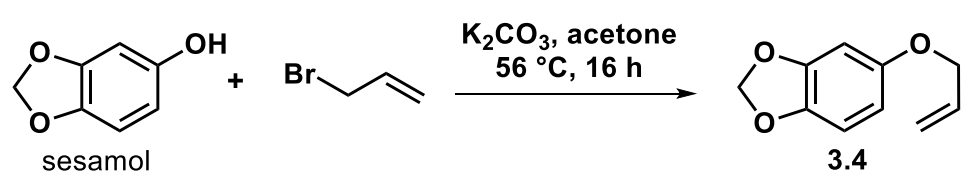

Compound 3.4 was prepared using a modified literature procedure. ${ }^{165}$

Sesamol (19.79 g, $140.4 \mathrm{mmol})$ was dissolved in anhydrous acetone (400 mL), then potassium carbonate $(20.35 \mathrm{~g}, 147.4 \mathrm{mmol})$ and allyl bromide $(17.3 \mathrm{~mL}, 193.7 \mathrm{mmol})$ were added. The mixture was heated at $56{ }^{\circ} \mathrm{C}$ until complete consumption of the starting material, judged by TLC analysis $(16 \mathrm{~h})$. The mixture was then diluted with $\mathrm{CH}_{2} \mathrm{Cl}_{2}(400 \mathrm{~mL})$ and washed with $2 \mathrm{M} a q$. $\mathrm{NaOH}(400 \mathrm{~mL})$. The organic layer was dried over anhydrous $\mathrm{Na}_{2} \mathrm{SO}_{4}$ and the solvent was removed under reduced pressure to afford allyl ether as a colourless oil (25.0 g, $140.3 \mathrm{mmol}, 99 \%$ crude yield). The crude material was deemed sufficiently pure ( $\left(\mathrm{see}^{1} \mathrm{H}\right.$ and ${ }^{13} \mathrm{C}$ NMR spectra on pages 379 and 380 ) to be used in the next step. All data for compound $\mathbf{3 . 4}$ matched literature values. ${ }^{165}$

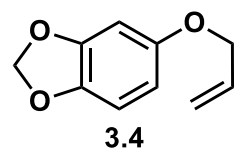

$\mathbf{R}_{\mathbf{f}}$ 0.54 (70:30 petroleum spirit 40-60 / diethyl ether);

${ }^{1} \mathbf{H}$ NMR $\left(500 \mathrm{MHz}, \mathrm{CDCl}_{3}\right) \delta 6.70(\mathrm{~d}, J=8.5 \mathrm{~Hz}, 1 \mathrm{H}), 6.52(\mathrm{~d}, J=2.5 \mathrm{~Hz}, 1 \mathrm{H}), 6.34$ $(\mathrm{dd}, J=8.5,2.5 \mathrm{~Hz}, 1 \mathrm{H}), 6.03$ (ddt, $J=17.3,10.6,5.3 \mathrm{~Hz}, 1 \mathrm{H}), 5.91$ (s, 2H), 5.42-5.36 (m, 1H), 5.29-5.25 (m, 1H), $4.46(\mathrm{dt}, J=5.3,1.5 \mathrm{~Hz}, 2 \mathrm{H}) \mathrm{ppm}$;

${ }^{13}$ C NMR (126 MHz, $\left.\mathrm{CDCl}_{3}\right) \delta 154.3,148.4,141.9,133.6,117.8,108.1,106.2,101.3$, 98.5, 70.0 ppm. 


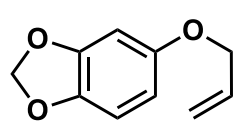

3.4

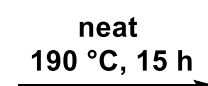

$190^{\circ} \mathrm{C}, 15 \mathrm{~h}$<smiles>C=CCc1cc2c(cc1O)OCO2</smiles>

3.5

Compound 3.5 was prepared using a modified literature procedure. ${ }^{165}$

Crude allyl ether $3.4(25.0 \mathrm{~g}, 140.3 \mathrm{mmol})$ was heated, neat, to $190{ }^{\circ} \mathrm{C}$ for $15 \mathrm{~h}$. The crude material was directly subjected to the next step without further purification. Analytically pure samples could be obtained via flash chromatography (70:30 petroleum spirit 40-60 / diethyl ether) to afford phenol 3.5 as a pale yellow crystalline solid. All data for compound $\mathbf{3 . 5}$ matched literature values. ${ }^{165}$

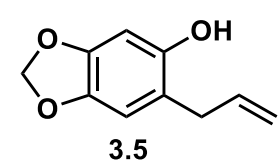

Rf 0.27 ( $70: 30$ petroleum spirit 40-60 / diethyl ether);

${ }^{1} \mathbf{H}$ NMR $\left(500 \mathrm{MHz}, \mathrm{CDCl}_{3}\right) \delta 6.58(\mathrm{~s}, 1 \mathrm{H}), 6.43(\mathrm{~s}, 1 \mathrm{H}), 5.97(\mathrm{ddt}, J=17.6,9.7,6.3$ $\mathrm{Hz}, 1 \mathrm{H}), 5.88$ (s, 2H), 5.18-5.15 (m, 1H), 5.14-5.12 (m, 1H), 4.65 (s, 1H), 3.31 (dt, $J$ $=6.3,1.6 \mathrm{~Hz}, 2 \mathrm{H}) \mathrm{ppm}$;

${ }^{13}$ C NMR $\left(126 \mathrm{MHz}, \mathrm{CDCl}_{3}\right) \delta 148.8,146.9,141.7,136.6,116.9,116.6,109.7,101.2$, 98.8, $35.2 \mathrm{ppm}$. 


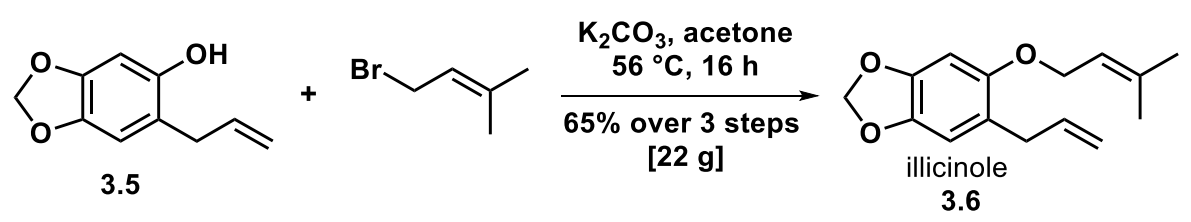

Compound 3.6 was prepared using a modified literature procedure. ${ }^{120}$

Crude phenol 3.5 (24.7 g) was dissolved in anhydrous acetone $(500 \mathrm{~mL})$, then potassium carbonate $(95.7 \mathrm{~g}, 693 \mathrm{mmol})$ and prenyl bromide $(20.2 \mathrm{~mL}, 166.4 \mathrm{mmol})$ were added. The mixture was heated at $56{ }^{\circ} \mathrm{C}$ until complete consumption of the starting material, judged by TLC analysis $(16 \mathrm{~h})$. The mixture was then diluted with $\mathrm{CH}_{2} \mathrm{Cl}_{2}(500 \mathrm{~mL})$ and washed with $1 \mathrm{M} a q$. $\mathrm{NaOH}(600 \mathrm{~mL})$. The organic layer was dried over anhydrous $\mathrm{Na}_{2} \mathrm{SO}_{4}$ and the solvent was removed under reduced pressure. Flash chromatography (95:5 petroleum spirit 40-60 / ethyl acetate) afforded ether 3.6 as a pale-yellow oil which solidified when stored at $-20{ }^{\circ} \mathrm{C}(22.15 \mathrm{~g}, 89.9 \mathrm{mmol})$ in $65 \%$ yield over 3 steps from sesamol. All data for compound 3.6 matched literature values. $^{120}$

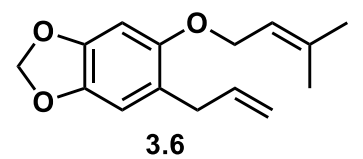

$\mathbf{R}_{\mathbf{f}}$ 0.42 (90:10 petroleum spirit 40-60 / ethyl acetate);

${ }^{1}$ H NMR $\left(500 \mathrm{MHz}, \mathrm{CDCl}_{3}\right) \delta 6.65(\mathrm{~s}, 1 \mathrm{H}), 6.53(\mathrm{~s}, 1 \mathrm{H}), 5.93$ (ddt, $J=16.8,10.1,6.6$ $\mathrm{Hz}, 1 \mathrm{H}), 5.88$ (s, 2H), 5.47-5.44 (m, 1H), 5.07-4.99 (m, 2H), $4.44(\mathrm{dm}, J=6.7 \mathrm{~Hz}$, $2 \mathrm{H}), 3.31(\mathrm{dm}, J=6.6 \mathrm{~Hz}, 2 \mathrm{H}), 1.79-1.78(\mathrm{~m}, 3 \mathrm{H}), 1.72-1.71(\mathrm{~m}, 3 \mathrm{H}) \mathrm{ppm}$;

${ }^{13}$ C NMR (126 MHz, $\left.\mathrm{CDCl}_{3}\right) \delta 151.4,146.3,141.3,137.5,137.5,121.7,120.4,115.4$, $109.7,101.1,96.8,66.9,34.2,25.9,18.4$ ppm. 

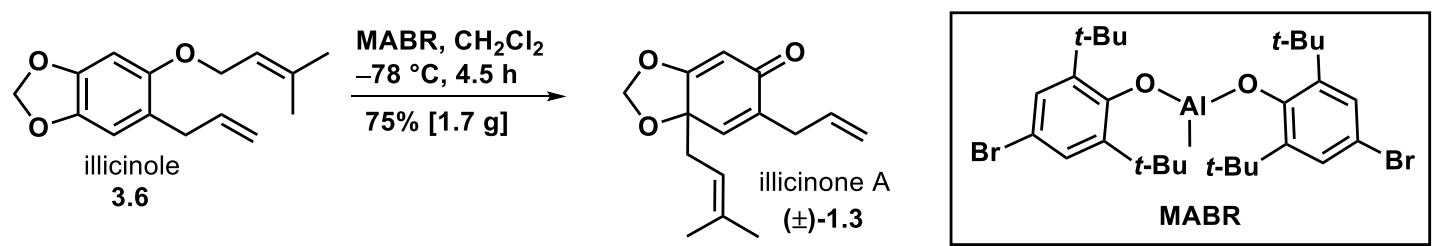

$( \pm)$-Illicinone A 1.3 was prepared using a modified literature procedure. ${ }^{120}$

4-Bromo-2,6-di-tertbutyl phenol (11.68 g, $40.0 \mathrm{mmol})$ was dissolved in anhydrous $\mathrm{CH}_{2} \mathrm{Cl}_{2}(80 \mathrm{~mL})$. Trimethylaluminium $(10.0 \mathrm{~mL}, 20.0 \mathrm{mmol}, 2 \mathrm{M}$ in hexane) was added dropwise at $\mathrm{rt}$ and the mixture was stirred for $1 \mathrm{~h}$. A solution of illicinole $\mathbf{3 . 6}$ $(2.35 \mathrm{~g}, 9.52 \mathrm{mmol})$ in anhydrous $\mathrm{CH}_{2} \mathrm{Cl}_{2}(10 \mathrm{~mL})$ was then added within $30 \mathrm{~min}$ at $-78{ }^{\circ} \mathrm{C}$. The mixture was stirred at the same temperature until complete consumption of the starting material, judged by ${ }^{1} \mathrm{H}$ NMR analysis $(3 \mathrm{~h})$. The reaction was then carefully quenched adding $1 \mathrm{M} a q . \mathrm{HCl}(50 \mathrm{~mL})$, then the mixture was extracted with diethyl ether $(3 \times 50 \mathrm{~mL})$. The combined organic layers were washed with brine $(150$ $\mathrm{mL}$ ), dried over anhydrous $\mathrm{Na}_{2} \mathrm{SO}_{4}$ and the solvent was removed under reduced pressure. Flash chromatography (90:10 petroleum spirit 40-60 / ethyl acetate) afforded ( \pm )-illicinone A 1.3 as a pale orange oil (1.74 g, $7.06 \mathrm{mmol}, 75 \%$ yield). All data for compound $( \pm)$-1.3 matched literature values. ${ }^{120}$

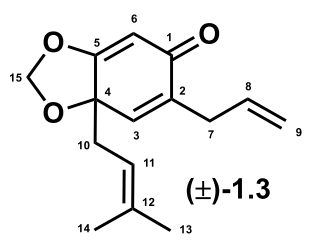

$\mathbf{R}_{\mathbf{f}} 0.30$ (90:10 petroleum spirit 40-60 / ethyl acetate);

${ }^{1} \mathbf{H}$ NMR $\left(500 \mathrm{MHz}, \mathrm{CDCl}_{3}\right) \delta 6.60(\mathrm{t}, J=1.5 \mathrm{~Hz}, 1 \mathrm{H}, \mathrm{H} 3), 5.81$ (ddt, $J=16.4,10.8$, $6.8 \mathrm{~Hz}, 1 \mathrm{H}, \mathrm{H} 8), 5.62$ (s, 1H, H15), 5.59 (s, 1H, H15), 5.54 (s, 1H, H6), 5.13-5.05 (m, 2H, H9), 5.03-4.94 (m, 1H, H11), 3.12-2.98 (m, 2H, H7), 2.46 (dm, J=14.2 Hz, 2H, H10), 1.68 (d, $J=1.4$ Hz, 3H, H13 or H14), 1.54 (d, J = 1.4 Hz, 3H, H13 or H14) ppm;

${ }^{13}$ C NMR (126 MHz, $\left.\mathrm{CDCl}_{3}\right) \delta 187.0(\mathrm{C} 1), 173.9$ (C5), 139.5 (C2), 137.8 (C12), 135.0 (C8), 134.5 (C3), 117.4 (C9), 116.1 (C11), 98.5 (C6), 98.2 (C15), 82.2 (C4), 35.0 (C10), 33.6 (C7), 26.0 (C13 or C14), 18.0 (C13 or C14) ppm. 

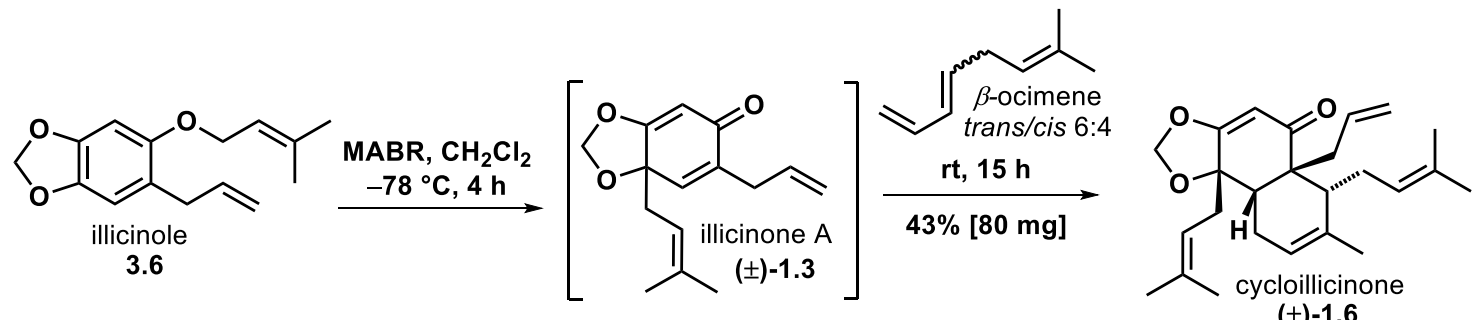

4-Bromo-2,6-di-tertbutyl phenol $(539 \mathrm{mg}, 1.85 \mathrm{mmol})$ was dissolved in anhydrous $\mathrm{CH}_{2} \mathrm{Cl}_{2}(3.5 \mathrm{~mL})$. Trimethylaluminium $(460 \mu \mathrm{L}, 0.92 \mathrm{mmol}, 2 \mathrm{M}$ in hexane) was added dropwise at $\mathrm{rt}$ and the mixture was stirred for $1 \mathrm{~h}$. A solution of illicinole 3.6 (114 mg, $0.46 \mathrm{mmol})$ in anhydrous $\mathrm{CH}_{2} \mathrm{Cl}_{2}(1.5 \mathrm{~mL})$ was then added within $30 \mathrm{~min}$ at $-78{ }^{\circ} \mathrm{C}$. The mixture was stirred at the same temperature until complete consumption of the starting material, judged by by ${ }^{1} \mathrm{H}$ NMR analysis $(2.5 \mathrm{~h}) . \beta$-Ocimene $(580 \mu \mathrm{L}, 2.1$ mmol, 60:40 trans/cis mixture of isomers) was then added and the mixture was slowly allowed to warm to the rt and stirred for $15 \mathrm{~h}$. The reaction was then carefully quenched adding $1 \mathrm{M} a q . \mathrm{HCl}(4 \mathrm{~mL})$, then the mixture was extracted with diethyl ether $(3 \times 5$ $\mathrm{mL})$. The combined organic layers were washed with brine $(10 \mathrm{~mL})$, dried over anhydrous $\mathrm{Na}_{2} \mathrm{SO}_{4}$ and the solvent was removed under reduced pressure. Flash chromatography (90:10 petroleum spirit 40-60 / ethyl acetate) afforded $( \pm)$-cycloillicinone $\mathbf{1 . 6}$ as a single diastereoisomer (pale yellow crystalline solid, 77 $\mathrm{mg}, 0.20 \mathrm{mmol}, 44 \%$ yield).

All data for compound $( \pm)-\mathbf{1 . 6}$ matched literature values. ${ }^{127}$

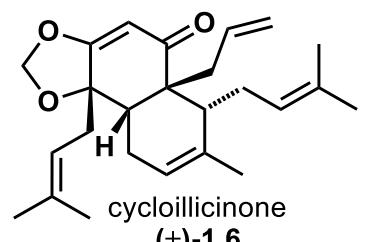

$( \pm)-1.6$

Rf 0.26 (90:10 petroleum spirit 40-60 / ethyl acetate);

${ }^{1} \mathbf{H}$ NMR $\left(600 \mathrm{MHz}, \mathrm{CDCl}_{3}\right) \delta 5.74$ (dddd, $\left.J=16.9,10.0,8.0,6.8 \mathrm{~Hz}, 1 \mathrm{H}\right), 5.60-5.56$ (m, 1H), $5.54(\mathrm{~s}, 2 \mathrm{H}), 5.40(\mathrm{~s}, 1 \mathrm{H}), 5.18-5.13(\mathrm{~m}, 1 \mathrm{H}), 5.05-5.01(\mathrm{~m}, 1 \mathrm{H}), 5.01-4.95$ (m, $2 \mathrm{H}), 2.55(\mathrm{dd}, J=13.9,8.0 \mathrm{~Hz}, 1 \mathrm{H}), 2.51(\mathrm{dd}, J=11.5,5.7 \mathrm{~Hz}, 1 \mathrm{H}), 2.52-2.45(\mathrm{~m}$, $1 \mathrm{H}), 2.39-2.32(\mathrm{~m}, 1 \mathrm{H}), 2.22(\mathrm{ddd}, J=15.2,7.5,5.7 \mathrm{~Hz}, 1 \mathrm{H}), 2.16(\mathrm{dd}, J=15.8,7.7$ $\mathrm{Hz}, 1 \mathrm{H}), 2.11(\mathrm{dd}, J=10.8,4.4 \mathrm{~Hz}, 1 \mathrm{H}), 2.01-1.90$ (m, 3H), 1.72 (s, 3H), 1.67 (apparent $\mathrm{t}, J=1.9 \mathrm{~Hz}, 3 \mathrm{H}), 1.62(\mathrm{~s}, 3 \mathrm{H}), 1.57(\mathrm{~s}, 3 \mathrm{H}), 1.50(\mathrm{~s}, 3 \mathrm{H}) \mathrm{ppm}$ 
${ }^{13}$ C NMR $\left(150 \mathrm{MHz}, \mathrm{CDCl}_{3}\right) \delta 201.6,172.7,142.3,136.5,135.0,132.3,123.6,121.8$, 118.1, 116.7, 99.5, 98.0, 84.1, 54.1, 51.4, 45.4, 41.0, 37.4, 29.8, 26.0, 25.8, 24.5, 24.1, 18.5, 17.9 ppm;

HRMS $\left(\mathrm{ESI}^{+}\right) 382.2504$ (calculated $[\mathrm{M}+\mathrm{H}]^{+}$382.2503);

IR: (film, cm ${ }^{-1}$ ) 2961, 2911, 1667, 1636;

m.p. $54-55^{\circ} \mathrm{C}\left(\mathrm{CH}_{2} \mathrm{Cl}_{2}\right)$. 


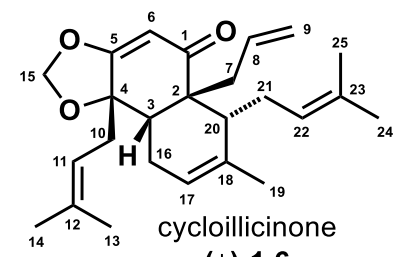

( \pm -1.6

\begin{tabular}{|c|c|c|c|c|c|c|}
\hline \multirow[t]{2}{*}{ Atom } & \multicolumn{3}{|c|}{ Synthetic $( \pm)$-Cycloillicinone $1.6\left(\mathrm{CDCl}_{3}\right)$} & \multicolumn{3}{|c|}{$\begin{array}{l}\text { Natural ( } \pm \text { )-Cycloillicinone 1.6 (Fukuyama) })^{127} \\
\left(\mathrm{CDCl}_{3}\right)\end{array}$} \\
\hline & $\delta^{1} \mathrm{H}(600 \mathrm{MHz})$ & $\mathbf{m} J(\mathrm{~Hz})$ & $\delta^{13} \mathrm{C}(150 \mathrm{MHz})$ & $\delta^{1} \mathrm{H}(600 \mathrm{MHz})$ & $\mathbf{m} J(\mathbf{H z})$ & $\delta^{13} \mathrm{C}(150 \mathrm{MHz})$ \\
\hline 1 & - & - & 201.6 & - & - & 201.5 \\
\hline 2 & - & - & 54.1 & - & - & 53.9 \\
\hline 3 & 2.51 & $\begin{array}{c}\mathrm{dd}, 11.5 \\
5.7\end{array}$ & 40.9 & 2.51 & $\begin{array}{c}\mathrm{dd}, 11.5 \\
6.0\end{array}$ & 40.7 \\
\hline 4 & - & - & 84.1 & - & - & 83.9 \\
\hline 5 & - & - & 172.7 & - & - & 172.6 \\
\hline 6 & $5.54^{\dagger}$ & $\mathrm{s}$ & 99.5 & 5.55 & $\mathrm{~s}$ & 99.4 \\
\hline 7 & 2.55 & $\begin{array}{l}\mathrm{dd}, 13.9 \\
\quad 8.0\end{array}$ & 45.4 & 2.56 & $\begin{array}{l}\mathrm{dd}, 14.0 \\
8.0\end{array}$ & 45.2 \\
\hline 8 & 5.74 & $\begin{array}{c}\text { dddd, } 16.9 \\
10.0,8.0 \\
6.8\end{array}$ & 135.0 & 5.75 & $\begin{array}{c}\text { dddd, } 17.0 \\
8.0,7.9 \\
6.9\end{array}$ & 134.9 \\
\hline \multirow{2}{*}{9} & $5.05-5.01$ & $\mathrm{~m}$ & \multirow{2}{*}{118.1} & 5.04 & $\mathrm{~d}, 17.0$ & \multirow{2}{*}{118.0} \\
\hline & $5.01-4.95^{\dagger \dagger}$ & $\mathrm{m}$ & & 5.00 & $\mathrm{~d}, 7.9$ & \\
\hline \multirow{2}{*}{10} & $2.52-2.45$ & $\mathrm{~m}$ & \multirow{2}{*}{37.4} & 2.49 & $\begin{array}{c}\mathrm{dd}, 15.4 \\
5.8\end{array}$ & \multirow{2}{*}{37.1} \\
\hline & 2.16 & $\begin{array}{c}\mathrm{dd}, 15.8 \\
7.7\end{array}$ & & 2.17 & $\begin{array}{c}\mathrm{dd}, 15.4 \\
7.8\end{array}$ & \\
\hline 11 & $5.18-5.13$ & $\mathrm{~m}$ & 116.7 & 5.16 & $\mathrm{dd}, 7.8,5.8$ & 116.5 \\
\hline 12 & - & - & 136.5 & - & - & 136.4 \\
\hline 13 & 1.57 & $\mathrm{~S}$ & 18.5 & 1.56 & $\mathrm{~S}$ & 18.4 \\
\hline 14 & 1.72 & $\mathrm{~s}$ & 26.0 & 1.72 & $\mathrm{~S}$ & 25.9 \\
\hline \multirow{2}{*}{15} & $5.54^{\dagger}$ & $\mathrm{s}$ & \multirow{2}{*}{98.0} & 5.55 & $\mathrm{~S}$ & \multirow{2}{*}{97.9} \\
\hline & 5.40 & $\mathrm{~s}$ & & 5.41 & $\mathrm{~s}$ & \\
\hline \multirow{2}{*}{16} & 2.22 & $\begin{array}{c}\text { ddd, } 15.2 \\
7.5,5.7\end{array}$ & \multirow{2}{*}{24.1} & 2.23 & $\begin{array}{c}\text { ddd, } 15.1 \\
7.4,6.0\end{array}$ & \multirow{2}{*}{24.0} \\
\hline & $2.01-1.90^{\dagger \dagger \dagger}$ & $\mathrm{m}$ & & 1.97 & $\begin{array}{c}\mathrm{ddd}, 15.1 \\
11.5,5.5\end{array}$ & \\
\hline 17 & $5.60-5.56$ & $\mathrm{~m}$ & 121.8 & 5.59 & $\begin{array}{c}\mathrm{ddq}, 7.4 \\
5.5,1.6 \\
\end{array}$ & 121.6 \\
\hline 18 & - & - & 142.3 & - & - & 142.1 \\
\hline 19 & 1.67 & app. t, 1.9 & 24.5 & 1.68 & $\mathrm{~d}, 1.6$ & 24.4 \\
\hline 20 & 2.11 & $\begin{array}{c}\mathrm{dd}, 10.8 \\
4.4\end{array}$ & 51.4 & 2.12 & $\begin{array}{c}\mathrm{dd}, 10.4 \\
4.7\end{array}$ & 51.3 \\
\hline 21 & $2.01-1.90^{\dagger \dagger \dagger}$ & $\mathrm{m}$ & 29.8 & 1.93 & $\mathrm{~m}$ & 29.7 \\
\hline 22 & $5.01-4.95^{\dagger \dagger}$ & $\mathrm{m}$ & 123.6 & 4.99 & $\mathrm{~m}$ & 123.4 \\
\hline 23 & - & - & 132.3 & - & - & 132.2 \\
\hline 24 & 1.50 & $\mathrm{~s}$ & 17.9 & 1.50 & $\mathrm{~s}$ & 17.8 \\
\hline 25 & 1.62 & $\mathrm{~S}$ & 25.8 & 1.63 & $\mathrm{~s}$ & 25.6 \\
\hline
\end{tabular}

$\dagger, \dagger, \dagger \dagger$ overlapping signals. 

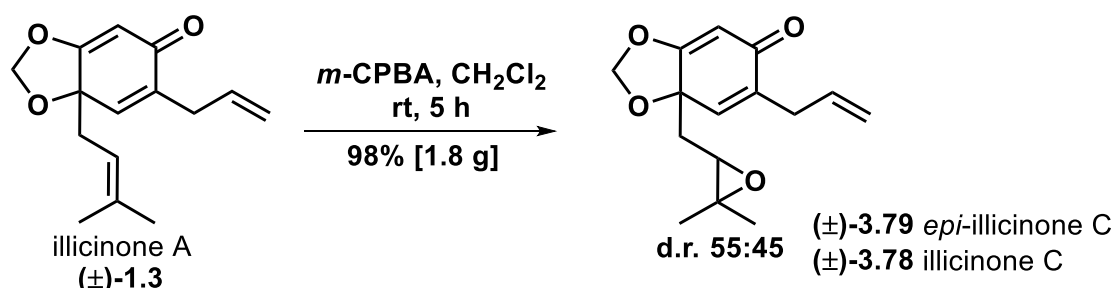

( \pm )-Illicinone A $1.3(1.70 \mathrm{~g}, 6.90 \mathrm{mmol})$ was dissolved in anhydrous $\mathrm{CH}_{2} \mathrm{Cl}_{2}(60 \mathrm{~mL})$, then $m$-CPBA $(2.01 \mathrm{~g}, 8.97 \mathrm{mmol})$ was added as a solid. The mixture was stirred at $\mathrm{rt}$ for $5 \mathrm{~h}$, then cooled to $0{ }^{\circ} \mathrm{C}$ until precipitation of a white solid ( $m$-CPBA and $m$-chlorobenzoic acid) was observed and the reaction was quenched with sat. $a q$. $\mathrm{Na}_{2} \mathrm{~S}_{2} \mathrm{O}_{3}(40 \mathrm{~mL})$. The mixture was filtered, washed with sat. $a q . \mathrm{NaHCO}_{3}(3 \times 50$ $\mathrm{mL}$ ) and the organic layer was dried over anhydrous $\mathrm{Na}_{2} \mathrm{SO}_{4}$. Removal of the solvent under reduced pressure afforded epoxide as a 55:45 mixture of diastereoisomers (colourless oil, $1.77 \mathrm{~g}, 6.75 \mathrm{mmol}, 98 \%$ crude yield) (see ${ }^{1} \mathrm{H}$ NMR spectrum on page 393). Flash chromatography (90:10 to 70:30 petroleum spirit 40-60 / ethyl acetate) afforded epoxide $( \pm$ )-epi-illicinone C $\mathbf{3 . 7 9}$ as a single diastereoisomer (colourless oil, $619 \mathrm{mg}, 2.36 \mathrm{mmol}, 34 \%$ yield) along with its stereoisomer ( \pm -illicinone C $\mathbf{3 . 7 9}$ as a colourless oil (313 $\mathrm{mg}, 1.19 \mathrm{mmol}, 17 \%$ yield). It was possible to isolate further quantities of both epoxides $( \pm)$-3.79 and $( \pm)$-3.78 purifying combined mixed fractions. All data for compounds $( \pm)-\mathbf{3 . 7 9}$ and $( \pm)$-3.78 matched literature values. ${ }^{135}$

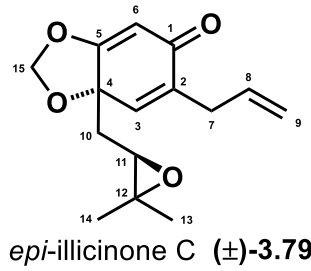

$\mathbf{R}_{\mathbf{f}} 0.24$ (70:30 petroleum spirit 40-60 / ethyl acetate);

${ }^{1} \mathbf{H}$ NMR $\left(500 \mathrm{MHz}, \mathrm{CDCl}_{3}\right) \delta 6.74(\mathrm{t}, J=1.6 \mathrm{~Hz}, 1 \mathrm{H}, \mathrm{H} 3), 5.84$ (ddt, $J=17.2,10.5$, $6.9 \mathrm{~Hz}, 1 \mathrm{H}, \mathrm{H} 8), 5.65$ (s, 1H, H15), 5.59 (s, 1H, H6), 5.59 (s, 1H, H15), 5.17-5.10 (m, 2H, H9), 3.10 (dd, $J=6.9,1.4$ Hz, 2H, H7), 2.73 (dd, $J=6.5,5.6$ Hz, 1H, H11), 2.22 (dd, $J=14.3,5.6 \mathrm{~Hz}, 1 \mathrm{H}, \mathrm{H} 10), 1.82$ (dd, $J=14.3,6.6 \mathrm{~Hz}, 1 \mathrm{H}, \mathrm{H} 10), 1.27$ (s, 3H, H13 or H14), 1.19 (s, 3H, H13 or H14) ppm; 
${ }^{13}$ C NMR (126 MHz, $\left.\mathrm{CDCl}_{3}\right) \delta 186.6(\mathrm{C} 1), 173.8$ (C5), 140.2 (C2), 134.6 (C8), 134.0 (C3), 118.0 (C9), 98.6 (C6), 98.3 (C15), 80.0 (C4), 59.2 (C11), 58.4 (C12), 35.8 (C10), 33.5 (C7), 24.6 (C13 or C14), 18.9 (C13 or C14) ppm.

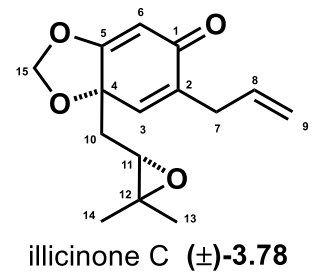

$\mathbf{R}_{\mathbf{f}} 0.22$ (70:30 petroleum spirit 40-60 / ethyl acetate);

${ }^{1} \mathbf{H}$ NMR $\left(500 \mathrm{MHz}, \mathrm{CDCl}_{3}\right) \delta 6.82(\mathrm{t}, J=1.5 \mathrm{~Hz}, 1 \mathrm{H}, \mathrm{H} 3)$, 5.89-5.80 (m, 1H, H8), 5.65 (s, 1H, H15), 5.60 (s, 1H, H6), 5.59 (s, 1H, H15), 5.17-5.09 (m, 2H, H9), 3.123.06 (m, 2H, H7), 2.82 (dd, $J=7.3,4.7 \mathrm{~Hz}, 1 \mathrm{H}, \mathrm{H} 11), 2.24$ (dd, $J=14.4,4.7 \mathrm{~Hz}, 1 \mathrm{H}$, H10), 1.74 (dd, $J=14.5,7.3$ Hz, 1H, H10), 1.28 (s, 3H, H13 or H14), 1.21 (s, 3H, H13 or H 14) ppm;

${ }^{13}$ C NMR (126 MHz, $\left.\mathrm{CDCl}_{3}\right) \delta 186.8$ (C1), 173.9 (C5), 139.3 (C2), 134.8 (C8), 134.7 (C3), 117.8 (C9), 98.8 (C6), 98.2 (C15), 80.3 (C4), 59.1 (C11), 58.4(C12), 36.5 (C10), 33.5 (C7), 24.7 (C13 or C14), 19.0 (C13 or C14) ppm. 

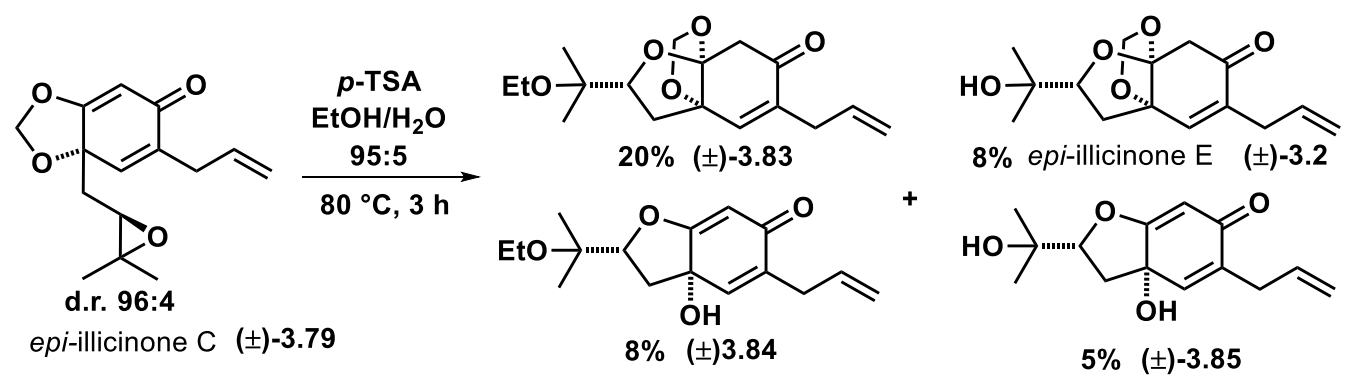

Epoxide ( \pm )-epi-illicinone C 3.79 (d.r. 96:4, 22 mg, $84 \mu \mathrm{mol}$ ) was dissolved in a 95:5 mixture of ethanol/water $(4 \mathrm{~mL})$. $p$-TSA $(1 \mathrm{mg}, 4 \mu \mathrm{mol})$ was added and the mixture was hated to $80^{\circ} \mathrm{C}$ until complete consumption of the starting material, judged by TLC analysis $(3 \mathrm{~h})$. The mixture was then extracted with $\mathrm{CH}_{2} \mathrm{Cl}_{2}(3 \times 5 \mathrm{~mL})$, the combined organic layers were dried over anhydrous $\mathrm{Na}_{2} \mathrm{SO}_{4}$ and the solvent was removed under reduced pressure. Flash chromatography (70:30 petroleum spirit 40-60 / ethyl acetate) afforded ether $( \pm)$-3.83 as a colourless oil ( $5 \mathrm{mg}, 16 \mu \mathrm{mol}, 20 \%$ yield), alcohol $( \pm)$-epiillicinone E 3.2 as a colourless oil ( $2 \mathrm{mg}, 7 \mu \mathrm{mol}, 8 \%$ yield $)$, ether $( \pm)-3.84$ as a colourless oil (2 mg, $7 \mu \mathrm{mol}, 8 \%$ yield $)$ and alcohol $( \pm)-\mathbf{3 . 8 5}$ as a colourless oil (1 mg, $4 \mu \mathrm{mol}, 5 \%$ yield).

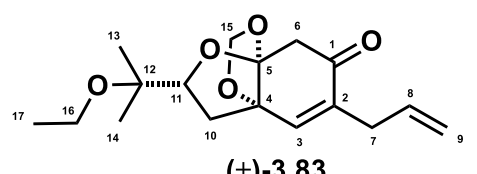

$( \pm)-3.83$

$\mathbf{R}_{\mathbf{f}} 0.40$ (70:30 petroleum spirit 40-60 / ethyl acetate);

${ }^{1} \mathbf{H}$ NMR $\left(500 \mathrm{MHz}, \mathrm{CDCl}_{3}\right) \delta 6.52(\mathrm{t}, J=1.4 \mathrm{~Hz}, 1 \mathrm{H}, \mathrm{H} 3), 5.80$ (ddt, $J=16.9,10.1$, $6.8 \mathrm{~Hz}, 1 \mathrm{H}, \mathrm{H} 8), 5.19$ (s, 1H, H15), 5.14-5.05 (m, 2H, H9), 4.95 (s, 1H, H15), 4.29 (dd, $J=10.3,5.3 \mathrm{~Hz}, 1 \mathrm{H}, \mathrm{H} 11), 3.53-3.38$ (m, 2H, H16), 3.24 (d, $J=17.0 \mathrm{~Hz}, 1 \mathrm{H}$, H6), 3.00 (ddd, $J=6.8,2.3,1.3 \mathrm{~Hz}, 2 \mathrm{H}, \mathrm{H} 7$ ), 2.89 (d, $J=17.0 \mathrm{~Hz}, 1 \mathrm{H}, \mathrm{H} 6$ ), 2.39 (dd, $J=13.5,5.3 \mathrm{~Hz}, 1 \mathrm{H}, \mathrm{H} 10), 2.17$ (dd, $J=13.5,10.3 \mathrm{~Hz}, 1 \mathrm{H}, \mathrm{H} 10), 1.20$ (s, 3H, H13 or H14), 1.11 (s, 3H, H13 or H14), 1.11 (t, $J=7.0$ Hz, 3H, H17) ppm;

${ }^{13}$ C NMR (126 MHz, $\mathrm{CDCl}_{3}$ ) $\delta 194.7$ (C1), 138.7 (C3), 138.4 (C2), 134.5 (C8), 117.7 (C9), 111.3 (C5), 94.9 (C15), 86.3 (C11), 85.5 (C4), 74.6 (C12), 57.5 (C16), 46.2 (C6), 37.4 (C10), 33.2 (C7), 22.5 (C13 or C14), 21.7 (C13 or C14), 16.3 (C17) ppm;

HRMS $\left(\mathrm{ESI}^{+}\right) 331.1521$ (calculated $[\mathrm{M}+\mathrm{Na}]^{+} 331.1521$ );

IR: Due to sample contamination, the IR spectrum was not recorded. 


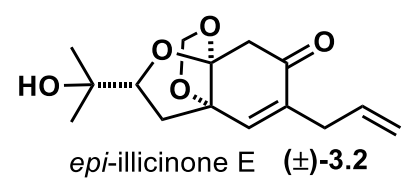

Rf 0.16 (70:30 petroleum spirit 40-60 / ethyl acetate);

${ }^{1} \mathbf{H}$ NMR $\left(500 \mathrm{MHz}, \mathrm{CDCl}_{3}\right) \delta 6.52(\mathrm{t}, J=1.4 \mathrm{~Hz}, 1 \mathrm{H}), 5.78(\mathrm{td}, J=10.2,6.8 \mathrm{~Hz}, 1 \mathrm{H})$, $5.21(\mathrm{~s}, 1 \mathrm{H}), 5.14-5.06(\mathrm{~m}, 2 \mathrm{H}), 4.94(\mathrm{~s}, 1 \mathrm{H}), 4.26(\mathrm{dd}, J=10.7,4.8 \mathrm{~Hz}, 1 \mathrm{H}), 3.28(\mathrm{~d}$, $J=16.7 \mathrm{~Hz}, 1 \mathrm{H}), 3.00(\mathrm{dd}, J=6.8,1.4 \mathrm{~Hz}, 2 \mathrm{H}), 2.79(\mathrm{~d}, J=16.7 \mathrm{~Hz}, 1 \mathrm{H}), 2.38(\mathrm{dd}, J$ $=13.3,4.8 \mathrm{~Hz}, 1 \mathrm{H}), 2.13(\mathrm{dd}, J=13.3,10.7 \mathrm{~Hz}, 1 \mathrm{H}), 1.73(\operatorname{broad} \mathrm{s}, 1 \mathrm{H}), 1.31(\mathrm{~s}, 3 \mathrm{H})$, 1.12 (s, 3H) ppm;

${ }^{13} \mathbf{C}$ NMR $\left(126 \mathrm{MHz}, \mathrm{CDCl}_{3}\right) \delta 194.2,139.0,138.1,134.3,117.9,111.1,95.0,86.3$, 85.8, 70.4, 46.5, 37.5, 33.3, 27.7, 24.4 ppm;

HRMS $\left(\mathrm{ESI}^{+}\right)$281.1400, (calculated $[\mathrm{M}+\mathrm{H}]^{+}$281.1384);

IR: (film, cm ${ }^{-1}$ ) 3497 (broad), 2976, 2886, 1685, 1643. 


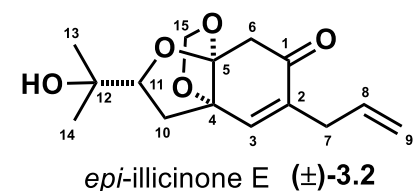

\begin{tabular}{|c|c|c|c|c|c|c|}
\hline \multirow{2}{*}{ Atom } & \multicolumn{3}{|c|}{ Synthetic $( \pm)$-epi-illicinone E $3.2\left(\mathrm{CDCl}_{3}\right)$} & \multicolumn{3}{|c|}{ Natural $( \pm)$-epi-illicinone E $3.2\left(\mathrm{Kuono}^{119}\left(\mathrm{CDCl}_{3}\right)\right.$} \\
\hline & $\delta^{1} \mathrm{H}(600 \mathrm{MHz})$ & $\mathbf{m} J(\mathbf{H z})$ & $\delta^{13} \mathrm{C}(150 \mathrm{MHz})$ & $\delta^{1} \mathrm{H}(600 \mathrm{MHz})$ & $\mathbf{m} J(\mathbf{H z})$ & $\delta^{13} \mathrm{C}(150 \mathrm{MHz})$ \\
\hline 1 & - & - & 194.2 & - & - & 194.1 \\
\hline 2 & - & - & 139.0 & - & - & 138.8 \\
\hline 3 & 6.52 & $\mathrm{t}, 1.4$ & 138.1 & 6.52 & $\mathrm{~S}$ & 138.0 \\
\hline 4 & - & - & 85.8 & - & - & 85.6 \\
\hline 5 & - & - & 111.1 & - & - & 110.9 \\
\hline \multirow{2}{*}{6} & 2.79 & $\mathrm{~d}, 16.7$ & \multirow{2}{*}{46.5} & 2.80 & $\mathrm{~d}, 17$ & \multirow{2}{*}{46.3} \\
\hline & 3.28 & $\mathrm{~d}, 16.7$ & & 3.28 & $\mathrm{~d}, 17$ & \\
\hline 7 & 3.00 & $\mathrm{dd}, 6.8,1.4$ & 33.3 & 3.00 & $\mathrm{dd}, 12,3$ & 33.1 \\
\hline 8 & 5.74 & $\begin{array}{c}\mathrm{td}, 10.2 \\
6.8\end{array}$ & 134.3 & 5.78 & $\begin{array}{c}\text { ddd, } 17,10 \\
3\end{array}$ & 134.1 \\
\hline \multirow{2}{*}{9} & \multirow{2}{*}{$5.14-5.06$} & \multirow{2}{*}{$\mathrm{m}$} & \multirow{2}{*}{117.9} & 5.09 & $\mathrm{dd}, 17,1$ & \multirow{2}{*}{117.8} \\
\hline & & & & 5.12 & $\mathrm{dd}, 10,1$ & \\
\hline \multirow{2}{*}{10} & 2.13 & $\begin{array}{c}\mathrm{dd}, 13.3 \\
10.7\end{array}$ & \multirow{2}{*}{37.5} & 2.14 & $\mathrm{dd}, 14,11$ & \multirow{2}{*}{37.3} \\
\hline & 2.38 & $\begin{array}{c}\mathrm{dd}, 13.3 \\
4.8\end{array}$ & & 2.38 & $\mathrm{dd}, 14,5$ & \\
\hline 11 & 4.26 & $\begin{array}{c}\mathrm{dd}, 10.7 \\
4.8\end{array}$ & 86.3 & 4.26 & $\mathrm{dd}, 11,5$ & 86.1 \\
\hline 12 & - & - & 70.4 & - & - & 70.2 \\
\hline 13 & 1.12 & $\mathrm{~s}$ & 24.4 & 1.12 & $\mathrm{~s}$ & 24.3 \\
\hline 14 & 1.31 & $\mathrm{~s}$ & 27.7 & 1.31 & $\mathrm{~s}$ & 27.5 \\
\hline \multirow{2}{*}{15} & 4.94 & $\mathrm{~s}$ & \multirow{2}{*}{95.0} & 4.94 & $\mathrm{~s}$ & \multirow{2}{*}{94.8} \\
\hline & 5.21 & $\mathrm{~s}$ & & 5.21 & $\mathrm{~s}$ & \\
\hline $\begin{array}{l}\mathrm{OH} \text { at } \\
\mathrm{C} 12 \\
\end{array}$ & 1.73 & s (br.) & - & - & - & - \\
\hline
\end{tabular}

$\dagger,{ }^{\dagger}, \dagger \dagger$ overlapping signals. 


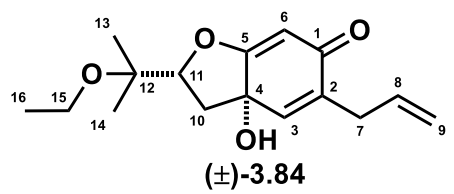

$\mathbf{R}_{\mathbf{f}}$ 0.22 (70:30 petroleum spirit 40-60 / ethyl acetate);

${ }^{1} \mathbf{H}$ NMR $\left(600 \mathrm{MHz}, \mathrm{CDCl}_{3}\right) \delta 6.59(\mathrm{t}, J=1.5 \mathrm{~Hz}, 1 \mathrm{H}, \mathrm{H} 3), 5.84$ (ddt, $J=17.0,10.3$, $6.9 \mathrm{~Hz}, 1 \mathrm{H}, \mathrm{H} 8$ ), 5.53 (s, 1H, H6), 5.14-5.12 (m, 1H, H9), 5.12-5.09 (m, 1H, H9), 4.87 (dd, $J=10.0,4.6 \mathrm{~Hz}, 1 \mathrm{H}, \mathrm{H} 11), 3.53-3.41$ (m, 2H, H15), 3.07 (ddd, $J=6.8,3.5,1.4$ Hz, 2H, H7), 2.27 (dd, $J=12.8,4.6 \mathrm{~Hz}, 1 \mathrm{H}, \mathrm{H} 10), 2.05$ (broad d, $J=2.6 \mathrm{~Hz}, 1 \mathrm{H}, \mathrm{OH}$ at C4), 2.02 (d, $J=12.6 \mathrm{~Hz}, 1 \mathrm{H}, \mathrm{H} 10), 1.26$ (s, 3H, H13 or H14), 1.19 (s, 3H, H13 or H14), 1.12 (t, $J=7.0 \mathrm{~Hz}, 3 \mathrm{H}, \mathrm{H} 15) \mathrm{ppm}$;

${ }^{13}$ C NMR (151 MHz, $\left.\mathrm{CDCl}_{3}\right) \delta 187.2(\mathrm{C} 1), 178.3$ (C5), 139.6 (C2), 135.3 (C3), 135.0 (C8), 117.6 (C9), 99.4 (C6), 91.2 (C11), 74.6 (C12), 73.0 (C4), 57.9 (C15), 36.3 (C10), 33.5 (C7), 22.6 (C13 or C14), 21.4 (C13 or C14), 16.3 (C16) ppm;

HRMS $\left(\mathrm{ESI}^{+}\right) \quad 279.1601,301.1408$ (calculated $[\mathrm{M}+\mathrm{H}]^{+}$279.1591, $[\mathrm{M}+\mathrm{Na}]^{+}$ 301.1410);

IR: due to insufficient quantities of material, the IR spectrum of compound ( \pm )-3.84 was not recorded. 


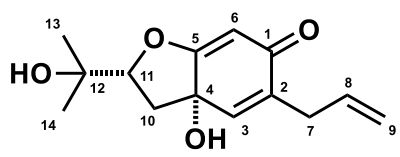

$( \pm)-3.85$

$\mathbf{R}_{\mathbf{f}}$ 0.14 (70:30 petroleum spirit 40-60 / ethyl acetate);

${ }^{1}$ H NMR $\left(500 \mathrm{MHz}, \mathrm{CDCl}_{3}\right) \delta 6.60$ (t, $\left.J=1.6 \mathrm{~Hz}, 1 \mathrm{H}, \mathrm{H} 3\right), 5.83$ (ddt, $J=16.8,10.4$, $6.9 \mathrm{~Hz}, 1 \mathrm{H}, \mathrm{H} 8$ ), 5.54 (s, 1H, H6), 5.14-5.08 (m, 2H, H9), 4.82 (dd, $J=9.9,4.6 \mathrm{~Hz}$, 1H, H11), 3.06 (dm, $J=6.9$ Hz, 2H, H7), 2.27 (dd, $J=12.6,4.7$ Hz, 1H, H10), 2.06 (dd, $J=12.8,10.1 \mathrm{~Hz}, 1 \mathrm{H}, \mathrm{H} 10), 2.04($ broad s, 1H, OH at C4 or C12), 1.65 (broad s, $1 \mathrm{H}, \mathrm{OH}$ at $\mathrm{C} 4$ or $\mathrm{C} 12), 1.40$ (s, 3H, H13 or H 14), 1.18 (s, 3H, H13 or H14) ppm;

${ }^{13}$ C NMR: due to sample contamination, the ${ }^{13} \mathrm{C}$ NMR spectrum of compound $( \pm)$-3.85 was not recorded.

IR: due to sample contamination, the IR spectrum of compound ( \pm )-3.85 was not recorded. 

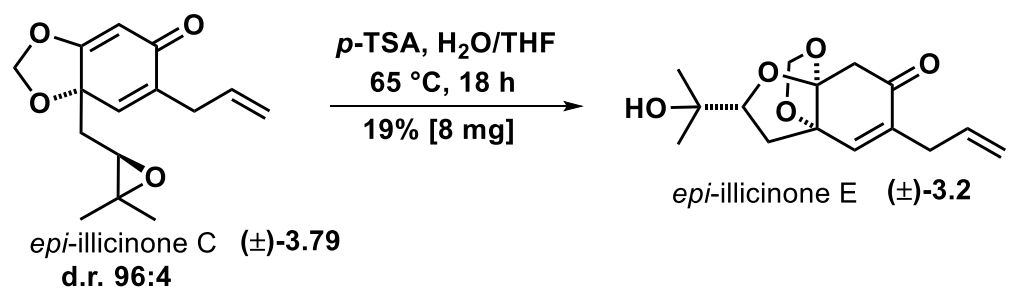

Epoxide ( \pm )-epi-illicinone C 3.79 (39 mg, $0.15 \mathrm{mmol}$ ) was dissolved in THF (7 mL). Water $(2 \mathrm{~mL})$ and $p$-TSA $(2 \mathrm{mg}, 0.01 \mathrm{mmol})$ was added and the mixture was heated to $65^{\circ} \mathrm{C}$ until complete consumption of the starting material, judged by TLC analysis (22 h). The mixture was then diluted with water $(8 \mathrm{~mL})$ and extracted with $\mathrm{CH}_{2} \mathrm{Cl}_{2}(3 \times 12$ $\mathrm{mL}$ ). The combined organic layers were dried over anhydrous $\mathrm{Na}_{2} \mathrm{SO}_{4}$ and the solvent was removed under reduced pressure. Flash chromatography (80:20 petroleum spirit 40-60 / ethyl acetate) afforded alcohol ( \pm )-epi-illicinone E $\mathbf{3 . 2}$ as a colourless oil (8 $\mathrm{mg}, 28 \mu \mathrm{mol}, 19 \%$ yield). See page 227 for characterisation data. 

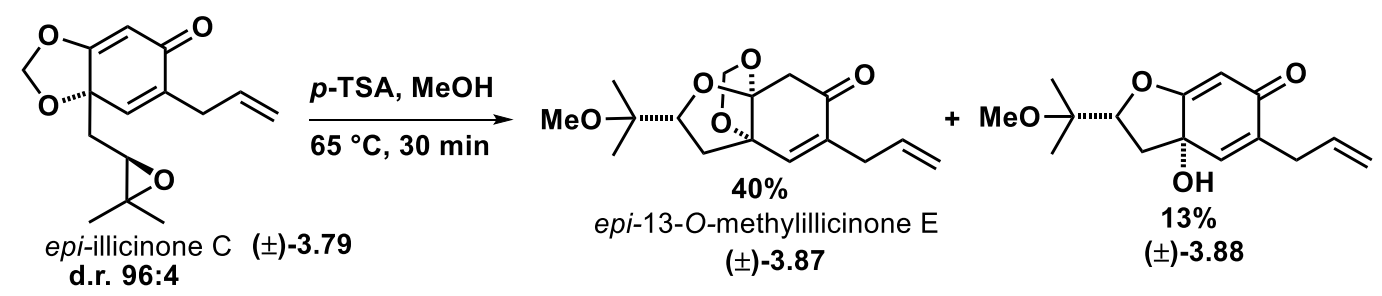

Epoxide ( \pm )-epi-illicinone C 3.79 (37 $\mathrm{mg}, 0.14 \mathrm{mmol})$ was dissolved in anhydrous methanol ( $7 \mathrm{~mL}) \cdot p$-TSA $(2 \mathrm{mg}, 0.01 \mathrm{mmol})$ was added and the mixture was heated to $65^{\circ} \mathrm{C}$ until complete consumption of the starting material, judged by TLC analysis (20 min). The mixture was then diluted with water $(10 \mathrm{~mL})$ and extracted with $\mathrm{CH}_{2} \mathrm{Cl}_{2}(3$ $\times 10 \mathrm{~mL}$ ). The combined organic layers were dried over anhydrous $\mathrm{Na}_{2} \mathrm{SO}_{4}$ and the solvent was removed under reduced pressure. Flash chromatography $(80: 20$ petroleum spirit 40-60 / ethyl acetate) afforded ether ( \pm )-3.87 as a colourless oil (16 mg, $56 \mu \mathrm{mol}$, $40 \%$ yield) along with alcohol ( \pm )-3.87 as a colourless oil (5 mg, $18 \mu \mathrm{mol}, 13 \%$ yield).

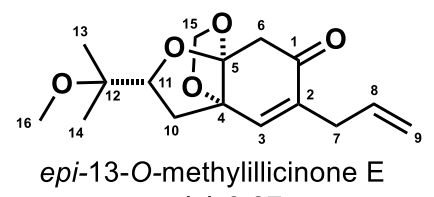

$( \pm)-3.87$

$\mathbf{R}_{\mathbf{f}} 0.56$ (60:40 petroleum spirit 40-60 / ethyl acetate);

${ }^{1} \mathbf{H}$ NMR $\left(600 \mathrm{MHz}, \mathrm{CDCl}_{3}\right) \delta 6.51$ (t, $\left.J=1.5 \mathrm{~Hz}, 1 \mathrm{H}, \mathrm{H} 3\right), 5.79$ (ddt, $J=17.0,10.1$, $6.8 \mathrm{~Hz}, 1 \mathrm{H}, \mathrm{H} 8), 5.18$ (s, 1H, H15), 5.15-5.03 (m, 2H, H9), 4.94 (s, 1H, H15), 4.30 (dd, $J=10.5,5.2 \mathrm{~Hz}, 1 \mathrm{H}, \mathrm{H} 11), 3.25$ (d, $J=17.0 \mathrm{~Hz}, 1 \mathrm{H}, \mathrm{H} 6), 3.24$ (s, 3H, H16), 3.022.96 (m, 2H, H7), 2.85 (d, $J=17.0 \mathrm{~Hz}, 1 \mathrm{H}, \mathrm{H} 6), 2.38$ (dd, $J=13.5,5.2 \mathrm{~Hz}, 1 \mathrm{H}, \mathrm{H} 10)$, $2.10(\mathrm{dd}, J=13.5,10.5 \mathrm{~Hz}, 1 \mathrm{H}, \mathrm{H} 10), 1.18$ (s, 3H, H13 or H14), 1.11 (s, 3H, H13 or H14) ppm;

${ }^{13}$ C NMR (151 MHz, $\left.\mathrm{CDCl}_{3}\right) \delta 194.6(\mathrm{C} 1), 138.6(\mathrm{C} 2), 138.5$ (C3), 134.5 (C8), 117.8 (C9), 111.2 (C5), 94.9 (C15), 86.0 (C11), 85.4 (C4), 74.9 (C12), 50.2 (C16), 46.2 (C6), 37.6 (C10), 33.3 (C7), 21.5 (C13 or C14), 21.0 (C13 or C14) ppm;

HRMS $\left(\mathrm{ESI}^{+}\right) \quad 295.1579,317.1392$ (calculated $[\mathrm{M}+\mathrm{H}]^{+}$295.1540, $[\mathrm{M}+\mathrm{Na}]^{+}$ $317.1360)$;

IR: (film, $\mathrm{cm}^{-1}$ ) 2976, 2941, 2895, 1686, 1641. 


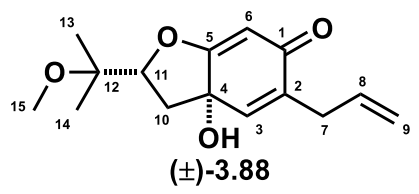

$\mathbf{R}_{\mathbf{f}} 0.42$ (60:40 petroleum spirit 40-60 / ethyl acetate);

${ }^{1} \mathbf{H}$ NMR $\left(600 \mathrm{MHz}, \mathrm{CDCl}_{3}\right) \delta 6.58(\mathrm{t}, J=1.6 \mathrm{~Hz}, 1 \mathrm{H}, \mathrm{H} 3), 5.84(\mathrm{ddt}, J=17.2,10.4$, $6.9 \mathrm{~Hz}, 1 \mathrm{H}, \mathrm{H} 8$ ), 5.53 (s, 1H, H6), 5.13-5.08 (m, 2H, H9), 4.87 (dd, $J=10.0,4.7 \mathrm{~Hz}$, 1H, H11), 3.26 (s, 3H, H15), 3.05 (dm, $J=6.9 \mathrm{~Hz}, 2 \mathrm{H}, \mathrm{H} 7), 2.26$ (dd, $J=12.8,4.7 \mathrm{~Hz}$, 1H, H10), 2.24 (broad d, $J=2.5 \mathrm{~Hz}, 1 \mathrm{H}, \mathrm{OH}$ at C4), 2.01 (ddd, $J=12.6,10.0,2.4 \mathrm{~Hz}$, 1H, H10), 1.26 (s, 3H, H13 or H14), 1.17 (s, 3H, H13 or H14) ppm;

${ }^{13}$ C NMR (151 MHz, $\mathrm{CDCl}_{3}$ ) $\delta 187.3$ (C1), 178.2 (C5), 139.6 (C2), 135.3 (C3), 135.0 (C8), 117.6 (C9), 99.5 (C6), 91.1 (C12), 74.8 (C4), 73.0 (C12), 50.4 (C15), 36.2 (C10), 33.5 (C7), 21.6 (C13 or C14), 20.8 (C13 or C14) ppm;

HRMS $\left(\mathrm{ESI}^{+}\right) \quad 265.1458,287.1275$ (calculated $[\mathrm{M}+\mathrm{H}]^{+}$265.1434, $[\mathrm{M}+\mathrm{Na}]^{+}$ 287.1254);

IR: (film, cm cm $^{-1} 3366$ (broad), 2978, 2941, 1667, 1614. 


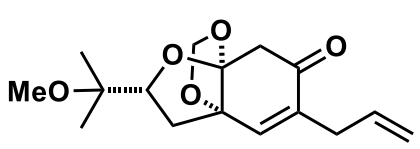

$( \pm)-3.88$

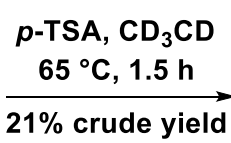

$21 \%$ crude yield

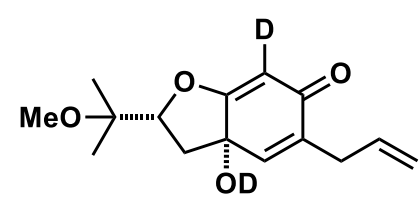

$( \pm)-3.89$

Ether $( \pm)$-3.88 $(5 \mathrm{mg}, 17 \mu \mathrm{mol})$ was dissolved in deuterated methanol $(0.6 \mathrm{~mL})$ in an NMR tube. $p$-TSA (a small crystal, $>1 \mathrm{mg}$ ) was added and mixture was heated to 55 ${ }^{\circ} \mathrm{C}$ and monitored by ${ }^{1} \mathrm{H}$ NMR. Analysis of the crude ${ }^{1} \mathrm{H}$ NMR spectrum after $1 \mathrm{~h}$ of reaction time indicated an approximatively $21 \%$ conversion into compound $( \pm)-3.89$. 


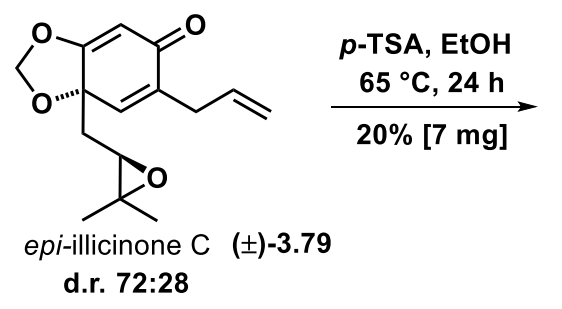

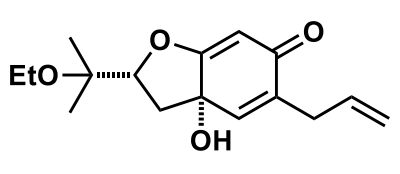

$( \pm)-3.84$

Epoxide ( \pm )-3.79 (d.r. 72:28) (32 mg, $0.12 \mathrm{mmol}$ ) was dissolved in anhydrous ethanol $(6 \mathrm{~mL}) \cdot p$-TSA $(2 \mathrm{mg}, 0.01 \mathrm{mmol})$ was added and the mixture was heated to $65^{\circ} \mathrm{C}$ for $24 \mathrm{~h}$. The mixture was then diluted with water $(8 \mathrm{~mL})$ and extracted with $\mathrm{CH}_{2} \mathrm{Cl}_{2}(3 \times$ $10 \mathrm{~mL}$ ). The combined organic layers were dried over anhydrous $\mathrm{Na}_{2} \mathrm{SO}_{4}$ and the solvent was removed under reduced pressure. Flash chromatography (80:20 petroleum spirit 40-60 / ethyl acetate) afforded ether $( \pm)$-3.84 as a colourless oil (7 mg, $25 \mu \mathrm{mol}$, $20 \%$ yield). See page 229 for characterisation data. 
4.4 NMR Spectra for Chapter 2

${ }^{1} \mathrm{H}$ NMR Spectrum of Compound 2.24

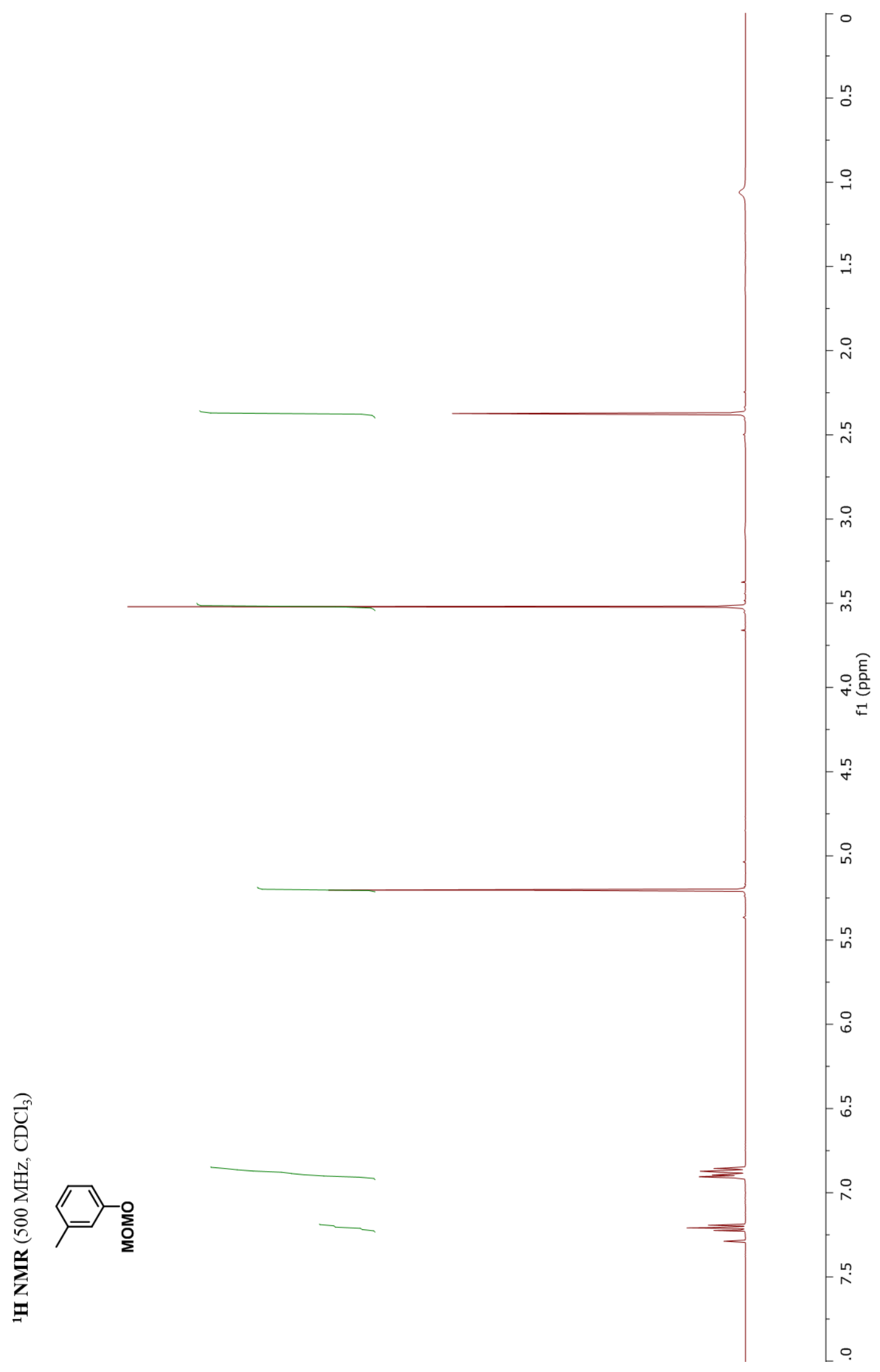


${ }^{13}$ C NMR Spectrum of Compound 2.24

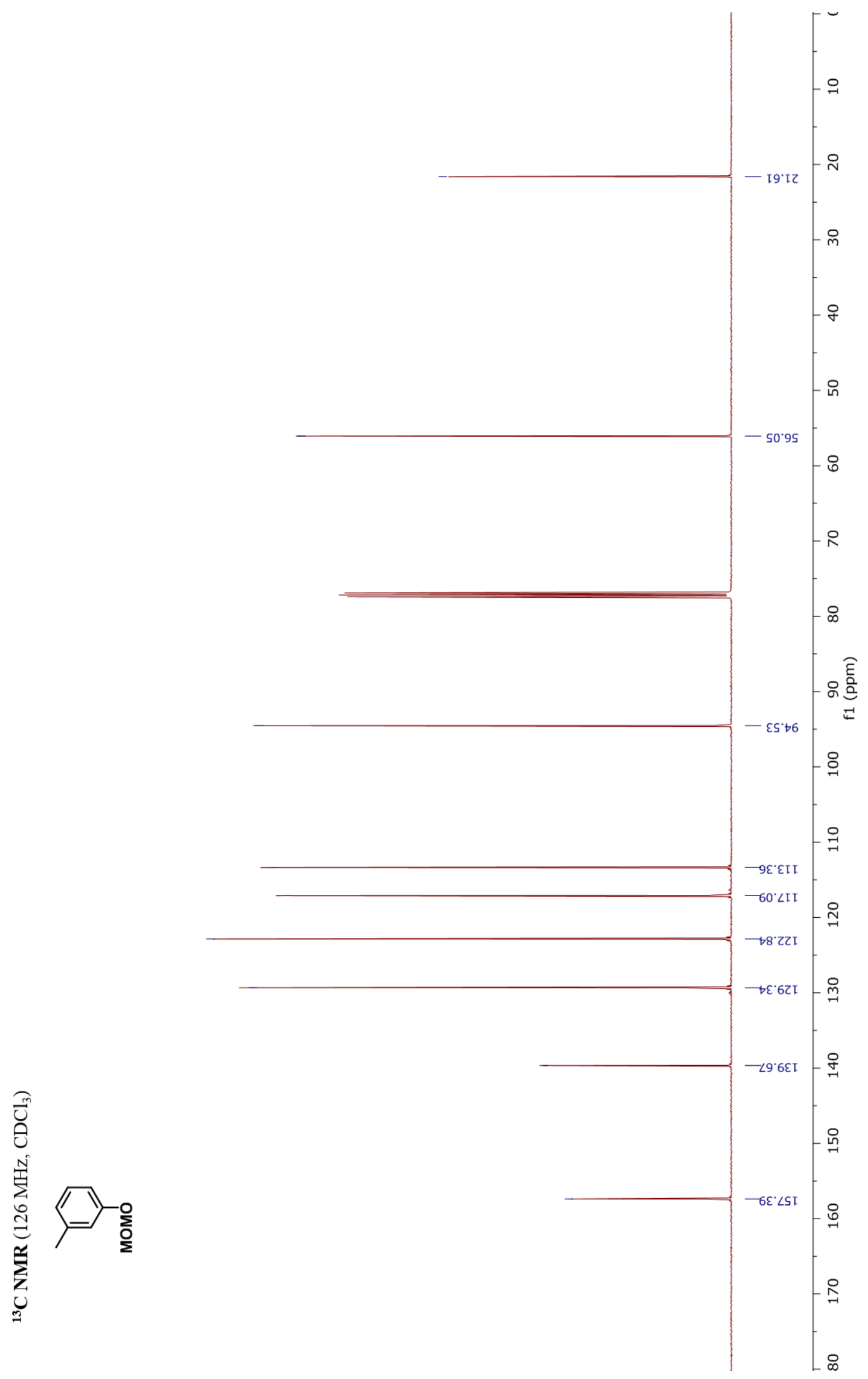


${ }^{1} \mathrm{H}$ NMR Spectrum of Compound 2.25

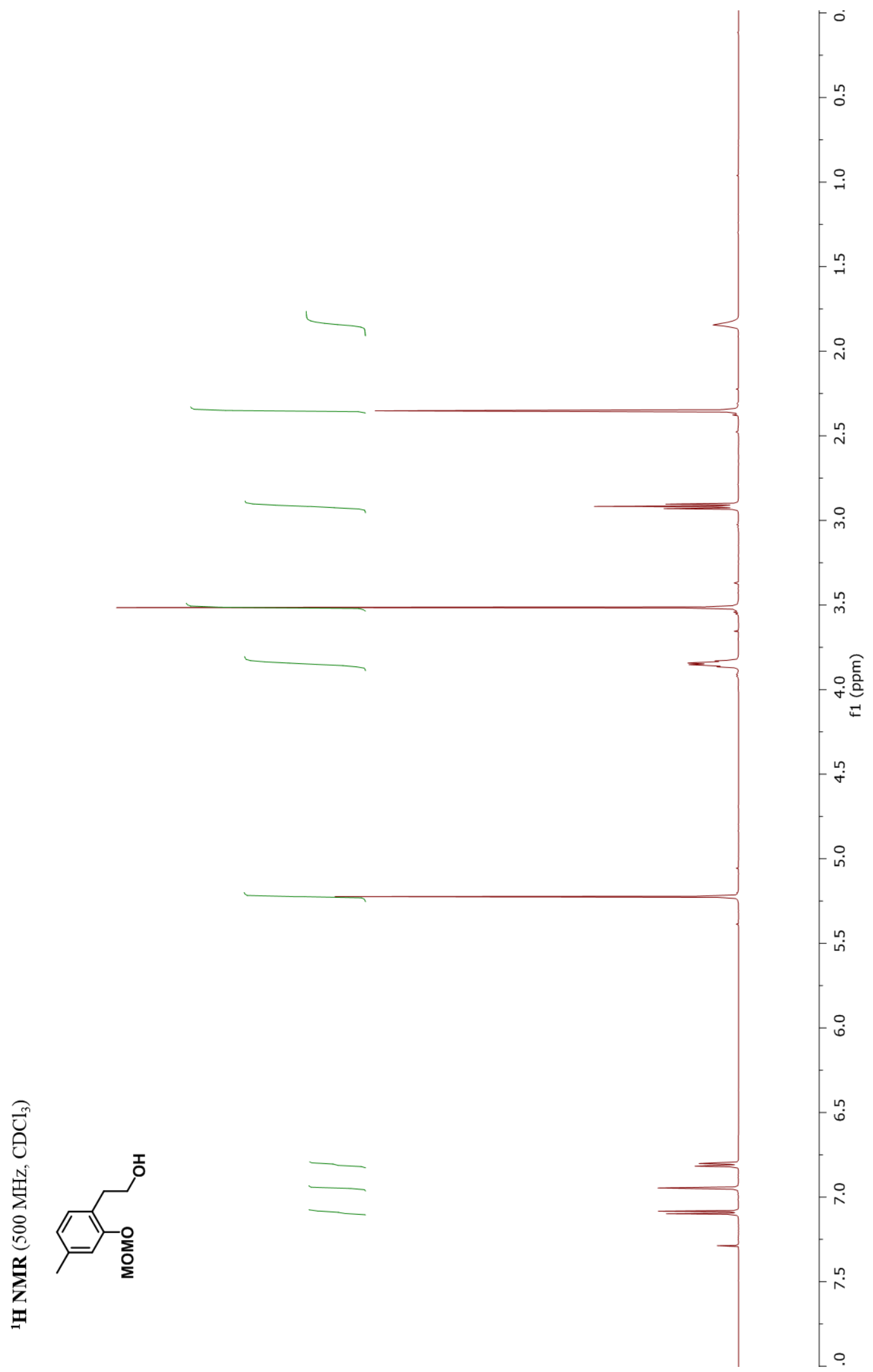


${ }^{13} \mathrm{C}$ NMR Spectrum of Compound 2.25

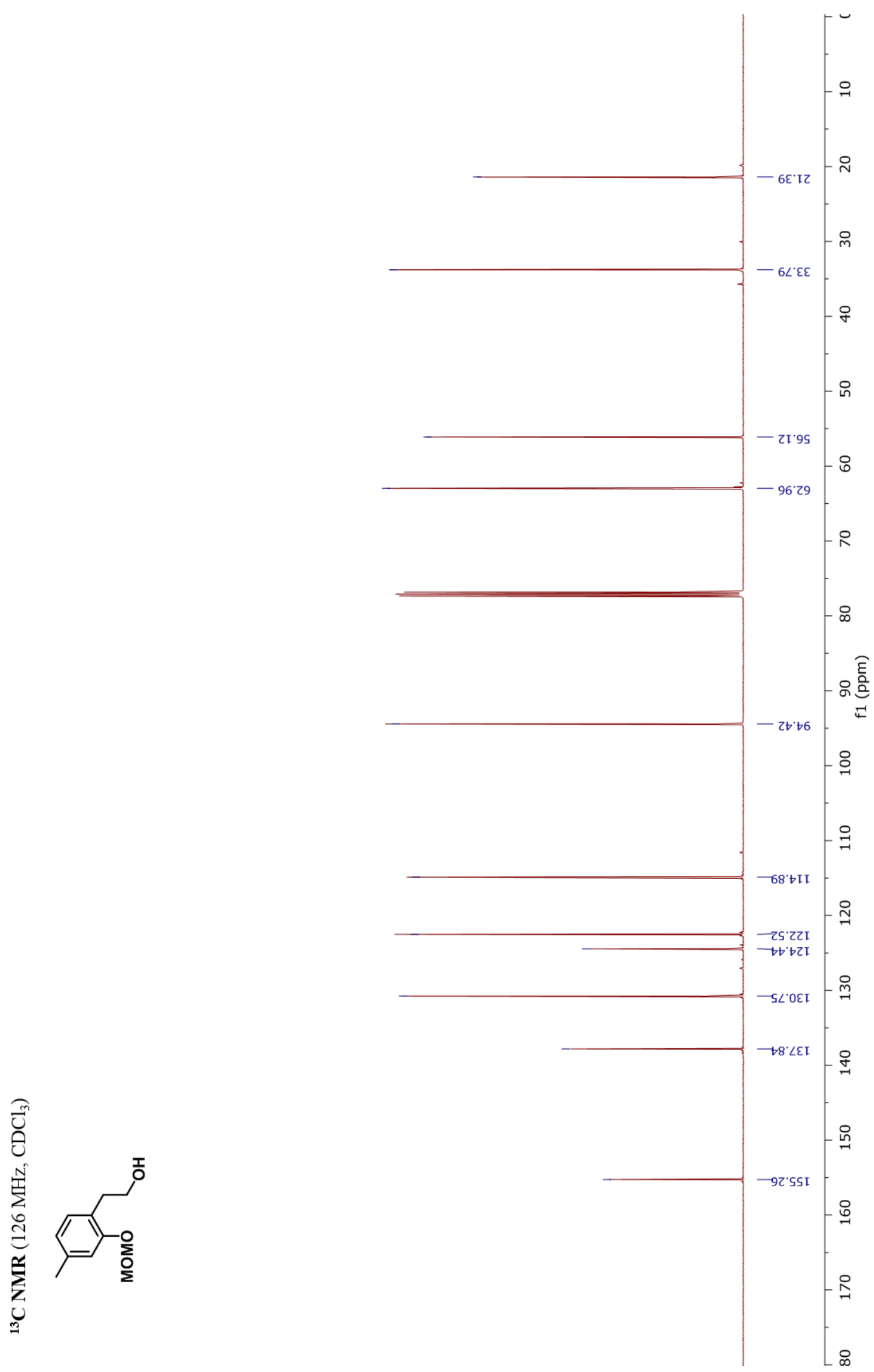


${ }^{1} \mathrm{H}$ NMR Spectrum of Compound 2.27

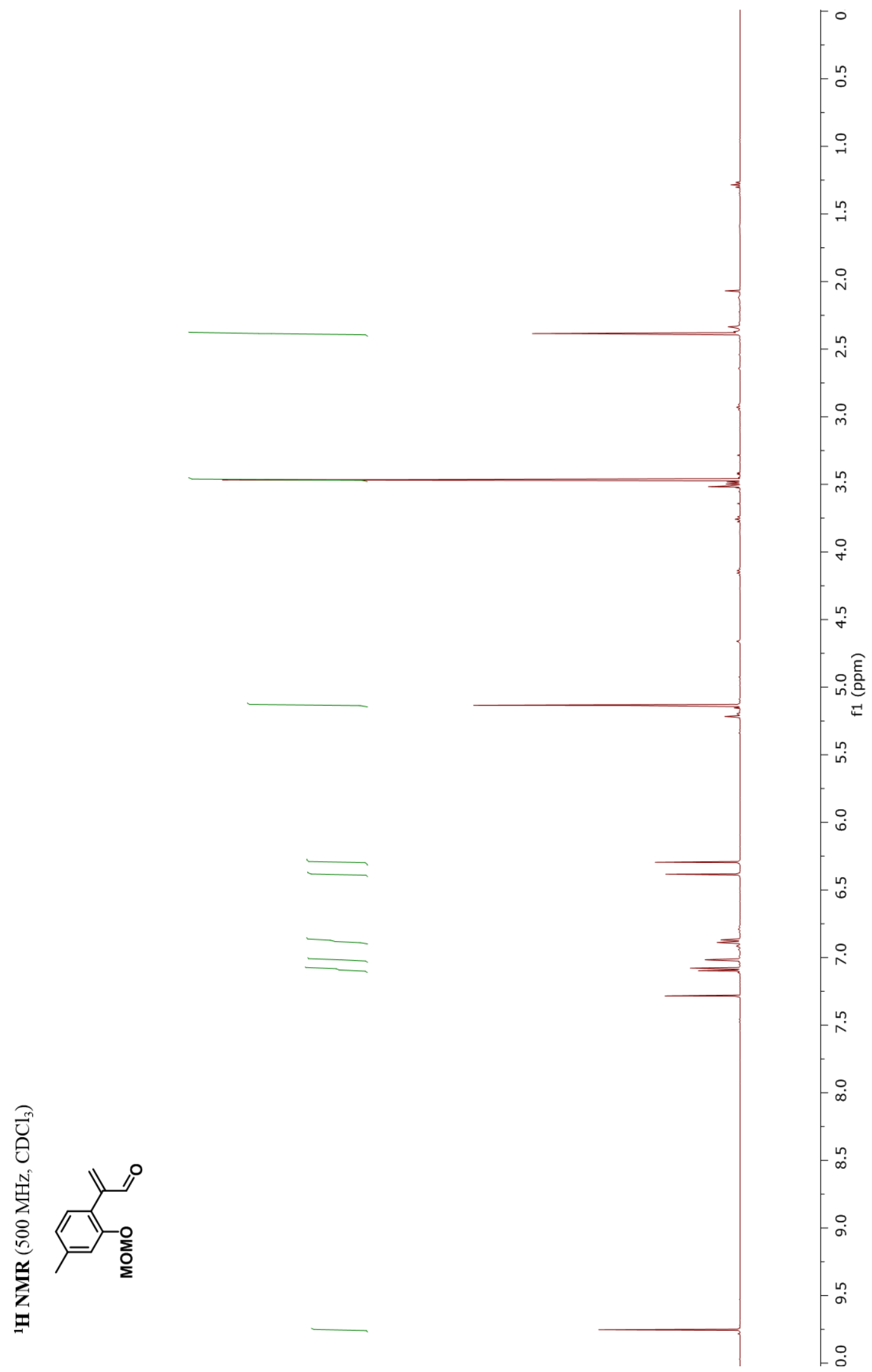


${ }^{13} \mathrm{C}$ NMR Spectrum of Compound 2.27

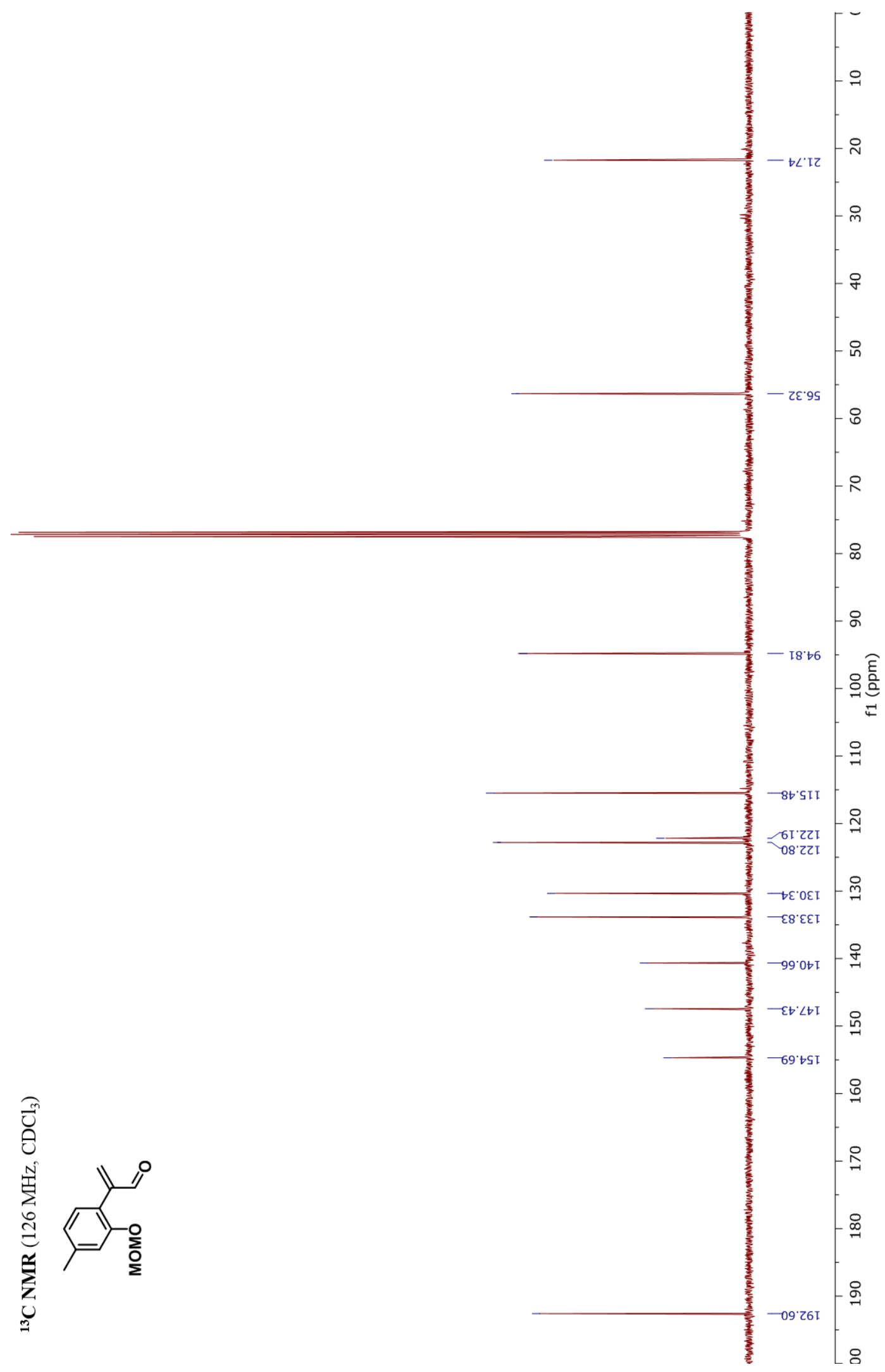


${ }^{1} \mathrm{H}$ NMR Spectrum of Compound ( \pm )-2.28

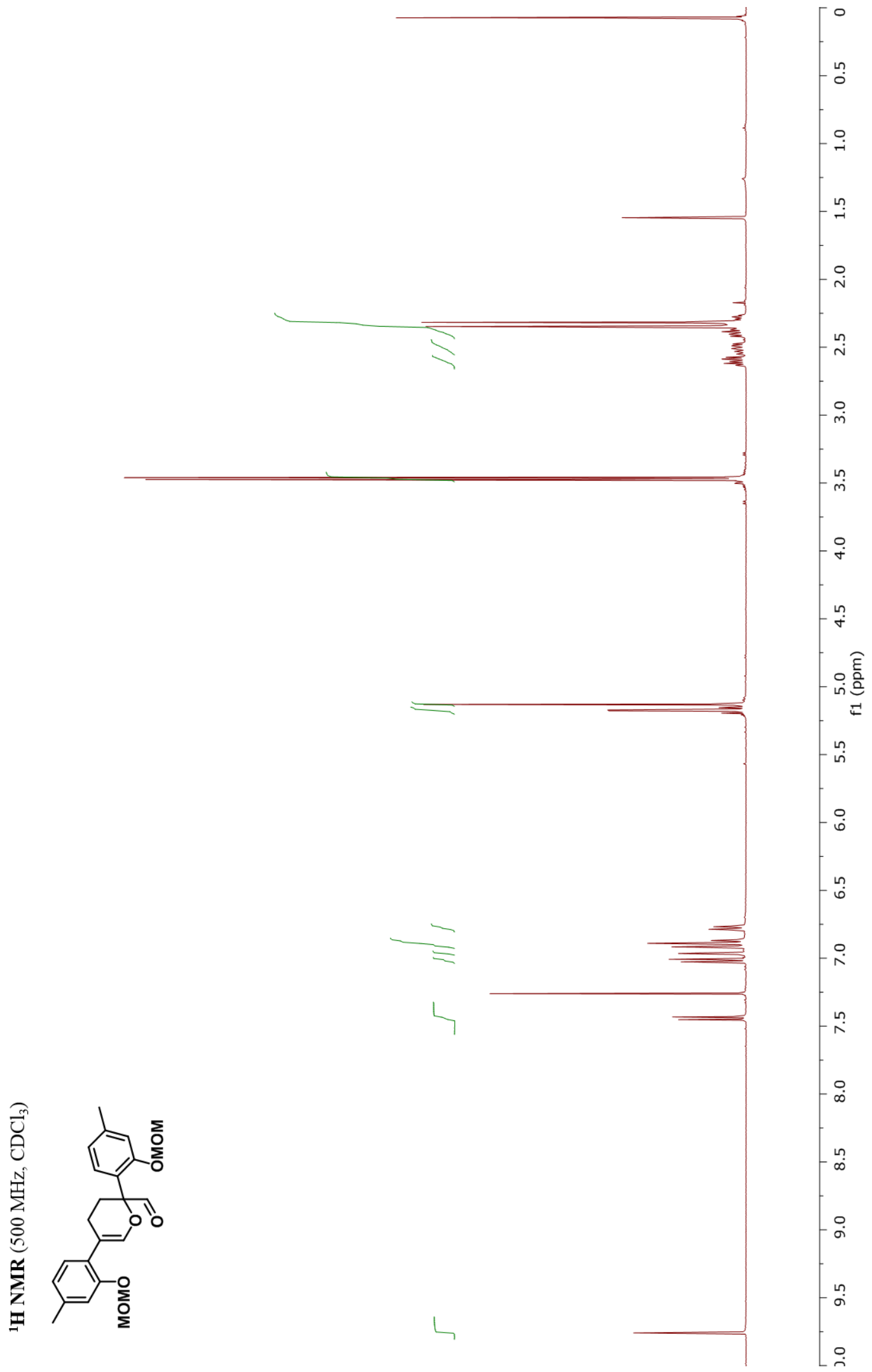


${ }^{13}$ C NMR Spectrum of Compound ( \pm )-2.28

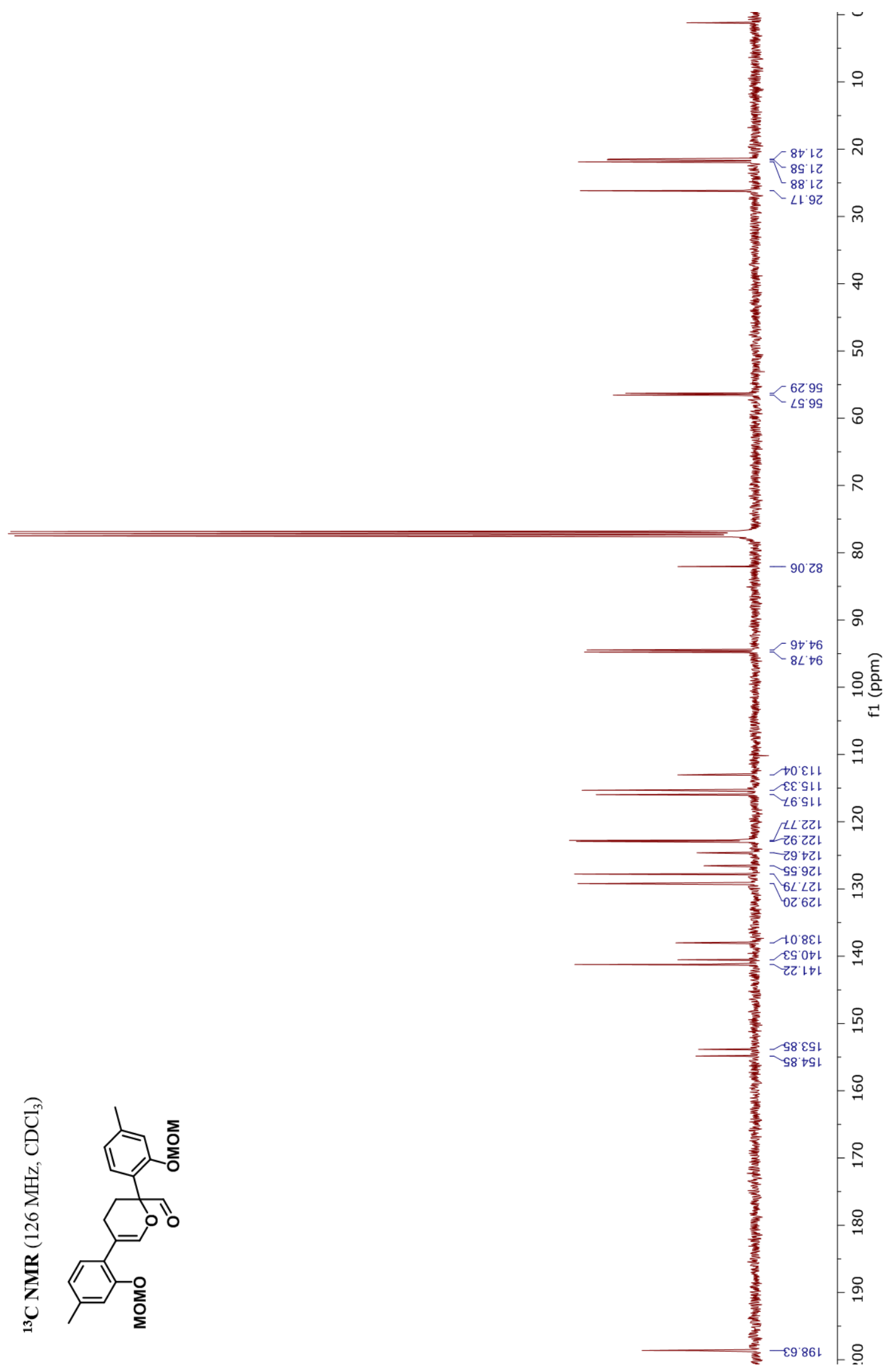


${ }^{1}$ H NMR Spectrum of Compound ( \pm )-2.29

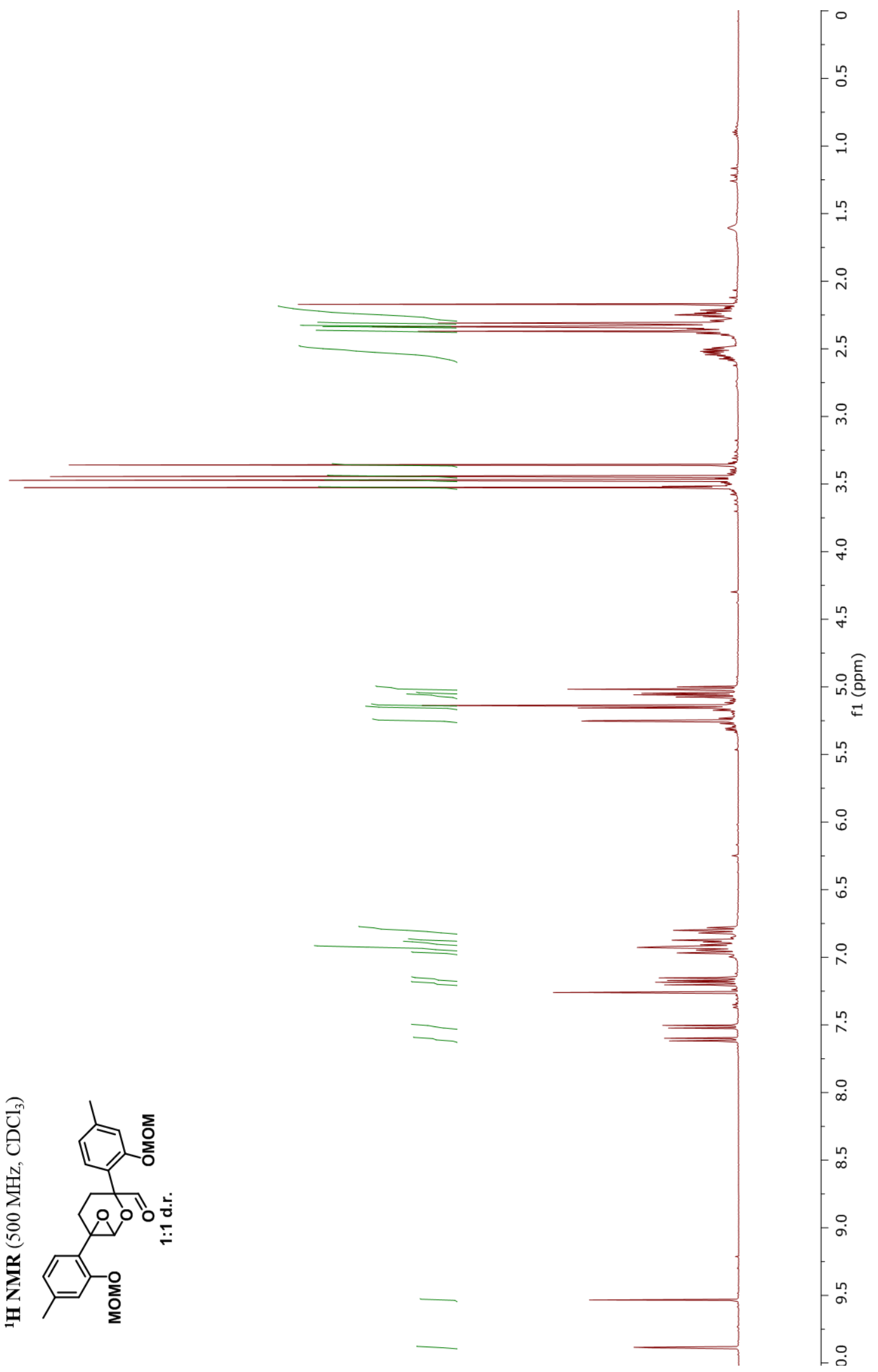


${ }^{13}$ C NMR Spectrum of Compound ( \pm )-2.29

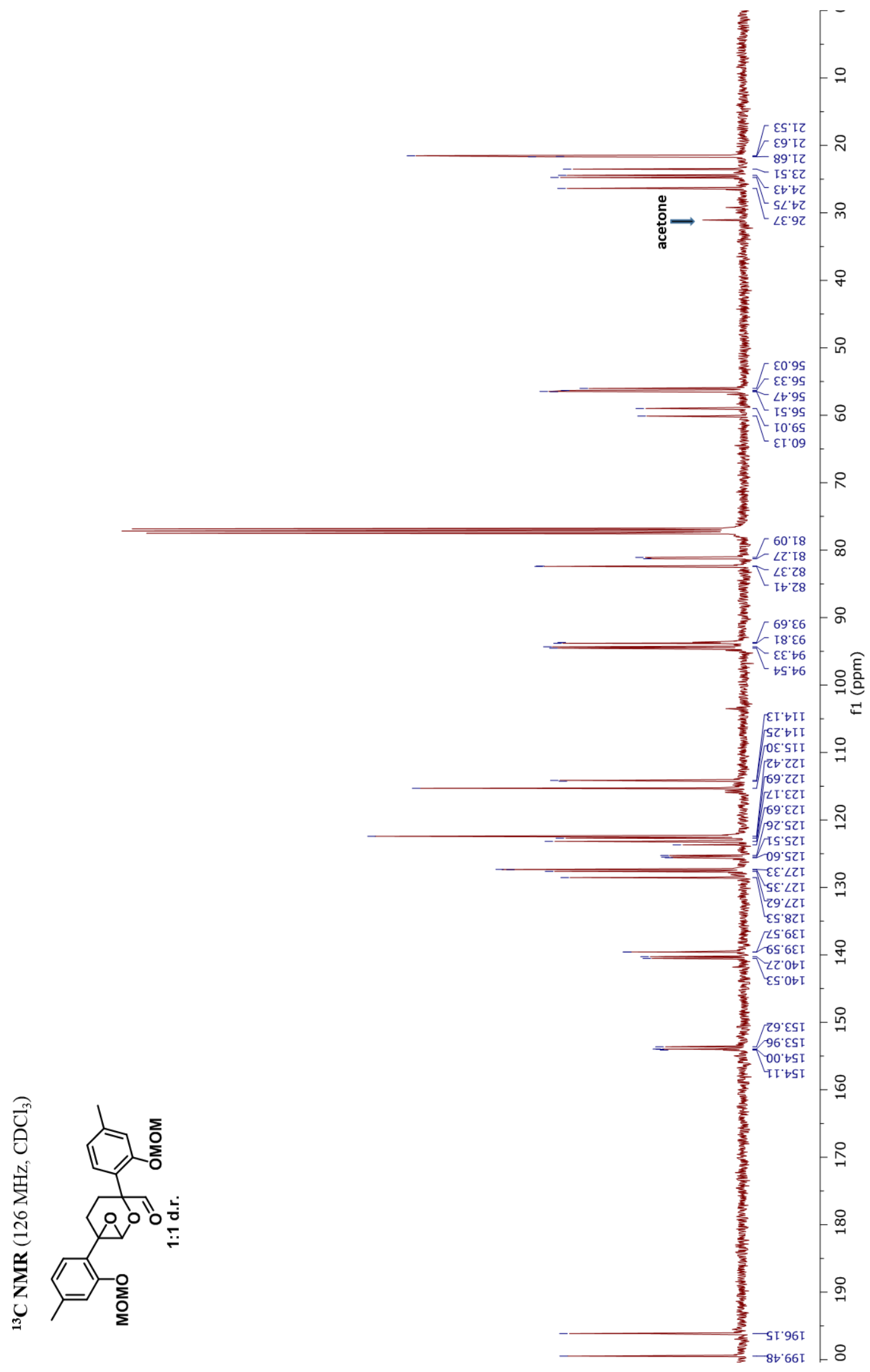


${ }^{1}$ H NMR Spectrum of Compound ( \pm )-2.30 (single diastereoisomer)

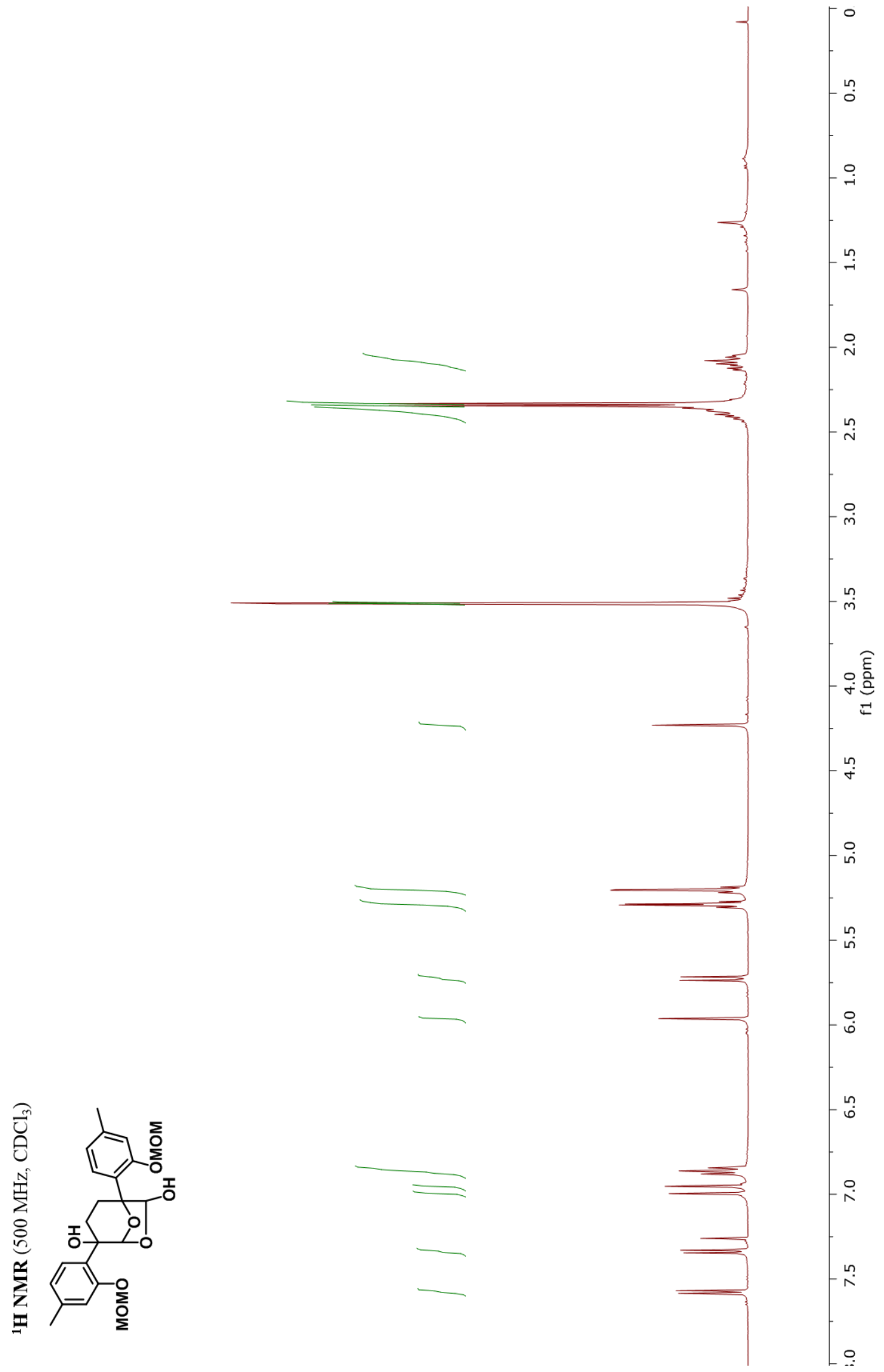


${ }^{13} \mathrm{C}$ NMR Spectrum of Compound ( \pm )-2.30 (single diastereoisomer)

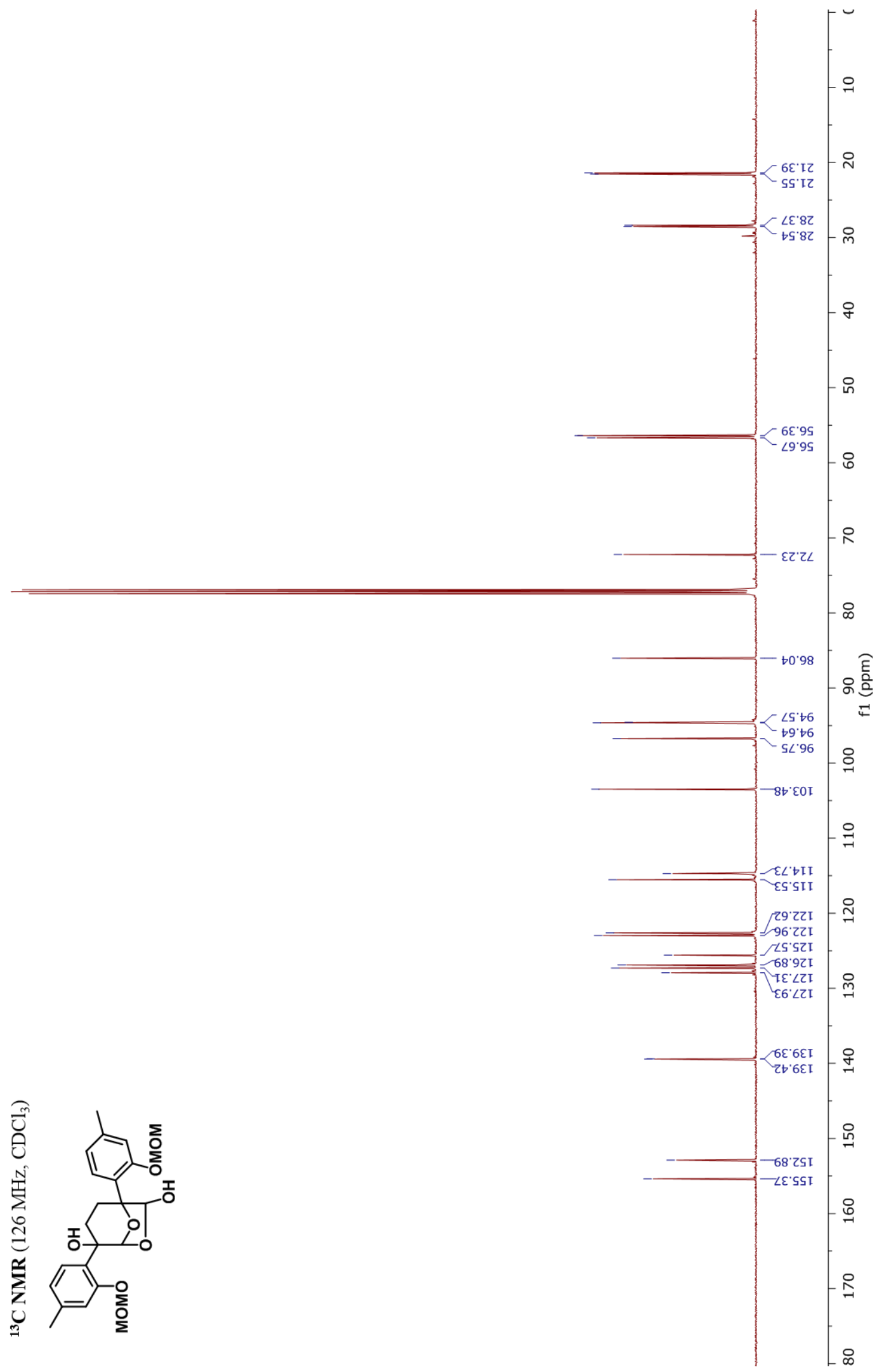


${ }^{1}$ H NMR Spectrum of Compound 2.31 (d.r. 1:1)

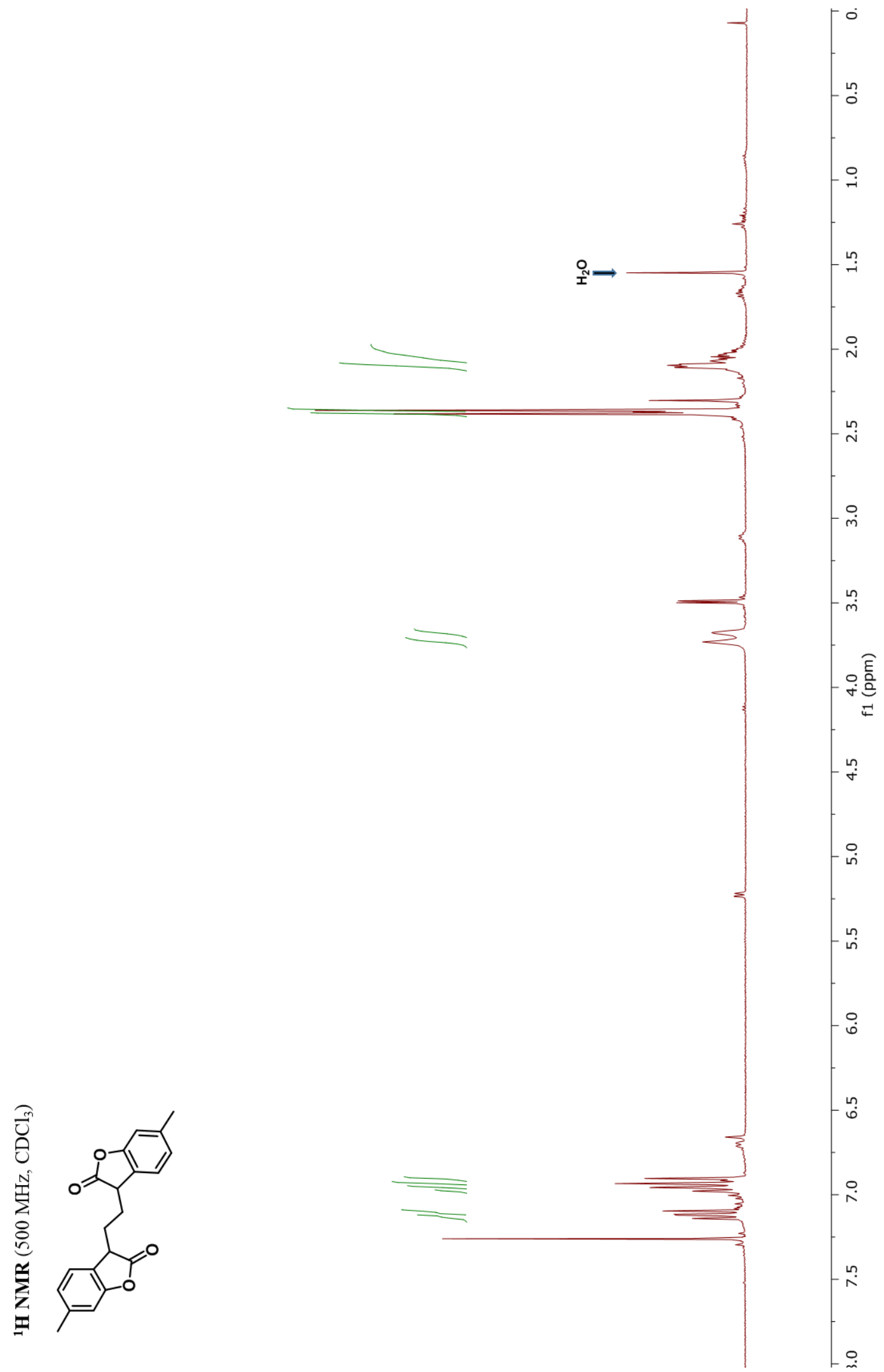


${ }^{1}$ H NMR Spectrum of Compound meso-2.31

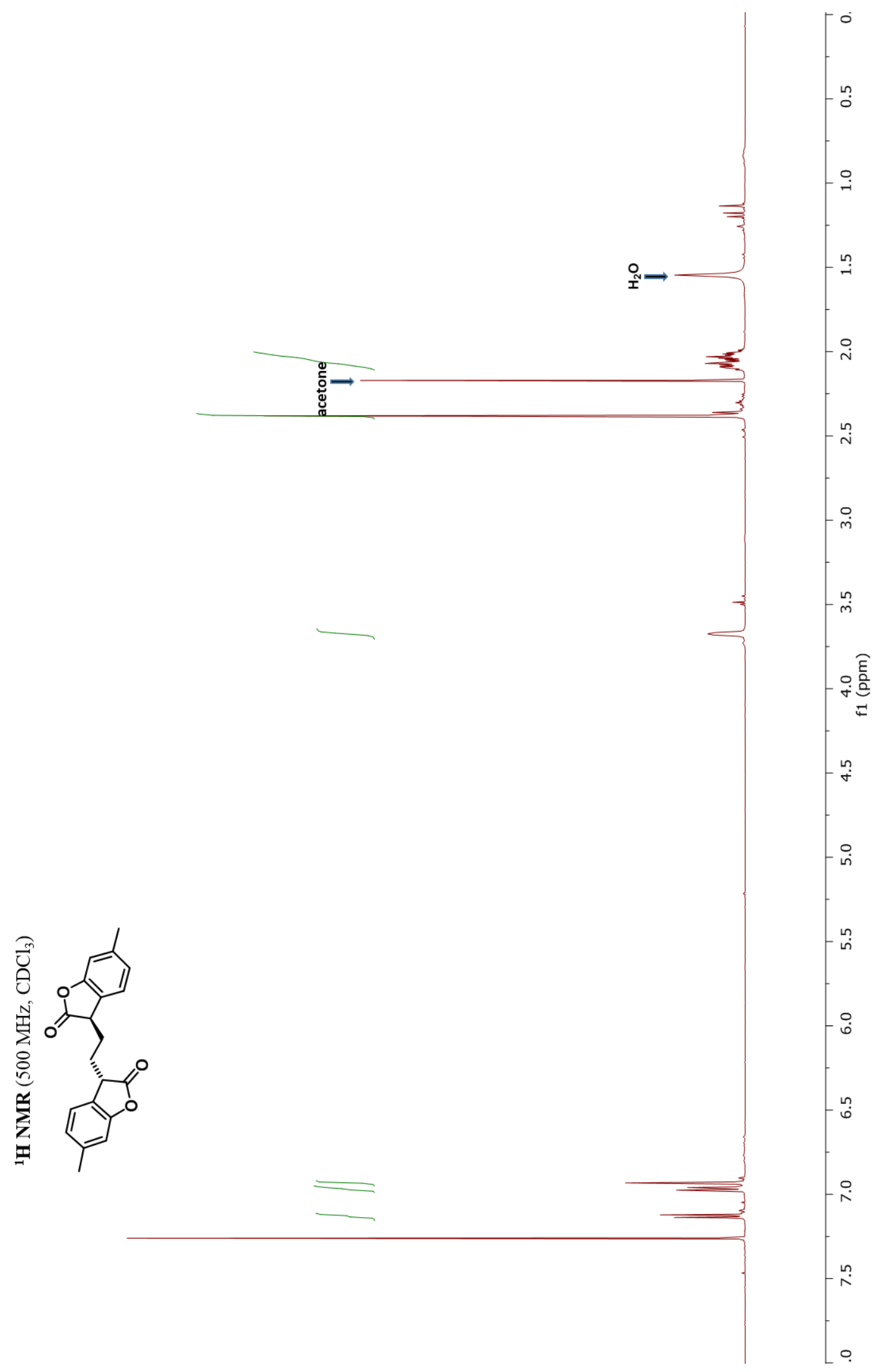


${ }^{13} \mathrm{C}$ NMR Spectrum of Compound meso-2.31

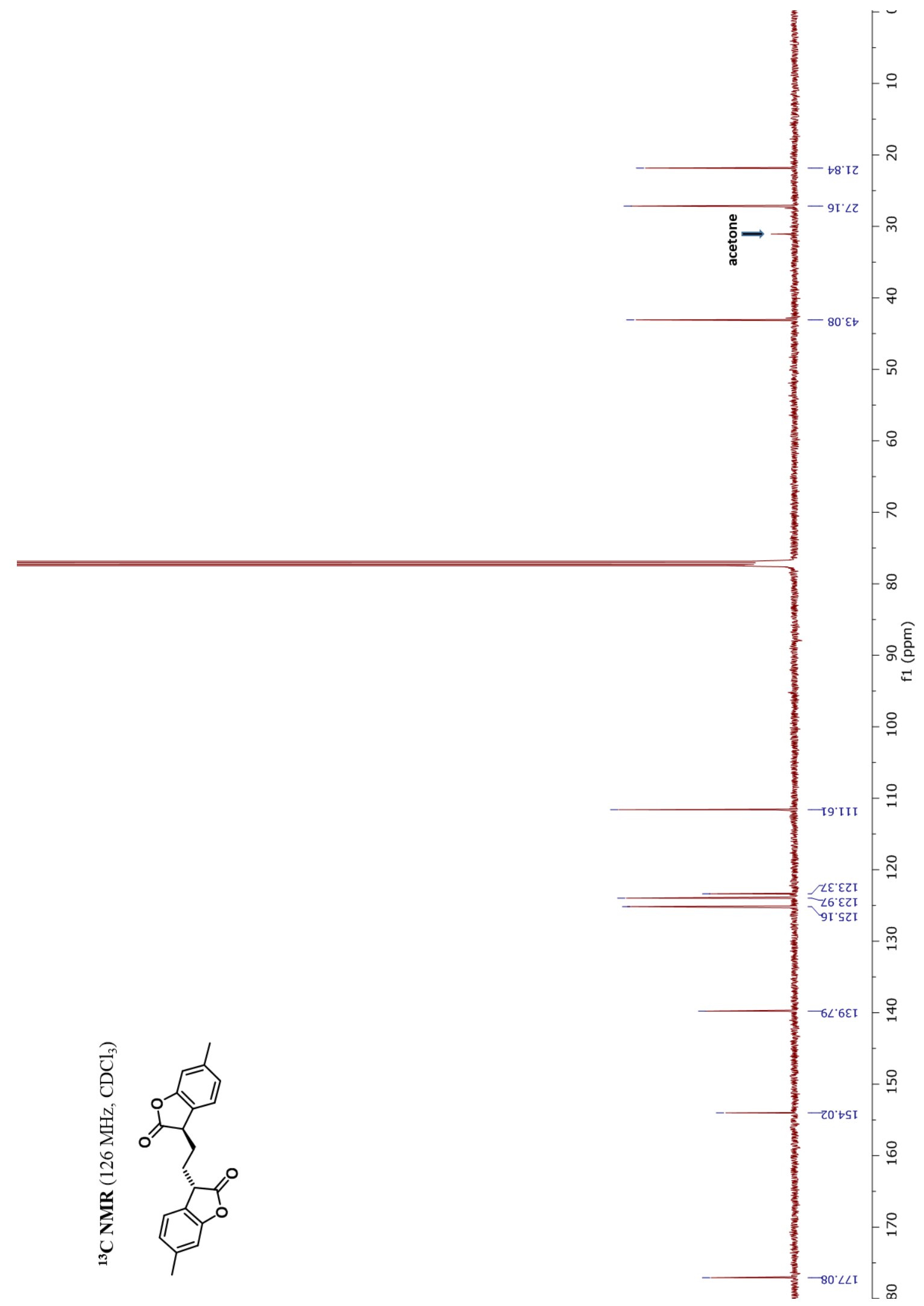


${ }^{1}$ H NMR Spectrum of Compound ( \pm )-2.35

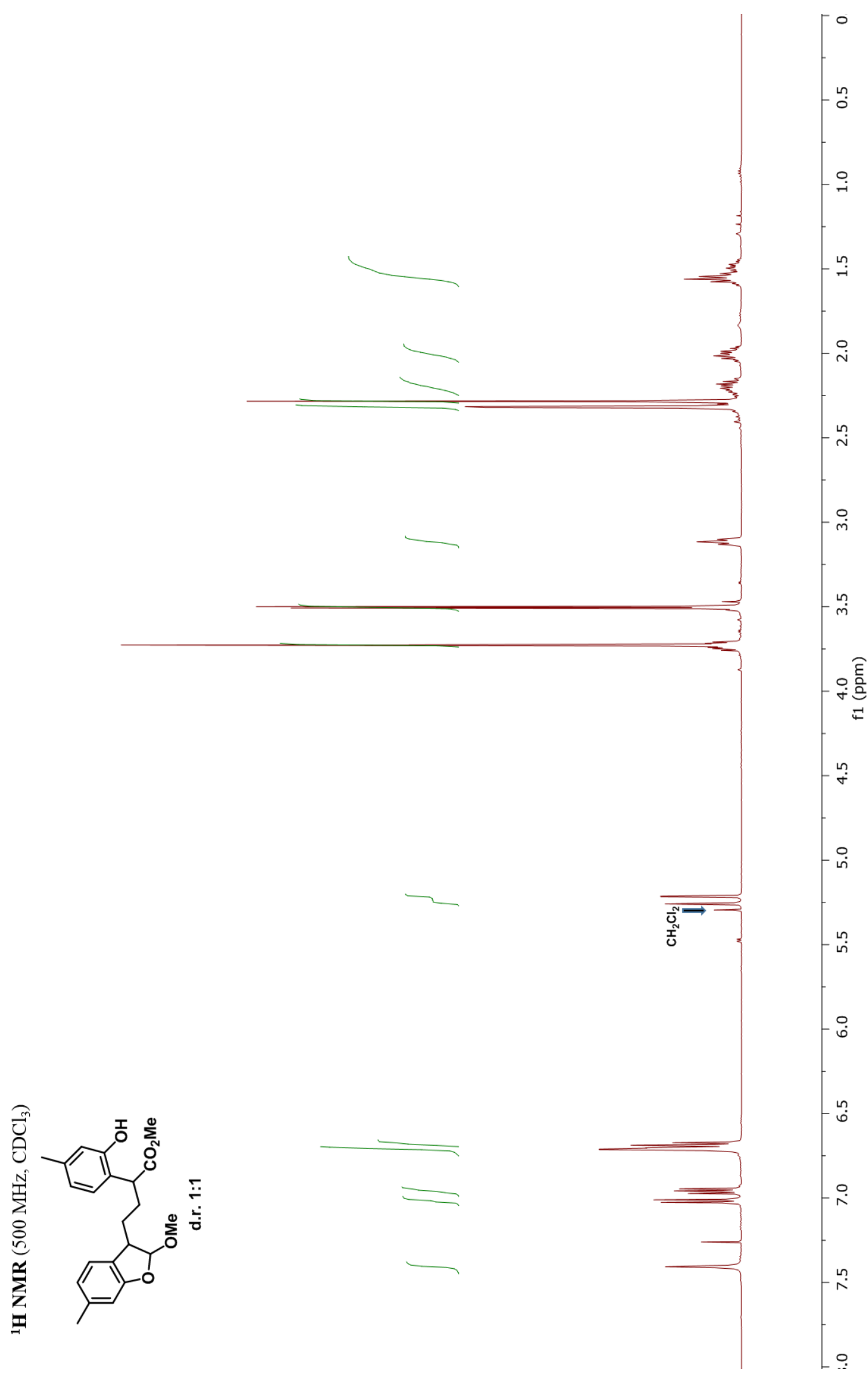


${ }^{13}$ C NMR Spectrum of Compound ( \pm )-2.35

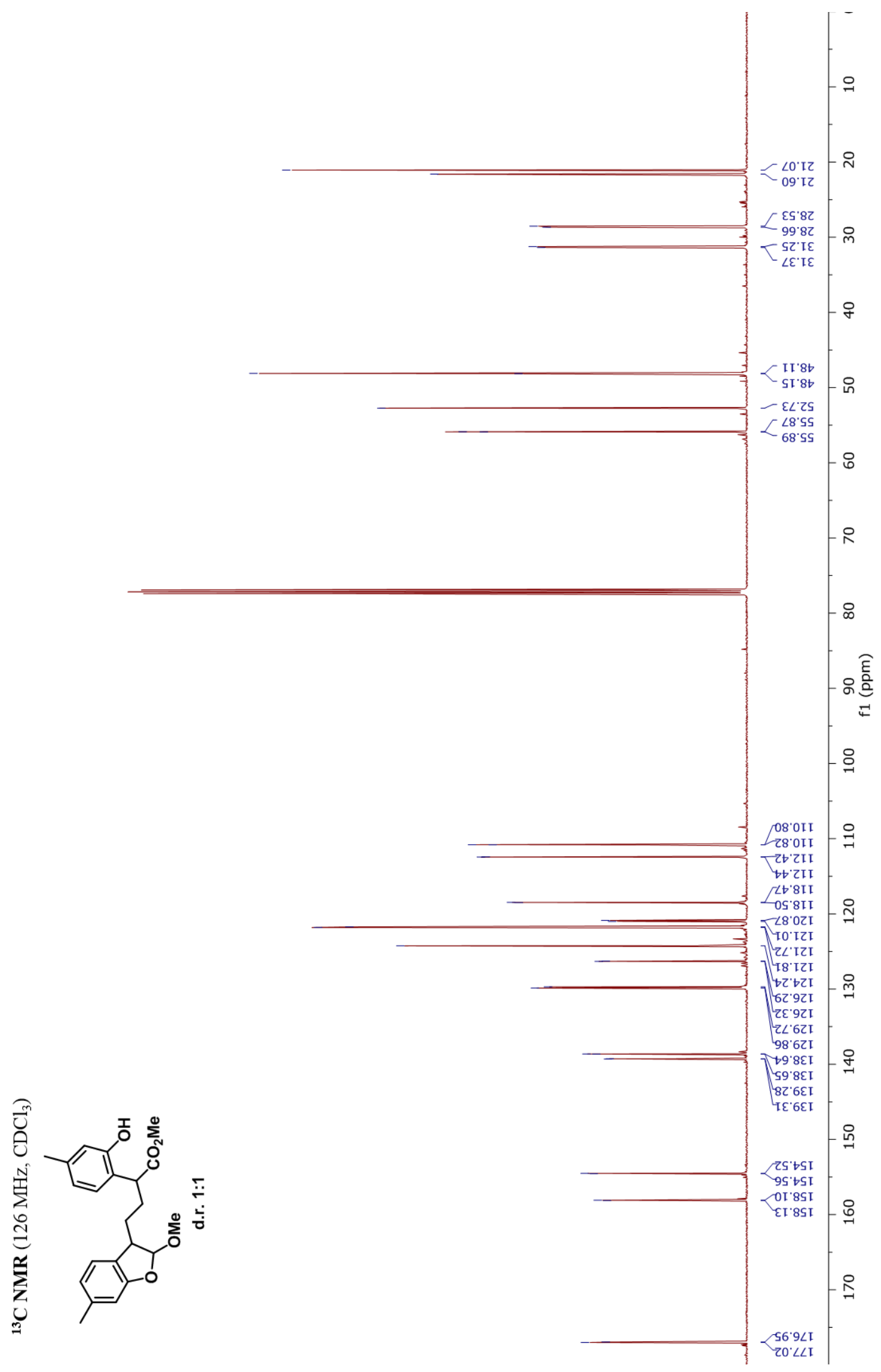


${ }^{1}$ H NMR Spectrum of Compound ( \pm )-2.38

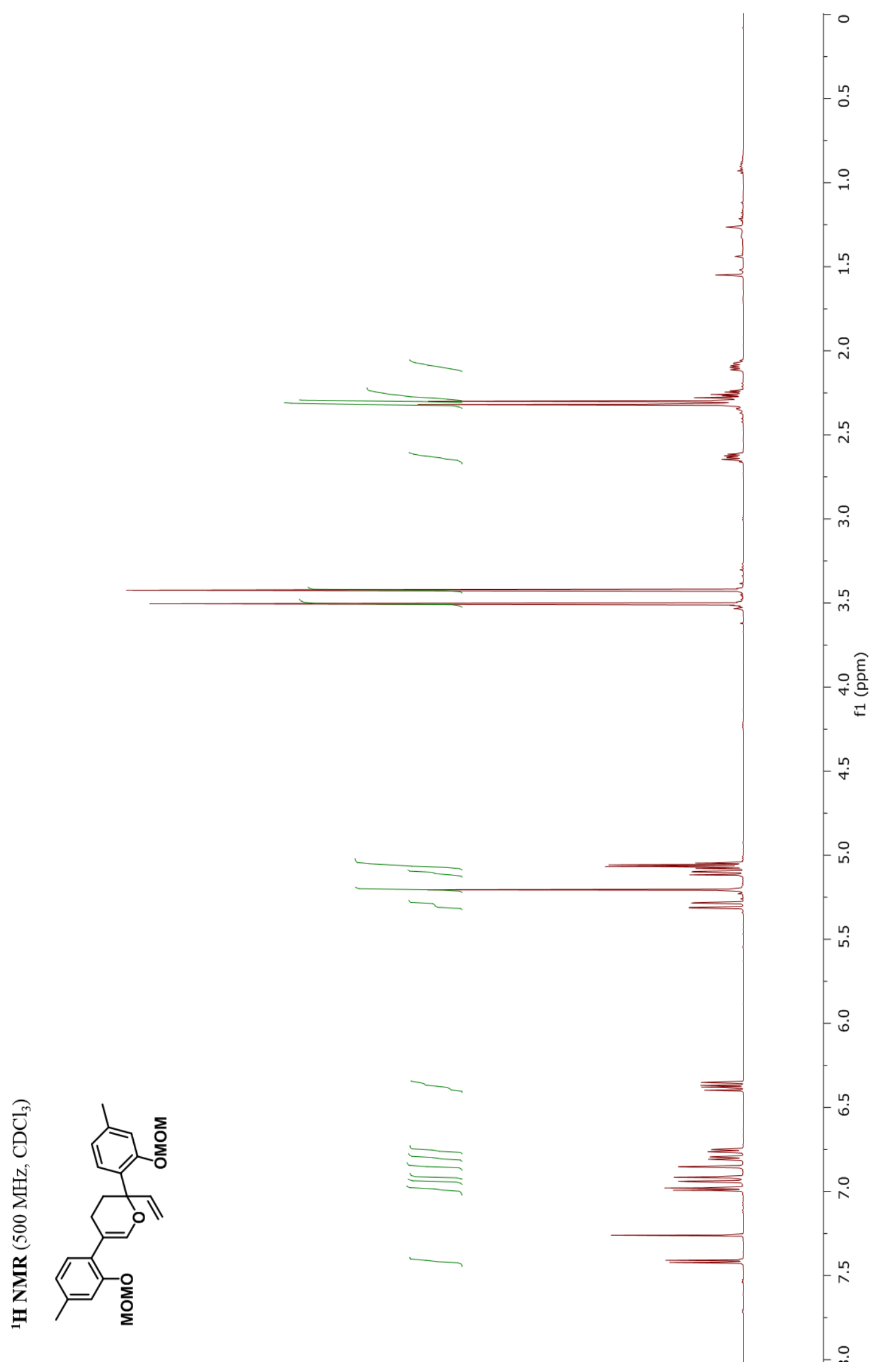


${ }^{13}$ C NMR Spectrum of Compound ( \pm )-2.38

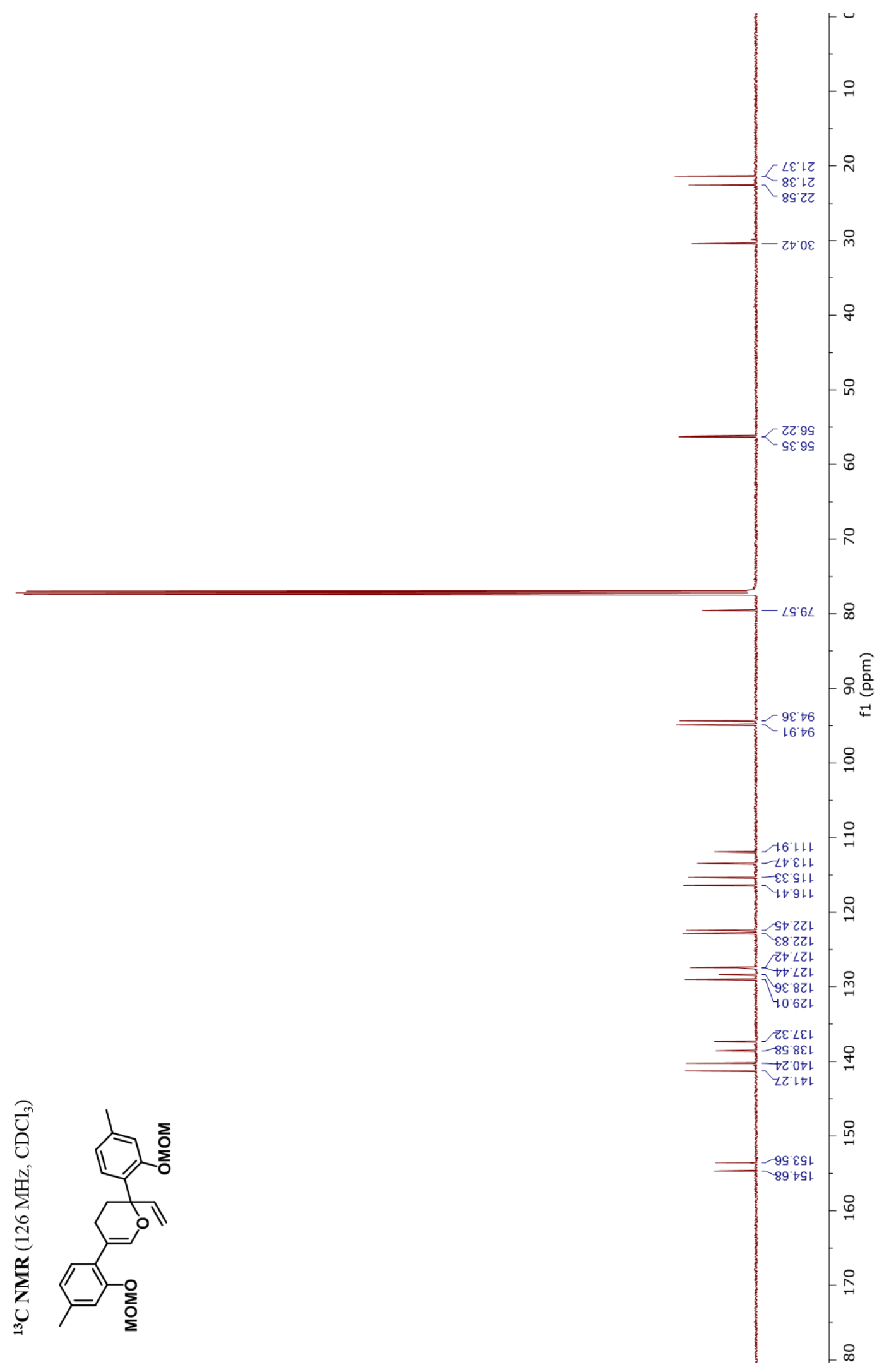


${ }^{1}$ H NMR Spectrum of Compound ( \pm )-2.39

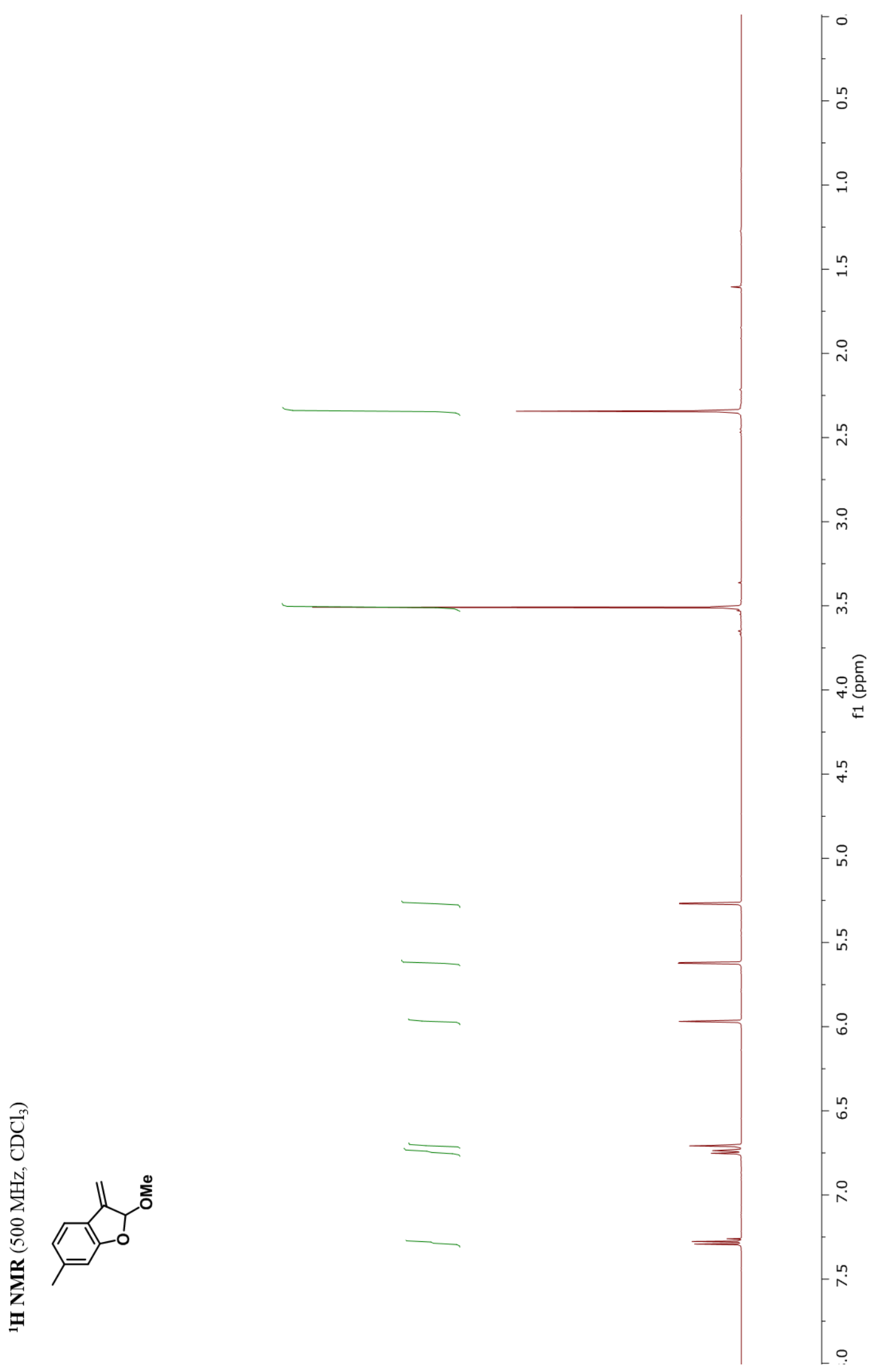


${ }^{13}$ C NMR Spectrum of Compound ( \pm )-2.39

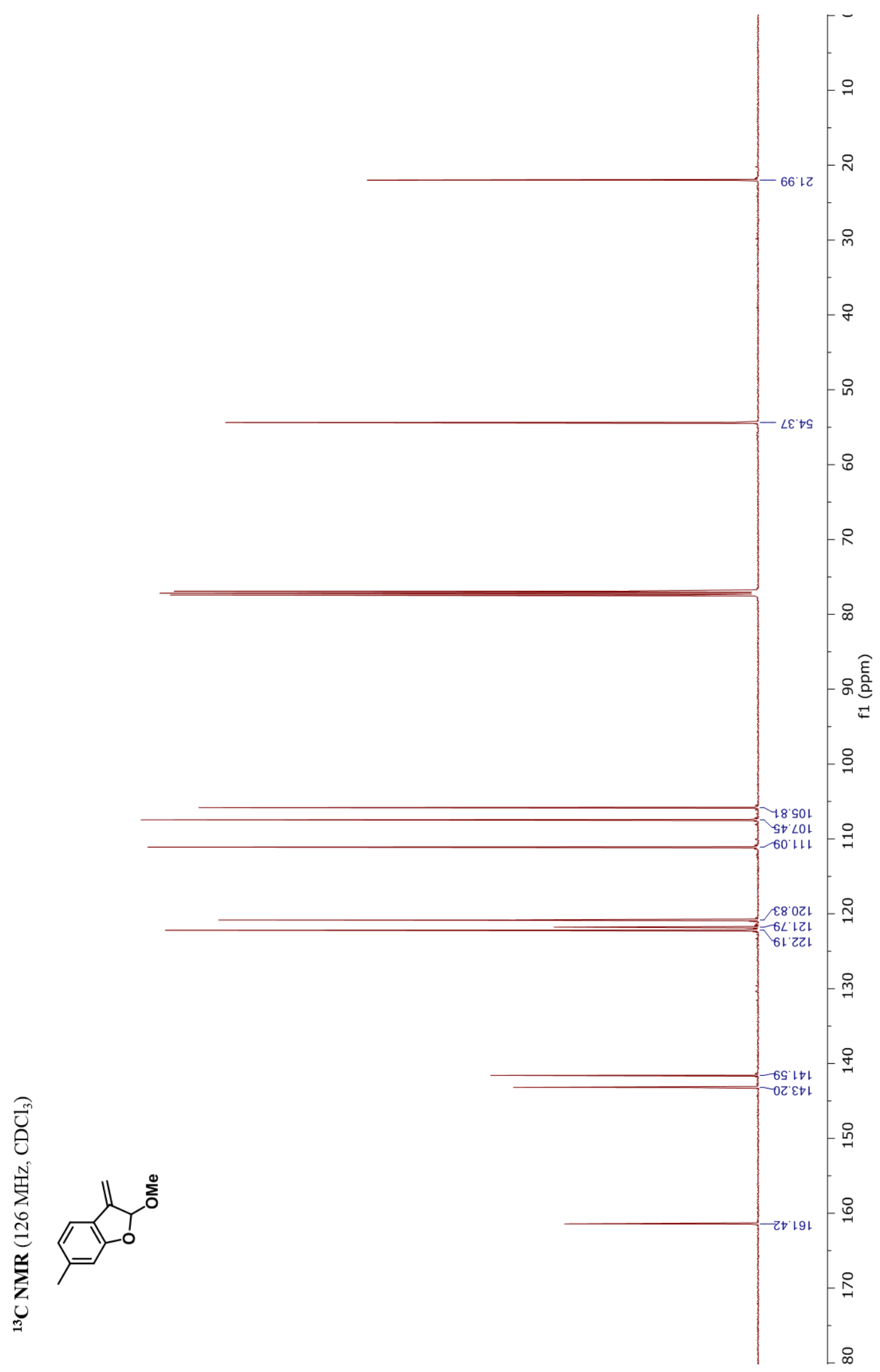


${ }^{1} \mathrm{H}$ NMR Spectrum of Compound 2.40

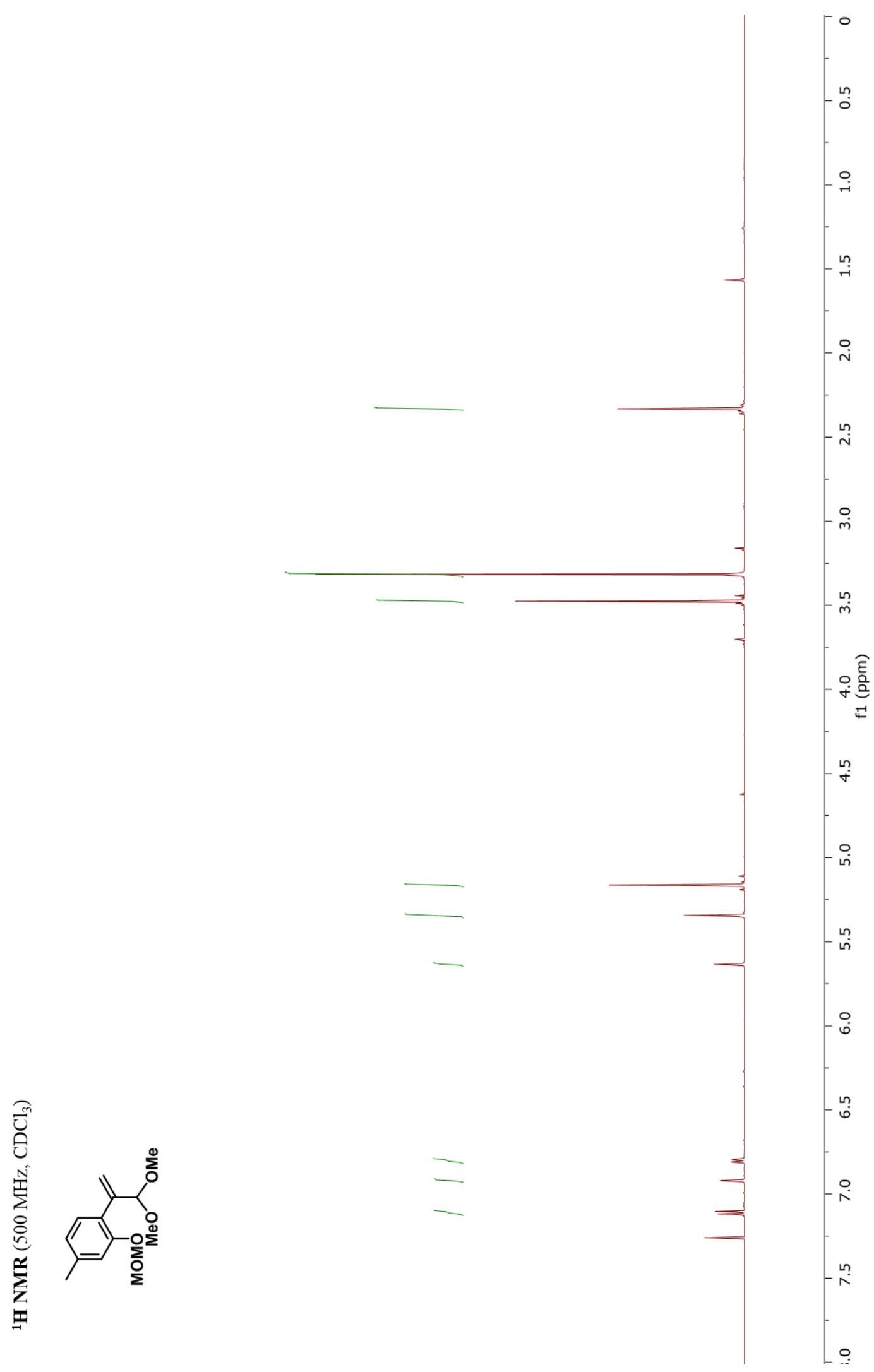


${ }^{13}$ C NMR Spectrum of Compound 2.40

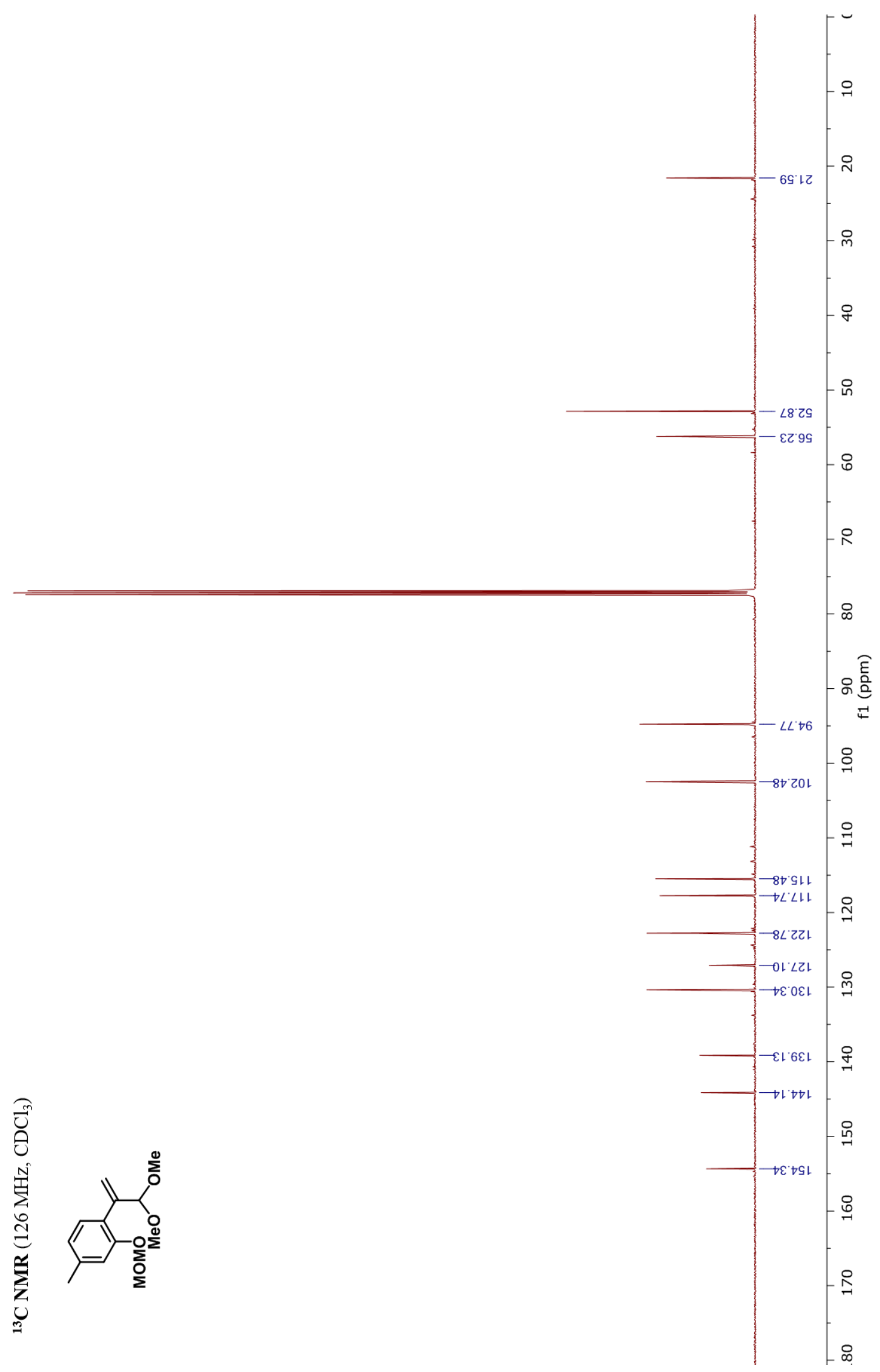


${ }^{1}$ H NMR Spectrum of Compound 2.41

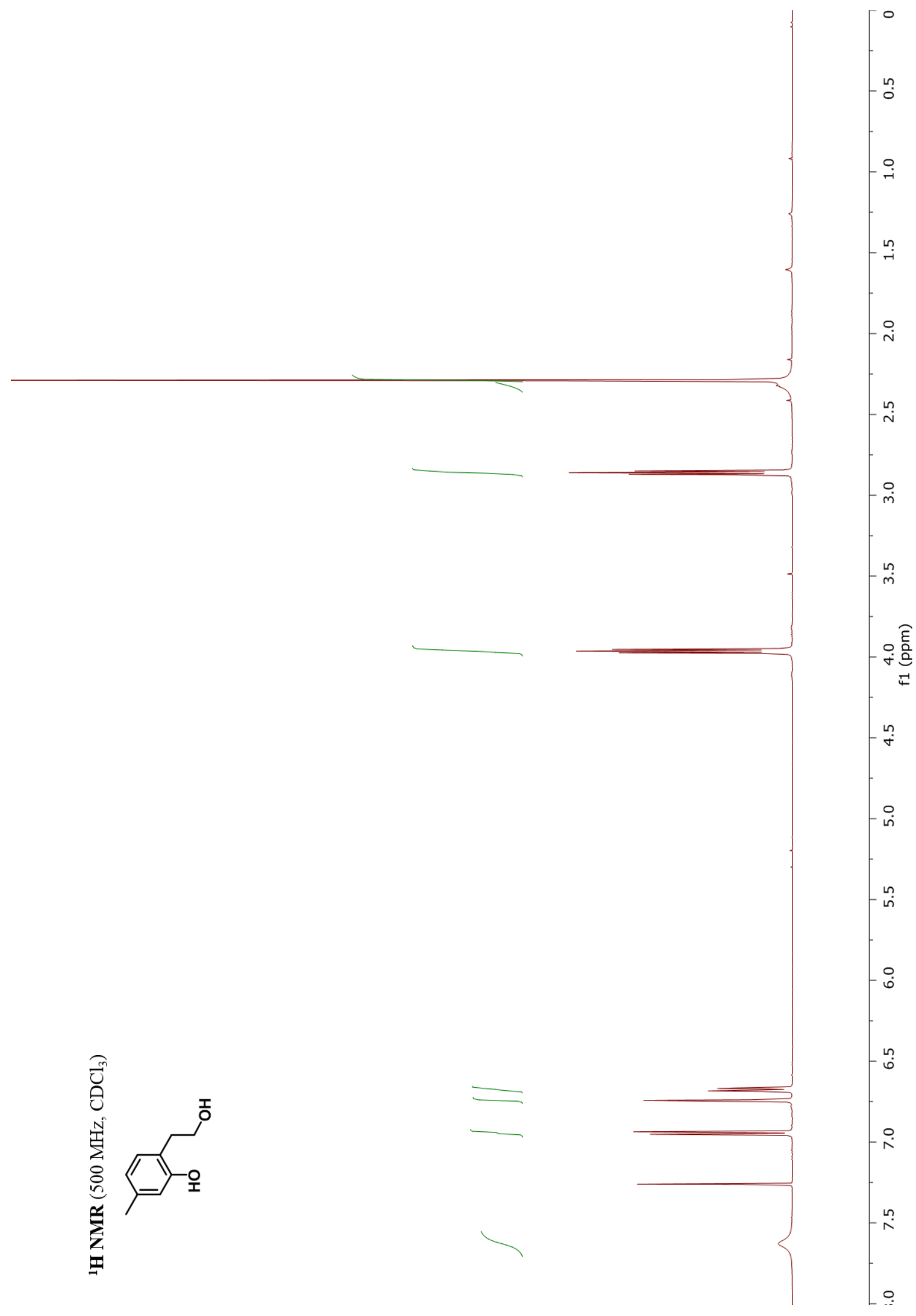


${ }^{13}$ C NMR Spectrum of Compound 2.41

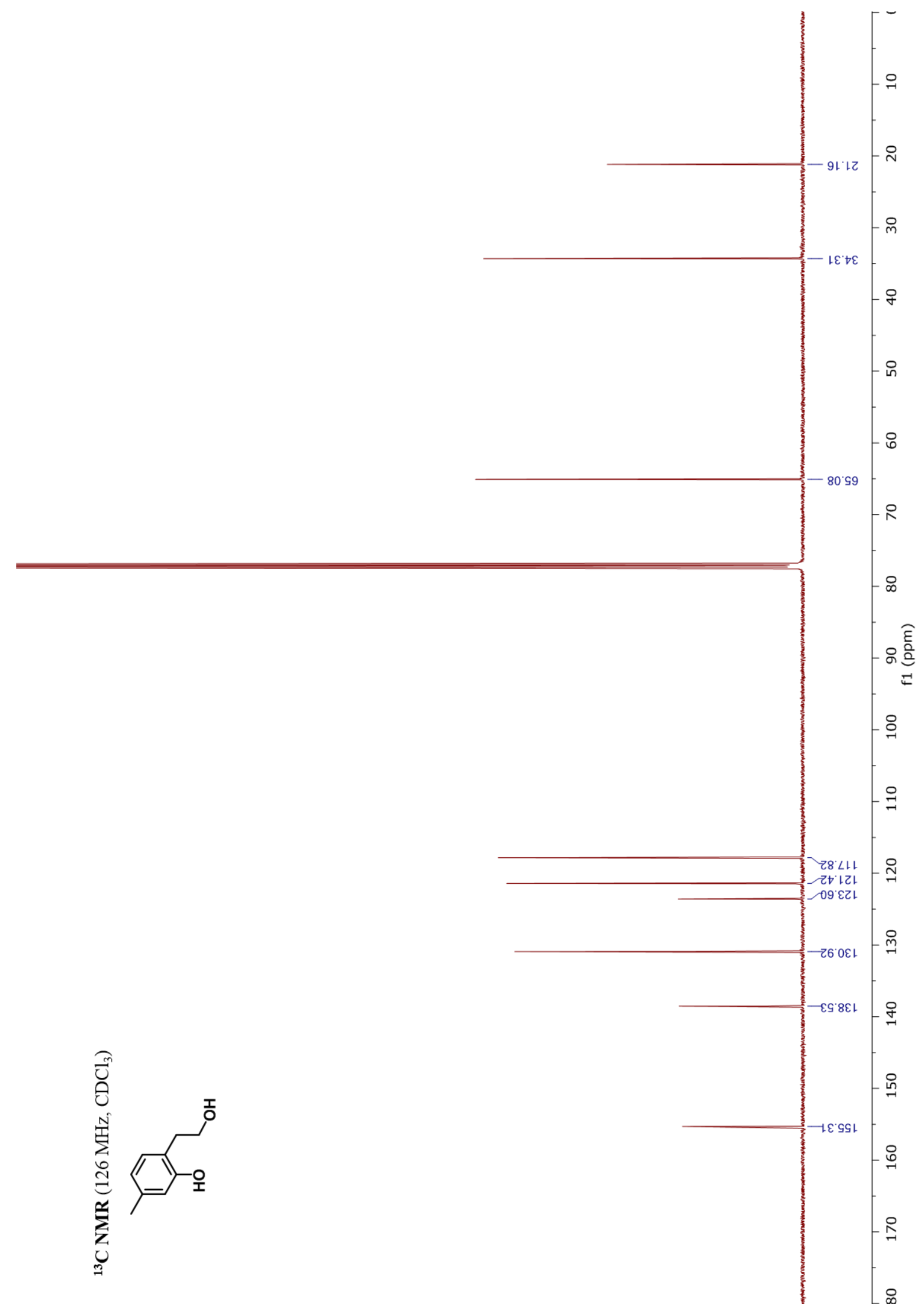


${ }^{1}$ H NMR Spectrum of Compound 2.42

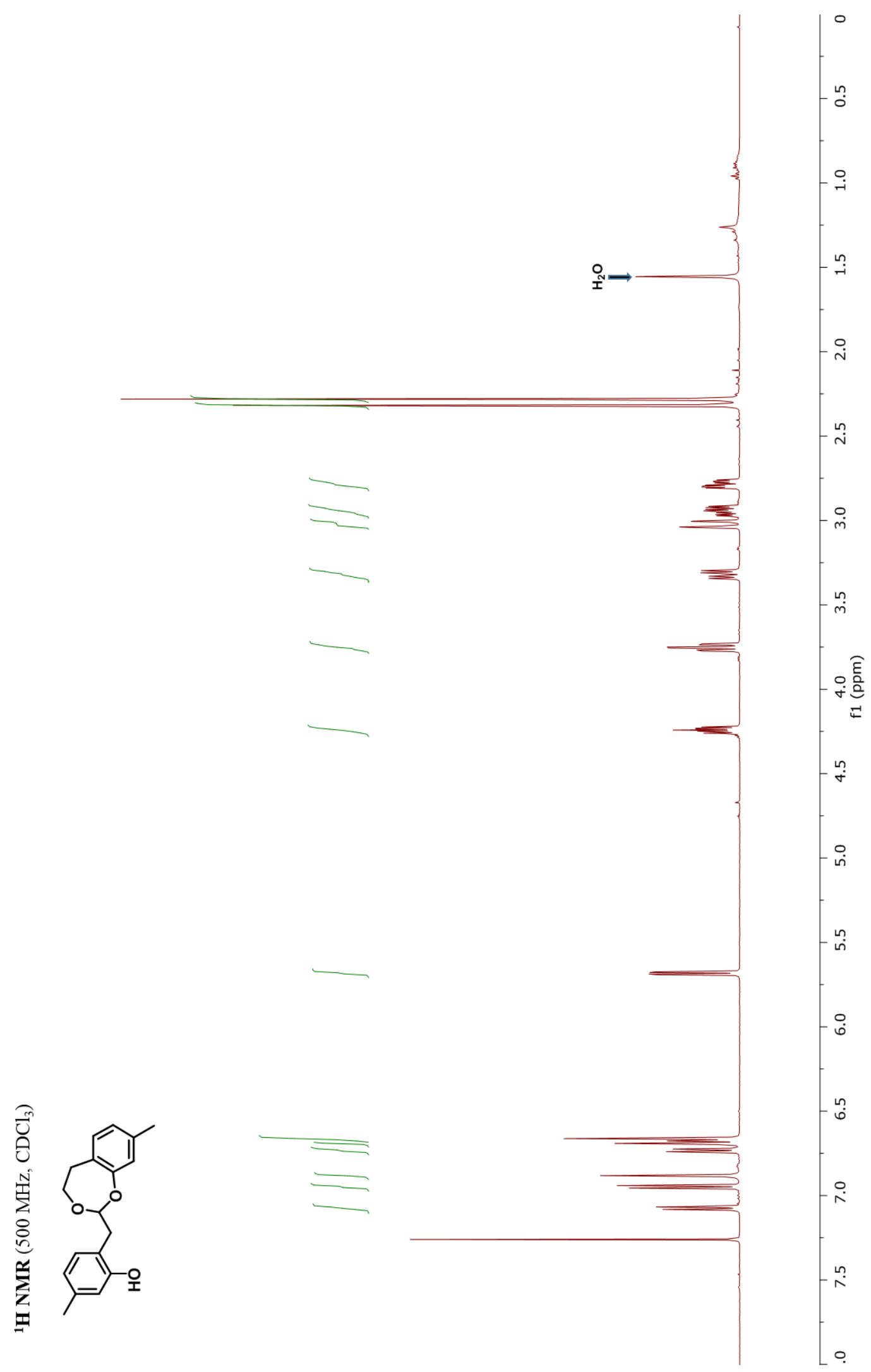


${ }^{13}$ C NMR Spectrum of Compound 2.42

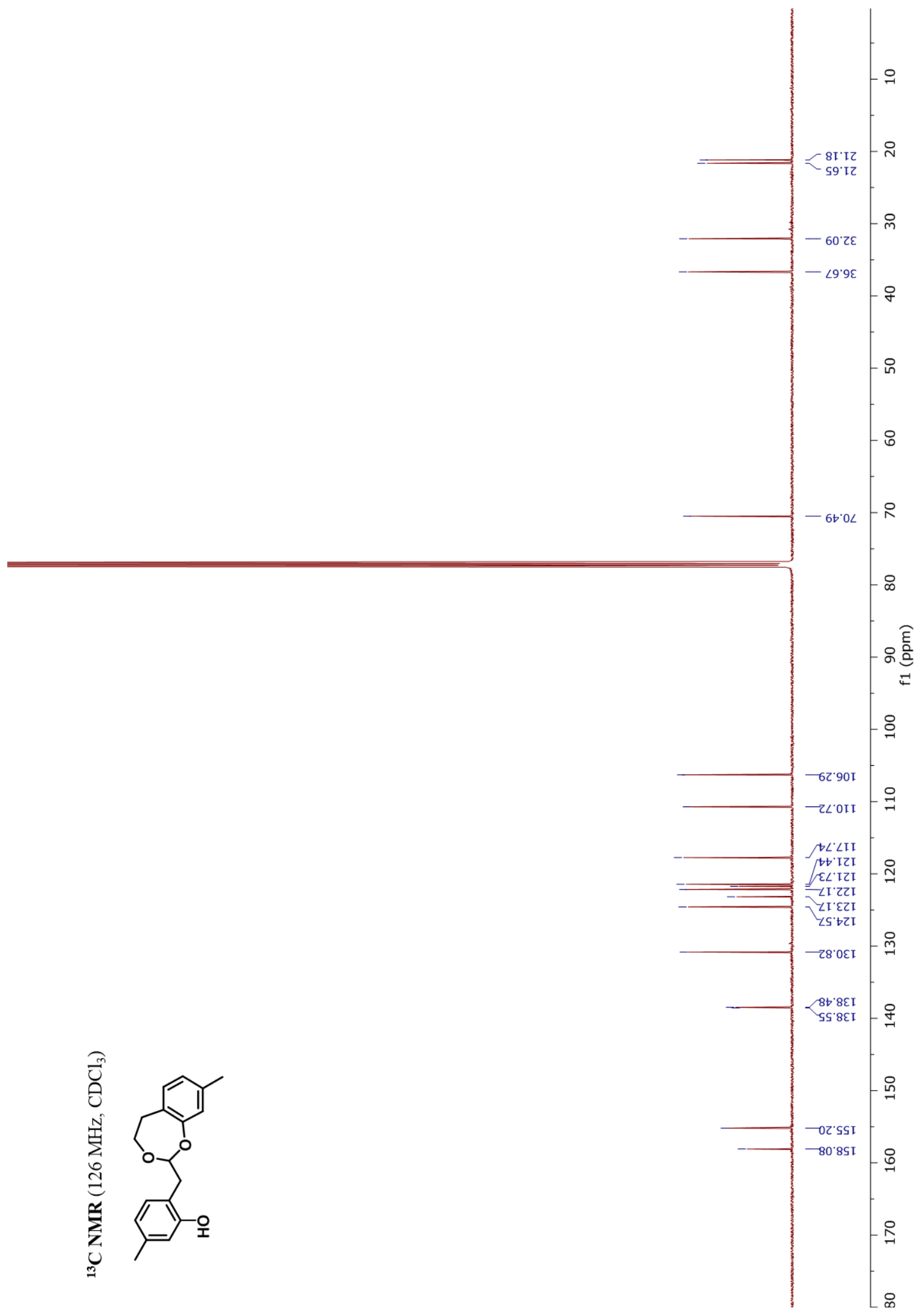


${ }^{1}$ H NMR Spectrum of Compound 2.1

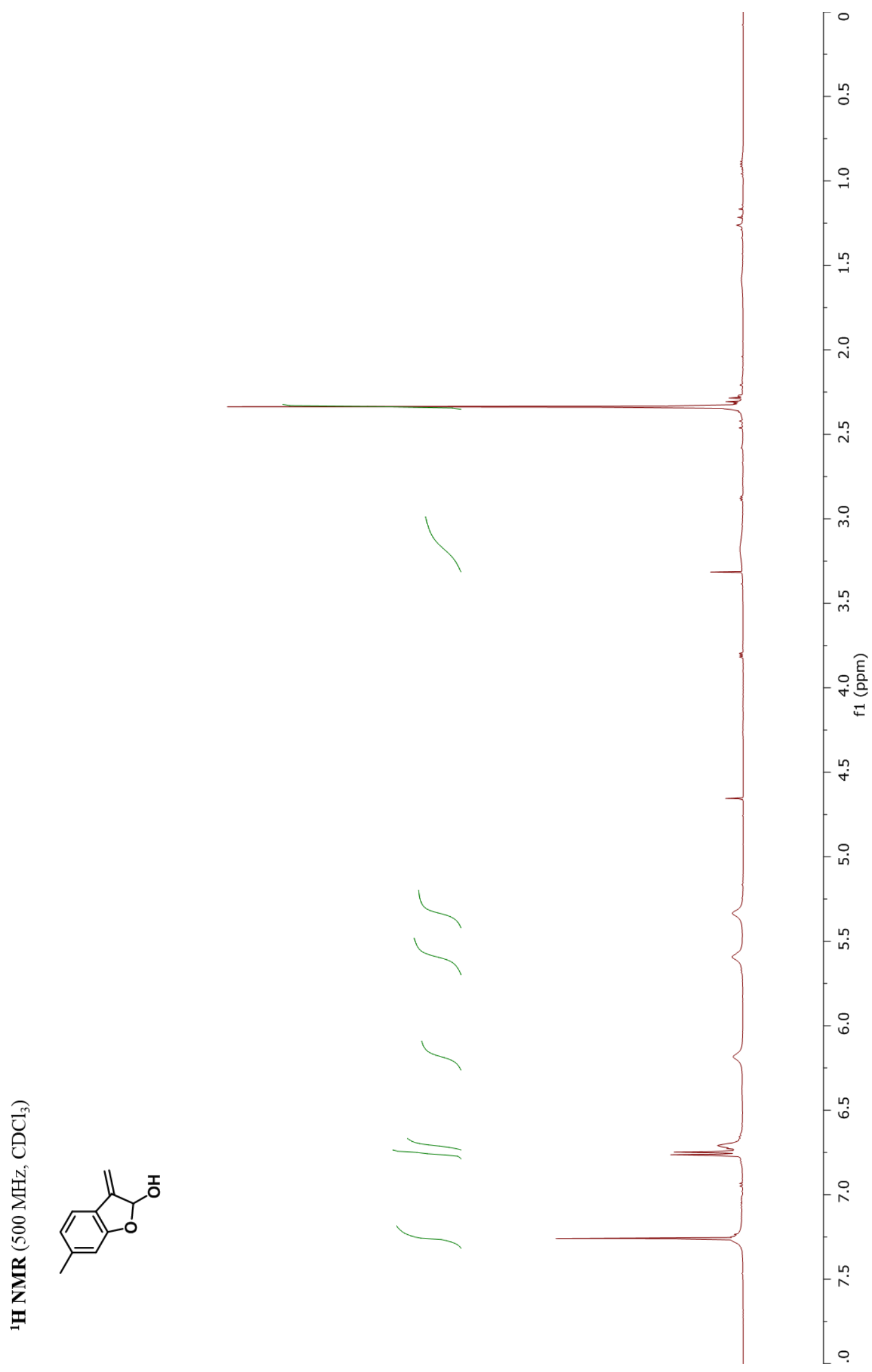




\section{Comparison of ${ }^{1} \mathrm{H}$ NMR Spectra of Compound 2.1}

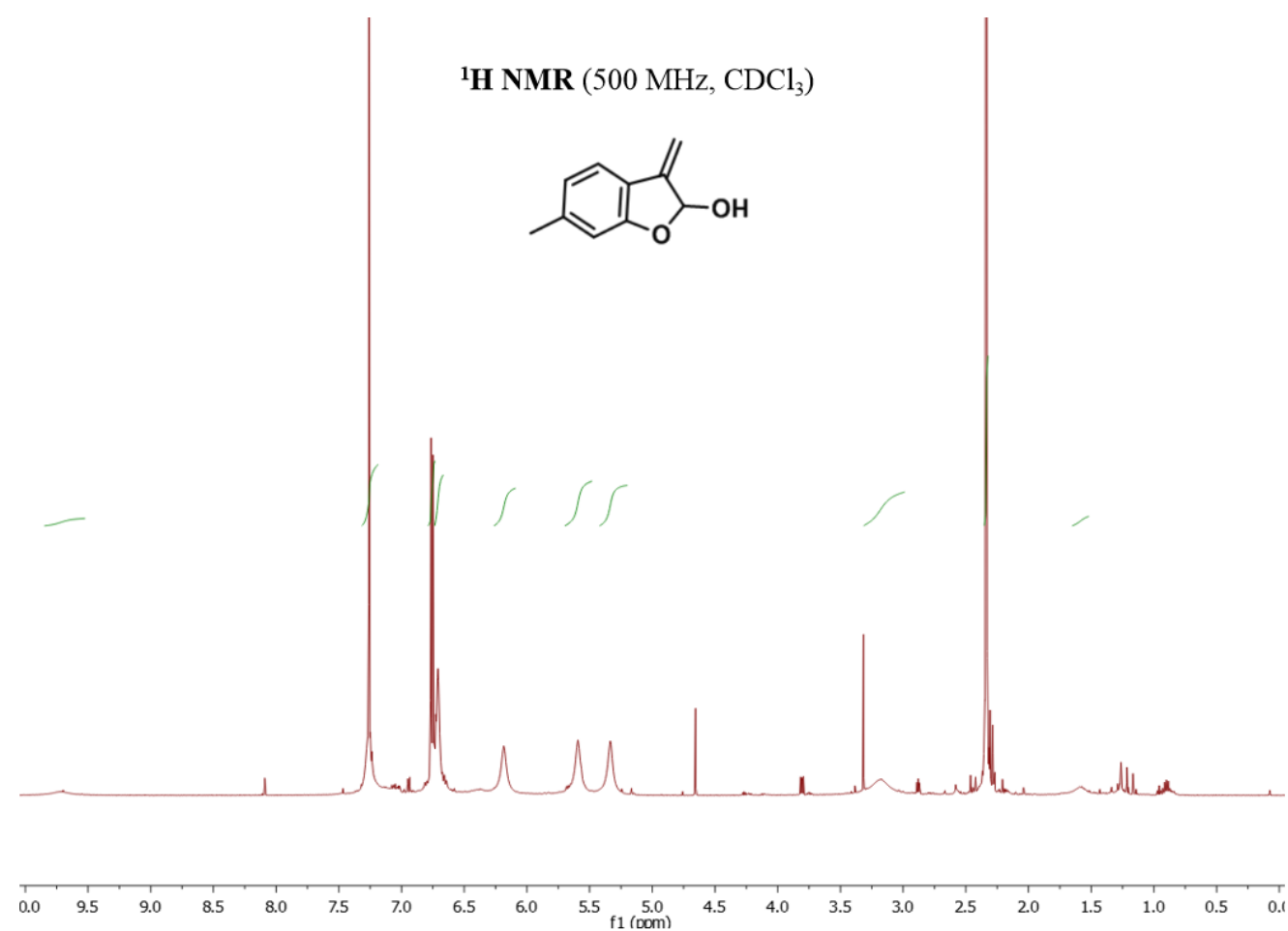

Literature ${ }^{1} \mathrm{H}$ NMR Spectrum (300 MHz, $\mathrm{CDCl}_{3}$ )

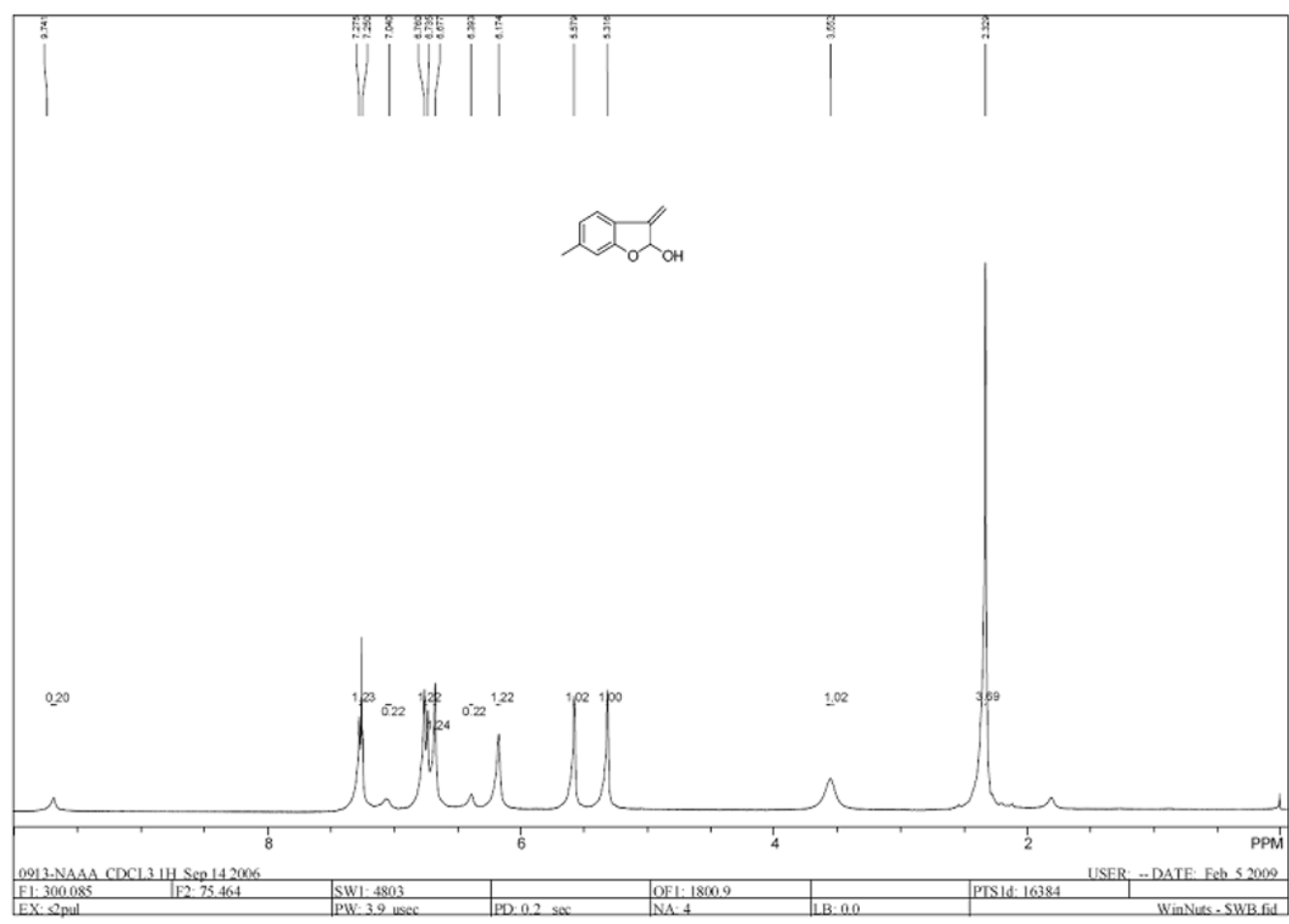


${ }^{1}$ H NMR Analysis for Dimerization of Compound 2.1

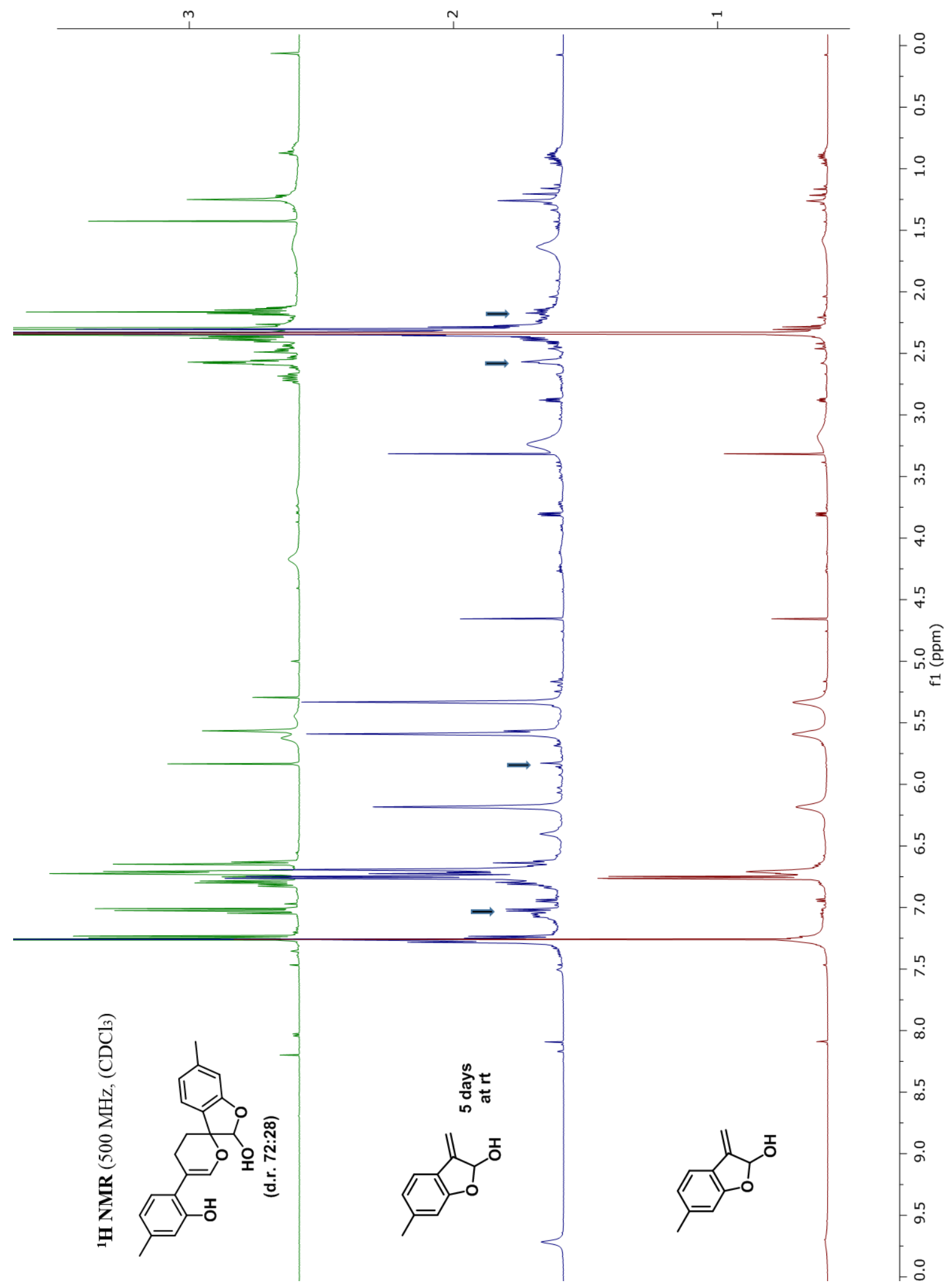


${ }^{1} \mathrm{H}$ NMR Spectrum of Compound 2.43

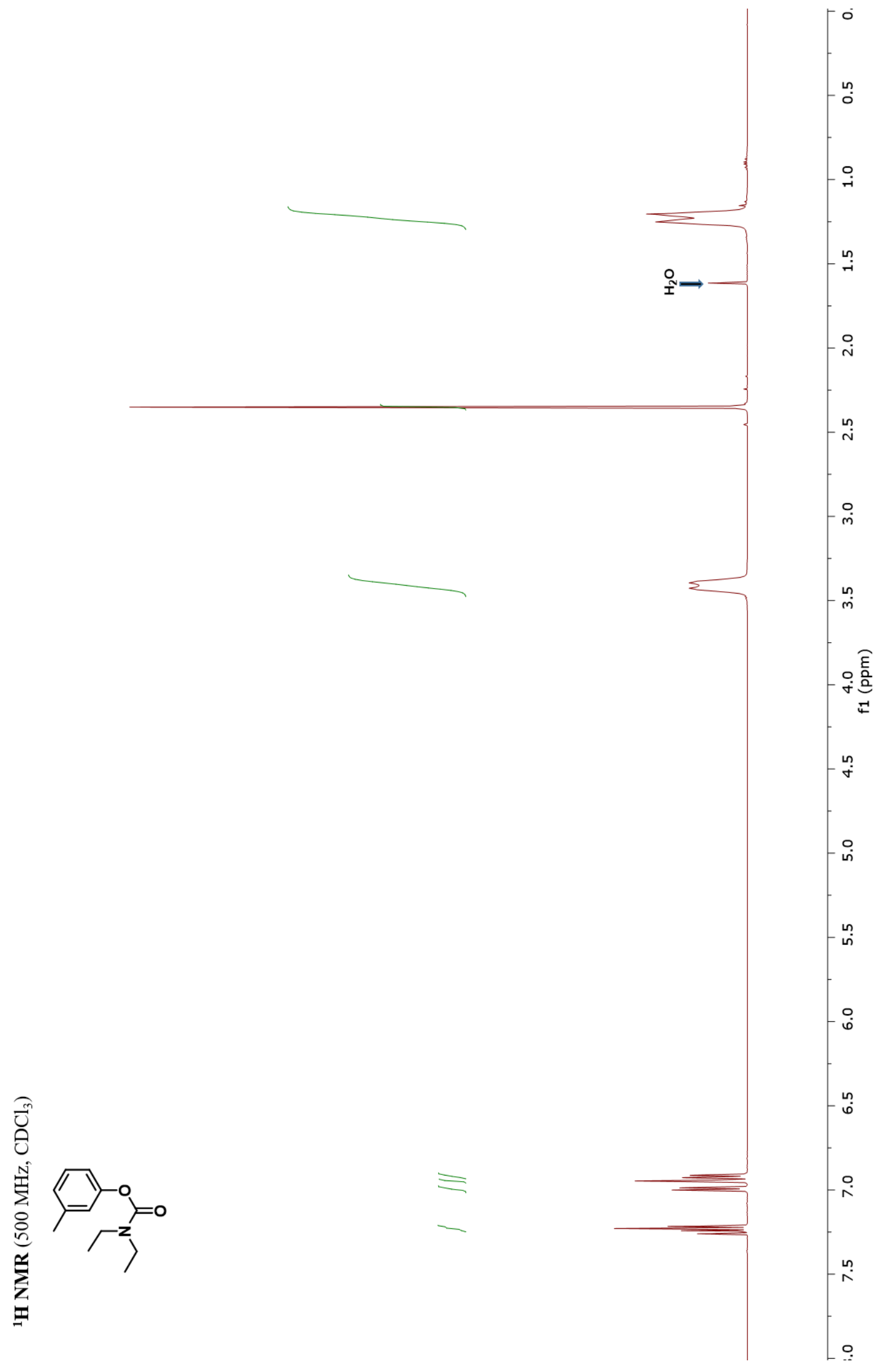


${ }^{13}$ C NMR Spectrum of Compound 2.43

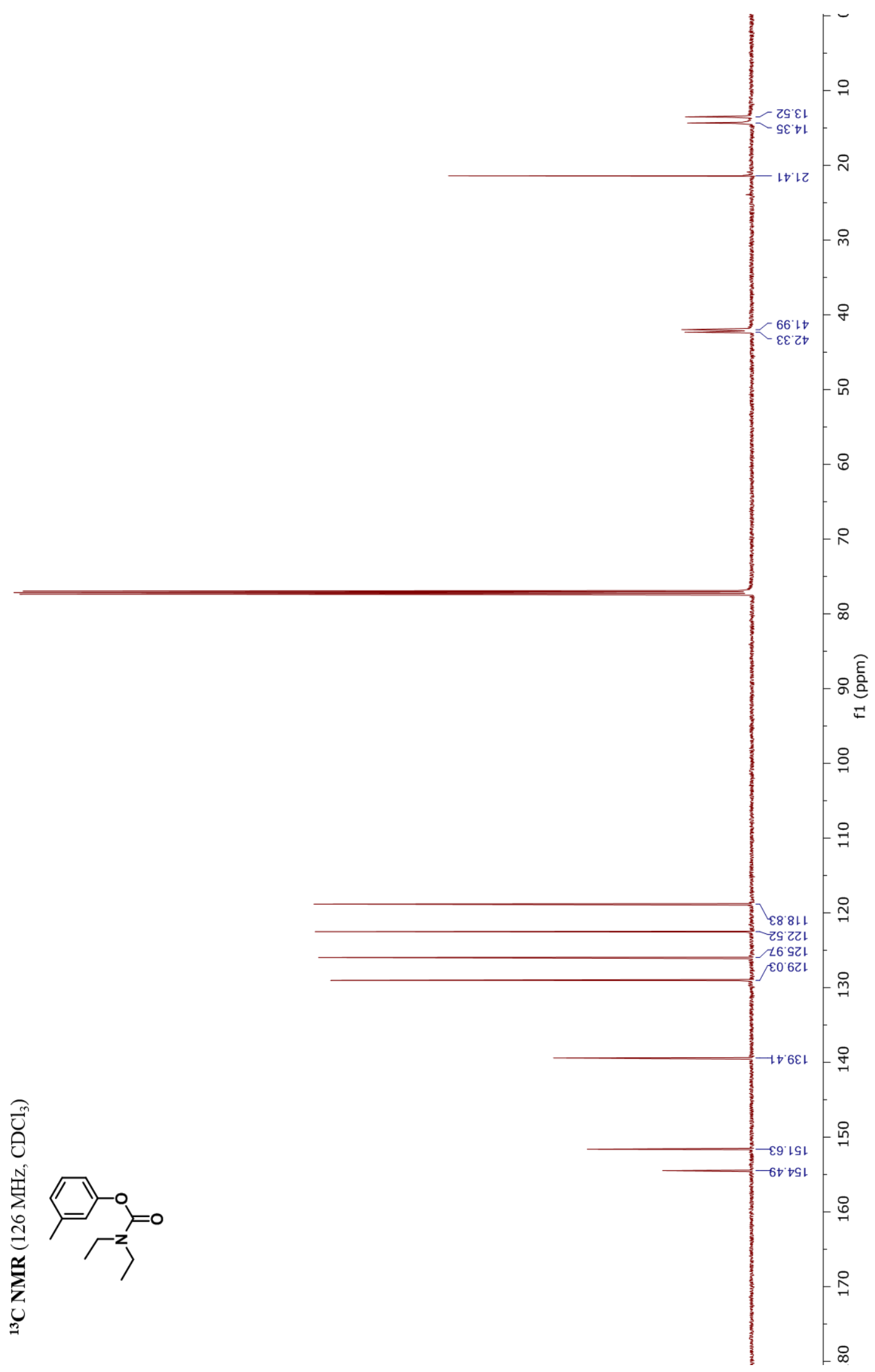


${ }^{1} \mathrm{H}$ NMR Spectrum of Compound 2.45

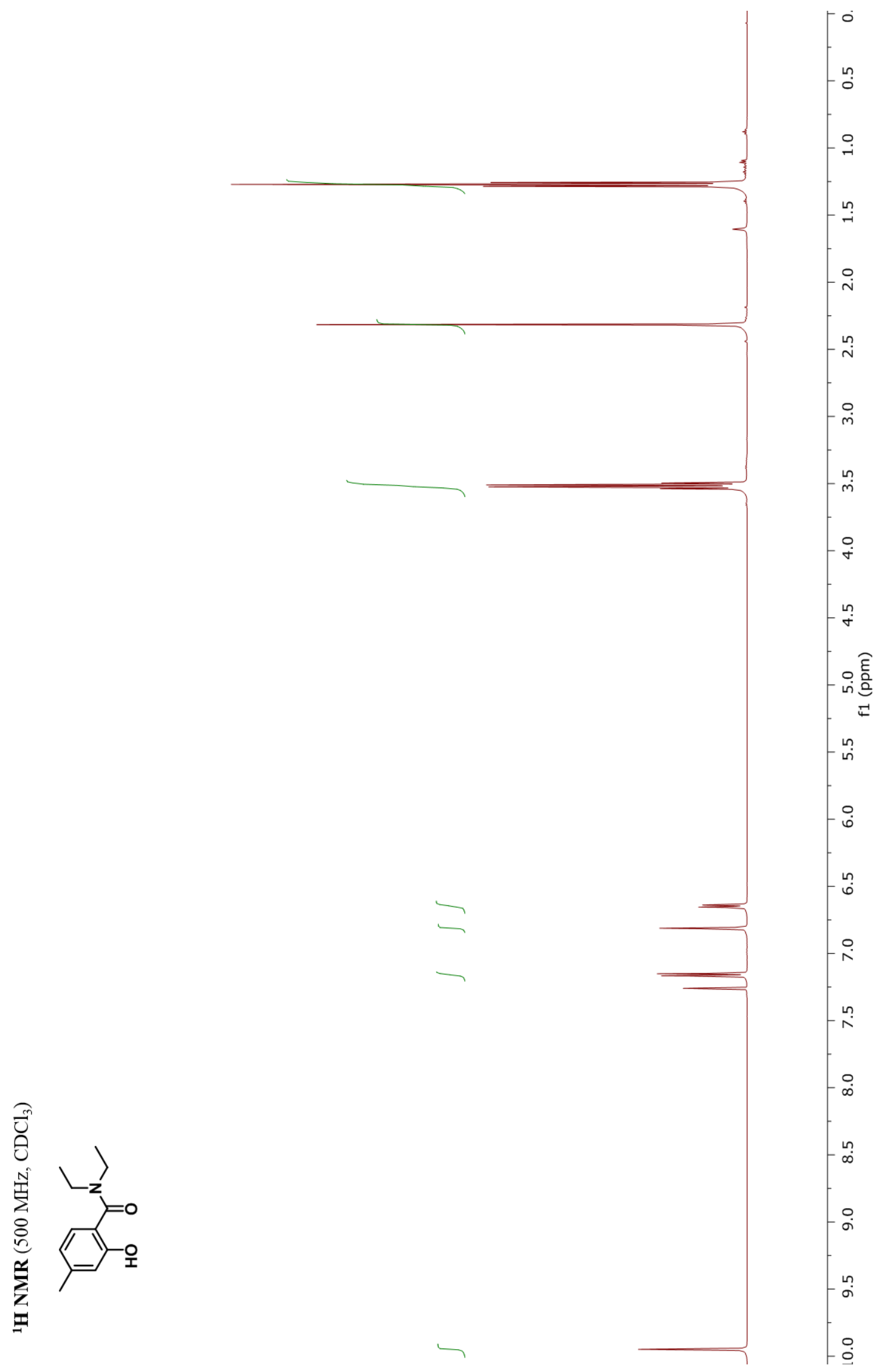


${ }^{13}$ C NMR Spectrum of Compound 2.45

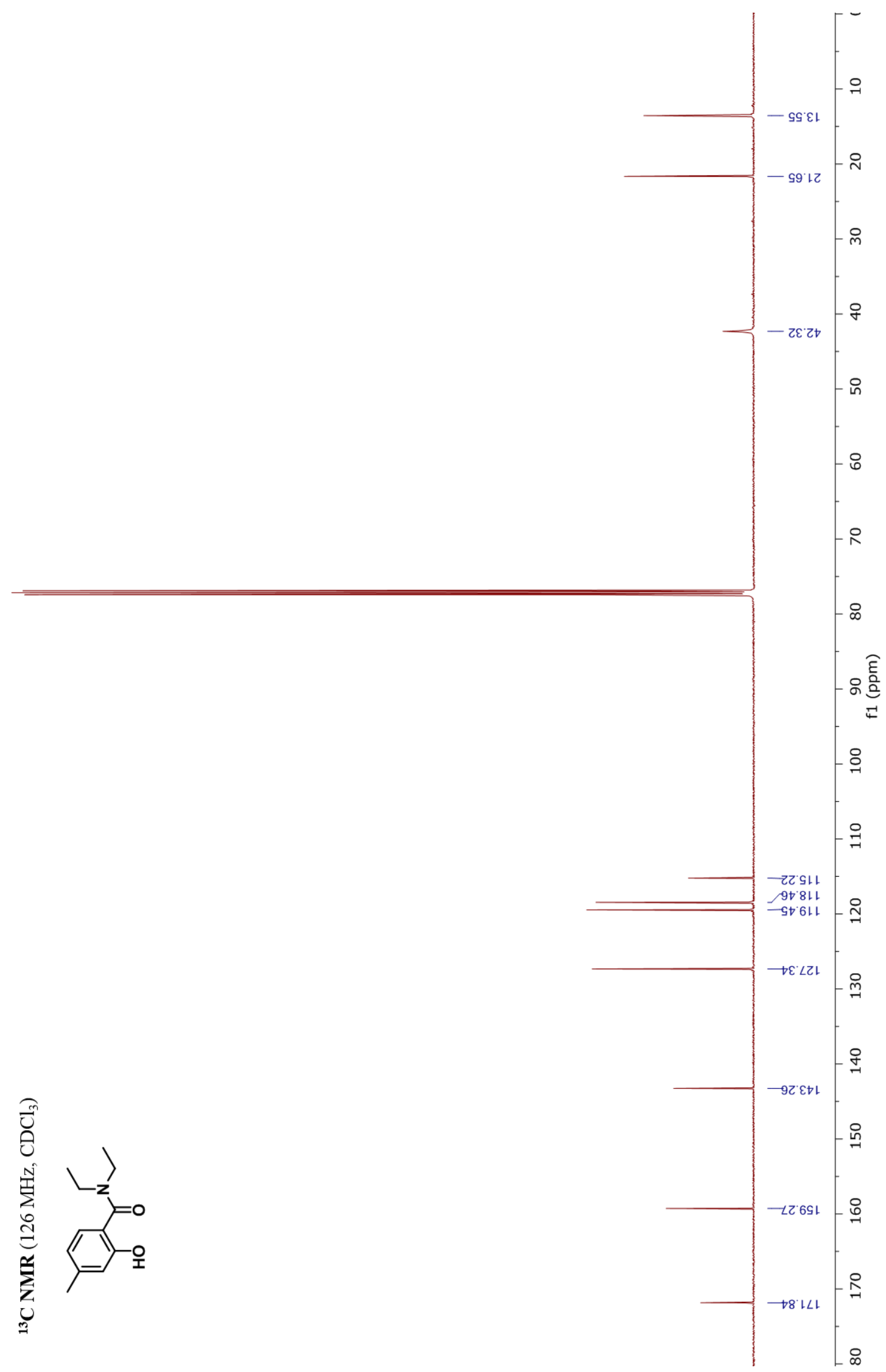


${ }^{1} \mathrm{H}$ NMR Spectrum of Compound 2.51

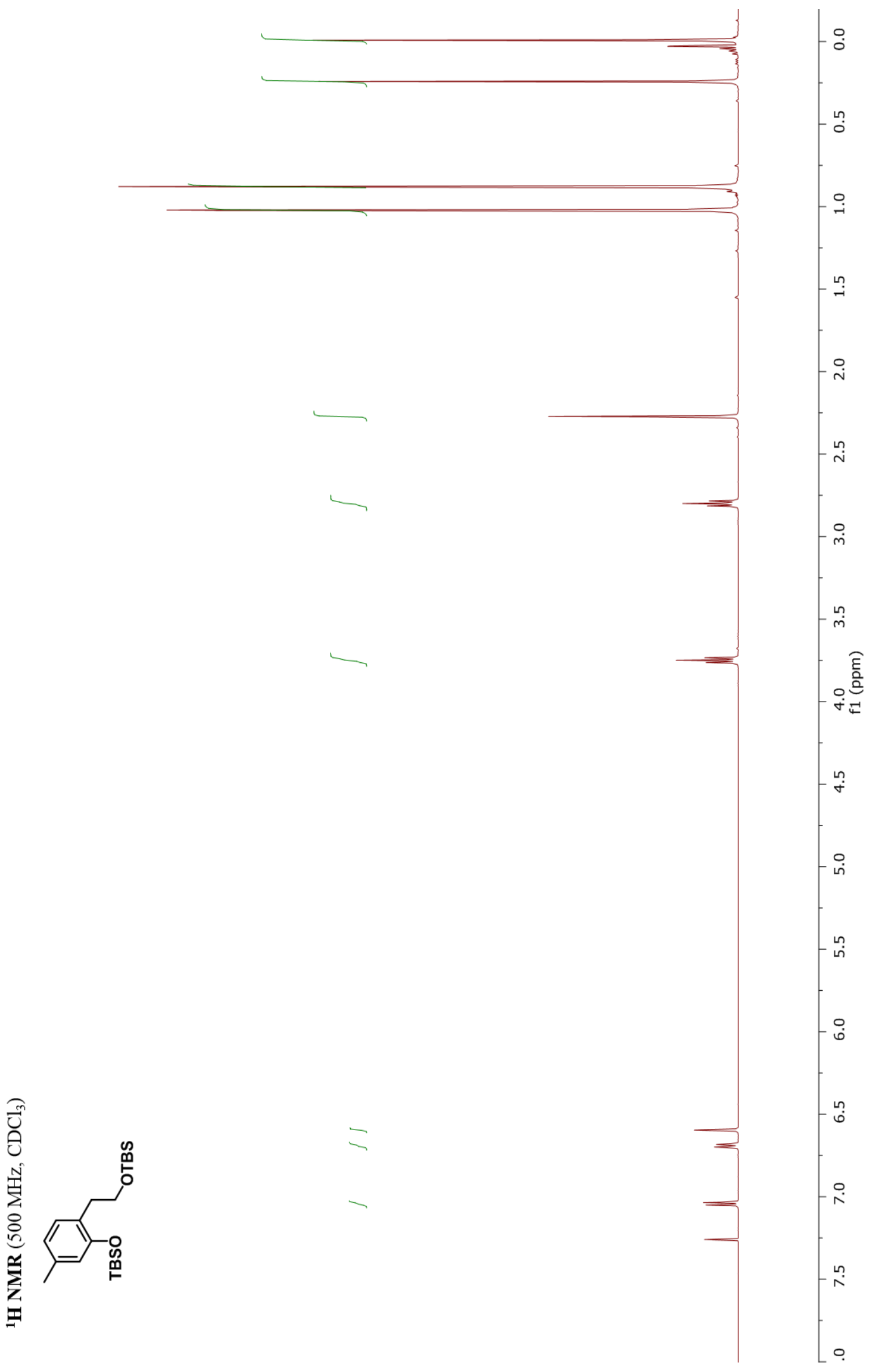


${ }^{13}$ C NMR Spectrum of Compound 2.51

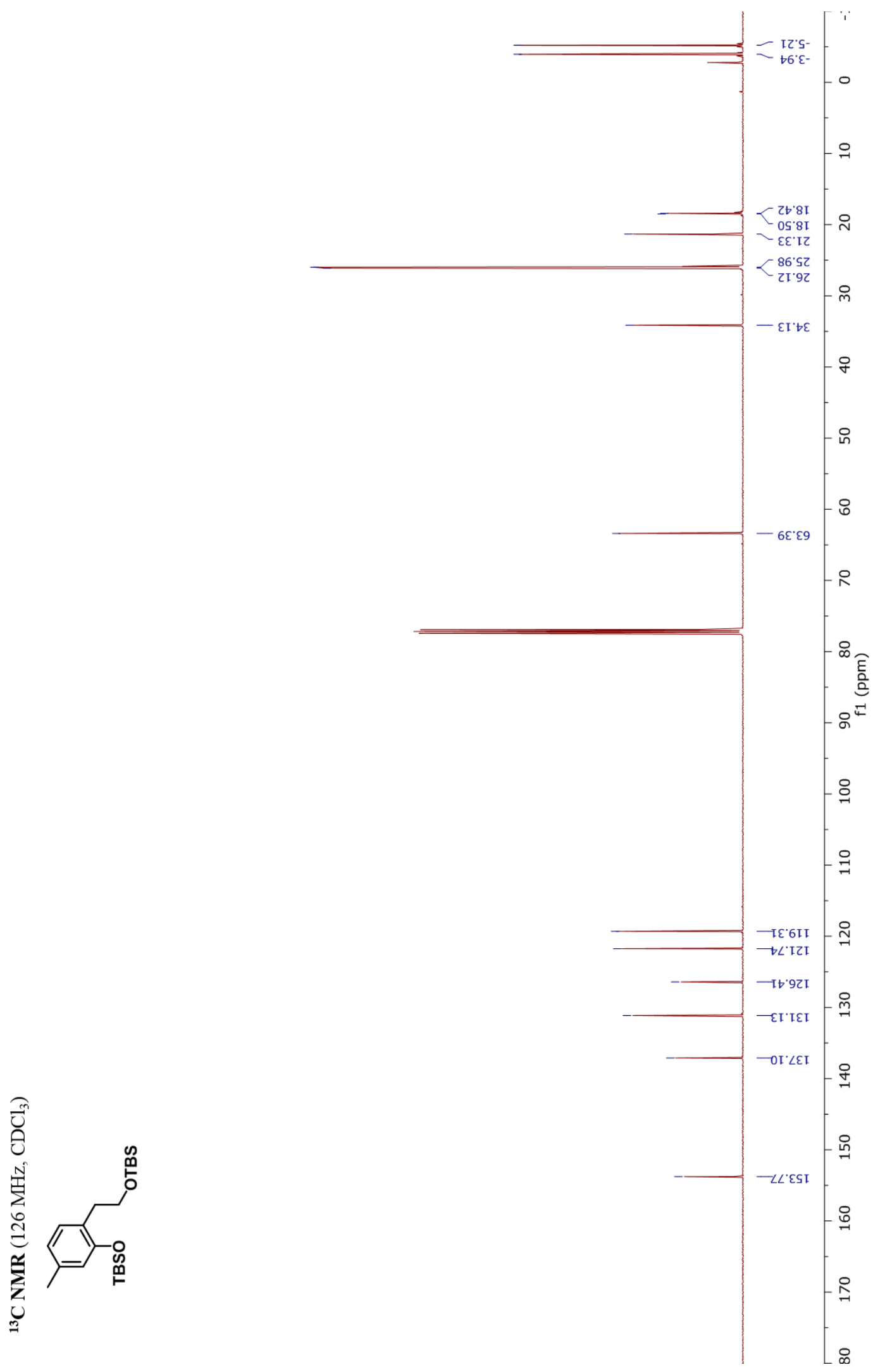


${ }^{1} \mathrm{H}$ NMR Spectrum of Compound 2.49

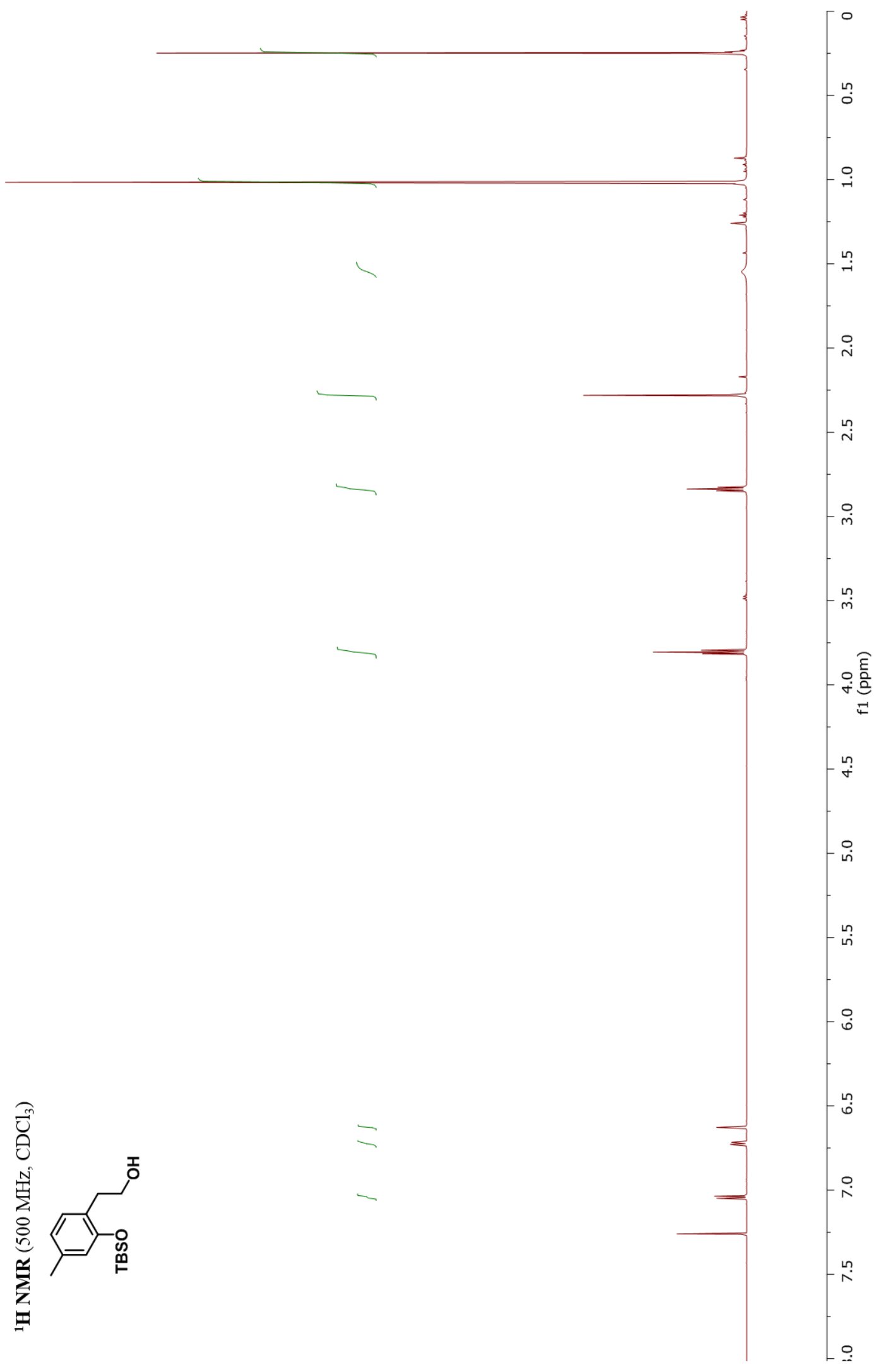


${ }^{13} \mathrm{C}$ NMR Spectrum of Compound 2.49

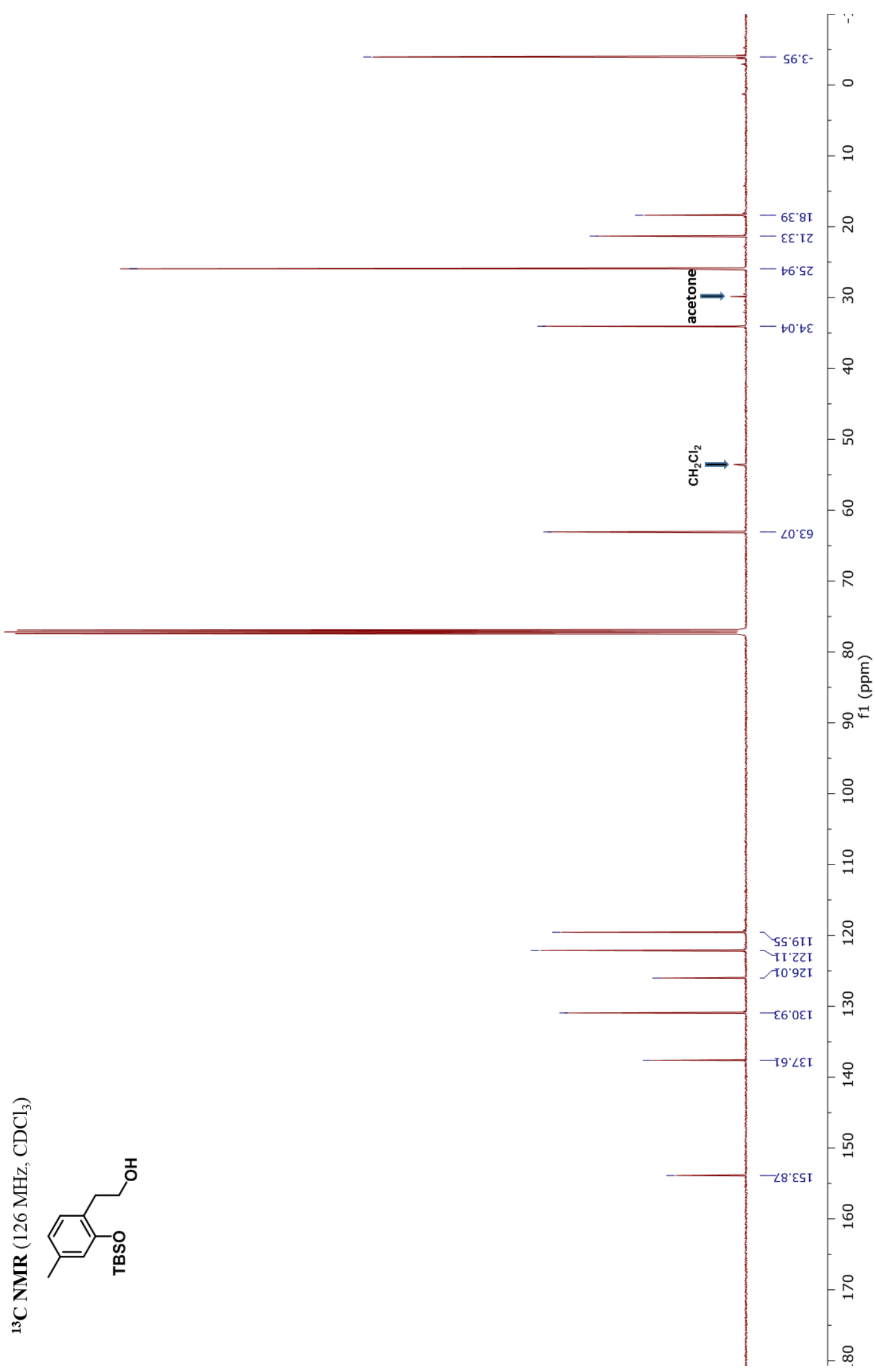


${ }^{1} \mathrm{H}$ NMR Spectrum of Compound 2.47

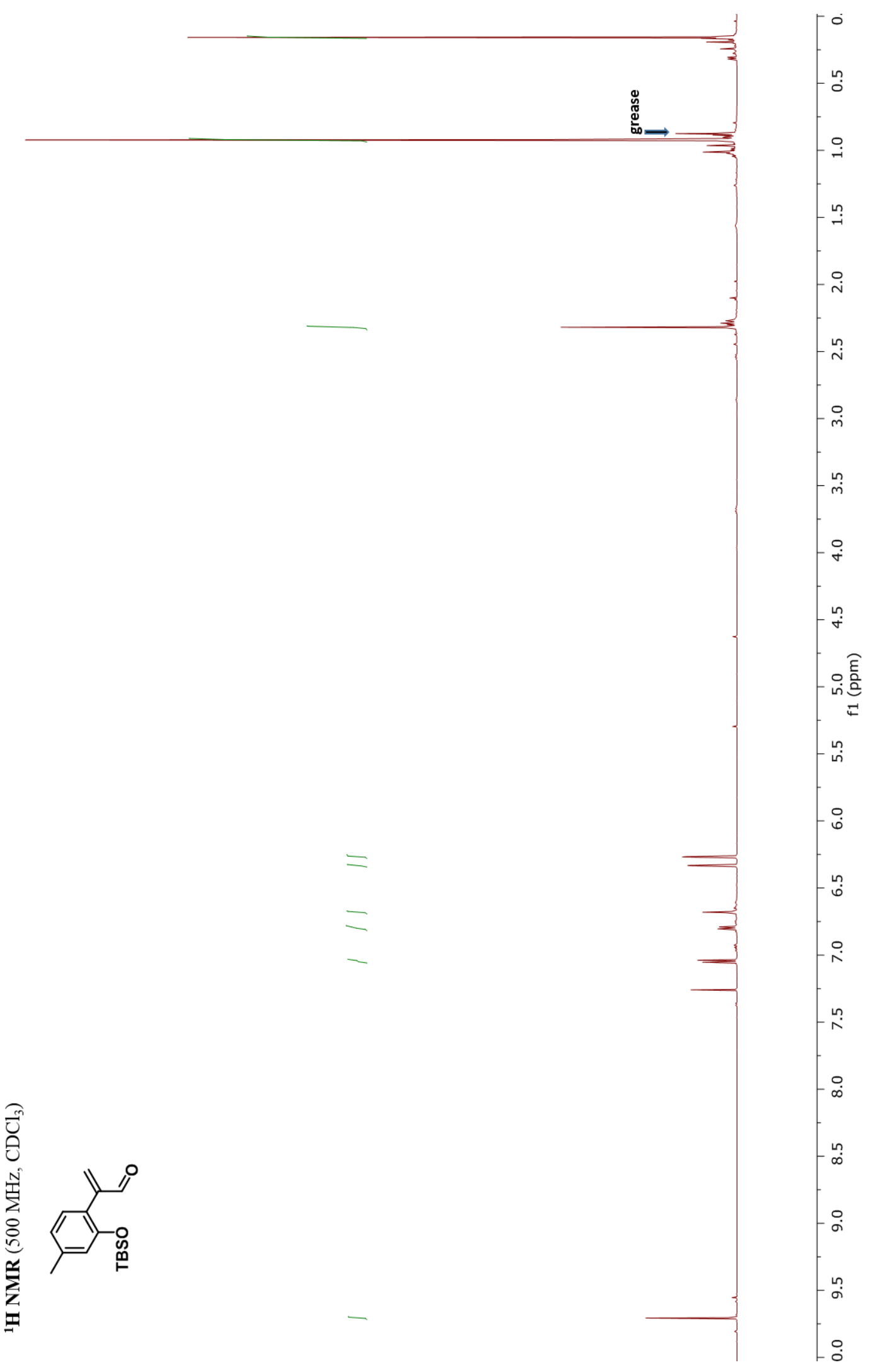


${ }^{13} \mathrm{C}$ NMR Spectrum of Compound 2.47

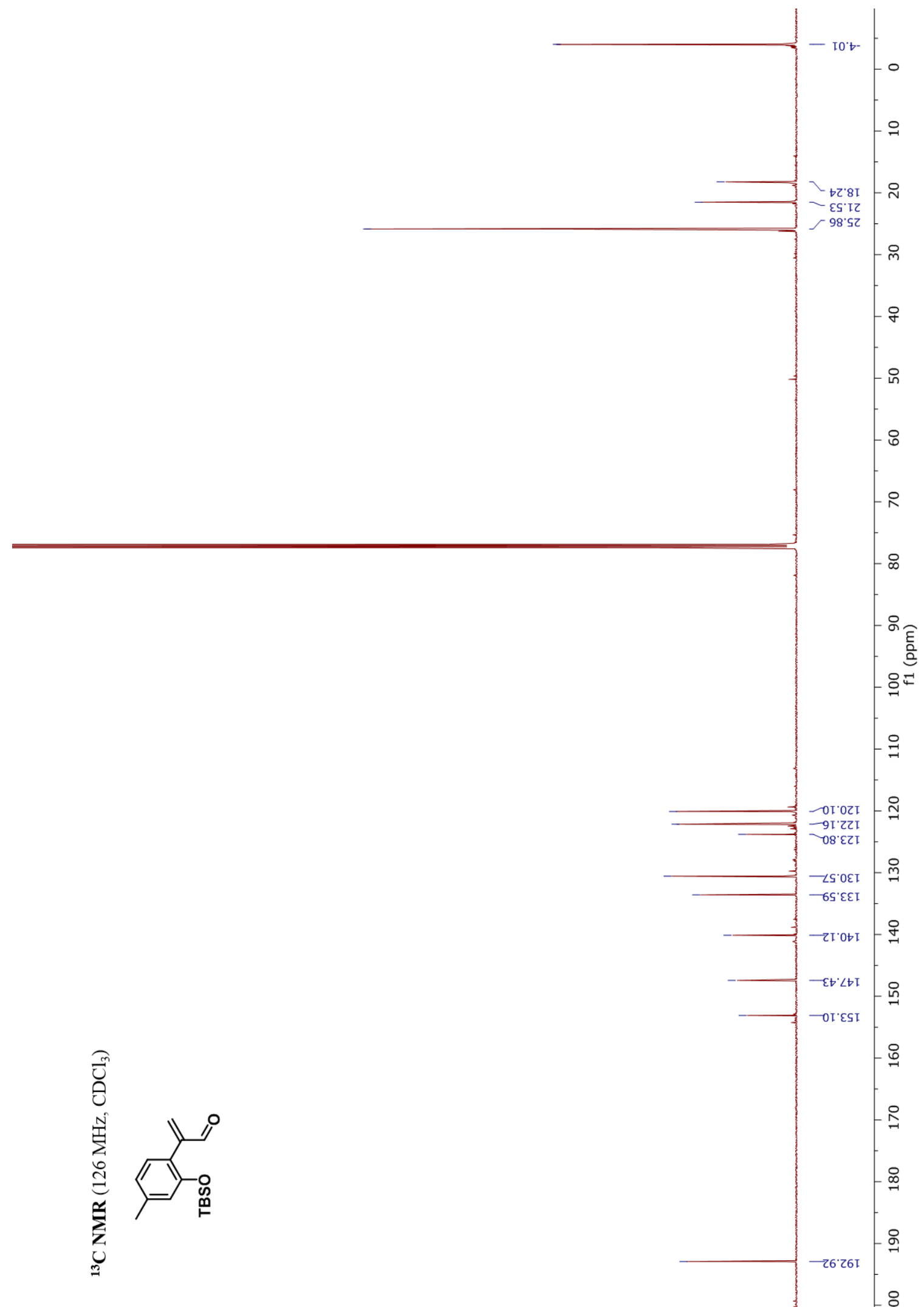


${ }^{1} \mathrm{H}$ NMR Spectrum of Compound 2.52

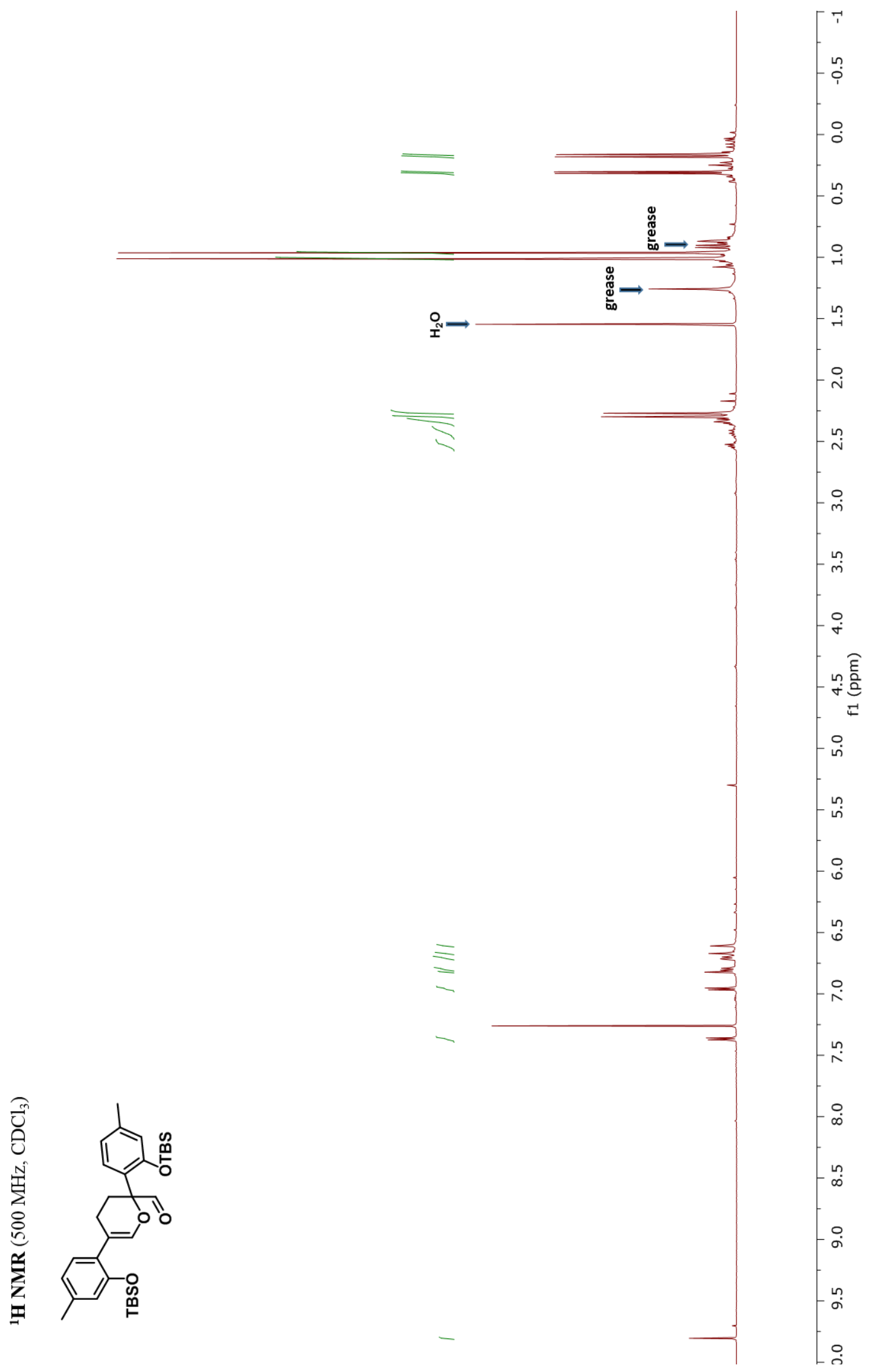


${ }^{13}$ C NMR Spectrum of Compound 2.52

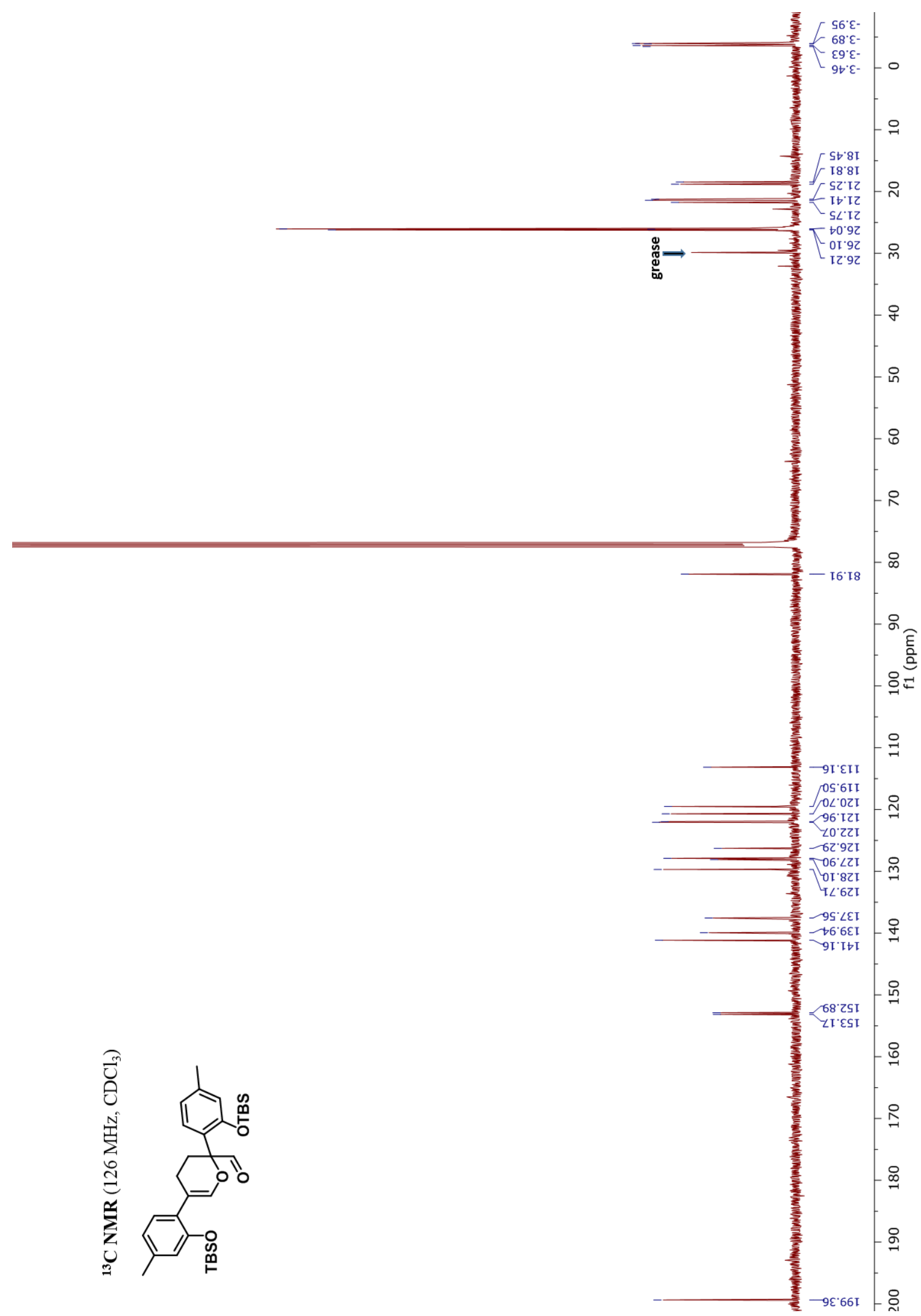


${ }^{1} \mathrm{H}$ NMR Spectrum of Compound 2.53

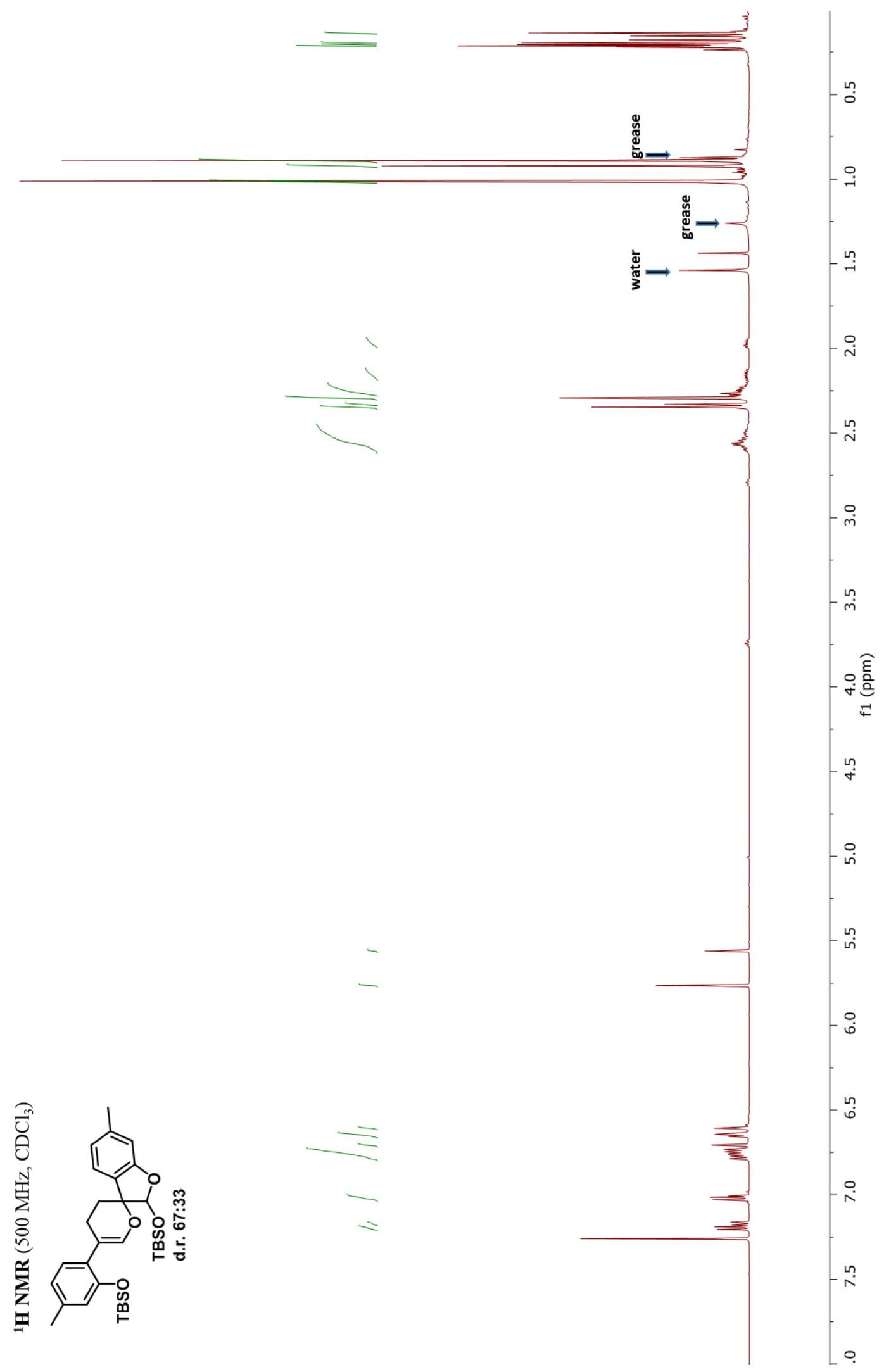


${ }^{13} \mathrm{C}$ NMR Spectrum of Compound 2.53

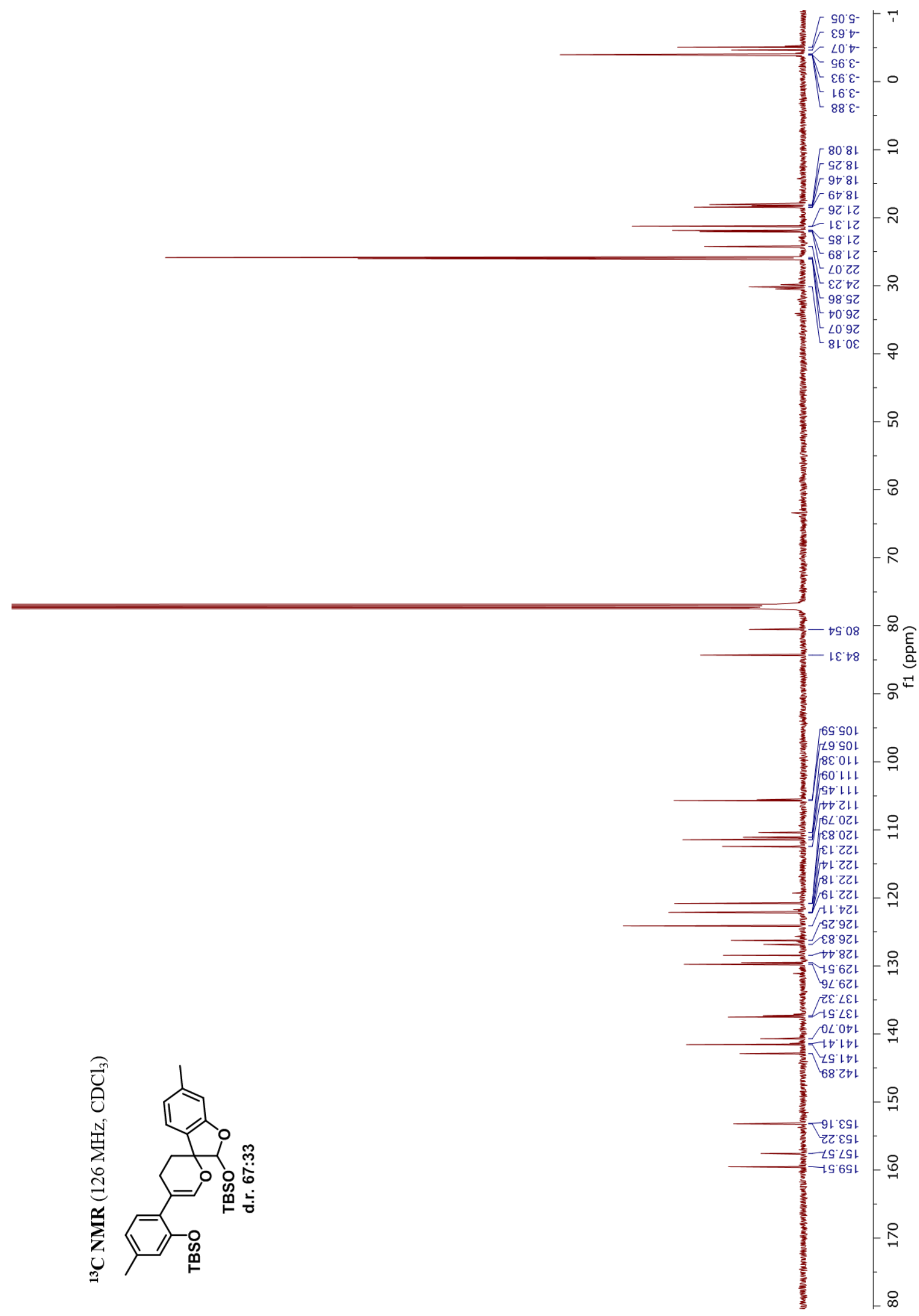


${ }^{1} \mathrm{H}$ NMR Spectrum of Compound 2.55

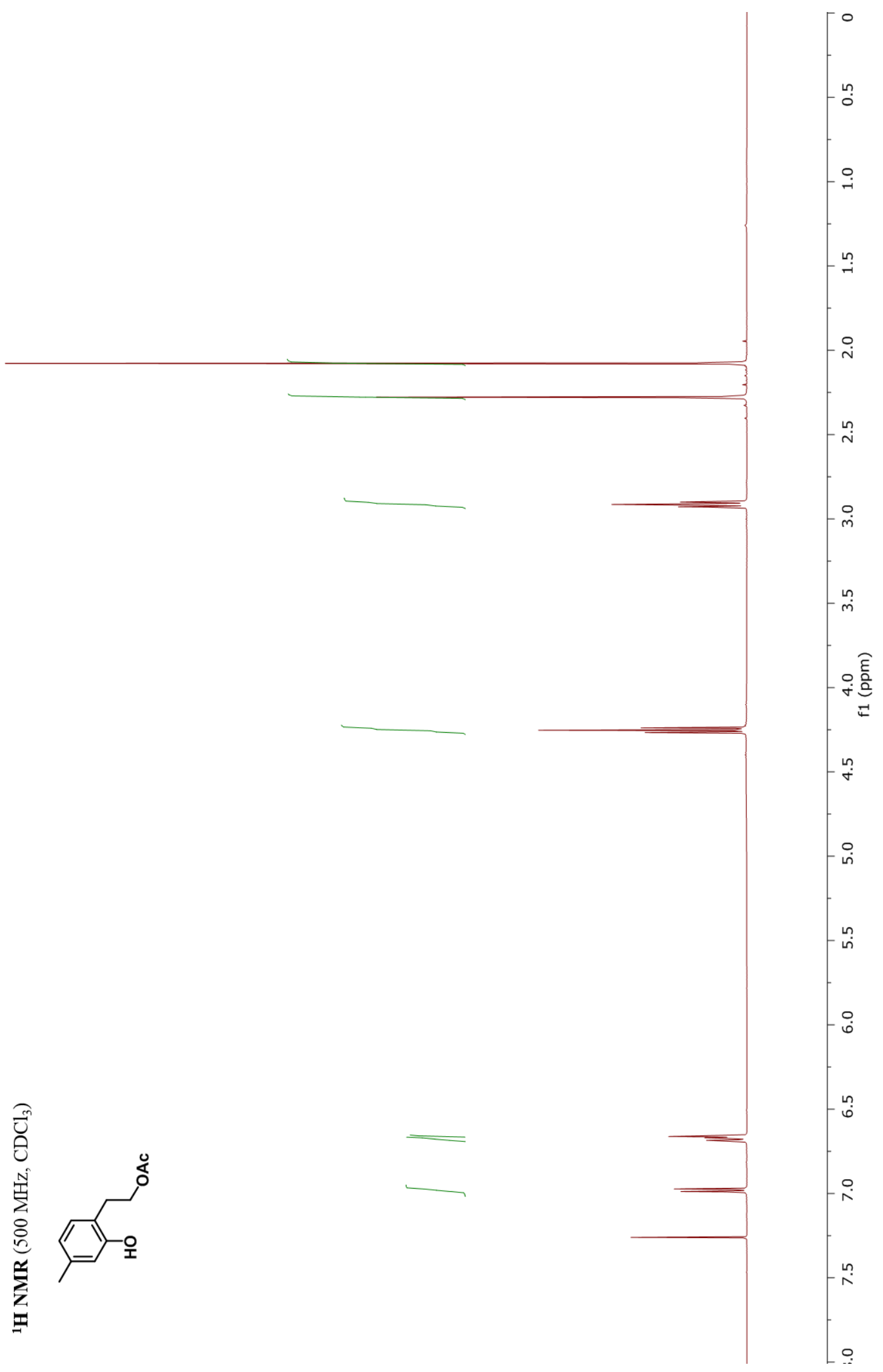


${ }^{13}$ C NMR Spectrum of Compound 2.55

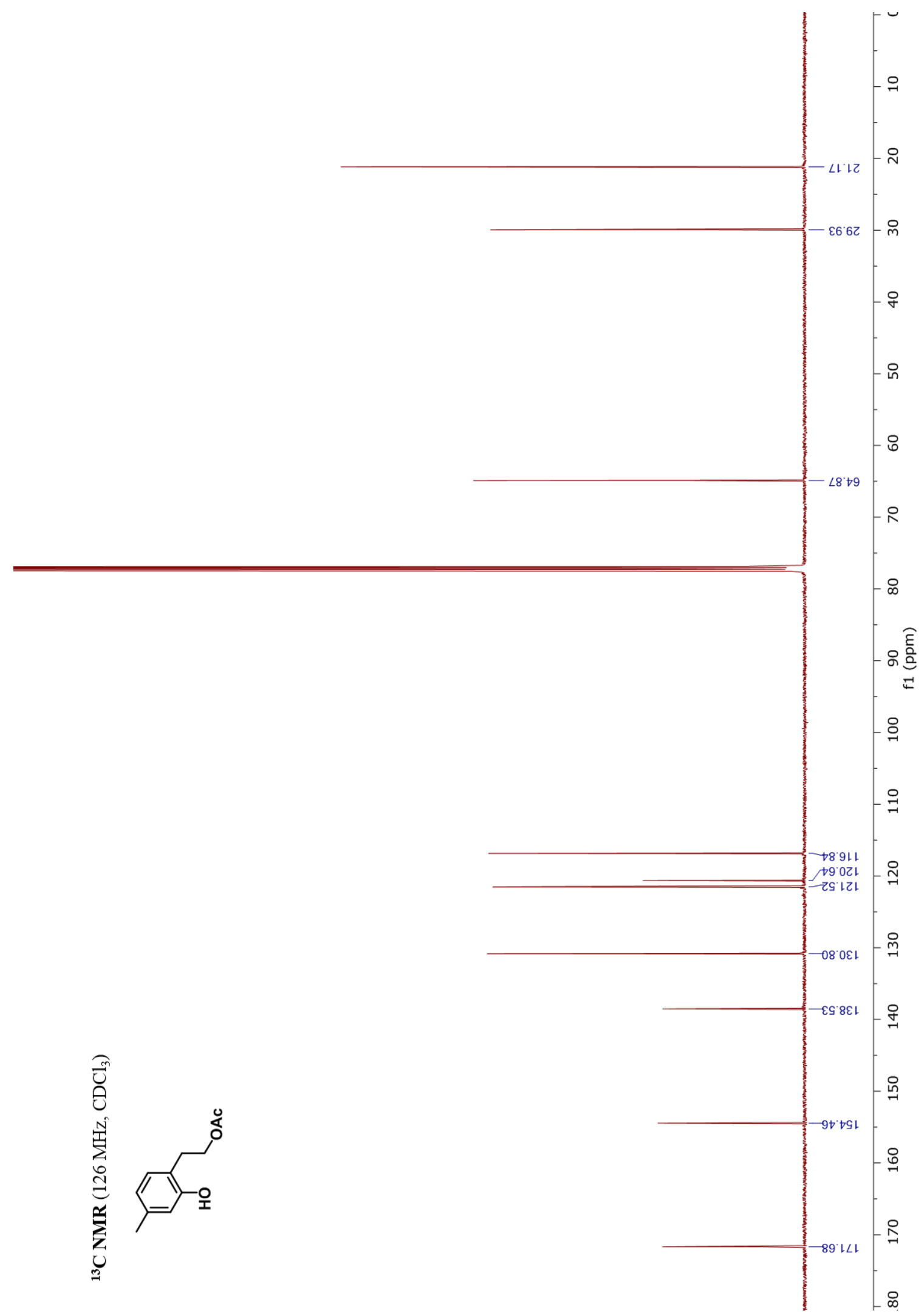


${ }^{1} \mathrm{H}$ NMR Spectrum of Compound 2.56

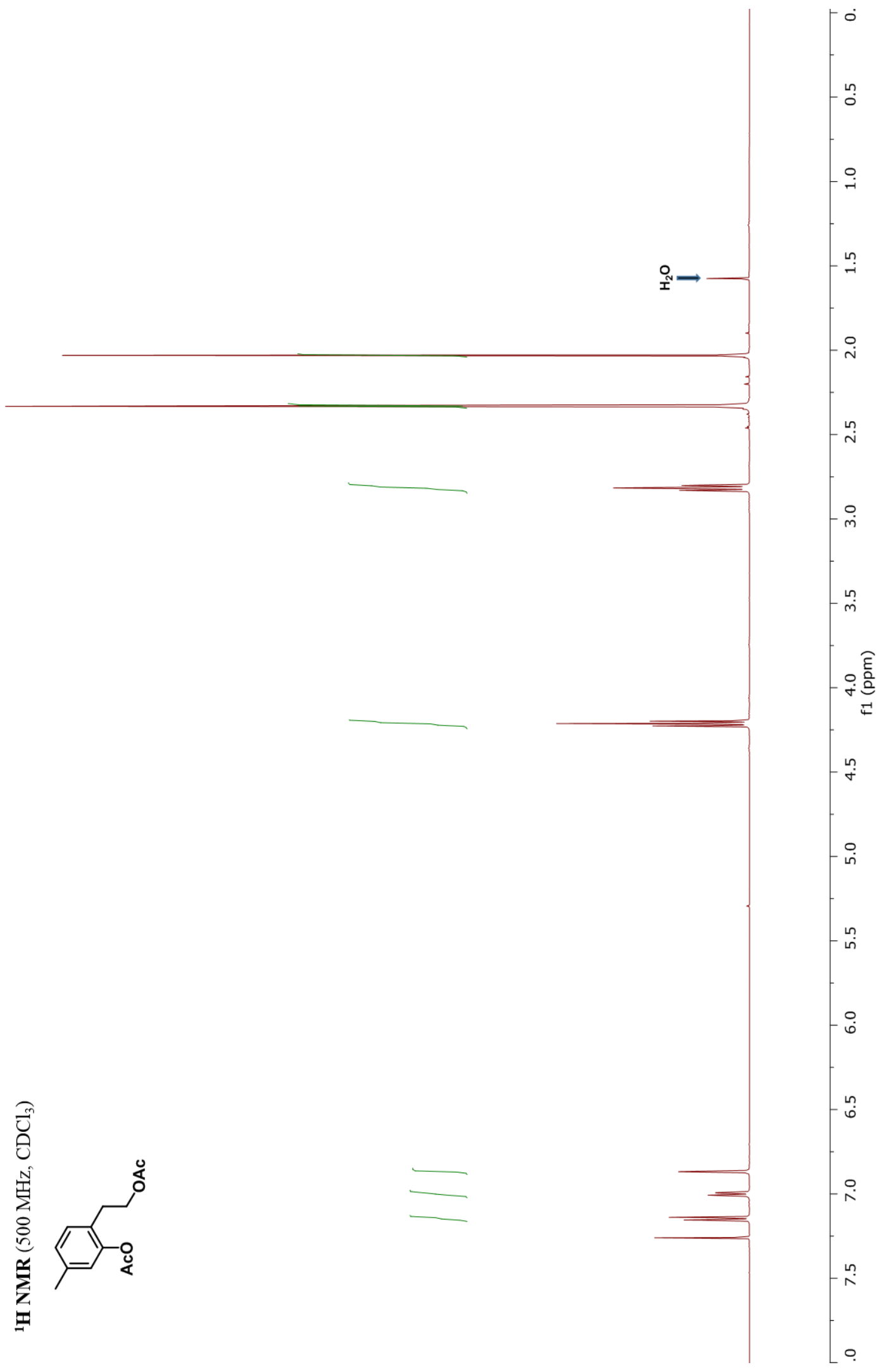


${ }^{13}$ C NMR Spectrum of Compound 2.56

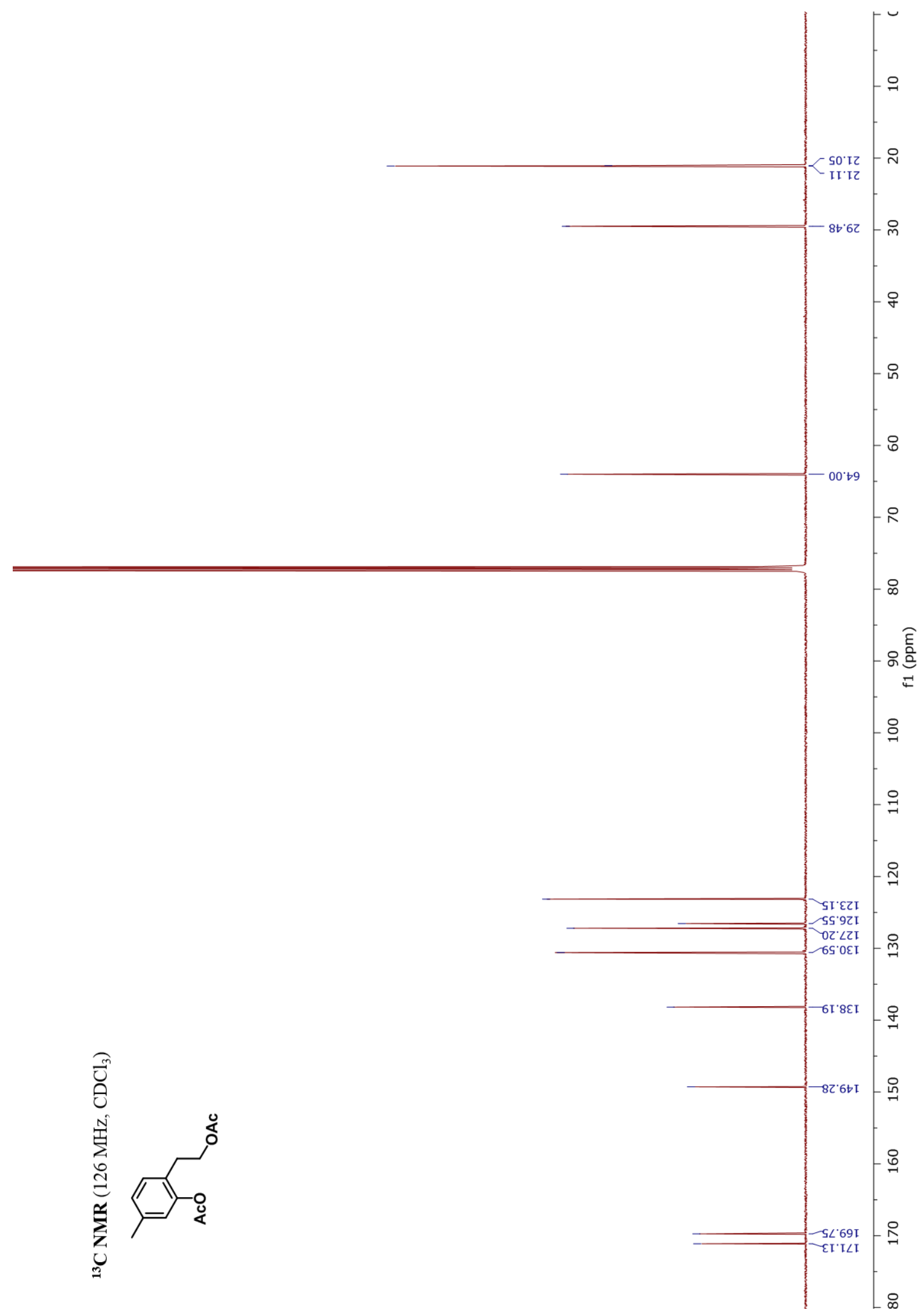


${ }^{1} \mathrm{H}$ NMR Spectrum of Compound 2.57

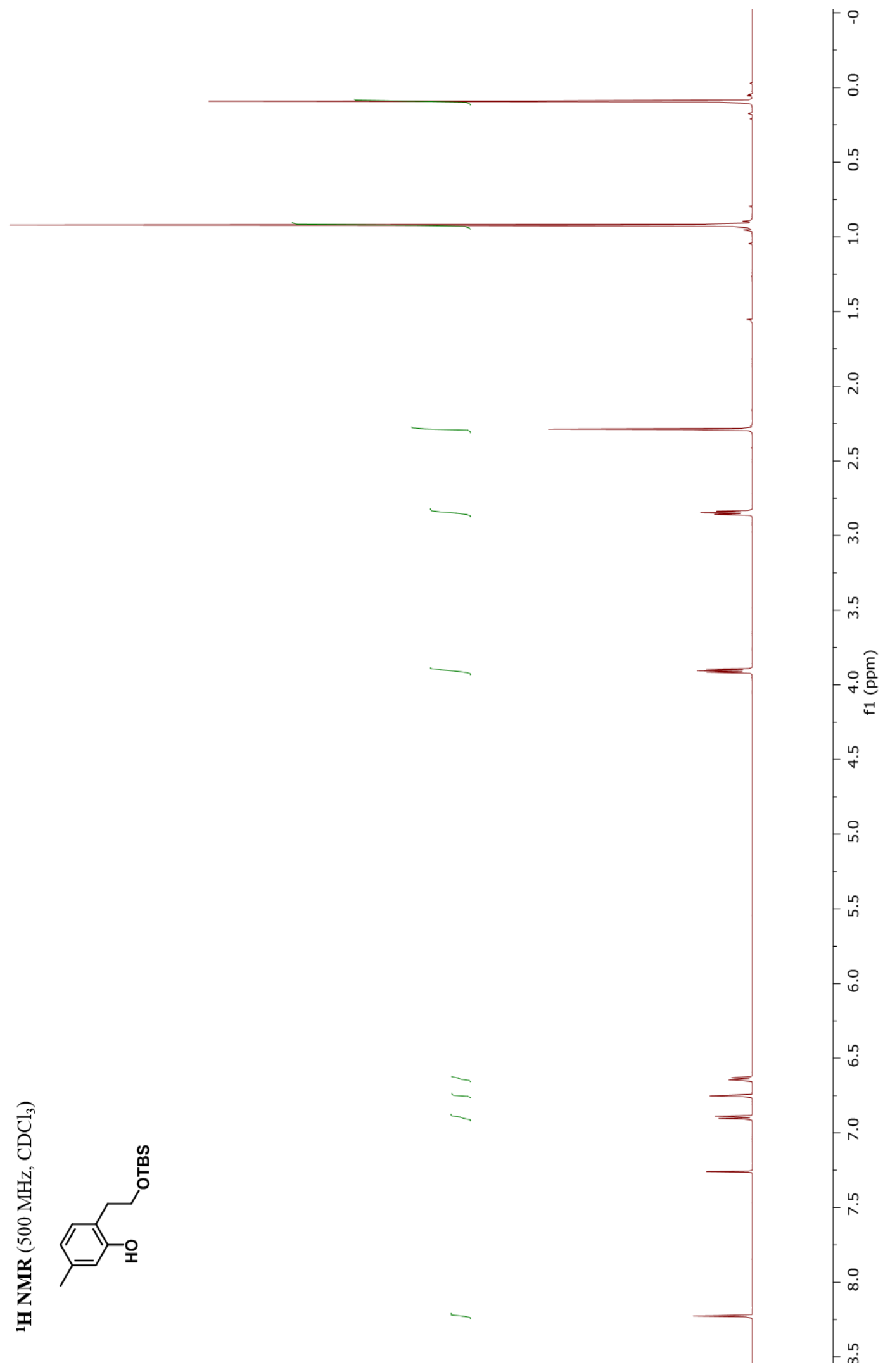


${ }^{13} \mathrm{C}$ NMR Spectrum of Compound 2.57

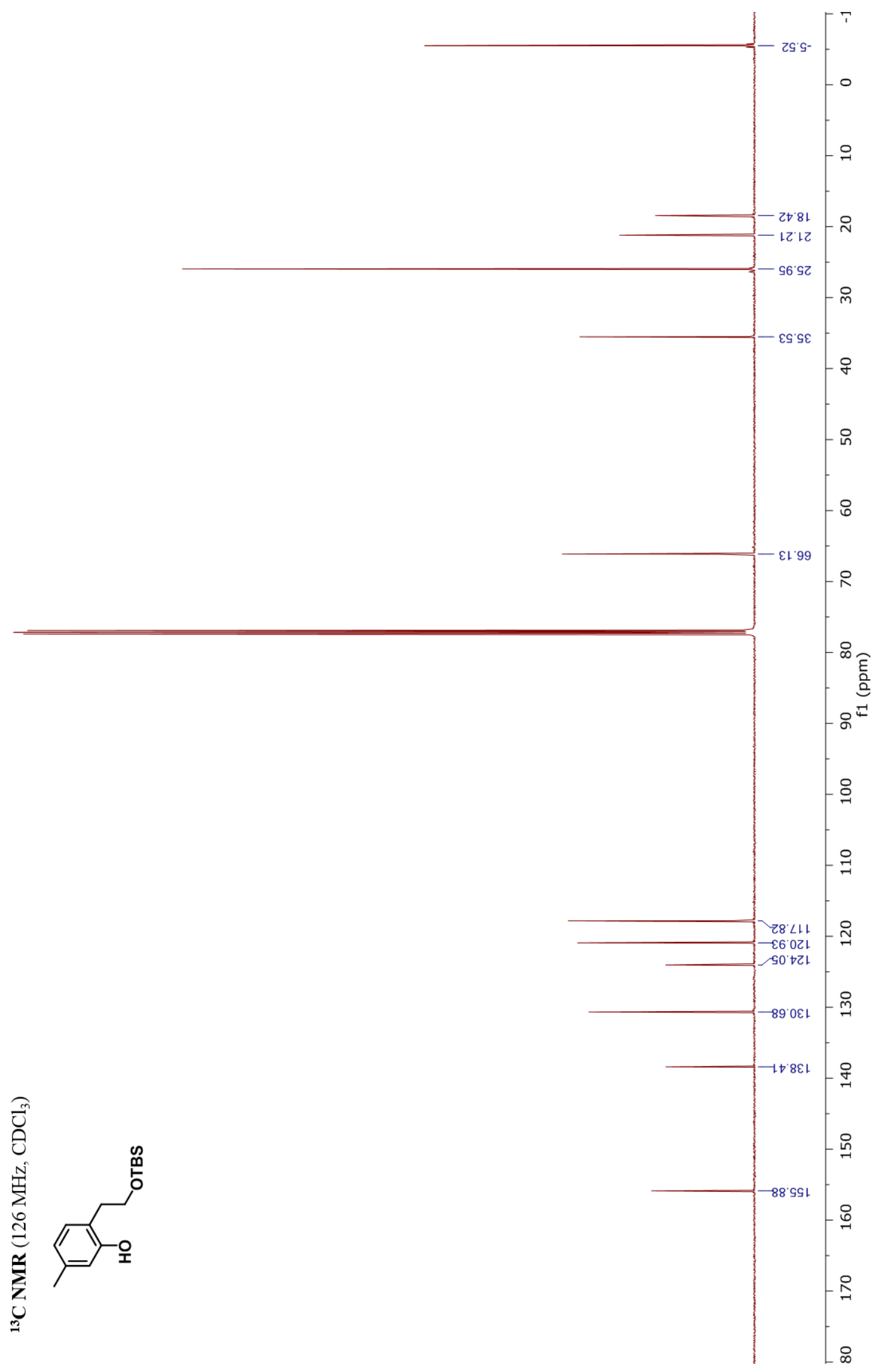


${ }^{1} \mathrm{H}$ NMR Spectrum of Compound 2.58

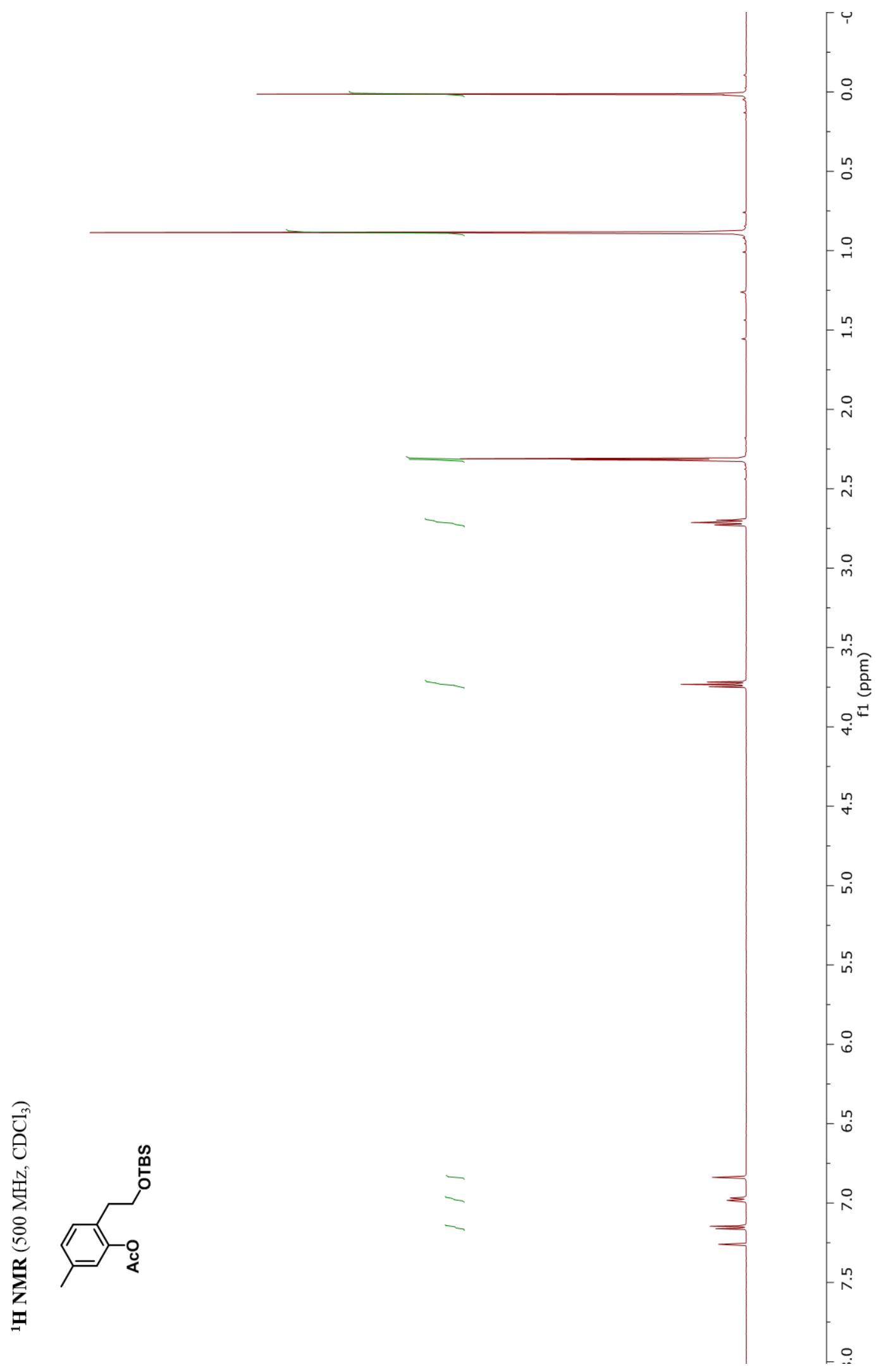


${ }^{13}$ C NMR Spectrum of Compound 2.58

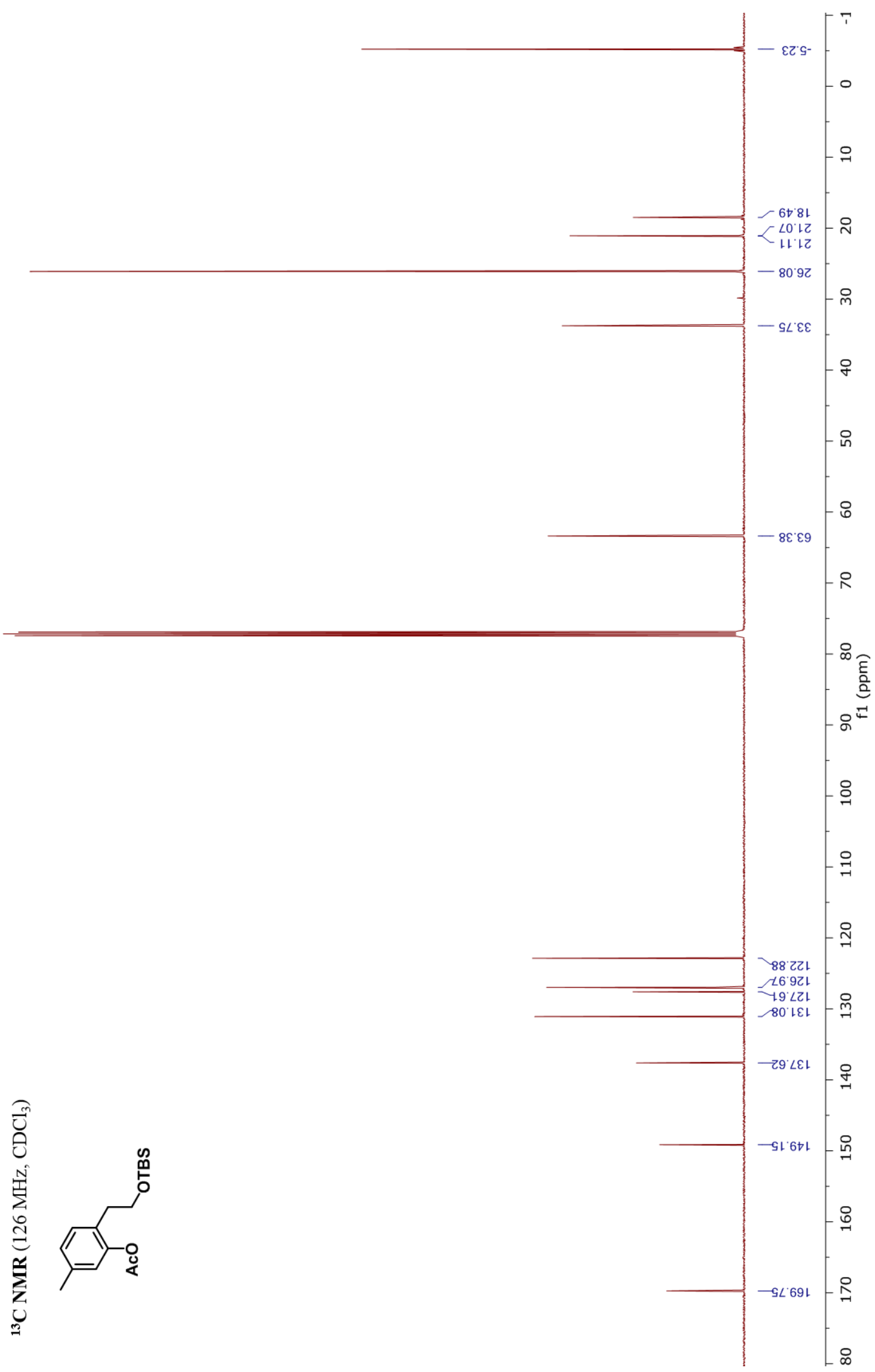


${ }^{1} \mathrm{H}$ NMR Spectrum of Compound 2.50

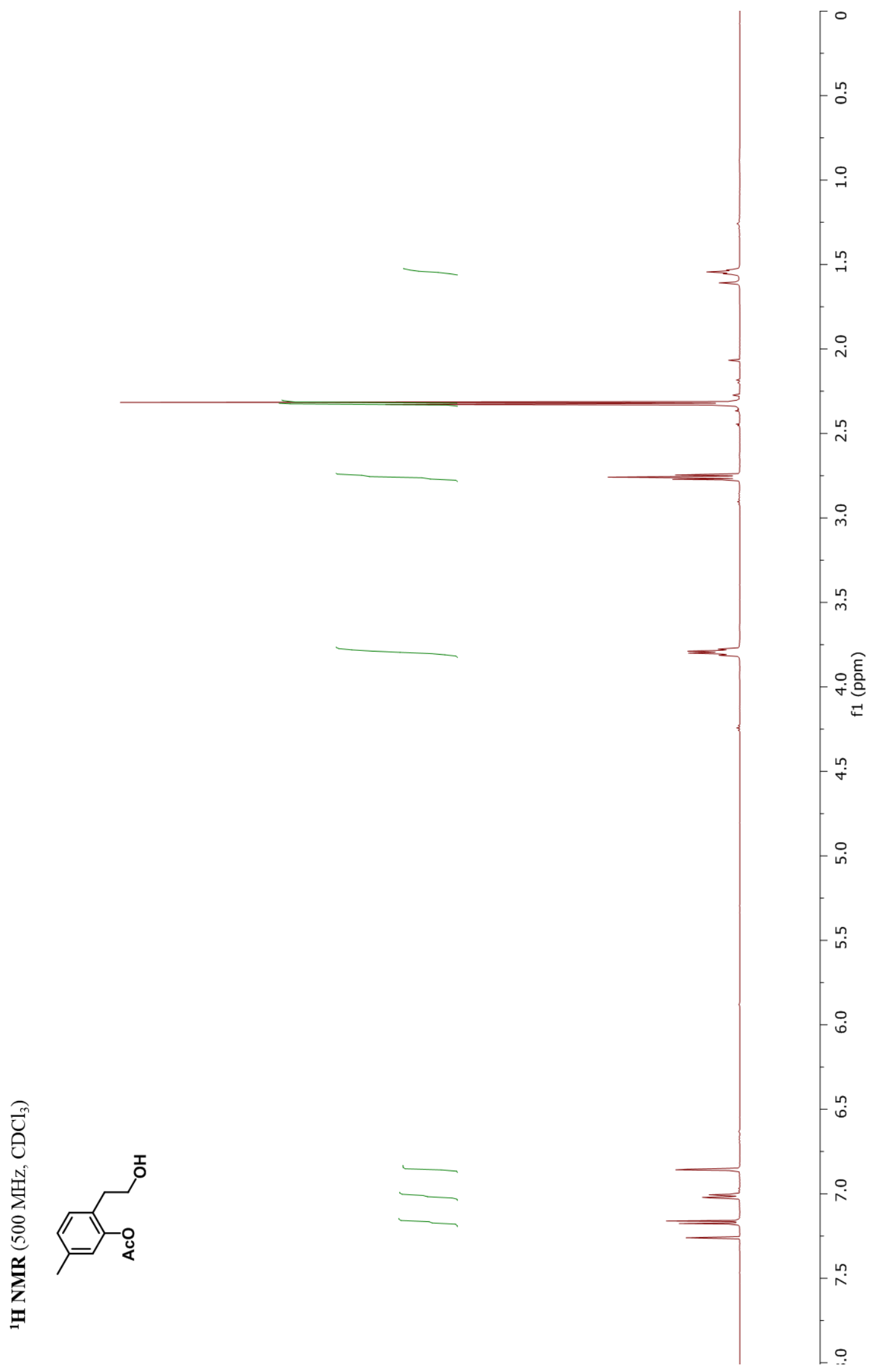


${ }^{13} \mathrm{C}$ NMR Spectrum of Compound 2.50

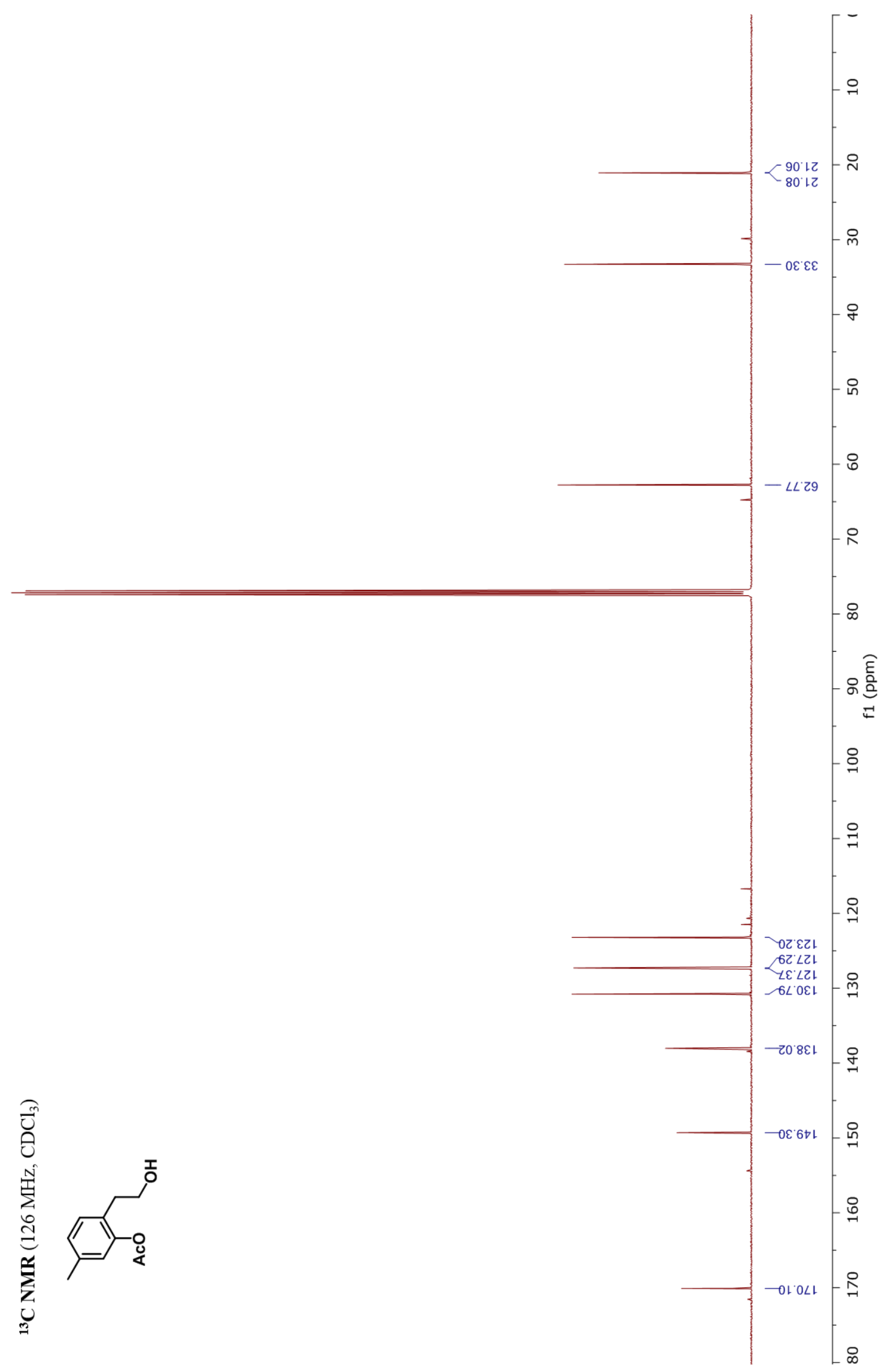


${ }^{1}$ H NMR Spectrum of Compound 2.48

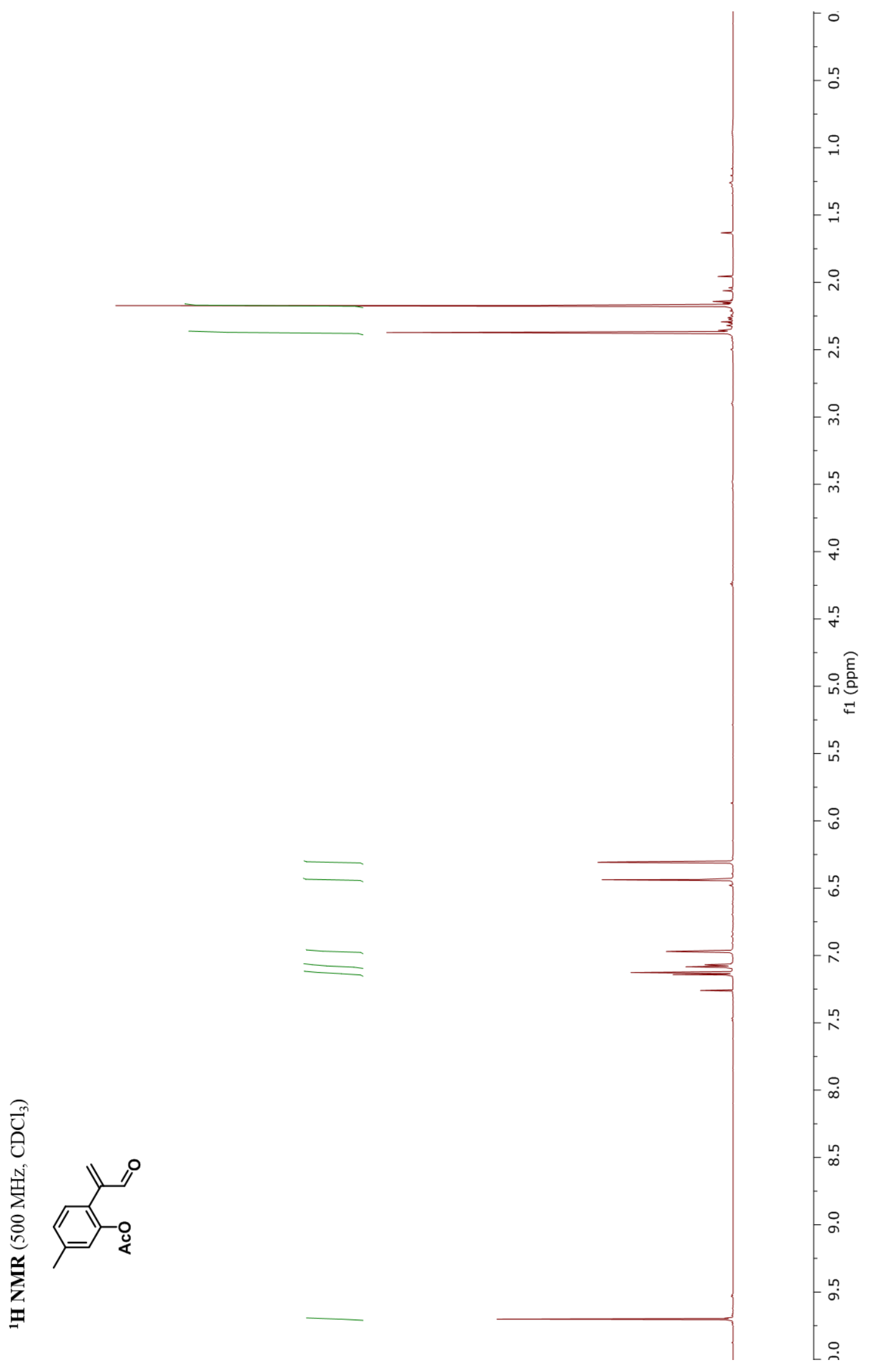


${ }^{13} \mathrm{C}$ NMR Spectrum of Compound 2.48

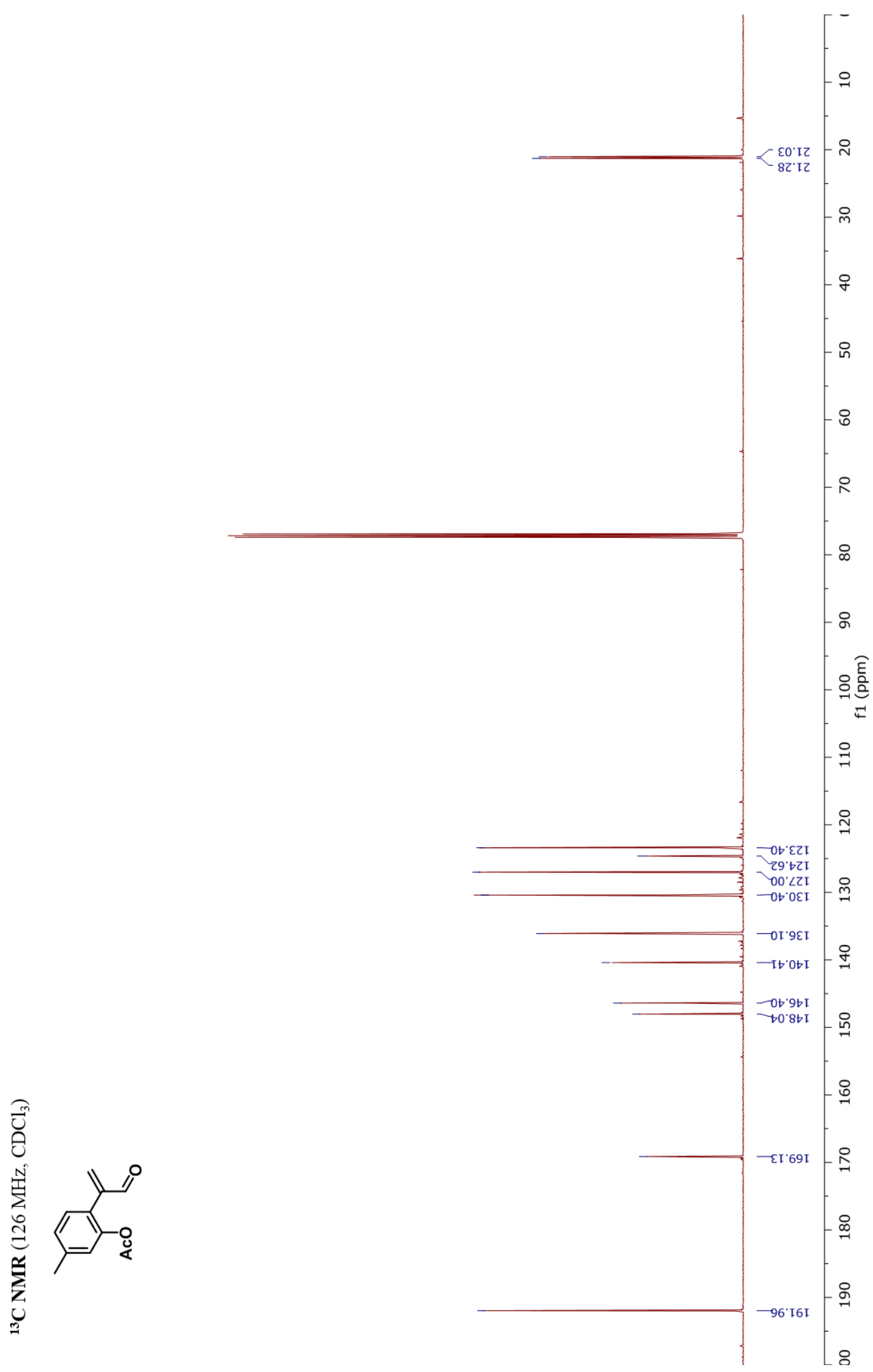


${ }^{1} \mathrm{H}$ NMR Spectrum of Compound 2.60

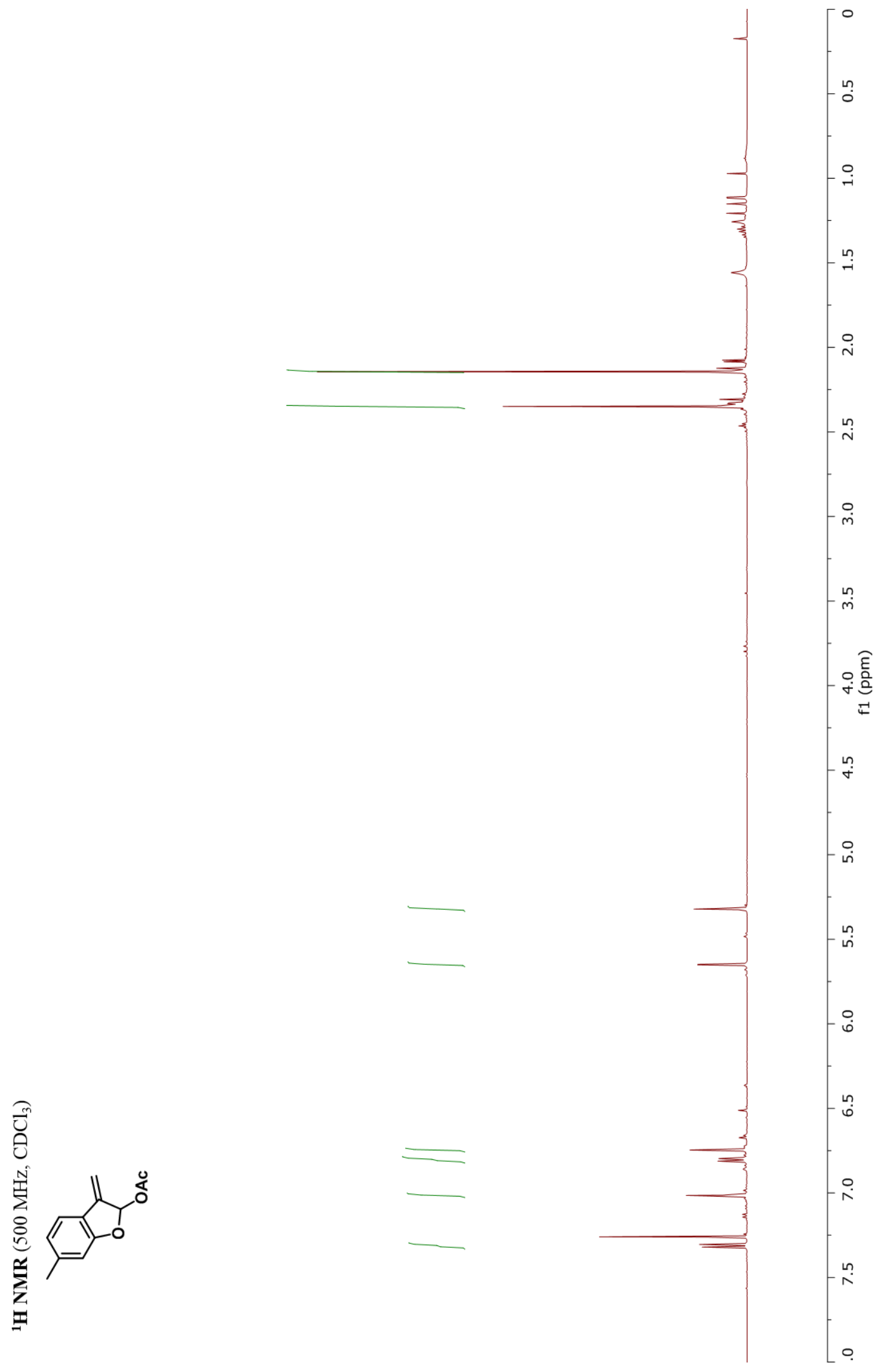


${ }^{13} \mathrm{C}$ NMR Spectrum of Compound 2.60

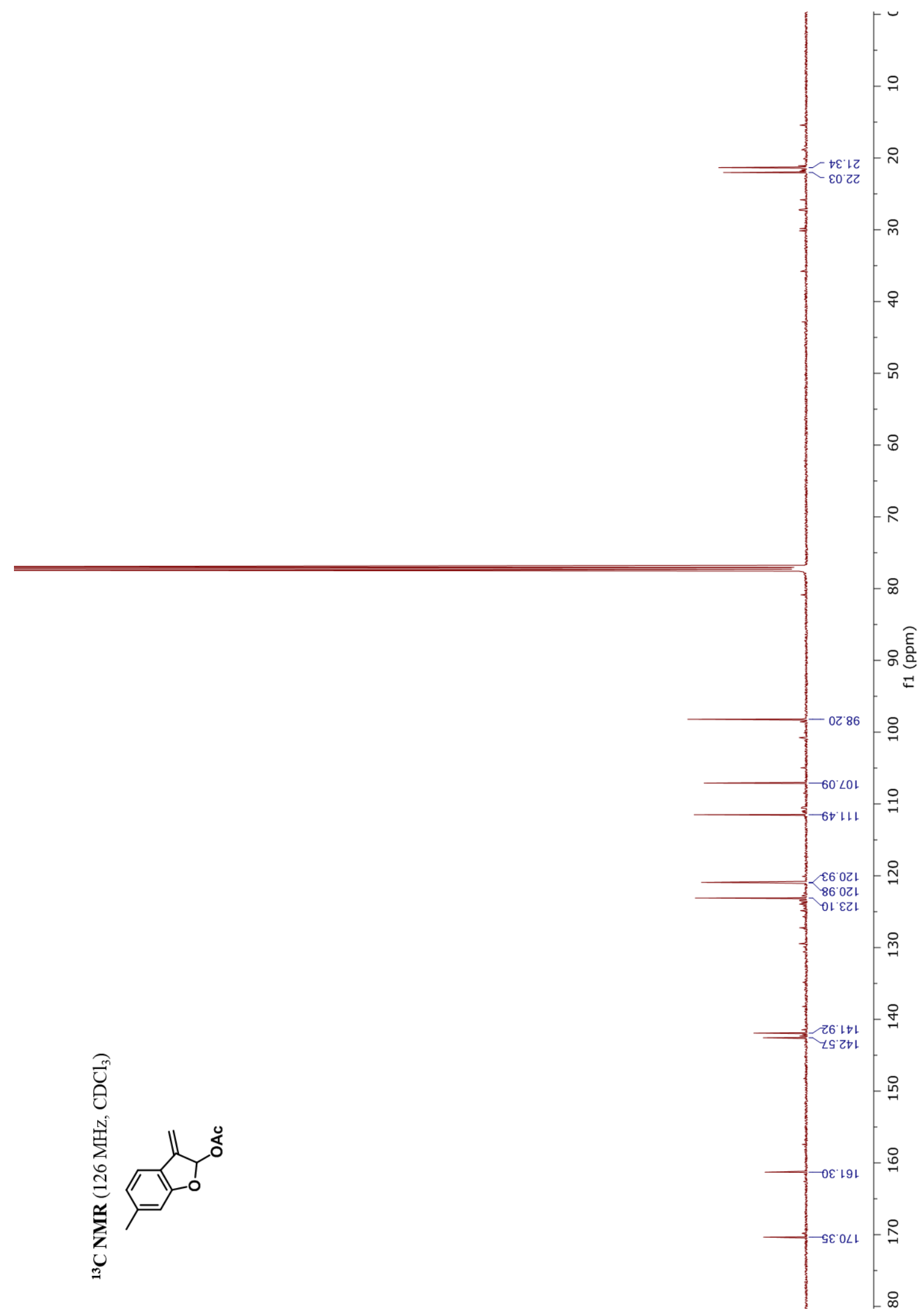


${ }^{1} \mathrm{H}$ NMR Spectrum of Compound ( \pm )-2.61

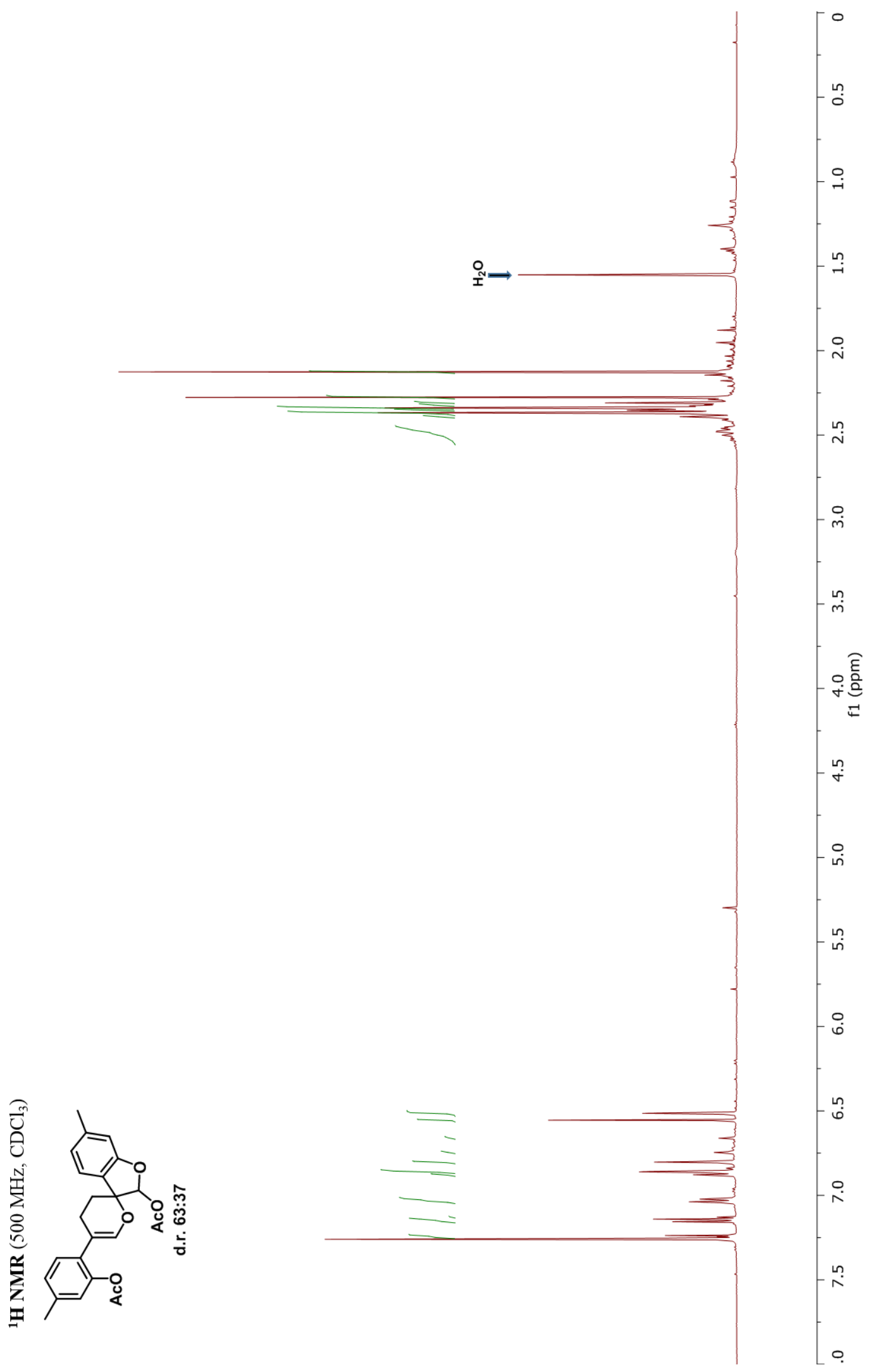


${ }^{13}$ C NMR Spectrum of Compound ( \pm )-2.61

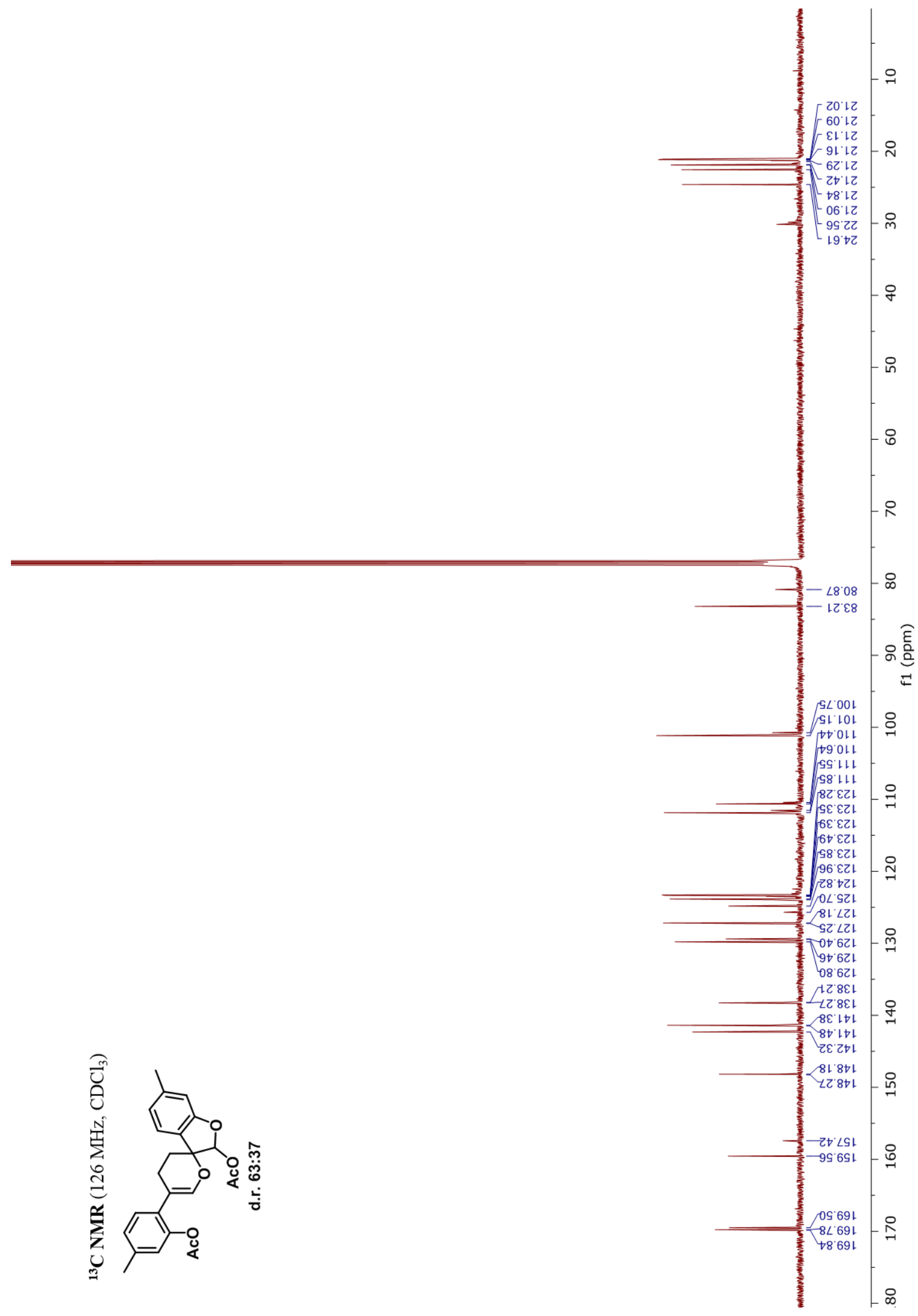


${ }^{1}$ H NMR Spectrum of Compound ( \pm )-2.59

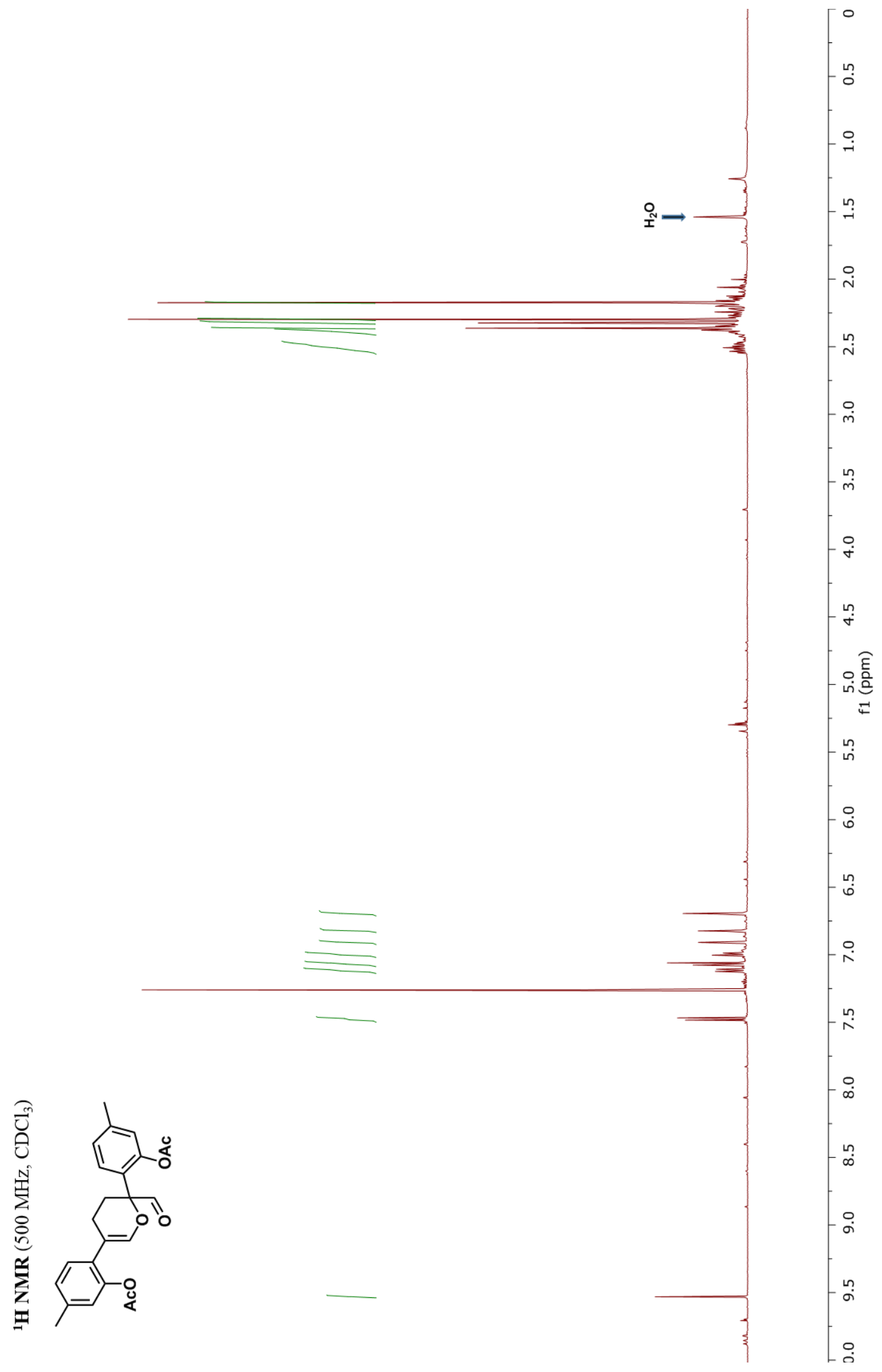


${ }^{13}$ C NMR Spectrum of Compound ( \pm )-2.59

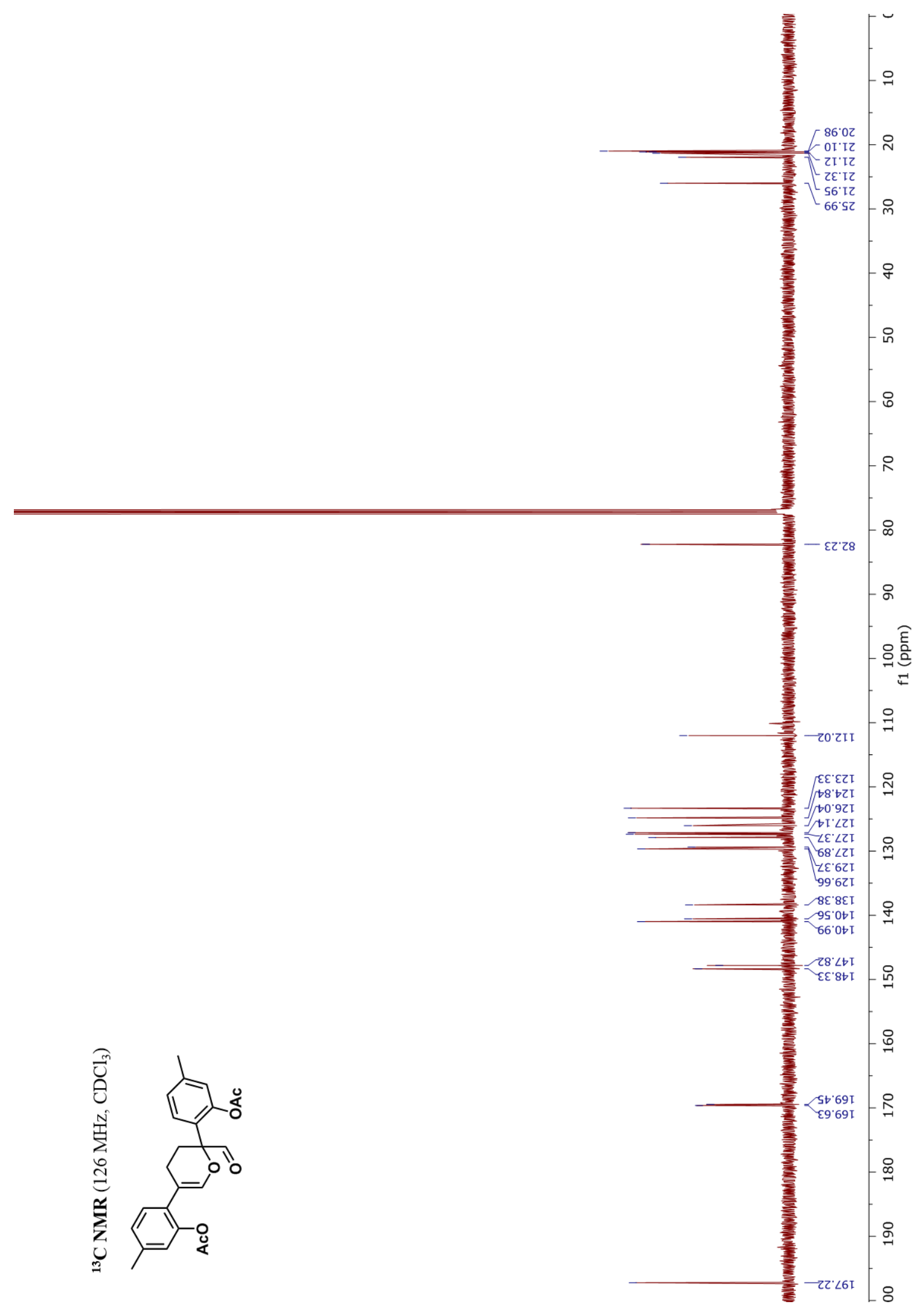


${ }^{1}$ H NMR Spectrum of Compound ( \pm )-2.62

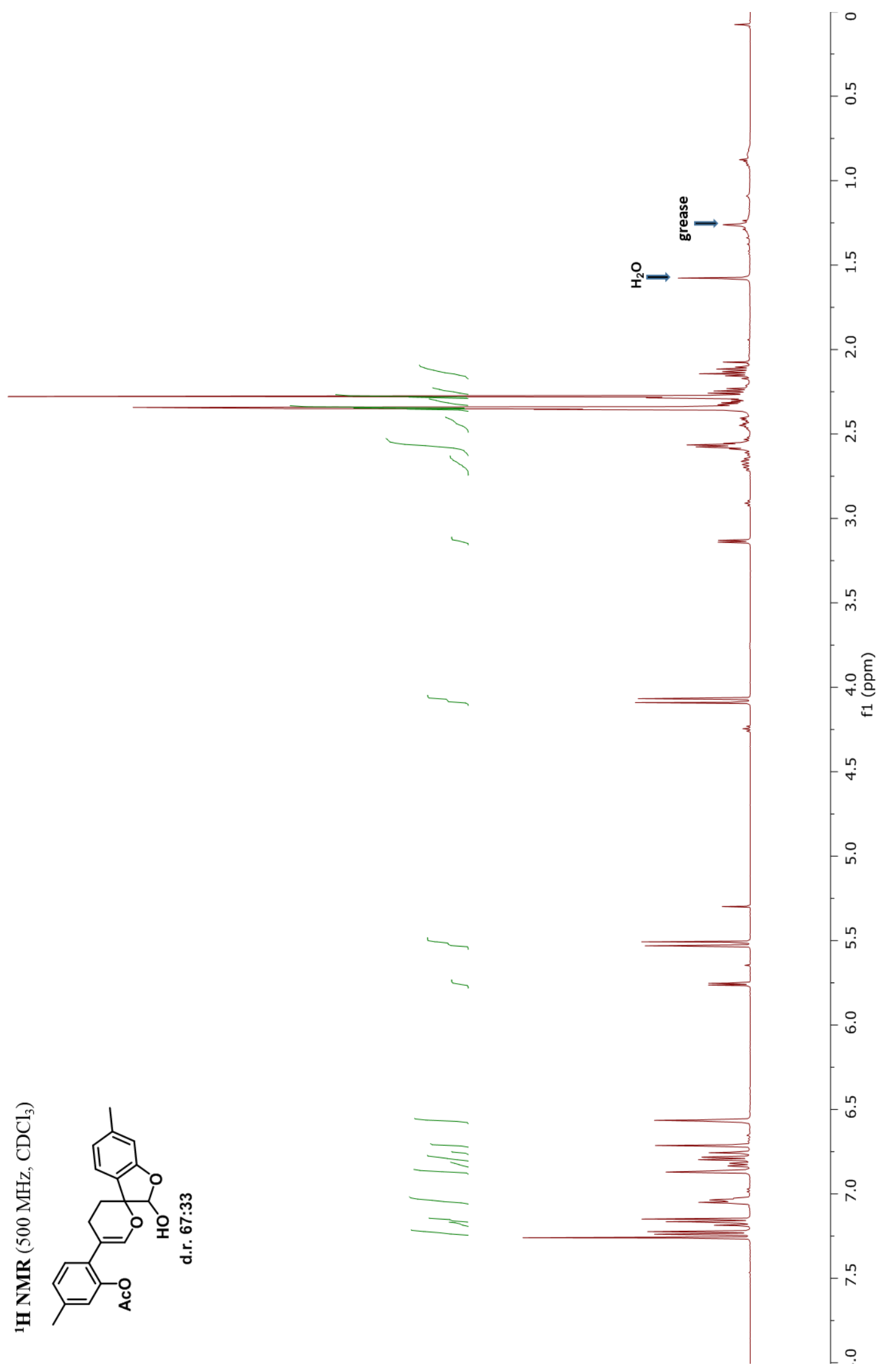


${ }^{13}$ C NMR Spectrum of Compound ( \pm )-2.62

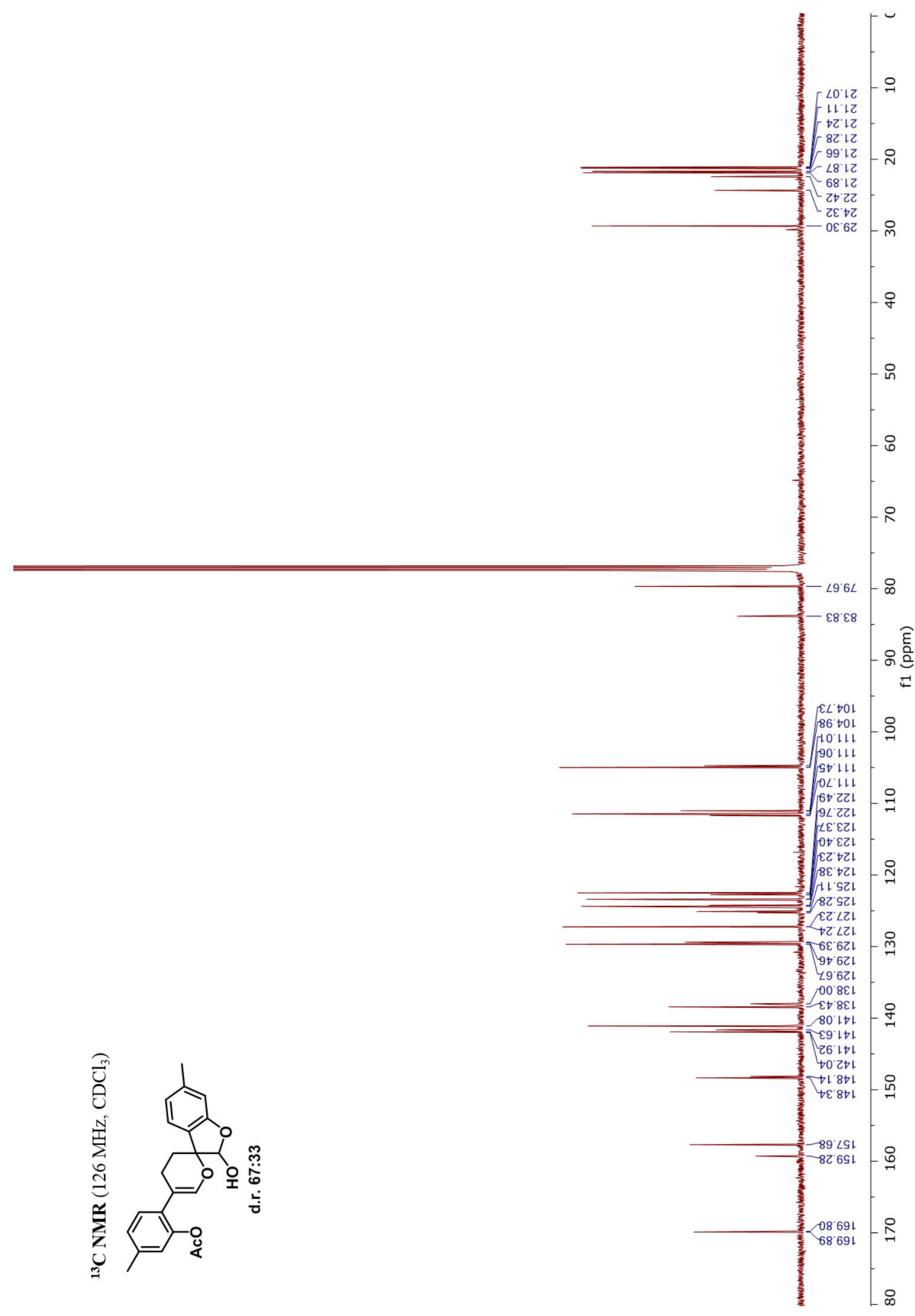


${ }^{1}$ H NMR Spectrum of Compound ( \pm )-2.9

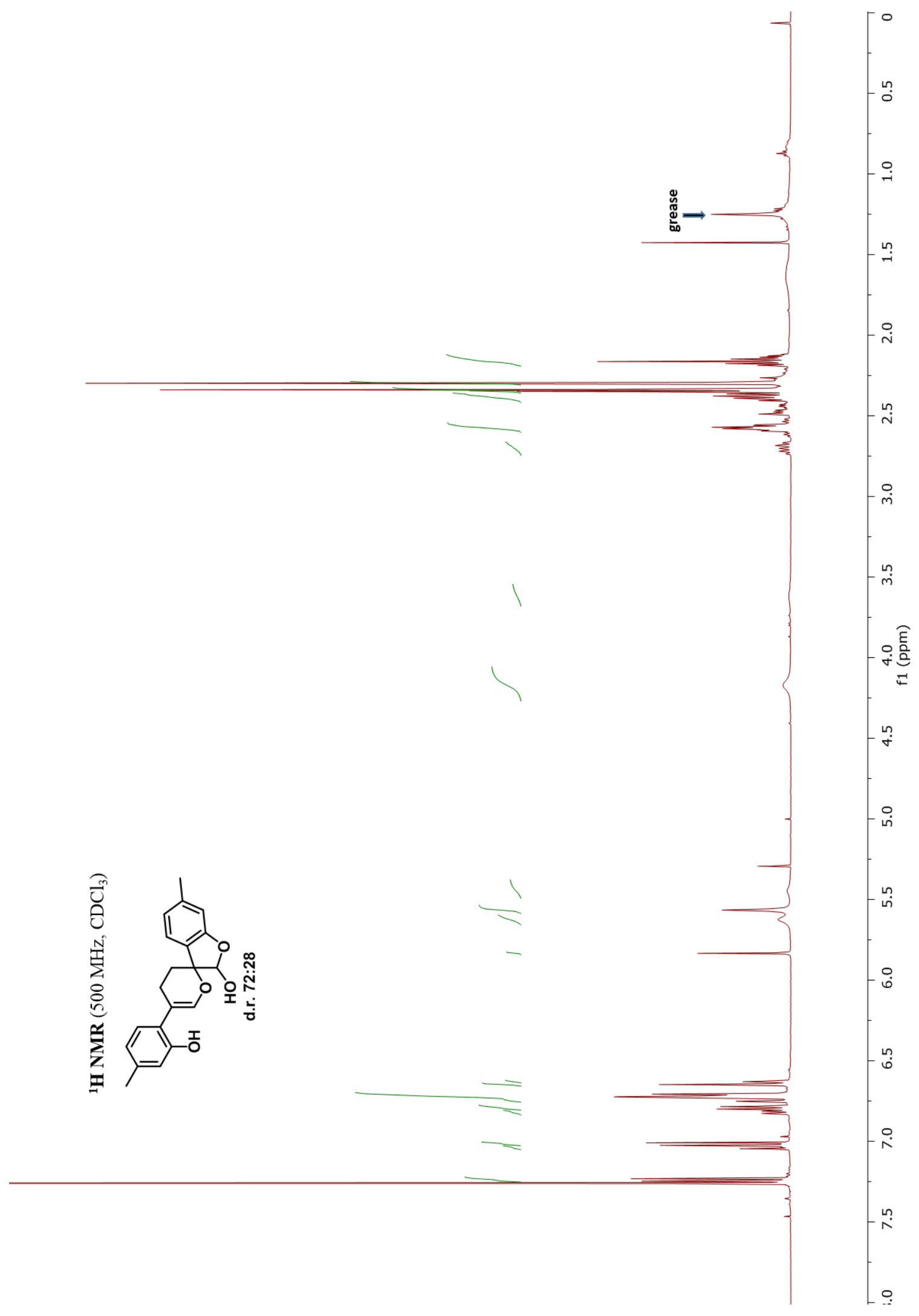


${ }^{13}$ C NMR Spectrum of Compound ( \pm )-2.9

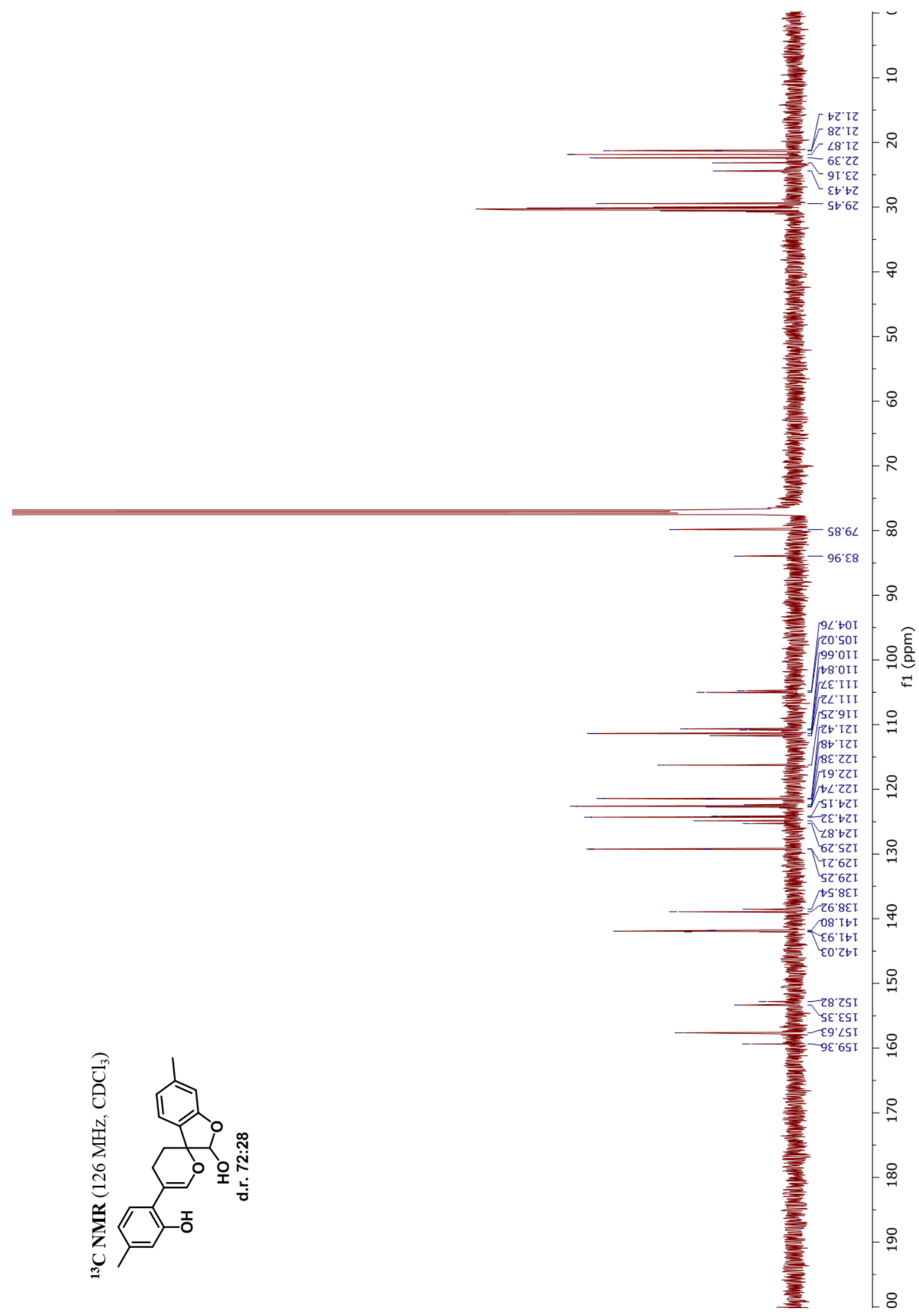


${ }^{1} \mathrm{H}$ NMR Spectrum of Compound 2.64

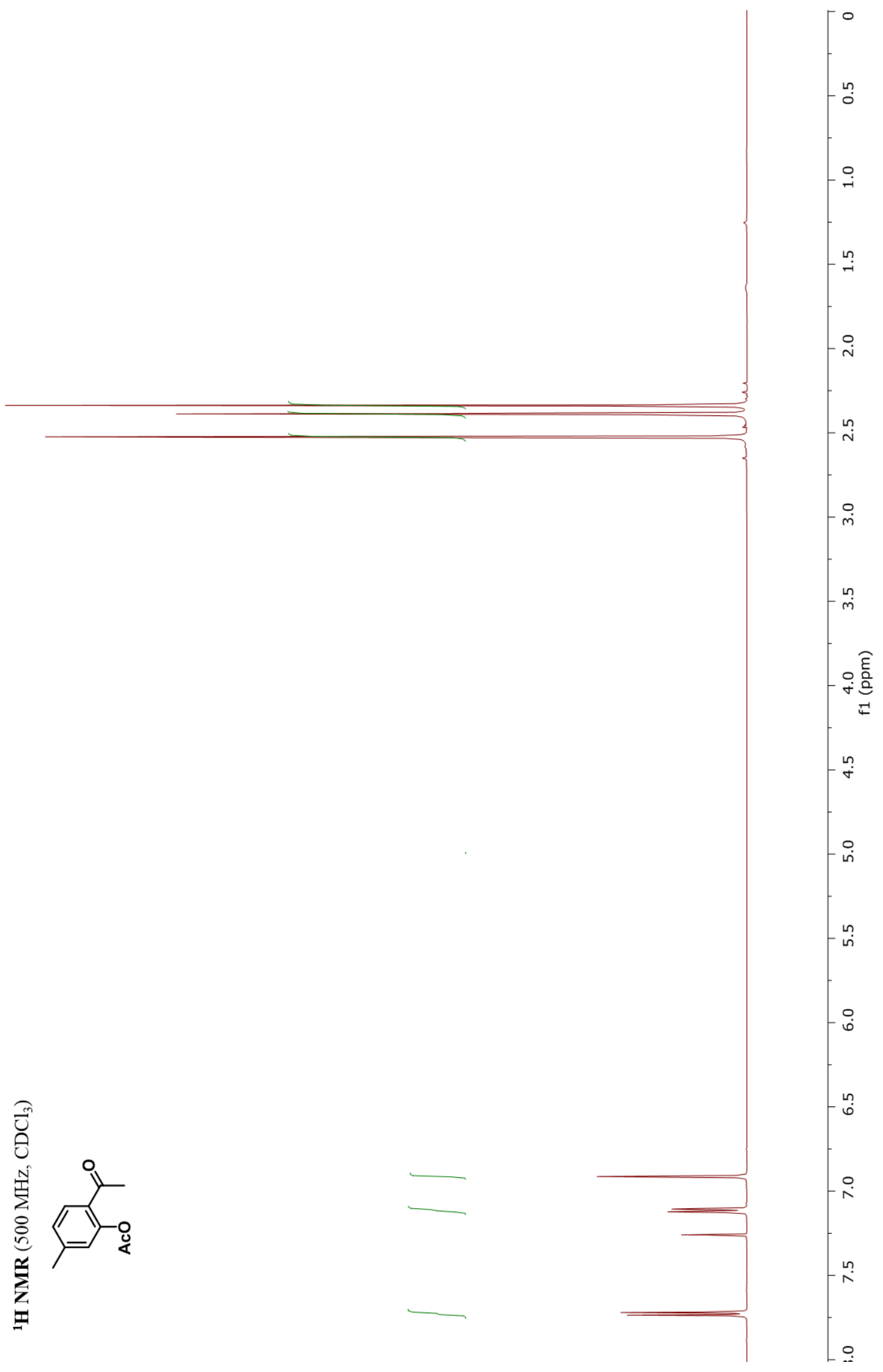


${ }^{13}$ C NMR Spectrum of Compound 2.64

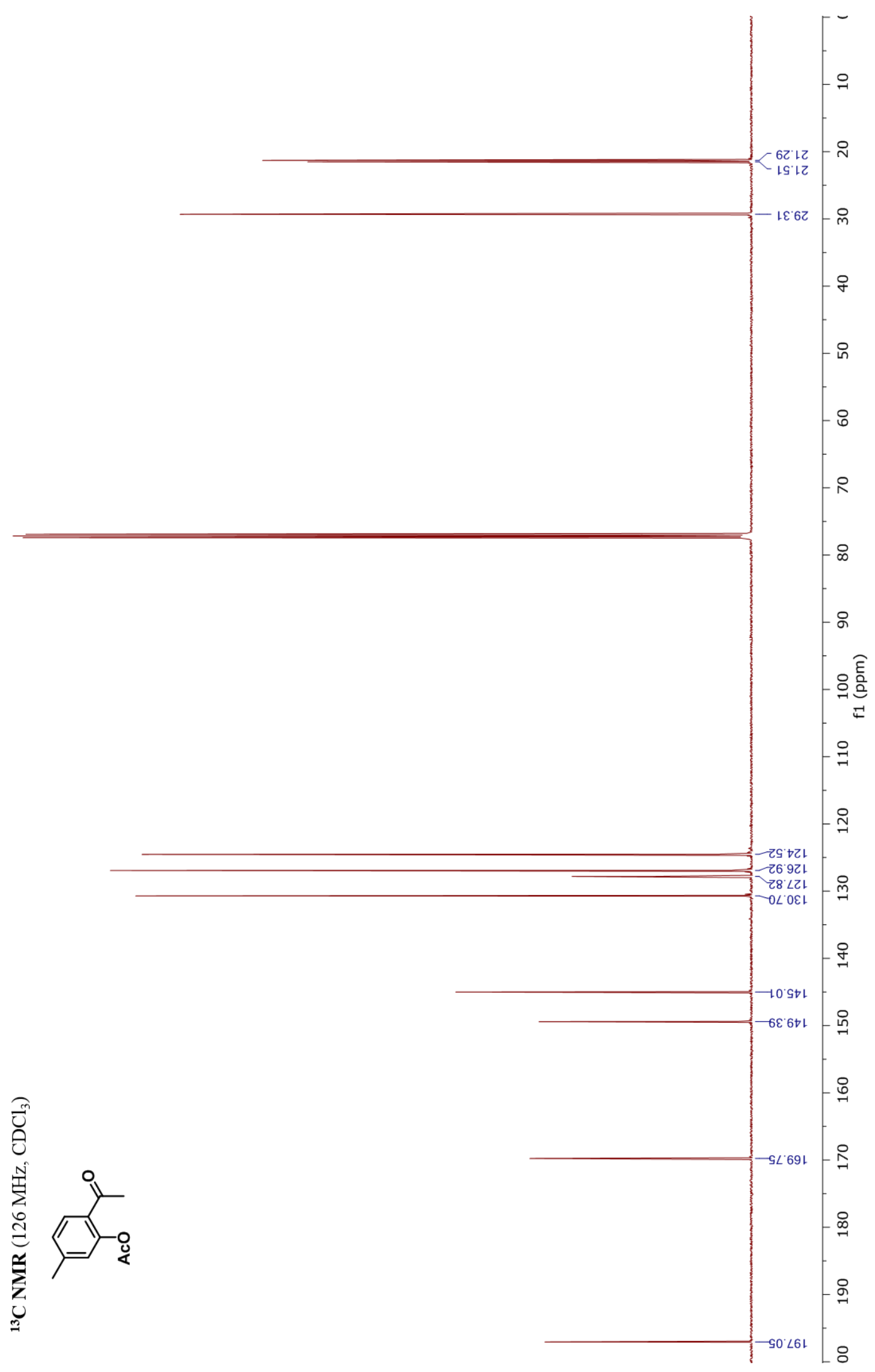


${ }^{1} \mathrm{H}$ NMR Spectrum of Compound 2.68

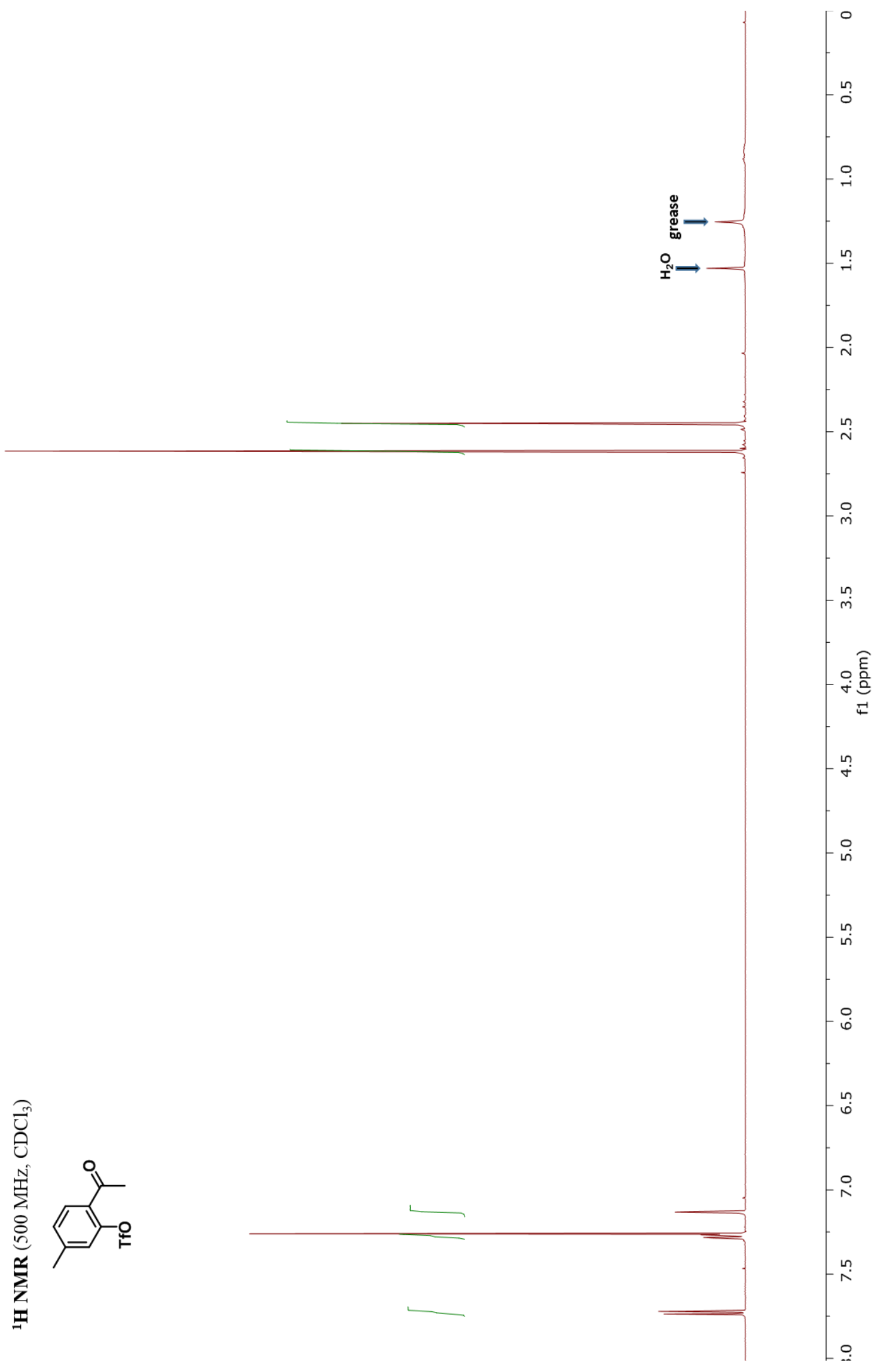


${ }^{13} \mathrm{C}$ NMR Spectrum of Compound 2.68

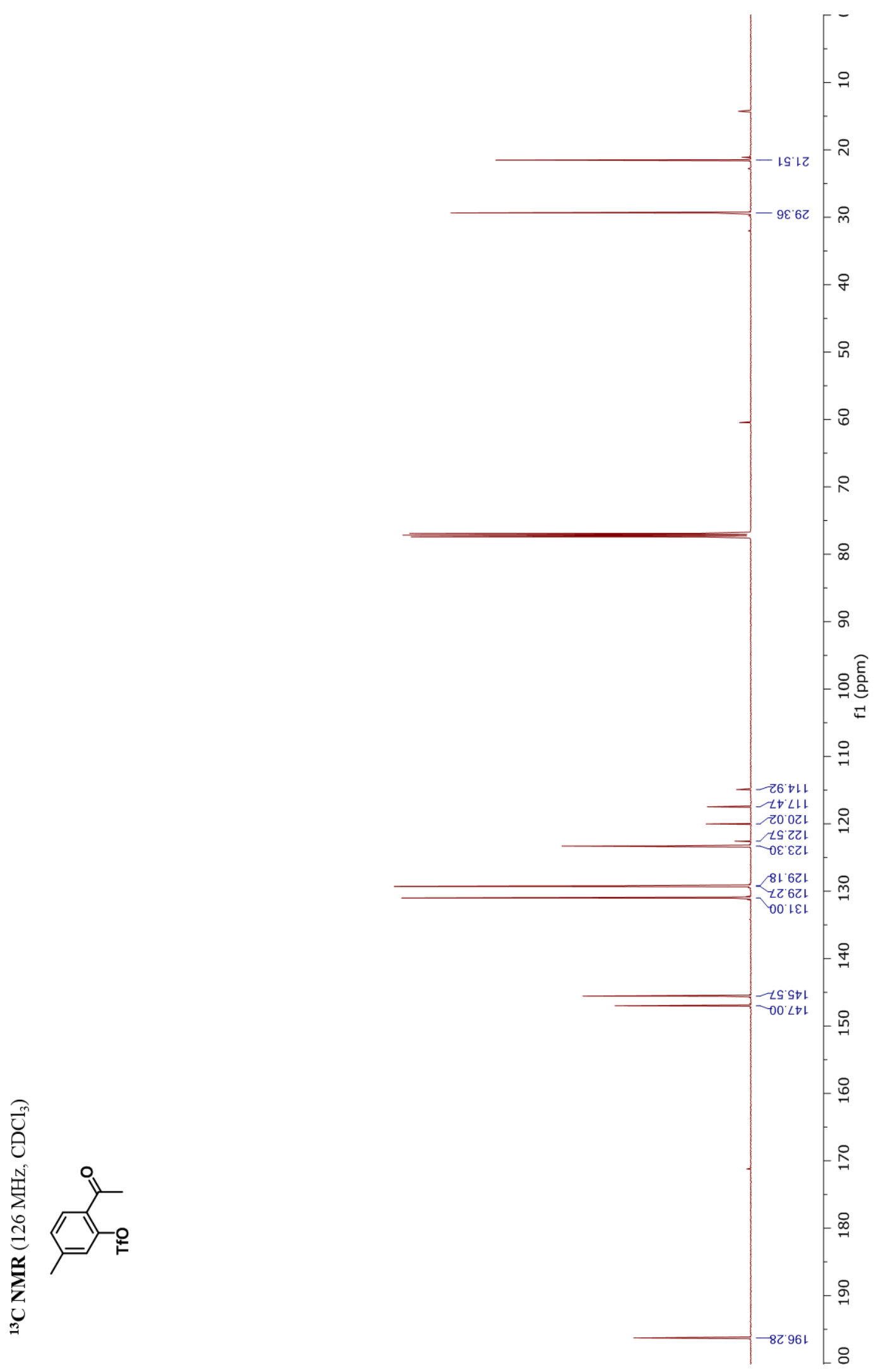


${ }^{1}$ H NMR Spectrum of Compound 2.69

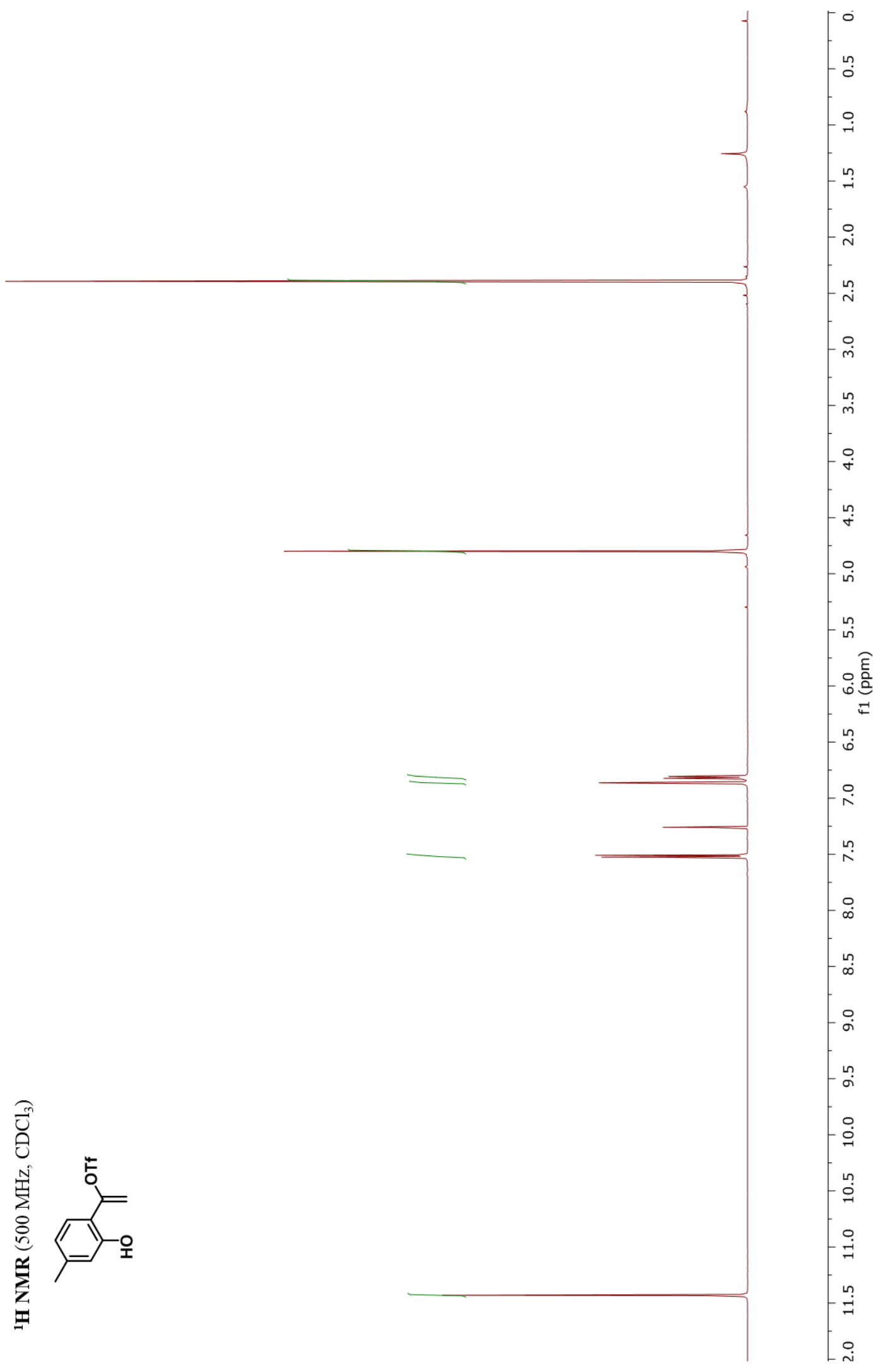


${ }^{13} \mathrm{C}$ NMR Spectrum of Compound 2.69
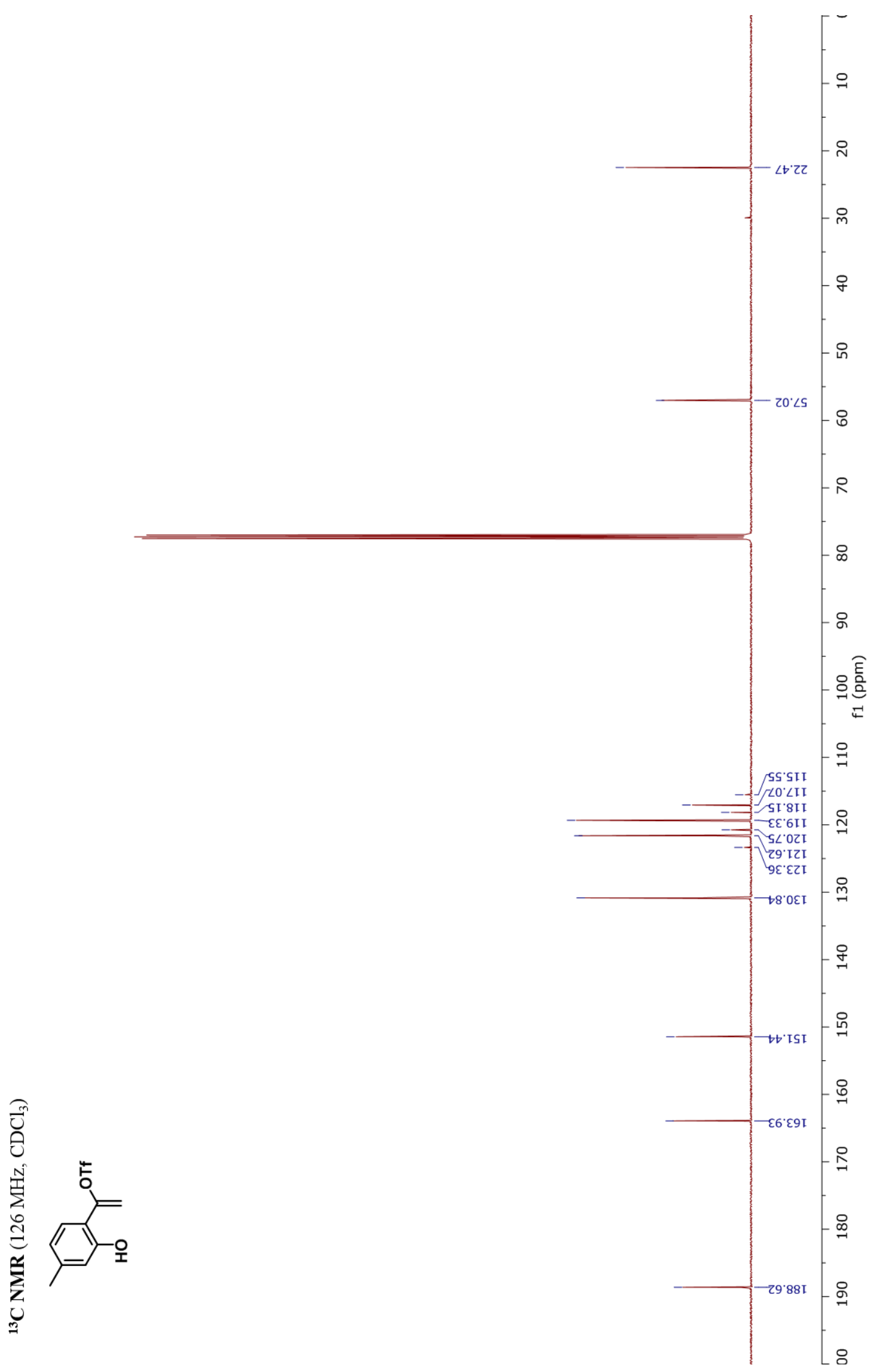
${ }^{1} \mathrm{H}$ NMR Spectrum of Compound 2.73

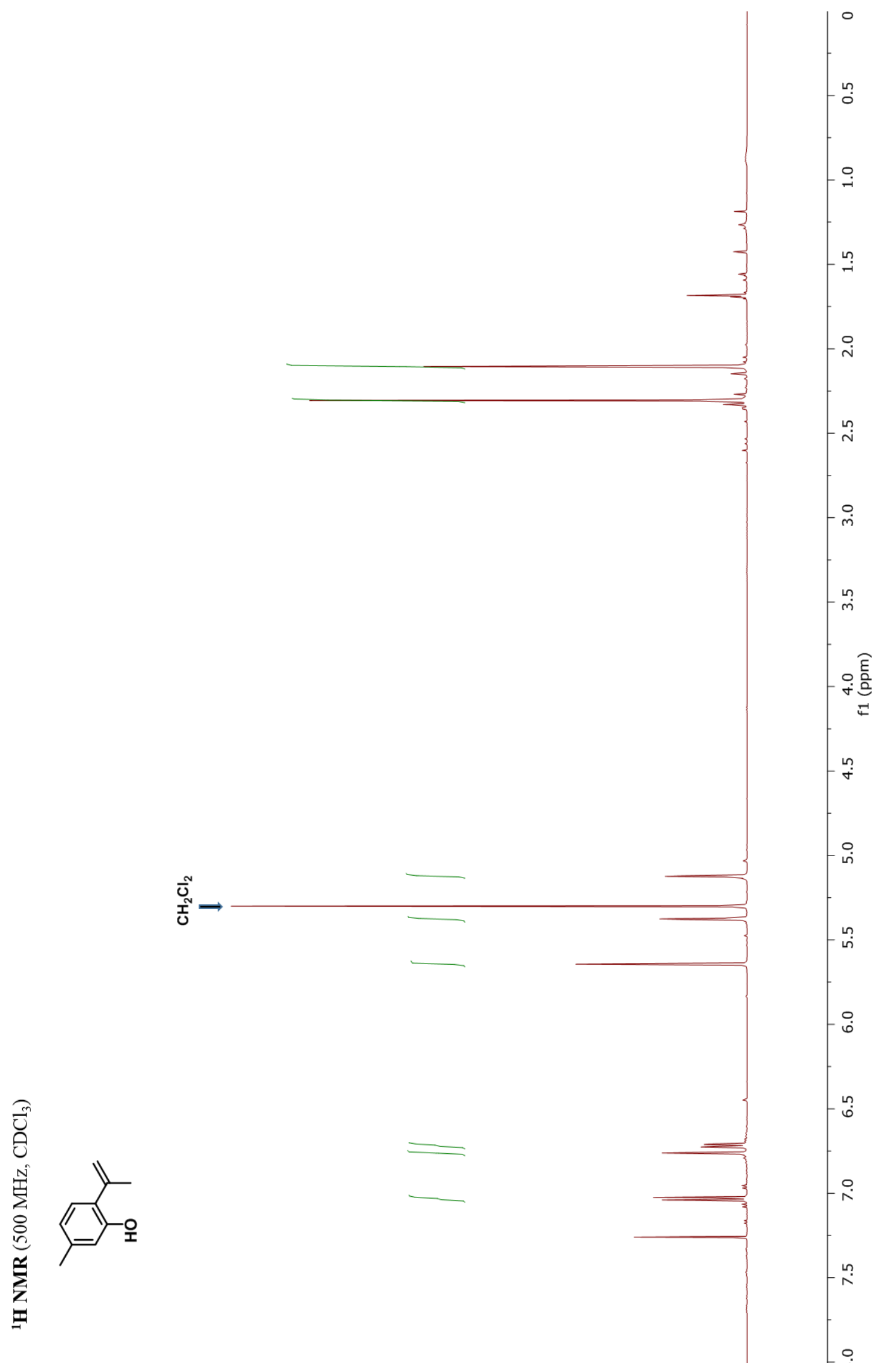


${ }^{13} \mathrm{C}$ NMR Spectrum of Compound 2.73

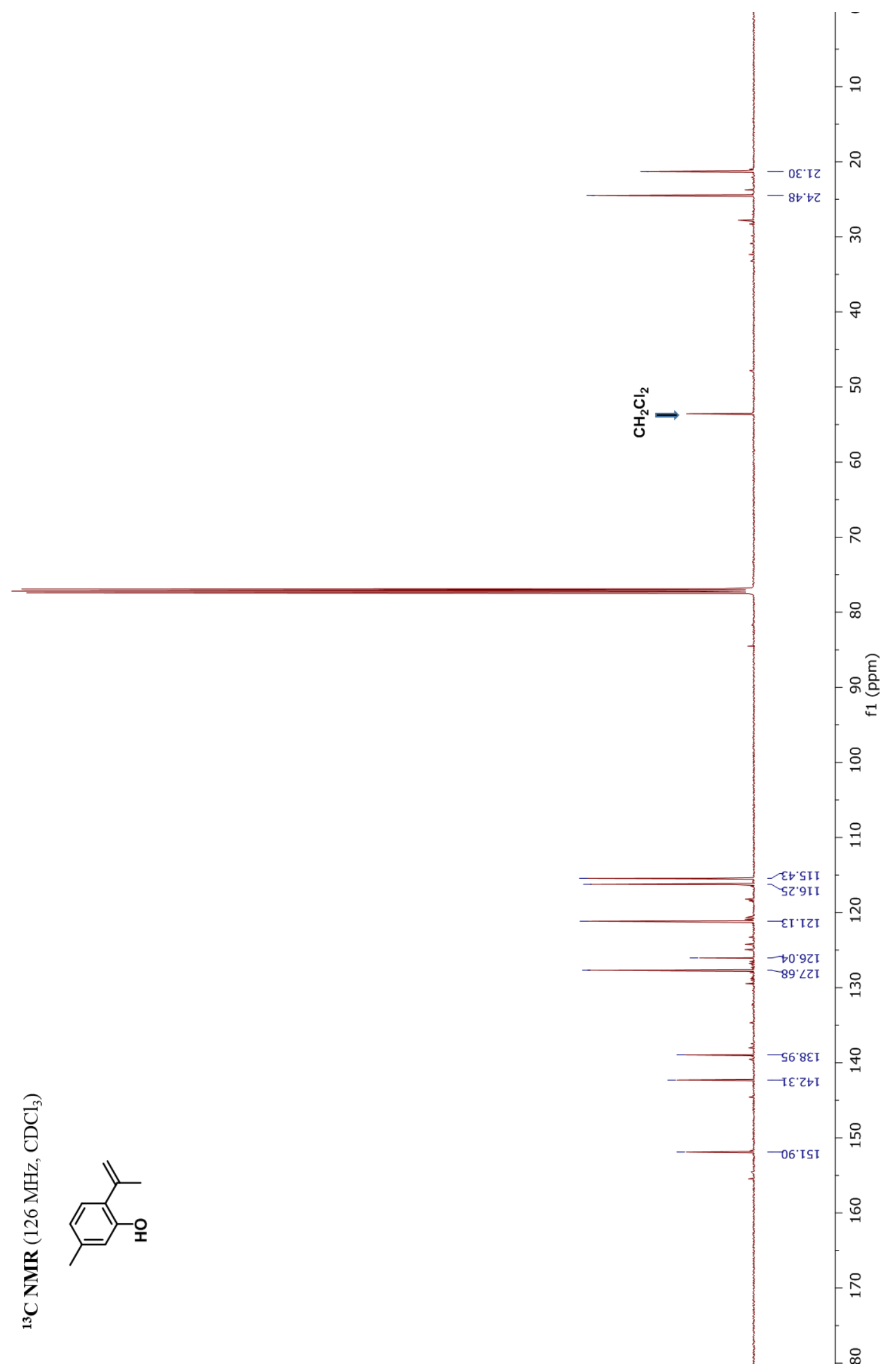


${ }^{1} \mathrm{H}$ NMR Spectrum of Compound 2.70

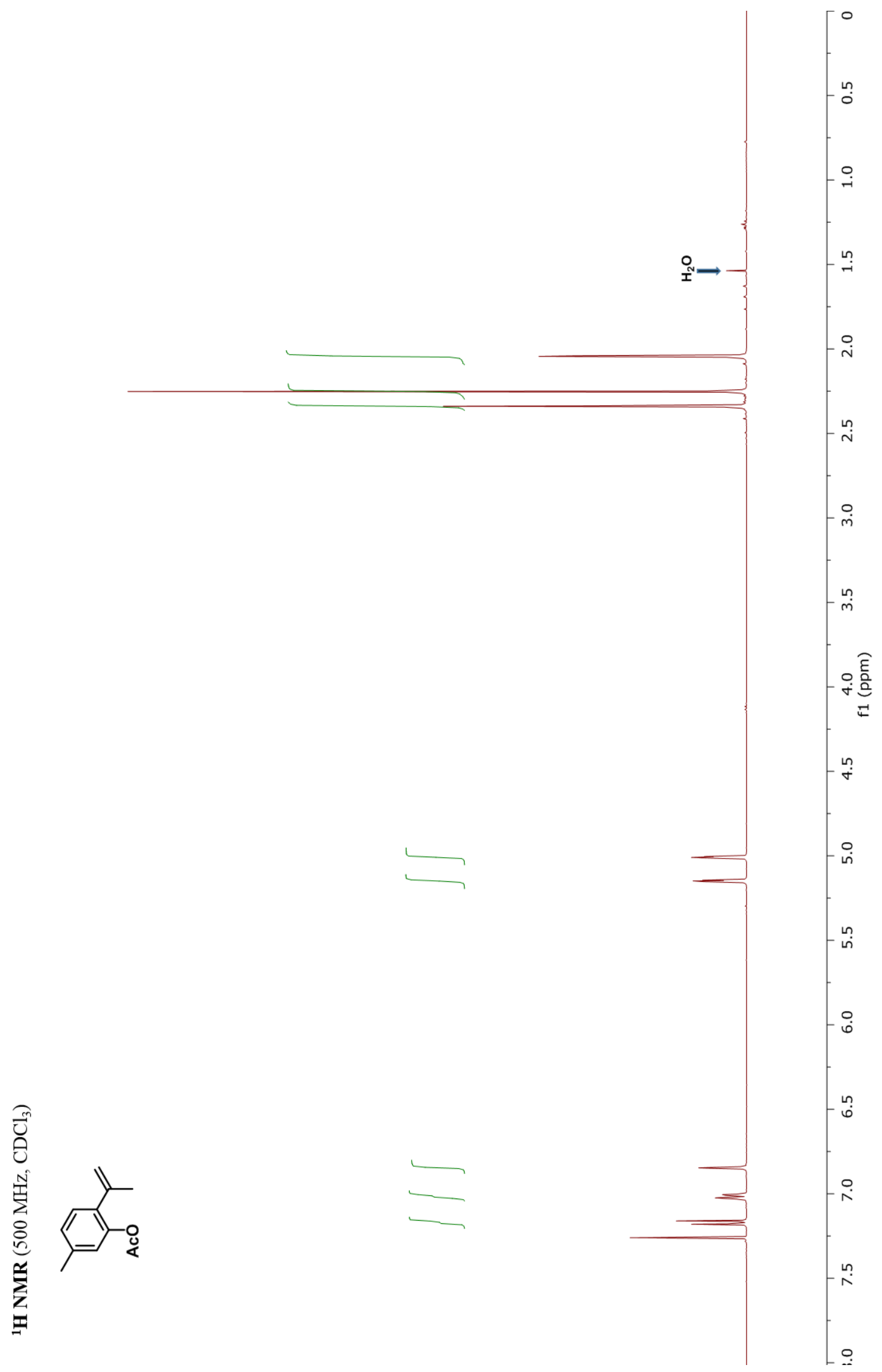


${ }^{13} \mathrm{C}$ NMR Spectrum of Compound 2.70

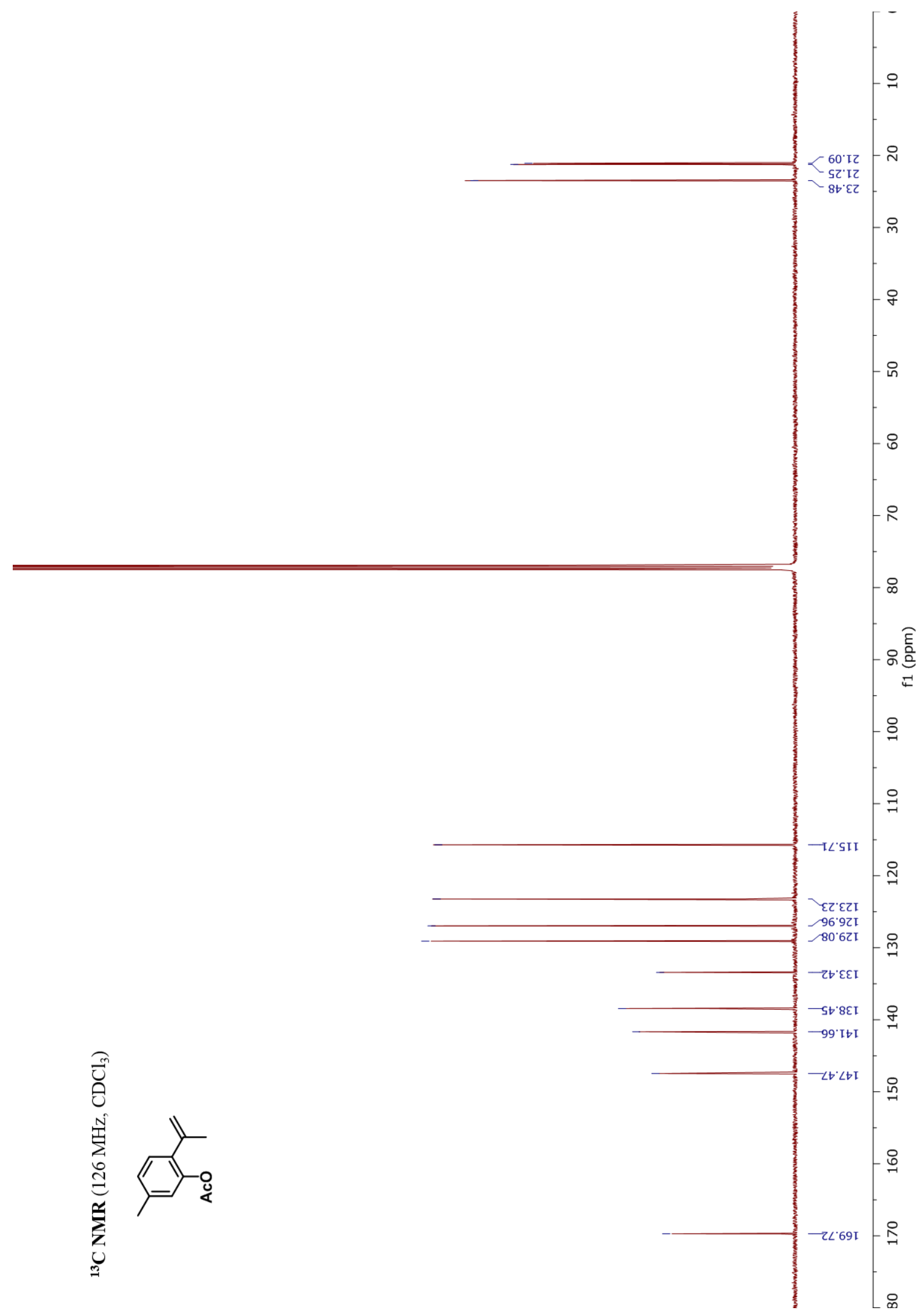


${ }^{1} \mathrm{H}$ NMR Spectrum of Compound 2.72

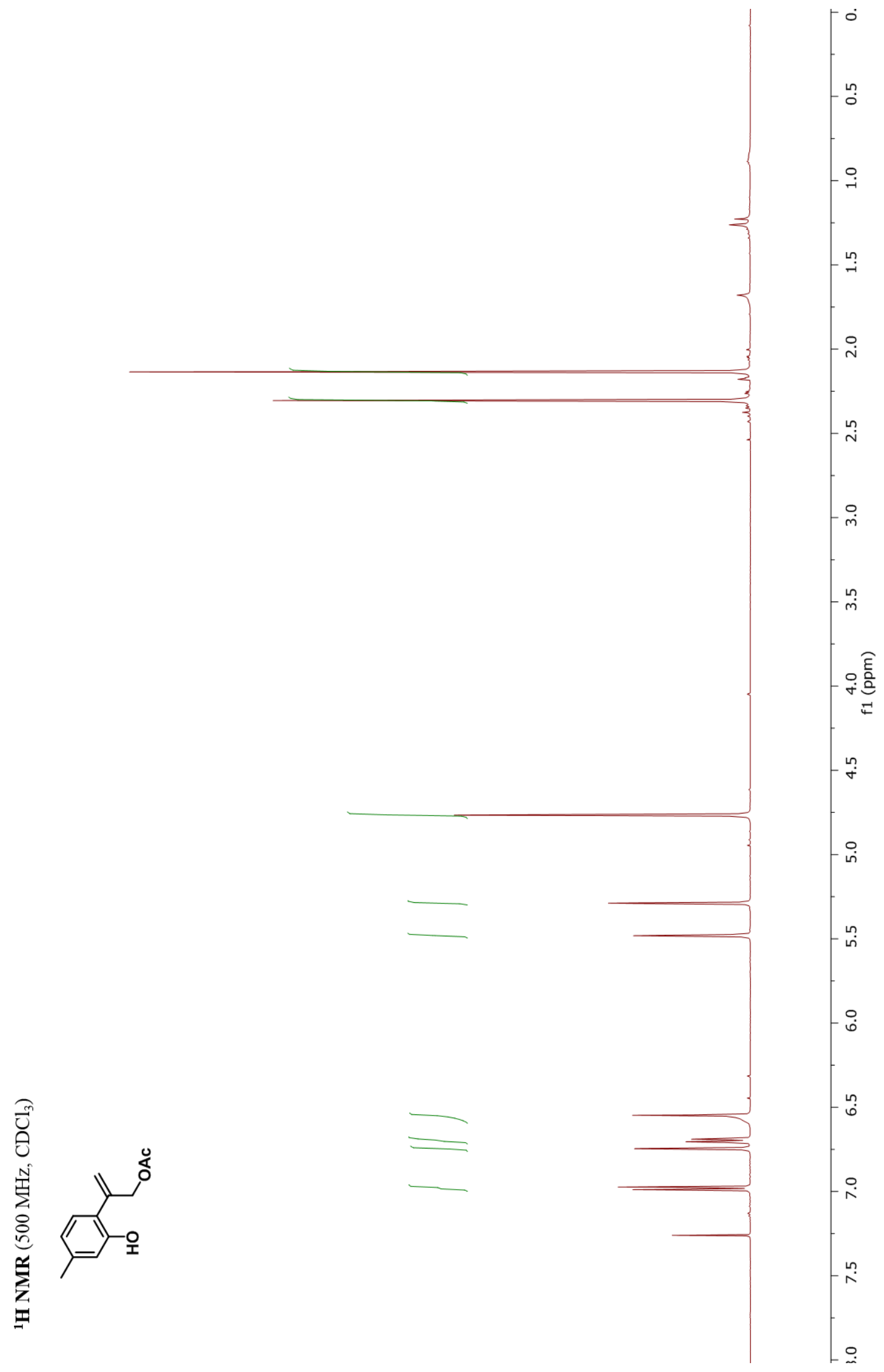


${ }^{13}$ C NMR Spectrum of Compound 2.72

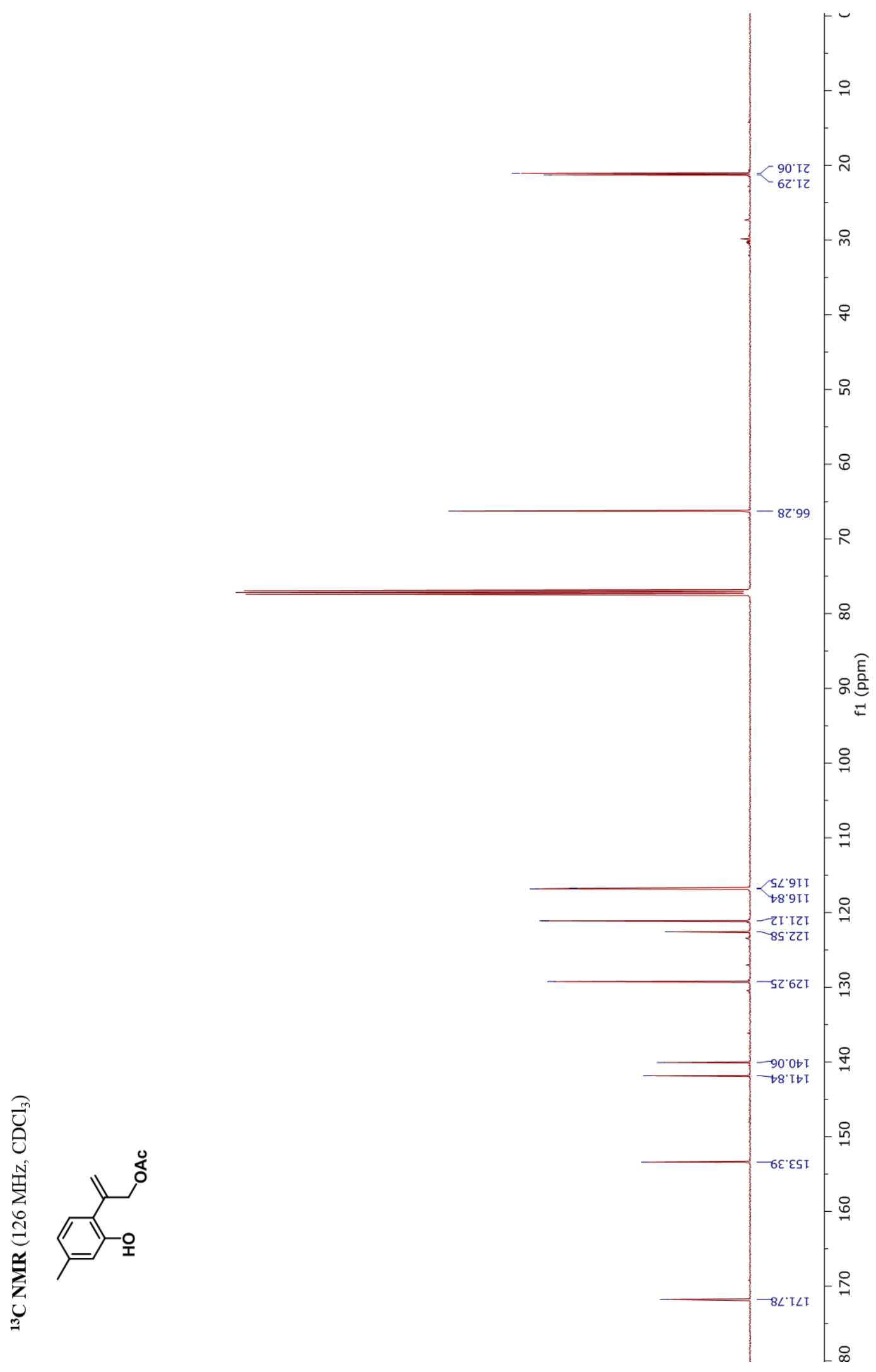


${ }^{1} \mathrm{H}$ NMR Spectrum of Compound ( \pm )-2.76

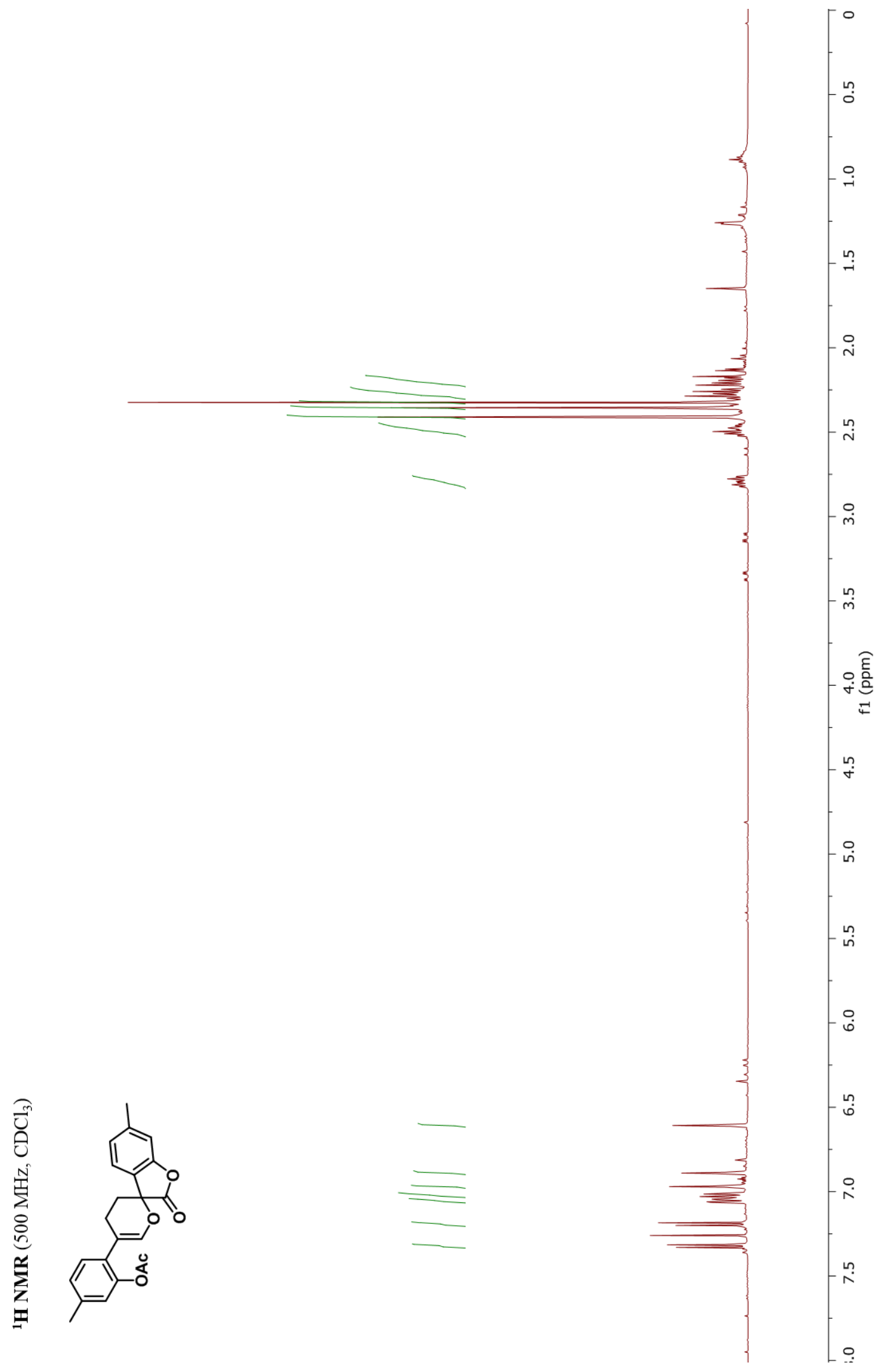


${ }^{13}$ C NMR Spectrum of Compound ( \pm )-2.76
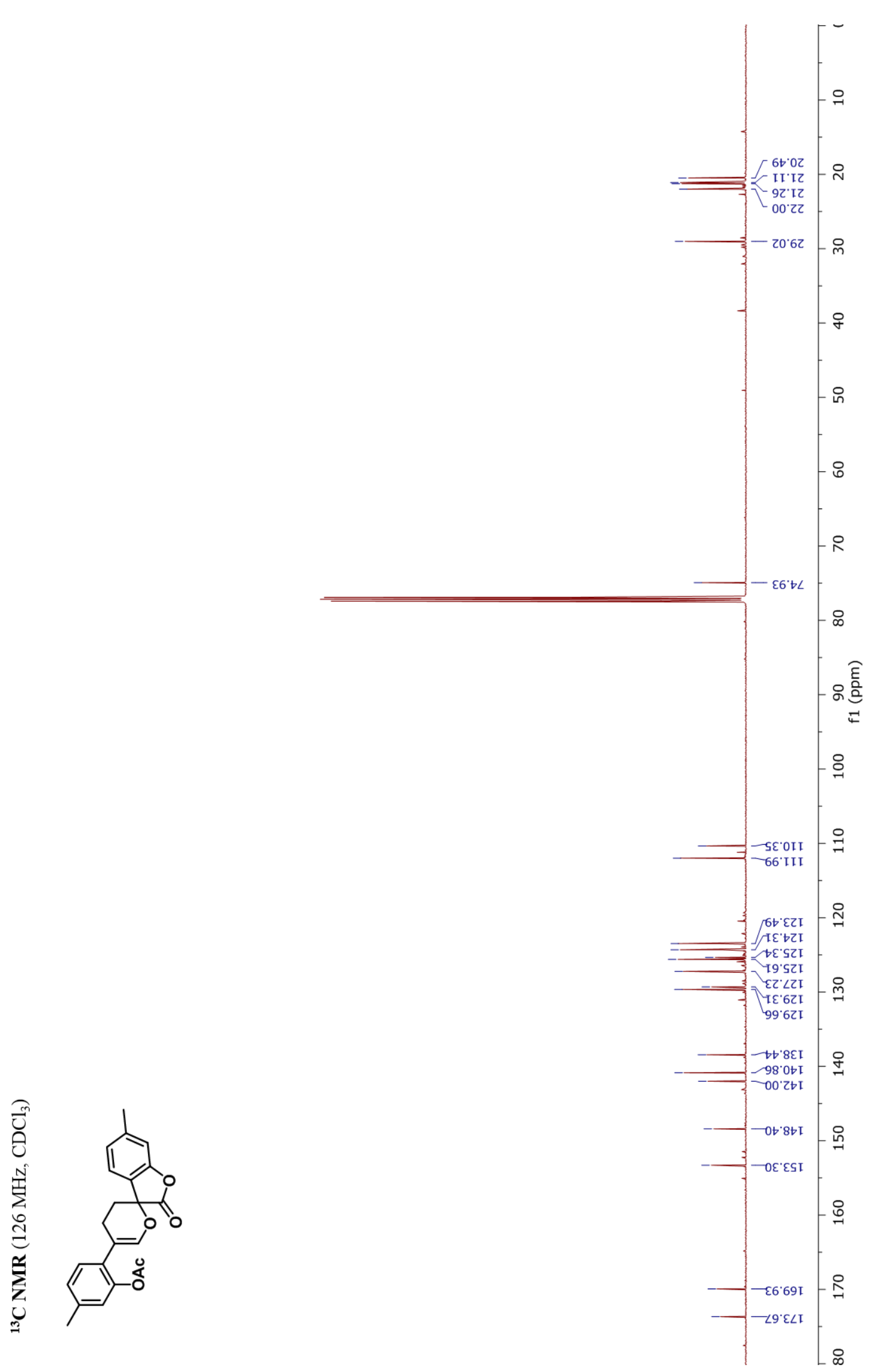
${ }^{1} \mathrm{H}$ NMR Spectrum of Compound 2.77

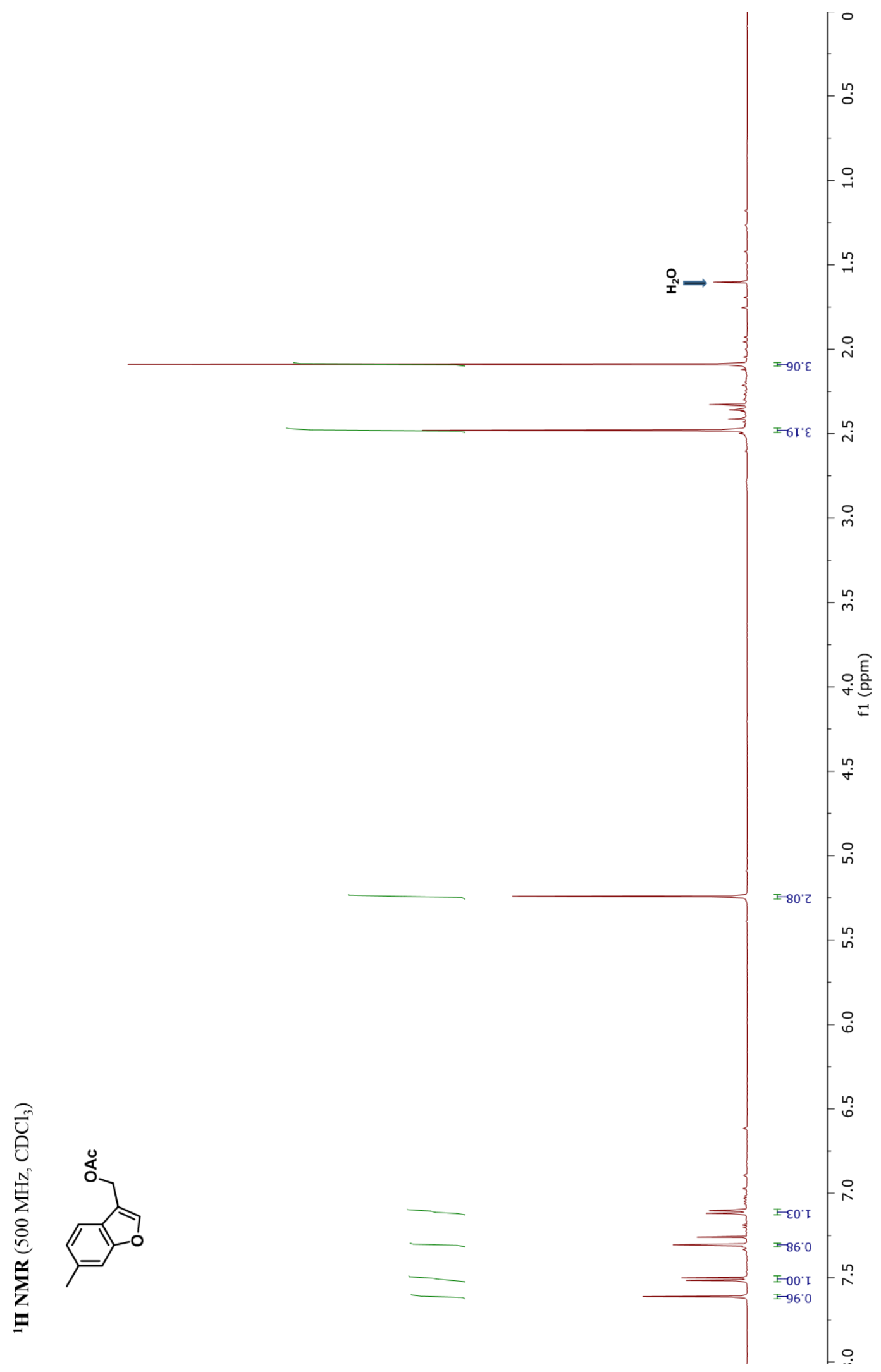


${ }^{13} \mathrm{C}$ NMR Spectrum of Compound 2.77

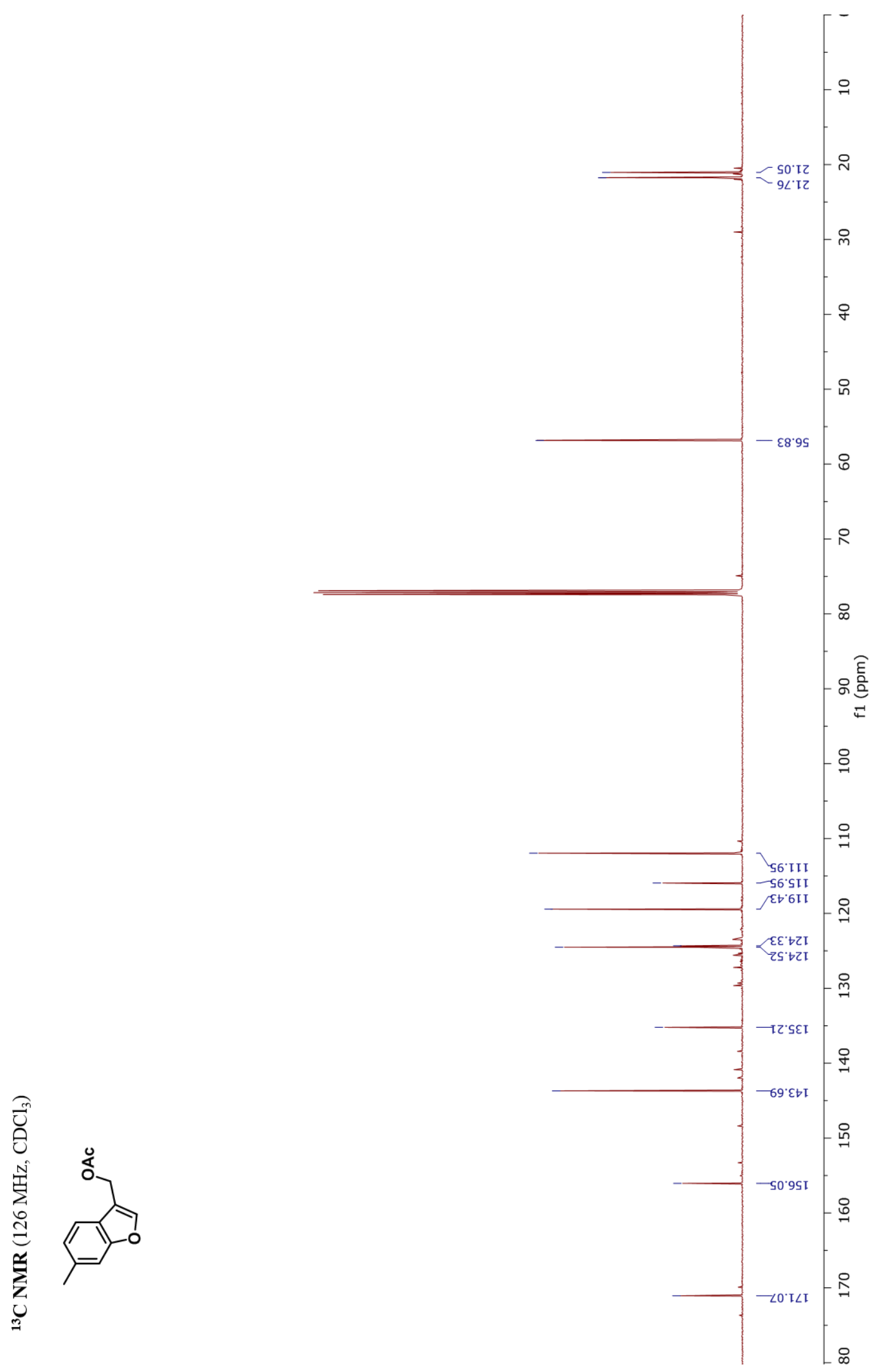


${ }^{1}$ H NMR Spectrum of Compound ( \pm )-2.78 (d.r. 1:1)

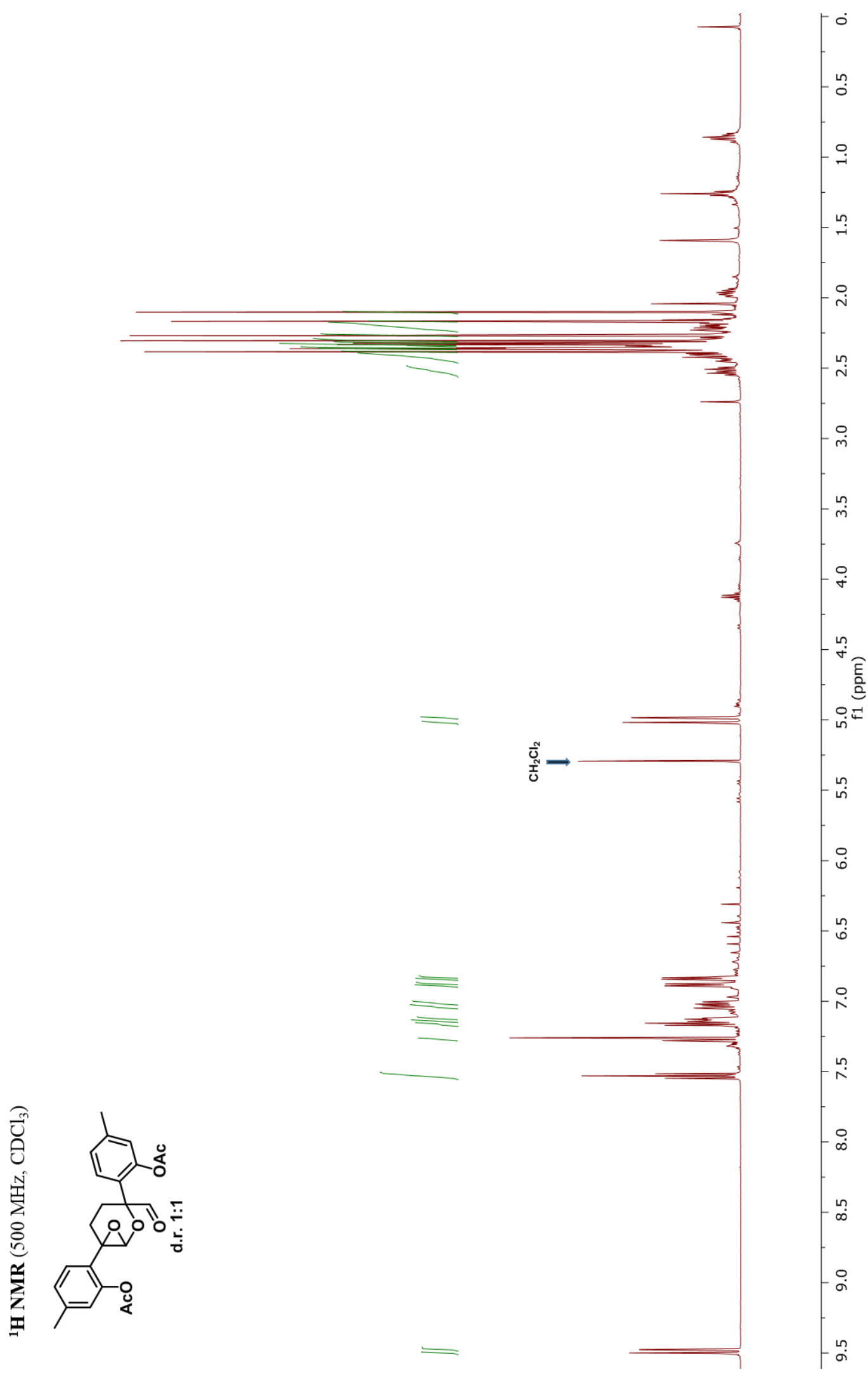


${ }^{1}$ H NMR Spectrum of Compound ( \pm )-2.78 (single diastereoisomer)

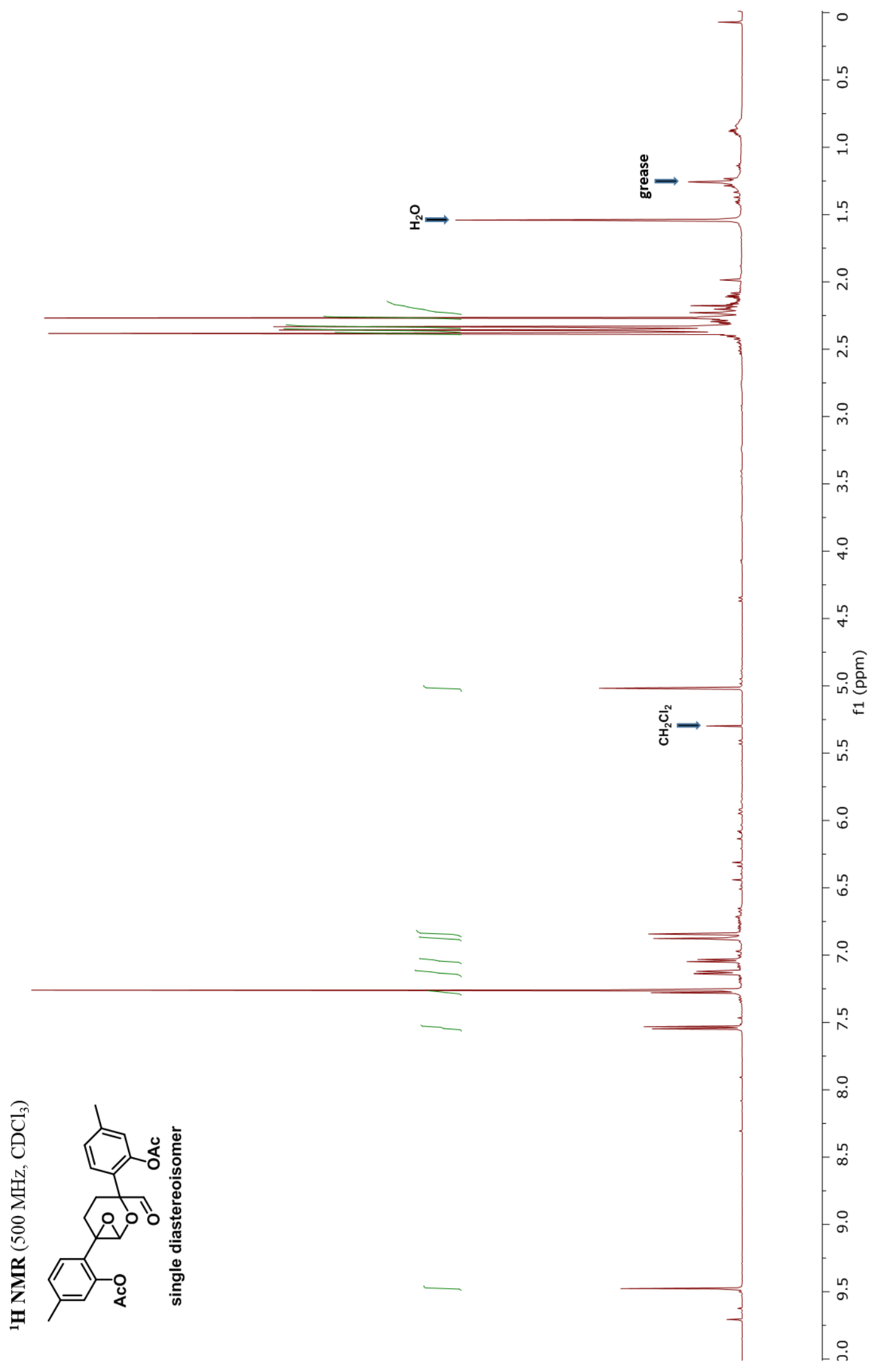


${ }^{13}$ C NMR Spectrum of Compound ( \pm )-2.78 (single diastereoisomer)

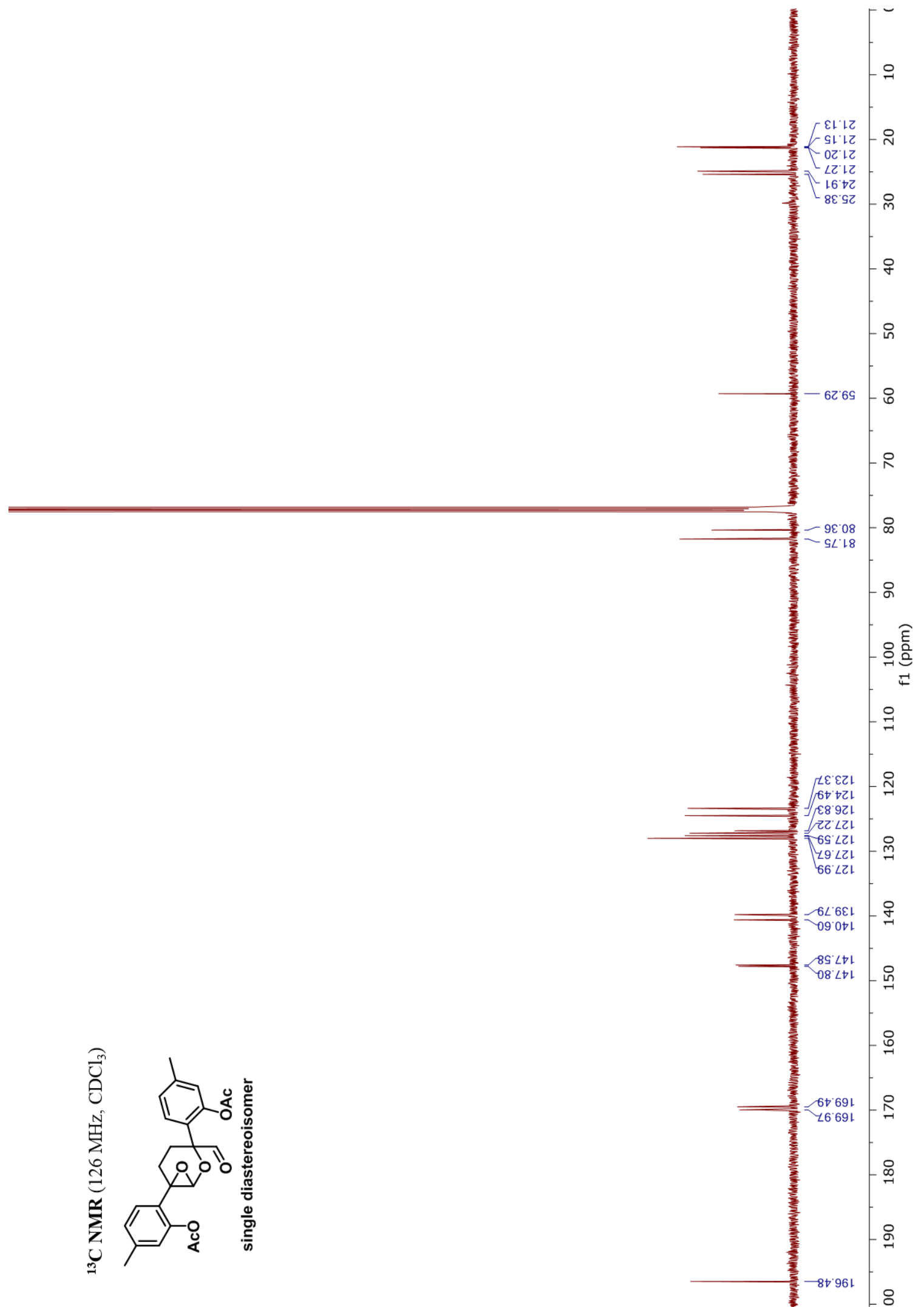


${ }^{1}$ H NMR Spectra of ( \pm )-Thymarnicol 1.1 and Crude Reaction Mixture

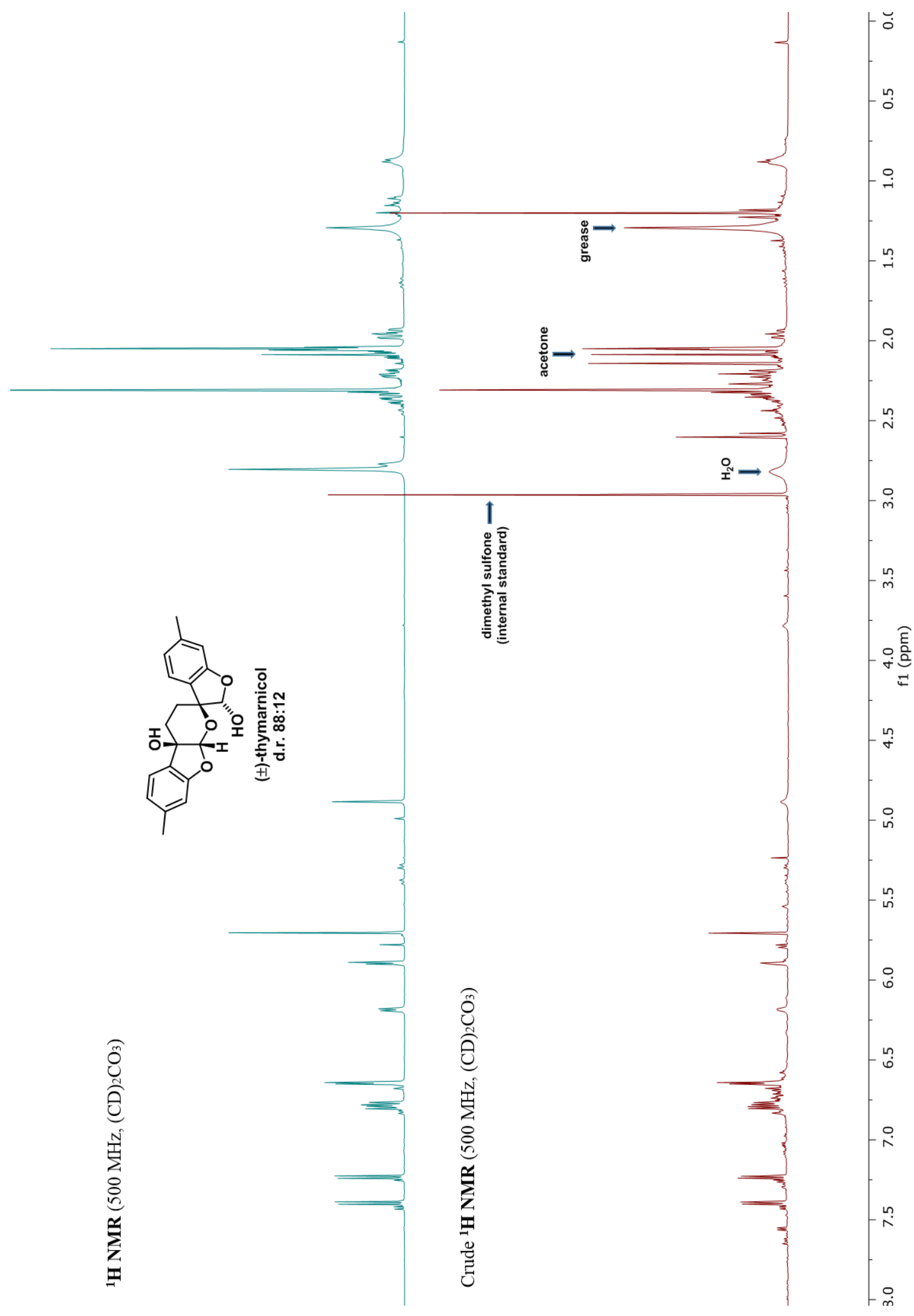


${ }^{1}$ H NMR Spectrum of ( \pm )-Thymarnicol 1.1

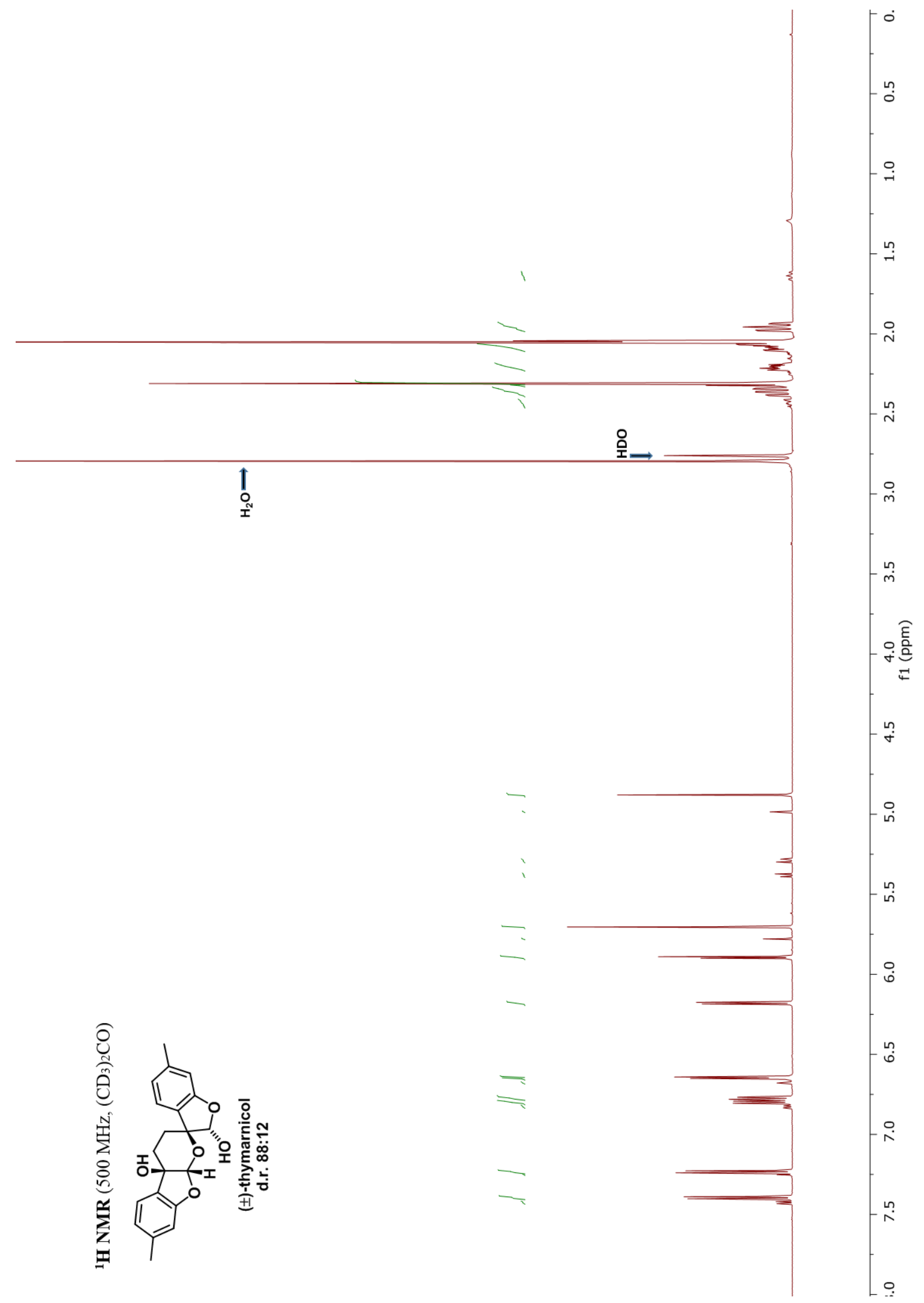


${ }^{13}$ C NMR Spectrum of ( \pm )-Thymarnicol 1.1

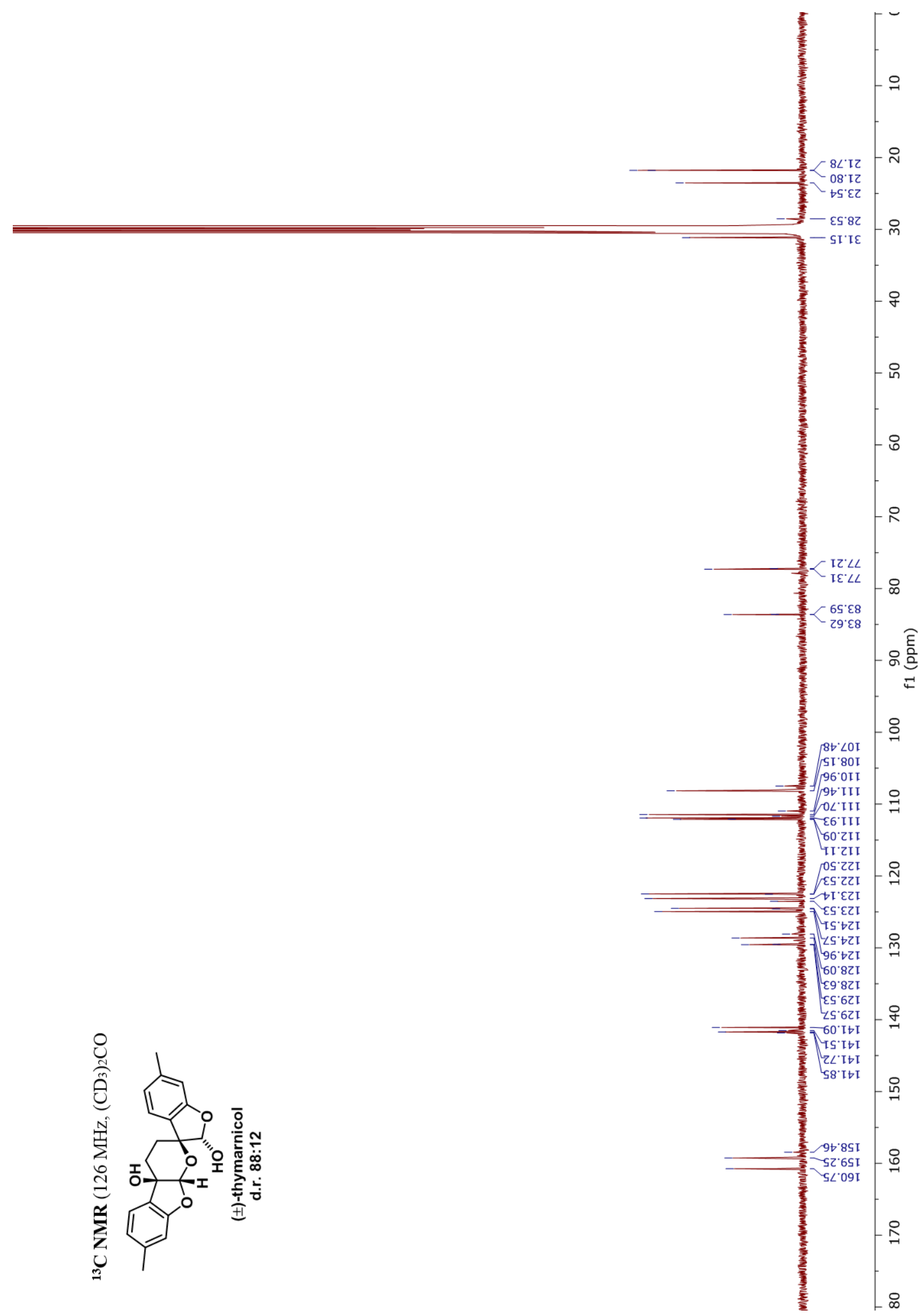


${ }^{1} \mathrm{H}-{ }^{1} \mathrm{H}$ COSY Spectrum of ( \pm )-Thymarnicol 1.1

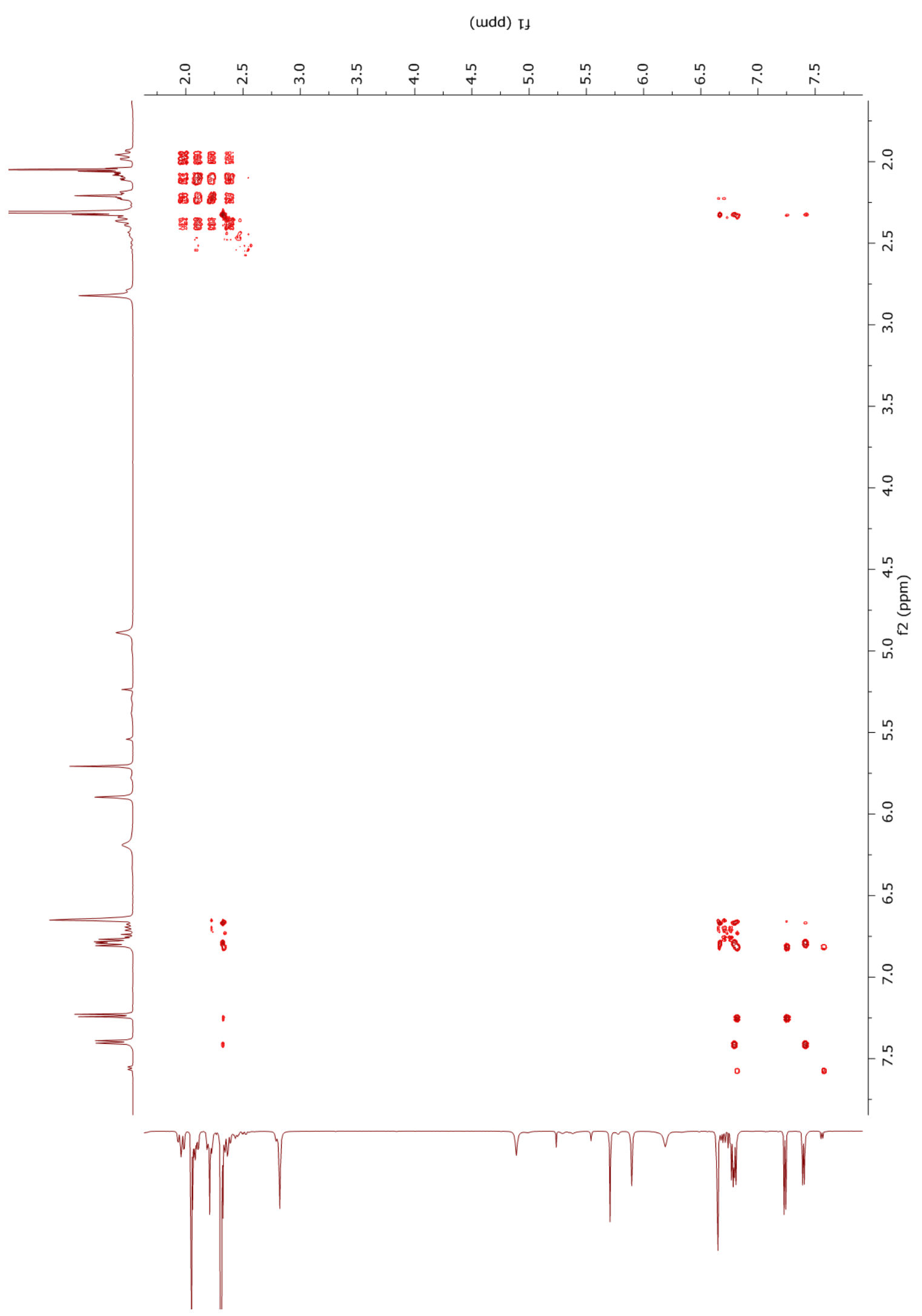

324 
${ }^{1} \mathrm{H}-{ }^{13} \mathrm{C}$ HSQC Spectrum of ( \pm )-Thymarnicol 1.1

(udd) it

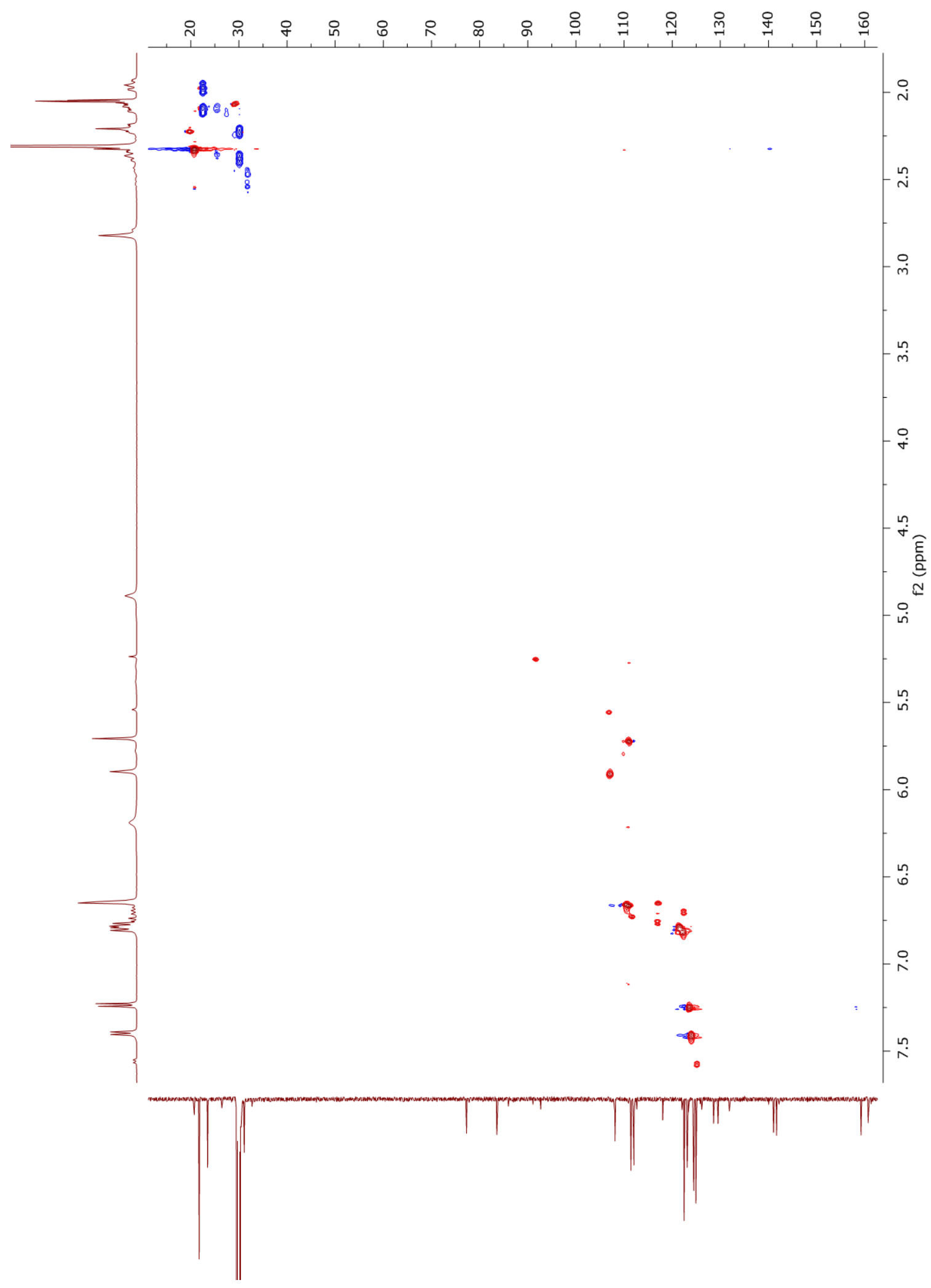


${ }^{1} \mathrm{H}-{ }^{13} \mathrm{C}$ HMBC Spectrum of ( \pm )-Thymarnicol 1.1

(udd) It

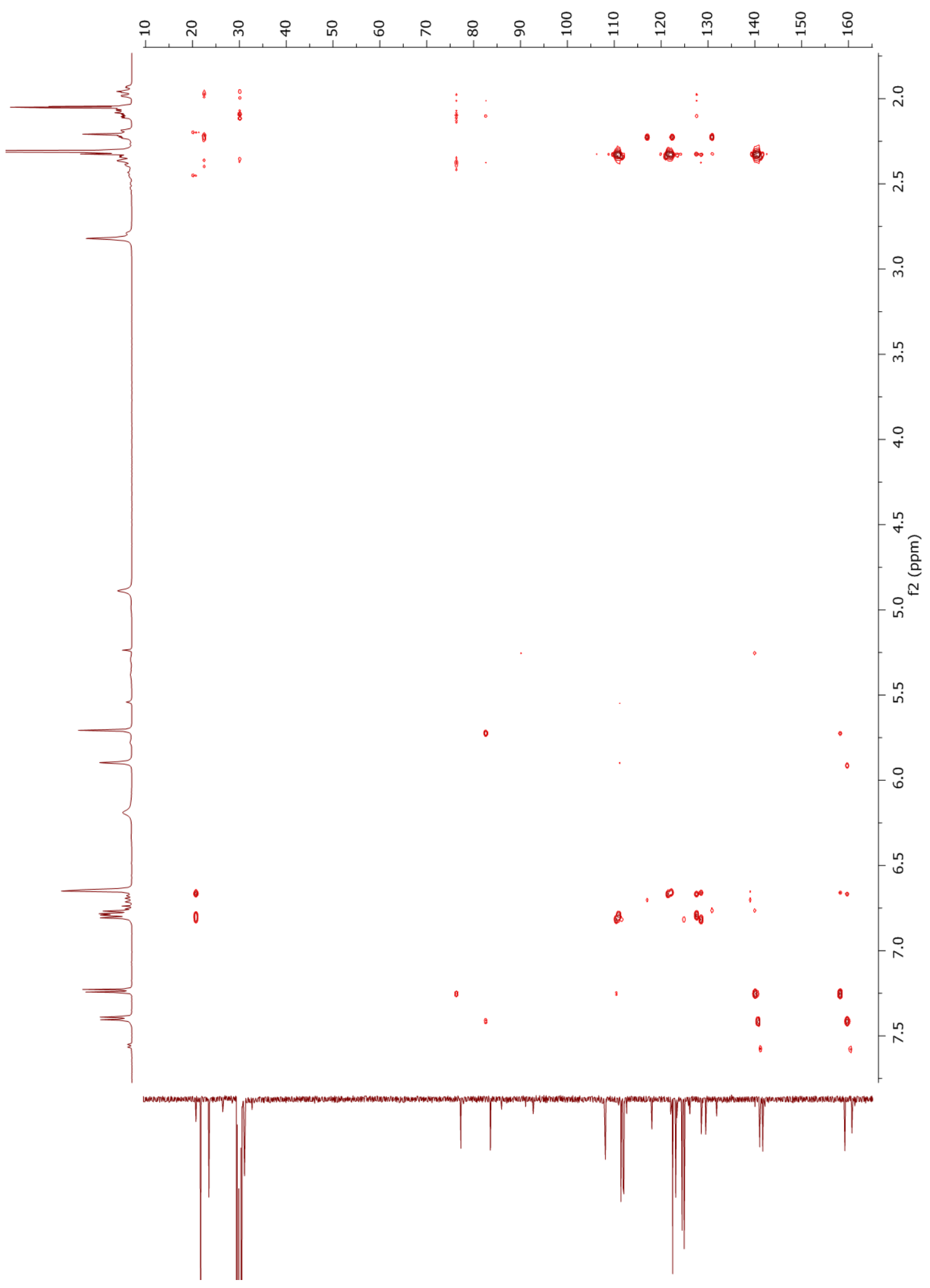


${ }^{1}$ H NMR Spectrum of Compound ( \pm )-2.79

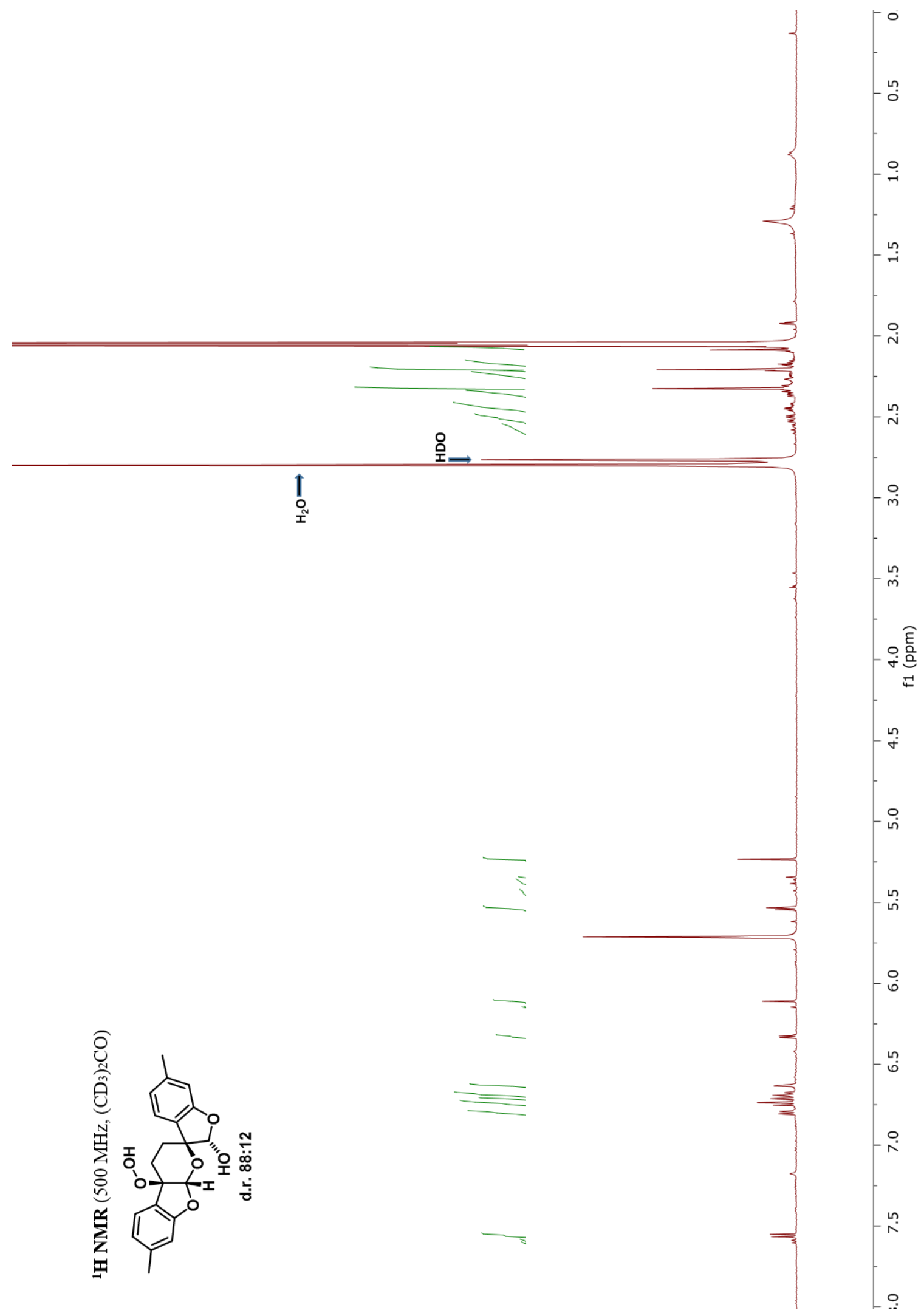


${ }^{13}$ C NMR Spectrum of Compound ( \pm )-2.79

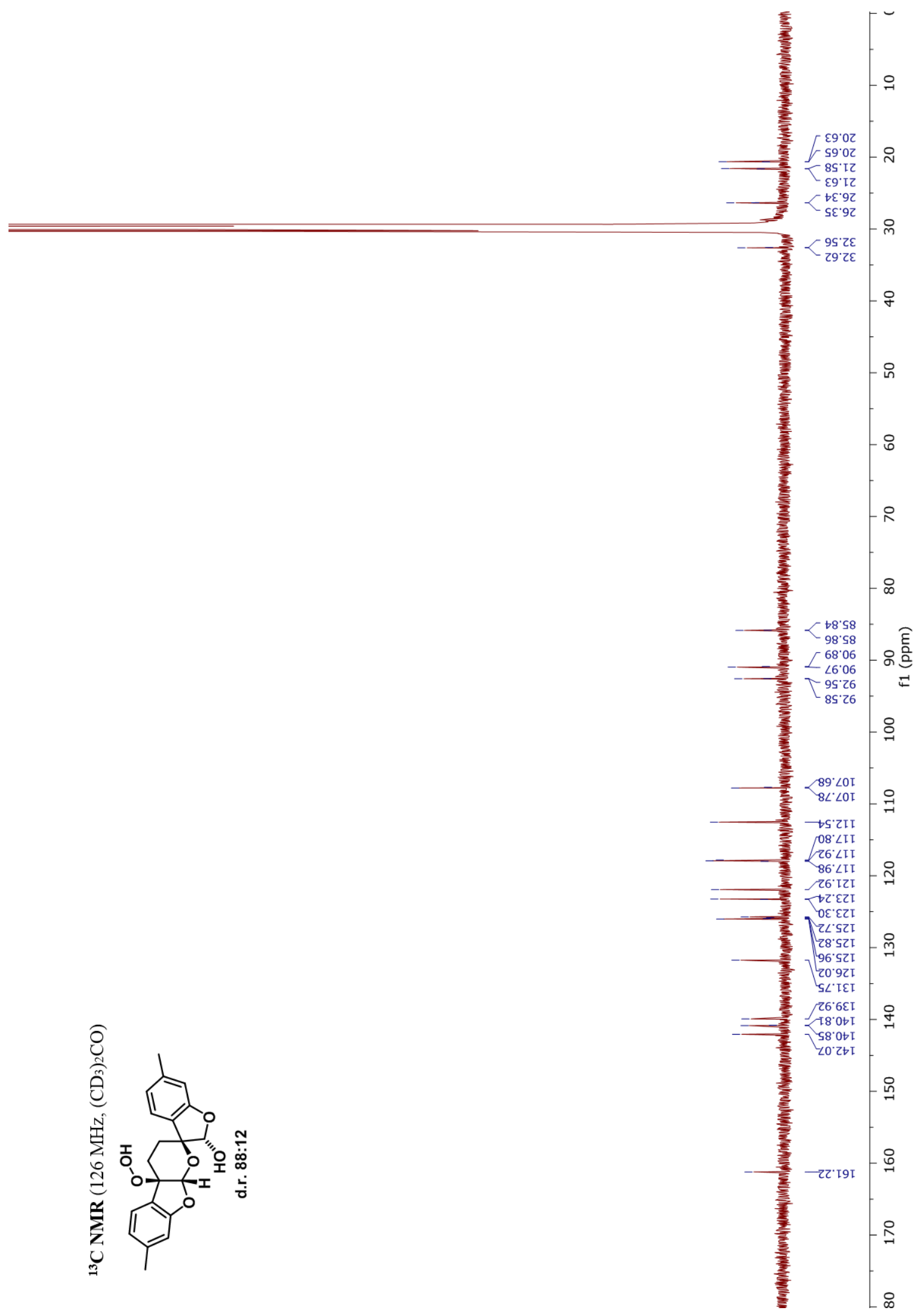




\subsection{NMR Spectra for Chapter 3}

${ }^{1}$ H NMR Spectrum of Compound 3.42

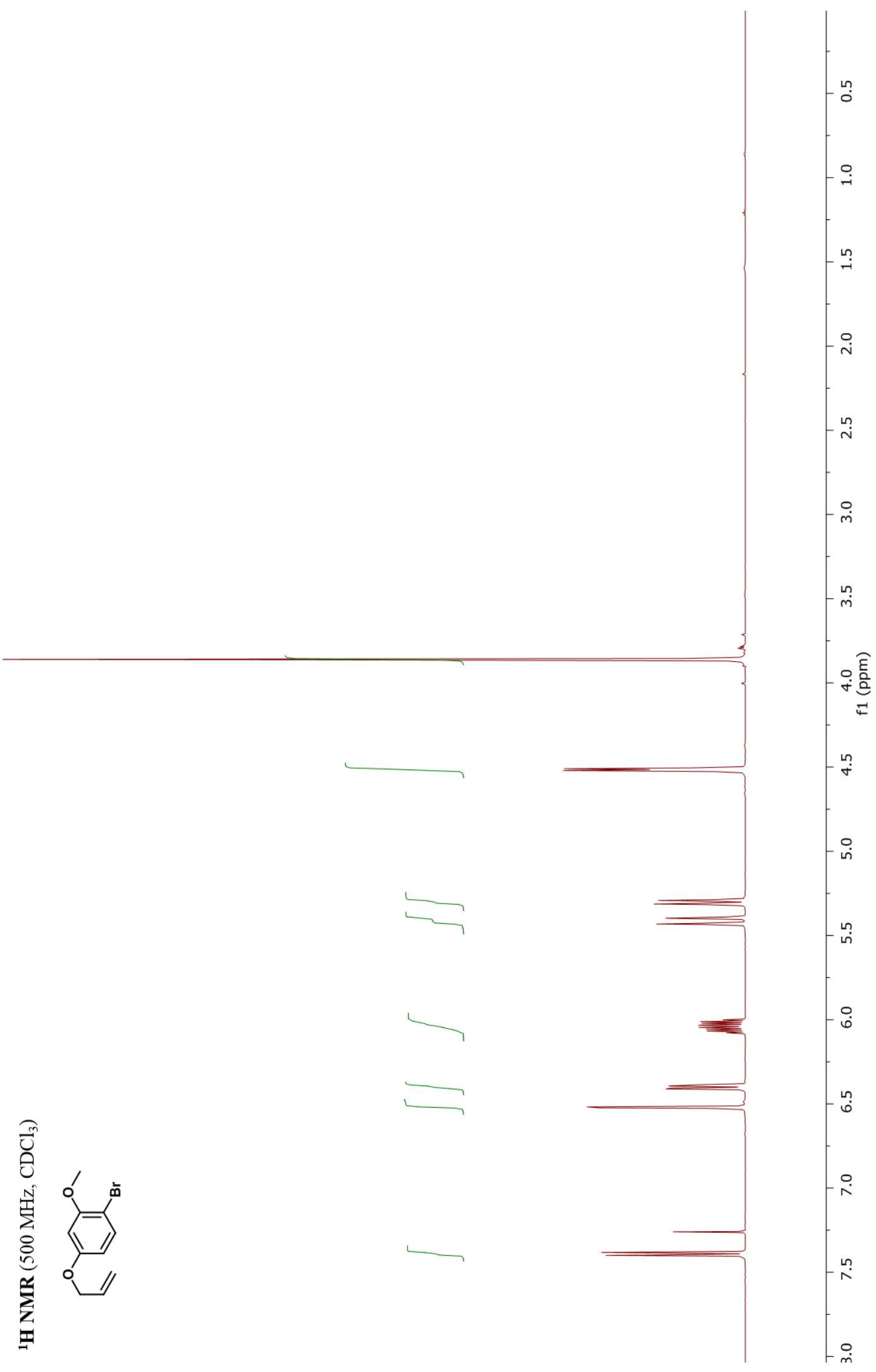


${ }^{13}$ C NMR Spectrum of Compound 3.42

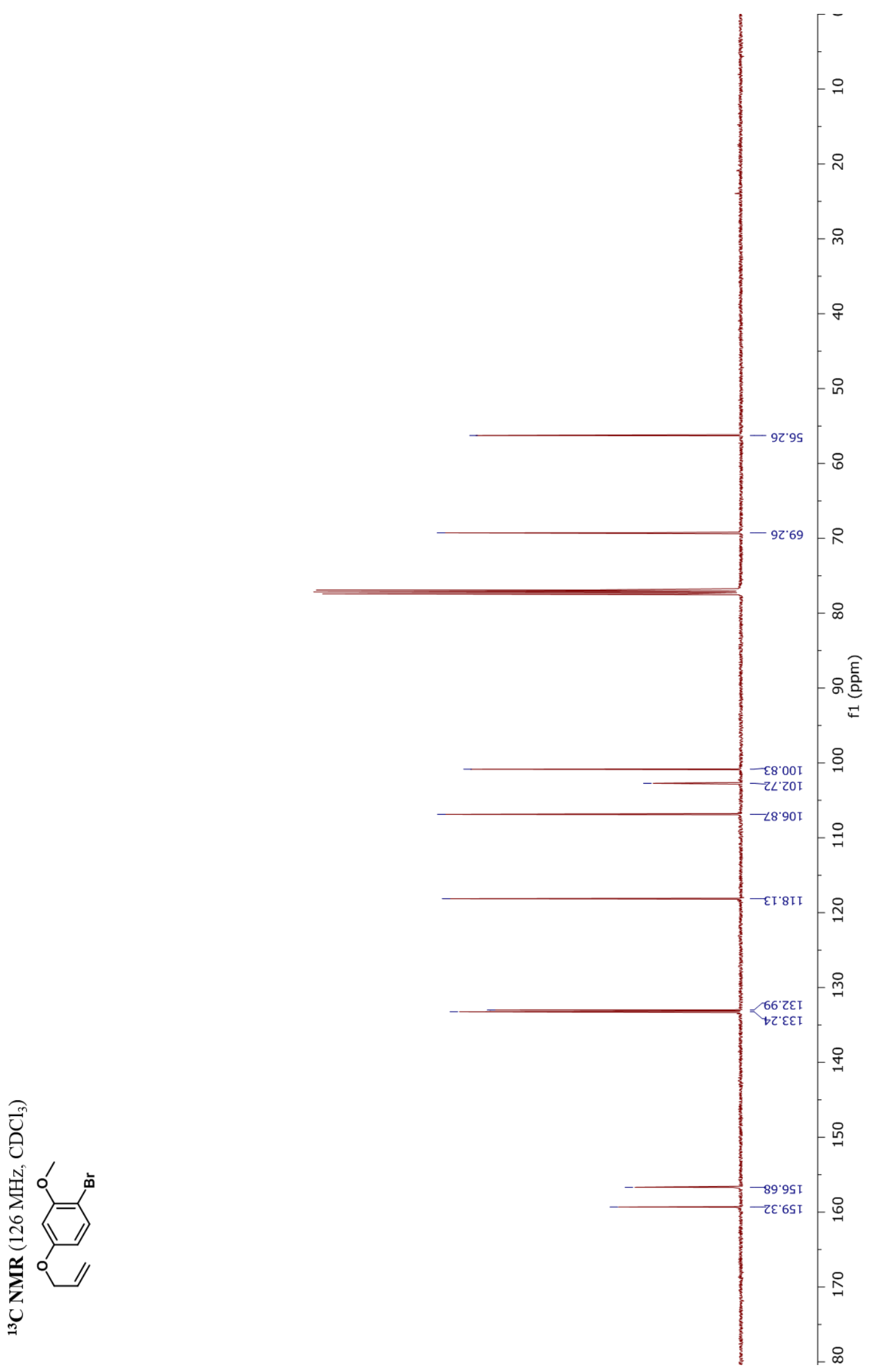


${ }^{1} \mathrm{H}$ NMR Spectrum of Compound 3.52

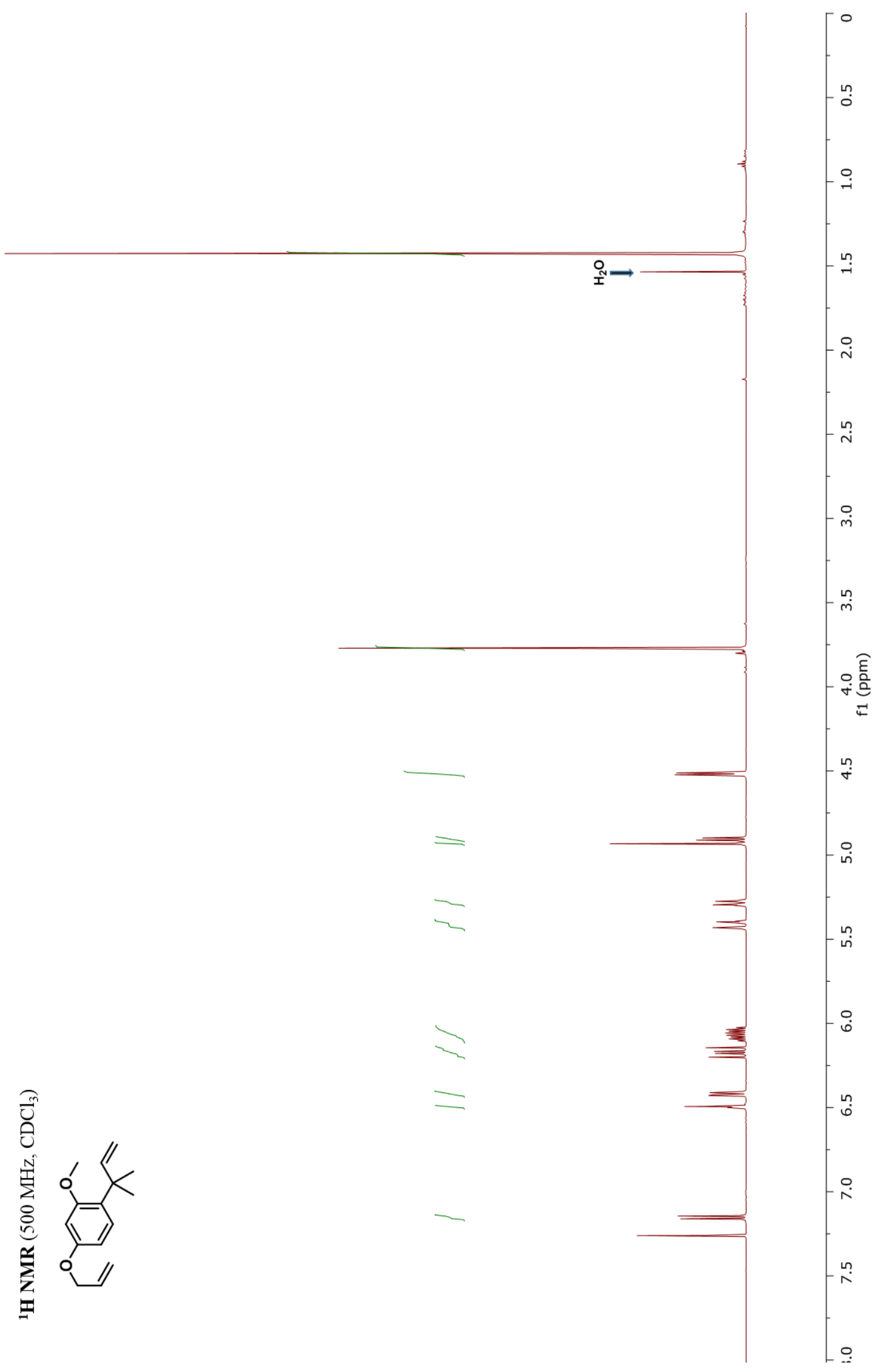


${ }^{13}$ C NMR Spectrum of Compound 3.52

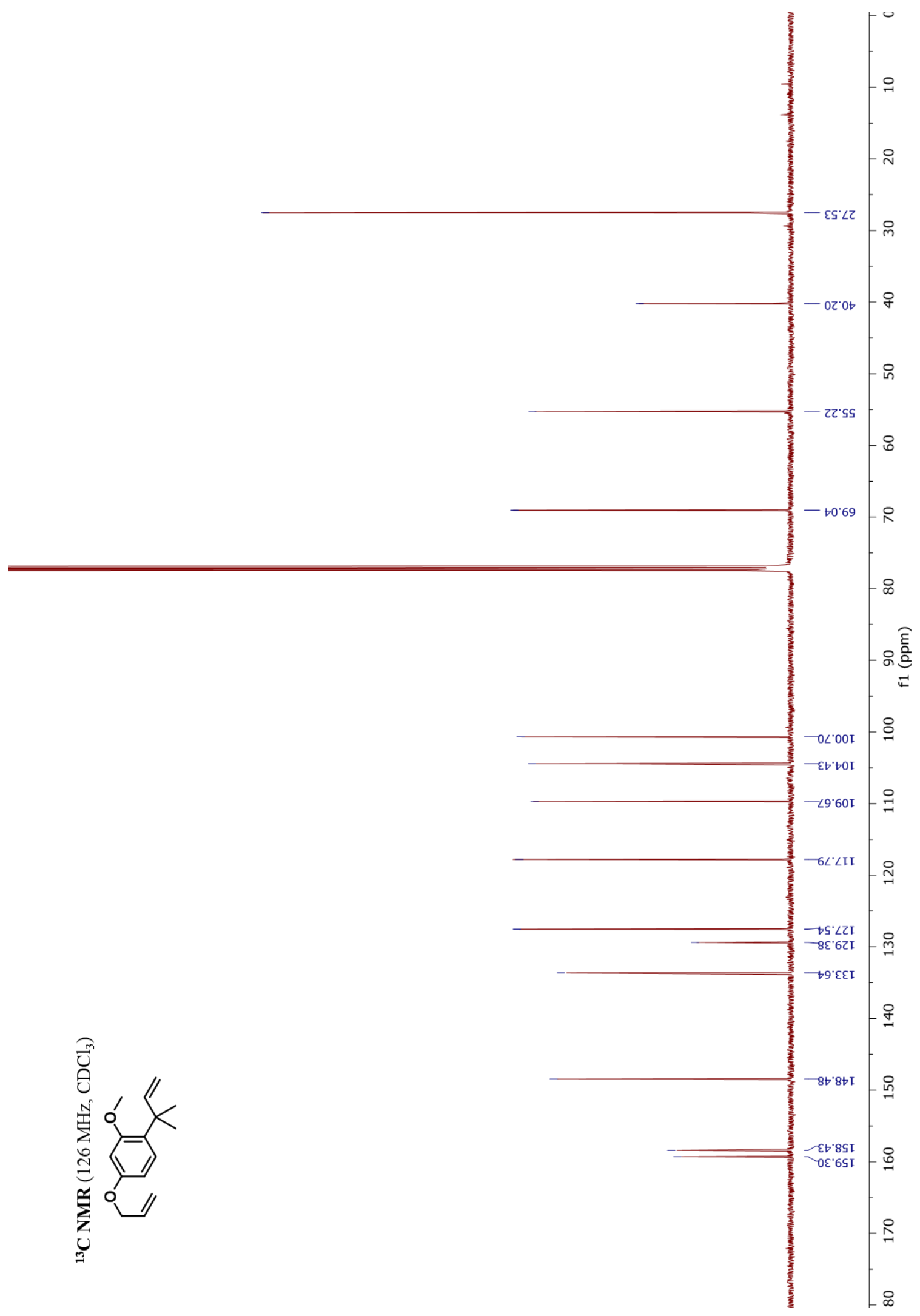


${ }^{1} \mathrm{H}$ NMR Spectrum of Compound 3.44

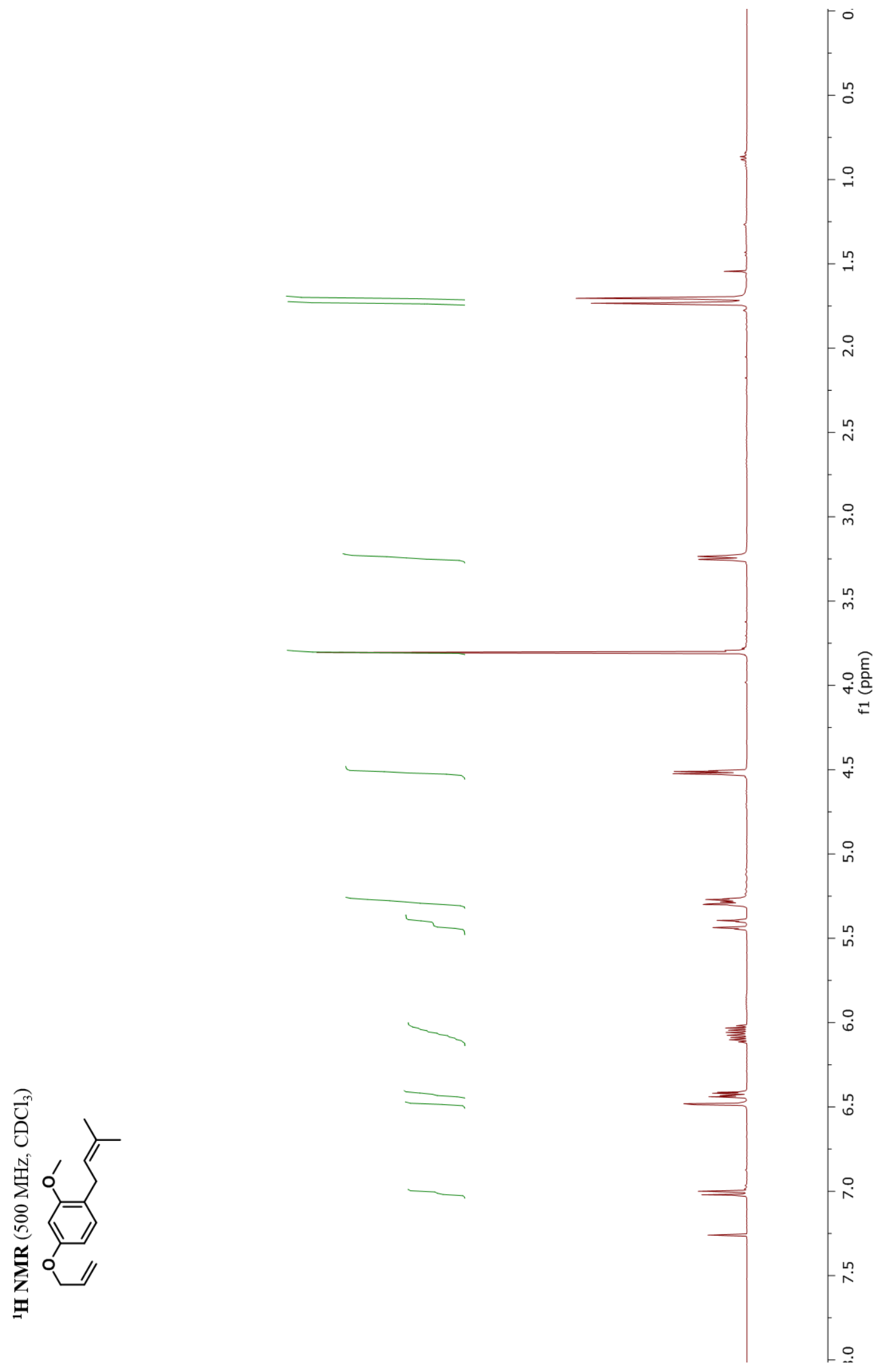


${ }^{13}$ C NMR Spectrum of Compound 3.44

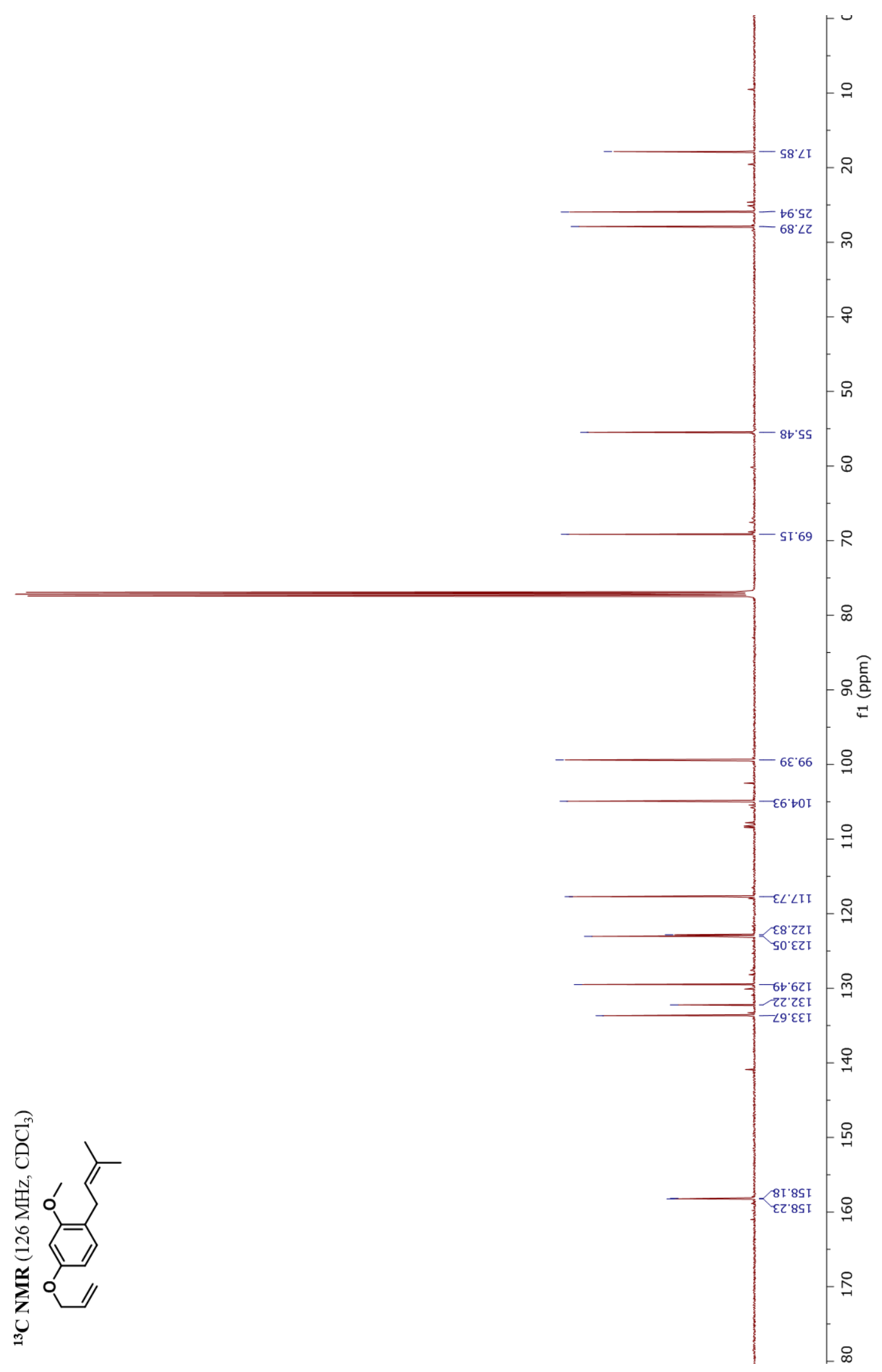


${ }^{1} \mathrm{H}$ NMR Spectrum of Compound 3.55

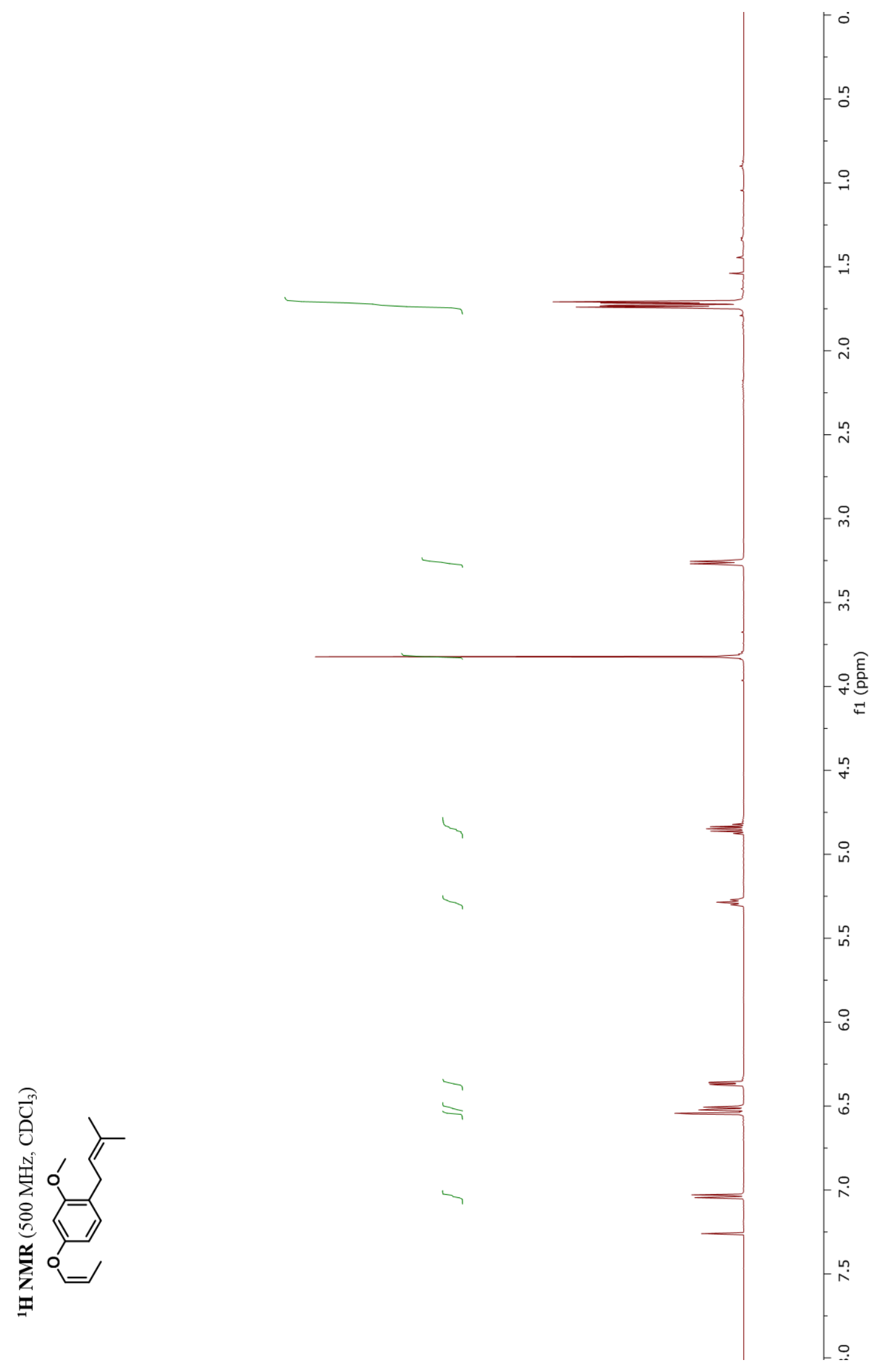


${ }^{13}$ C NMR Spectrum of Compound 3.55

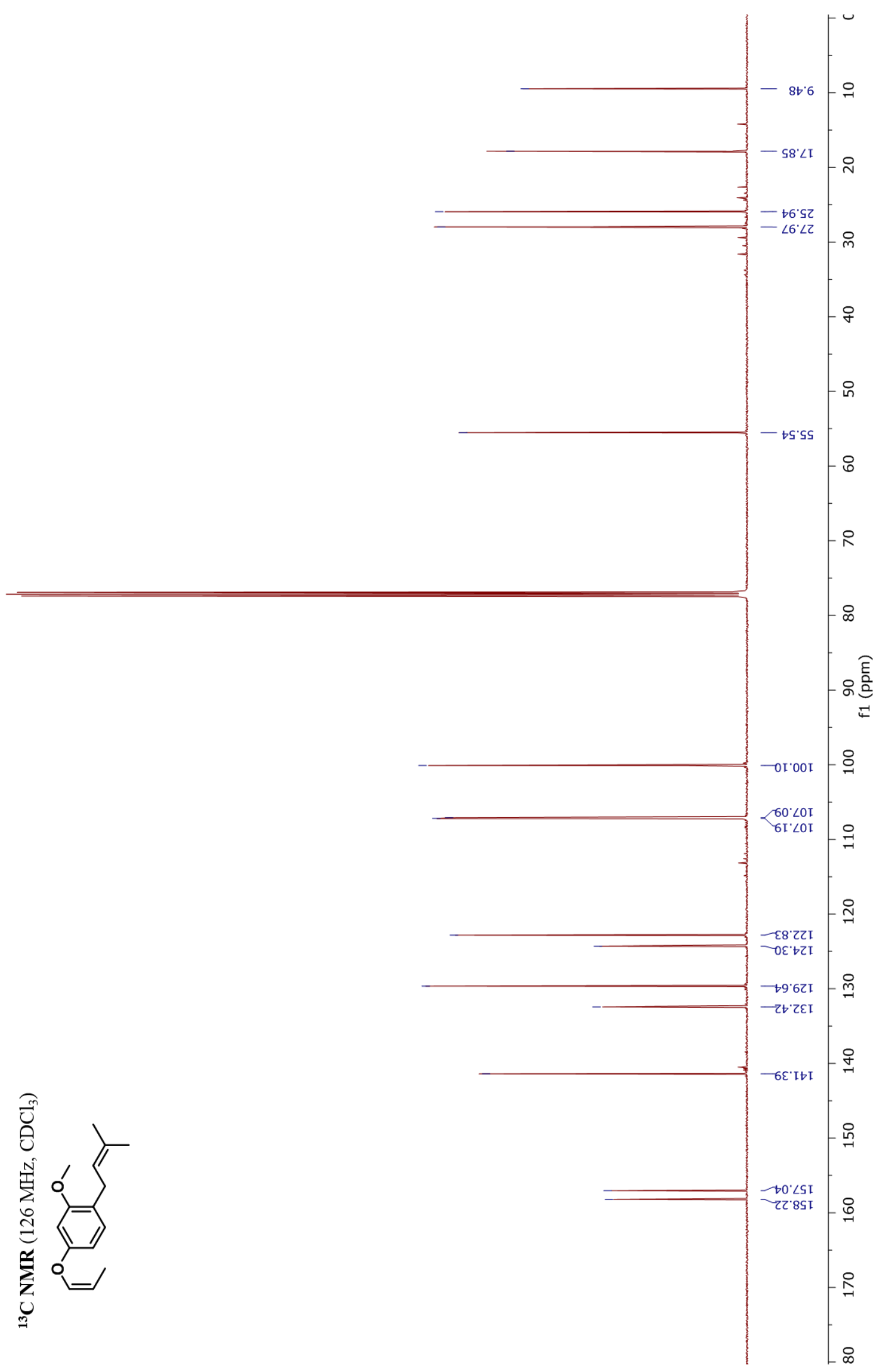


${ }^{1} \mathrm{H}$ NMR Spectrum of Compound 3.56

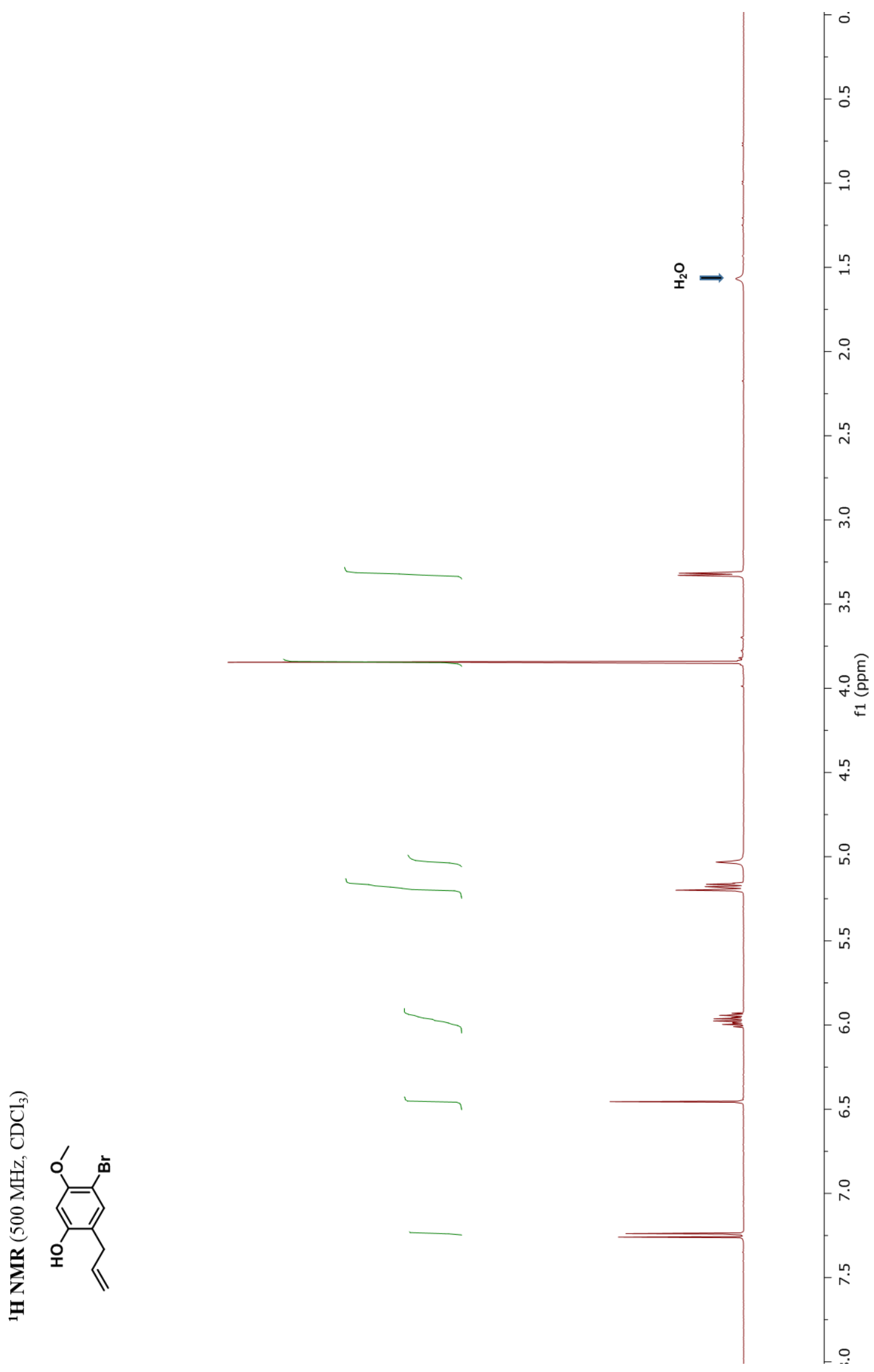


${ }^{13}$ C NMR Spectrum of Compound 3.56

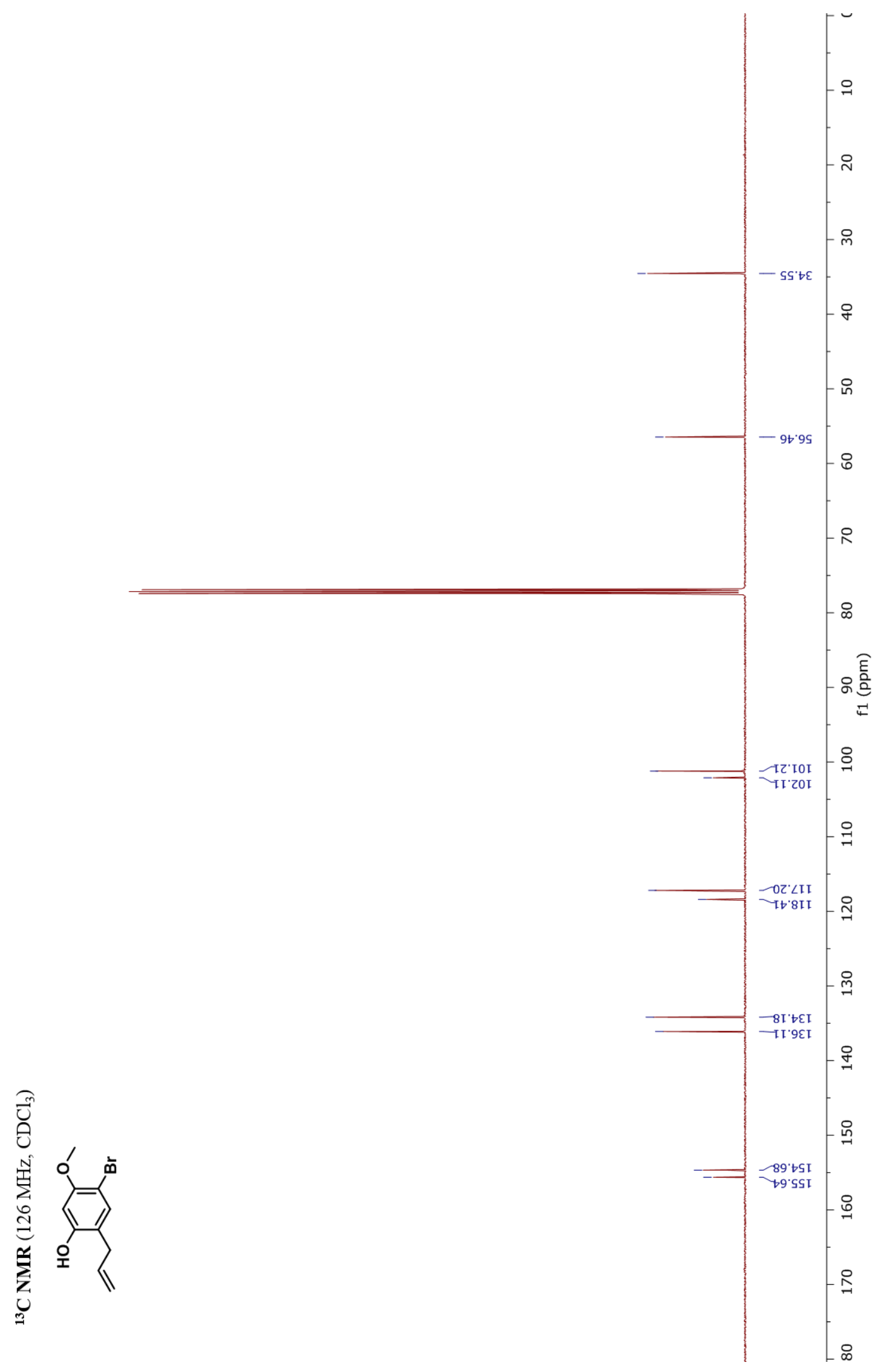


${ }^{1} \mathrm{H}$ NMR Spectrum of Compound 3.57

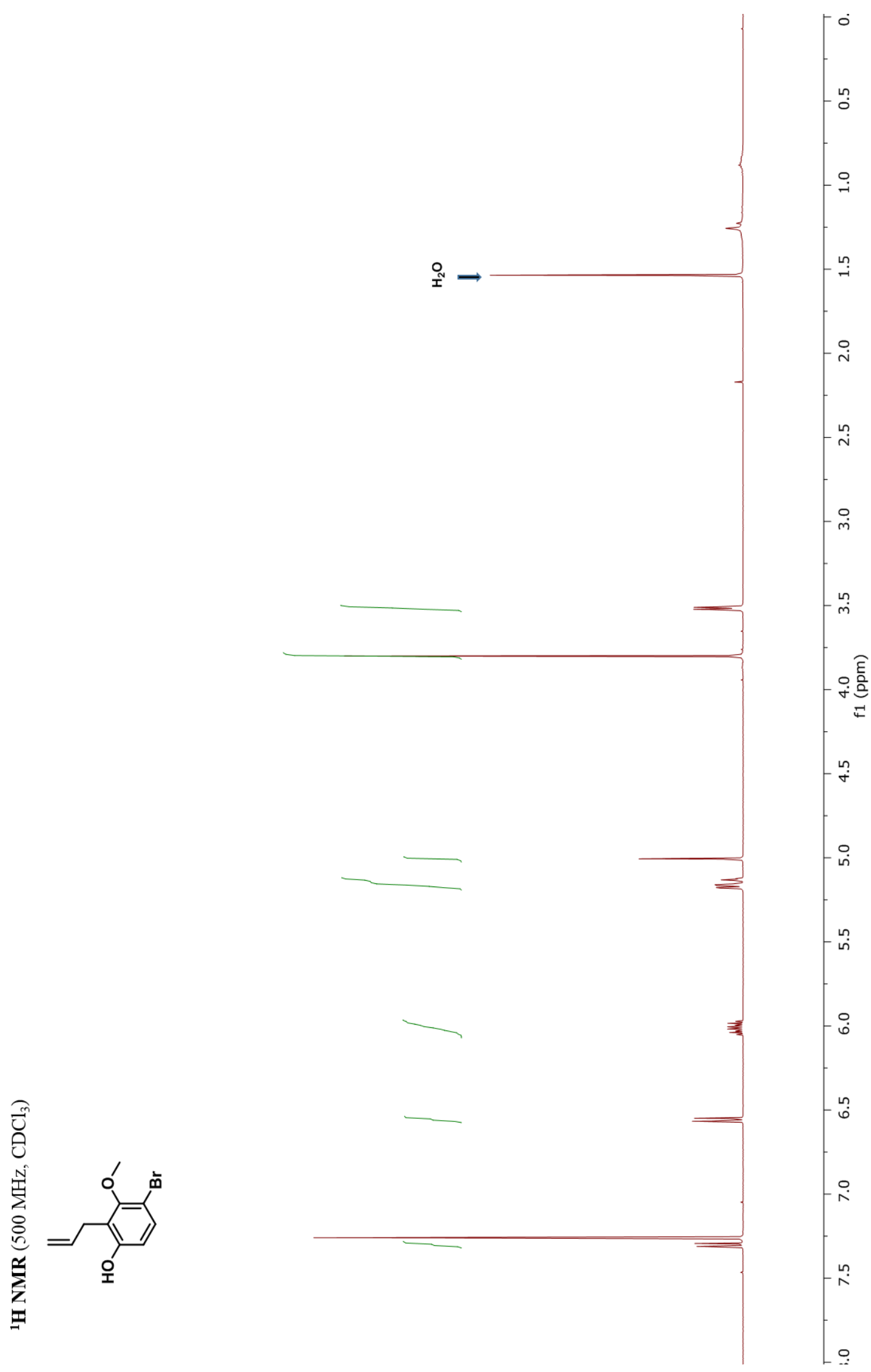


${ }^{13}$ C NMR Spectrum of Compound 3.57

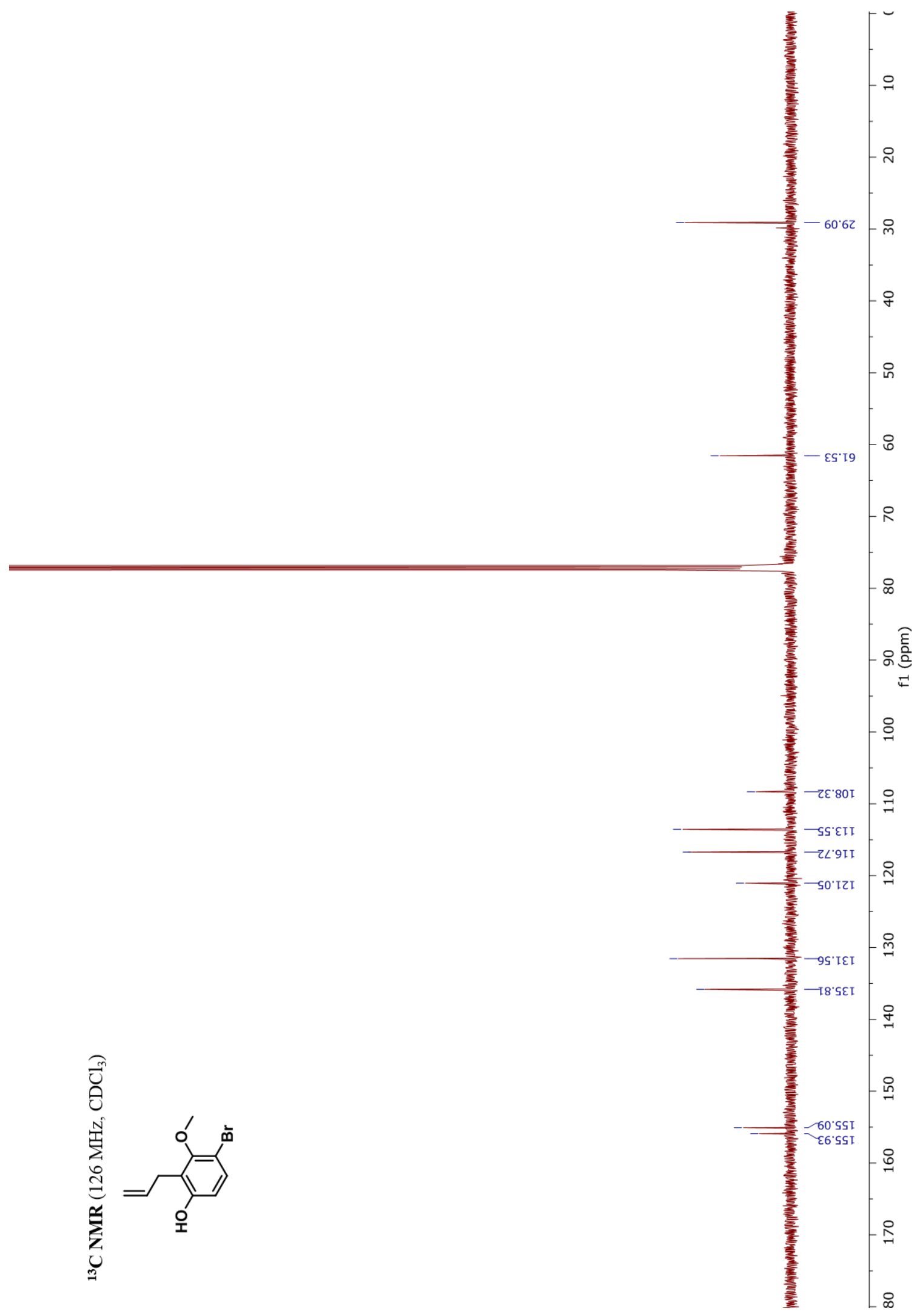


${ }^{1} \mathrm{H}$ NMR Spectrum of Compound 3.40

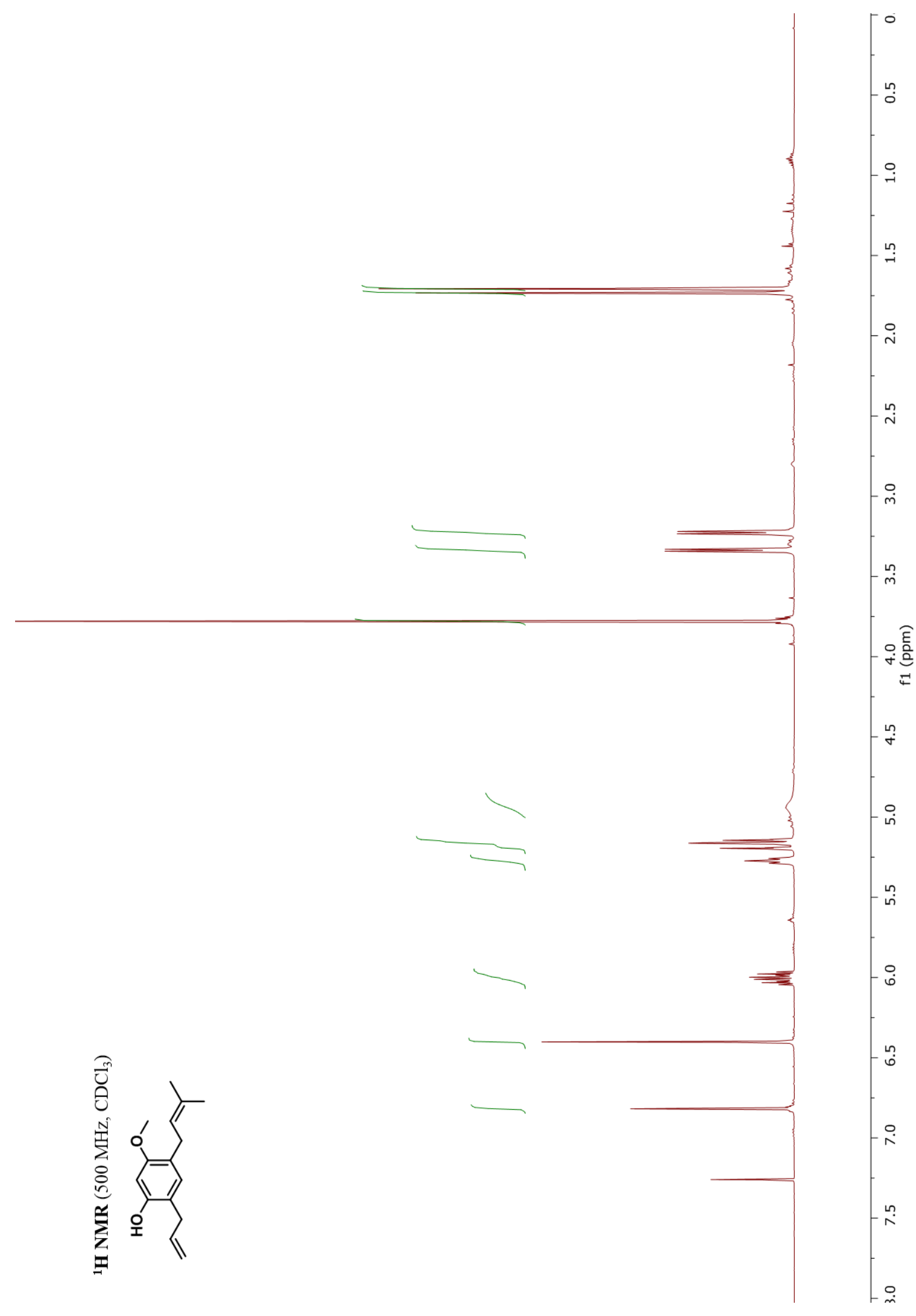


${ }^{13}$ C NMR Spectrum of Compound 3.40

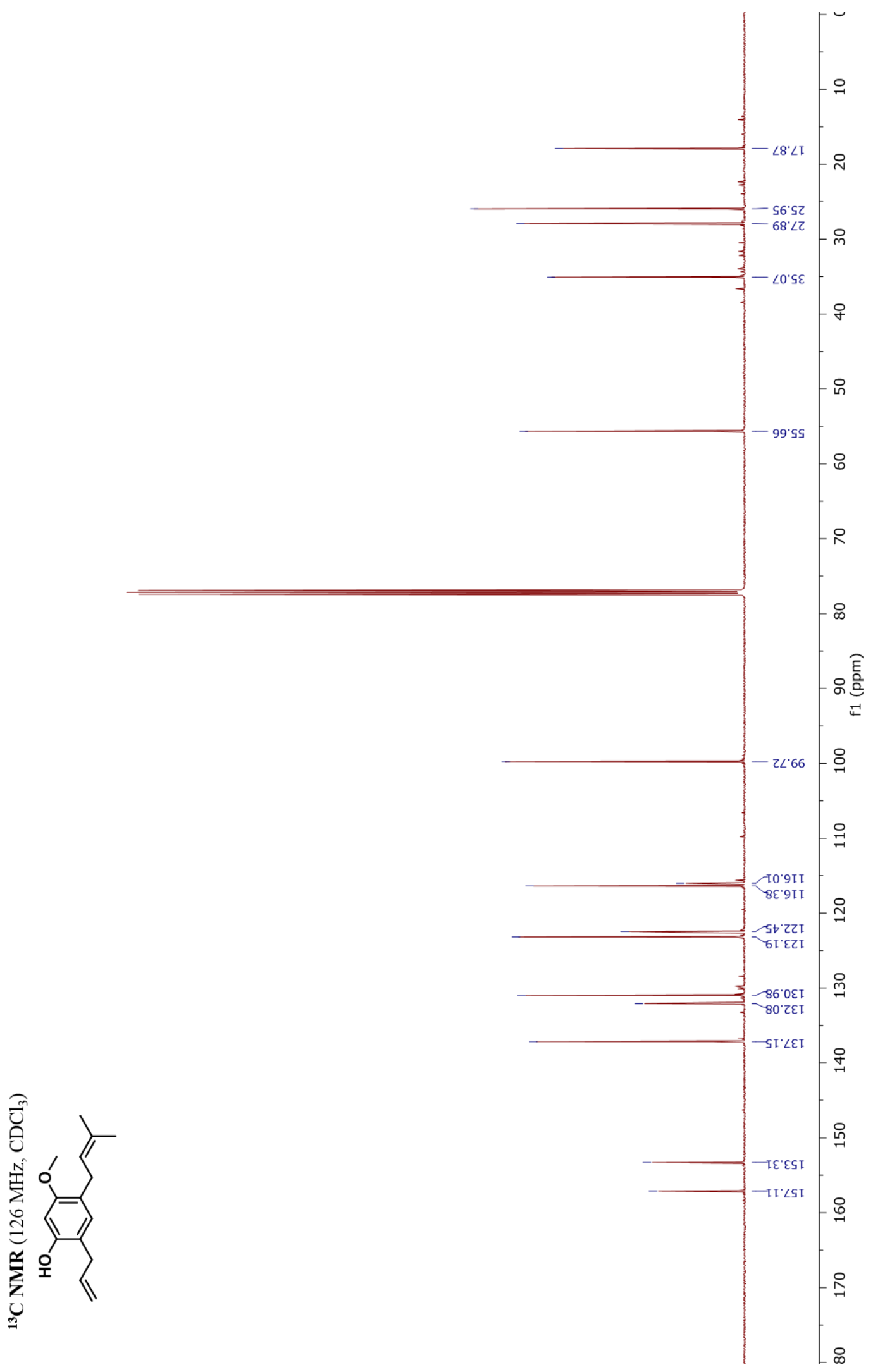


${ }^{1} \mathrm{H}$ NMR Spectrum of Compound 3.58

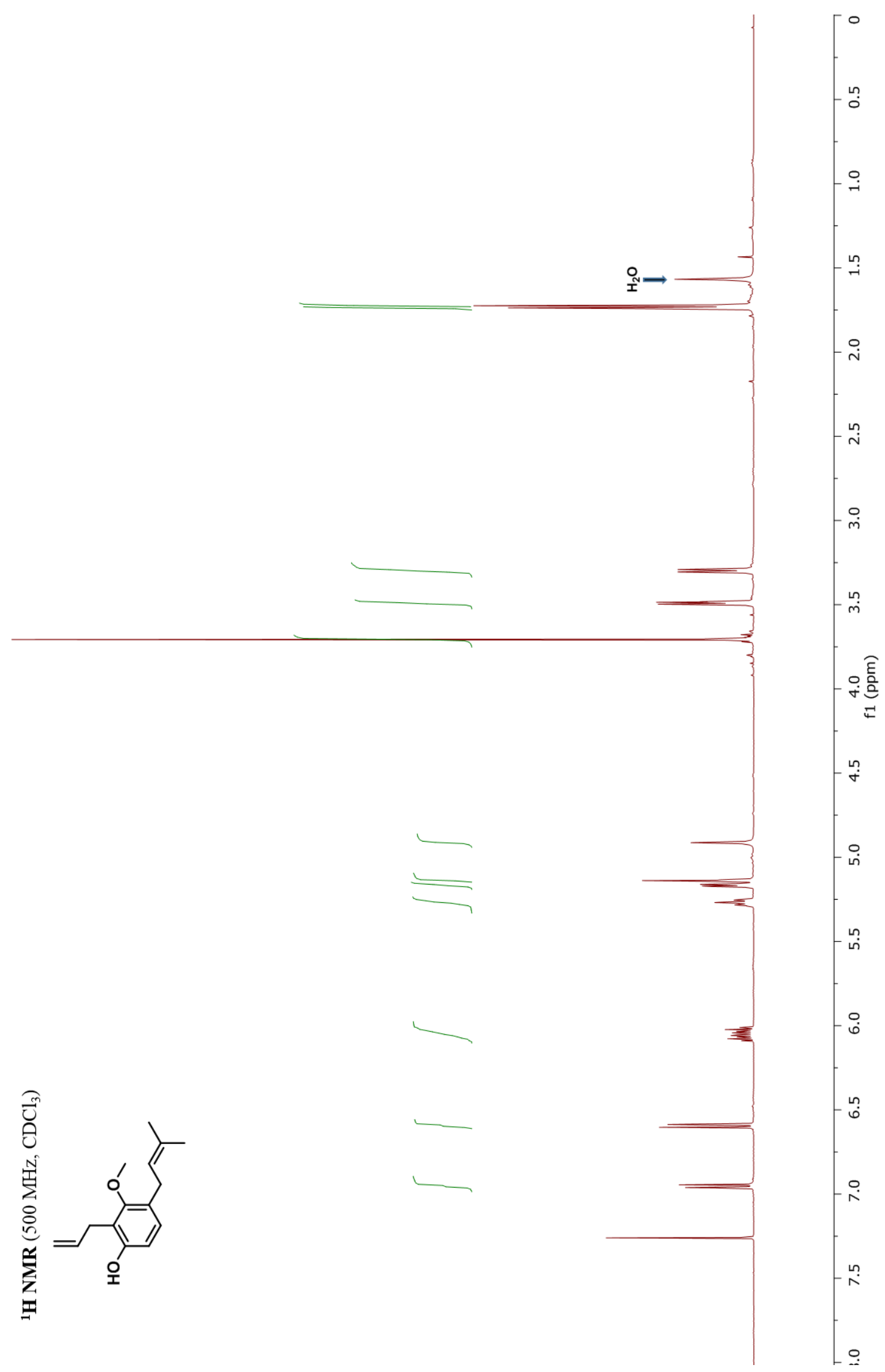


${ }^{13} \mathrm{C}$ NMR Spectrum of Compound 3.58

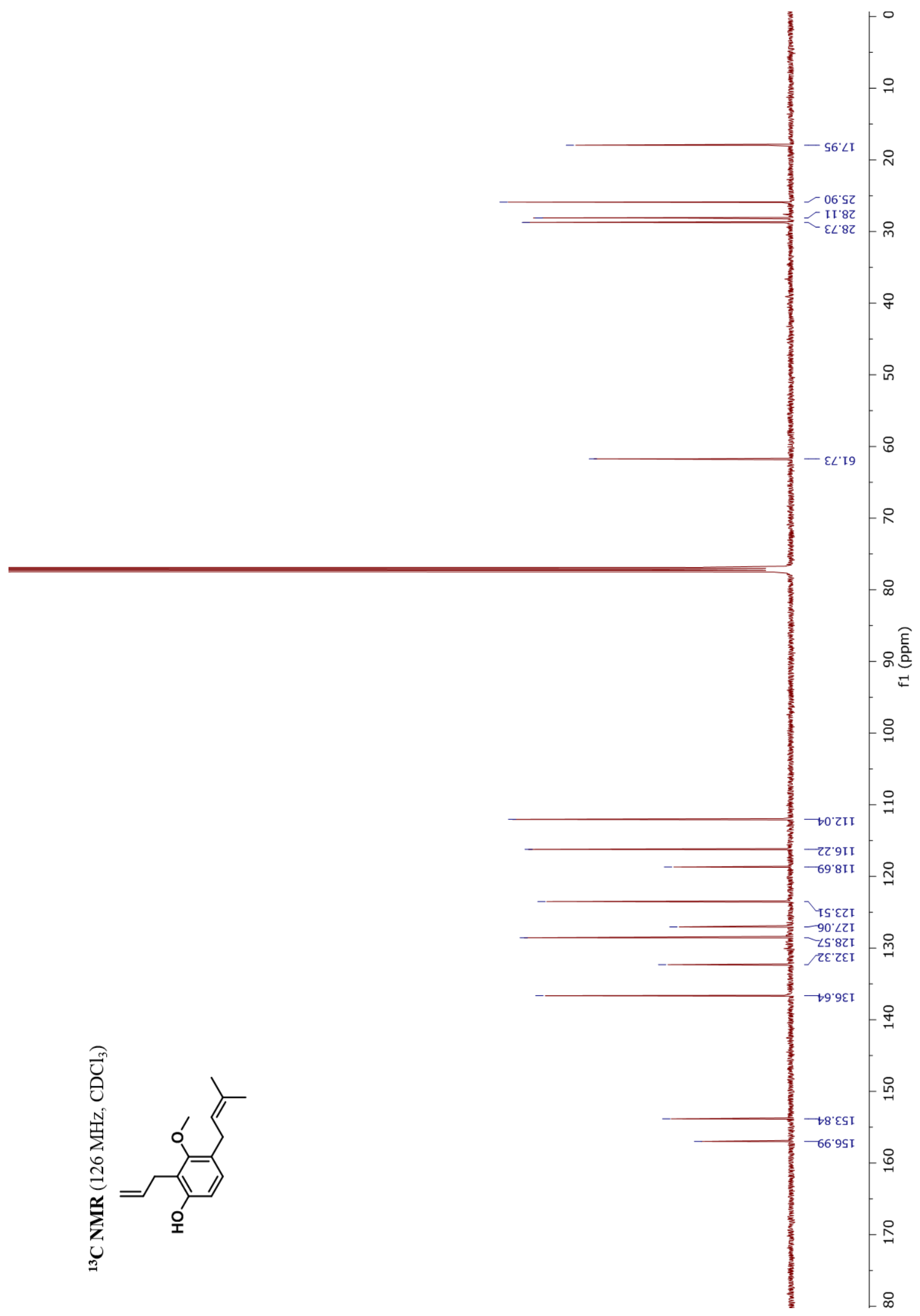


${ }^{1} \mathrm{H}$ NMR Spectrum of Compound 3.59

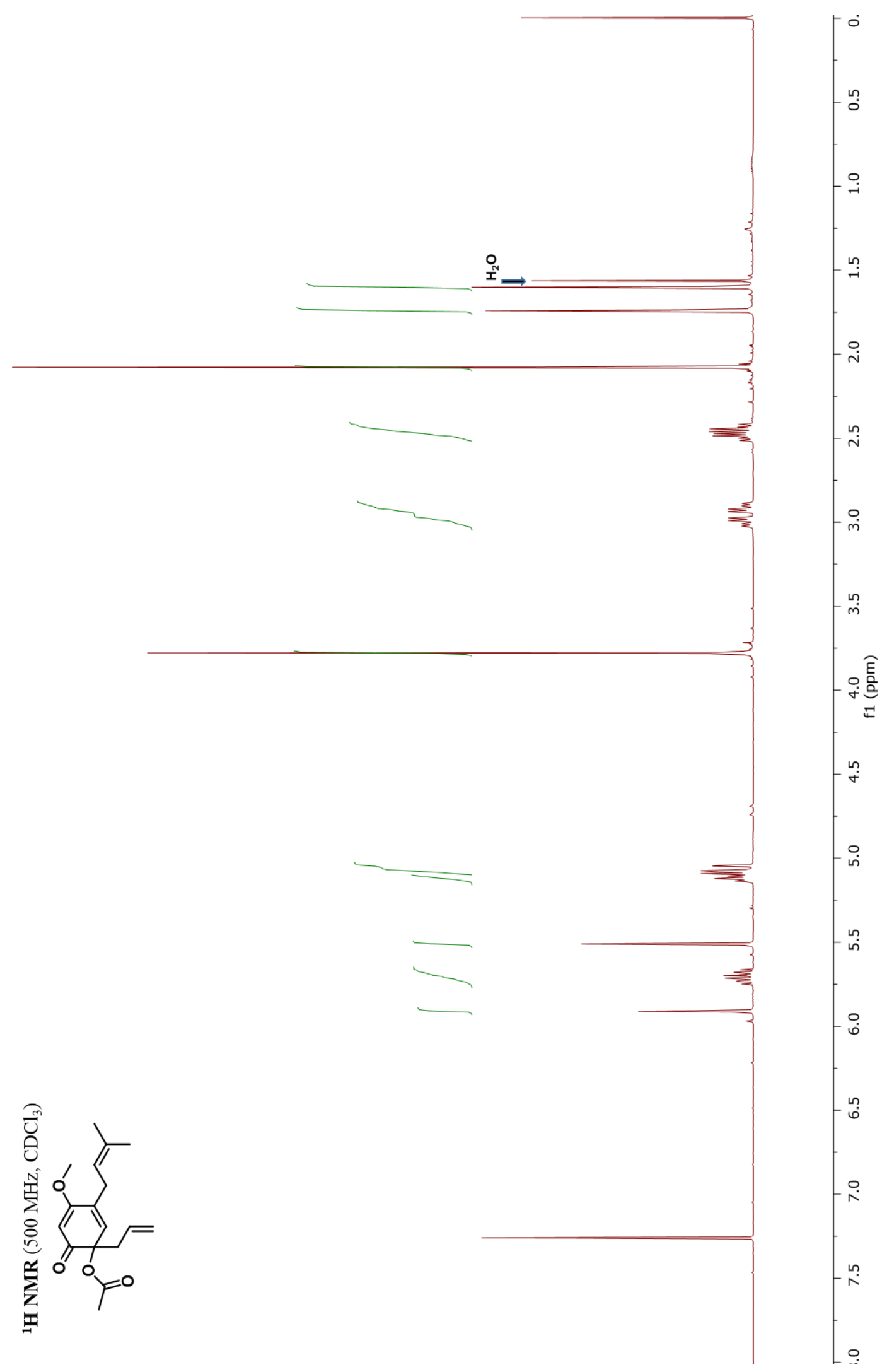


${ }^{13}$ C NMR Spectrum of Compound 3.59

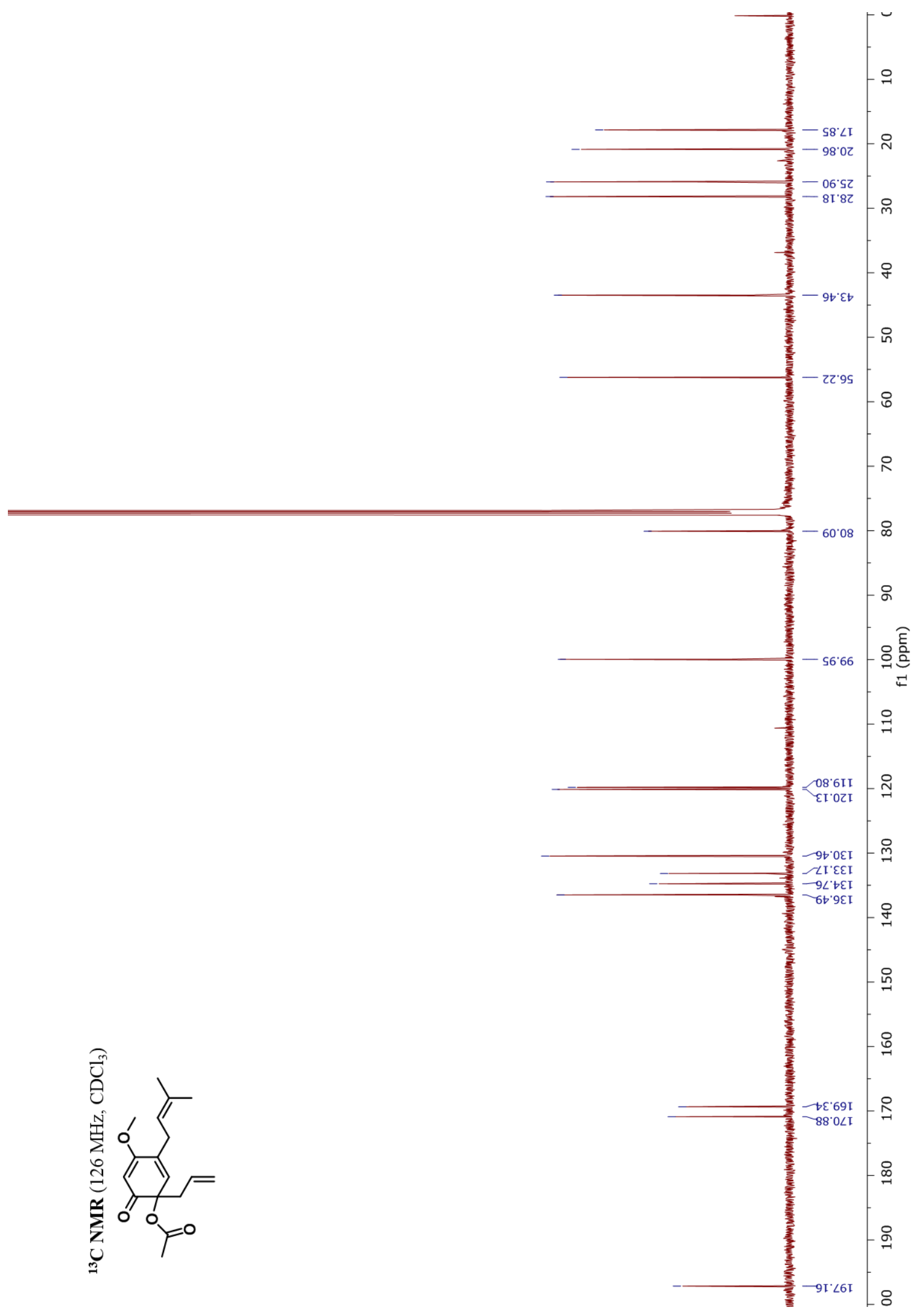


${ }^{1}$ H NMR Spectrum of Compound 3.61

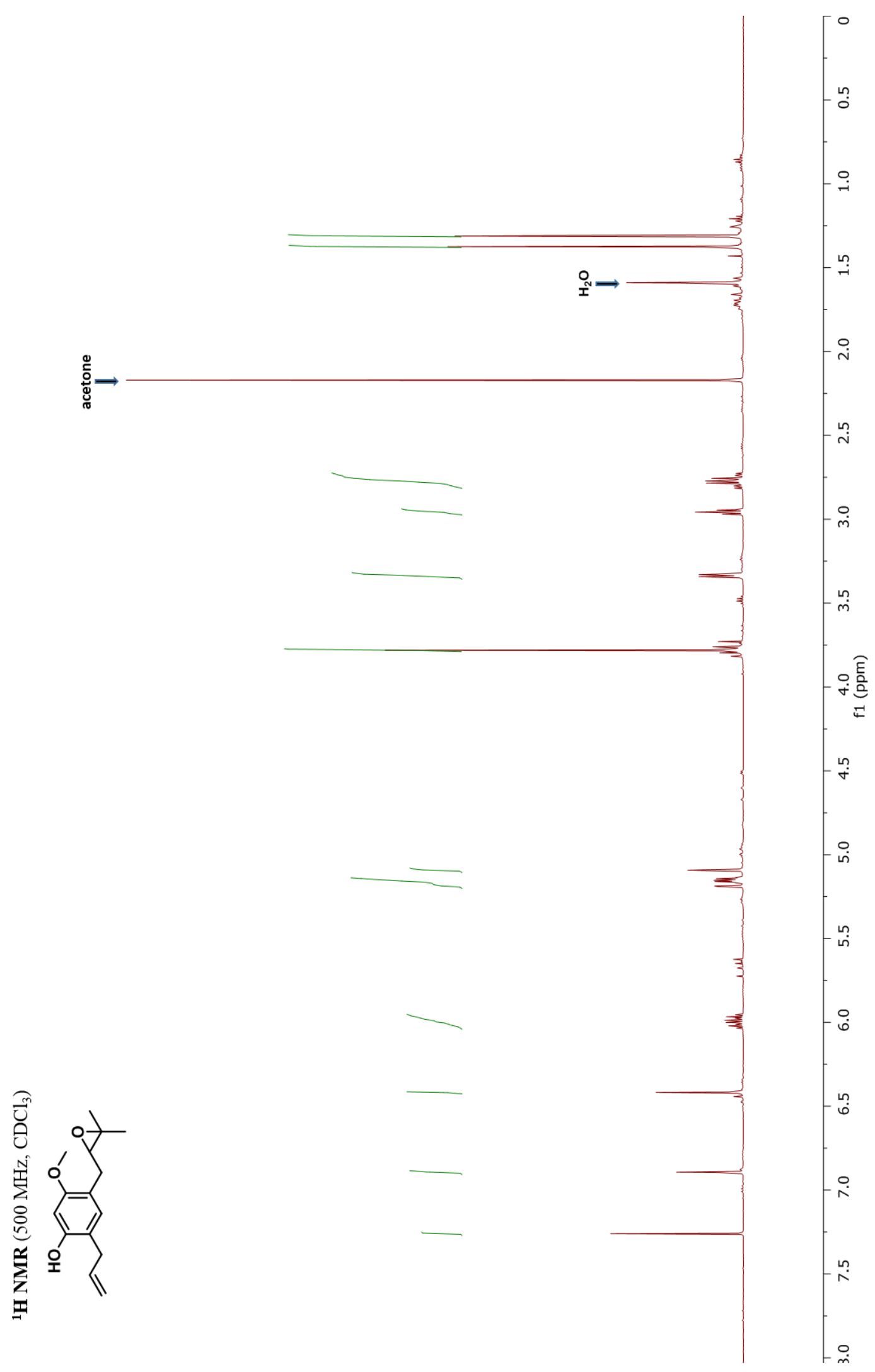


${ }^{13}$ C NMR Spectrum of Compound 3.61

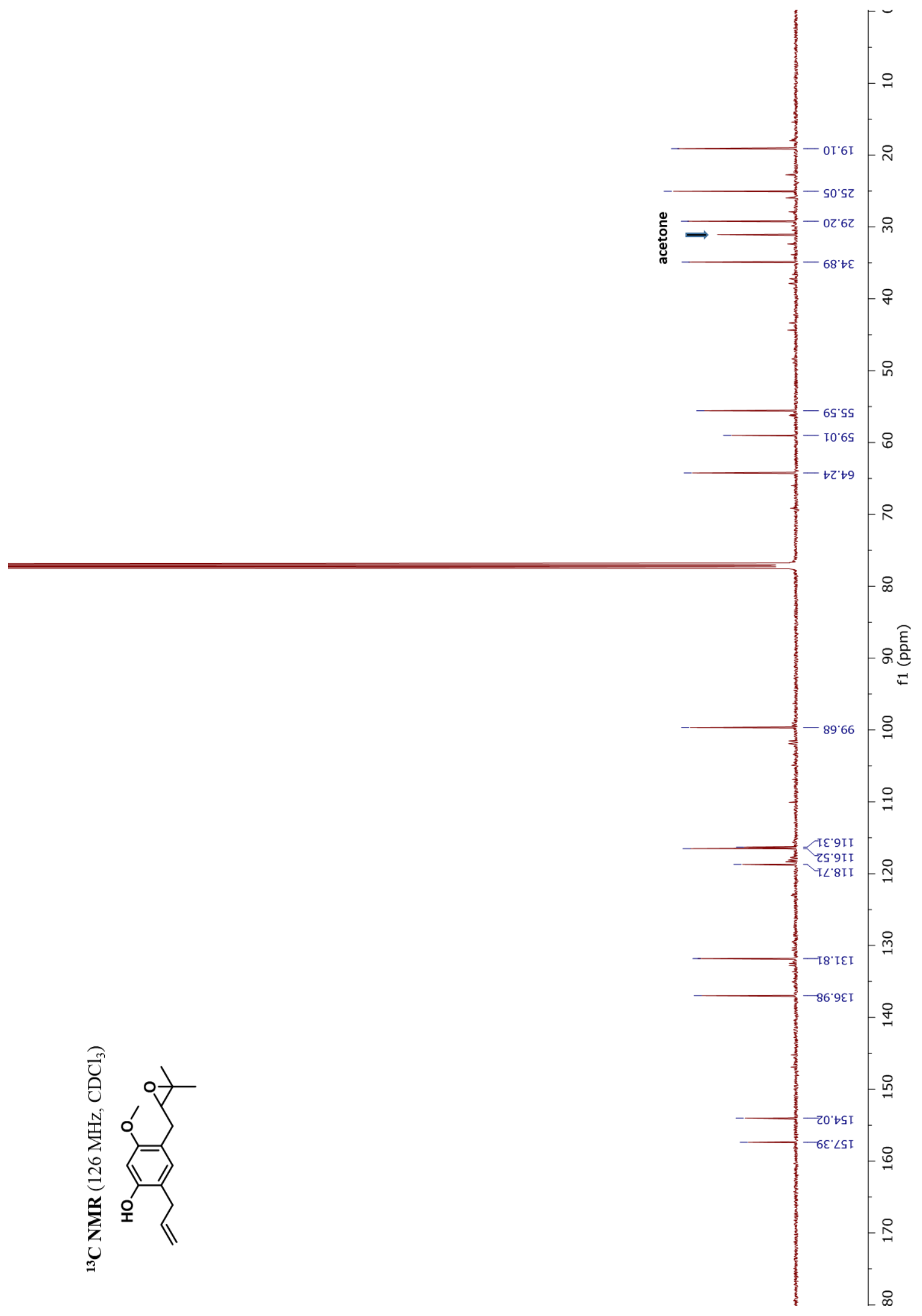


${ }^{1} \mathrm{H}$ NMR Spectrum of Compound ( \pm )-3.35

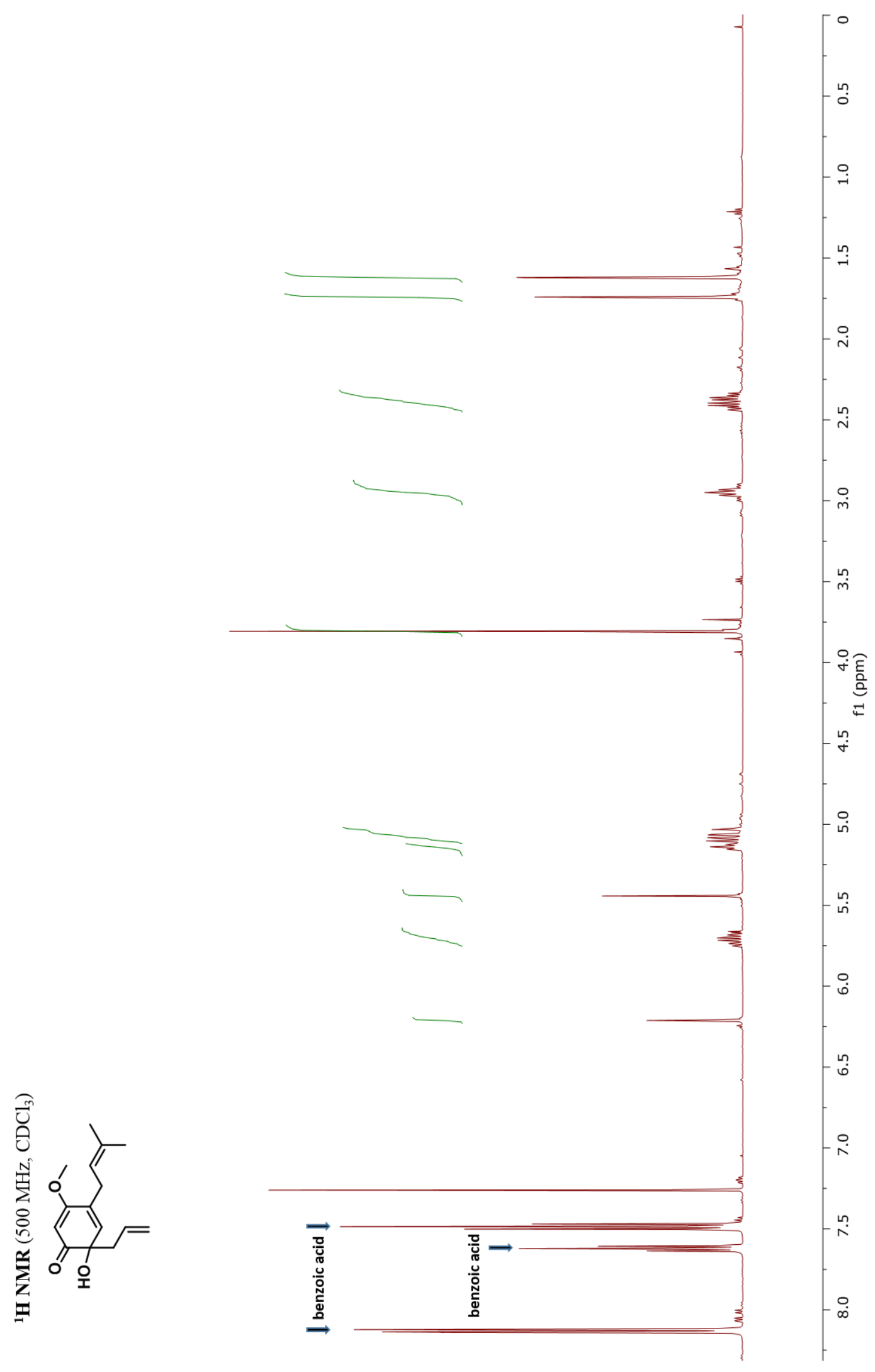


${ }^{13}$ C NMR Spectrum of Compound ( $( \pm)-3.35$

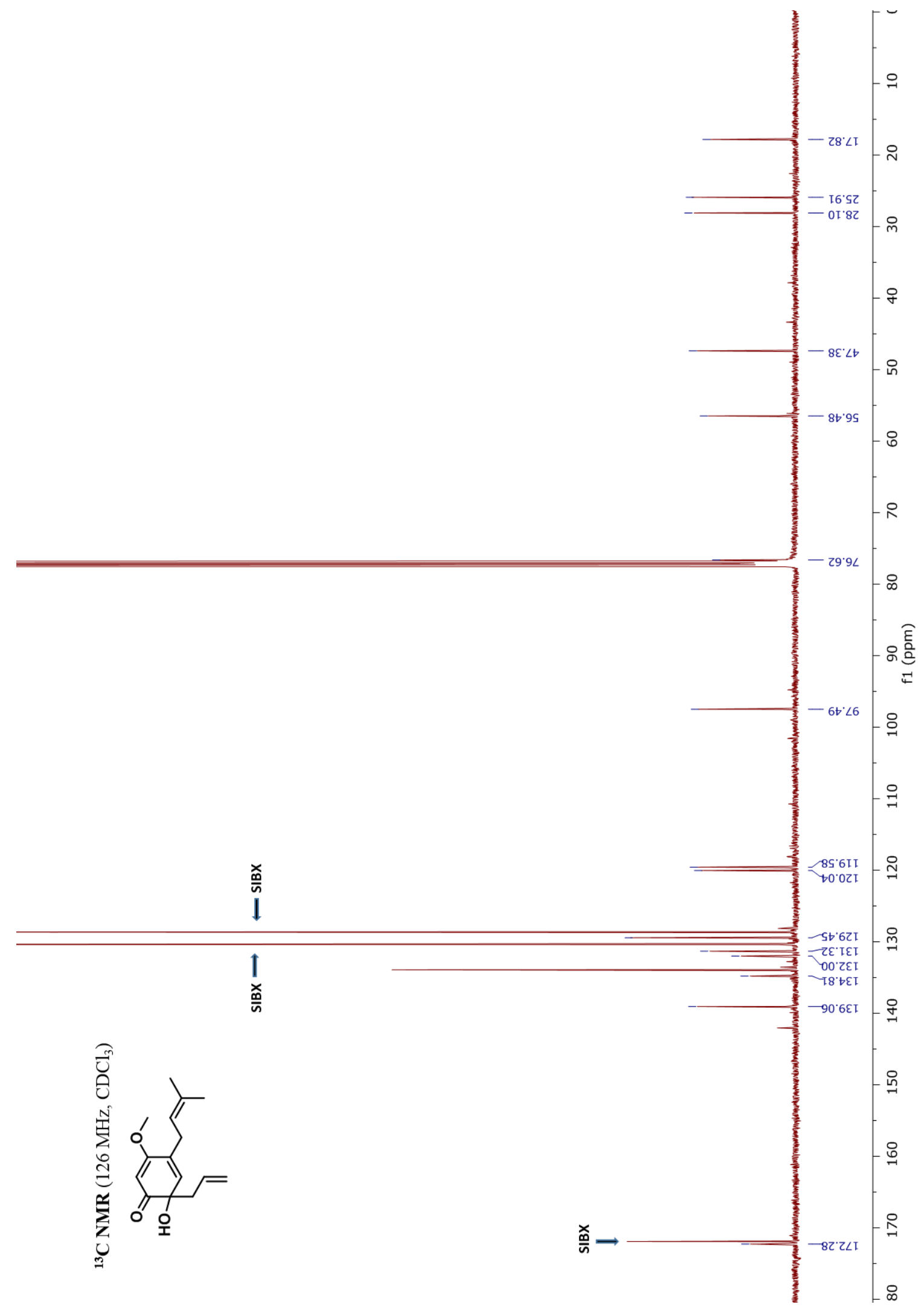


${ }^{1} \mathrm{H}$ NMR Spectrum of Compound ( \pm )-3.38

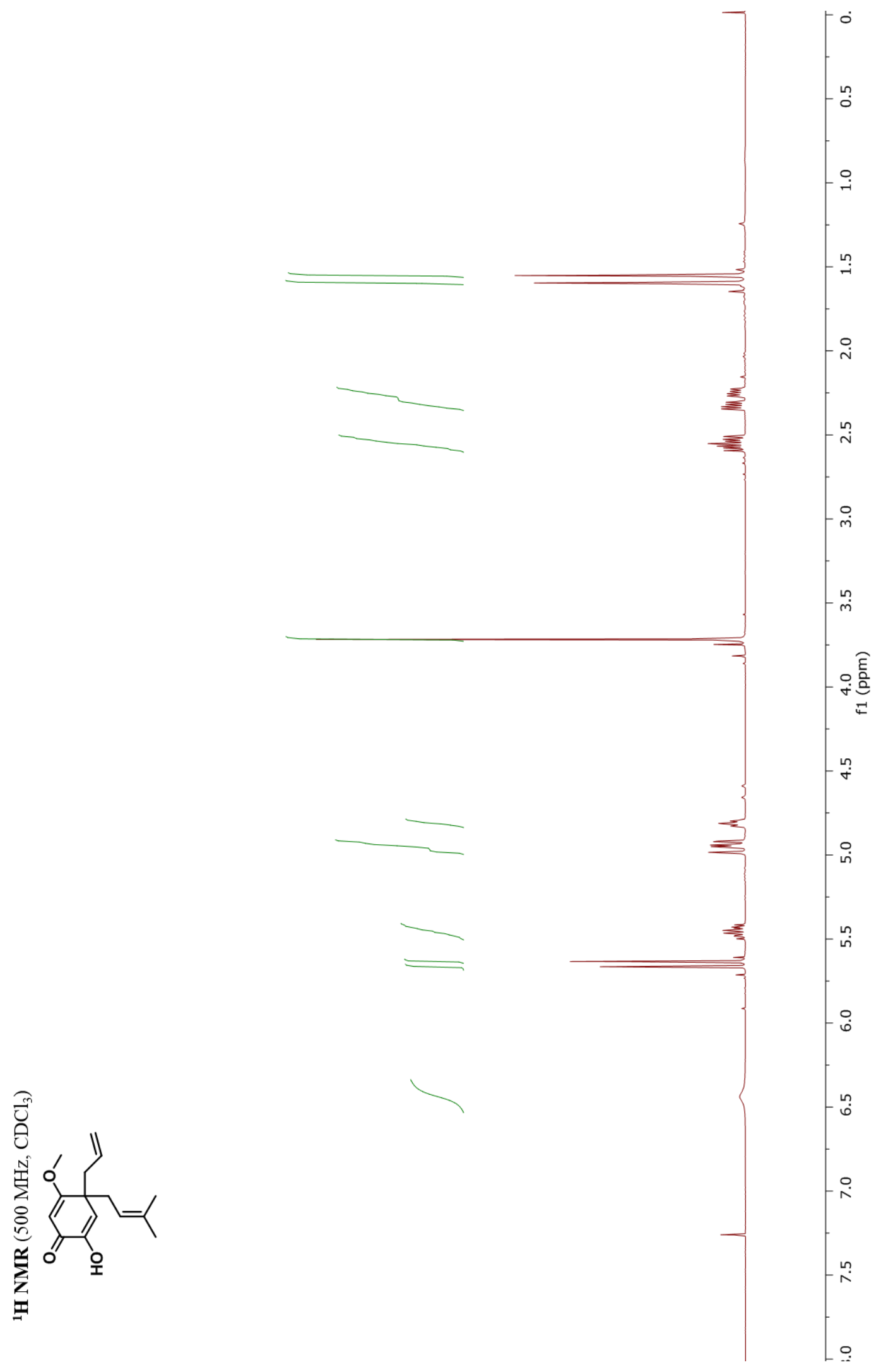


${ }^{13}$ C NMR Spectrum of Compound ( $( \pm)-3.38$

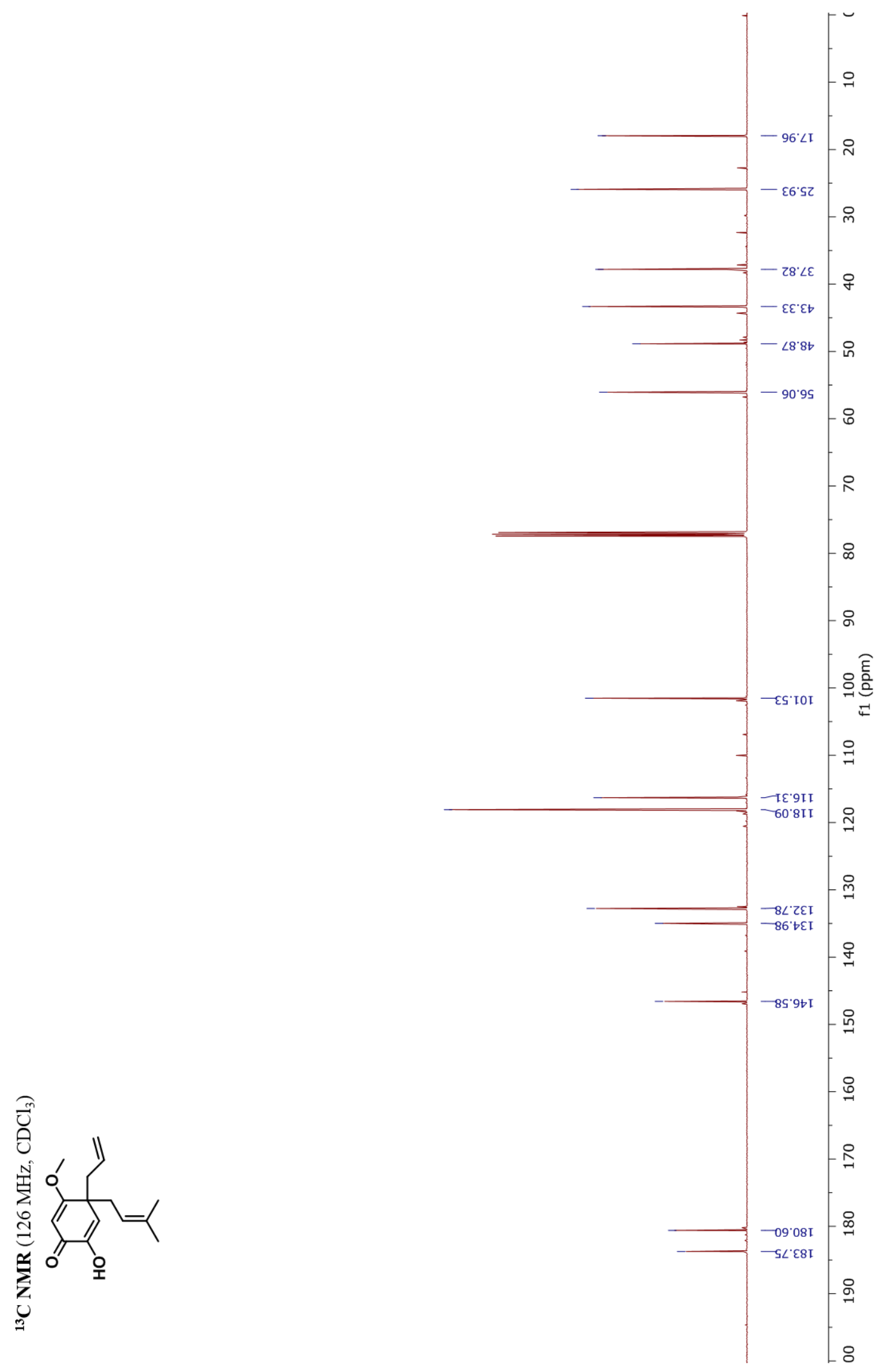


${ }^{1} \mathrm{H}$ NMR Spectrum of Compound 3.62

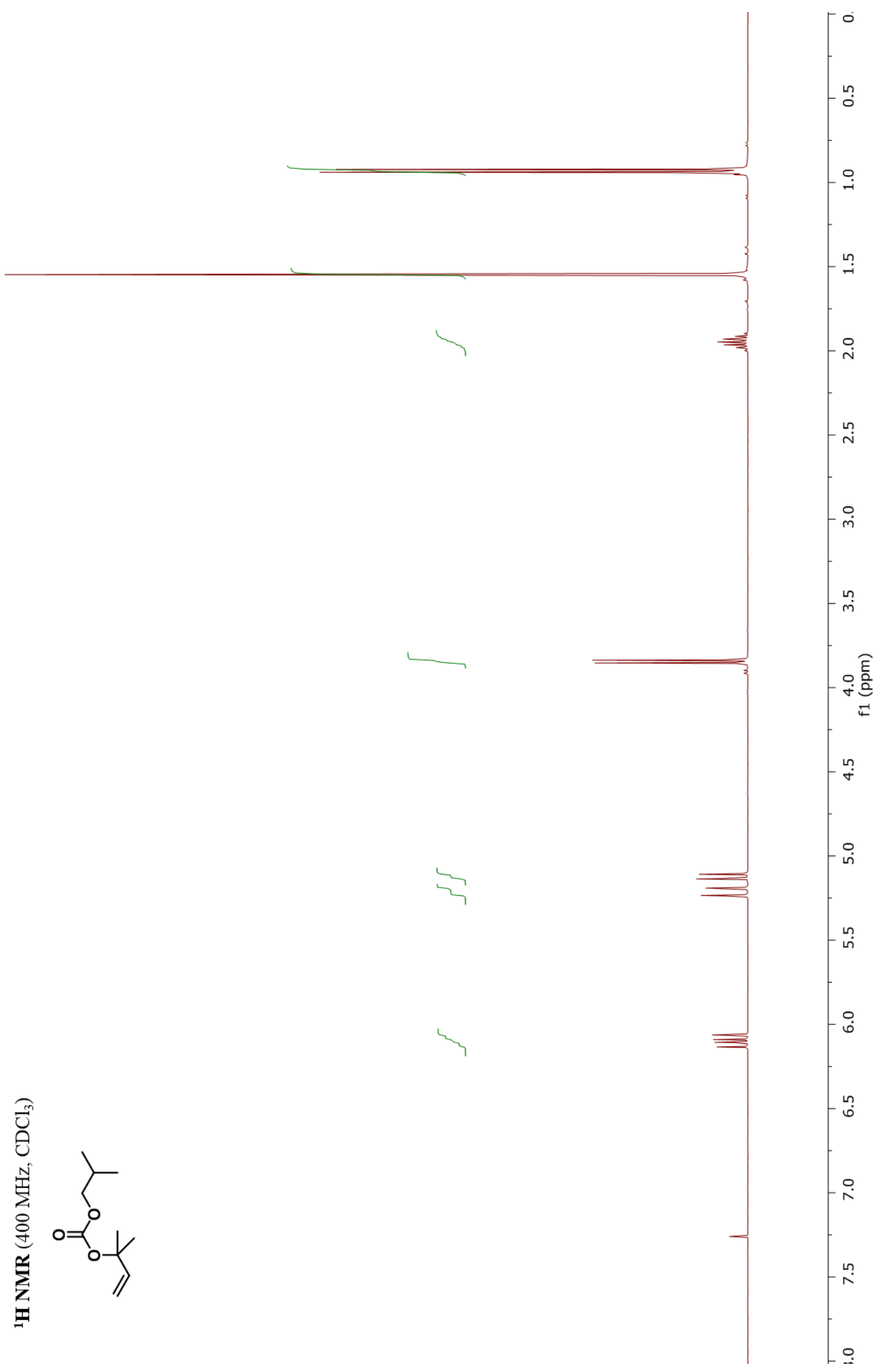


${ }^{13}$ C NMR Spectrum of Compound 3.62

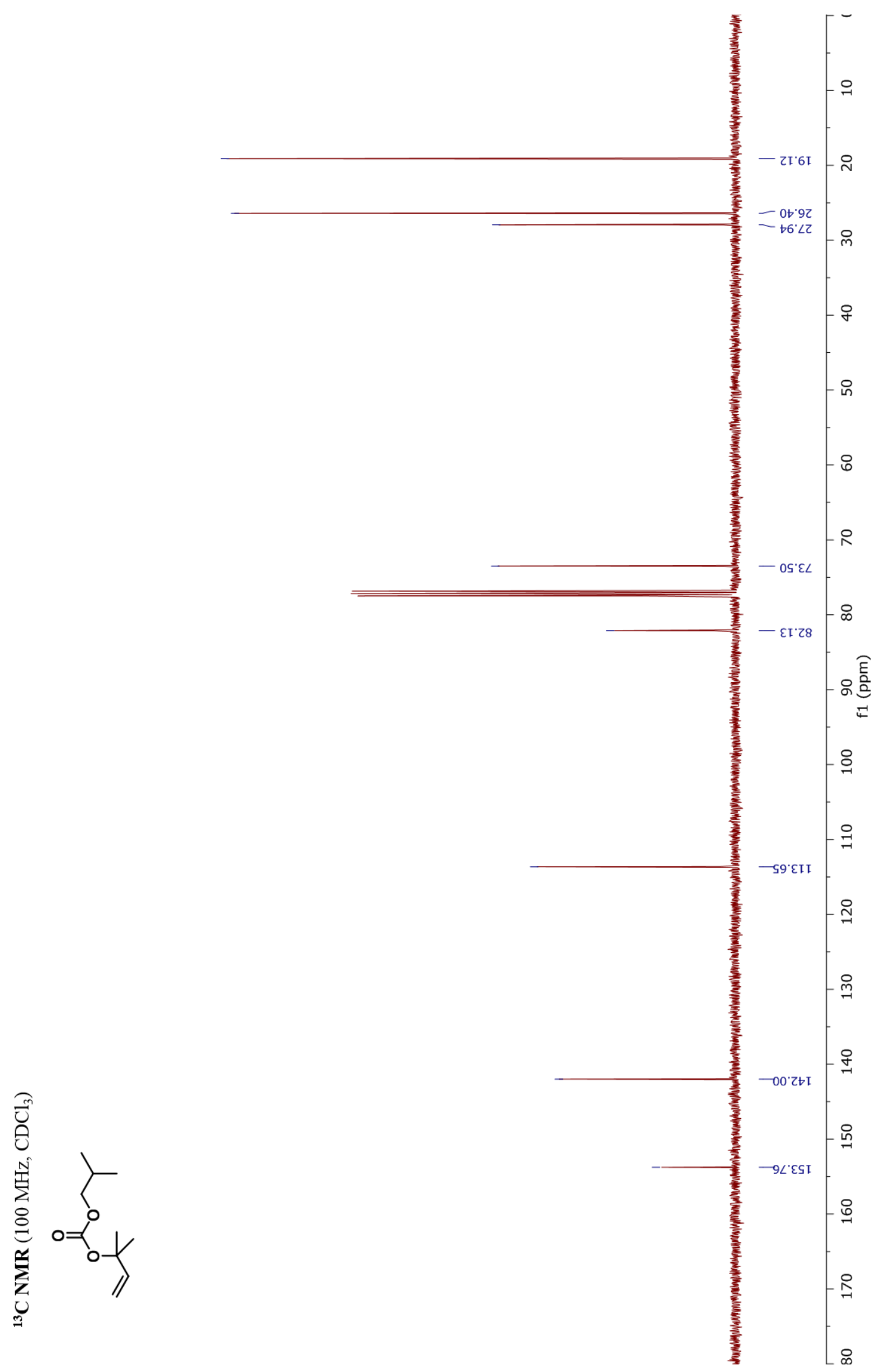


${ }^{1} \mathrm{H}$ NMR Spectrum of Compound 3.65

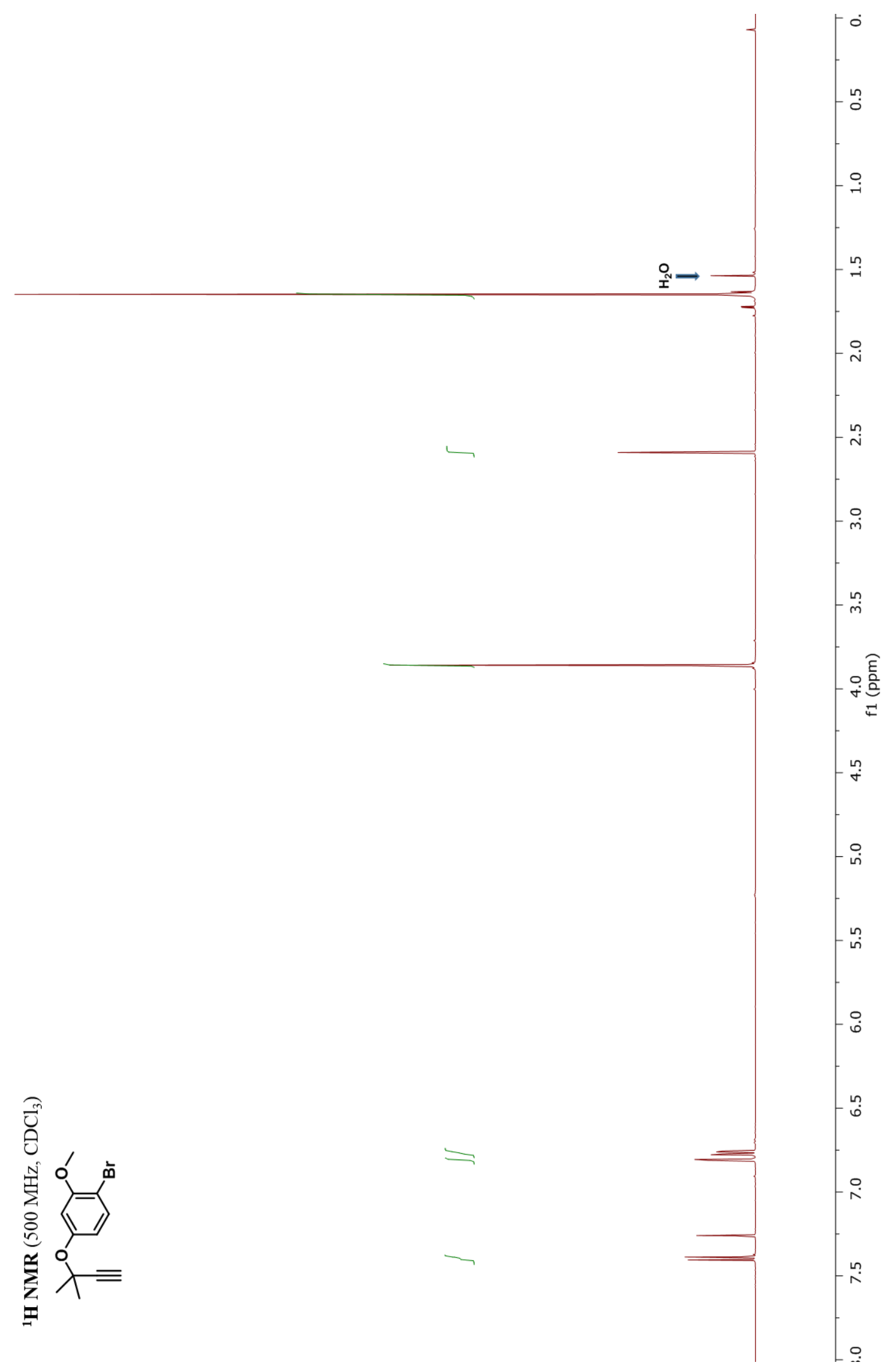


${ }^{13}$ C NMR Spectrum of Compound 3.65

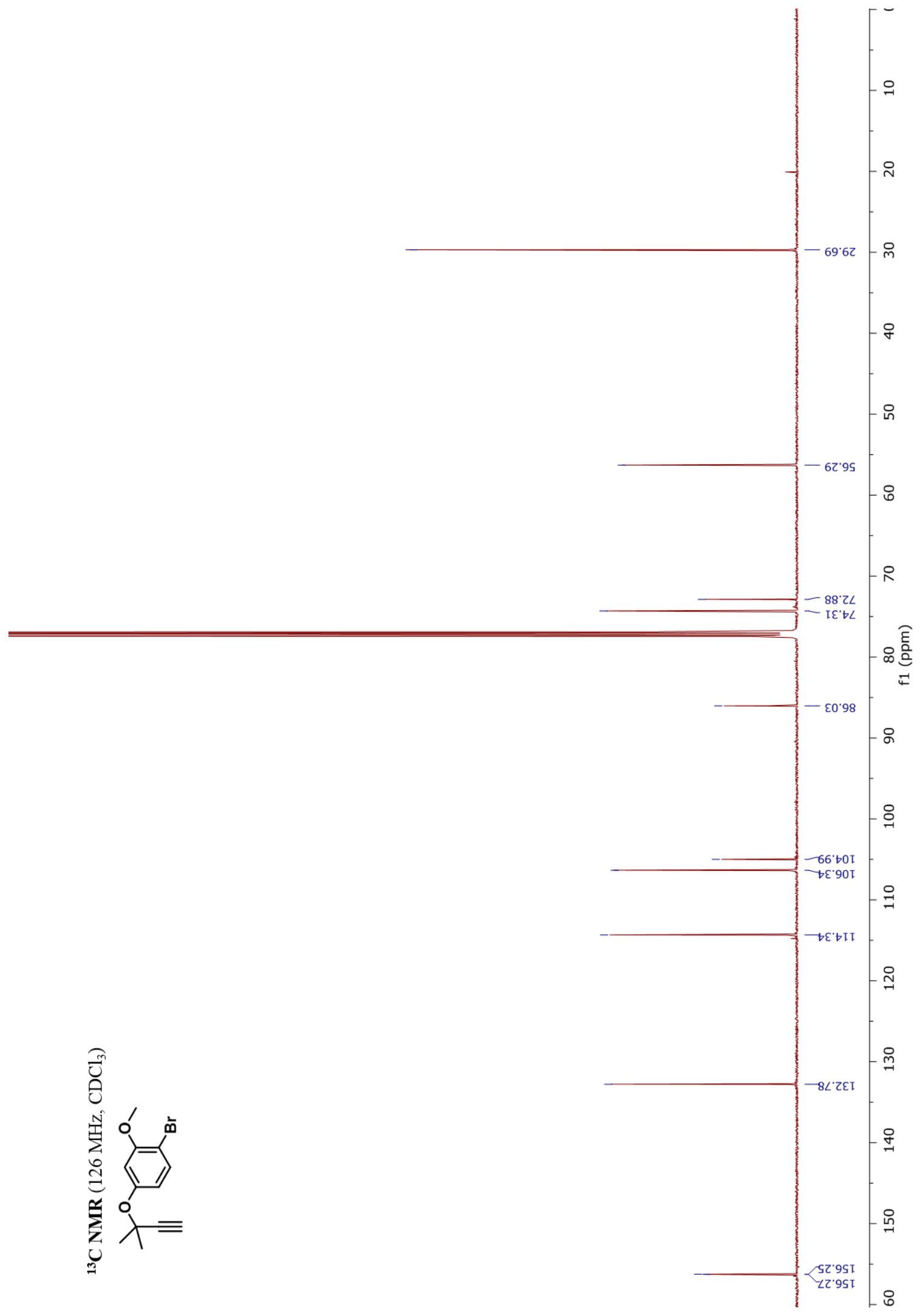


${ }^{1} \mathrm{H}$ NMR Spectrum of Compound 3.66

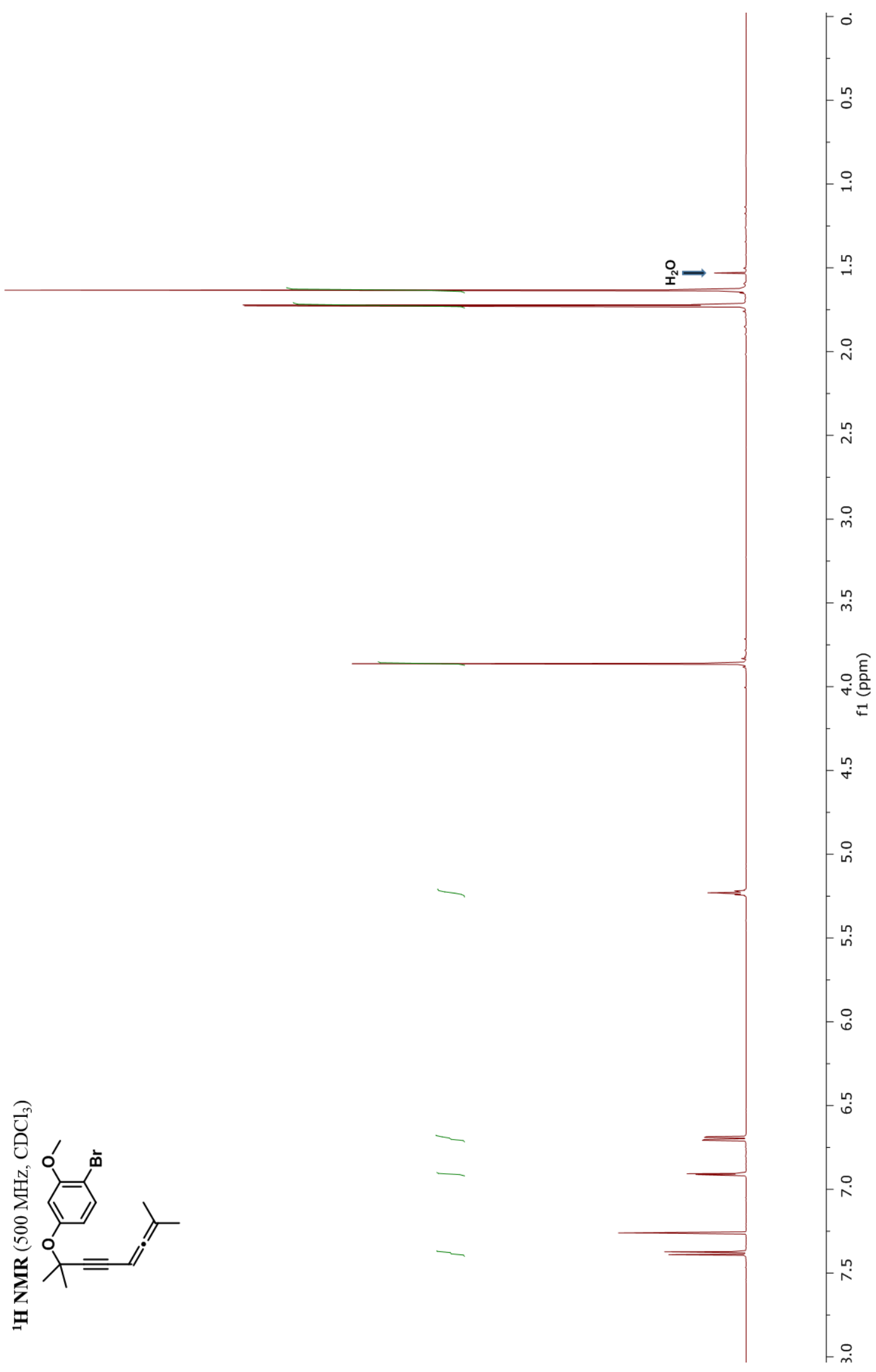


${ }^{13}$ C NMR Spectrum of Compound 3.66

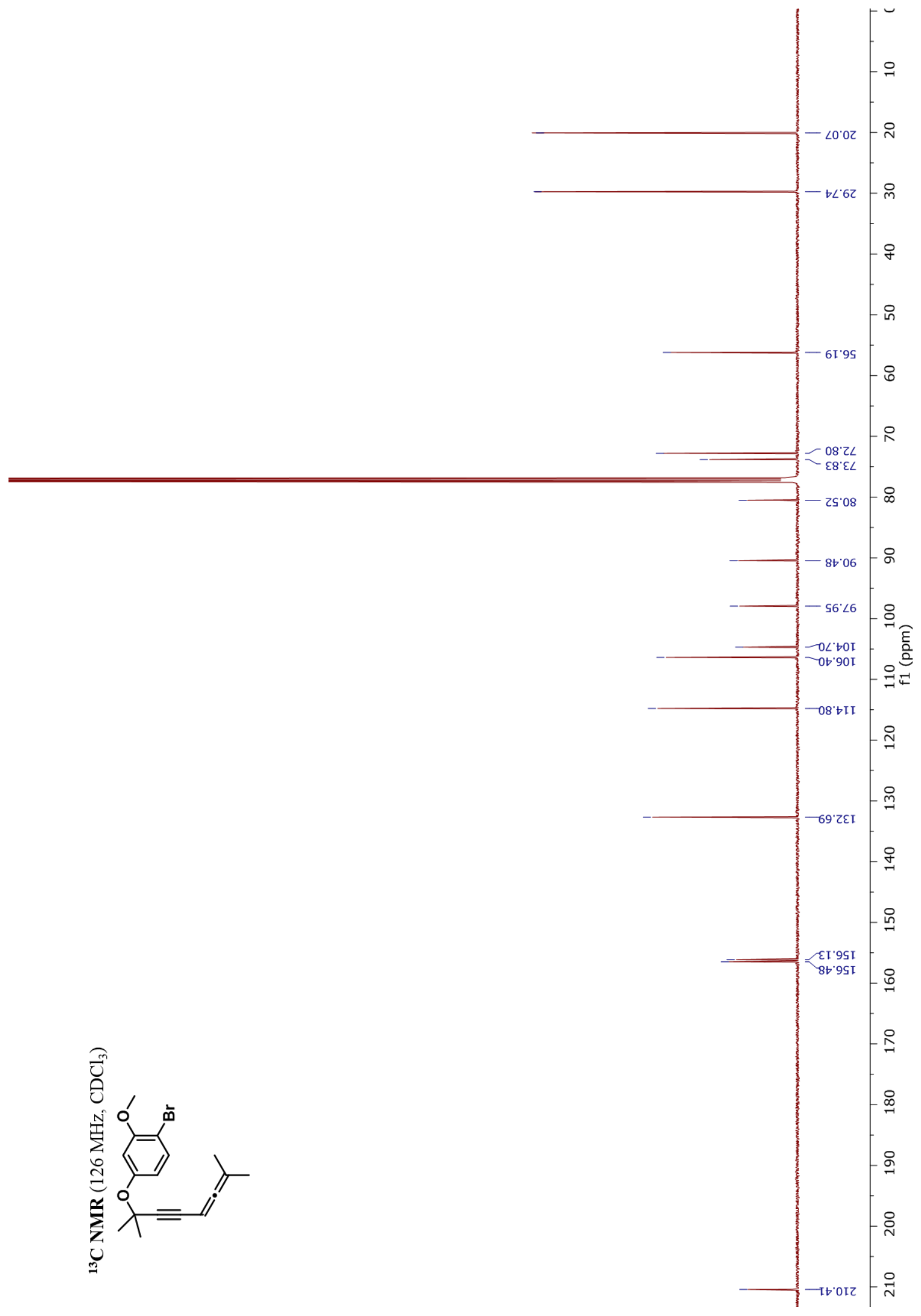


${ }^{1} \mathrm{H}$ NMR Spectrum of Compound 3.43

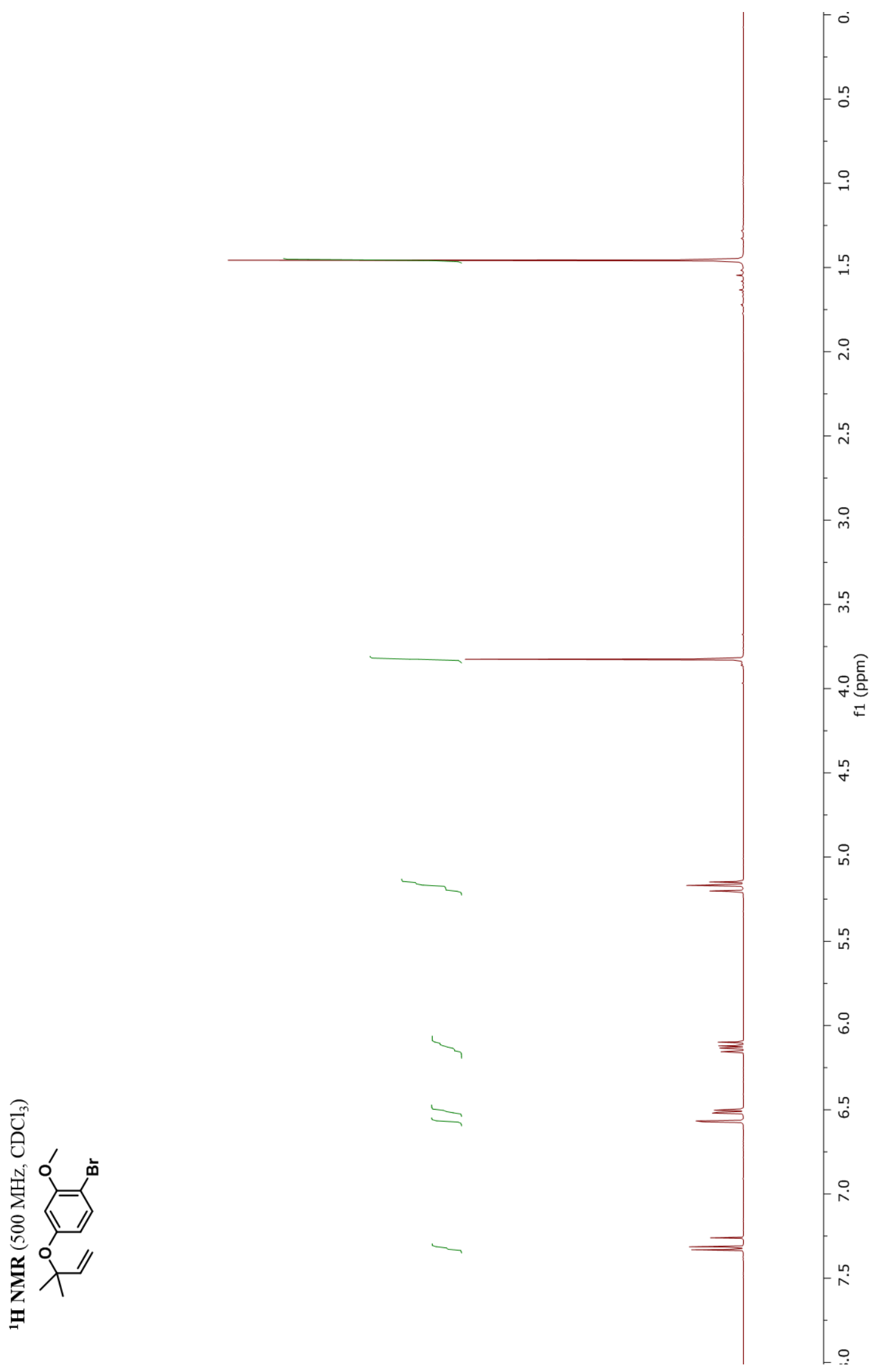


${ }^{13}$ C NMR Spectrum of Compound 3.43

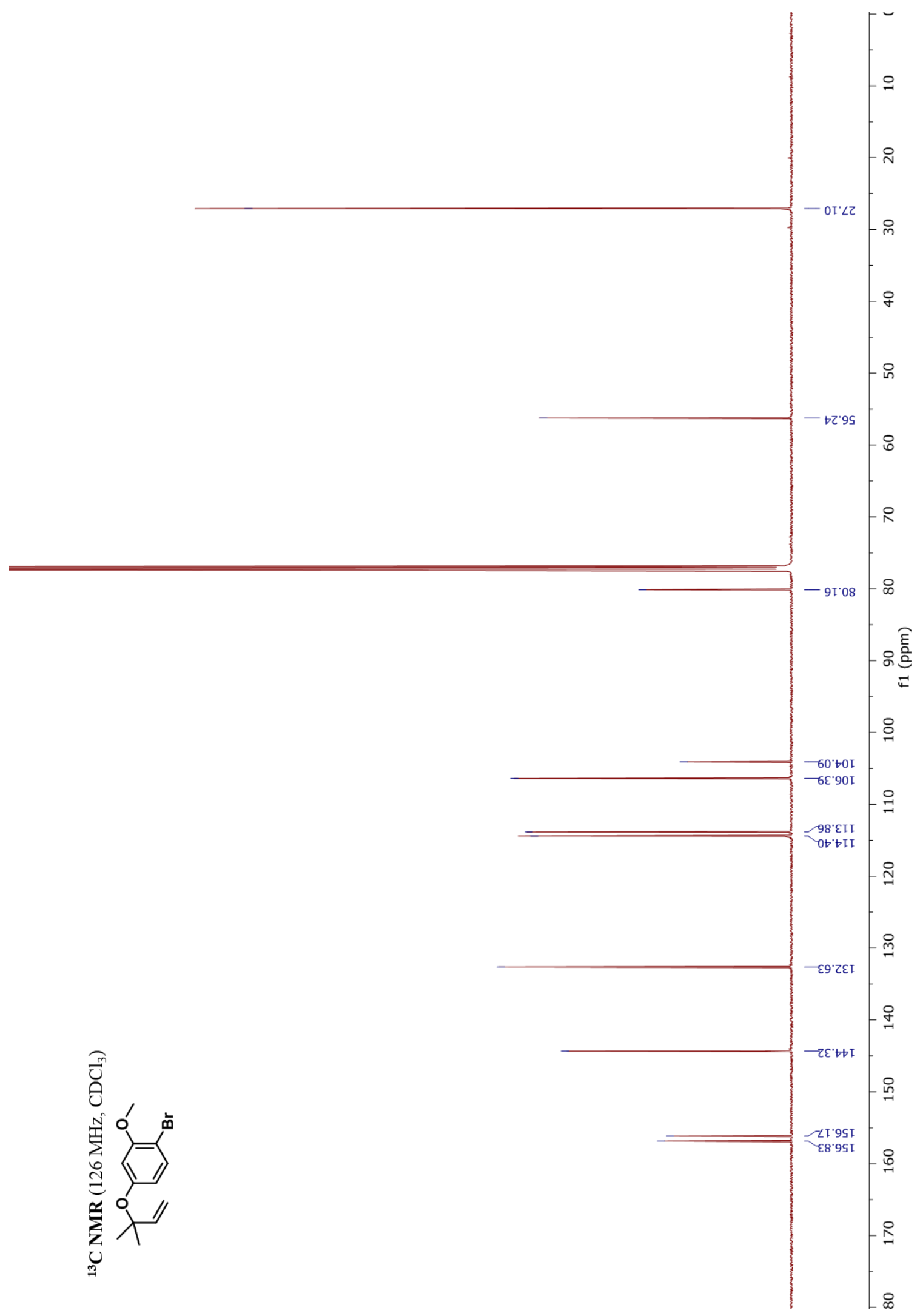


${ }^{1} \mathrm{H}$ NMR Spectrum of Compound 3.68

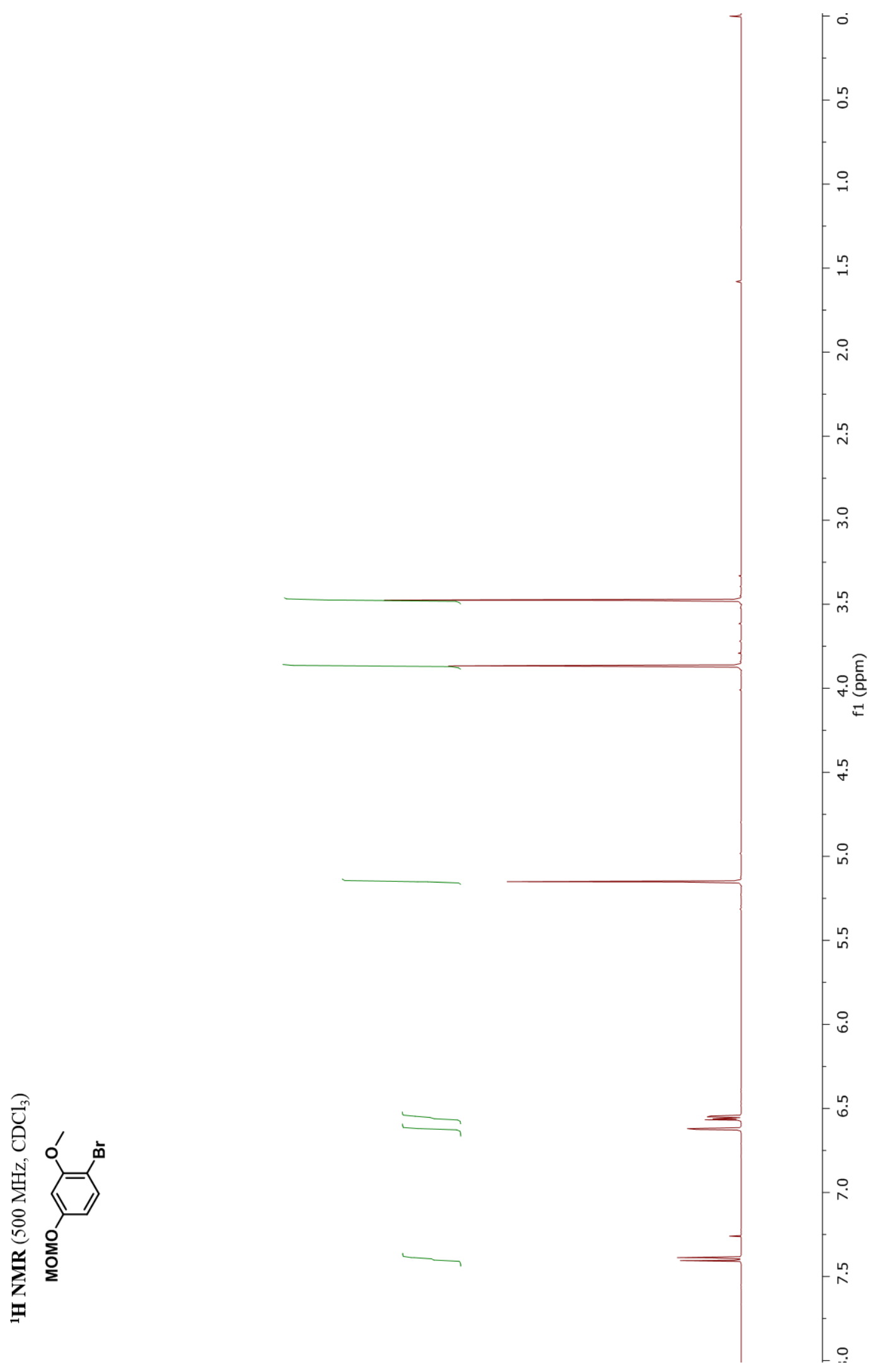


${ }^{13}$ C NMR Spectrum of Compound 3.68

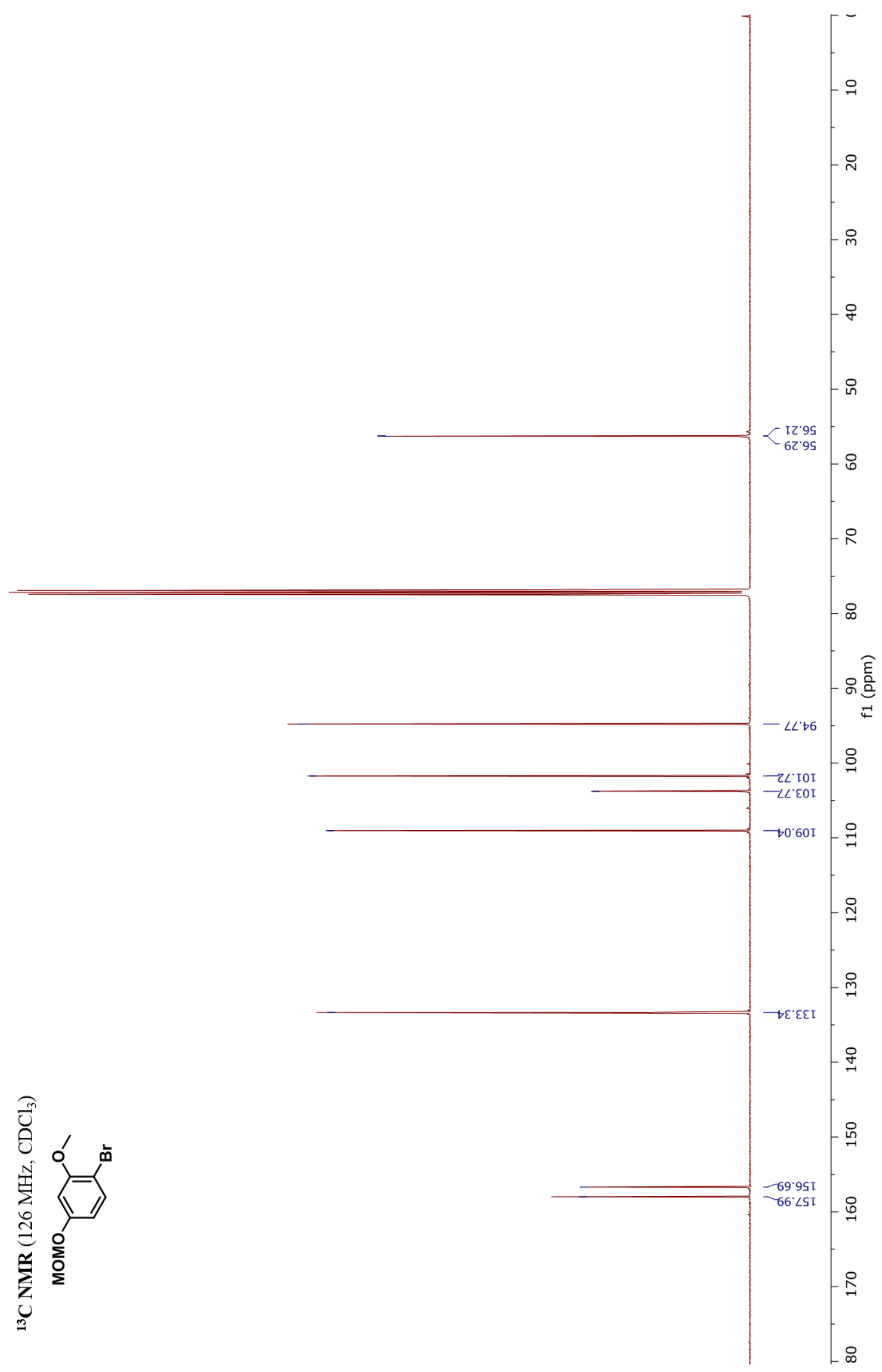


${ }^{1} \mathrm{H}$ NMR Spectrum of Compound 3.69

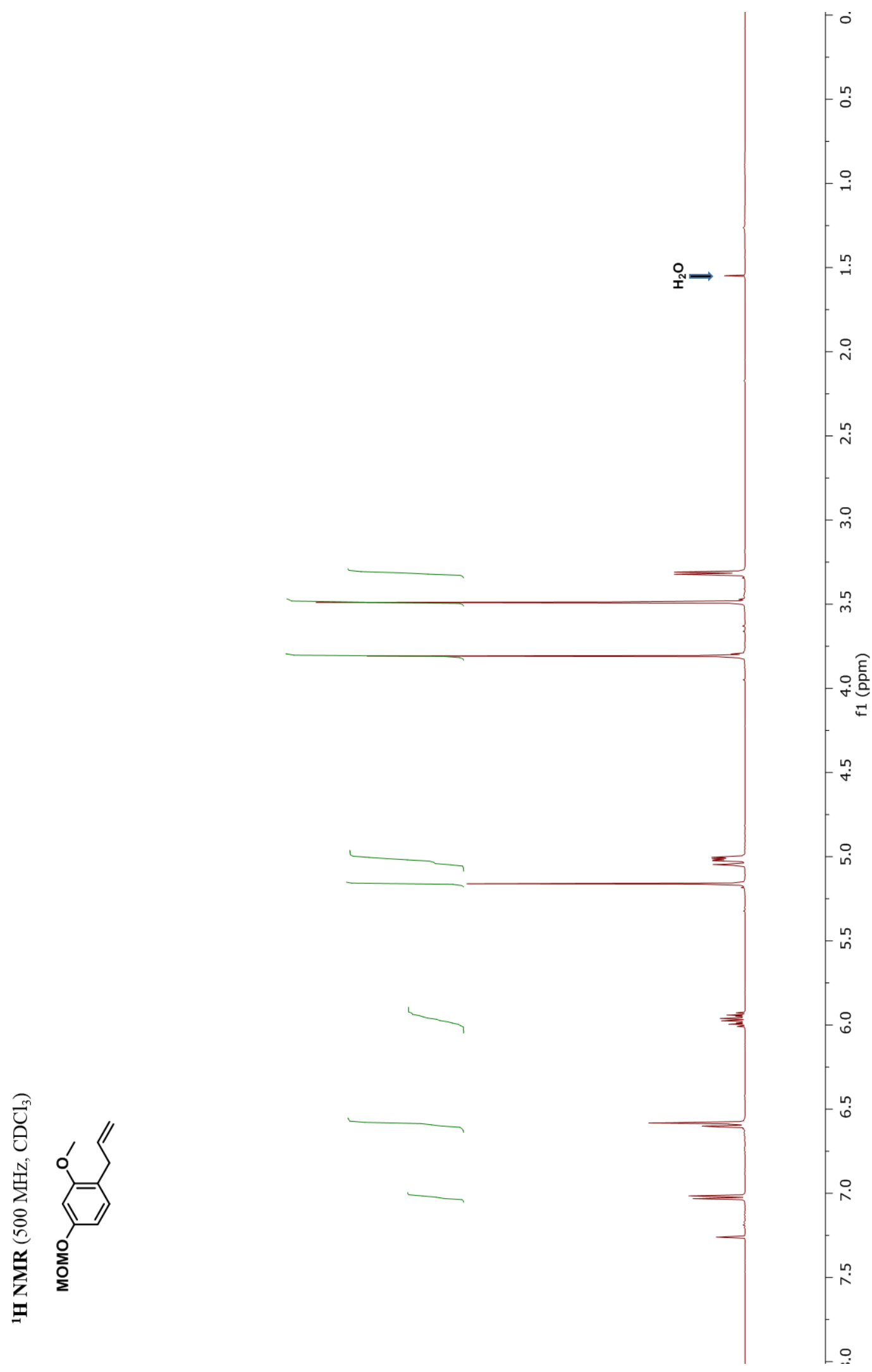


${ }^{13}$ C NMR Spectrum of Compound 3.69

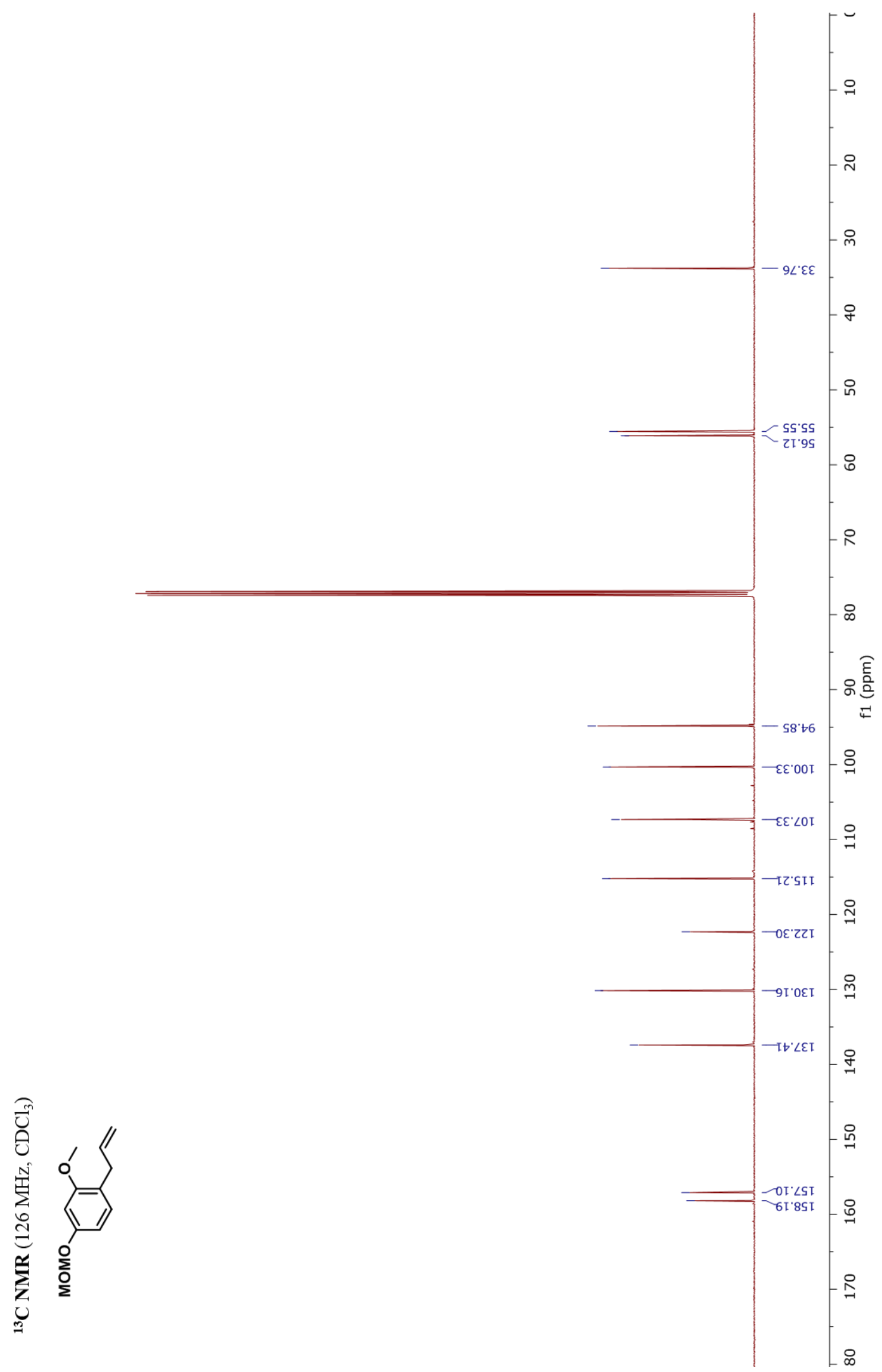


${ }^{1} \mathrm{H}$ NMR Spectrum of Compound 3.70

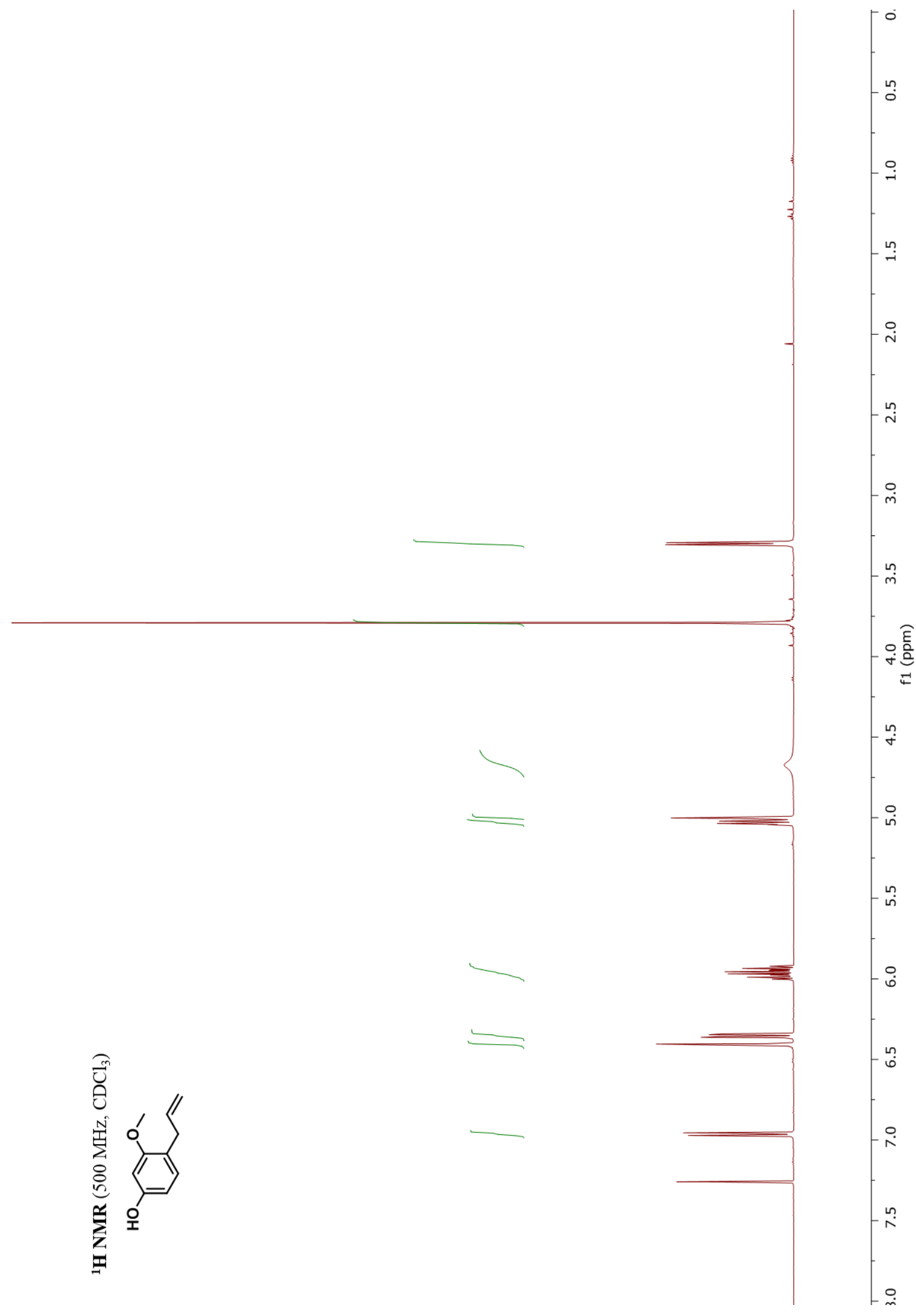


${ }^{13}$ C NMR Spectrum of Compound 3.70

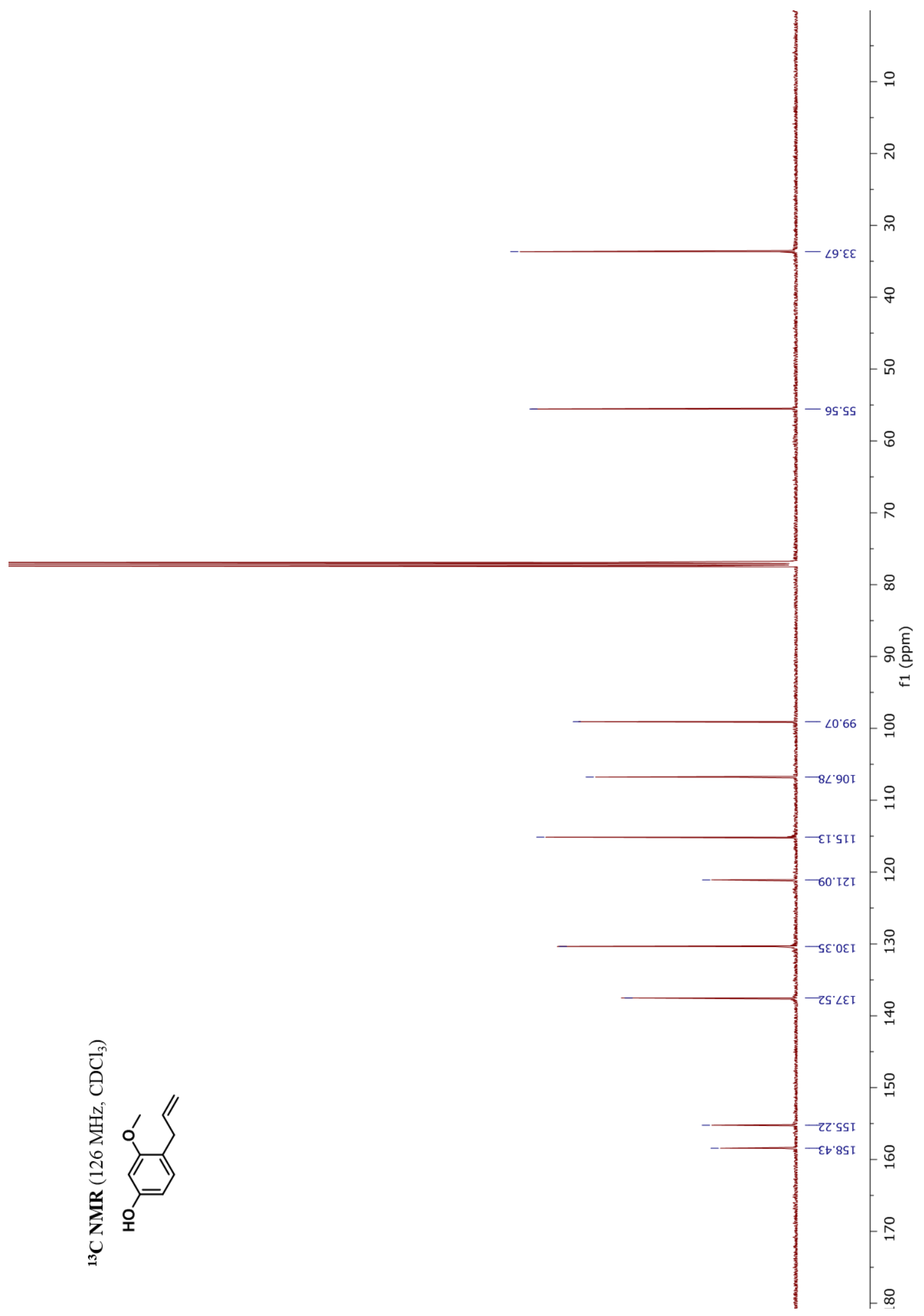


${ }^{1}$ H NMR Spectrum of Compound 3.71

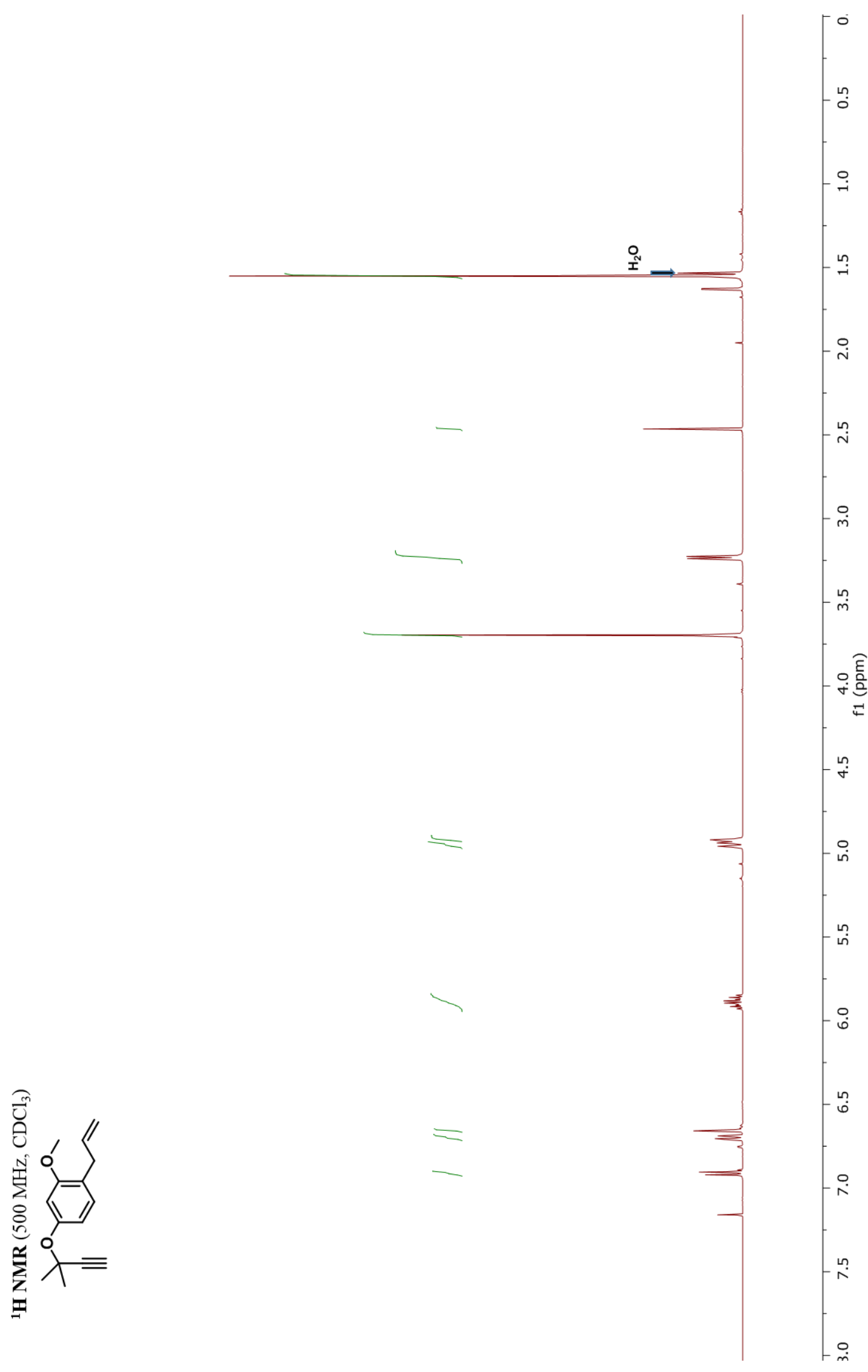


${ }^{13}$ C NMR Spectrum of Compound 3.71

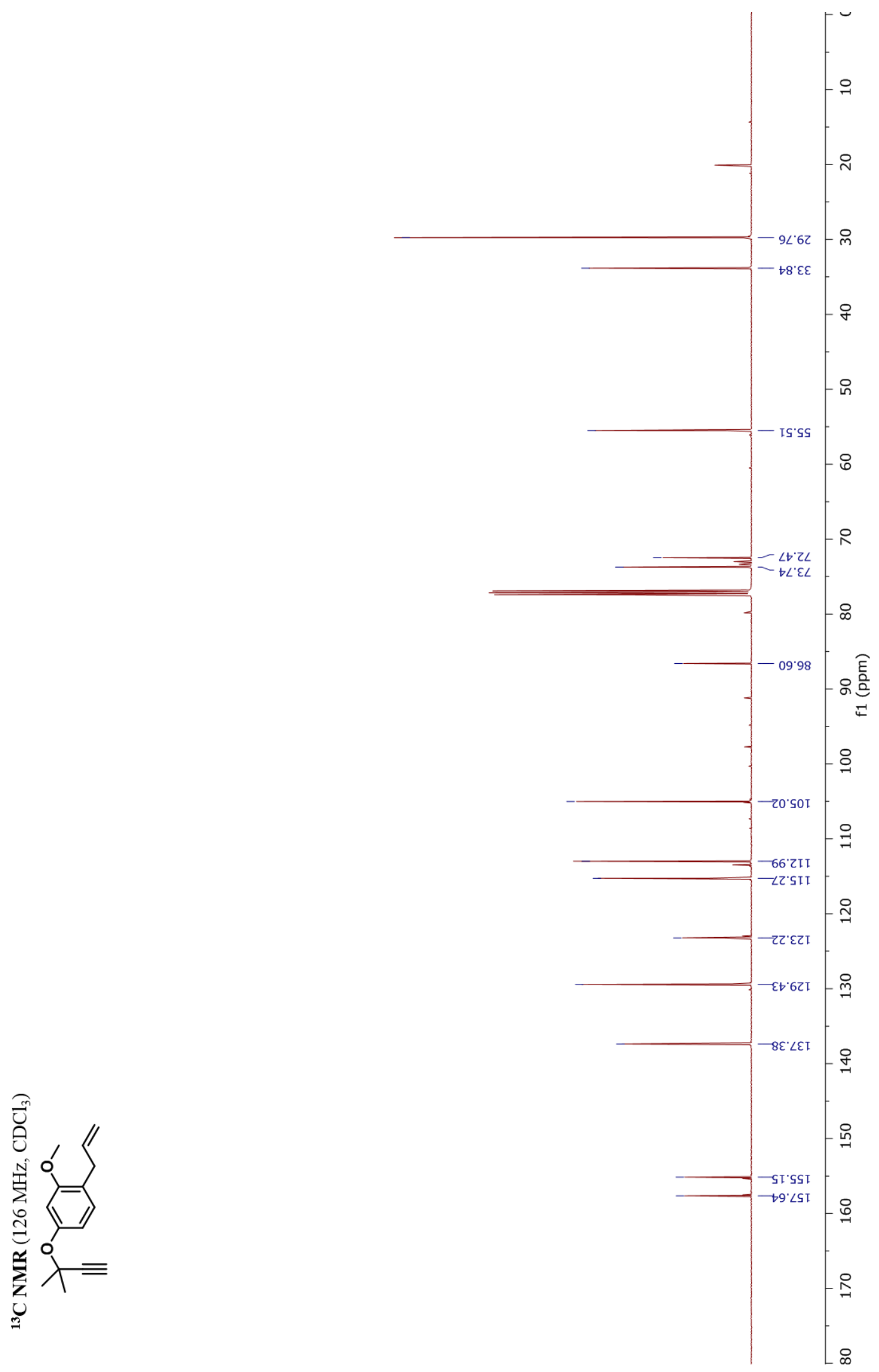


${ }^{1} \mathrm{H}$ NMR Spectrum of Compound 3.72

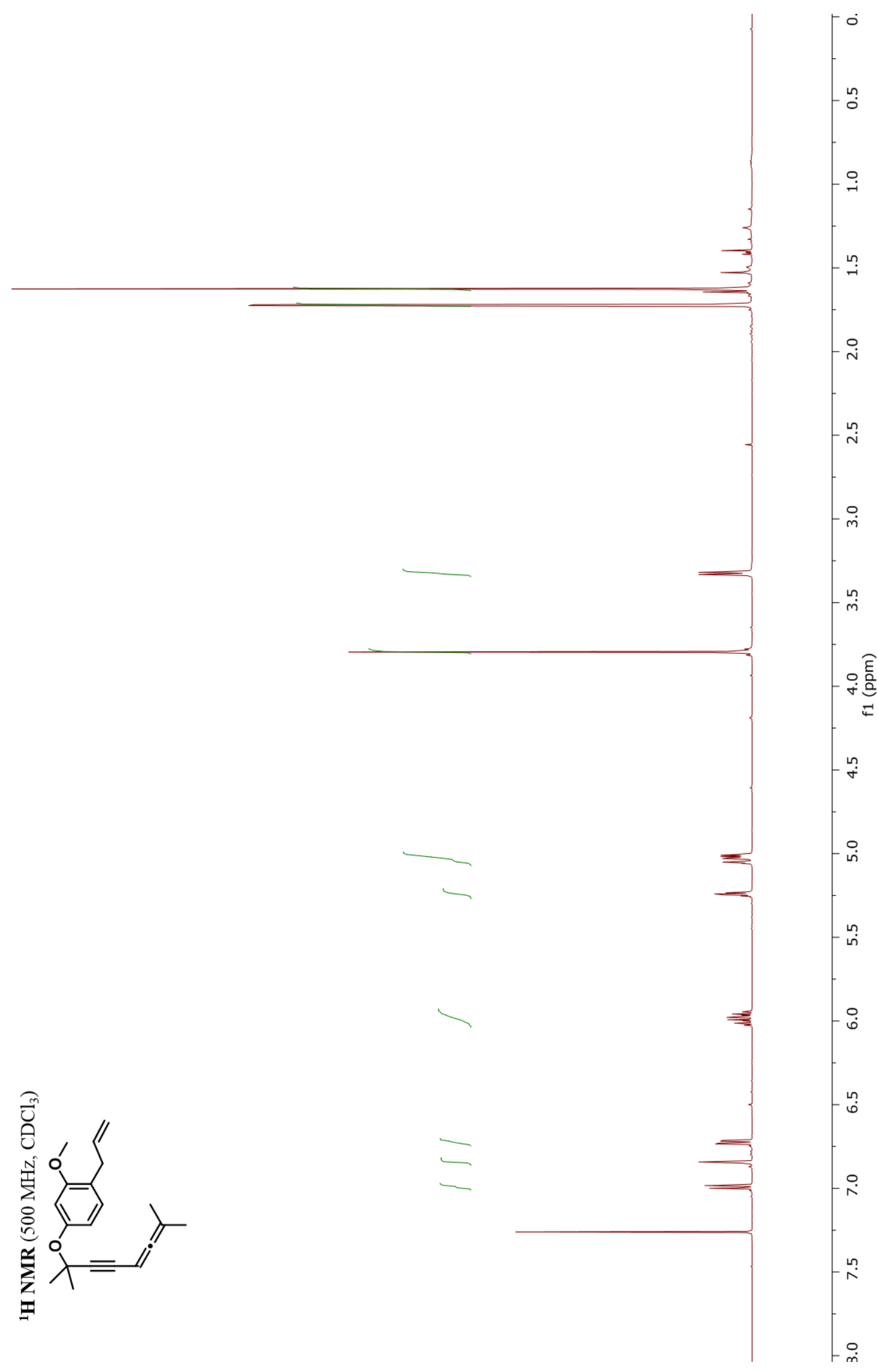


${ }^{13}$ C NMR Spectrum of Compound 3.72

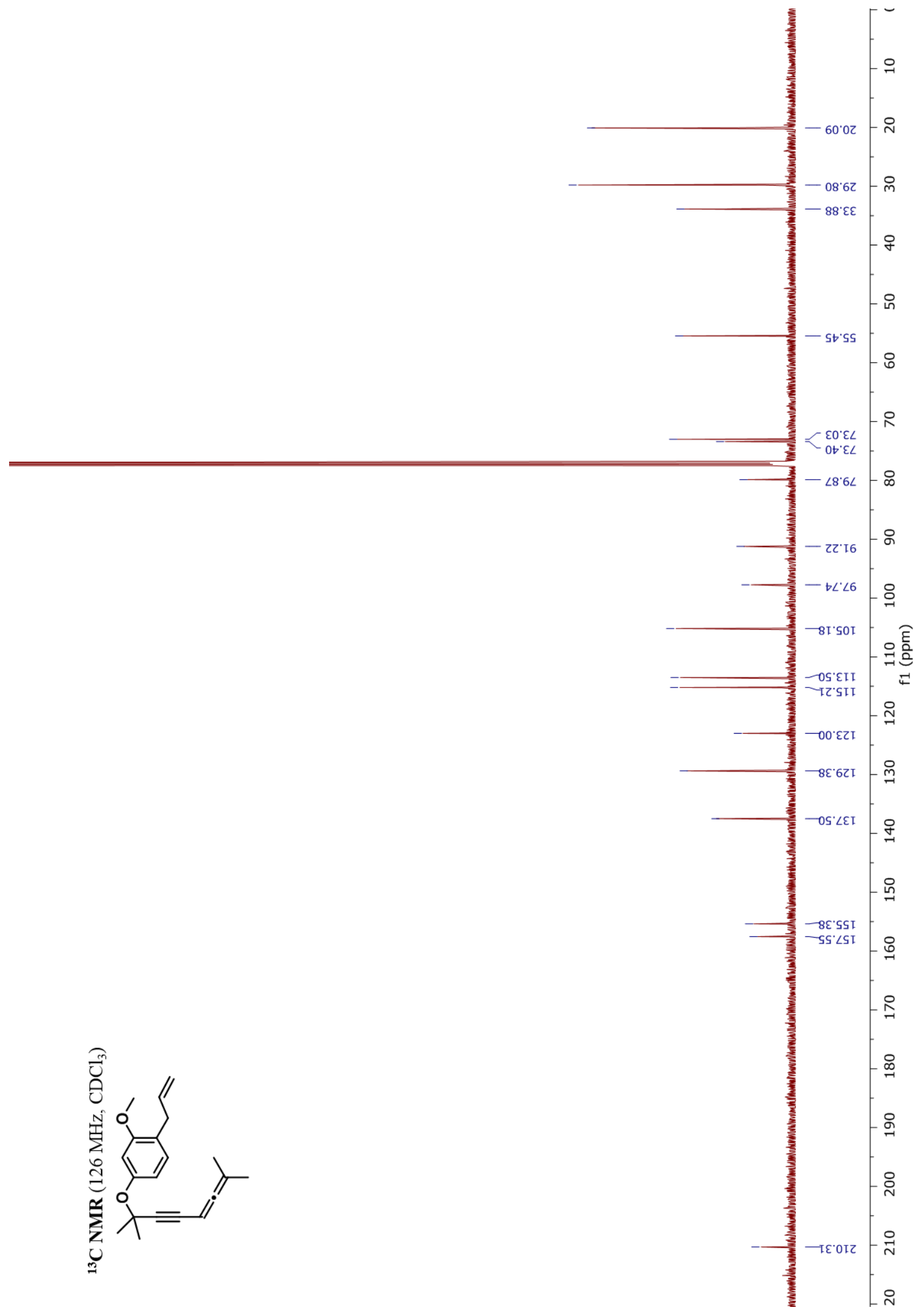


${ }^{1} \mathrm{H}$ NMR Spectrum of Compound 3.45

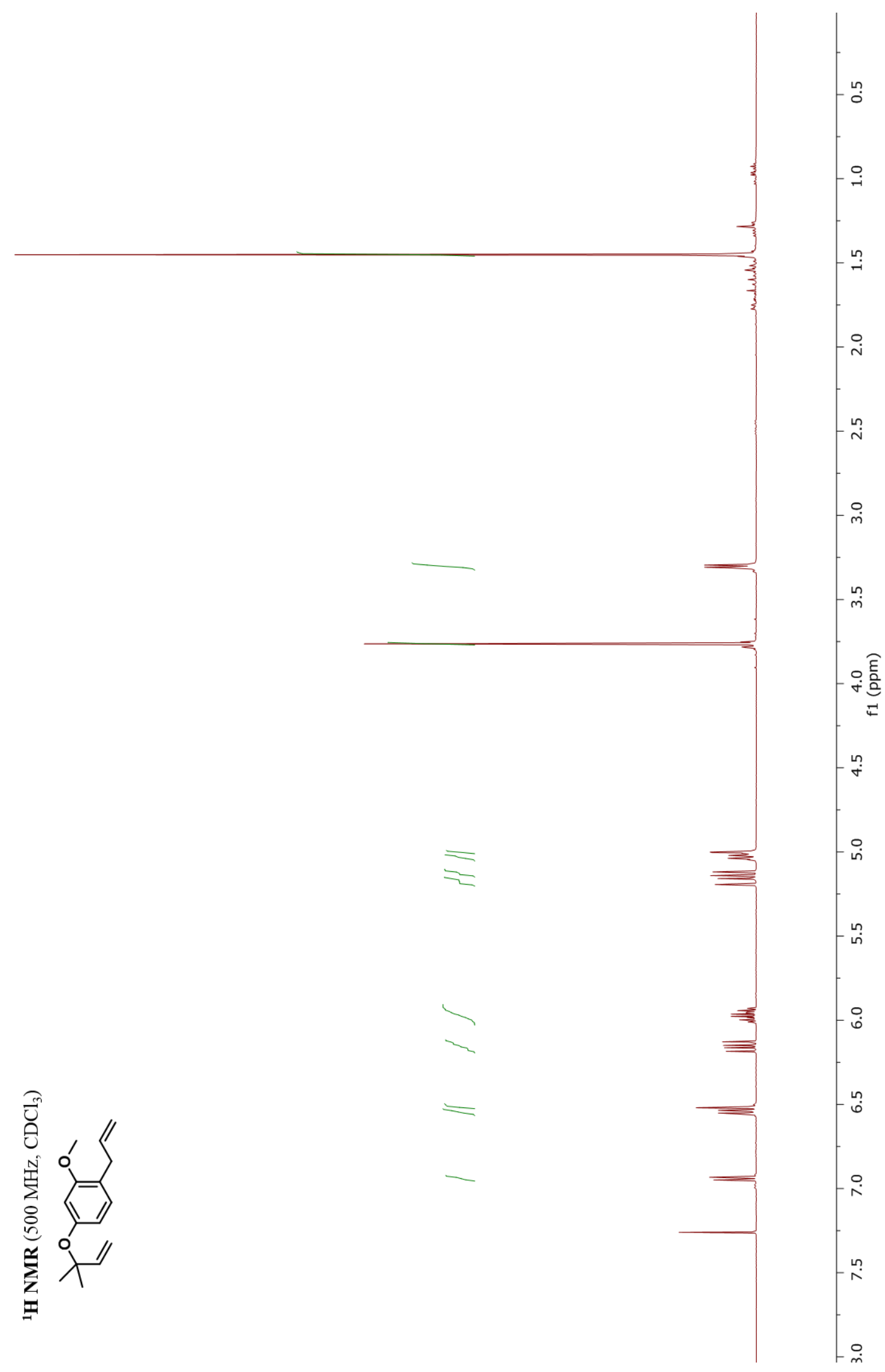


${ }^{13}$ C NMR Spectrum of Compound 3.45

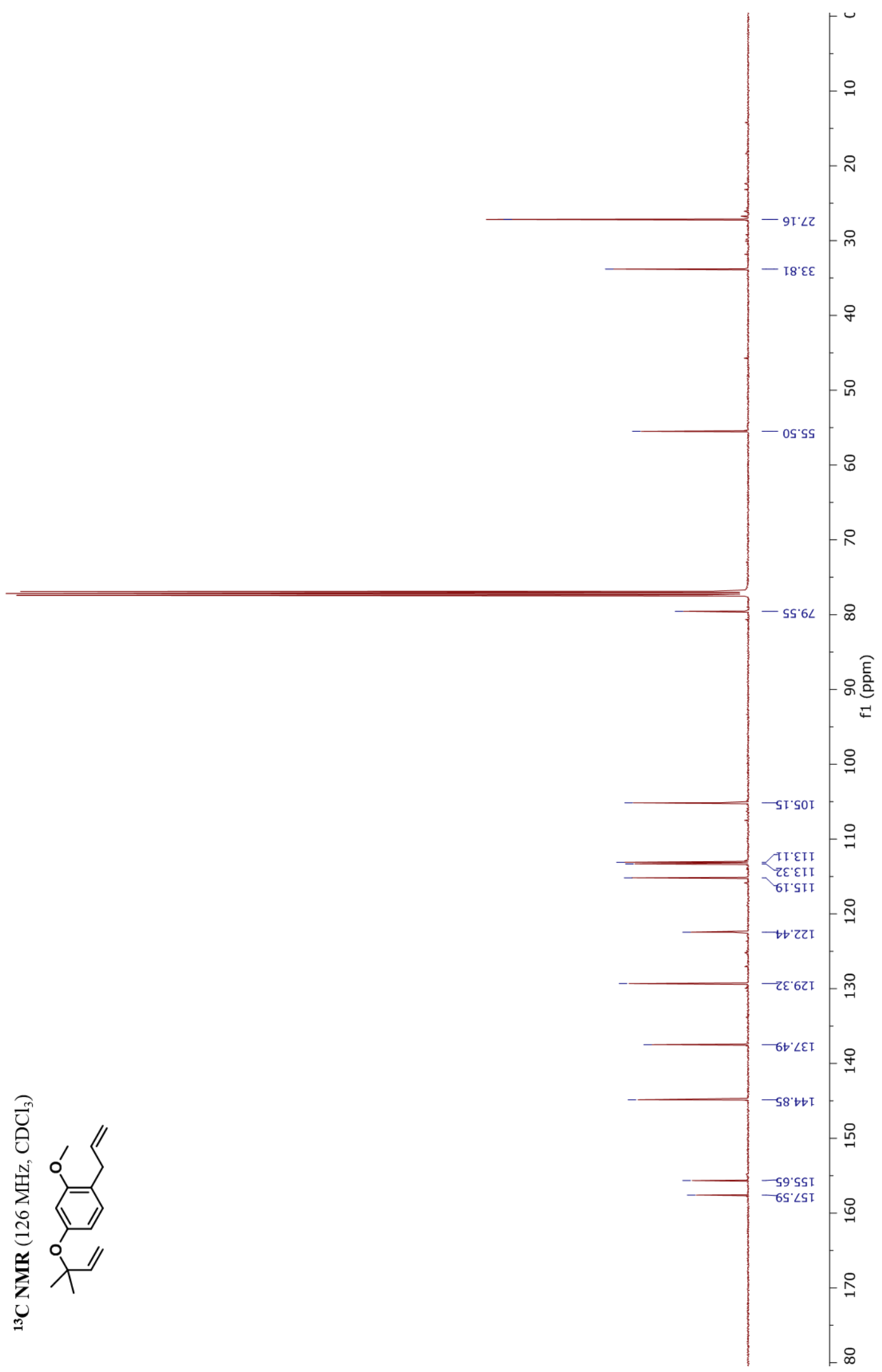


${ }^{1}$ H NMR Spectrum of Compound 3.41

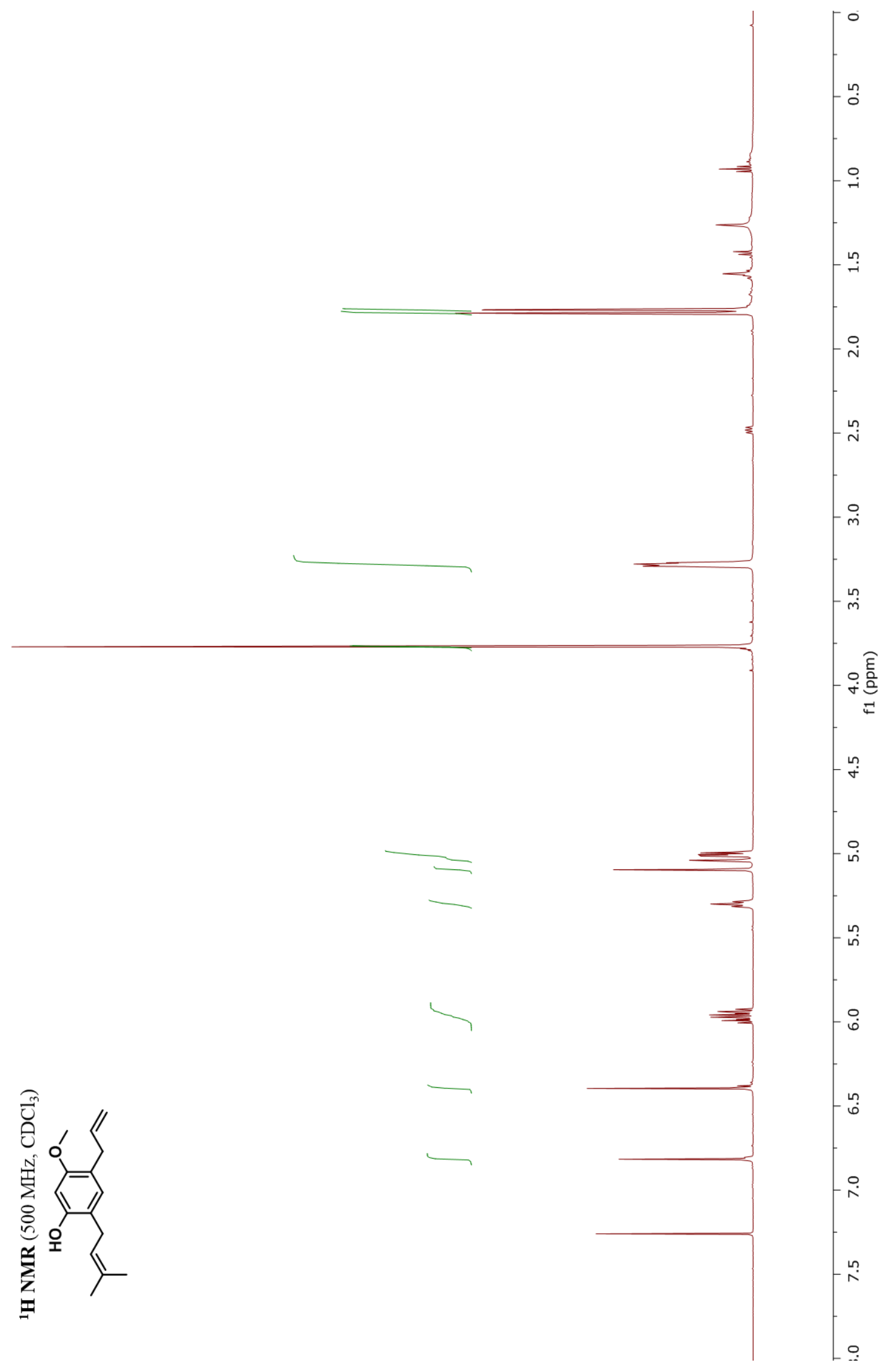


${ }^{13}$ C NMR Spectrum of Compound 3.41

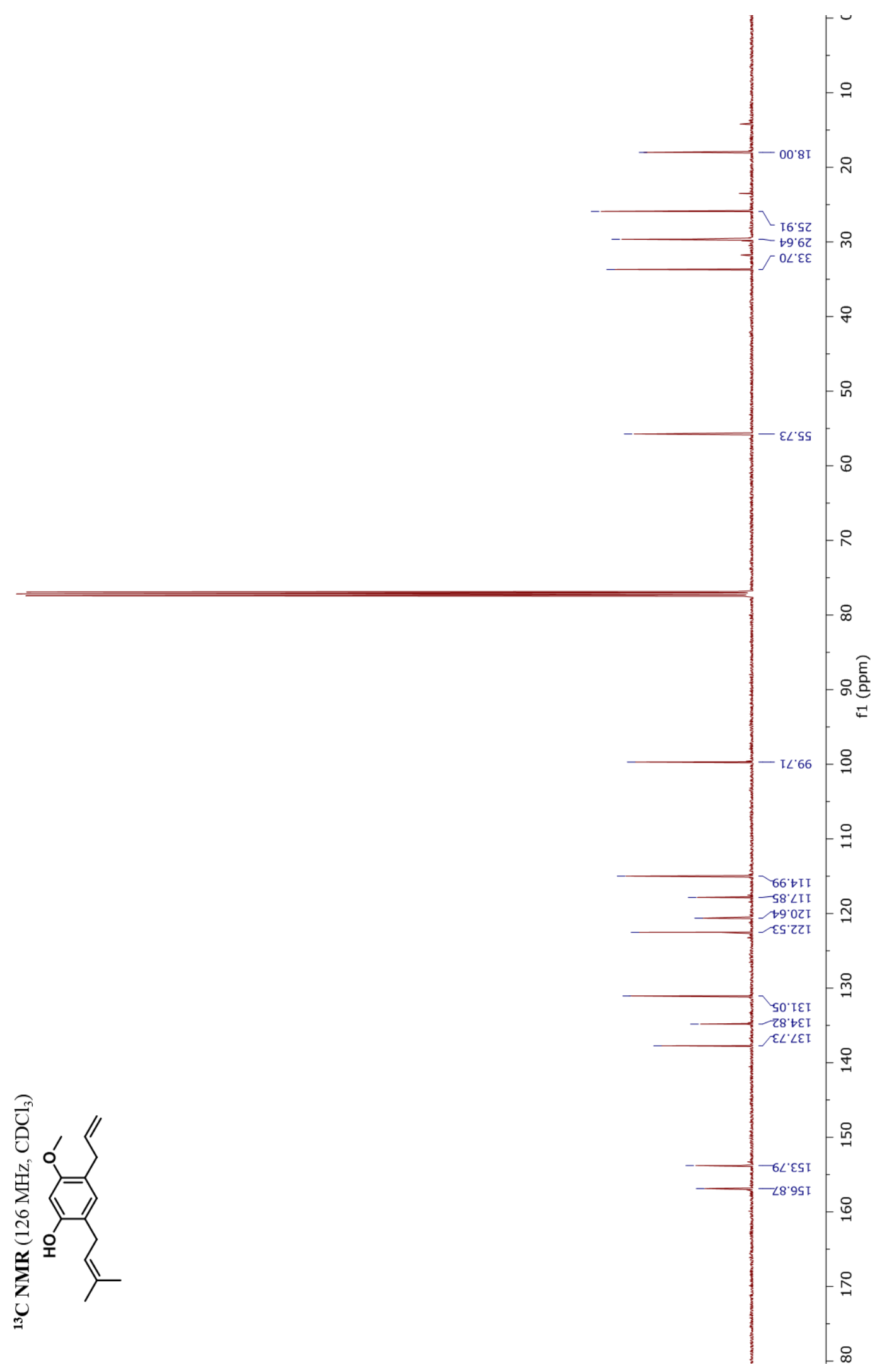


${ }^{1} \mathrm{H}$ NMR Spectrum of Compound 3.73

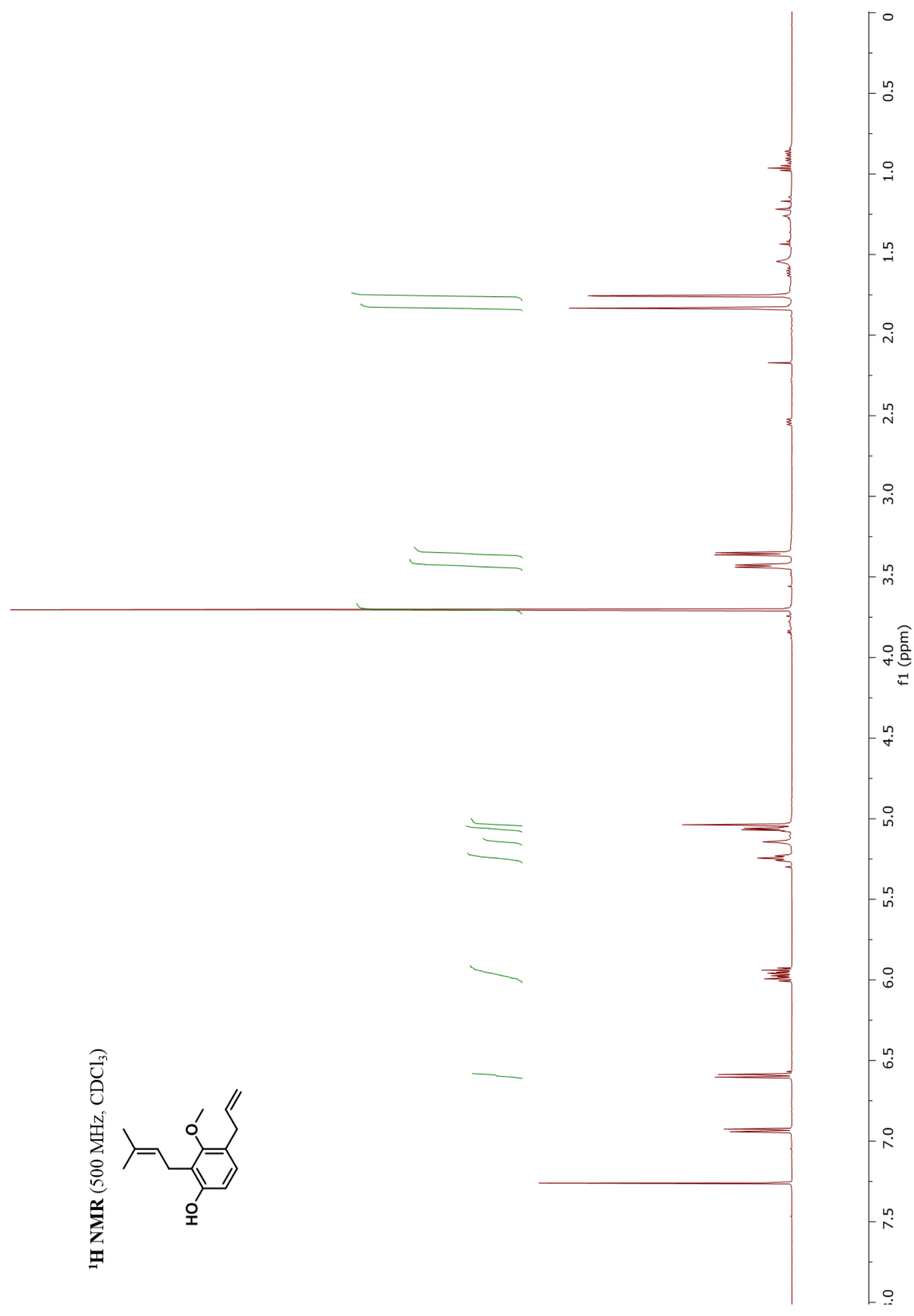


${ }^{13}$ C NMR Spectrum of Compound 3.73

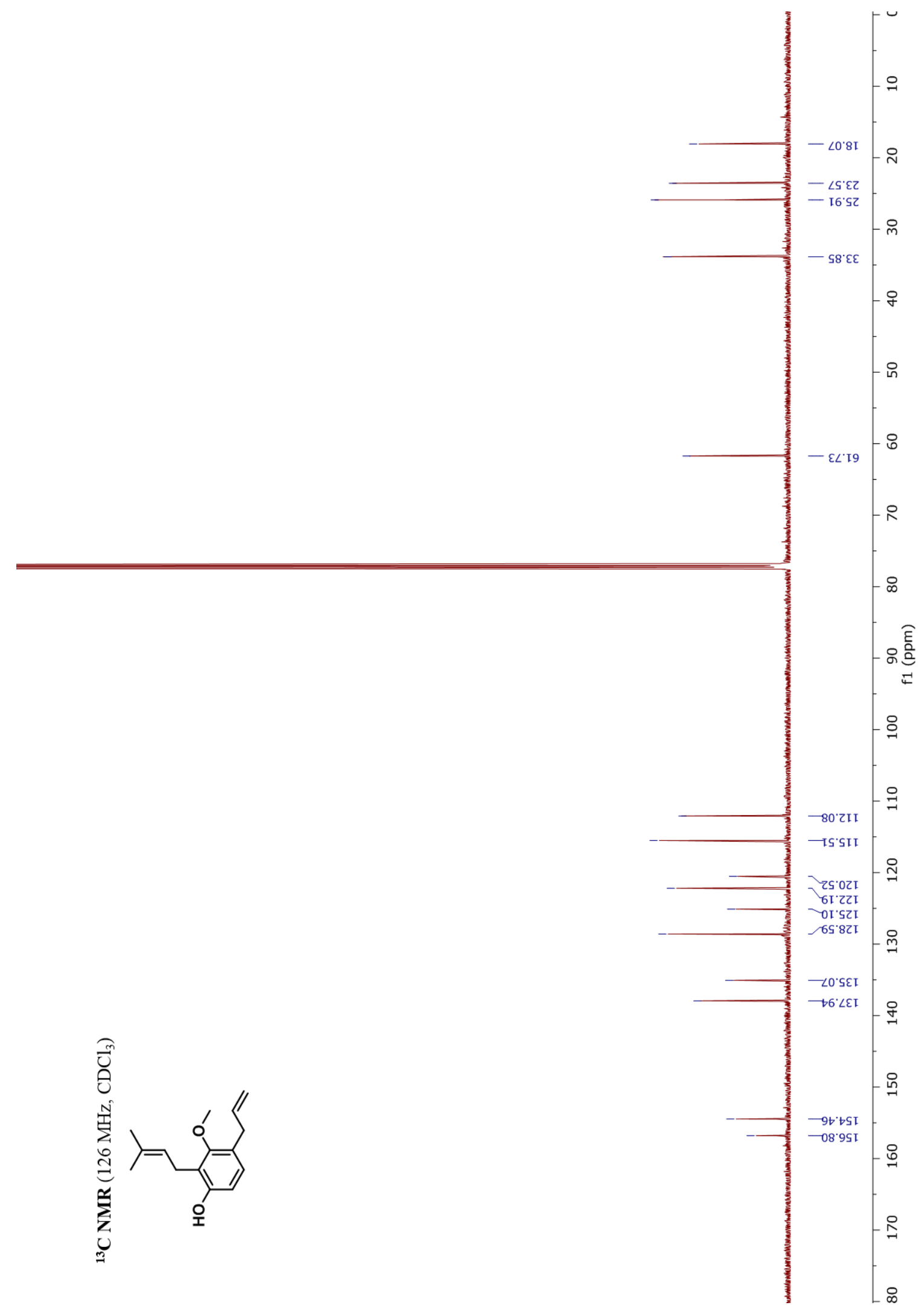


${ }^{1}$ H NMR Spectrum of Crude Compound ( \pm )-3.37

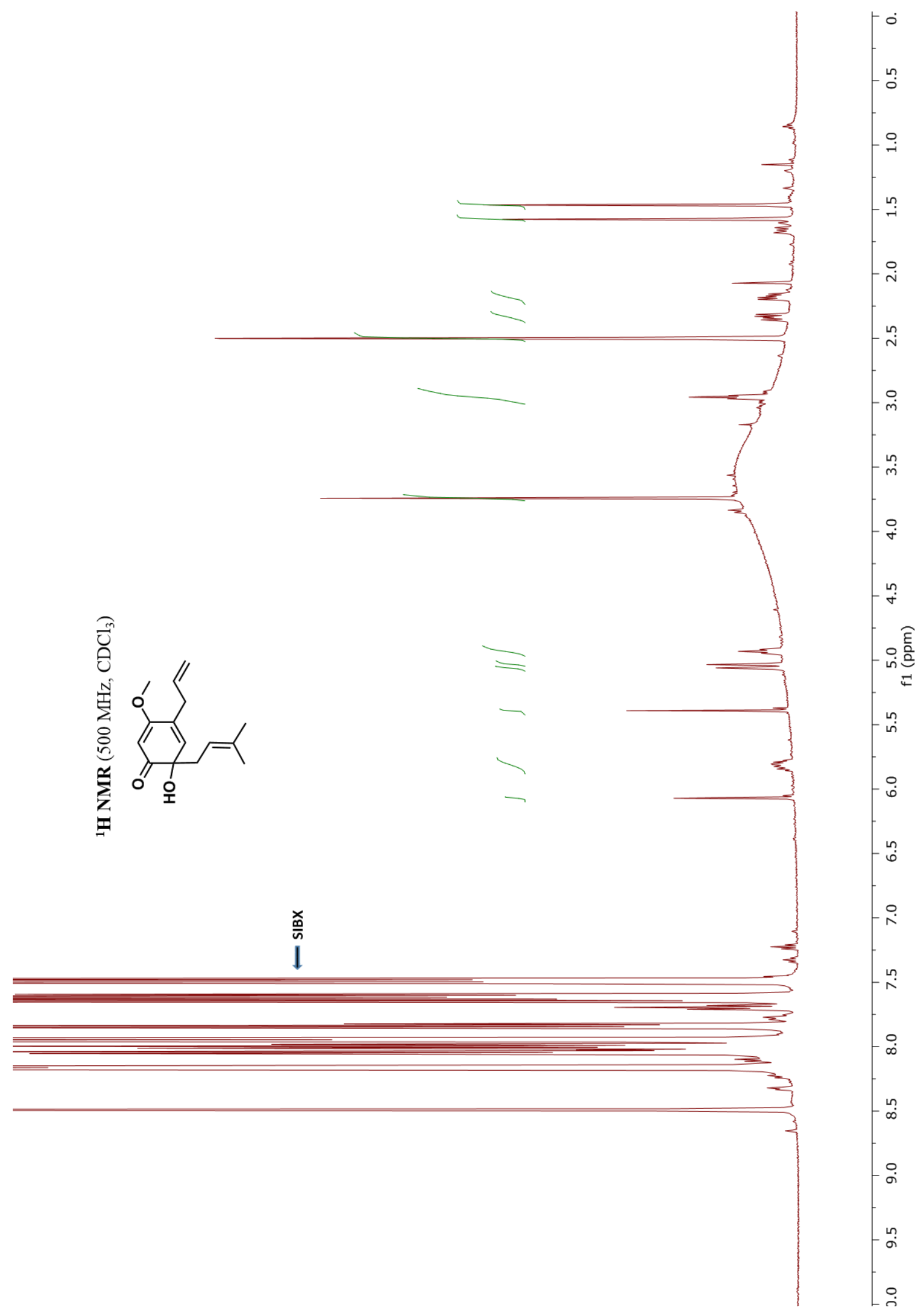


${ }^{1}$ H NMR Timed Experiment for the Formation of Compound ( \pm )-3.37

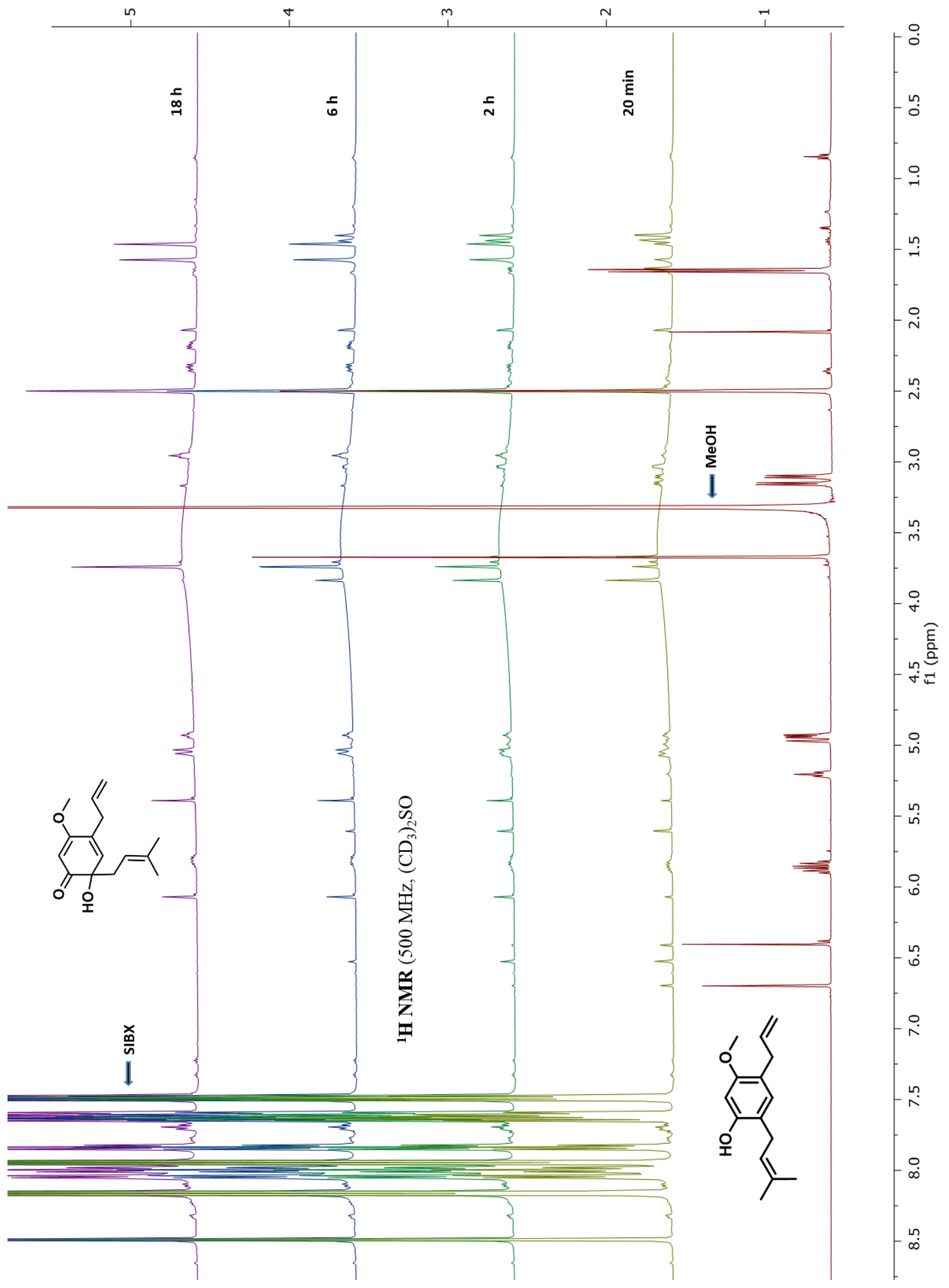


${ }^{1}$ H NMR Spectrum of Compound 3.4

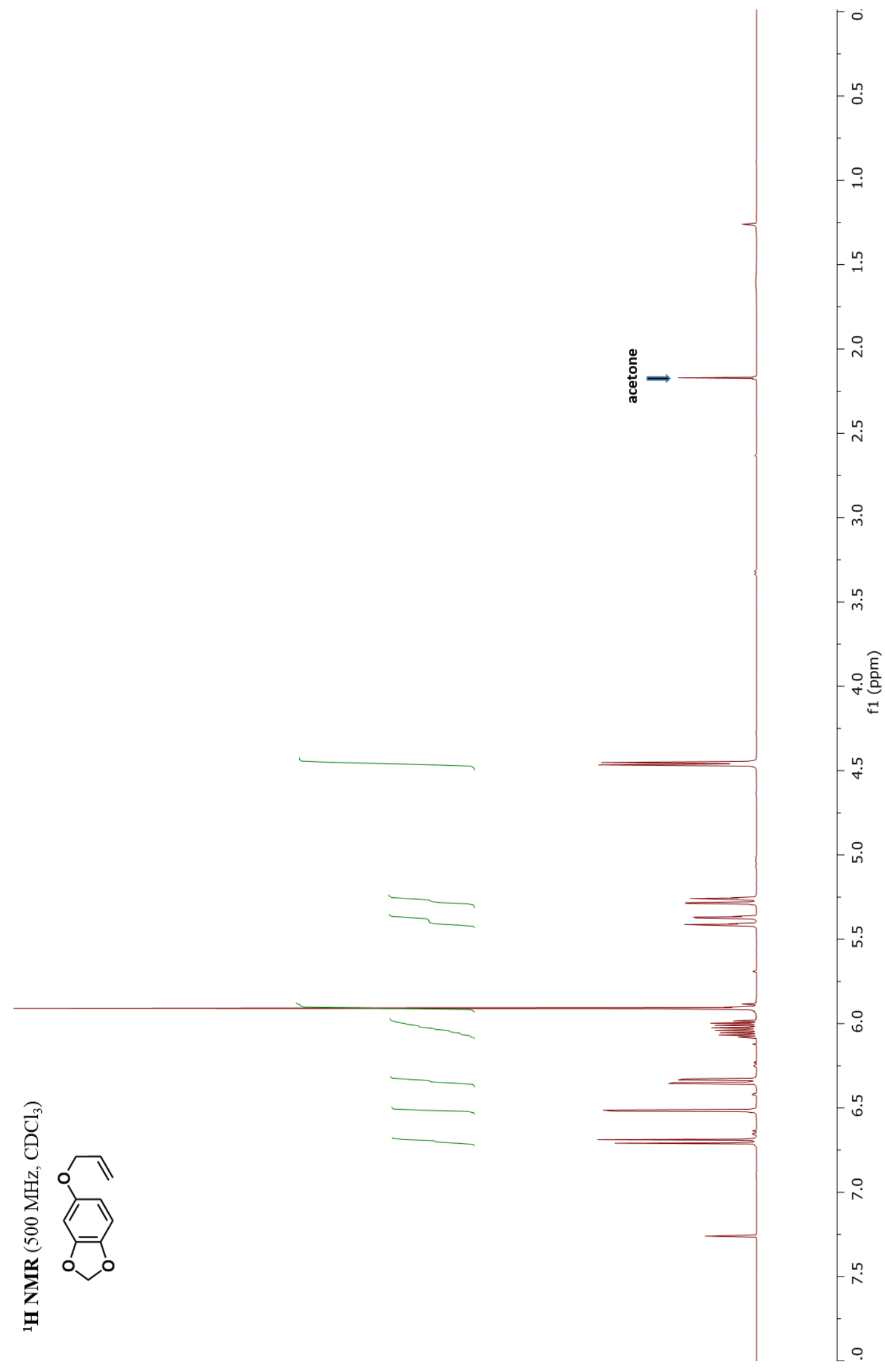


${ }^{13}$ C NMR Spectrum of Compound 3.4

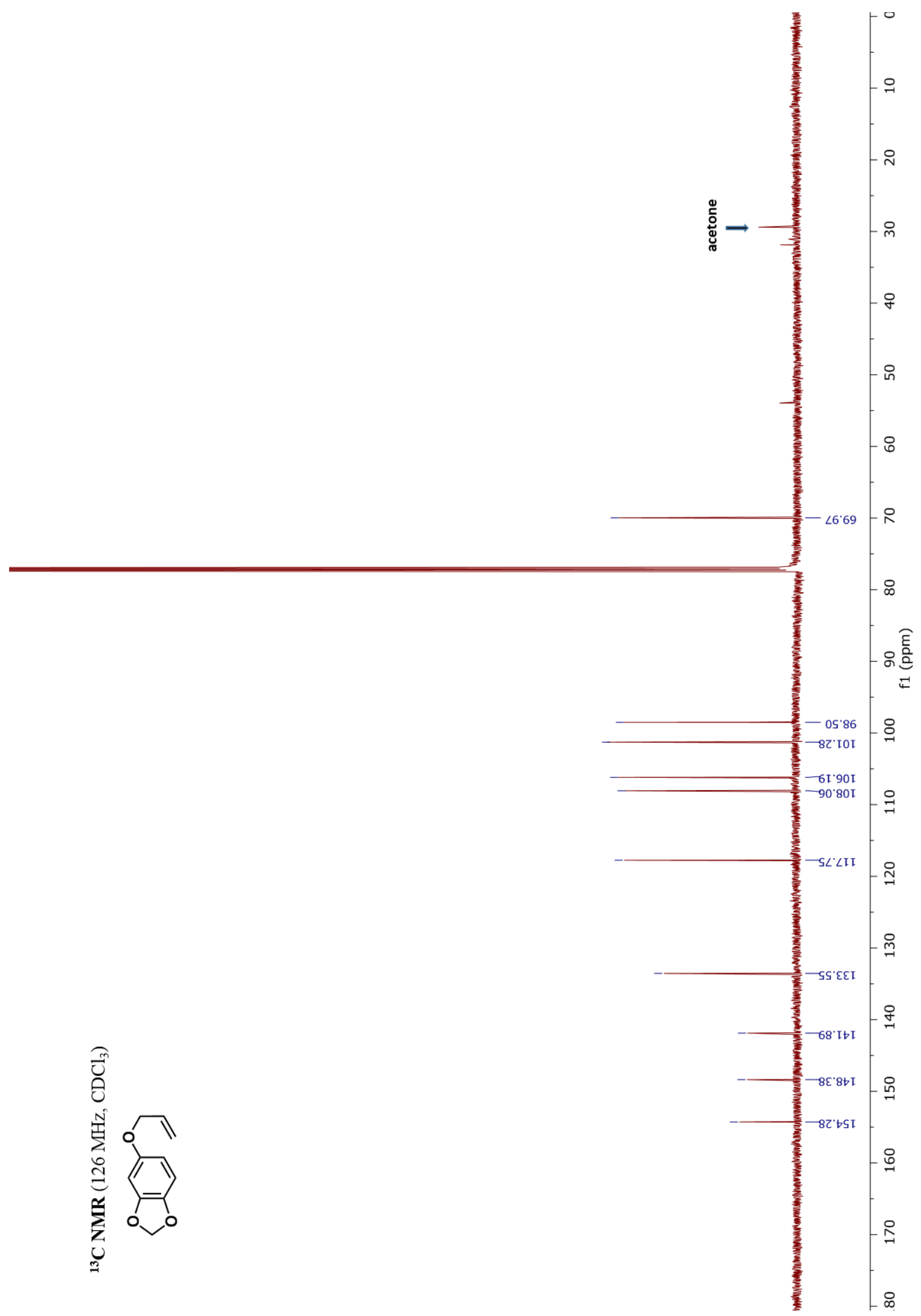


${ }^{1}$ H NMR Spectrum of Compound 3.5

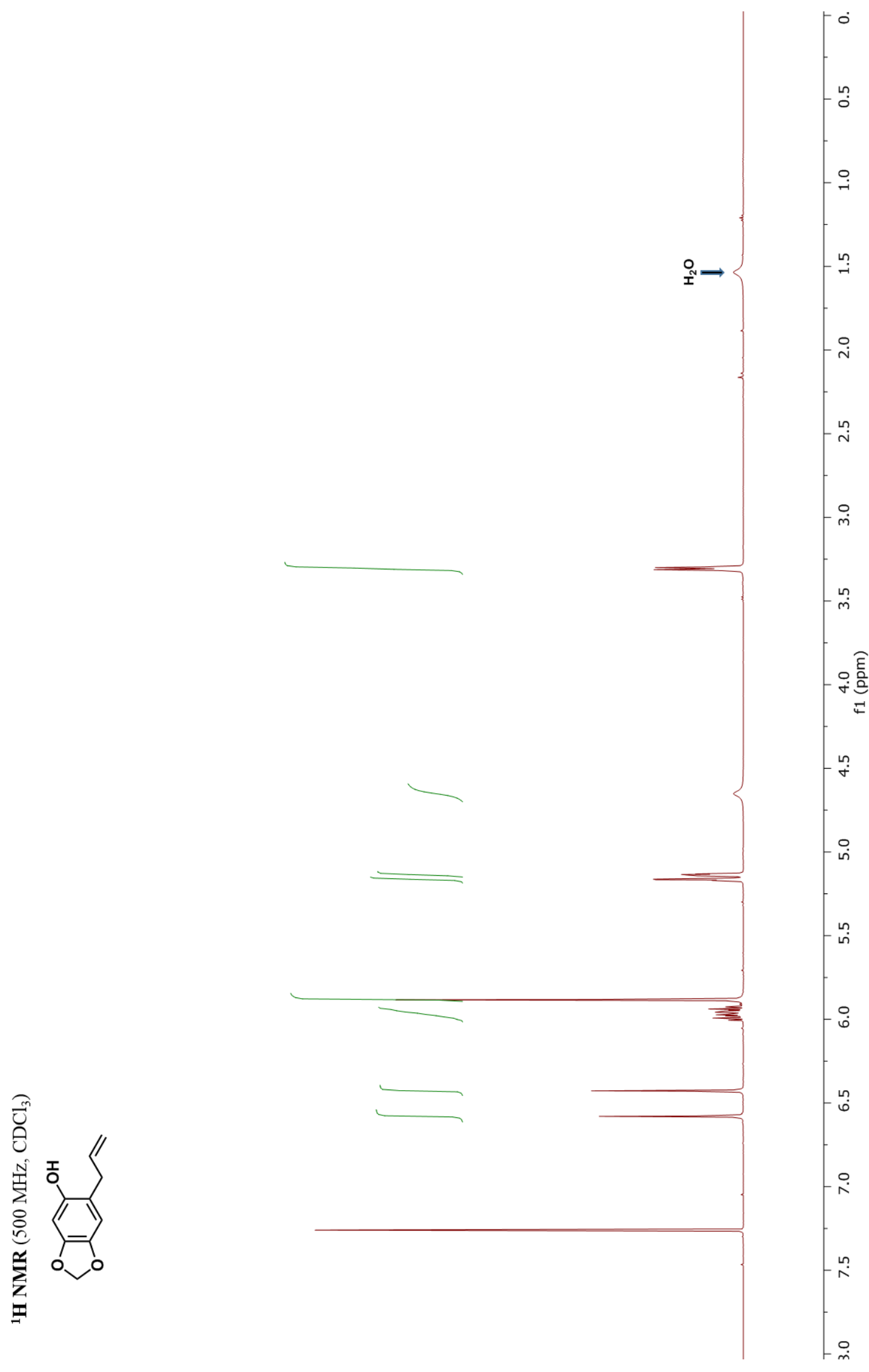


${ }^{13}$ C NMR Spectrum of Compound 3.5

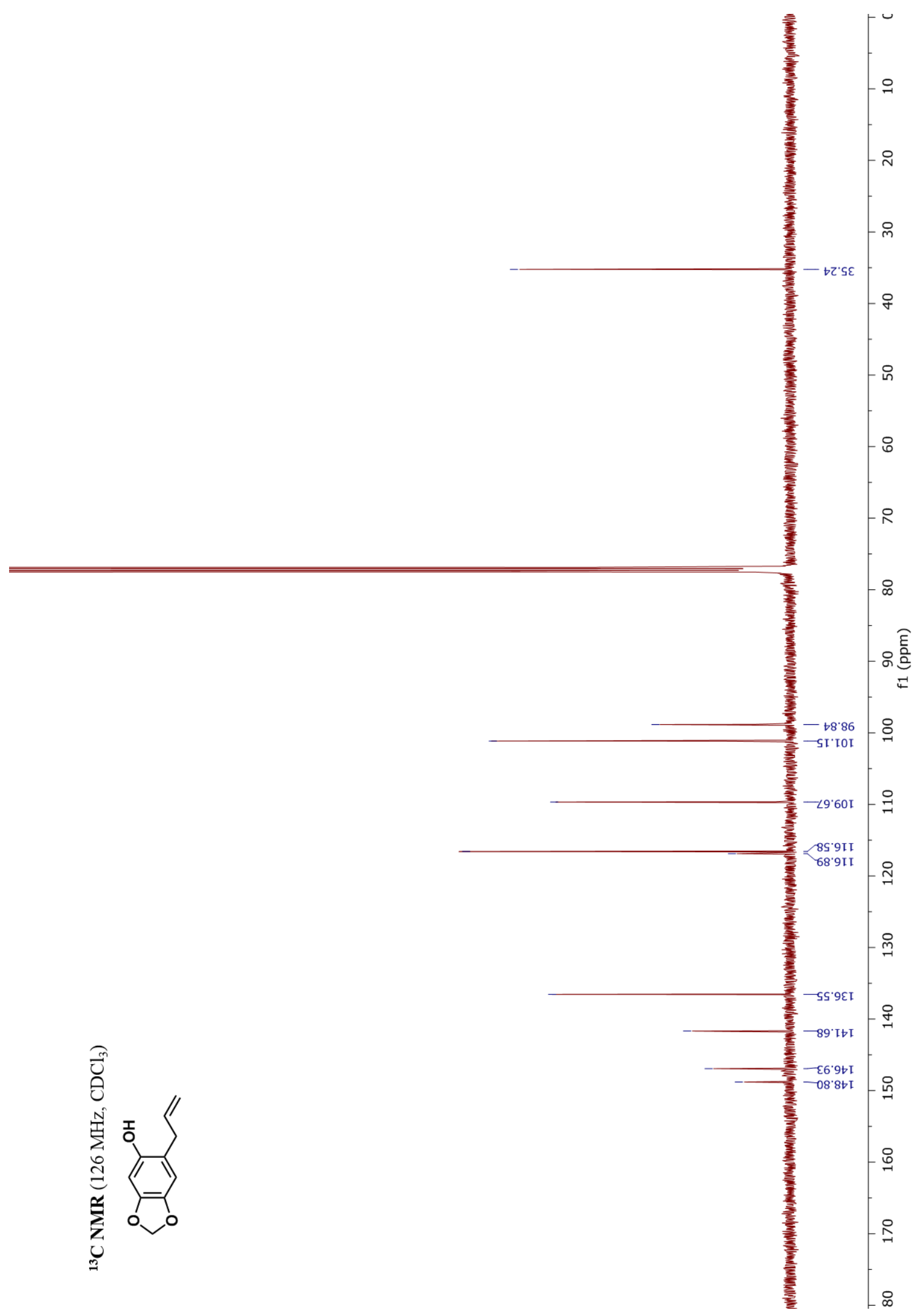


${ }^{1}$ H NMR Spectrum of Illicinole 3.6

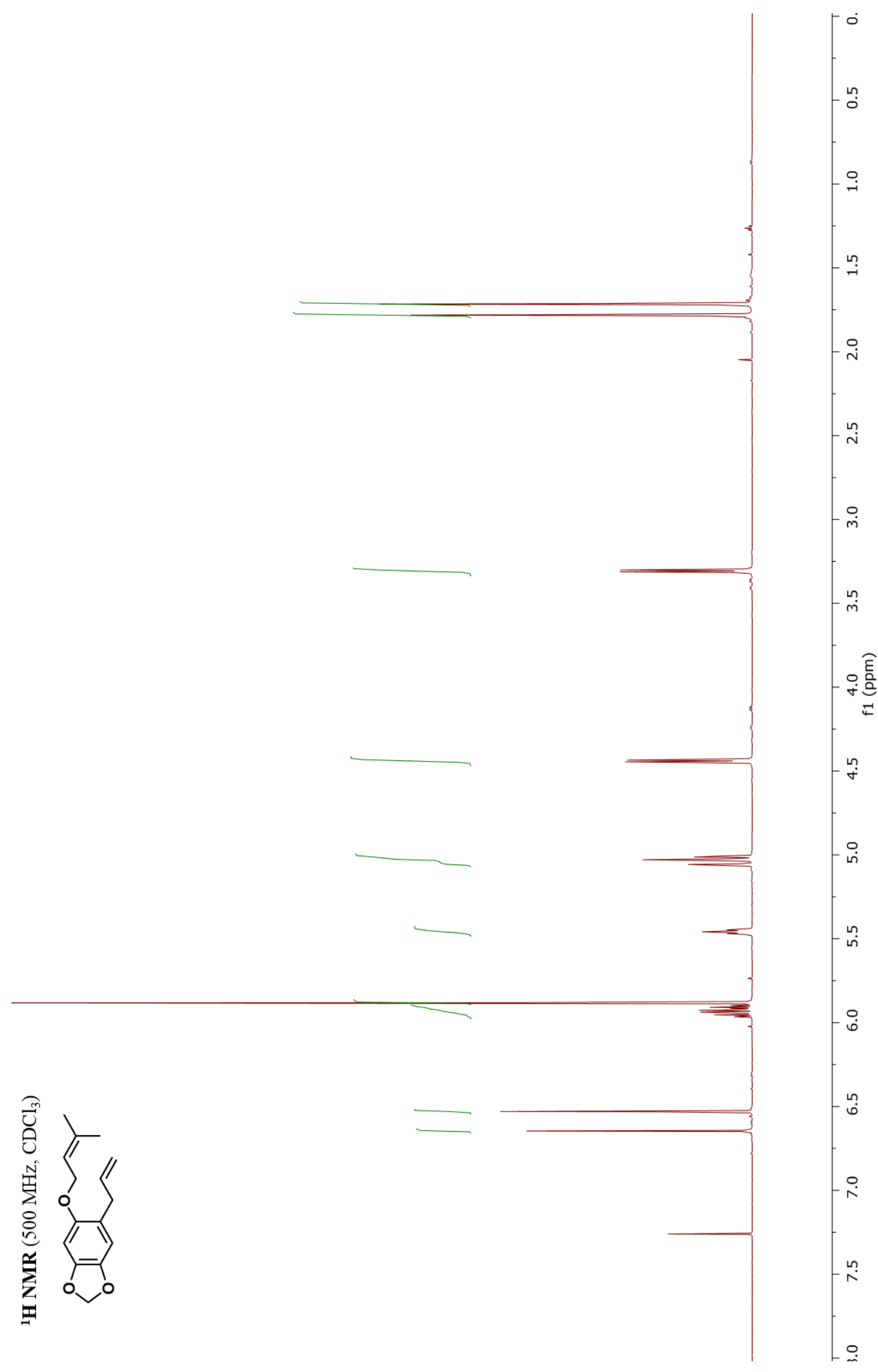


${ }^{13}$ C NMR Spectrum of Illicinole 3.6

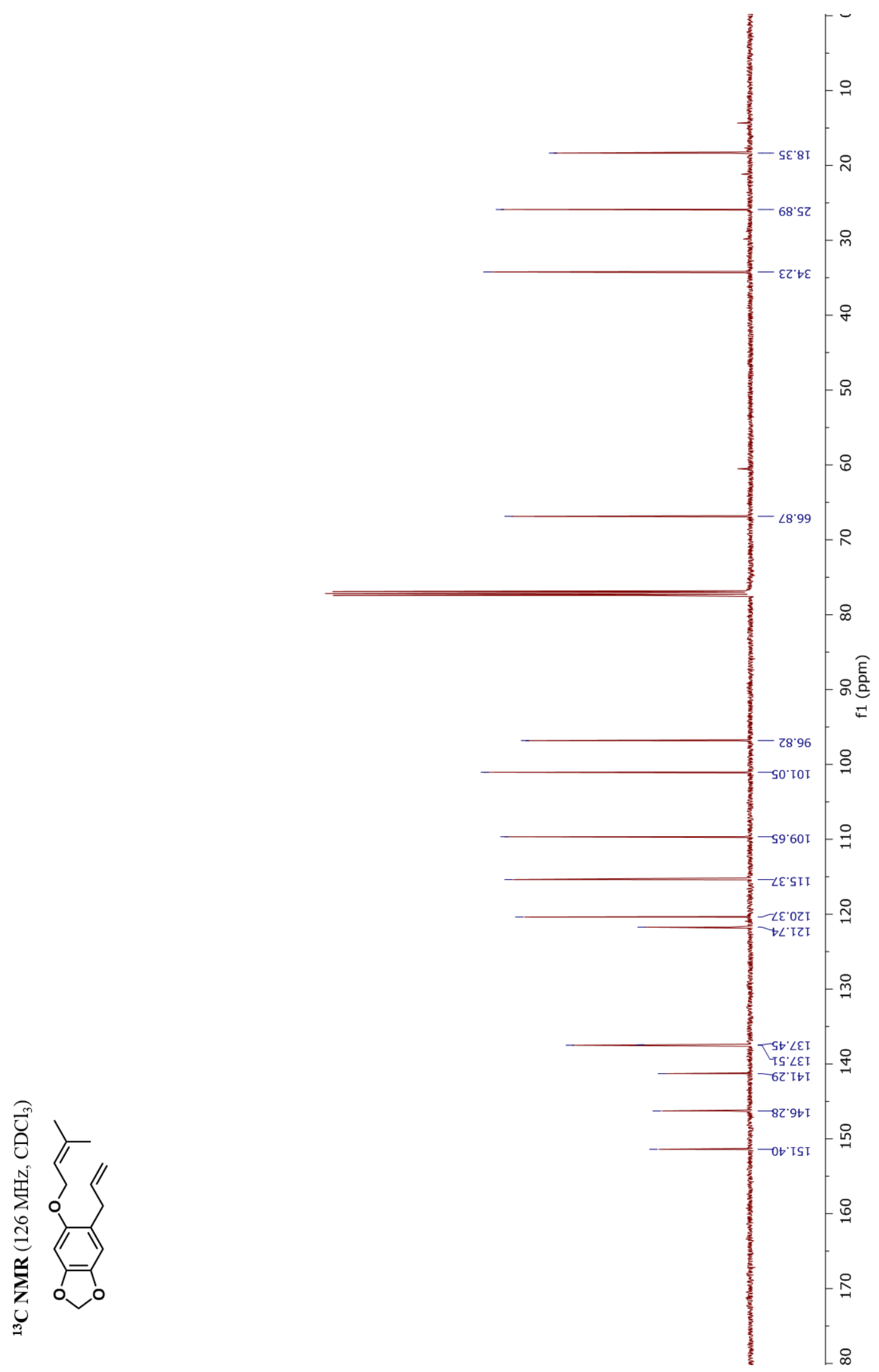


${ }^{1}$ H NMR Spectrum of ( \pm )-Illicinone A 1.3

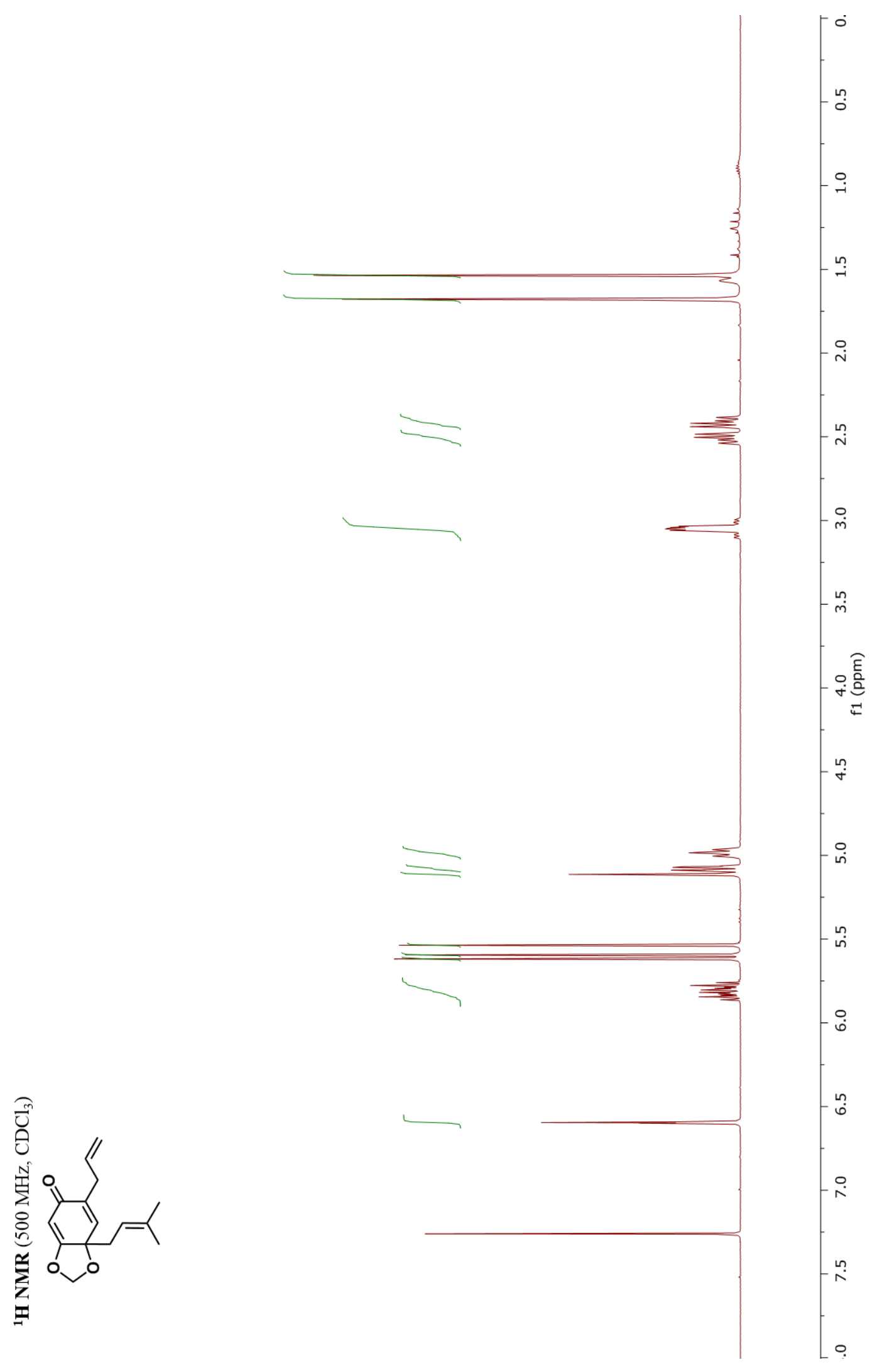


${ }^{13}$ C NMR Spectrum of ( \pm )-Illicinone A 1.3

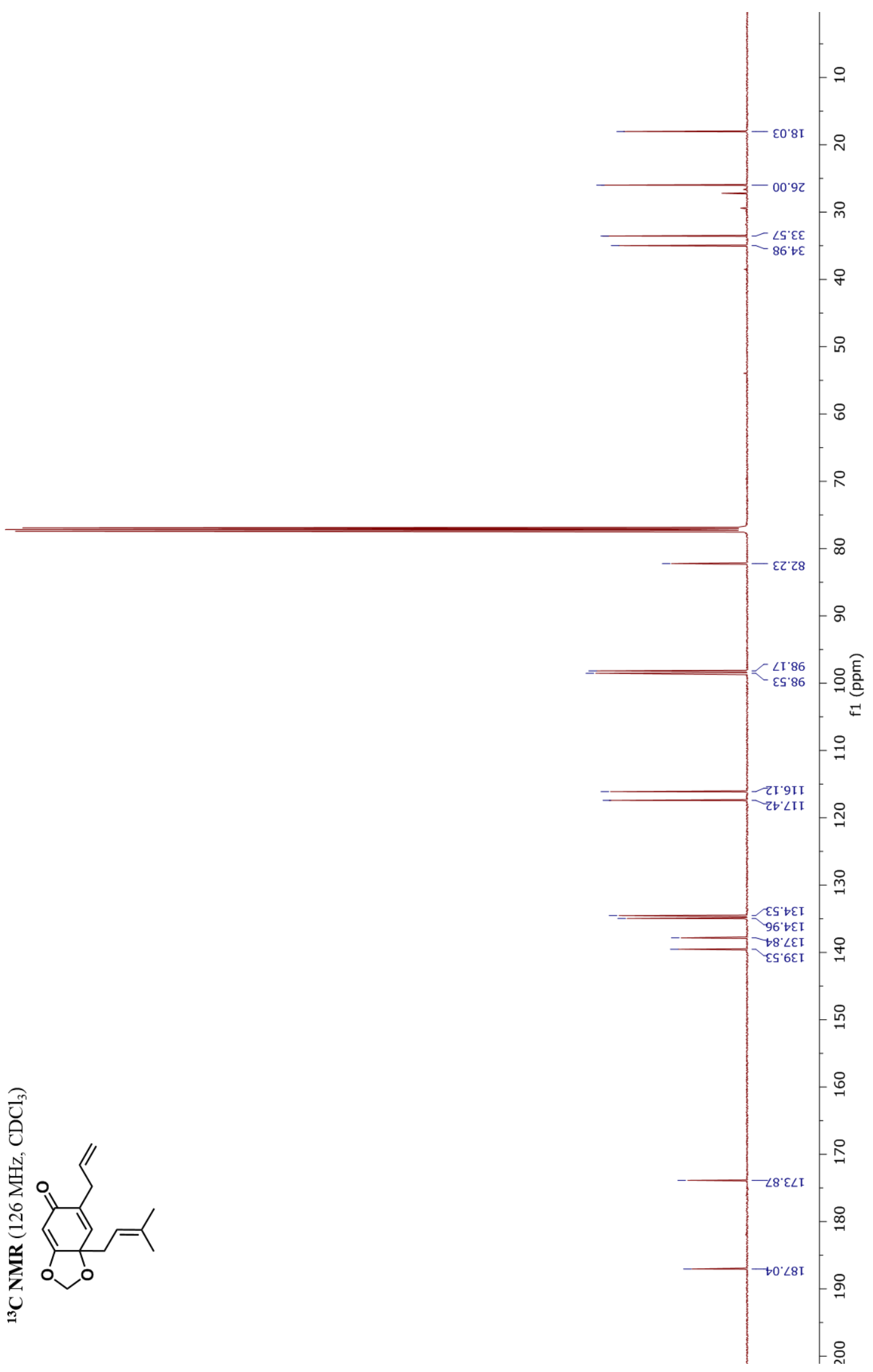


${ }^{1}$ H NMR Spectrum of ( \pm -Cycloillicinone 1.6

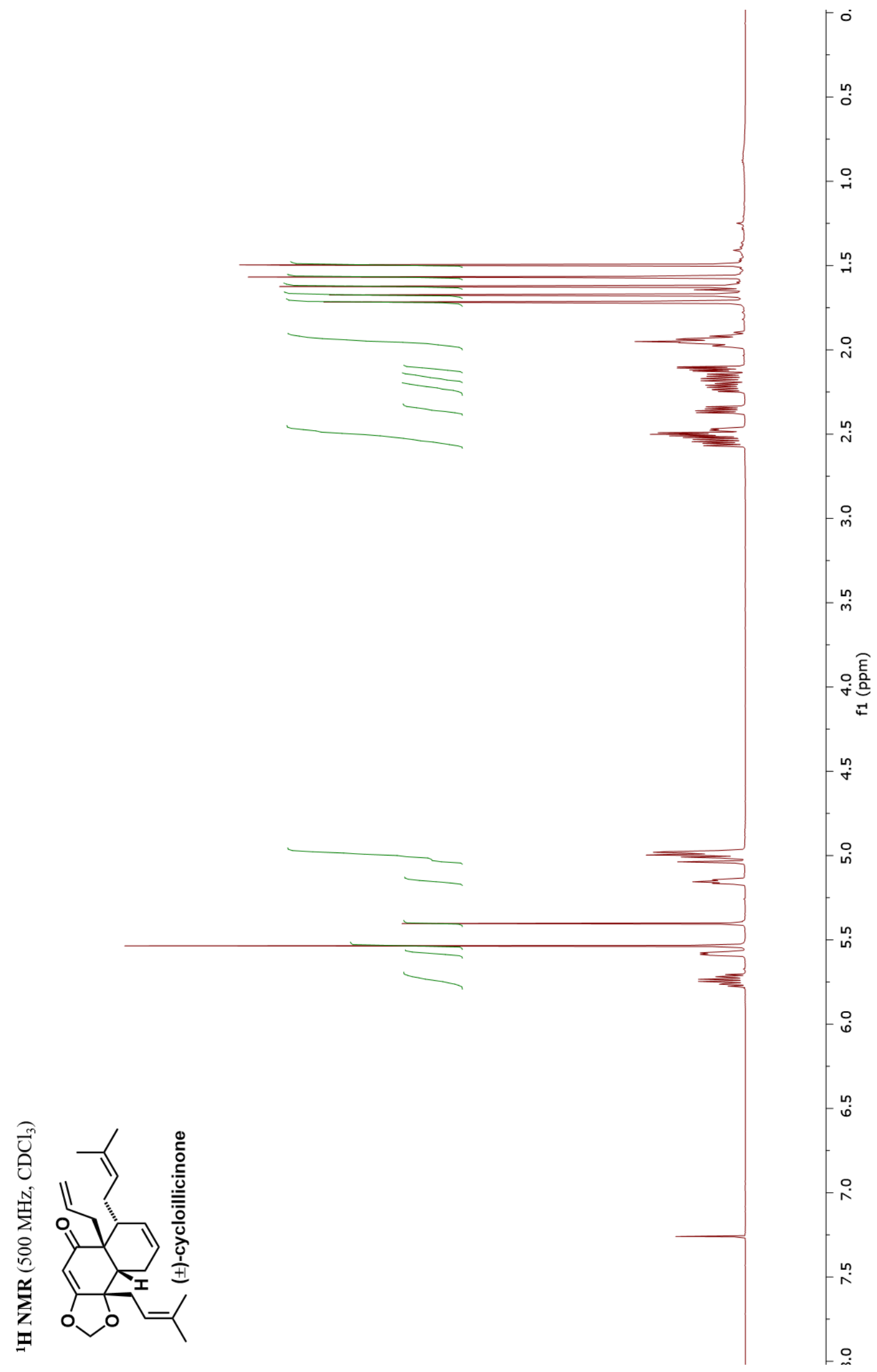


${ }^{13}$ C NMR Spectrum of ( \pm )-Cycloillicinone 1.6

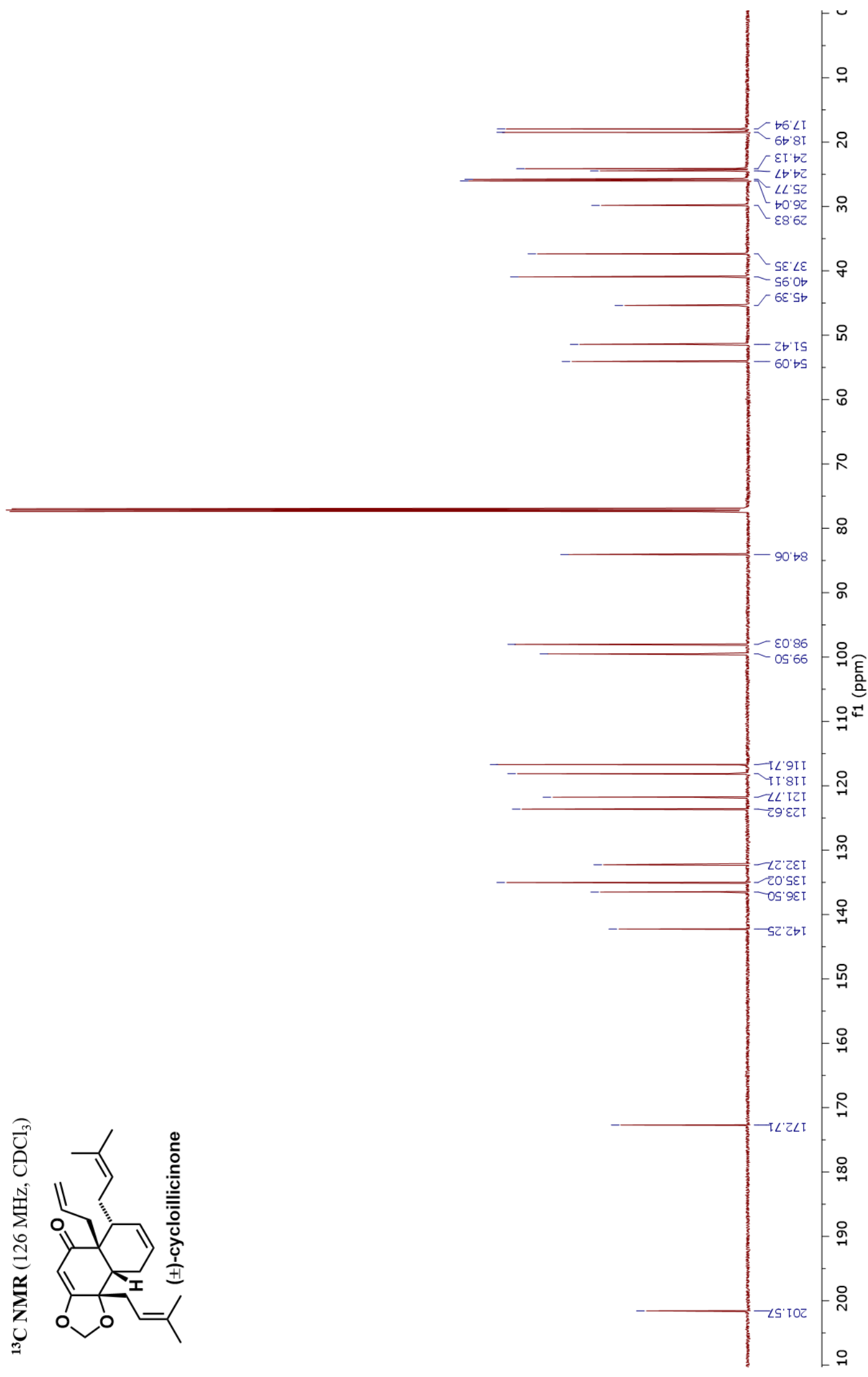


${ }^{1} \mathrm{H}-{ }^{1} \mathrm{H}$ COSY Spectrum of ( \pm )-Cycloillicinone $\mathbf{1 . 6}$

(udd) If

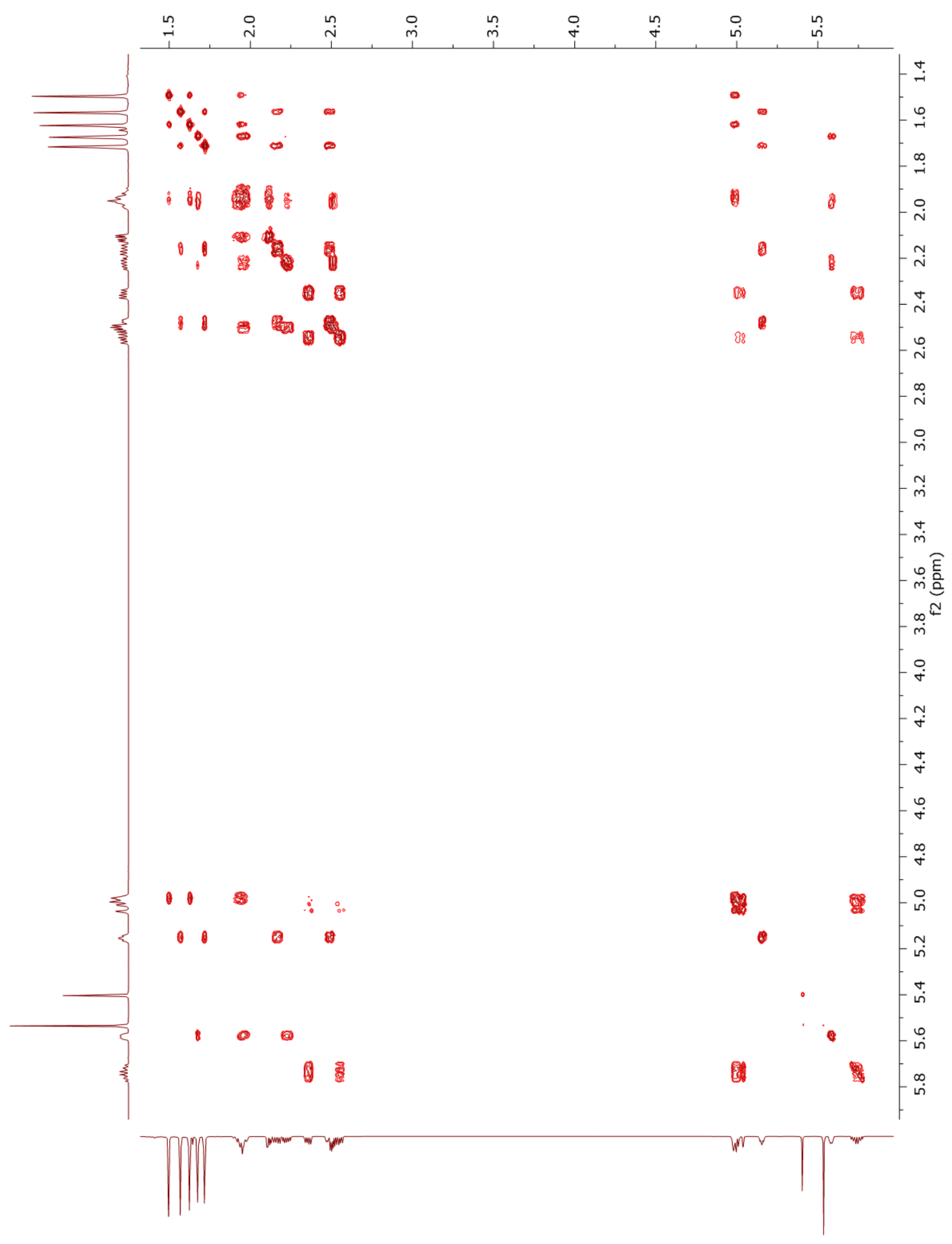


${ }^{1} \mathrm{H}-{ }^{13} \mathrm{C}$ HSQC Spectrum of ( \pm )-Cycloillicinone 1.6

(udd) If

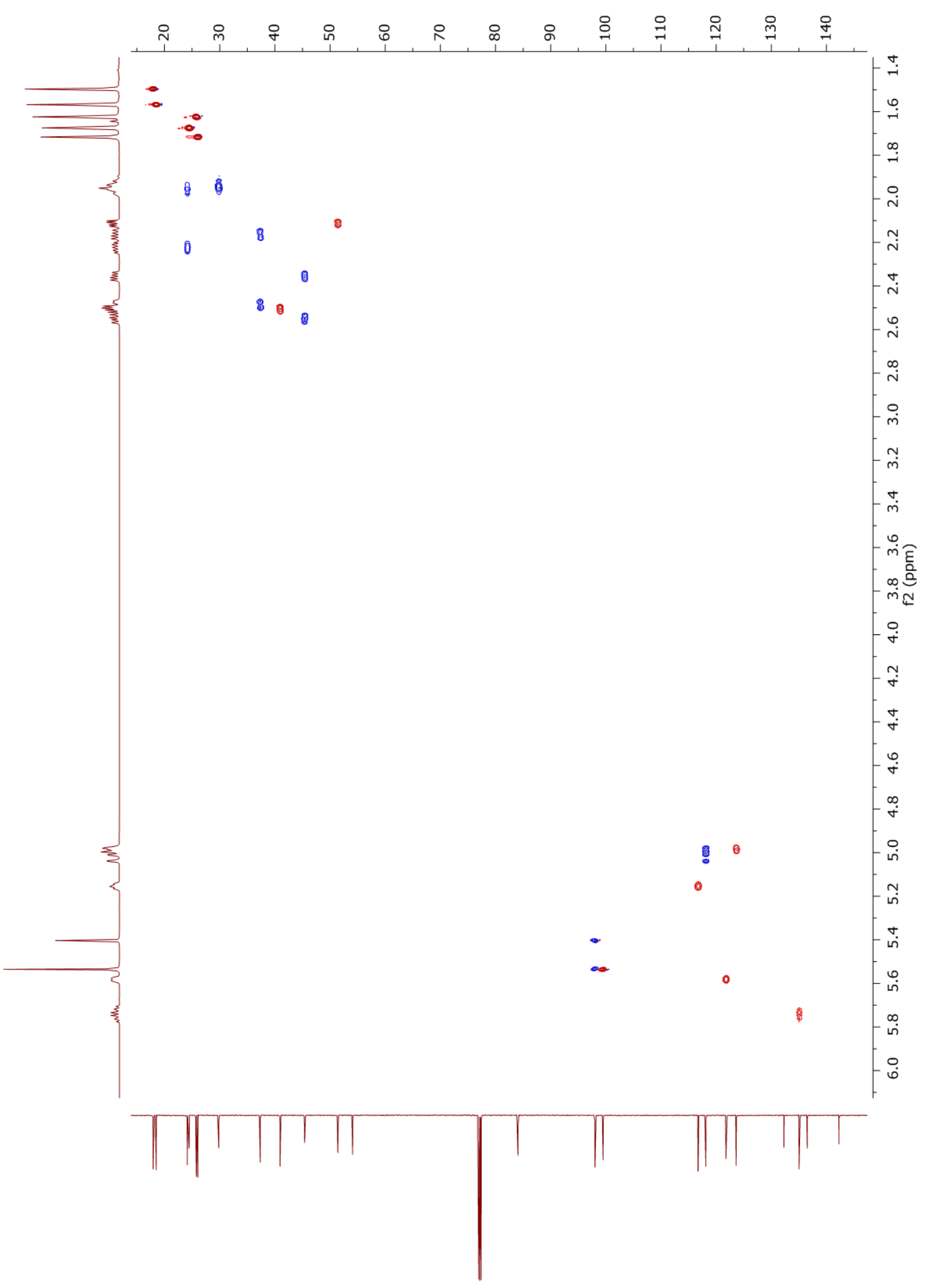


${ }^{1} \mathrm{H}-{ }^{13} \mathrm{C}$ HMBC Spectrum of $( \pm)$-Cycloillicinone 1.6

(udd) If

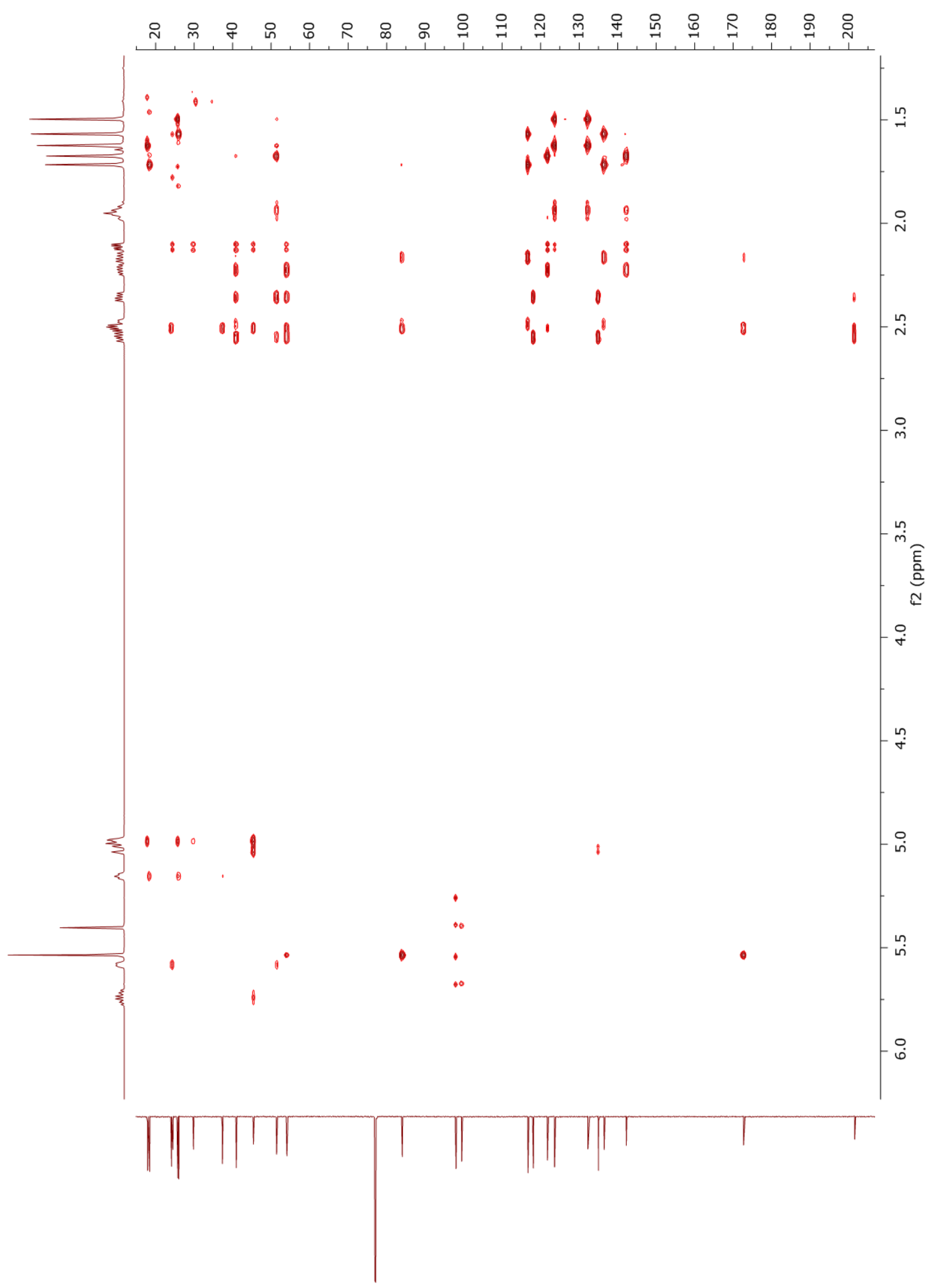


${ }^{1}$ H NMR Spectrum of ( \pm )-epi-Illicinone C 3.79 and ( \pm )-Illicinone C 3.78

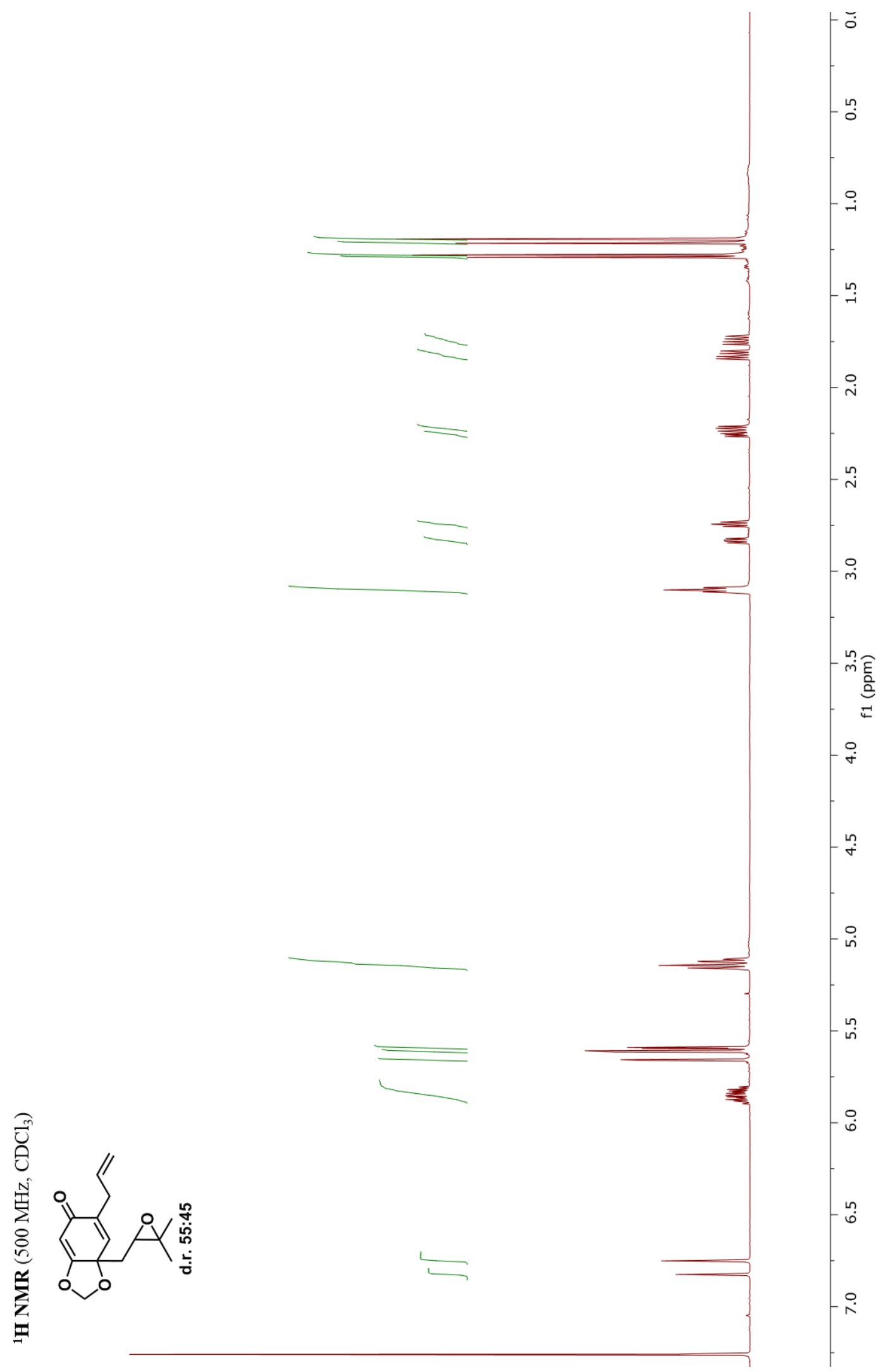


${ }^{1}$ H NMR Spectrum of ( \pm )-epi-Illicinone C 3.79

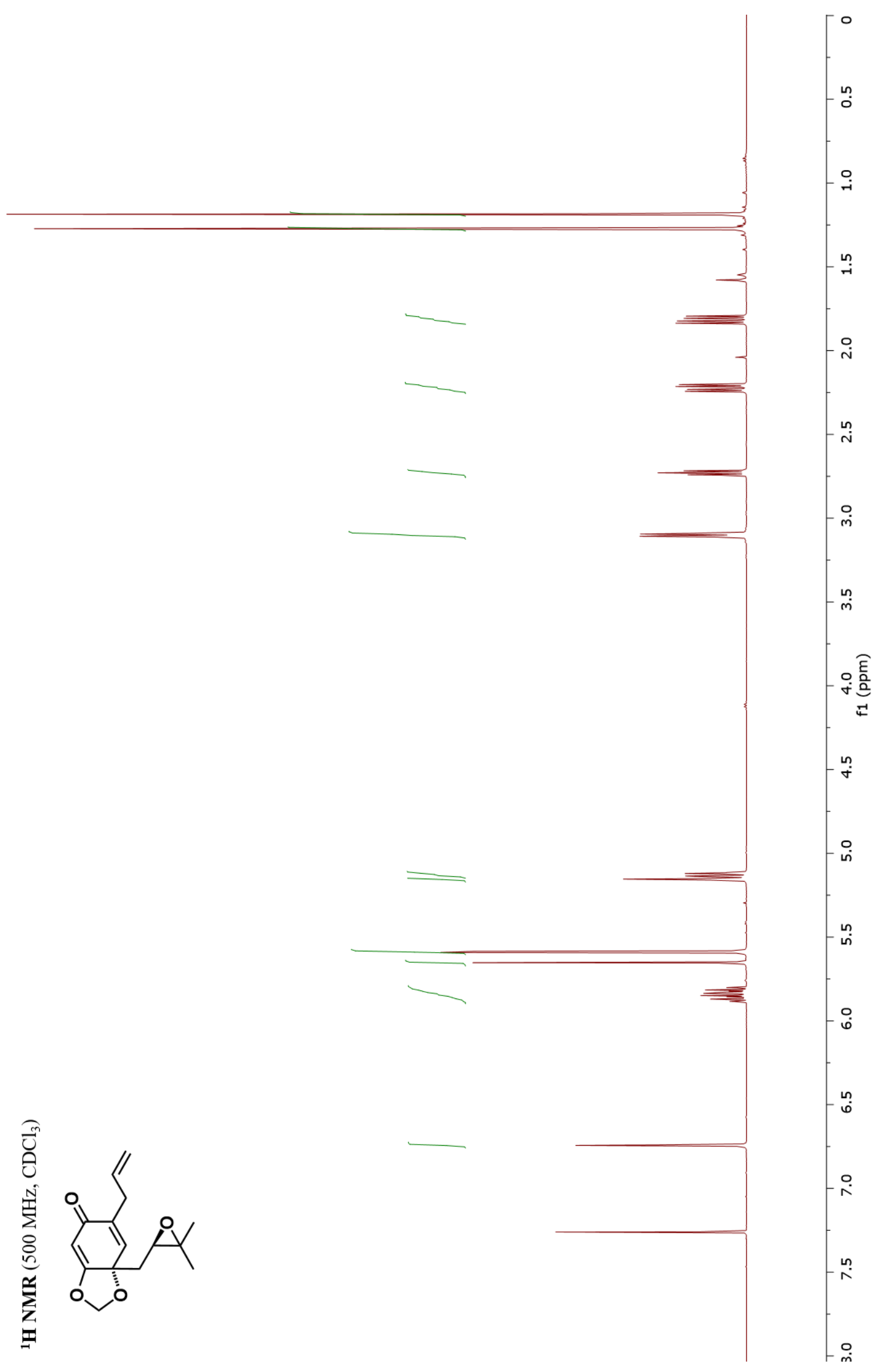


${ }^{13}$ C NMR Spectrum of ( \pm )-epi-Illicinone C 3.79

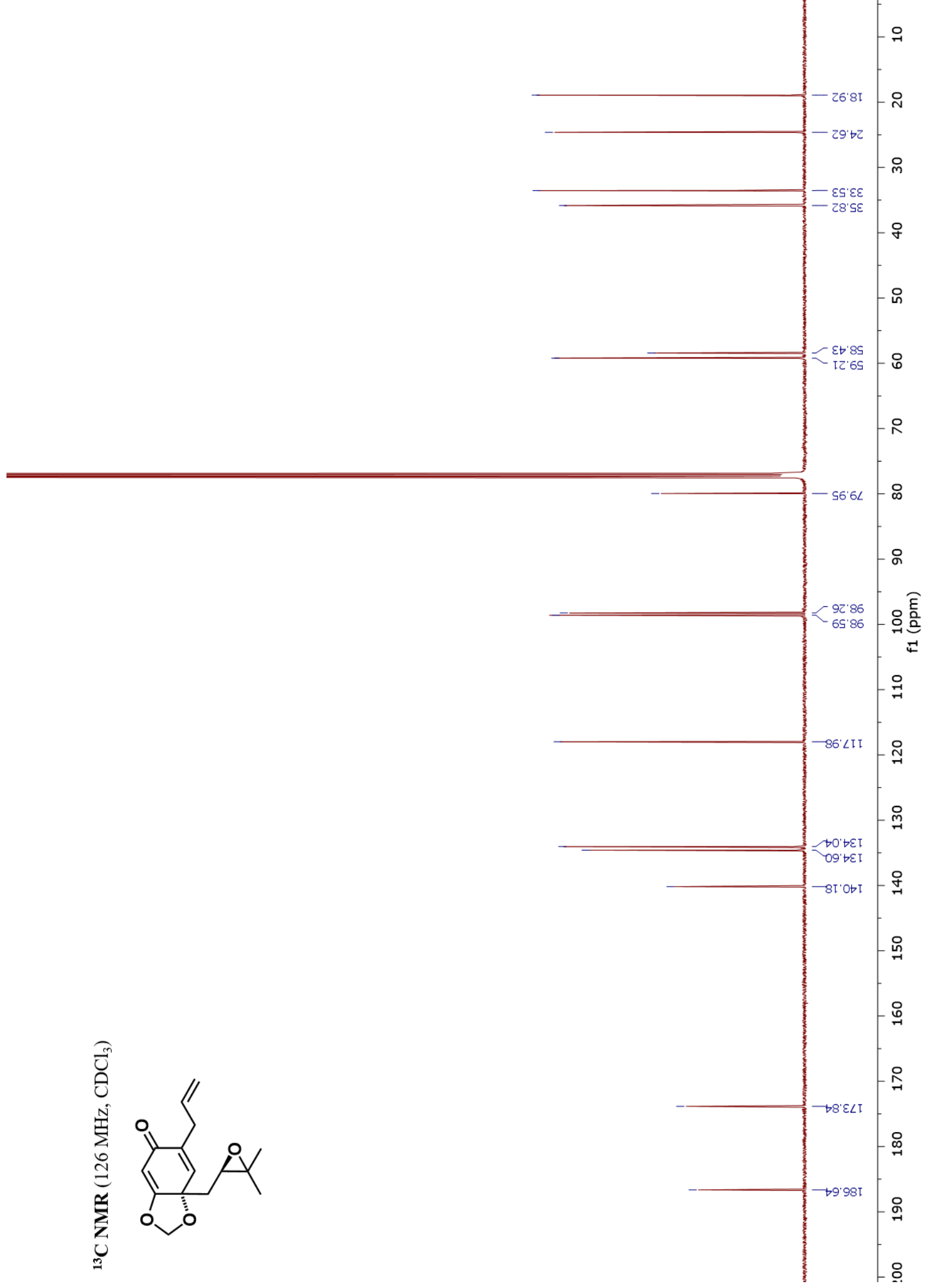


${ }^{1} \mathrm{H}$ NMR Spectrum of ( \pm )-Illicinone C 3.78

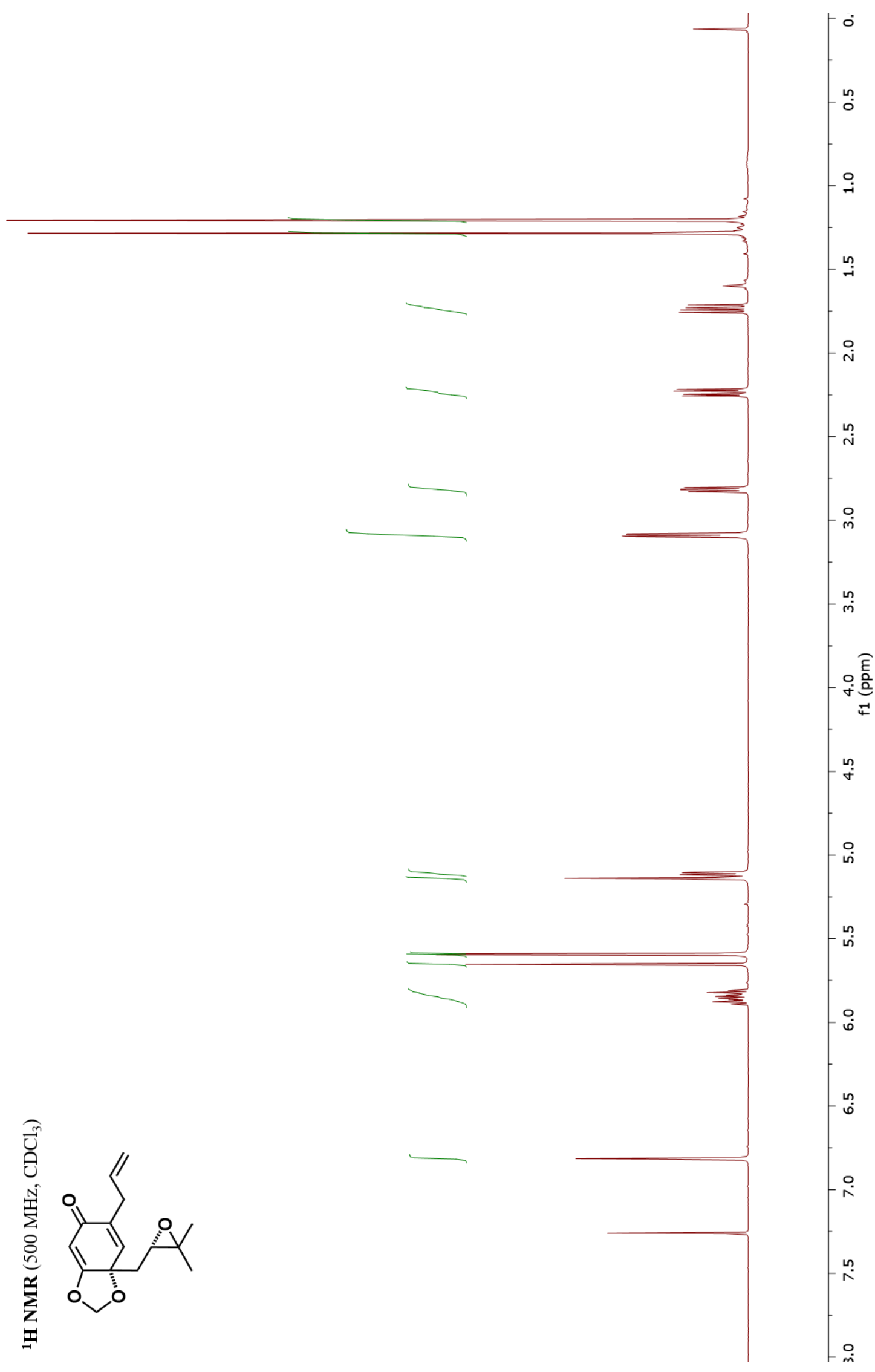


${ }^{13}$ C NMR Spectrum of ( \pm )-Illicinone C 3.78
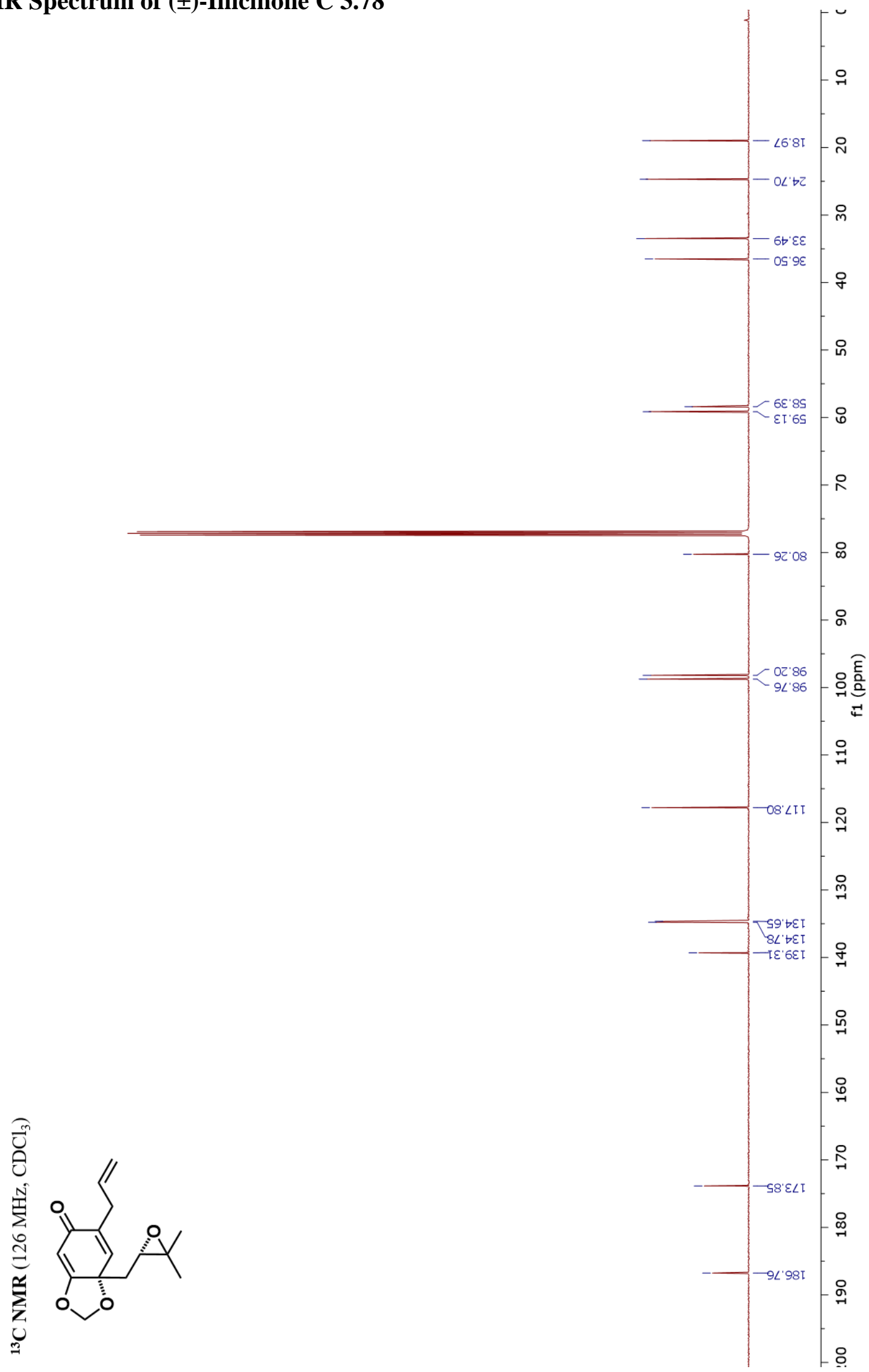
${ }^{1} \mathrm{H}$ NMR Spectrum of Compound ( \pm )-3.83

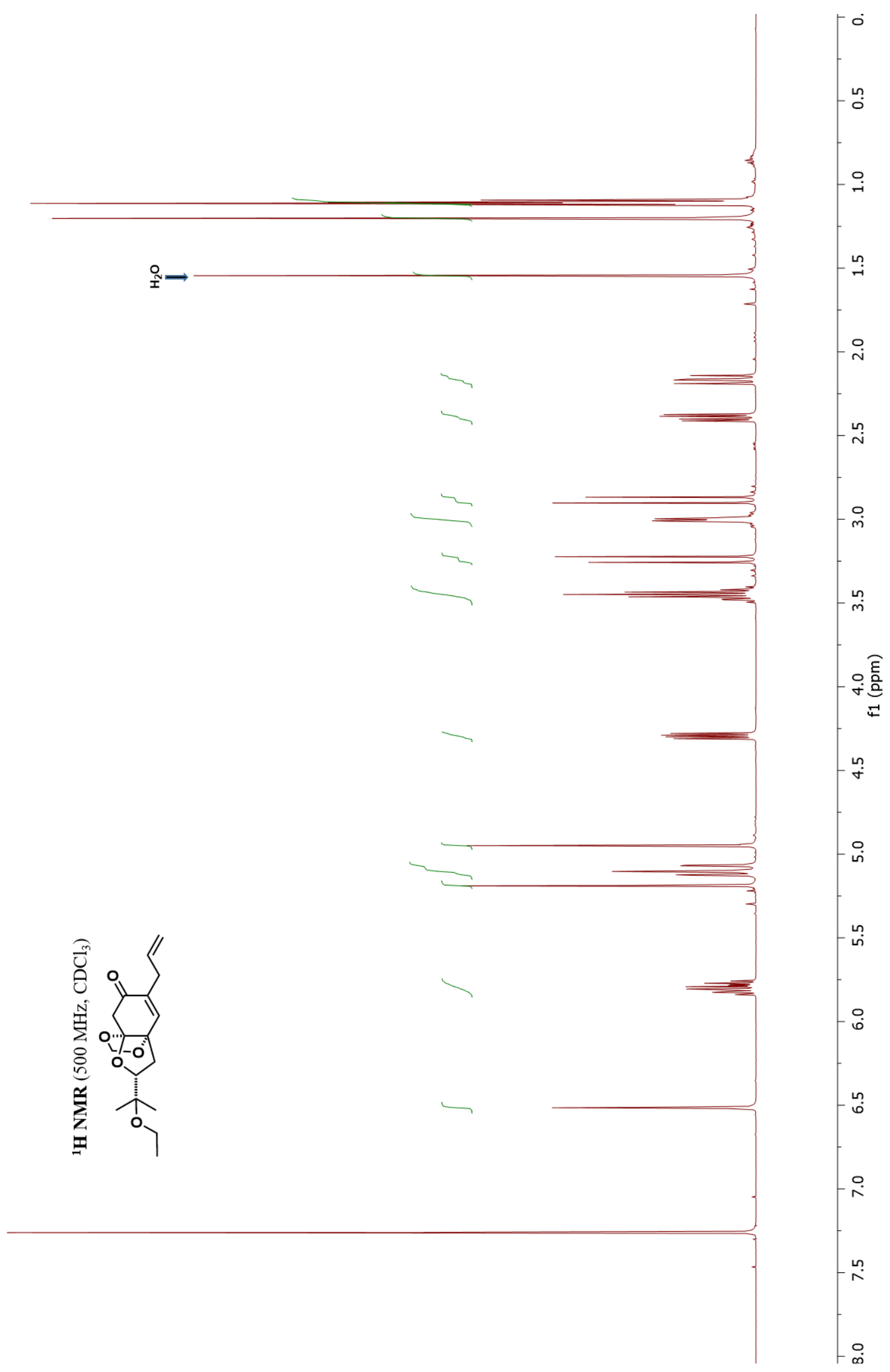


${ }^{13}$ C NMR Spectrum of Compound ( \pm )-3.83

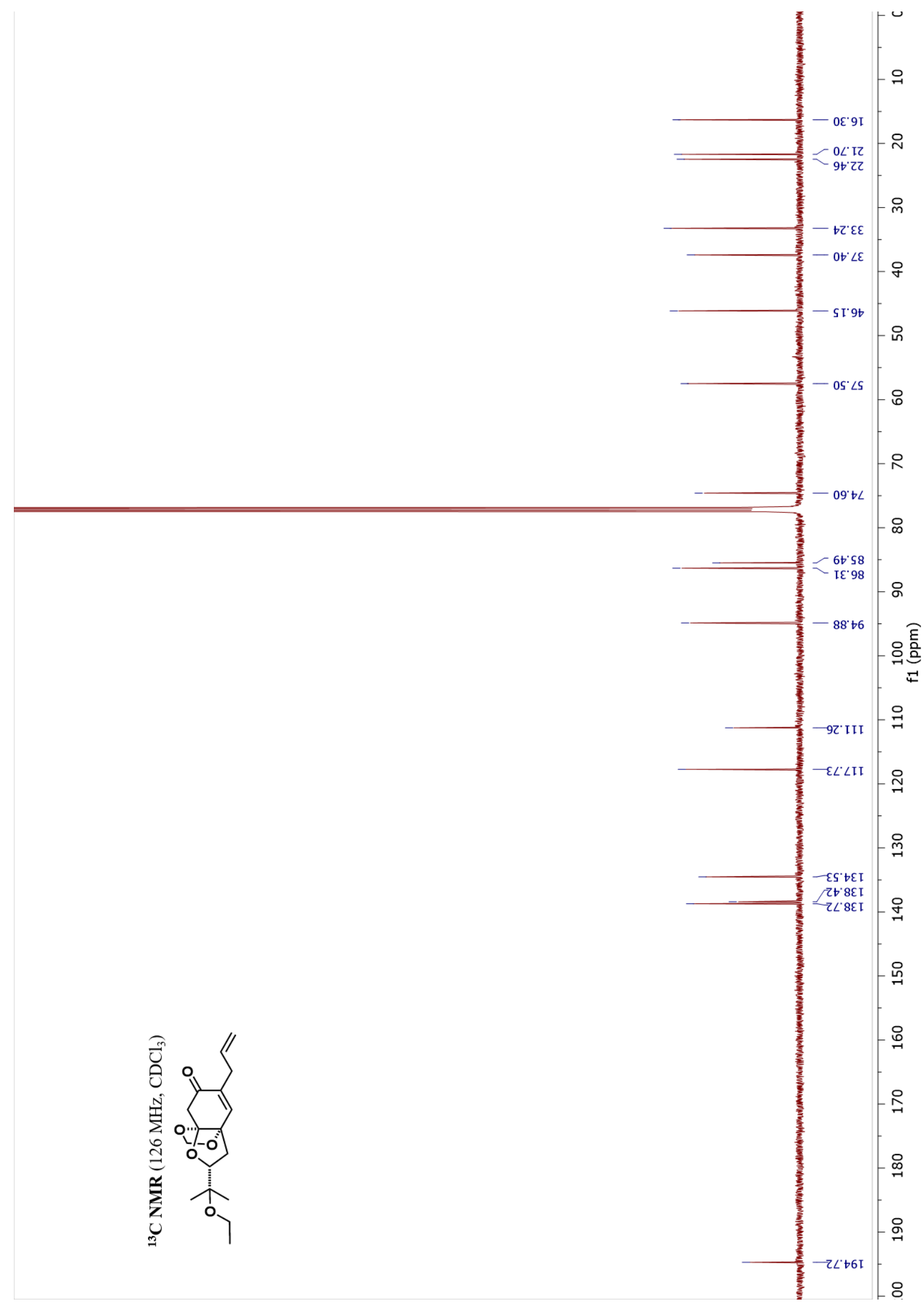


${ }^{1}$ H NMR Spectrum of ( \pm -epi-Illicinone E 3.2

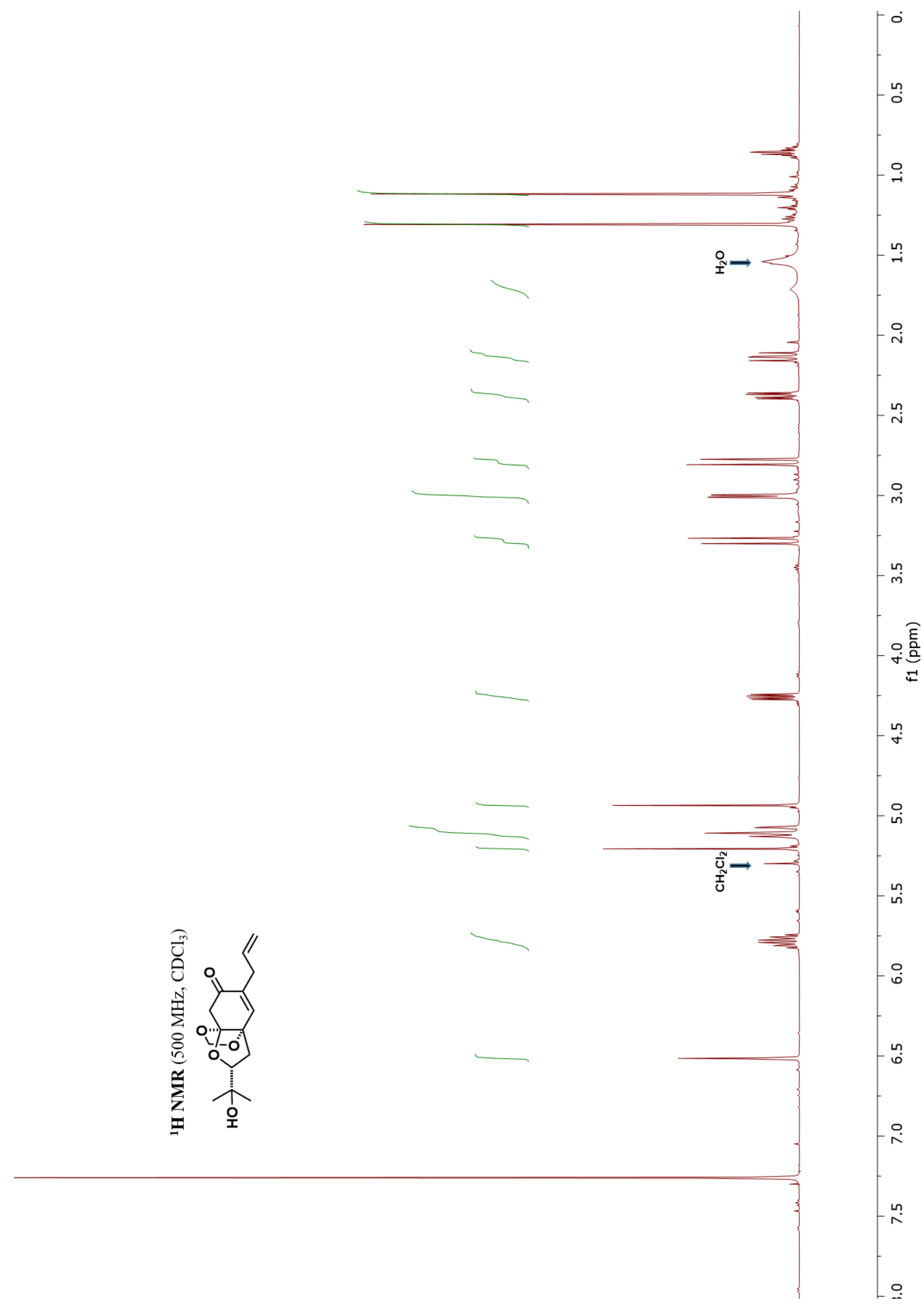


${ }^{13}$ C NMR Spectrum of ( \pm )-epi-Illicinone E 3.2

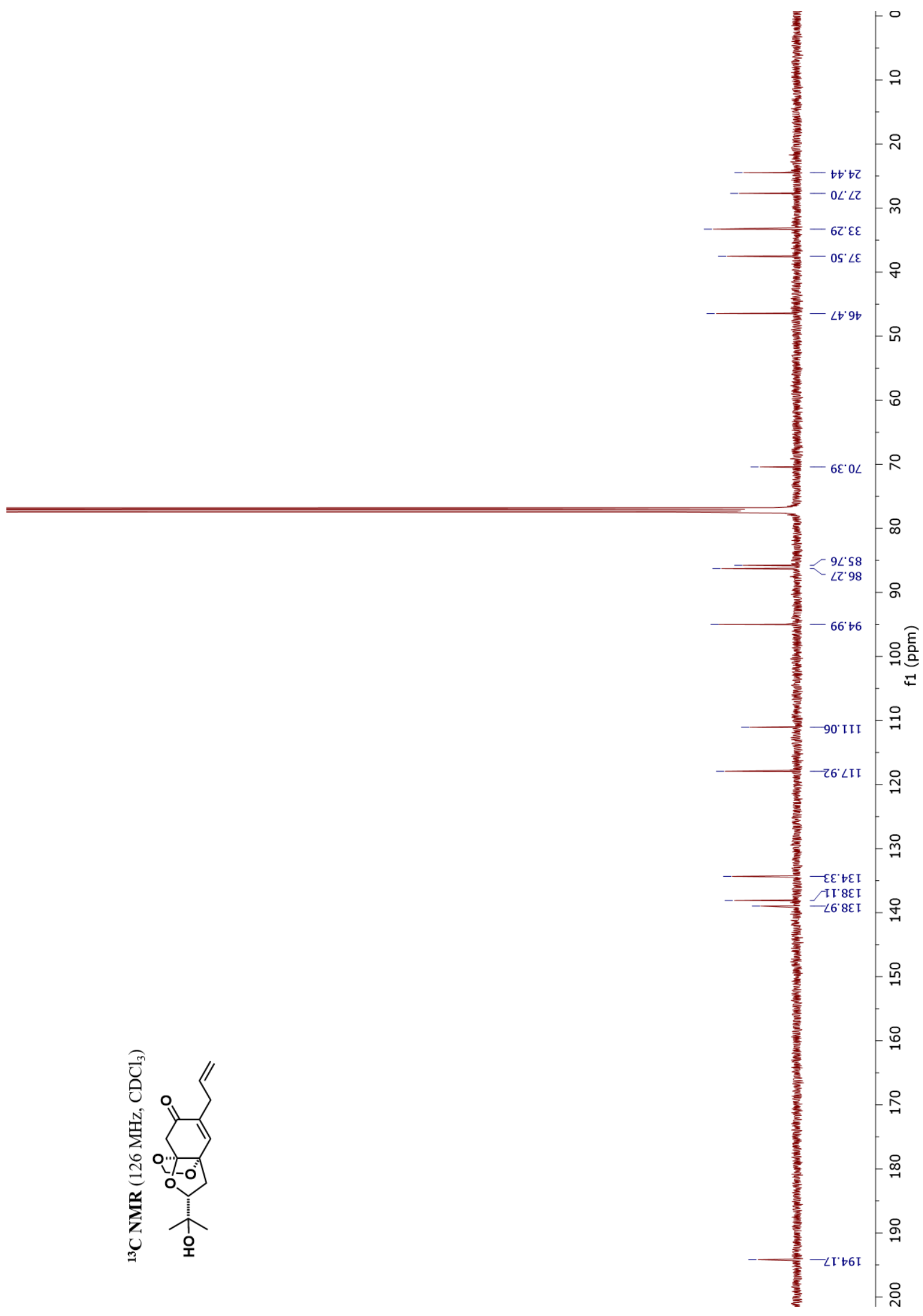


${ }^{1} \mathrm{H}-{ }^{1} \mathrm{H}$ COSY Spectrum of ( \pm )-epi-Illicinone E 3.2

(udd) It

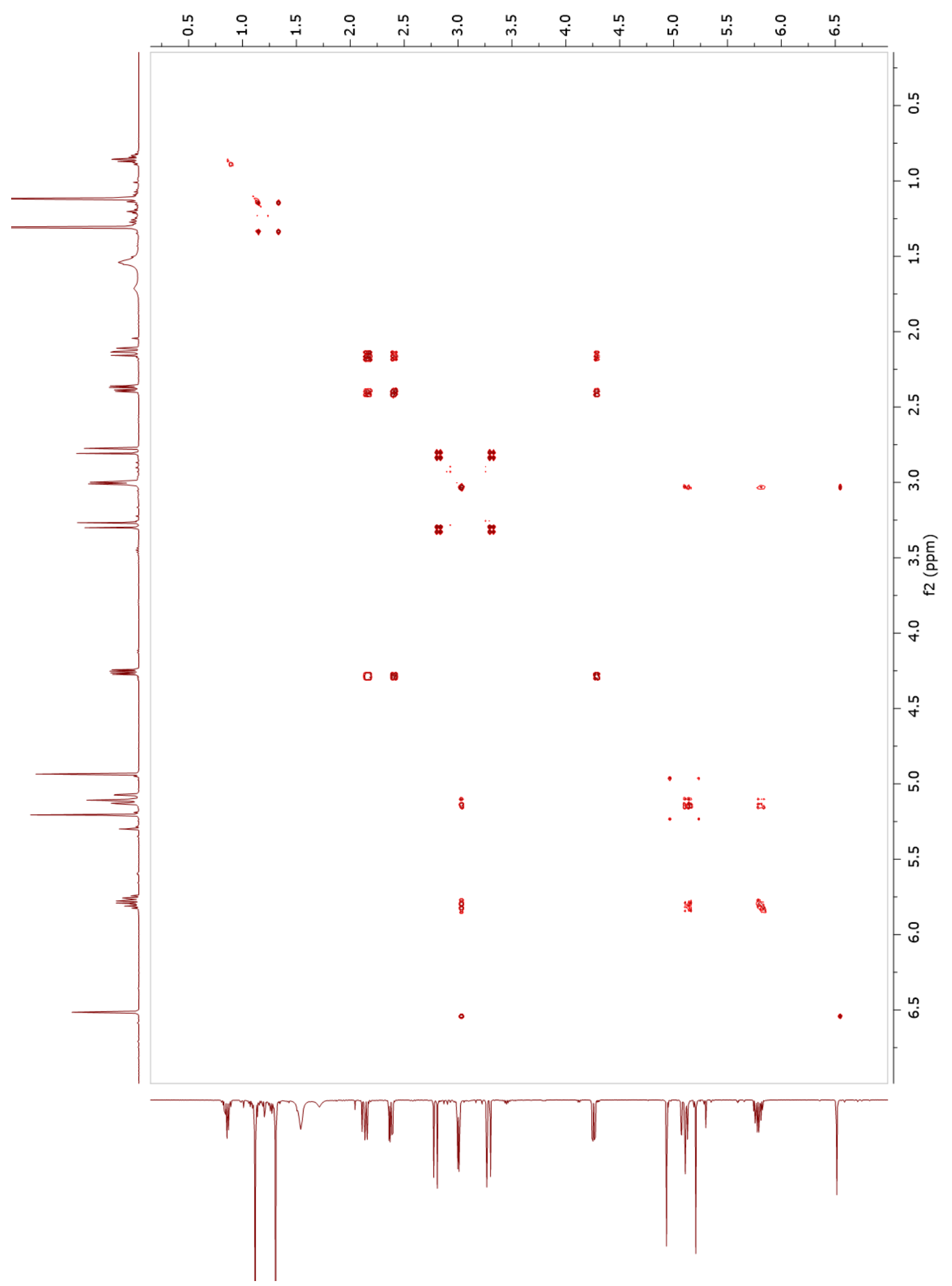


${ }^{1} \mathrm{H}-{ }^{13} \mathrm{C}$ HSQC Spectrum of ( \pm )-epi-Illicinone E 3.2

(udd) If

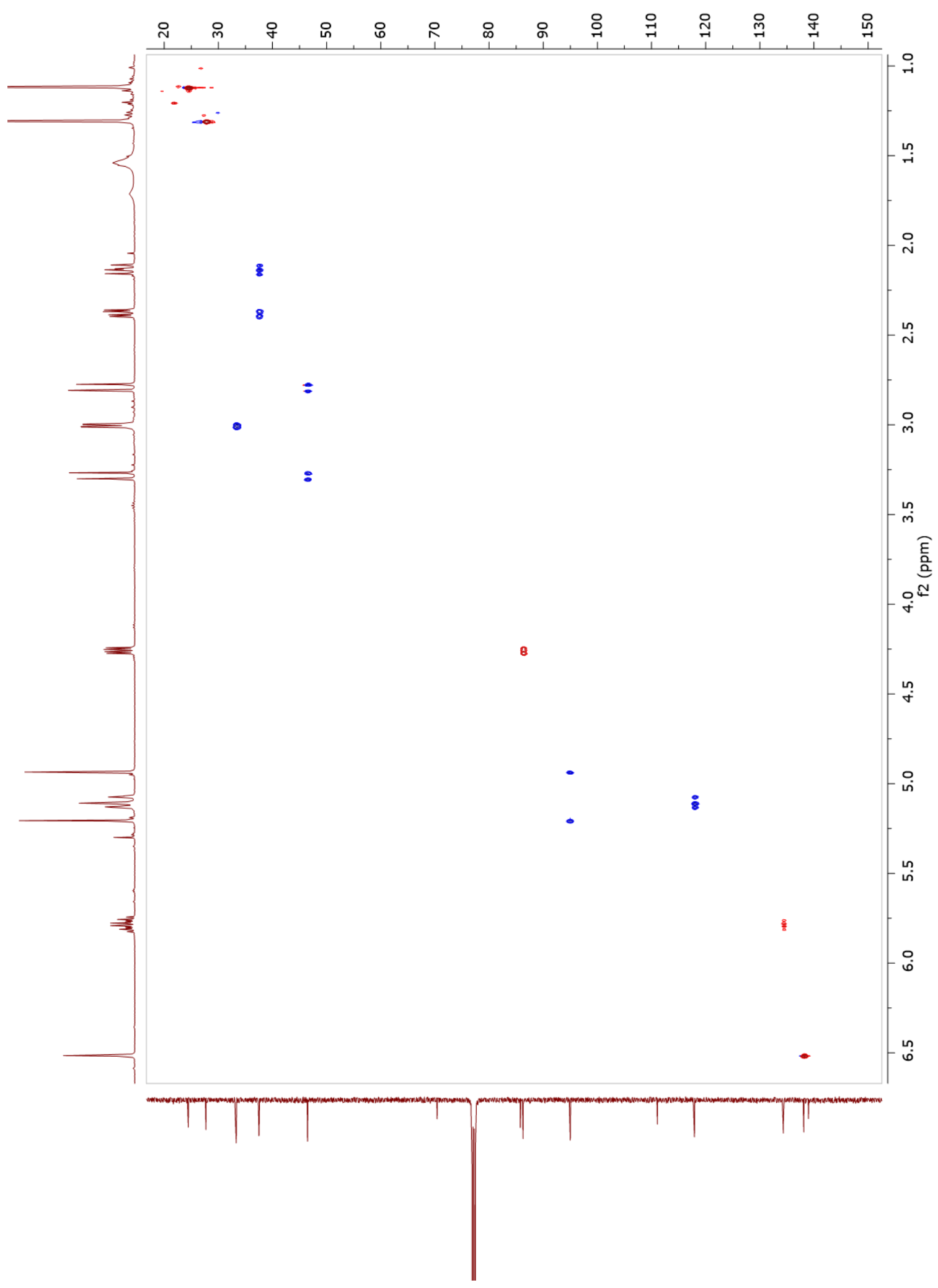


${ }^{1} \mathrm{H}-{ }^{13} \mathrm{C}$ HMBC Spectrum of ( \pm -epi-Illicinone E 3.2

(udd) It

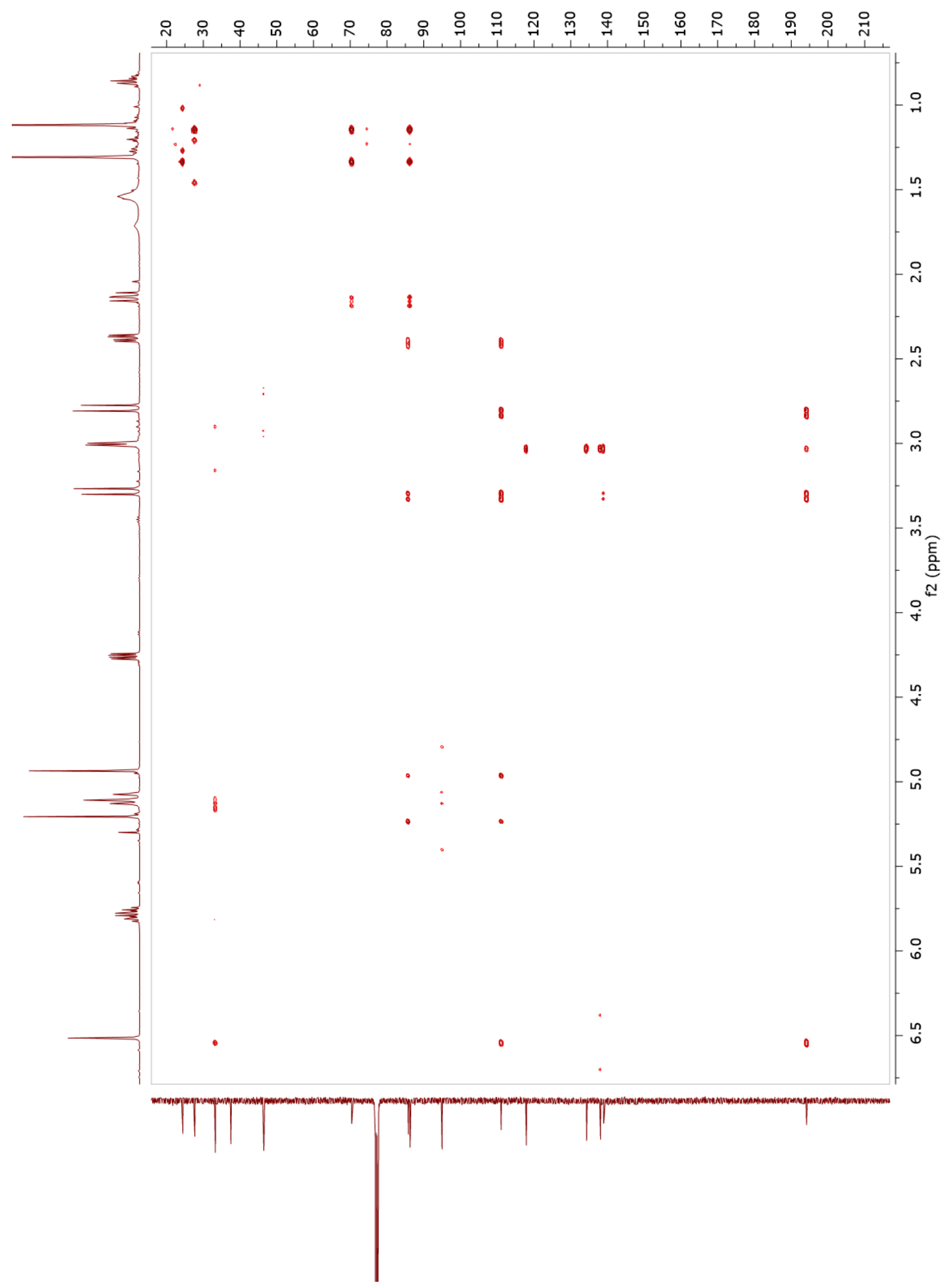


${ }^{1}$ H NMR Spectrum of Compound ( \pm )-3.84

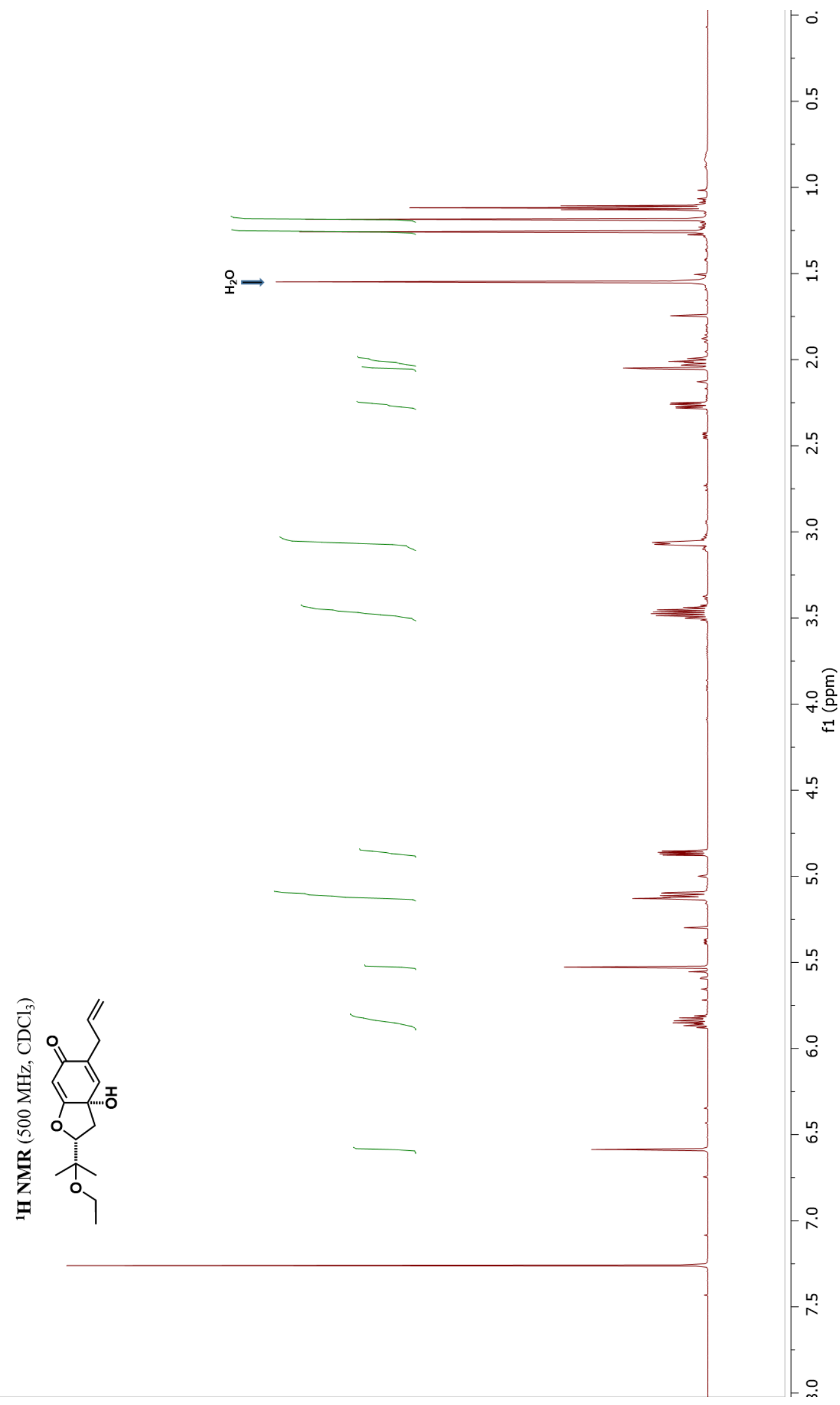


${ }^{13}$ C NMR Spectrum of Compound ( \pm )-3.84

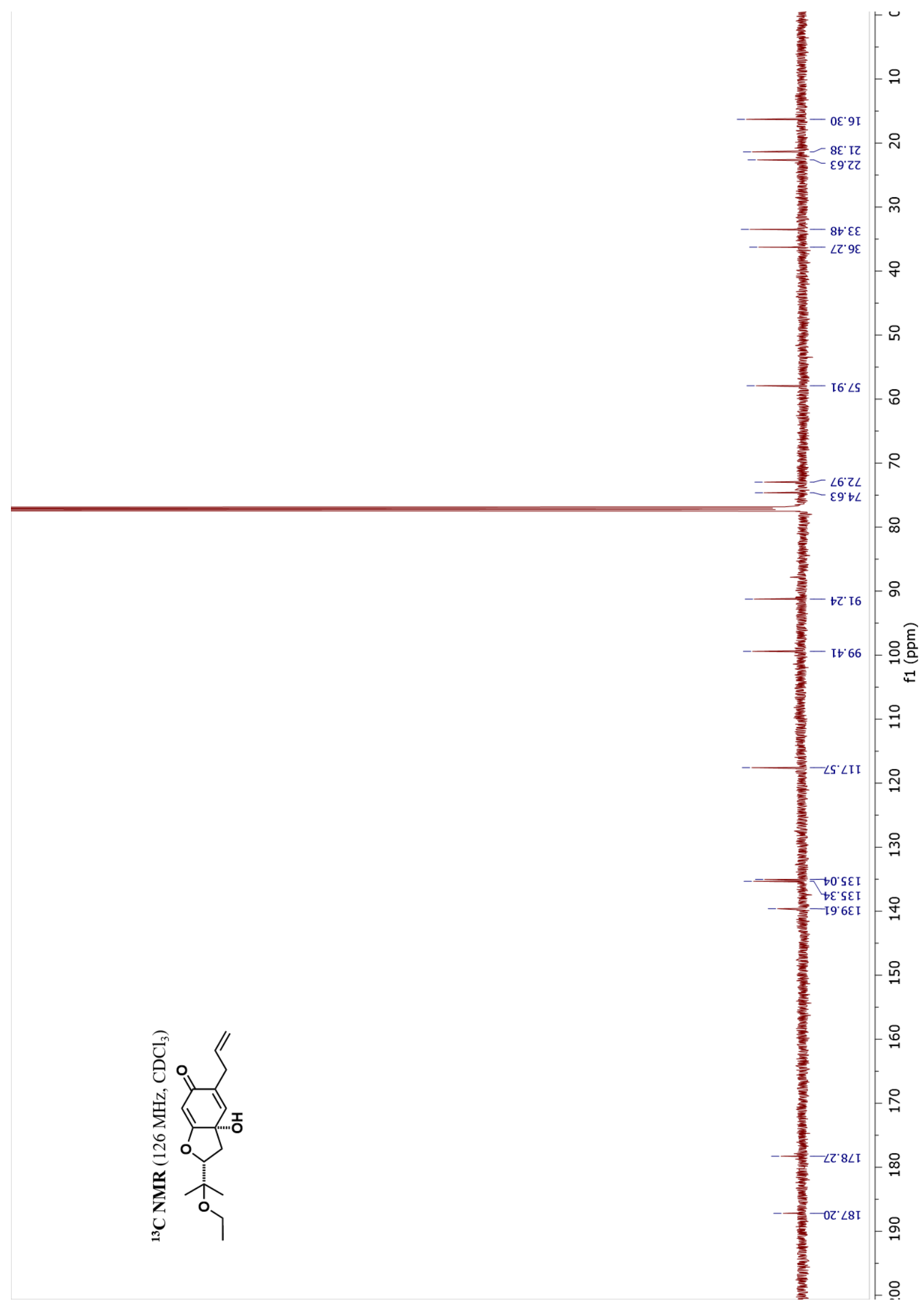


${ }^{1} \mathrm{H}$ NMR Spectrum of Compound ( \pm )-3.85

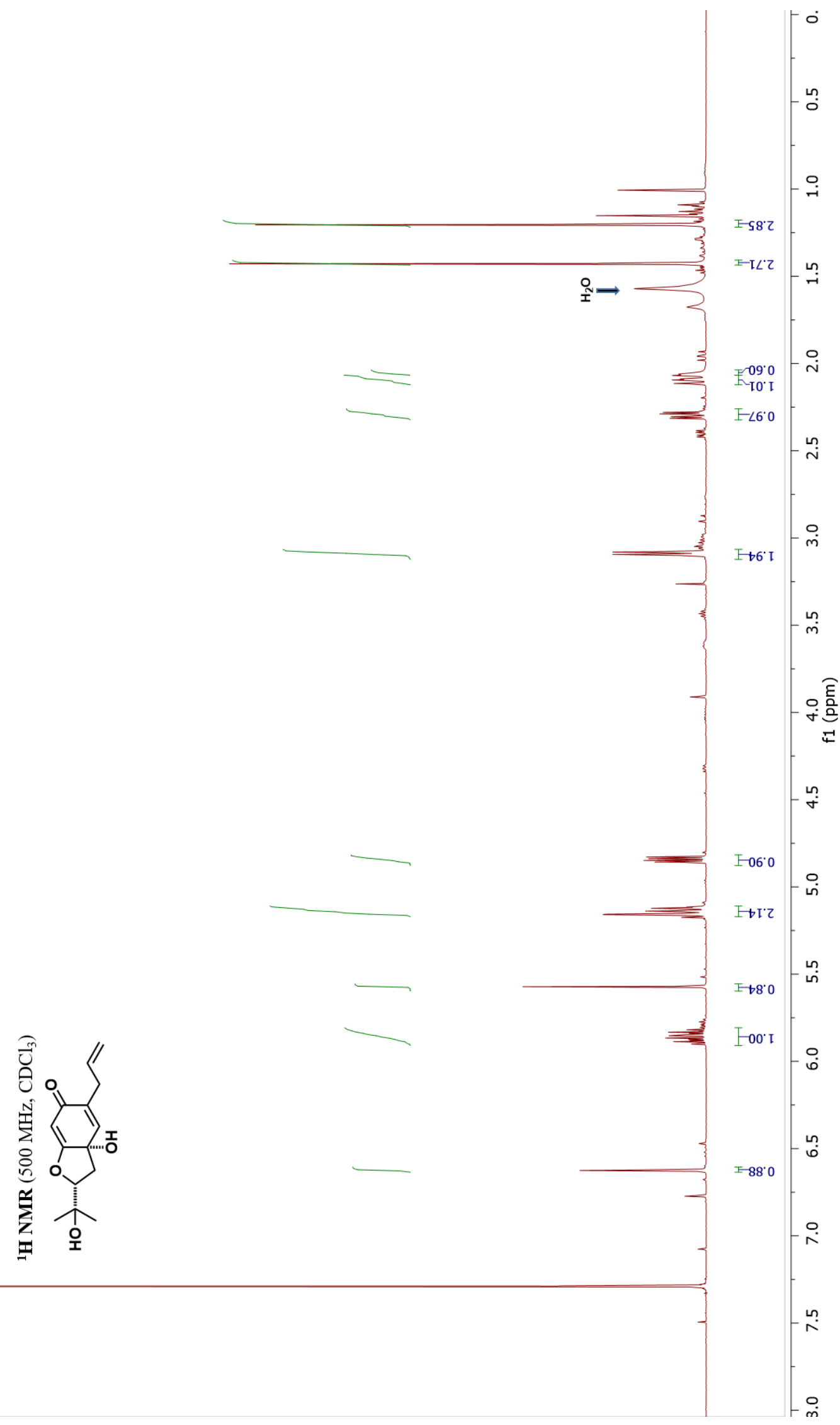


${ }^{1} \mathrm{H}$ NMR Spectrum of Compound ( \pm )-3.87

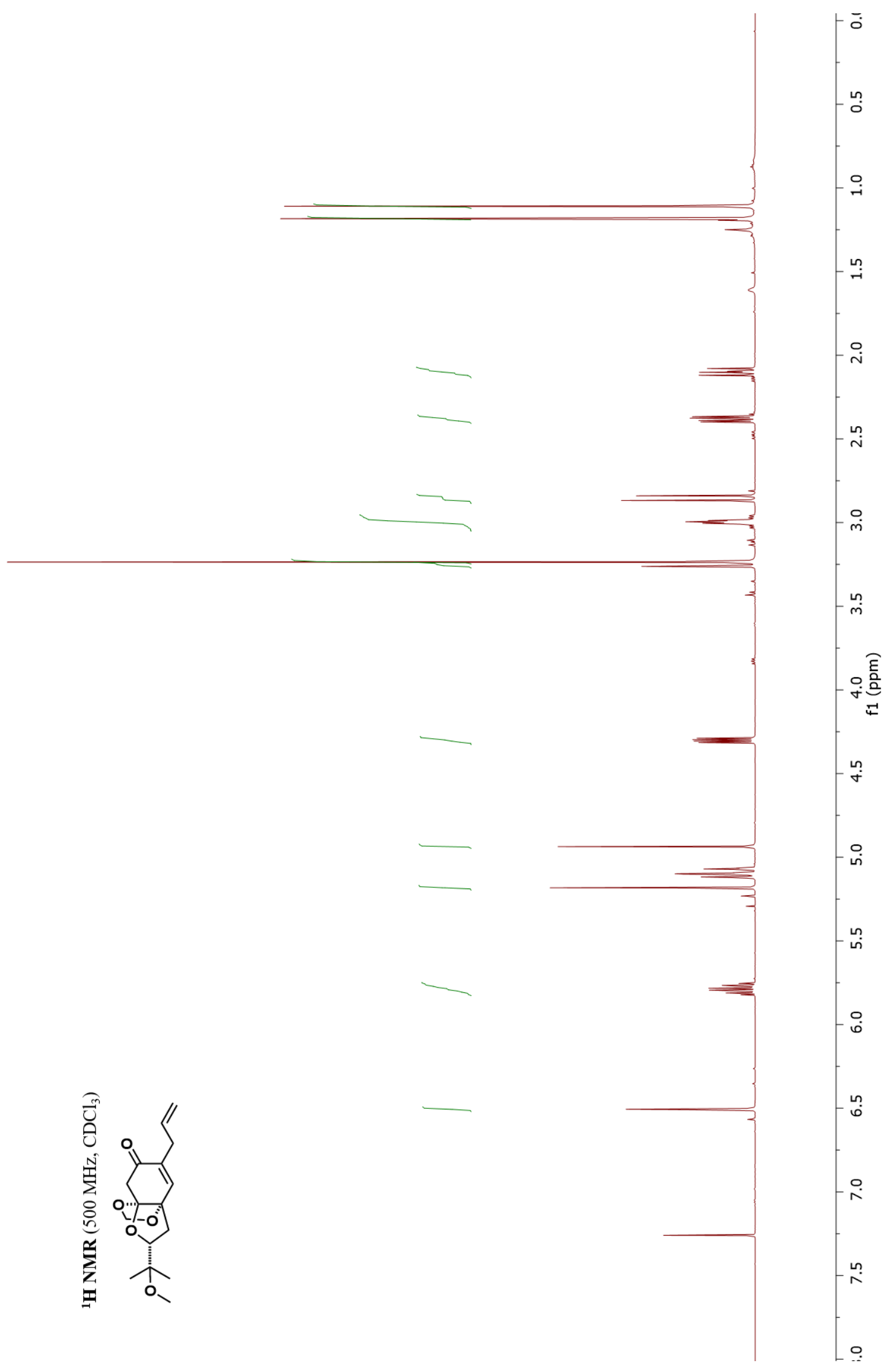


${ }^{13}$ C NMR Spectrum of Compound ( \pm )-3.87

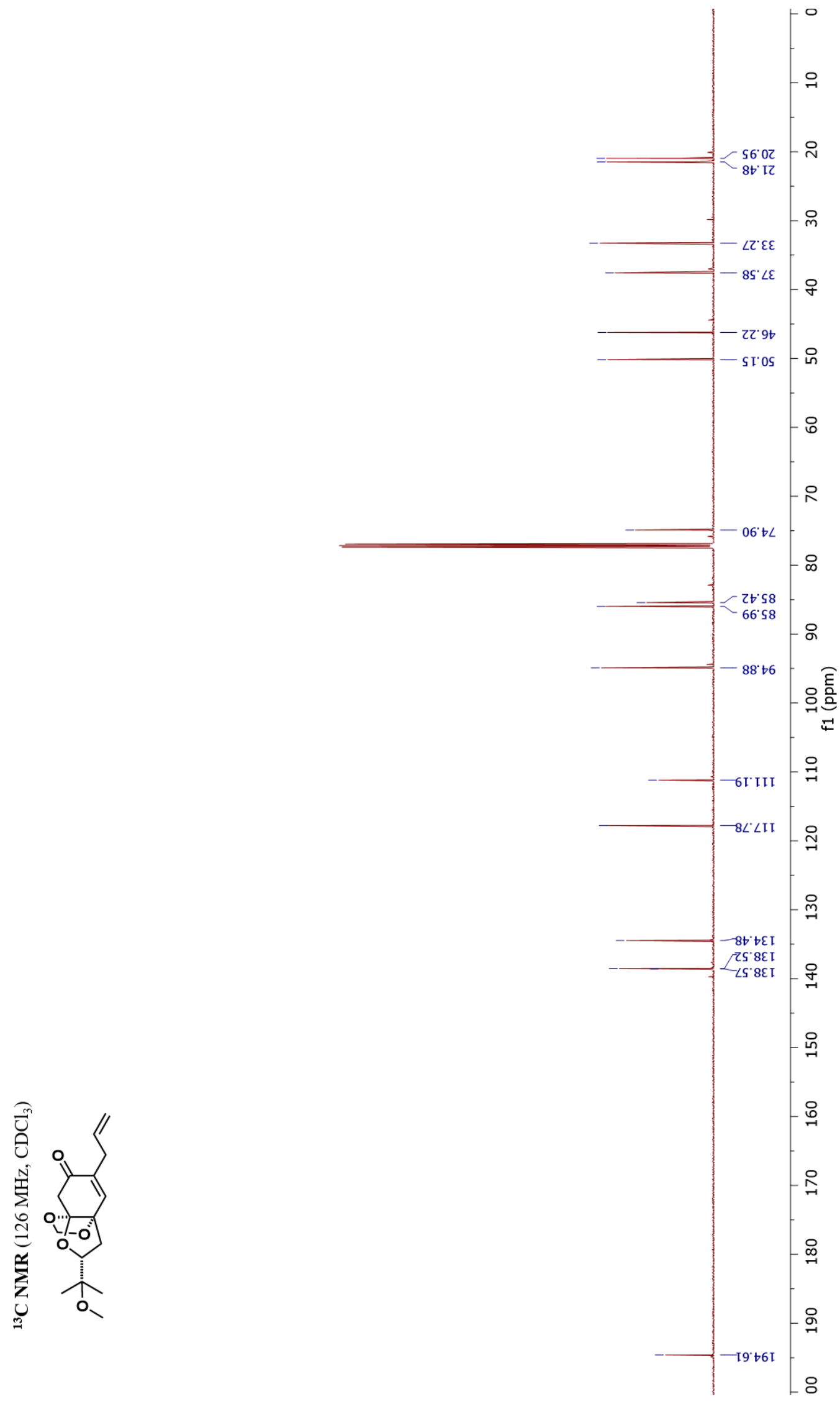




\subsection{Computational Studies (Dr Fernanda Duarte)}

\subsubsection{Computational Methods}

Calculations were performed with Gaussian09 rev. E. $01^{166}$ using the long-range corrected hybrid $\omega$ B97X-D density functional, ${ }^{167}$ which incorporates a force-field-like pairwise dispersion correction term developed by Grimme, ${ }^{168}$ in combination with the 6-31+G(d) basis set. Calculations were repeated using the 6-311++G(d,p) basis set to ensure that geometries and energies are not affected by the selection of a smaller basis set. All stationary points were verified as either minima or saddle points by the presence of zero or a single imaginary harmonic vibrational frequency, respectively. In all cases, a quasi-rigid-rotor-harmonic-oscillator approximation was used in which the treatment of vibrational entropies switches from the standard rigid-rotor/harmonicoscillator (RRHO) model to a free rotor description for frequencies below $100 \mathrm{~cm}^{-1}$, as first proposed by Grimme, ${ }^{169}$ and implemented in Python. ${ }^{170}$ Relative free energies were evaluated at $353 \mathrm{~K}$.

A two-dimensional scan of the potential energy surface (PES) was performed on the model $p$ - $N$-cis system, constraining the forming $\mathrm{C} \cdots \mathrm{C}$ and $\mathrm{C} \cdots \mathrm{O}$ bonds in increments of $0.1 \AA$ and $0.2 \AA$, respectively. This surface allowed us to locate the bis-pericyclic Diels-Alder and the [3,3]-sigmatropic rearrangement transition states $\left(\mathrm{TS}_{1}\right.$ and $\mathrm{TS}_{2}$, respectively), which were then optimised without any constraint to obtain the corresponding transition state (TS), reactant (RS) and product (PS) complexes.

\subsubsection{Benchmark Study}

Using a simplified model bearing no ortho-substituent (hereinafter referred to as $\mathbf{2 . 8 1}$ ) we explored the transition state (TS) structures for each regioisomeric outcome of the Diels-Alder (DA) dimerisation. For this system, there are eight distinct TSs possible; Described using a notation where the regiochemical-orientation is meta $(\mathrm{m})$ or para $(p)$, the Alder-Stein mode is endo $(N)$ or exo $(X)$, and the dienophile adopts an s-cis (cis) or s-trans (trans) conformation. Energies obtained at the $\omega \mathrm{B} 97 \mathrm{X}-\mathrm{D} / 6-31+\mathrm{G}(\mathrm{d})$, were compared to those obtained using the $6-311++\mathrm{G}(\mathrm{d}, \mathrm{p})$ basis set (optimisation and energy calculations). Both sets of energies lead to the same preference and relative 
ordering, with the latter being about $1.5 \mathrm{kcal} \mathrm{mol}^{-1}$ higher in energy. Single point calculations using the $6-311++\mathrm{G}(\mathrm{d}, \mathrm{p})$ basis set on the lower level optimised structures lead to slightly higher values to the ones obtained at the $\omega \mathrm{B} 97 \mathrm{X}-\mathrm{D} / 6$ $311++\mathrm{G}(\mathrm{d}, \mathrm{p}) / / \omega \mathrm{B} 97 \mathrm{X}-\mathrm{D} / 6-311++\mathrm{G}(\mathrm{d}, \mathrm{p})$, but once again leading to the same preference. Given these results, and the computational cost associated with them, we

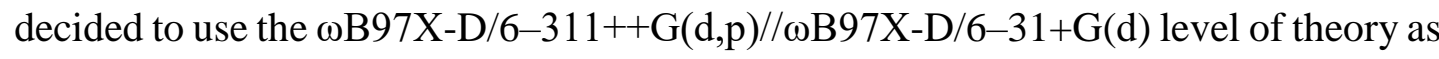
a good compromise between accuracy and computational cost for further analysis.

Table 4.1: Reaction free energies for the dimerisation of the model enal 2.81 (endo arrangement) at different levels of theory using the $\omega \mathrm{B} 97 \mathrm{X}-\mathrm{D}$ functional and the $6-31+\mathrm{G}(\mathrm{d})$ and $6-311++\mathrm{G}(\mathrm{d}, \mathrm{p})$ basis sets. TS and PS denote transition and product states, respectively. OPT and SP denote optimisation and single point energy using the indicated basis set. All energies are in $\mathrm{kcal} \mathrm{mol}^{-1}$ relative to the independent trans-reactant).

\begin{tabular}{|c|c|c|c|c|}
\hline Systems & & $\begin{array}{c}\mathrm{OPT}+\mathrm{SP} \\
6-31+\mathrm{G}(\mathrm{d}) \\
\end{array}$ & $\begin{array}{c}\mathrm{SP} \\
6-311++\mathrm{G}(\mathrm{d}, \mathrm{p}) \\
\end{array}$ & $\begin{array}{c}\mathrm{OPT}+\mathrm{SP} \\
6-311++\mathrm{G}(\mathrm{d}, \mathrm{p}) \\
\end{array}$ \\
\hline \multirow{2}{*}{$p$-N-trans } & $\Delta \mathrm{G}^{\ddagger}$ & 35.7 & 37.2 & 36.5 \\
\hline & $\Delta \mathrm{G}^{0}$ & -7.8 & -5.4 & -6.2 \\
\hline \multirow{2}{*}{$p-N$-cis } & $\Delta \mathrm{G}^{\ddagger}$ & 29.3 & 31.1 & 31.9 \\
\hline & $\Delta \mathrm{G}^{0}$ & -7.8 & -5.4 & -6.1 \\
\hline \multirow{2}{*}{$m$-N-trans } & $\Delta \mathrm{G}^{\ddagger}$ & 42.6 & 44.1 & 43.6 \\
\hline & $\Delta \mathrm{G}^{0}$ & -1.2 & 0.8 & -2.1 \\
\hline \multirow{2}{*}{$m-N-c i s$} & $\Delta \mathrm{G}^{\ddagger}$ & 41.5 & 43.0 & 42.3 \\
\hline & $\Delta \mathrm{G}^{0}$ & -4.1 & -1.8 & -2.7 \\
\hline
\end{tabular}

\subsubsection{Regioselectivity}

Eight different potential TS were obtained and characterised at the $\omega \mathrm{B} 97 \mathrm{X}-\mathrm{D} / 6$ $31+\mathrm{G}(\mathrm{d}) / / \omega \mathrm{B} 97 \mathrm{X}-\mathrm{D} / 6-31+\mathrm{G}(\mathrm{d})$ level of theory (figure 4.1 and table 4.2). The regioselectivity for this process takes place under kinetic control, in view of the lower activation barrier computed for the formation of the $\mathrm{p}-\mathrm{N}$-cis adduct. The energy barrier for this pathway is $\Delta \mathrm{G}^{+}=31.0 \mathrm{kcal} \mathrm{mol}^{-1}$ and is $2.2 \mathrm{kcal} \mathrm{mol}^{-1}$ lower than the analogous $p$-X-cis pathway. This value agree well with the one obtained for the dimerisation of acrolein under similar conditions with the experimental $\left(\Delta \mathrm{G}^{*}=31.6 \mathrm{kcal} \mathrm{mol}^{-1}\right) .{ }^{171}$ 


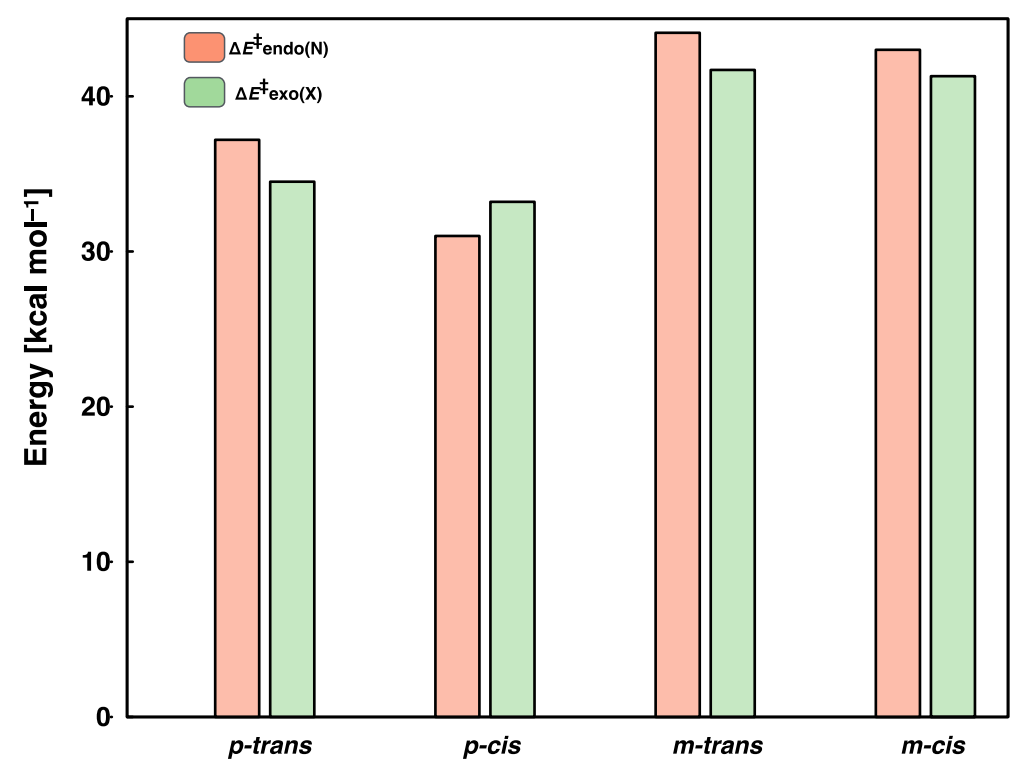

Figure 4.1: Activation free energies $\left(\mathrm{kcal} \mathrm{mol}^{-1}\right)$ at the $\omega \mathrm{B} 97 \mathrm{X}-\mathrm{D} / 6-311++\mathrm{G}(\mathrm{d}, \mathrm{p}) / / \omega \mathrm{B} 97 \mathrm{X}-\mathrm{D} / 6-$ $31+\mathrm{G}(\mathrm{d})$ level of theory for the eight different potential TSs obtained for the hetero-Diels-Alder dimerisation of the model enal 2.81. Values are provided in table $4.2\left(\Delta \mathrm{G}^{\ddagger}\right.$ corr $)$.

Table 4.2: Reaction energies for the dimerisation process of the model enal 2.81 at the $\omega B$ 97X-D/6$31+\mathrm{G}(\mathrm{d})$ level of theory and at the $\omega \mathrm{B} 97 \mathrm{X}-\mathrm{D} / 6-311++\mathrm{G}(\mathrm{d}, \mathrm{p}) / / \omega \mathrm{B} 97 \mathrm{X}-\mathrm{D} / 6-31+\mathrm{G}(\mathrm{d})$ level of theory $\left(\Delta \mathrm{G}_{\text {corr }}^{\dagger}\right.$ ). All energies are in $\mathrm{kcal} \mathrm{mol}^{-1}$ relative to the independent lower energy reactant at $353 \mathrm{~K}$. See table 4.7 for absolute values.

\begin{tabular}{|c|c|c|c|c|c|c|c|c|}
\hline & $\Delta \mathbf{E}^{*}$ el & ZPE & $\Delta \mathbf{H}^{*}$ & $\mathbf{T} \Delta \mathbf{S}^{*}$ & $\Delta \mathbf{G}^{\ddagger}$ & $\Delta \mathbf{G}^{\mathbf{0}}$ & $\Delta \mathbf{G}^{\ddagger}$ corr & $\Delta \mathbf{G}^{\mathbf{0}}$ corr \\
\hline$p$-N-trans & 15.3 & 2.3 & 16.9 & -18.9 & 35.7 & -7.8 & 37.2 & -5.4 \\
\hline$p$-X-trans & 12.6 & 2.3 & 14.1 & -19.2 & 33.3 & -7.7 & 34.5 & -5.2 \\
\hline$p-N-c i s$ & 9.0 & 2.0 & 10.3 & -19.0 & 29.3 & -7.8 & 31.0 & -5.4 \\
\hline$p$-X-cis & 10.8 & 2.6 & 12.5 & -19.5 & 32.0 & -7.3 & 33.2 & -4.8 \\
\hline$m$-N-trans & 22.0 & 2.1 & 23.3 & -19.3 & 42.6 & -1.2 & 44.1 & 0.8 \\
\hline$m$-X-trans & 19.3 & 2.6 & 20.8 & -20.1 & 40.9 & -1.5 & 41.7 & 0.6 \\
\hline$m-N$-cis & 20.8 & 2.2 & 22.3 & -19.2 & 41.5 & -4.1 & 43.0 & -1.8 \\
\hline$m-X-c i s$ & 18.9 & 2.5 & 20.4 & -19.9 & 40.4 & -3.9 & 41.3 & -1.6 \\
\hline
\end{tabular}

Noteworthy, the lowest energy TS $p$-N-cis, which displays a $C_{2}$-symetry, correspond to a bis-pericyclic TS as first discovery by Caramella et al. ${ }^{172-175}$ figure 4.2 shows the relative positions of the bis-pericyclic Diels-Alder and [3,3]-sigmatropic rearrangement transition states $\left(\mathrm{TS}_{1}\right.$ and $\mathrm{TS}_{2}$, respectively) for the model 2.81. At $\mathrm{TS}_{1}$ the $\mathrm{C} \cdots \mathrm{C}$ bond at the end of the diene moieties is $1.9 \AA$, while the symmetrical $\mathrm{C} \cdots \mathrm{O}$ 4,2 and 2,4 bonds are still rather long ( $2.84 \AA$ ). In this regard, $\mathrm{TS}_{1}$ closely follows the bis-pericyclic TS found by Caramella et al. for the dimerisation of methacrolein. ${ }^{172}$ 
After $\mathrm{TS}_{1}$ the intrinsic reaction coordinate (IRC) calculation leads toward the $[3,3]-$ rearrangement transition state $\left(\mathrm{TS}_{2}\right.$, figure 4.2b) for the interconversion of the two products. This TS is only $0.8 \mathrm{kcal} \mathrm{mol}^{-1}$ higher in energy (after inclusion of ZPE and entropy effects) and it shows a much shorter $\mathrm{C} \cdots \mathrm{C}$ bond $(1.60 \AA)$ and $\mathrm{C} \cdots \mathrm{O}$ bonds of equal length ( $2.60 \AA$ ). Given the symmetry of the system, the system can exit via either of the two degenerate products channels $[4+2] /[2+4]$.
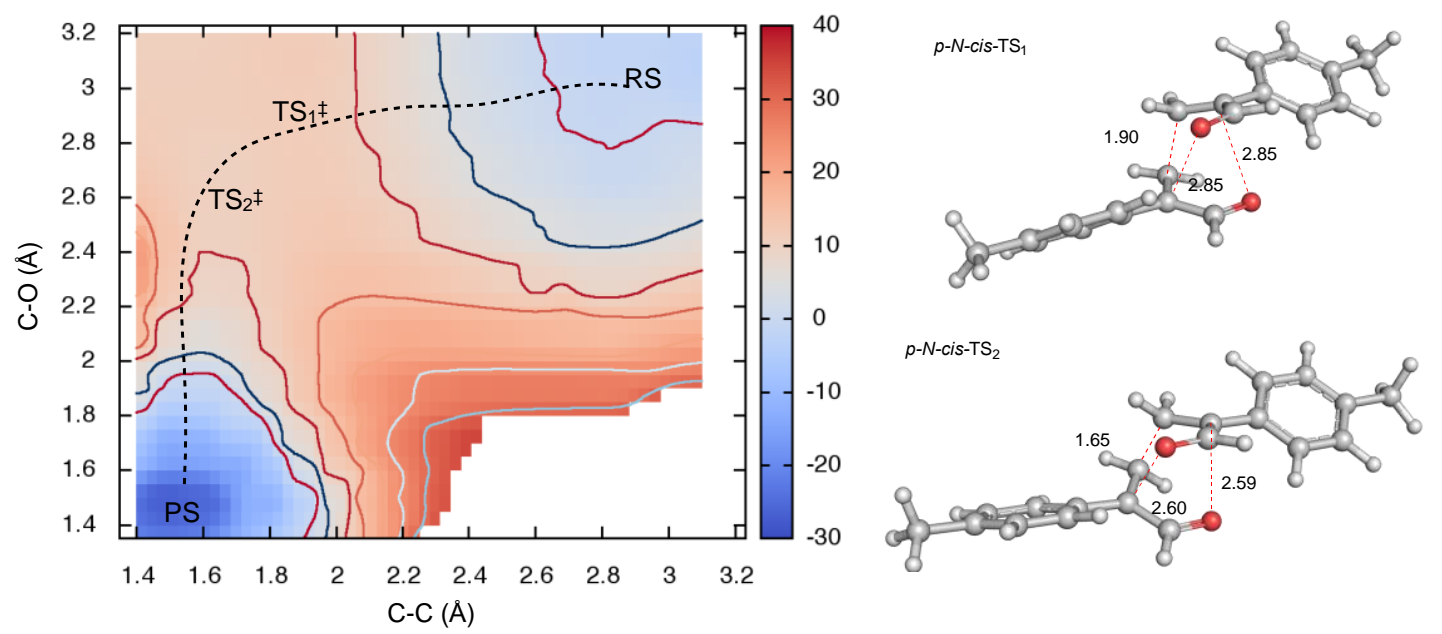

Figure 4.2: (left) Calculated potential energy landscapes, at the $\omega \mathrm{B} 97 \mathrm{X}-\mathrm{D} / 6-311++\mathrm{G}(\mathrm{d}, \mathrm{p}) / / \omega \mathrm{B} 97 \mathrm{X}-$ $\mathrm{D} / 6-31+\mathrm{G}(\mathrm{d})$ level of theory, for the Diels-Alder reaction of the model enal 2.81 via $p$ - $N$-cis TS. Approximate transition state position for the bis-pericyclic Diels-Alder and the [3,3]-sigmatropic rearrangement transition states $\left(\mathrm{TS}_{1}\right.$ and $\mathrm{TS}_{2}$ ) are indicated. (right) Optimised $\mathrm{TS}_{1}$ and $\mathrm{TS}_{2}$ and relevant distances obtained at the $\omega \mathrm{B} 97 \mathrm{X}-\mathrm{D} / 6-31+\mathrm{G}(\mathrm{d})$ level of theory.

Without exception, the $p$-TSs were found to be significantly lower in energy than the $m$-TSs, the $m$-N/X-cis being 10.3 and $12.0 \mathrm{kcal} \mathrm{mol}^{-1}$ higher in energy than the analogous $p$ - $N$-cis. This is due to better orbital overlap and lower distortion penalties. Regarding exo/endo-selectivity, most of the TSs showed exo-selectivity, except the $p$-cis-TS, where the endo configuration is the preferred form. The exo-selectivity may sound counterintuitive given the well-known preference for the endo cycloadduct in Diels-Alder reactions involving maleic anhydride and cyclopentadiene or butadiene. Fernandez and Bickelhaupt ${ }^{176}$ have shown that endo preference is mainly caused by unfavourable steric arrangements in the exo pathway, which translates into a more destabilising activation strain and a preference for the endo form.

In order to rationalise these observations we performed a similar distortion/interaction analysis, ${ }^{177,178}$ which has been previously applied in understanding 1,3-dipolar and Diels-Alder cycloadditions. Here, the activation energy of a process $\left(\Delta E^{\ddagger}\right)$ is 
decomposed into the energy required for the reactants to reach the transition state geometry $\left(\Delta E_{\mathrm{dist}}{ }^{\star}\right)$ and the energy of interaction between these distorted fragments $\left(\Delta E_{\text {int }}\right)$. The interaction energy term encompasses all of the stabilising and repulsive interactions between the diene and dienophile fragments at the TS. ${ }^{179,180}$

Table 4.3. Distortion/interaction analysis at the $\omega \mathrm{B} 97 \mathrm{X}-\mathrm{D} / 6-311++\mathrm{G}(\mathrm{d}, \mathrm{p}) / / \omega \mathrm{B} 97 \mathrm{X}-\mathrm{D} / 6-31+\mathrm{G}(\mathrm{d})$ level of theory for the different TSs obtained for the hetero-Diels-Alder dimerisation of the model enal 2.81. Shown here are the distortion energies of the diene $\left(\Delta E^{\ddagger}\right.$ dist_diene $)$ and dienophile $\left(\Delta E^{*}\right.$ dist_dienophile $)$, the interaction energies $\left(\Delta E_{\text {int }}^{*}\right)$ and the activation energies $\left(\Delta E^{*}\right)$. All energies are given in $\mathrm{kcal} \mathrm{mol}^{-1}$.

\begin{tabular}{ccccccccc}
\hline & \multicolumn{2}{c}{$p$-trans } & \multicolumn{2}{c}{$p$-cis } & \multicolumn{2}{c}{ m-trans } & \multicolumn{2}{c}{ m-cis } \\
& $N$ & $X$ & $N$ & $X$ & $N$ & $X$ & $N$ & $X$ \\
\hline$\Delta E_{\text {dist_diene }}^{\ddagger}$ & 15.5 & 17.8 & 12.3 & 14.1 & 17.6 & 16.5 & 15.2 & 14.8 \\
$\Delta E^{\ddagger}$ dist_dienophile & 10.3 & 13.3 & 12.3 & 12.6 & 11.7 & 14.7 & 15.0 & 15.4 \\
$\Delta E_{\text {dist }}^{\ddagger}$ & 25.8 & 31.0 & 24.7 & 26.7 & 29.4 & 31.2 & 30.2 & 30.3 \\
$\Delta E_{\text {int }}^{\ddagger}$ & -9.0 & -17.3 & -13.9 & -14.7 & -5.9 & -11.1 & -7.9 & -10.5 \\
$\Delta E^{\ddagger}$ & 16.7 & 13.8 & 10.7 & 12.0 & 23.4 & 20.1 & 22.3 & 19.8 \\
\hline
\end{tabular}

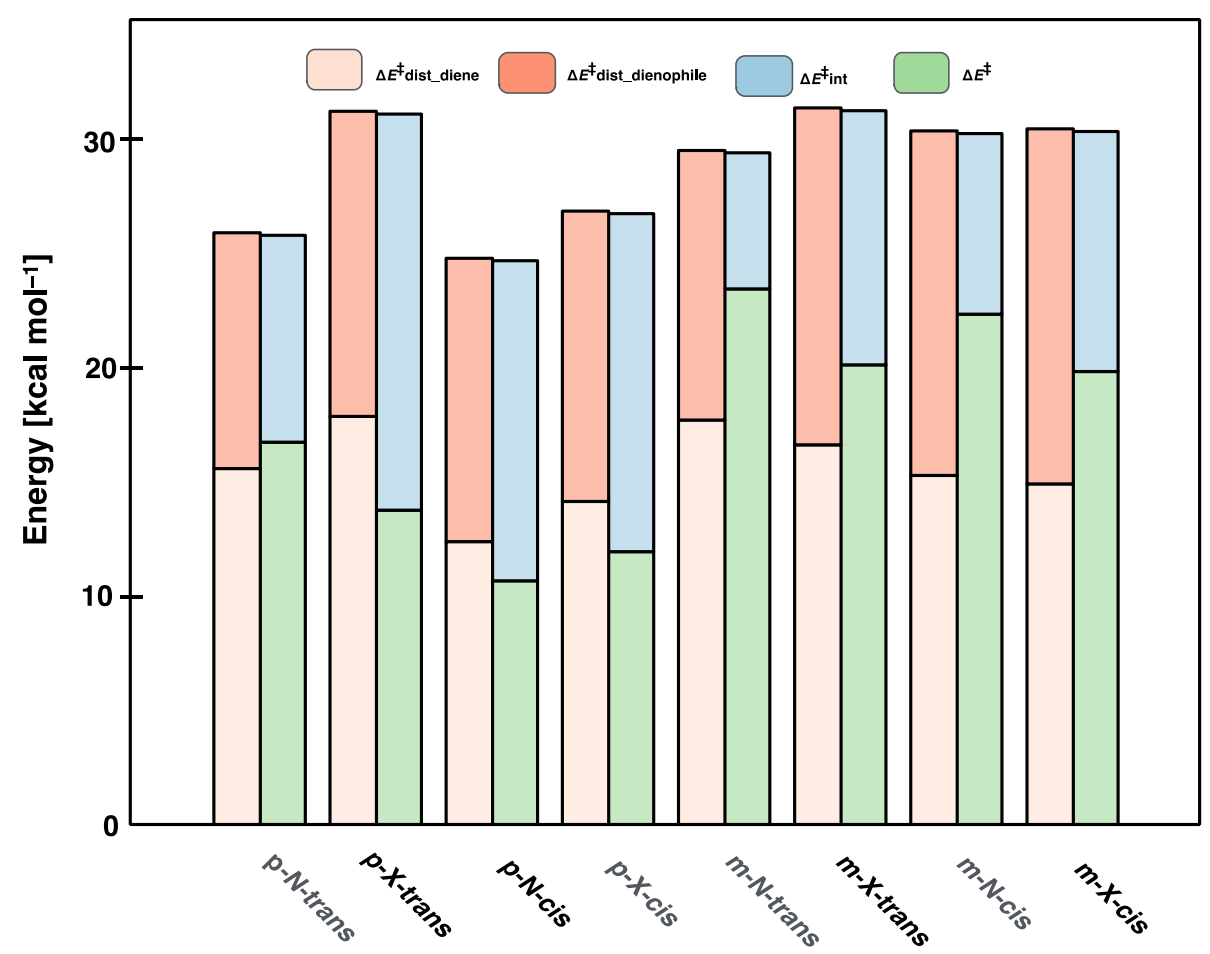

Figure 4.3: Distortion, interaction and activation energy components for the different TSs obtained for the hetero-Diels-Alder dimerisation of the model enal 2.81. The length of the light/dark pink bars represents the distortion energy of the diene $\left(\Delta E_{\text {dist_diene }}^{\ddagger}\right)$ and dienophile $\left(\Delta E^{\ddagger}\right.$ dist_dienophile), respectively. The blue bars represent the interaction energy $\left(\Delta E_{\text {int }}^{\ddagger}\right)$ and the green bar the activation energy $\left(\Delta E^{\ddagger}\right)$. All energies in $\mathrm{kcal} \mathrm{mol}^{-1}$. 
As graphically shown in figure 4.3, the exo pathway shows in all cases a higher distortion penalty, in line with the results previously obtained by Fernandez and Bickelhaupt. However, this is overcome by a much larger stabilising interaction contribution along this pathway. The nature of these interactions has been explored by comparing the noncovalent interaction $(\mathrm{NCI})^{179,180}$ index for each of the TSs (figure 4.4). The NCI isosurface shows regions in space corresponding to overlapping atomic densities, which may be associated with non-covalent interactions. In this case, green regions indicate van der Waals/dispersion interactions, blue regions show strong polar interactions and red regions indicate steric repulsion.

As can be seen from figure 4.4 the exo arrangement leads to attractive non-bonding (dispersion) interactions between the aromatic fragments (and in some cases a $\mathrm{CH}-\pi$ interaction), which are not present in the $N$ configuration.

A different scenario is observed when comparing the $p$-N/X-cis TSs, in this case the energy to distort the reactant is the determining factor and the interaction energy between the two fragments at the TS is of similar strength. In this case the bispericyclic nature of the $p$-cis TS allows for a secondary interaction between the lonepair of oxygen with the $\mathrm{C}-\mathrm{C} \pi$-bond is comparable in magnitude with the dispersion interactions present in the exo TS (figure 4.4). This interaction is also clear when visualising the HOMO of the $p$-cis-TS, which shows an orbital (figure 4.5). Therefore, the favorable steric arrangement (23.6 vs $\left.25.8 \mathrm{kcal} \mathrm{mol}^{-1}\right)$ and the presence of secondary orbital interactions between the lone-pair of oxygen with the $\mathrm{C}-\mathrm{C} \pi$ lead to a preference for the $p$ - $N$-cis pathway. 

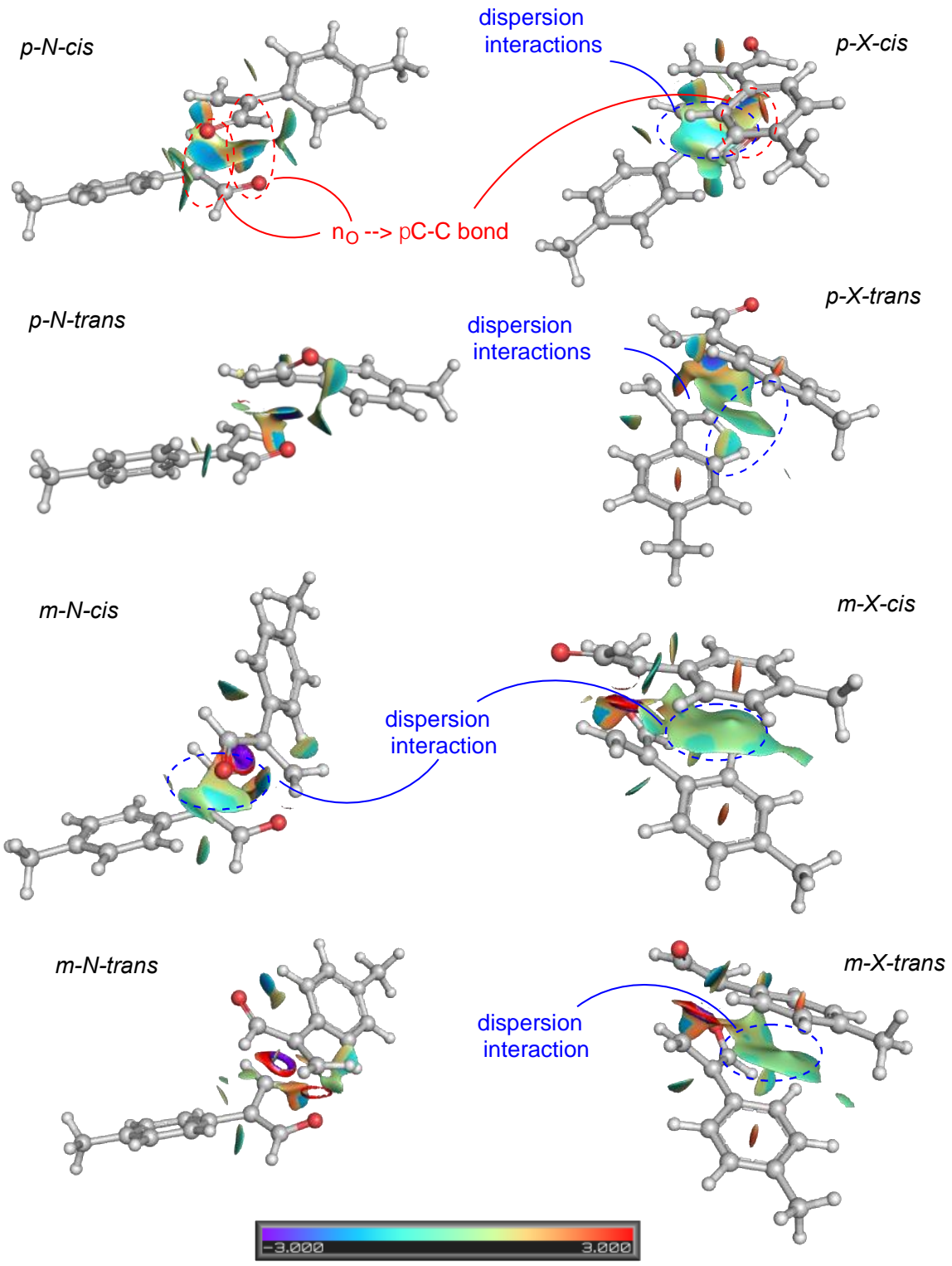

Figure 4.4: Non-Covalent Interaction (NCI) surfaces showing the key NCI interactions at the TS for the hetero-Diels-Alder dimerisation of enal 2.81. NCI surfaces correspond to $\mathrm{s}=0.5$ and a colour scale of $-0.03<\rho<+0.03$ au.

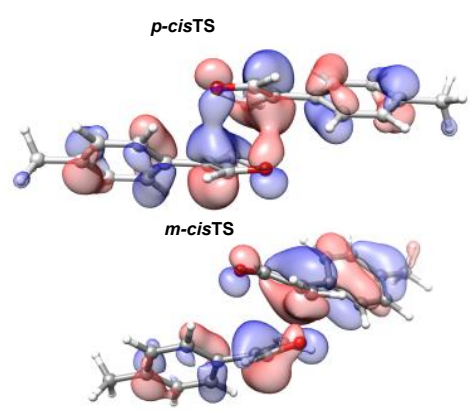

Figure 4.5: HOMO of the $p$ - $N$-cis TS (top) and $m$ - $N$-cis TS (bottom). The former one shows an orbital interaction of the lone-pair of oxygen with the $\mathrm{C}-\mathrm{C} \pi$-bond, while the $m-N$-cis TS lacks such secondary orbital interaction. 


\subsubsection{Substituent Effect}

The same regioselectivity was obtained for hetero-Diels-Alder dimerisation of enal 2.48. Here several potential TS state geometries were explored by considering the variable rotations along the aryl and acetal bonds. The activation free energies and geometries for the lowest energy TS for each configuration are shown in figure 4.6 and figure 4.7 .

The lowest energy TS was similarly found to be a $p-N$-cis TS, which although not $\mathrm{C}_{2-}$ symmetric still has bis-pericyclic character (figure 4.7). Once again a larger distortion penalty is generally observed for the exo pathway, however this is compensated by an additional interaction contribution due to non-covalent interactions (table 4.5). The preference for the $\mathrm{p}-\mathrm{N}$-cis TS is the result of a lower distortion penalty and an almost equal interaction energy, although different in nature.

Table 4.4: Reaction energies for the dimerisation process of enal 2.48 at the $\omega B 97 X-D / 6-31+G(d)$ level of theory and at the $\omega \mathrm{B} 97 \mathrm{X}-\mathrm{D} / 6-311++\mathrm{G}(\mathrm{d}, \mathrm{p}) / \omega \mathrm{B} 97 \mathrm{X}-\mathrm{D} / 6-31+\mathrm{G}(\mathrm{d})$ level of theory $\left(\Delta \mathrm{G}^{\ddagger}\right.$ corr $)$. All energies are in $\mathrm{kcal} \mathrm{mol}^{-1}$ relative to the independent lower energy reactant at $353 \mathrm{~K}$. See table 4.8 for absolute numbers.

\begin{tabular}{ccccccc}
\hline & $\mathbf{\Delta} \mathbf{E}_{\text {el }}^{*}$ & $\mathbf{Z P E}$ & $\mathbf{\Delta} \mathbf{H}^{*}$ & $\mathbf{T} \mathbf{\Delta} \mathbf{S}^{*}$ & $\mathbf{\Delta} \mathbf{G}^{*}$ & $\boldsymbol{\Delta} \mathbf{G}_{\text {corr }}^{*}$ \\
\hline$p$ - $N$-trans & 16.0 & 2.6 & 17.7 & -20.0 & 37.6 & 38.6 \\
\hline$p$-X-trans & 13.8 & 2.9 & 15.6 & -21.0 & 36.6 & 37.0 \\
\hline$p-N$-cis & 11.2 & 2.2 & 12.7 & -19.5 & 32.2 & 33.4 \\
\hline$p$-X-cis & 12.7 & 2.5 & 14.3 & -20.0 & 34.3 & 35.3 \\
\hline$m-N$-trans & 24.2 & 2.5 & 25.7 & -20.4 & 46.1 & 47.2 \\
\hline$m-X$-trans & 19.6 & 2.5 & 21.0 & -21.0 & 42.0 & 42.1 \\
\hline$m-N$-cis & 20.6 & 2.3 & 22.0 & -20.0 & 42.0 & 43.1 \\
\hline$m-X$-cis & 20.4 & 2.1 & 21.6 & -19.7 & 41.3 & 42.1 \\
\hline
\end{tabular}




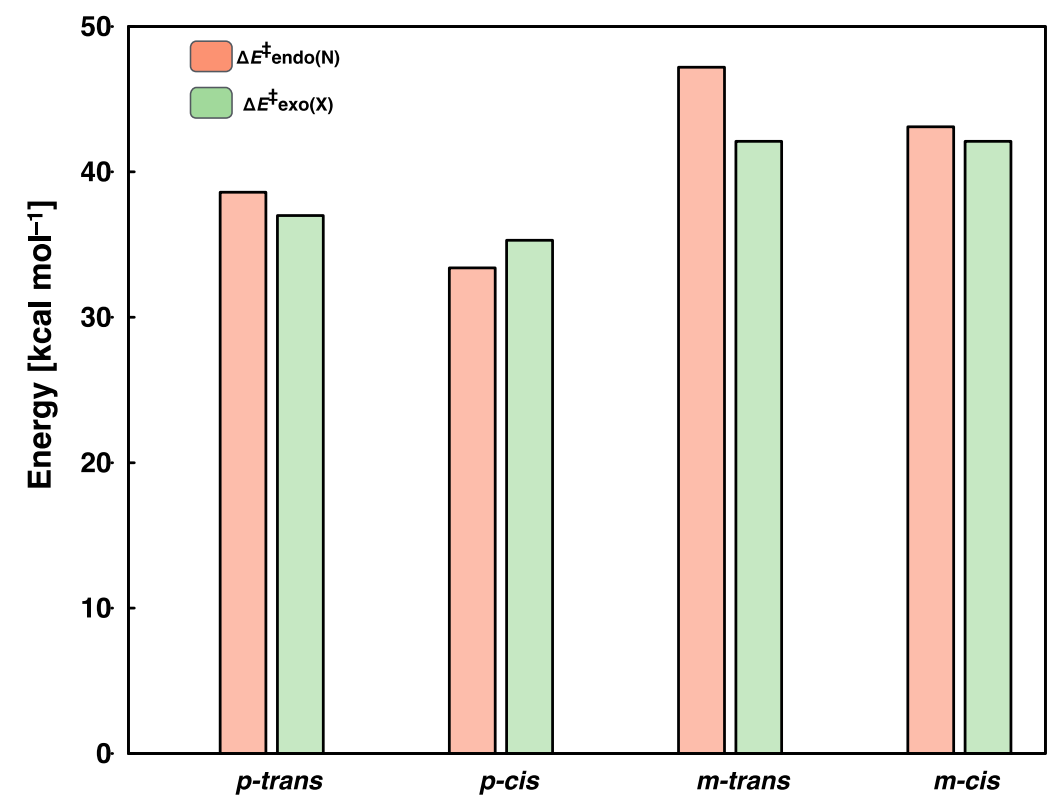

Figure 4.6: Reaction free energies for the hetero-Diels-Alder dimerisation of enal $\mathbf{2 . 4 8}$ (in $\mathrm{kcal} \mathrm{mol}^{-1}$ ). Calculations were carried out at the $\omega$ B97X-D/6-31++G(d,p)// $\omega$ B97X-D/6-31+G(d) level of theory. For absolute values see table 4.7.

Table 4.5. Distortion/Interaction Analysis at the $\omega B 97 X-D / 6-311++G(d, p) / / \omega B 97 X-D / 6-31+G(d)$ level of theory for the different TS for the hetero-Diels-Alder dimerisation of enal 2.48. Shown here are the distortion energies of the diene $\left(\Delta E^{\ddagger}\right.$ dist_diene $)$ and dienophile ( $\Delta E_{\text {dist_dienophile }}^{*}$, the interaction energies $\left(\Delta E_{\text {int }}^{\ddagger}\right)$ and the activation energies $\left(\Delta E^{\ddagger}\right)$. All energies are given in $\mathrm{kcal} \mathrm{mol}^{-1}$.

\begin{tabular}{ccccccccc}
\hline & \multicolumn{2}{c}{$p$-trans } & \multicolumn{2}{c}{$p$-cis } & \multicolumn{2}{c}{ m-trans } & \multicolumn{2}{c}{ m-cis } \\
& $N$ & $X$ & $N$ & $X$ & $N$ & $X$ & $N$ & $X$ \\
\hline$\Delta E_{\text {dist_diene }}^{\ddagger}$ & 17.9 & 18.4 & 15.5 & 15.7 & 18.0 & 17.5 & 16.3 & 15.6 \\
$\Delta E_{\text {dist_dienophile }}^{*}$ & 12.9 & 16.2 & 12.0 & 13.8 & 14.4 & 14.9 & 14.3 & 15.6 \\
\hline$\Delta E^{\ddagger}$ dist & 30.8 & 34.6 & 27.5 & 29.4 & 32.4 & 32.4 & 30.7 & 31.2 \\
$\Delta E_{\text {int }}^{\ddagger}$ & -18.1 & -20.4 & -15.1 & -15.7 & -10.8 & -12.7 & -9.0 & -10.1 \\
$\Delta E^{\ddagger}$ & 12.7 & 14.2 & 12.4 & 13.7 & 21.7 & 19.7 & 21.7 & 21.1
\end{tabular}




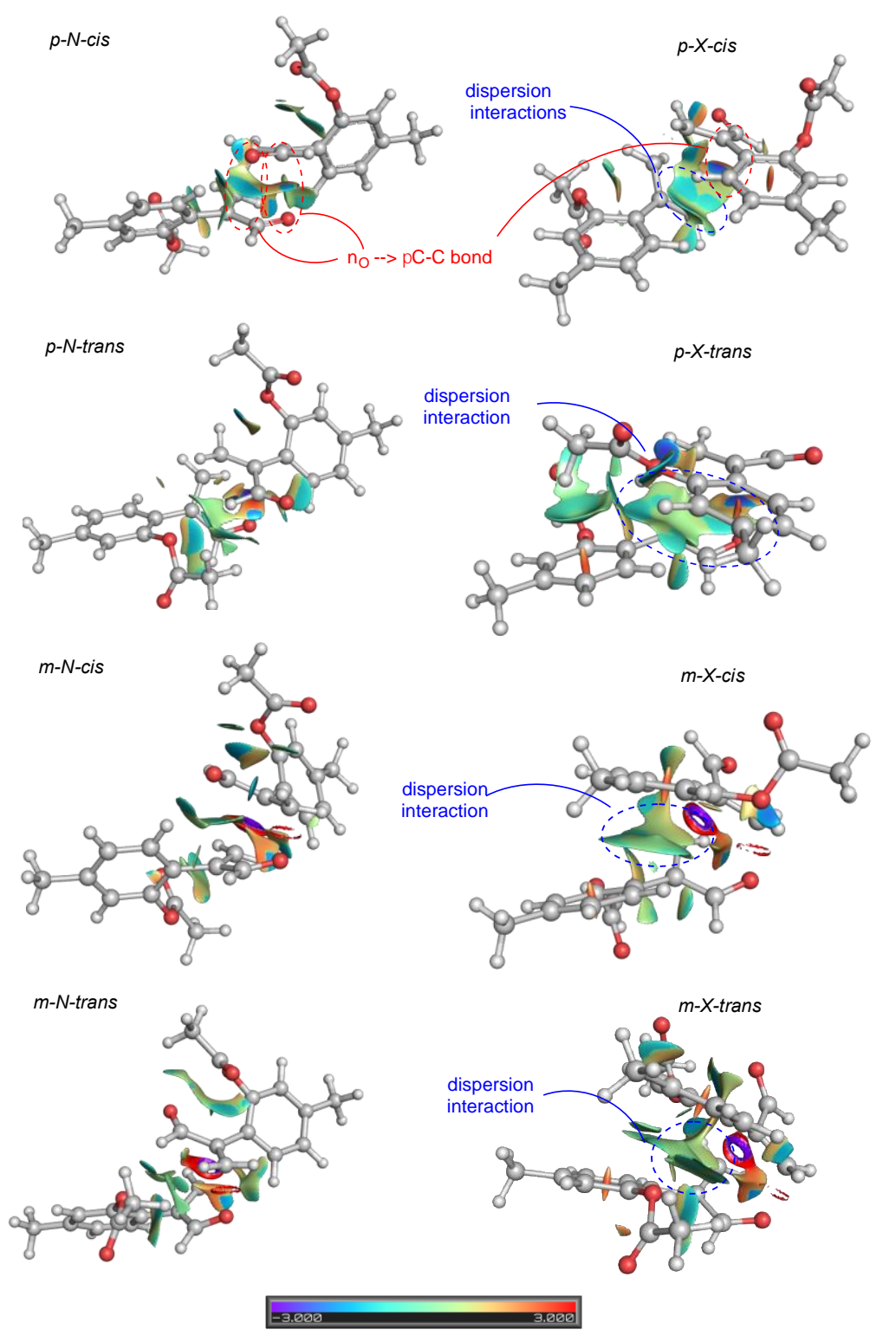

Figure 4.7: TS geometry and non-Covalent Interaction (NCI) surface for $p$ - $N$-cis TS for the TS for the hetero-Diels-Alder dimerisation of enal 2.48. NCI surfaces correspond to $s=0.5$ and a colour scale of $-0.03<\rho<+0.03$ au. 


\subsubsection{Tables with Absolute Energies}

Table 4.6: Absolute electronic energies $\left(\mathrm{E}_{\mathrm{el}}\right)$, zero-point energy $(\mathrm{ZPE})$, enthalpy $(\mathrm{H})$, quasi-harmonic entropies (Tqh-S) and quasi-harmonic Gibbs energy (qh-G) in atomic units for the dimerisation process. RS, TS and PS denote reactant, transition and product states respectively. Reaction free energies are given in $\mathrm{kcal} \cdot \mathrm{mol}^{-1}$ relative to the independent trans specie. Calculations were carried out at the $\mathbf{\omega B} \mathbf{B} 9 \mathbf{X}$ D/6-311++G(d,p) level of theory.

\begin{tabular}{|c|c|c|c|c|c|c|}
\hline & $\mathbf{E}_{\text {el }}$ & ZPE & $\mathbf{H}$ & Tqh-S & qh-G & $v\left(\mathrm{~cm}^{-1}\right)$ \\
\hline Sub-cis & -462.24299 & 0.17115 & -462.05677 & 0.05915 & -462.11593 & 53.5 \\
\hline Sub-trans & 462.24496 & 0.17145 & -462.05855 & 0.05890 & -462.11745 & 60.2 \\
\hline$p$-N-trans-RS & -924.49921 & 0.34417 & -924.12383 & 0.09378 & -924.21762 & 18.1 \\
\hline$p$-N-trans-TS & -924.46314 & 0.34577 & -924.08835 & 0.08845 & -924.17680 & -441.4 \\
\hline$p$-N-trans-PS & -924.53603 & 0.35016 & -924.15757 & 0.08714 & -924.24471 & 19.1 \\
\hline$p-N-c i s-T S_{l}$ & -924.47277 & 0.34551 & -924.09821 & 0.08835 & -924.18655 & -338.9 \\
\hline$p-N-c i s-T S_{2}$ & -924.47462 & 0.34795 & -924.09878 & 0.08532 & -924.18410 & -53.8 \\
\hline$p-N-c i s-P S$ & -924.53606 & 0.35022 & -924.15762 & 0.08698 & -924.24460 & 19.5 \\
\hline$m$ - $N$-trans- $R S$ & -924.50323 & 0.34499 & -924.12741 & 0.09226 & -924.21968 & 23.2 \\
\hline$m$-N-trans-TS & -924.45249 & 0.34582 & -924.07792 & 0.08748 & -924.16540 & -605.0 \\
\hline$m$-N-trans-PS & -924.53094 & 0.35092 & -924.15192 & 0.08625 & -924.23817 & 34.0 \\
\hline$m-N$-cis-RS & -924.49815 & 0.34430 & -924.12259 & 0.09457 & -924.21717 & 4.8 \\
\hline$m-N-c i s-T S$ & -924.45424 & 0.34575 & -924.07962 & 0.08780 & -924.16742 & -592.0 \\
\hline \multirow[t]{2}{*}{$m-N-c i s-P S$} & -924.53105 & 0.35052 & -924.15213 & 0.08705 & -924.23918 & 27.1 \\
\hline & $\Delta \mathbf{E}_{\mathrm{el}}^{\ddagger}$ & ZPE & $\Delta \mathbf{H}^{*}$ & $\mathbf{T} \Delta \mathbf{S}^{\star}$ & $\Delta \mathbf{G}^{*}$ & \\
\hline$p$-N-trans-RS & -5.8 & 0.8 & -4.2 & -15.1 & 10.8 & \\
\hline$p$-N-trans-TS & 16.8 & 1.8 & 18.0 & -18.4 & 36.5 & \\
\hline$p$-N-trans-PS & -28.9 & 4.6 & -25.4 & -19.2 & -6.2 & \\
\hline$p-N-c i s-T S$ & 9.6 & 3.2 & 11.5 & -20.4 & 31.9 & \\
\hline$p-N-c i s-P S$ & -29.0 & 4.6 & -25.4 & -19.3 & -6.1 & \\
\hline$m$ - $N$-trans- $T S$ & 23.5 & 1.8 & 24.6 & -19.0 & 43.6 & \\
\hline$m$-N-trans-PS & -25.7 & 5.0 & -21.9 & -19.8 & -2.1 & \\
\hline$m-N-c i s-T S$ & 22.4 & 1.8 & 23.5 & -18.8 & 42.3 & \\
\hline$m-N-c i s-P S$ & -25.8 & 4.8 & -22.0 & -19.3 & -2.7 & \\
\hline
\end{tabular}


Table 4.7: Absolute electronic energies $\left(\mathrm{E}_{\mathrm{el}}\right)$, zero-point energy (ZPE), enthalpy $(\mathrm{H})$, quasi-harmonic entropies (Tqh-S), quasi-harmonic Gibbs energy (qh-G) (in atomic units) and the lowest frequency value $\left(v, \mathrm{~cm}^{-1}\right)$ for the dimerisation of $\mathbf{2 . 8 1}$ process. Reaction free energies are given in $\mathrm{kcal} \mathrm{mol}^{-1}$ relative to the independent trans substrate specie at $353 \mathrm{~K}$. TS and PS denote transition and product states respectively. Calculations were carried out at the $\omega \mathrm{B} 97 \mathrm{X}-\mathrm{D} / 6-31+\mathrm{G}(\mathrm{d})$ level of theory. For comparison, free energies at the $\omega \mathrm{B} 97 \mathrm{X}-\mathrm{D} / 6-311++\mathrm{G}(\mathrm{d}, \mathrm{p}) / / \omega \mathrm{B} 97 \mathrm{X}-\mathrm{D} / 6-31+\mathrm{G}(\mathrm{d})$ level of theory are also provided ( $\Delta \mathrm{G}_{\text {corr }}^{\ddagger}$.

\begin{tabular}{|c|c|c|c|c|c|c|c|}
\hline & $\mathbf{E}_{\text {el }}$ & ZPE & $\mathbf{H}$ & Tqh-S & qh-G & $v\left(\mathrm{~cm}^{-1}\right)$ & Ecorr \\
\hline Sub-cis & -462.14142 & 0.17240 & -461.95400 & 0.05906 & -462.01306 & 54.4 & -462.24279 \\
\hline Sub-trans & -462.14310 & 0.17234 & -461.95572 & 0.05919 & -462.01491 & 40.5 & -462.24475 \\
\hline$p$-N-trans-TS & -924.26177 & 0.34830 & -923.88454 & 0.08832 & -923.97287 & -430.4 & -924.46282 \\
\hline$p$-N-trans- $P S$ & -924.33628 & 0.35260 & -923.95548 & 0.08682 & -924.04230 & 19.9 & -924.53572 \\
\hline$p$-X-trans-TS & -924.26617 & 0.34839 & -923.88898 & 0.08777 & -923.97675 & -361.8 & -924.46753 \\
\hline$p$-X-trans- $P S$ & -924.33598 & 0.35259 & -923.95507 & 0.08708 & -924.04215 & 22.9 & -924.53527 \\
\hline$p-N-c i s-T S_{l}$ & -924.27437 & 0.35011 & -923.89638 & 0.08539 & -923.983195 & -353.7 & -924.47244 \\
\hline$p-N-c i s-T S_{2}$ & -924.27186 & 0.347827 & -923.89504 & 0.093744 & -923.981769 & -70.5 & -924.47423 \\
\hline$p$-N-cis-PS & -924.33629 & 0.35262 & -923.95554 & 0.08673 & -924.042264 & 20.4 & -924.53575 \\
\hline$p-X$-cis-TS & -924.26900 & 0.34885 & -923.89149 & 0.08727 & -923.97875 & -407.3 & -924.47041 \\
\hline$p$-X-cis-PS & -924.33611 & 0.35289 & -923.95509 & 0.08638 & -924.04147 & 23.9 & -924.53536 \\
\hline$m$-N-trans-TS & -924.25113 & 0.34806 & -923.87433 & 0.08755 & -923.96188 & -588.3 & -924.45217 \\
\hline$m$-N-trans- $P S$ & -924.32658 & 0.35323 & -923.94531 & 0.08638 & -924.03169 & 19.7 & -924.52674 \\
\hline$m$-X-trans-TS & -924.25544 & 0.34878 & -923.87829 & 0.08634 & -923.96462 & -58.2 & -924.45744 \\
\hline$m$-X-trans-PS & -924.32622 & 0.35278 & -923.94522 & 0.08695 & -924.03217 & 17.4 & -924.52625 \\
\hline$m-N-c i s-T S$ & -924.25299 & 0.34821 & -923.87596 & 0.08775 & -923.96371 & -585.2 & -924.45393 \\
\hline$m-N$-cis-PS & -924.33120 & 0.35319 & -923.94986 & 0.08651 & -924.03638 & 19.7 & -924.53078 \\
\hline$m-X$-cis-TS & -924.25610 & 0.34867 & -923.87889 & 0.08660 & -923.96549 & -563.9 & -924.45790 \\
\hline \multirow[t]{2}{*}{$m-X$-cis-PS } & -924.33046 & 0.35318 & -923.94910 & 0.08687 & -924.03597 & 20.1 & -924.53015 \\
\hline & $\Delta \mathbf{E}^{\ddagger} \mathrm{el}$ & ZPE & $\Delta \mathbf{H}^{\ddagger}$ & $\mathbf{T} \Delta \mathbf{S}^{\ddagger}$ & $\Delta \mathbf{G}^{*}$ & $\Delta \mathbf{G}_{\text {corr }}^{\dagger}$ & \\
\hline$p$ - $N$-trans-TS & 15.3 & 2.3 & 16.9 & -18.9 & 35.7 & 37.2 & \\
\hline$p$ - $N$-trans- $P S$ & -31.4 & 5.0 & -27.6 & -19.8 & -7.8 & -5.4 & \\
\hline$p$-X-transTS & 12.6 & 2.3 & 14.1 & -19.2 & 33.3 & 34.5 & \\
\hline$p$ - $X$-trans $P S$ & -31.2 & 5.0 & -27.4 & -19.6 & -7.7 & -5.2 & \\
\hline$p-N$-cis-TS $S_{I}$ & 9.0 & 2.0 & 10.3 & -19.0 & 29.3 & 31.0 & \\
\hline$p-N$-cis-TS 2 & 7.4 & 3.4 & 9.5 & -20.7 & 30.1 & 32.3 & \\
\hline$p$-N-cis-PS & -31.4 & 5.0 & -27.7 & -19.9 & -7.8 & -5.4 & \\
\hline$p$-X-cis-TS & 10.8 & 2.6 & 12.5 & -19.5 & 32.0 & 33.2 & \\
\hline$p-X$-cis- $P S$ & -31.3 & 5.2 & -27.4 & -20.1 & -7.3 & -4.8 & \\
\hline$m$-N-trans-TS & 22.0 & 2.1 & 23.3 & -19.3 & 42.6 & 44.1 & \\
\hline$m$-N-trans-PS & -25.3 & 5.4 & -21.3 & -20.1 & -1.2 & 0.8 & \\
\hline$m$-X-trans-TS & 19.3 & 2.6 & 20.8 & -20.1 & 40.9 & 41.7 & \\
\hline$m$ - $X$-trans- $P S$ & -25.1 & 5.1 & -21.2 & -19.7 & -1.5 & 0.6 & \\
\hline$m-N$-cis-TS & 20.8 & 2.2 & 22.3 & -19.2 & 41.5 & 43.0 & \\
\hline$m-N$-cis-PS & -28.2 & 5.3 & -24.1 & -20.0 & -4.1 & -1.8 & \\
\hline$m$-X-cis-TS & 18.9 & 2.5 & 20.4 & -19.9 & 40.4 & 41.3 & \\
\hline$m-X$-cis-PS & -27.8 & 5.3 & -23.6 & -19.8 & -3.9 & -1.6 & \\
\hline
\end{tabular}


Table 4.8: Absolute electronic energies $\left(\mathrm{E}_{\mathrm{el}}\right)$, zero-point energy (ZPE), enthalpy $(\mathrm{H})$, quasi-harmonic entropies (Tqh-S) and quasi-harmonic Gibbs energy (qh-G) (in atomic units) and the lowest frequency value $\left(v, \mathrm{~cm}^{-1}\right)$ for the dimerisation of 2.48. Activation free energies are given relative to the independent cis-cis substrate specie at $353 \mathrm{~K}$. Calculations were carried out at the $\omega \mathrm{B} 97 \mathrm{X}-\mathrm{D} / 6-31+\mathrm{G}(\mathrm{d})$ level of theory. For comparison, free energies at the $\omega \mathrm{B} 97 \mathrm{X}-\mathrm{D} / 6-311++\mathrm{G}(\mathrm{d}, \mathrm{p}) / \omega \mathrm{B} 97 \mathrm{X}-\mathrm{D} / 6-31+\mathrm{G}(\mathrm{d})$ are also provided $\left(\Delta \mathrm{G}_{\text {corr }}^{\ddagger}\right)$.

\begin{tabular}{|c|c|c|c|c|c|c|c|}
\hline & Eel & ZPE & $\mathbf{H}$ & Tqh-S & qh-G & $v\left(\mathrm{~cm}^{-1}\right)$ & $\mathbf{E}_{\text {corr }}$ \\
\hline Sub-cis-cis & -689.95898 & 0.21465 & -689.72323 & 0.07225 & -689.79549 & 35.4 & -690.11692 \\
\hline Sub-cis-trans & -689.95775 & 0.21459 & -689.72204 & 0.07239 & -689.79444 & 36.3 & -690.11564 \\
\hline Sub-trans-cis & -689.95841 & 0.21476 & -689.72259 & 0.07249 & -689.79508 & 22.9 & -690.11658 \\
\hline Sub-trans-trans & -689.95778 & 0.21497 & -689.72186 & 0.07203 & -689.79389 & 30.4 & -690.11600 \\
\hline$p$-N-trans & -1379.89243 & 0.43339 & -1379.41832 & 0.11266 & -1379.53099 & -380.1 & -1379.54009 \\
\hline$p$-X-trans & -1379.89599 & 0.43398 & -1379.42156 & 0.11110 & -1379.53266 & -399.2 & -1379.54045 \\
\hline$p-N-c i s$ & -1379.90015 & 0.43287 & -1379.42625 & 0.11346 & -1379.53971 & -383.5 & -1379.54941 \\
\hline$p-X-c i s$ & -1379.89767 & 0.43323 & -1379.42365 & 0.11265 & -1379.53630 & -413.9 & -1379.54550 \\
\hline$m$-N-trans & -1379.87940 & 0.43335 & -1379.40551 & 0.11198 & -1379.51750 & -586.2 & -1379.52598 \\
\hline$m$-X-trans & -1379.88669 & 0.43332 & -1379.41296 & 0.11110 & -1379.52405 & -590.5 & -1379.53242 \\
\hline$m-N$-cis & -1379.88511 & 0.43298 & -1379.41133 & 0.11265 & -1379.52399 & -564.1 & -1379.53315 \\
\hline \multirow[t]{2}{*}{$m$-X-cis } & -1379.88553 & 0.43258 & -1379.41201 & 0.11312 & -1379.52513 & -586.5 & -1379.53514 \\
\hline & $\Delta \mathbf{E}^{\ddagger}$ el & ZPE & $\Delta \mathbf{H}^{*}$ & $\mathbf{T} \Delta \mathbf{S}^{\ddagger}$ & $\Delta \mathbf{G}^{\star}$ & $\Delta \mathbf{G}_{\text {corr }}^{\ddagger}$ & \\
\hline$p$-N-trans & 16.0 & 2.6 & 17.7 & -20.0 & 37.6 & 38.6 & \\
\hline$p$-X-trans & 13.8 & 2.9 & 15.6 & -21.0 & 36.6 & 37.0 & \\
\hline$p-N-c i s$ & 11.2 & 2.2 & 12.7 & -19.5 & 32.2 & 33.4 & \\
\hline$p-X-c i s$ & 12.7 & 2.5 & 14.3 & -20.0 & 34.3 & 35.3 & \\
\hline$m$-N-trans & 24.2 & 2.5 & 25.7 & -20.4 & 46.1 & 47.2 & \\
\hline$m$-X-trans & 19.6 & 2.5 & 21.0 & -21.0 & 42.0 & 42.1 & \\
\hline$m-N-c i s$ & 20.6 & 2.3 & 22.0 & -20.0 & 42.0 & 43.1 & \\
\hline$m-X$-cis & 20.4 & 2.1 & 21.6 & -19.7 & 41.3 & 42.1 & \\
\hline
\end{tabular}




\subsection{Crystal Structures}

\subsubsection{Crystal Structure of meso-2.31}

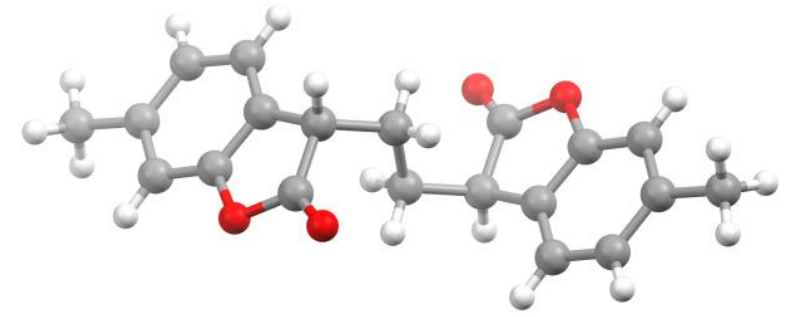

Experimental. Single colourless block-shaped crystals of meso-2.31 were recrystallised from a mixture of DCM and ethyl acetate by slow evaporation. A suitable crystal $(0.19 \times 0.06 \times 0.04)$ was selected and mounted on a MITIGEN holder in Paratone oil on a Agilent Technologies SuperNova diffractometer. The crystal was kept at $T=120.0 \mathrm{~K}$ during data collection. Using Olex2 (Dolomanov et al., 2009), the structure was solved with the ShelXS (Sheldrick, 2008) structure solution program, using the Direct Methods solution method. The model was refined with version of ShelXL (Sheldrick, 2008) using Least Squares minimisation.

Crystal Data. $\mathrm{C}_{20} \mathrm{H}_{18} \mathrm{O}_{4}, M_{r}=322.34$, monoclinic, $\mathrm{P} 2{ }_{1} / \mathrm{c}$ (No. 14), $\mathrm{a}=5.1224(2) \AA, \mathrm{b}=10.9427(4) \AA, \mathrm{c}=$ 13.8090(5) $\AA, \quad \beta=98.335(4)^{\circ}, \quad \alpha=\gamma=90^{\circ}, \quad V=$ 765.87(5) $\AA^{3}, T=120.0 \mathrm{~K}, Z=2, Z^{\prime}=0.5, \mu\left(\mathrm{MoK}_{\alpha}\right)=$ $0.097,32811$ reflections measured, 1344 unique $\left(R_{\text {int }}=0.0887\right)$ which were used in all calculations. The final $w R_{2}$ was 0.1419 (all data) and $R_{l}$ was 0.0793 (I > 2(I)).

\begin{tabular}{|c|c|}
\hline Compound & meso-2.31 \\
\hline Formula & $\mathrm{C}_{20} \mathrm{H}_{18} \mathrm{O}_{4}$ \\
\hline$D_{\text {calc }} / \mathrm{g} \mathrm{cm}^{-3}$ & 1.398 \\
\hline$\mu / \mathrm{mm}^{-1}$ & 0.097 \\
\hline Formula Weight & 322.34 \\
\hline Colour & colourless \\
\hline Shape & block \\
\hline Max Size/mm & 0.19 \\
\hline Mid Size/mm & 0.06 \\
\hline Min Size/mm & 0.04 \\
\hline$T / \mathrm{K}$ & 120.0 \\
\hline Crystal System & monoclinic \\
\hline Space Group & $\mathrm{P} 2{ }_{1} / \mathrm{c}$ \\
\hline$a / \AA$ & $5.1224(2)$ \\
\hline$b / \AA ̊$ & $10.9427(4)$ \\
\hline$c / \AA ̊$ & $13.8090(5)$ \\
\hline$\alpha l^{\circ}$ & 90 \\
\hline$\beta l^{\circ}$ & $98.335(4)$ \\
\hline$y y^{\circ}$ & 90 \\
\hline $\mathrm{V} / \AA^{3}$ & $765.87(5)$ \\
\hline$Z$ & 2 \\
\hline$Z^{\prime}$ & 0.5 \\
\hline$\Theta_{\min } 1^{\circ}$ & 2.982 \\
\hline$\Theta_{\max } x^{\circ}$ & 24.997 \\
\hline Measured Refl. & 32811 \\
\hline Independent Refl. & 1344 \\
\hline Reflections Used & 1322 \\
\hline$R_{\text {int }}$ & 0.0887 \\
\hline Parameters & 110 \\
\hline Restraints & 0 \\
\hline Largest Peak & 0.269 \\
\hline Deepest Hole & -0.247 \\
\hline GooF & 1.344 \\
\hline$w R_{2}$ (all data) & 0.1419 \\
\hline$w R_{2}$ & 0.1414 \\
\hline$R_{l}$ (all data) & 0.0807 \\
\hline$R_{1}$ & 0.0793 \\
\hline
\end{tabular}




\subsubsection{Crystal Structure of ( \pm -)-Thymarnicol 1.1}

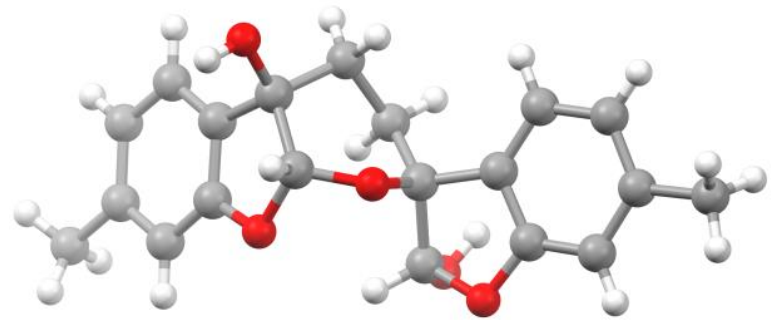

Experimental. Single colourless plate-shaped crystals of ( \pm )-1.1 were recrystallised from acetonitrile by slow evaporation. A suitable crystal $(0.51 \times 0.25 \times 0.06) \mathrm{mm}^{3}$ was selected and mounted on a MITIGEN holder in Paratone oil on a Rigaku Oxford Diffraction SuperNova diffractometer. The crystal was kept at $T=120.0 \mathrm{~K}$ during data collection. Using Olex2 (Dolomanov et al., 2009), the structure was solved with the ShelXS (Sheldrick, 2008) structure solution program, using the direct solution method. The model was refined with version 2016/6 of ShelXL (Sheldrick, 2015) using Least Squares minimisation.

Crystal Data. $\mathrm{C}_{20} \mathrm{H}_{20} \mathrm{O}_{5}, M_{r}=340.36$, monoclinic, $\mathrm{P} 2{ }_{1} / \mathrm{c}$ (No. 14), $\mathrm{a}=6.0345(2) \AA, \mathrm{b}=14.1721(5) \AA, \mathrm{c}=$ $19.3120(8) \AA, \quad \beta=93.383(4)^{\circ}, \quad \alpha=\gamma=90^{\circ}, \quad V=$ 1648.71(11) $\AA^{3}, \quad T=120.0 \mathrm{~K}, Z=4, \quad Z^{\prime}=1$, $\mu\left(\mathrm{MoK}_{\alpha}\right)=0.098,22767$ reflections measured, 4210 unique $\left(R_{\text {int }}=0.0425\right)$ which were used in all calculations. The final $w R_{2}$ was 0.1394 (all data) and $R_{l}$ was 0.0570 (I > 2(I)).
Compound

$( \pm)-1.1$

Formula

$D_{\text {calc. }} / \mathrm{g} \mathrm{cm}^{-3}$

$\mathrm{C}_{20} \mathrm{H}_{20} \mathrm{O}_{5}$

$\mu / \mathrm{mm}^{-1}$

1.371

0.098

Formula Weight

Colour

Shape

Size $/ \mathrm{mm}^{3}$

$T / \mathrm{K}$

Crystal System

Space Group

$a / \AA$

$b / \AA$

$c / \AA$

$\alpha l^{\circ}$

$\beta l^{\circ}$

$\gamma^{\circ}$

$\mathrm{V}$

Z

Wavelength/Å

Radiation type

$\Theta_{\min } l^{\circ}$

$\Theta_{\max } l^{\circ}$

Measured Refl. 22767

Independent Refl. $\quad 4210$

Reflections Used 3541

$R_{\text {int }}$

0.0425

234

2

0.768

$-0.282$

1.098

0.1394

0.1327

0.0693 


\section{$5 \quad$ Appendix}

\section{type $A_{1}$}<smiles>C=CC[C@]1(CC=C(C)C)C=C2OCOC2=CC1=O</smiles><smiles>C=CCC1(CC(O)C(C)(C)O)COC2=C(OCO2)C1=O</smiles><smiles>C=CCC1(CC(O)C(C)(C)OCC)C=C2OCOC2=CC1=O</smiles><smiles>C=CCC1(/C=C/C(C)CO)C=C2OCOC2=CC1=O</smiles><smiles>C=CCC1(CCC(=C)C)C=C2OCOC2=CC1=O</smiles>

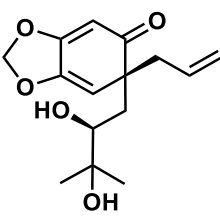

$\mathrm{R}=\mathrm{H}(-)$-illihenryone $\mathrm{A}$ $\mathrm{R}=\mathrm{Glu}(-)$-illihenryone $\mathrm{B}$

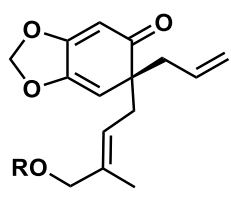

$\mathrm{R}=\mathrm{H}(-)$-illihenryone $\mathrm{E}$ $\mathrm{R}=$ Glu $(-)$-illihenryone $\mathrm{F}$

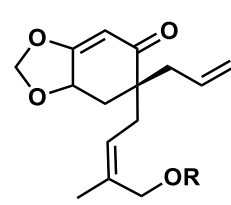

$\mathrm{R}=\mathrm{H}(+)$-illihenryone $\mathrm{C}$ $\mathrm{R}=\mathrm{Glu}(-)$-illihenryone D

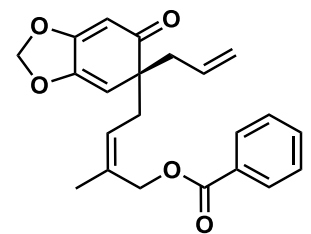

(+)-illioliganone D

\section{type $A_{2}$}

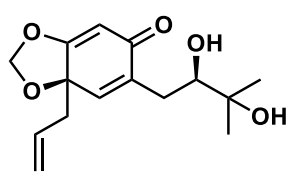

(-)-illioliganone B

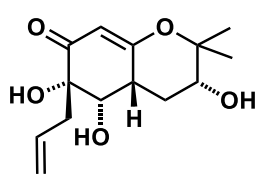

(+)-illihenrypyranone A

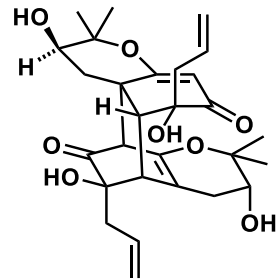

(+)-illihendione A

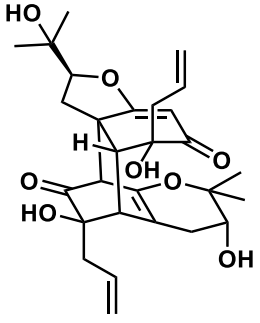

(+)-illicidione A<smiles>C=CC1(O)C(=O)C=C2OC(C(C)(C)O)CC2[C@H]1O</smiles>

(-)-illihenryfunone C<smiles>C=CC[C@]1(O)C[C@H]2CC(C(C)(C)O)OC2=CC1=O</smiles>

(-)-illicarborin B

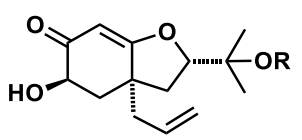

$\mathrm{R}=\mathrm{H}(+)$-illihenryfunone $\mathrm{A}$ $\mathrm{R}=\mathrm{OH}(+)$-illihenryfunone $\mathrm{B}$ 


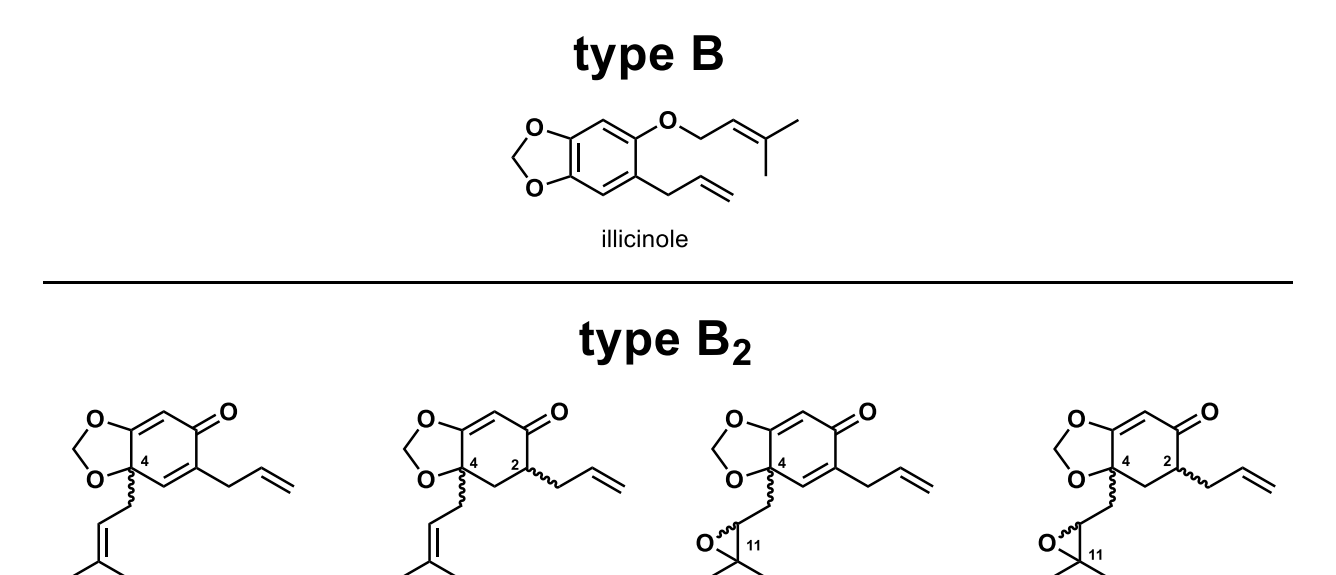

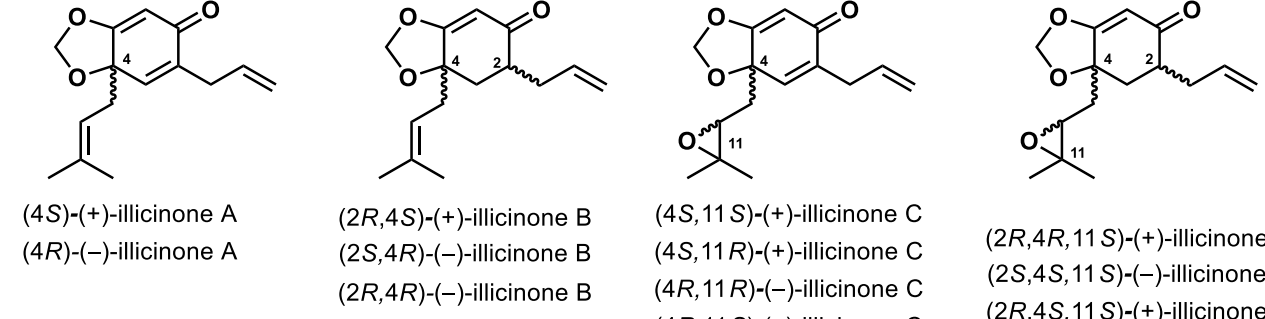

gre sto gro gra

工力n

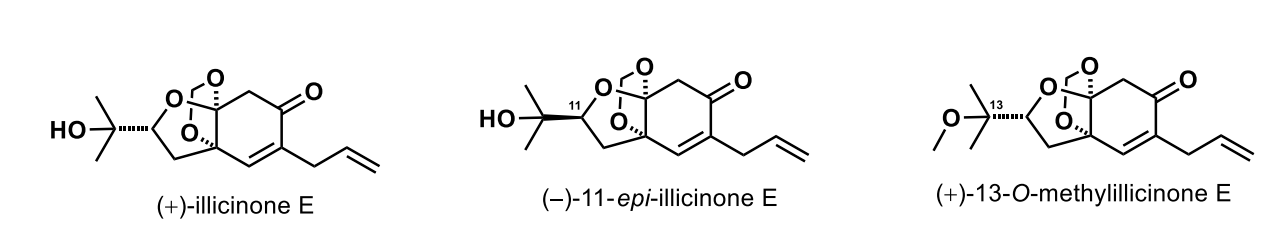

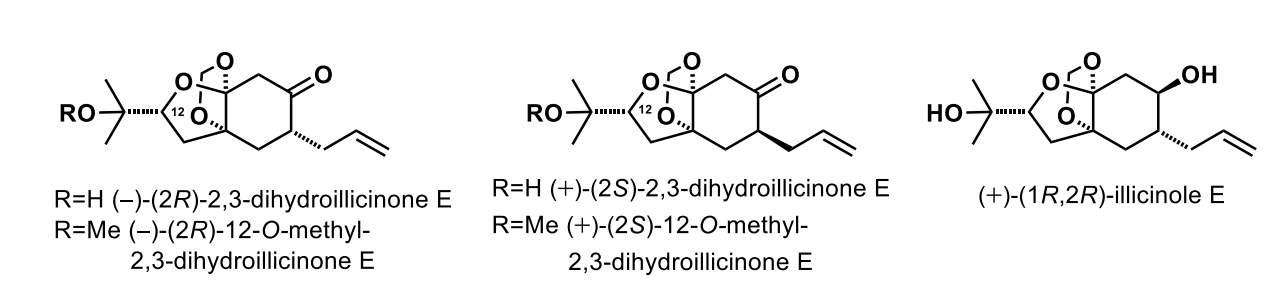

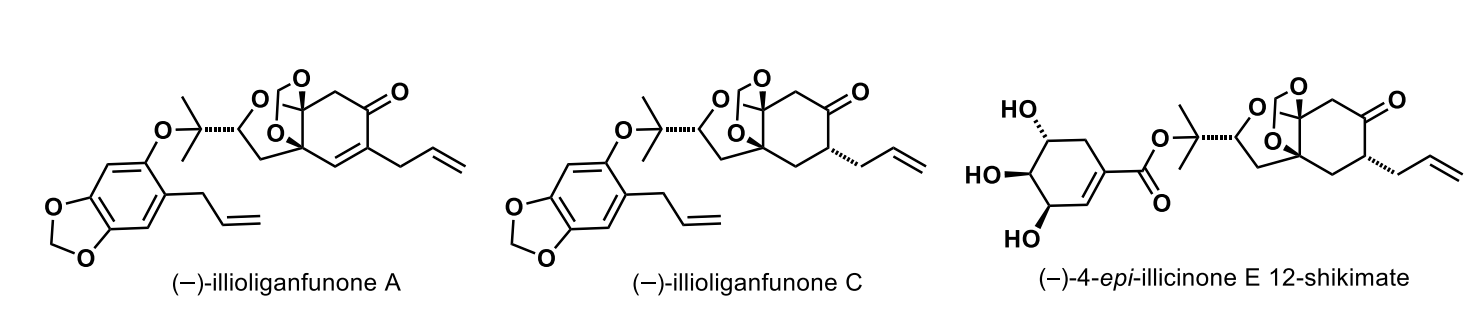

427 


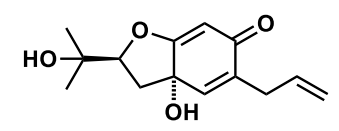

(+)-2,3-dehydroillifunone C

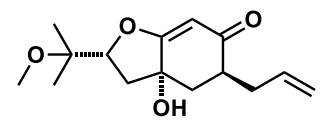

(-)-12-O-methylillifunone A

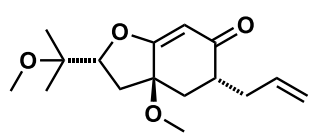

4,12-di-O-methylillicinone C

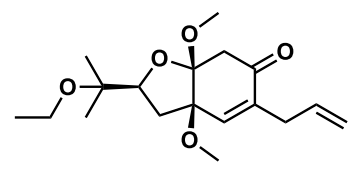

(-)-2,3-dehydro-4,5-di-O-methyl -12-O-ethylillifunone $\mathrm{E}$

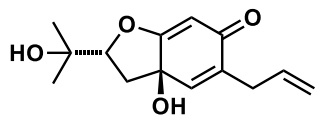

(-)-2,3-dehydroillifunone C

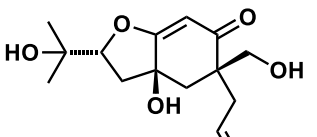

(-)-illicarborin A

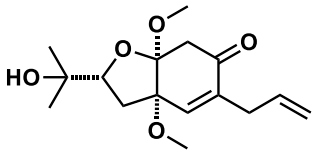

(+)-2,3-dehydro-5,6-di-Omethylillifunone $\mathrm{E}$
HO $\rightarrow$ <

(-)-3-hydroxyillifunone B

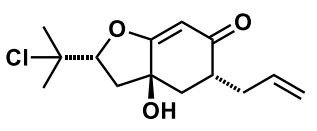

(-)-12-chloroillifunone C

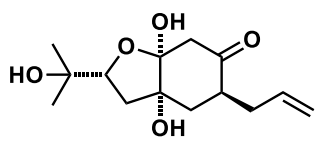

(+)-illifunone E

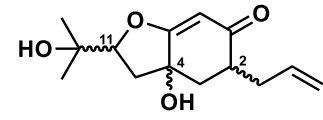

$(2 S, 4 R, 11 R)$-(-)-illifunone A $(2 R, 4 R, 11 R)-(-)$-illifunone $\mathrm{B}$ $(2 R, 4 S, 11 R)-(+)$-illifunone $C$ $(2 S, 4 S, 11 R)-(+)$-illifunone $D$

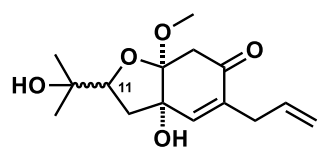

(11R)-(-)-2,3-didehydro-5-Omethy-11-epi-illifunone $\mathrm{E}$

(11S)-(-)-2,3-didehydro-5-Omethyillifunone $E$<smiles>[B]C1=CC2(O)C[C@H](C(C)(C)Oc3cc4c(cc3CC=C)OCO4)OC2=CC1=O</smiles>

(-)-illioliganfunone B<smiles>C=CCC1=CC2(O)C[C@H](C(=C)C)OC2=CC1=O</smiles>

(-)-illioliganfunone D<smiles>C=CCC1=C[C@@](CC=C(C)C)(OC)C(OC)=CC1=O</smiles>

(+)-illicinone I<smiles>C=CCC1=CC(/C=C/C(C)(C)O)(OC)C(OC)=CC1=O</smiles>

4,5-dimethoxyillicinone $\mathrm{G}$

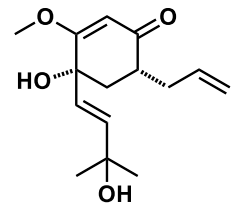

(+)-illicinone $\mathrm{H}$

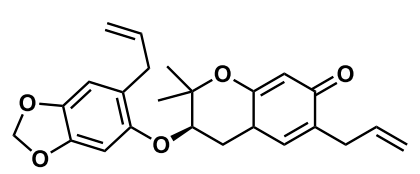

(+)-illioliganpyranone $\mathrm{A}$

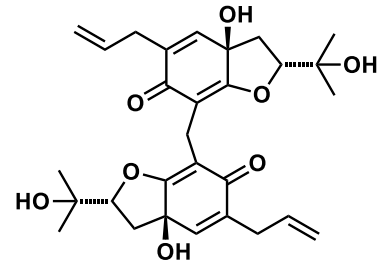

(-)-illicidione B<smiles>C=CCC1CC2(O)C[C@H](C(C)(C)O)OC2=CC1=O</smiles>

(-)-illioliganone I<smiles></smiles>

(-)-illioliganone $\mathrm{H}$

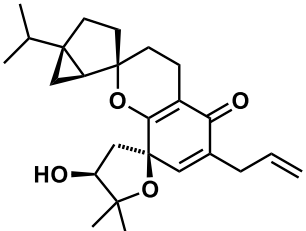

(-)-spirooliganone A<smiles>C=CCC1=CC23OC(C)(C)C(O)CC2(CCC2(CCCC2(C)C(C)C)O3)OC1=O</smiles>

(-)-spirooliganone B но $\rightarrow$

(+)-illicidione C

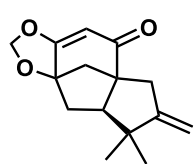<smiles>C=CCC1CC2(O)CCOC2=C(CC)C1=O</smiles>

$( \pm)$-tricycloillicinone 


\section{References}

(1) Haslam, E.; Cai, Y. Nat. Prod. Rep. 1994, 11, 41.

(2) Quideau, S.; Deffieux, D.; Douat-Casassus, C.; Pouységu, L. Angew. Chem., Int. Ed. 2011, 50, 586.

(3) Shi, J.; Pohorly, J. E.; Kakuda, Y. J. Med. Food 2003, 6, 291.

(4) Macheix, J.J.; Billot, J.; Fruit Phenolics CRC press, Inc: Boca Raton, FL, 1990.

(5) Scalbert, A.; Johnson, I. T.; Saltmarsh, M. Am. J. Clin. Nutr. 2005, 81, $215 \mathrm{~S}$.

(6) Ferrieres, J. Heart 2004, 90, 107.

(7) Goldberg, D. M.; Yan, J.; Soleas, G. J. Clin. Biochem. 2003, 36, 79.

(8) Shao, C.; Hristu-Varsakelis, D. Bioinspir. \& Biomim. 2006, 1, 1.

(9) Vincent, J. F. V.; Bogatyreva, O. A.; Bogatyrev, N. R.; Bowyer, A.; Pahl, A.K. J. R. Soc. Interface 2006, 3, 471.

(10) Van Tamelen, E. Fortsch. Chem. Org. Nat. 1961, 19.

(11) Dewick, P.M. Medicinal Natural Products; John Wiley \& Sons, Ltd: Chichester, UK, 2009.

(12) Collie, N., Myers W.S. J. Chem. Soc. Trans. 1893, 63.

(13) Ündeğer, Ü.; Başaran, A.; Degen, G. H.; Başaran, N. Food Chem. Toxicol. 2009, 47, 2037.

(14) Zarrini, G.; Delgosha, Z. B.; Moghaddam, K. M.; Shahverdi, A. R. Pharm. Biol. 2010, 48, 633.

(15) Yamazaki, M.; Usui, T.; Shibata, S. Chem. Pharm. Bull. 1963, 11, 363.

(16) Bueno, A. C.; Brandão, B. B. N. S.; Gusevskaya, E. V. Appl. Catal. A-Gen. 2008, 351, 226.

(17) Poulose, A.J. Arch. Biochem. Biophys. 1978, 187.

(18) Granger, R.; Verdier, R. Acad. Sci. 1964, 268, 5539.

(19) Bohlmann, J.; Meyer-Gauen, G.; Croteau, R. P. Natl. Acad. Sci. 1998, 95, 4126.

(20) Baunach, M.; Franke, J.; Hertweck, C. Angew. Chem., Int. Ed. 2015, 54, 2604.

(21) Rosales, V.; Zambrano, J.; Demuth, M. Eur. J. Org. Chem.2004, 1798.

(22) Wang, X.; Zheng, J.; Chen, Q.; Zheng, H.; He, Y.; Yang, J.; She, X. J. Org. Chem. 2010, 75, 5392 .

(23) Valla, A.; Andriamialisoa, Z.; Valla, B.; Labia, R.; Le Guillou, R.; Dufossé, L.; Cartier, D. Eur. J. Org. Chem. 2007, 711.

(24) Maeda, H.; Annu. Rev. Plant. Biol. 2012, 63, 73.

(25) Bender, S.L.; Medhi, S.; Knowles, J.R.; Biochemistry 1989, 28, 7555.

(26) Bender, S.L.; Widlansky, T.; Knowles, J.R. Biochemistry 1989, 28, 7560.

(27) Widlanski, T.; Bender, S. L.; Knowles, J.R.; Biochemistry 1989, 28, 7572.

(28) Chapman, O.L.; Springer, J.P.; Clardy, J.C. J. Am. Chem. Soc. 1971, 93, 6696.

(29) Brophy, G. C.; Mohandas, J.; Slaytor, M.; Sternhell, S.; Watson, T. R.; Wilson, L. A. Tetrahedron Lett. 1969, 10, 5159.

(30) Matsuda, Y.; Awakawa, T.; Mori, T.; Abe, I. Curr. Opin. Chem. Biol. 2016, 31,1 .

(31) Yudai, M.; Abe, I. Nat. Prod. Rep. 2016, 33, 26.

(32) Saleh, O.; Haagen, Y.; Seeger, K.; Heide, L. Phytochemistry 2009, 70, 1728.

(33) Lee, S. S.; Peng, F. C.; Chiou, C. M.; Ling, K. H. J. Nat. Prod. 1992, 55, 251.

(34) Tomoda, H.; Tabata, N.; Nakata, Y.; Nishida, H.; Kaneko, T.; Obata, R.; Sunazuka, T.; Ōmura, S. J. Org. Chem. 1996, 61, 882. 
(35) Lawrence, A. L.; Adlington, R. M.; Baldwin, J. E.; Lee, V.; Kershaw, J. A.; Thompson, A. L. Org. Lett. 2010, 12, 1676.

(36) Yang, X.-L.; Hsieh, K.-L.; Liu, J.-K. Org. Lett. 2007, 9, 5135.

(37) Voloshchuk, T.; Farina, N. S.; Wauchope, O. R.; Kiprowska, M.; Haberfield, P.; Greer, A. J. Nat. Prod. 2004, 67, 1141.

(38) Lian, G.; Yu, B. Chem. Biodiv. 2010, 7, 2660.

(39) Howard-Jones, A. R.; Walsh, C. T. J. Am. Chem. Soc. 2007, 129, 11016.

(40) Wezeman, T.; Masters, K.-S.; Bräse, S. Angew. Chem. Int. Ed. 2014, 53, 4524.

(41) Sherwood, T. C.; Trotta, A. H.; Snyder, S. A. J. Am. Chem. Soc. 2014, 136, 9743.

(42) Nicolaou, K. C.; Gray, D. Angew. Chem. Int. Ed. 2001, 40, 761.

(43) Ernst-Russell, M. A.; Elix, J. A.; Chai, C. L. L.; Willis, A. C.; Hamada, N.; Nash Iii, T. H. Tetrahedron Lett. 1999, 40, 6321.

(44) Yamamoto, Y.; Matsubara, H.; Kinoshita, Y.; Kinoshita, K.; Koyama, K.; Takahashi, K.; Ahmadjiam, V.; Kurokawa, T.; Yoshimura, I. Phytochemistry 1996, 43, 1239.

(45) Bohm, B. A. The Biology and the Chemistry of the Compositae; Academic Press: London, 1977.

(46) Passreiter, C. M.; Matthiesen, U.; Willuhn, G. Phytochemistry 1998, 49, 777.

(47) Bohlmann, F.; Schulz, J.; Bühmann, U. Tetrahedron Lett. 1969, 10, 4703.

(48) Passreiter, C. M.; Willuhn, G.; Weber, H.; Schleifer, K.-J. Tetrahedron 1999, 55, 2997.

(49) Willuhn G., J. I., Wendisch D. Planta Medica 1986, 349.

(50) De Mesmaeker, A.; Hoffmann, P.; Ernst, B. Tetrahedron Lett. 1988, 29, 6585.

(51) Roy, S. C.; Mandal, P. K. Tetrahedron 1996, 52, 12495.

(52) Bérard, D.; Giroux, M.-A.; Racicot, L.; Sabot, C.; Canesi, S. Tetrahedron 2008, 64, 7537.

(53) Passreiter, C. M.; Weber, H.; Bläser, D.; Boese, R. Tetrahedron 2002, 58, 279.

(54) Bohlmann, F.; Grenz, M. Phytochemistry 1979, 18, 179.

(55) Wang, Y.; Zhang, A. Tetrahedron 2009, 65, 6986.

(56) Seiichi, T.; Kohei, I.; Kiyohiro, S.; Shun'ichi, T.; Masashi, Y.; Mahito, S.;

Yoshiharu, I.; Takumichi, S.; Kunio, O. Chem. Lett. 1989, 18, 1283.

(57) Overman, L. E.; Freerks, R. L.; Petty, C. B.; Clizbe, L. A.; Ono, R. K.; Taylor, G. F.; Jessup, P. J. J. Am. Chem. Soc. 1981, 103, 2816.

(58) Siguara Bastos, L. S.; Della Torre, A.; De Carvalho J. E.; Tasca Gois Ruiz, A. L.; Silva, L. S. Molecules, 2015, 20, 1475.

(59) Lin, H.-C.; Tsai, R.-T.; Wu, H.-P.; Lee H.-J.; Lee G.-H. Tetrahedron, 2016, $72,184$.

(60) Laitalainen, T.; Kuronen, P.; Hesso, A. Org. Prep. Proced. Int. 1993, 25, 597.

(61) Davies, H. M. L.; Dai, X. J. Org. Chem. 2005, 70, 6680.

(62) Liu, G.; Wurst, J. M.; Tan, D. S. Org. Lett. 2009, 11, 3670.

(63) Vyvyan, J. R.; Loitz, C.; Looper, R. E.; Mattingly, C. S.; Peterson, E. A.; Staben, S. T. J. Org. Chem. 2004, 69, 2461.

(64) Ronald, R. C. Tetrahedron Lett. 1975, 16, 3973.

(65) Guanti, G.; Banfi, L.; Riva, R. Tetrahedron 1994, 50, 11945.

(66) Huang, Q.; Fazio, A.; Dai, G.; Campo, M. A.; Larock, R. C. J. Am. Chem. Soc. 2004, 126, 7460. 
(67) Tromp, R. A.; van Boom, S. S. G. E.; Marco Timmers, C.; van Zutphen, S.; van der Marel, G. A.; Overkleeft, H. S.; van Boom, J. H.; Reedijk, J. Bioorg. Med. Chem. Lett. 2004, 14, 4273.

(68) Crandall, J. K. In EROS; John Wiley \& Sons, Ltd: 2001.

(69) Denmark, S. E.; Forbes, D. C.; Hays, D. S.; DePue, J. S.; Wilde, R. G. J. Org. Chem. 1995, 60, 1391.

(70) Cheshev, P.; Marra, A.; Dondoni, A. Carbohyd. Res. 2006, 341, 2714.

(71) Tu, Y.; Wang, Z.-X.; Shi, Y. J. Am. Chem. Soc. 1996, 118, 9806.

(72) Wang, Z.-X.; Shi, Y. J. Org. Chem. 1998, 63, 3099.

(73) Gomes Jr, M.; Antunes, O. A. C. Catal. Comm. 2001, 2, 225.

(74) Ramesh, C.; Ravindranath, N.; Das, B. J. Org. Chem. 2003, 68, 7101.

(75) Breton, G. W. J. Org.Chem. 1997, 62, 8952.

(76) Hanessian, S.; Delorme, D.; Dufresne, Y. Tetrahedron Lett. 1984, 25, 2515.

(77) Saimoto, H.; Kusano, Y.; Hiyama, T. Tetrahedron Lett. 1986, 27, 1607.

(78) Abdel-Rahman M., E. H. W., Binks R., Kung W., Rapoport H. J. Med. Chem. 1966, $9,1$.

(79) Lim, K. S.; Lee, H.; Kim, S. E.; Ha, T.-H.; Ann, J.; Son, K.; Choi, S.; Sun, W., Pearce, L. V.; De Andrea-Lazarus, I. A.; Blumberg, P. M.; Lee, J. Eur. J. Med. Chem. 2013, 68, 233.

(80) Nicolaou, K. C.; Sasmal, P. K.; Xu, H. J. Am. Chem. Soc. 2004, 126, 5493.

(81) Divakar, K.J.; Kulkarni, B. D.; Rao, A.S. Indian J. Chem. 1977, 15B, 849.

(82) Schön, U.; Messinger, J.; Solodenko, W.; Kirschning, A. Synthesis 2012, 44, 3822.

(83) Sanz, R.; Castroviejo, M. P.; Fernández, Y.; Fañanás, F. J. J. Org. Chem. 2005, $70,6548$.

(84) Yamazaki, K.; Kawamorita, S.; Ohmiya, H.; Sawamura, M. Org. Lett. 2010, 12, 3978.

(85) Morin, J.; Zhao, Y.; Snieckus, V. Org. Lett. 2013, 15, 4102.

(86) Sibi M.P., S. V. J. Org. Chem. 1983, 48, 306.

(87) Wilson, N. S.; Keay, B. A. Tetrahedron Lett. 1996, 37, 153.

(88) Pérez-Vásquez, A.; Reyes, A.; Linares, E.; Bye, R.; Mata, R. J. Nat. Prod. 2005, 68, 959.

(89) Brown, P. D.; Lawrence, A. L. Angew. Chem. Int. Ed. 2016, 55, 8421.

(90) Vaino, A. R.; Szarek, W. A. Chem. Comm. 1996, 2351.

(91) Paradisi, M. P.; Zecchini, G. P.; Torrini, I. Tetrahedron Lett. 1986, 27, 5029.

(92) Baillargeon, V. P.; Stille, J. K. J. Am. Chem. Soc. 1986, 108, 452.

(93) Xiang, A. X.; Watson, D. A.; Ling, T.; Theodorakis, E. A. J. Org. Chem. 1998, 63,6774 .

(94) Almstead, J.-I. K.; Demuth Jr, T. P.; Ledoussal, B. Tetrahedron-Asymmetr. 1998, 9,3179 .

(95) Okutani, M.; Mori, Y. Chem. Pharm .Bull. 2015, 63, 393.

(96) Coe, J. W.; Bianco, K. E.; Boscoe, B. P.; Brooks, P. R.; Cox, E. D.; Vetelino, M. G. J. Org. Chem. 2003, 68, 9964.

(97) Salmond, W. G.; Barta, M. A.; Havens, J. L. J. Org. Chem. 1978, 43, 2057.

(98) González, M. A.; Zaragozá, R. J. J. Nat. Prod. 2014, 77, 2114.

(99) Angelaud, R.; Zhong, Y.-L.; Maligres, P.; Lee, J.; Askin, D. J. Org. Chem. 2005, 70, 1949.

(100) Yoo, S.-e.; Yi, K. Y. Synlett 1990, 1990, 697. 
(101) Divakar K.J., Kulkarni. B. D., Rao A.S. Indian J. Chem. 1977, 15B, 322.

(102) Stephenson, L. M.; Speth, D. R. J. Org. Chem. 1979, 44, 4683.

(103) Coxon, J. M.; Dansted, E.; Hartshorn, M. P. In Organic Syntheses; John Wiley \& Sons, Inc.: 2003.

(104) Tokuyasu, T.; Kunikawa, S.; Masuyama, A.; Nojima, M. Org. Lett. 2002, 4, 3595.

(105) Sy, L.-K.; Brown, G. D. Phytochemistry 2001, 58, 1159.

(106) Ngo, K.-S.; Brown, G. D. Tetrahedron 1999, 55, 15109.

(107) Faizi, S.; Naz, A. Helv. Chim. Acta 2004, 87, 46.

(108) Simic, M. G.; J. Chem. Ed. 1981, 58, 125.

(109) Barton, D. H. R.; Wang, T.-L. Tetrahedron Lett. 1994, 35, 5149.

(110) Drees, M.; Strassner, T. Inorg. Chem. 2011, 50, 5833.

(111) Bohacek, R. S.; McMartin, C.; Guida, W. C. Med. Res. Rev. 1996, 16, 3.

(112) Burke, M. D.; Schreiber, S. L. Angew. Chem. Int. Ed. 2004, 43, 46.

(113) Bauer, R. A.; Wenderski, T. A.; Tan, D. S. Nat. Chem. Biol. 2013, 9, 21.

(114) Griffith, D. R.; Botta, L.; St. Denis, T. G.; Snyder, S. A. J. Org. Chem. 2013, $79,88$.

(115) Pelish, H. E.; Westwood, N. J.; Feng, Y.; Kirchhausen, T.; Shair, M. D. J. Am. Chem. Soc. 2001, 123, 6740.

(116) Lee, A. S.; Liau, B. B.; Shair, M. D. J. Am. Chem. Soc. 2014, 136, 13442.

(117) Fukuyama, Y.; Shida, N.; Sakurai, T.; Kodama, M. Phytochemistry, 1992, 31, 3975.

(118) Fukuyama, Y.; Shida, N.; Hata, Y.; Anzaki, S.; Kodama, M. Phytochemistry 1994, 37, 1653.

(119) Kouno, I.; Shimamoto, S.; Jiang, Z.-H.; Tanaka, T. Phytochemistry 1997, 46, 1389.

(120) Lei, X.; Dai, M.; Hua, Z.; Danishefsky, S. J. Tetrahedron Lett. 2008, 49, 6383.

(121) Takaoka, S.; Takaoka, N.; Minoshima, Y.; Huang, J.-M.; Kubo, M.; Harada, K.; Hioki, H.; Fukuyama, Y. Tetrahedron 2009, 65, 8354.

(122) Tang, W.-Z.; Ma, S.-G.; Yu, S.-S.; Qu, J.; Liu, Y.-B.; Liu, J. J. Nat. Prod. 2009, $72,1017$.

(123) Wu, X.-F.; Li, Y.; Lu, H.-N.; Yu, S.-S.; Ma, S.-G.; Liu, J. J. Asian Nat. Prod. Res. 2009, 11, 1056.

(124) Lin, Y.-X.; Fazary, A. E.; Chen, S.-Y.; Chien, C.-T.; Kuo, Y.-H.; Sheu, S.-Y.; Shen, Y.-C. Food Chem. 2010, 123, 1105.

(125) Ma, S.-G.; Tang, W.-Z.; Liu, Y.-X.; Hu, Y.-C.; Yu, S.-S.; Zhang, Y.; Chen, X.G.; Qu, J.; Ren, J.-H.; Liu, Y.-B.; Xu, S.; Liu, J.; Liu, Y.-Y.; Li, Y.; Lü, H.-N.; $\mathrm{Wu}, \mathrm{X} . \mathrm{F}$. Phytochemistry 2011, 72, 115.

(126) Tang, W.-Z.; Ma, S.-G.; Qu, J.; Yu, S.-S.; Liu, Y.-B.; Su, D.-M.; Liu, J. J. Nat. Prod. 2011, 74, 1268.

(127) Kubo, M.; Shima, N.; Harada, K.; Hioki, H.; Fukuyama, Y. Chem. Pharm. Bull. 2011, 59, 898.

(128) Liaw, C.-C.; Chen, Y.-C.; Eid Fazary, A.; Hsieh, J.-L.; Chen, S.-Y.; Chien, C.T.; Sheu, S.-Y.; Kuo, Y.-H.; Chiang, B.-L.; Shen, Y.-C. Phytochemistry Lett. 2013, 6, 397.

(129) Ma, S.-G.; Gao, R.-M.; Li, Y.-H.; Jiang, J.-D.; Gong, N.-B.; Li, L.; Lü, Y.; Tang, W.-Z.; Liu, Y.-B.; Qu, J.; Lü, H.-N.; Li, Y.; Yu, S.-S. Org. Lett. 2013, 15,4450 . 
(130) Zhuang, P.-Y.; Zhang, G.-J.; Wang, X.-J.; Zhang, Y.; Yu, S.-S.; Ma, S.-G.; Liu, Y.-B.; Qu, J.; Li, Y.; Xu, S.; Lü, H.-N.; Chen, X.; Li, L.; Si, Y.-K.; Zhang, D. Phytochemistry 2013, 86, 176.

(131) Zhuang, P.-Y.; Ma, S.-G.; Zhang, G.-J.; Wang, X.-J.; Zhang, Y.; Yu, S.-S.; Liu, Y.-B.; Qu, J.; Li, Y.; Hou, Q. Phytochemistry Lett. 2013, 6, 444.

(132) Huang, D.; Zhu, H.; Chen, Y.; Chen, W.; Xue, D.; Sun, L. Fitoterapia 2015, 107, 22.

(133) Liaw, C-C.; Chen, Y-C.; Fazary, A. E.; Hsieh, J.-L.; Chen, S-Y.; Chien, C-T.; Sheu, S-Y.; Kuo, Y-H.; Chiang, B-L.; Shen, Y-C. Phytochemistry Lett. 2013, 6, 397.

(134) Fukuyama, Y.; Shida, N.; Kodama, M.; Chaki, H.; Yugami, T. Chem. Pharm. Bull. 1995, 43, 2270.

(135) Yakushijin, K.; Tohshima, T.; Kitagawa, E.; Suzuki, R.; Sekikawa, J.; Morishita, T.; Murata, H.; Lu, S.; Furukawa, H. Chem. Pharm. Bull. 1984, 32, 11.

(136) Yakushijin, K.; Furukawa, H.; McPhail, A. T. Chem. Pharm. Bull. 1984, 32, 23.

(137) Nonoshita, K.; Banno, H.; Maruoka, K.; Yamamoto, H. J. Am. Chem. Soc. 1990, 112, 316.

(138) Wei, L.; Xiao, M.; Xie, Z. Org. Lett. 2014, 16, 2784.

(139) Song, L.; Yao, H.; Tong, R. Org. Lett. 2014, 16, 3740.

(140) Zhao, N.; Ren, X.; Ren, J.; Lü, H.; Ma, S.; Gao, R.; Li, Y.; Xu, S.; Li, L.; Yu, S-S. Org. Lett. 2015, 17, 3118.

(141) Ren, X.-D.; Zhao, N.; Xu, S.; Lü, H.-N.; Ma, S.-G.; Liu, Y.-B.; Li, Y.; Qu, J.; $\mathrm{Yu}$, S.-S. Tetrahedron 2015, 71, 4821.

(142) Winkelblech, J.; Fan, A.; Lee, S.-M. Appl. Miocrobiol. Biotechnol. 2015, 99, 7379.

(143) Jiménez-González, L.; García-Muñoz, S.; Álvarez-Corral, M.; Muñoz-Dorado, M.; Rodríguez-García, I. Chem. Eur. J. 2006, 12, 8762.

(144) Netherton, M. R.; Fu, G. C. Org. Lett. 2001, 3, 4295.

(145) Barney, R. J.; Wasko, B. M.; Dudakovic, A.; Hohl, R. J.; Wiemer, D. F. Bioorg. Med. Chem. 2010, 18, 7212.

(146) Gozzo, F. C.; Fernandes, S. A.; Rodrigues, D. C.; Eberlin, M. N.; Marsaioli, A. J. J. Org. Chem. 2003, 68, 5493.

(147) Ito, F.; Fusegi, K.; Kumamoto, T.; Ishikawa, T. Synthesis 2007, 12, 1785.

(148) Barradas, S.; Hernández-Torres, G.; Urbano, A.; Carreño, M. C. Org. Lett. 2012, 14, 5952.

(149) Qi, C.; Qin, T.; Suzuki, D.; Porco, J. A. J. Am. Chem. Soc. 2014, 136, 3374.

(150) Gong, J.; Lin, G.; Li, C.-c.; Yang, Z. Org. Lett. 2009, 11, 4770.

(151) Brown, P. D.; Willis, A. C.; Sherburn, M. S.; Lawrence, A. L. Org. Lett. 2012, 14, 4537.

(152) Lebrasseur, N.; Gagnepain, J.; Ozanne-Beaudenon, A.; Leger, J-M.; Quideau, S. J. Org. Chem. 2007, 72, 6280.

(153) Niggemann, M.; Meel, M. J. Angew. Chem. Int. Ed. 2010, 49, 3684.

(154) Jiang, H.; Hamada, Y. Org. Biom. Chem. 2009, 7, 4173.

(155) Lykakis, I. N.; Efe, C.; Gryparis, C.; Stratakis, M. Eur. J. Org. Chem. 2011, 2334.

(156) Coombes, C. L.; Moody, C. J. J. Org. Chem. 2008, 73, 6758. 
(157) Kishuku, H.; Shindo, M.; Shishido, K. Chem. Comm. 2003, 350.

(158) Wateh, A. N.; Thy, C. K.; Chee, C. F.; Rahman, N. A. Synth. Comm. 2015, 45, 1421.

(159) Fukuyama, Y.; Shida, N.; Hata, Y.; Kodama, M. Phytochemistry 1994, 36, 1497.

(160) Bedford, R. B.; Webster, R. L.; Mitchell, C. J. Org. Biomol. Chem. 2009, 7, 4853.

(161) Nicolaou, K. C.; Becker, J.; Lim, Y. H.; Lemire, A.; Neubauer, T.; Montero, A. J. Am. Chem. Soc. 2009, 131, 14812.

(162) Tian, Y.; Qi, J.; Sun, C.; Yin, D.; Wang, X.; Xiao, Q. Org. Biomool. Chem. 2013, 11, 7262 .

(163) Ferguson, J.; Zeng, F.; Alper, H. Org. Lett. 2012, 14, 5602.

(164) González, A. G.; Barrera, J. B.; Yanes, A. C.; Díaz, J. G.; Pérez, E. M. R. Phytochemistry 1989, 28, 2520.

(165) Nguyen Van, T.; Debenedetti, S.; De Kimpe, N. Tetrahedron Lett. 2003, 44, 4199.

(166) Gaussian 09, R. E., M. J. Frisch, G. W. Trucks, H. B. Schlegel, G. E. Scuseria, M. A. Robb, J. R. Cheeseman, G. Scalmani, V. Barone, B. Mennucci, G. A. Petersson, H. Nakatsuji, M. Caricato, X. Li, H. P. Hratchian, A. F. Izmaylov, J. Bloino, G. Zheng, J. L. Sonnenberg, M. Hada, M. Ehara, K. Toyota, R. Fukuda, J. Hasegawa, M. Ishida, T. Nakajima, Y. Honda, O. Kitao, H. Nakai, T. Vreven, J. A. Montgomery, Jr., J. E. Peralta, F. Ogliaro, M. Bearpark, J. J. Heyd, E. Brothers, K. N. Kudin, V. N. Staroverov, R. Kobayashi, J. Normand, K. Raghavachari, A. Rendell, J. C. Burant, S. S. Iyengar, J. Tomasi, M. Cossi, N. Rega, J. M. Millam, M. Klene, J. E. Knox, J. B. Cross, V. Bakken, C. Adamo, J. Jaramillo, R. Gomperts, R. E. Stratmann, O. Yazyev, A. J. Austin, R. Cammi, C. Pomelli, J. W. Ochterski, R. L. Martin, K. Morokuma, V. G. Zakrzewski, G. A. Voth, P. Salvador, J. J. Dannenberg, S. Dapprich, A. D. Daniels, Ö. Farkas, J. B. Foresman, J. V. Ortiz, J. Cioslowski, and D. J. Fox, Gaussian, Inc., Wallingford CT, 2009.

(167) Chai, J.-D.; Head-Gordon, M. Phys. Chem. Chem. Phys. 2008, 10, 6615.

(168) Grimme, S. J. Comput. Chem. 2006, 27, 1787.

(169) Grimme, S. Chem. Eur. J. 2012, 18, 9955.

(170) I. Funes-Ardoiz, R. S. P. GoodVibes v1.0.0 DOI: 10.5281/zenodo.56091 (accessed 20th March 2017)

(171) Jenner, G.; Rimmelin, J.; Libs, S.; Antoni, F. Tetrahedron 1976, 32, 1107.

(172) Toma, L.; Romano, S.; Quadrelli, P.; Caramella, P. Tetrahedron Lett. 2001, 42, 5077.

(173) Caramella, P.; Quadrelli, P.; Toma, L. J. Am. Chem. Soc. 2002, 124, 1130.

(174) Quadrelli, P.; Romano, S.; Toma, L.; Caramella, P. Tetrahedron Lett. 2002, 43, 8785.

(175) Quadrelli, P.; Romano, S.; Toma, L.; Caramella, P. J. Org. Chem. 2003, 68, 6035.

(176) Fernández, I.; Bickelhaupt, F. M. J. Comput. Chem. 2014, 35, 371.

(177) Ess, D. H.; Houk, K. N. J. Am. Chem. Soc. 2008, 130, 10187.

(178) Ess, D. H.; Houk, K. N. J. Am. Chem. Soc. 2007, 129, 10646.

(179) Johnson, E. R.; Keinan, S.; Mori-Sánchez, P.; Contreras-García, J.; Cohen, A. J.; Yang, W. J. Am. Chem. Soc. 2010, 132, 6498. 
(180) Contreras-García, J.; Johnson, E. R.; Keinan, S.; Chaudret, R.; Piquemal, J.-P.; Beratan, D. N.; Yang, W. J. Chem. Theory Comput. 2011, 7, 625. 